Portland State University

PDXScholar

\title{
A Study of Differences between Social/HMO and Other Medicare Beneficiaries Enrolled in Kaiser Permanente under Capitation Contracts Regarding Intermediate Care Facility Use Rates and Expenditures
}

Lynn Allen Boose

Portland State University

Follow this and additional works at: https://pdxscholar.library.pdx.edu/open_access_etds Let us know how access to this document benefits you.

\section{Recommended Citation}

Boose, Lynn Allen, "A Study of Differences between Social/HMO and Other Medicare Beneficiaries Enrolled in Kaiser Permanente under Capitation Contracts Regarding Intermediate Care Facility Use Rates and Expenditures" (1993). Dissertations and Theses. Paper 1135.

https://doi.org/10.15760/etd.1134

This Dissertation is brought to you for free and open access. It has been accepted for inclusion in Dissertations and Theses by an authorized administrator of PDXScholar. Please contact us if we can make this document more accessible: pdxscholar@pdx.edu. 
A STUDY OF DIFFERENCES BETWEEN SOCIAL/HMO AND OTHER MEDICARE BENEFICIARIES ENROLLED IN KAISER PERMANENTE UNDER CAPITATION CONTRACTS REGARDING INTERMEDIATE CARE FACILITY USE RATES AND EXPENDITURES

by

LYNN ALLEN BOOSE

A dissertation submitted in partial fulfillment of the requirements for the degree of

\author{
DOCTOR OF PHILOSOPHY \\ in \\ URBAN STUDIES
}

Portland State University 1993 
TO THE OFFICE OF GRADUATE STUDIES:

The members of the Committee approve the dissertation of Lynn Allen Boose presented February 16, 1993.
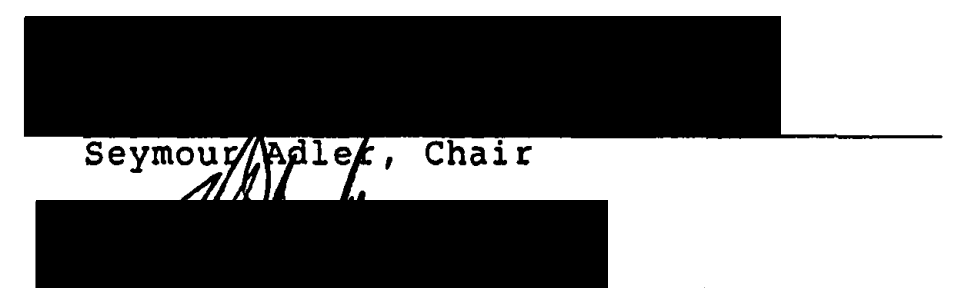

Meryy/h/Grejpicick
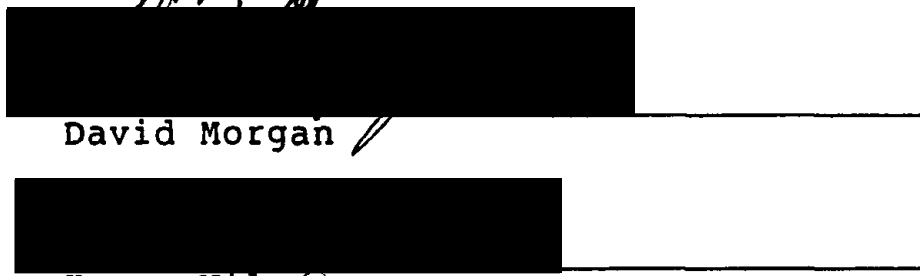

Reren wijson

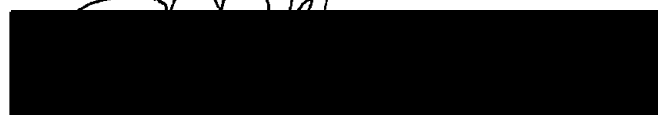

Edward $\backsim r u ̛ b b$

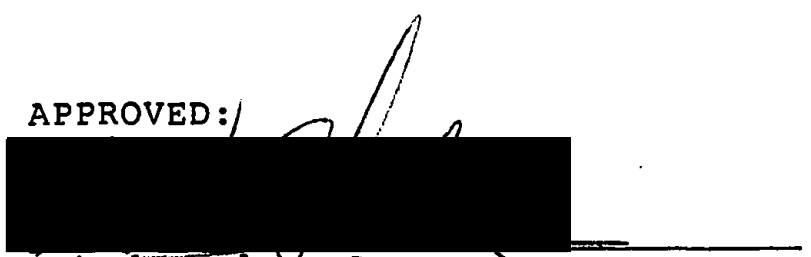

Nohad"Toulap, Dean, Schond of Urban and Public Affaiis

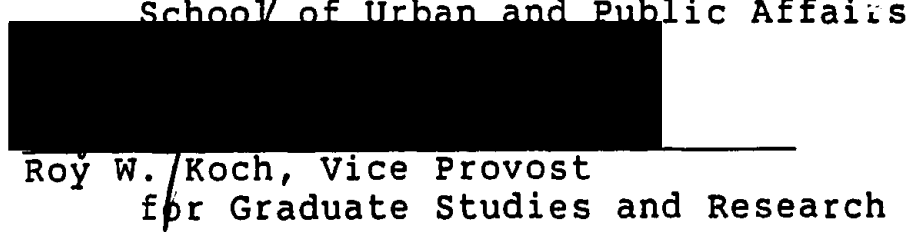


AN ABSTRACT OF THE DISSERTATION OF Lynn Allen Boose for the Doctor of Philosophy in Urban Studies presented February 16, 1993.

Title: A Study of Differences Between Social/HHO and Other Medicare Beneficiaries Enrolled In Kaiser Permanente Under Capitation Contracts Regarding Intermediate Care Facility Use Rates and Expenditures

APPROVED BY THE MEMBERS OF THE DISSERTATION COMMITTEE:

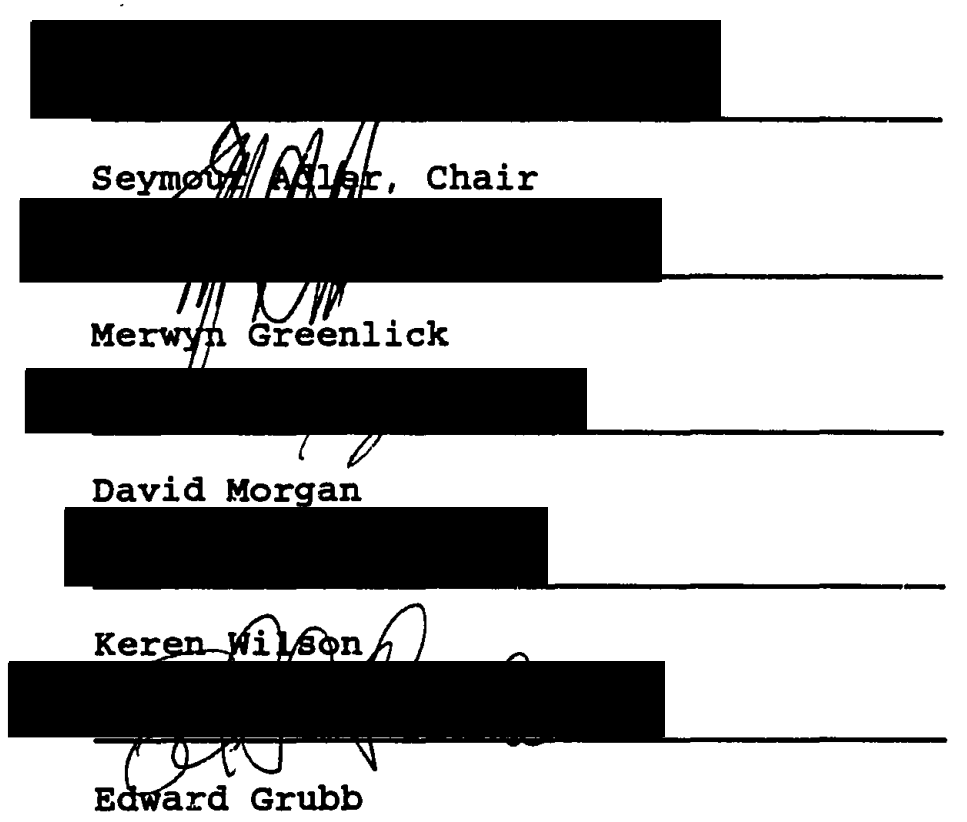

The Social/HMO Demonstration evaluates the feasibility of expanding Medicare Supplemental Insurance benefits to 
cover a limited amount of ICF and community based long-term care (ITC) services provided under a comprehensive HMO benefit package for capitated Medicare beneficiaries. The policy research question addressed by this study is whether adding an Expanded Care Benefit (ECB) to the capitated HMO benefit package offered by Kaiser Permanente (KP) changes utilization patterns and costs of ICF services, and the probability of becoming Medicaid eligible. This study provides descriptive information regarding this policy research question.

The research goal of this study is to measure the extent to which collective ICF use rates and expenditure patterns for $s / H M O$ members are consistently the same, greater or less than baseline data of Risk HMO Medicare members who do not have the S/HMO ECB. The purpose of such measurement is to determine if an empirical basis exists for postulating an ICF utilization and expenditures outcome effect which is influenced by the S/HMO ECB.

Utilization and financial data are collected from all SNF and ICF level nursing homes in Multnomah County for all Medicare beneficiaries ersolled in KP between June 1, 1986 and July 31, 1988. Eligibility data are assembled on all Medicare beneficiaries enrolled in KP during the same time period who were residents of Multnomah county. Nursing home use rates and rates for related expenditures are determined for all nursing home residents $(1,331)$ by their eligibility status in KP during the time of each nursing home stay. Days 
in an ICF are censored by transfers between Cost, Risk and s/HMO enrollment status. Rates are standardized by the age and gender distribution of research population members $(19,261)$ to adjust use rates for differences in age cohort distribution of Risk members and $\mathrm{s} / \mathrm{HMO}$ members. Risk rates and $\mathrm{S} / \mathrm{HMO}$ rates are compared and differences in utilization and expenditures are evaluated. Conclusions about such patterns are used to formulate hypotheses for testing and confirming descriptive observations.

Findings show that overall S/HMO member rates are less than Risk member rates for five of the six Research Questions addressed in this study. Specifically, the probability of admission to an ICF is substantially greater for $\mathrm{s} / \mathrm{HMO}$ members than for Risk members. However, $\mathrm{s} / \mathrm{HMO}$ members remained in ICFs fewer days than Risk members, over the two year study period, as measured by age adjusted rates for ICF days per member year of eligibility during the study period. Difference in the mean length of ICF stay is statistically significant between Risk and s/HMO.

The rate of total payments received by nursing homes for S/HMO ICF residents per $1000 \mathrm{~S} / \mathrm{HMO}$ members was substantially less than that for Risk members. The rate of spend-down to welfare status was substantially lower for $s /$ HO members than for Risk members who became ICF residents. Higher proportions of $\mathrm{s} / \mathrm{HMO}$ members were discharged from ICFs to home than were Risk members, which is consistent with S/HMO Expanded Care Benefit objectives. 
Findings infer that a case-managed, HMO based Expanded Care Benefit can be expected to reduce ICF days used and related nursing home revenues for $\mathrm{S} / \mathrm{HMO}$ members, and is likely to reduce $\mathrm{S} / \mathrm{HMO}$ member need for Medicaid assistance to pay for nursing home debts. This observation is encouraging regarding the prospects for adopting $s / H M O$ as a partial solution to the LTC policy problem in America. 


\section{ACKNOWLEDGMENTS}

This dissertation involves a career commitment to learning about operational outcomes of a health care system known as Kaiser Permanente, with which I have had a robust relationship for thirty years, concluding with this study.

Fulfilling this commitment was made possible by the abiding support of family, friends, KP associates, and PSU faculty where Sy Adler served as my model for scholarship.

Many of the RP Center for Health Research staff offered technical assistance and advice about the research process. Mitch Greenlick's support and tutoring is basic to completion of this dissertation. Michael Goodman's data management acumen made impossible programming possible, and Linda Phelps persevered while I learned SAS and 1032.

Patients and staff at Portland Adventist Convalescent Center kept me focused on what LTC nursing home use rates are all about; I am indebted to Harley Clendenen for that. During this study, Miles taught me to understand that enduring chronic illness requires great courage; Laurel kept me in touch with me, an essential attribute for surviving dissertation work and nursing. home care giving; my loving mother, Agnes, provided a consummate model of nursing home utilization: she lives actively and healthfully at home. 
TABLE OF CONTENTS

PAGE

ACKNOWLEDGEMENTS ........................

IIST OF TABLES ........................ vii

IIST OF FIGURES......................... xiv

CHAPTER

I INTRODUCTION, BACKGROUND INFORMATION,

RESEARCH ENVIRONMENT.................... 1

Introduction.................... 1

Background Information............. 12

Research Environment.............. 31

II POLICY ISSUES ADDRESSED BY THIS STUDY...... 40

III RESEARCH DESIGN AND METHODOLOGY......... 51

Setting Iimits on The Research Plan

For This study................... 52

Approach To Evaluation of Findings..... 97

ICF vs SNF vs Nursing Home........... 99

Issues Related To Generalization of

study Findings................... 101

IV OVERALL USE RATES FOR RESEARCH

QUESTIONS I AND II................... 103

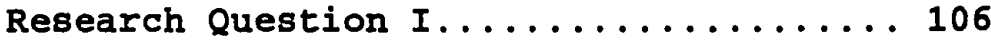

Research Question II.............. 118

Summary of Findings.............. 139 
Discussion: External Issues

Potentially Affecting Use Rates........ 141

Policy Research Observations

Regarding ICF Use Rates............... 144

$\checkmark$ USE RATE DIFFERENCES BASED ON SOURCE OF

ENROLLMENT. .................. 146

Research Question Ia............. 147

Research Question IIa............ 151

Summary of Observations About Rates

In This Chapter and Their Implications

Regarding Priox observations......... 156

Research Methodology Issues Related

To This Chapter................. 158

Policy Research Issues............ 159

VI DIFFERENCES BETWEEN INDIVIDUAL RISR AND

S/HMO RESIDENTS REGARDING ADMISSION AND

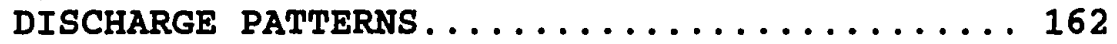

Policy Research Issues............ 180

VII REVENUES RECEIVED BY NURSING HOMES FOR

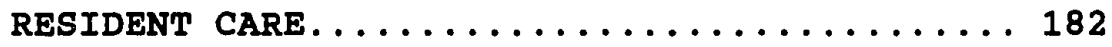

Research Question III............ 184

Summary of Observations............ 199

Policy Research Issues............. 199

VIII DIFFERENCES IN MEDICAID STATUS BETWEEN

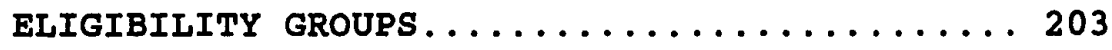

Research Question IV.............. 205

Research Question v.............206

Research Question VI.............. 214

Discussion About Findings.......... 230

Summary of Observations............ 234 
Policy Research Recommendations....... 234

IX MEDICAL STATUS OF NURSING HOME RESIDENTS.... 239

Policy Issues................. 256

$X$ SUMMARY OF FINDINGS AND POLICY

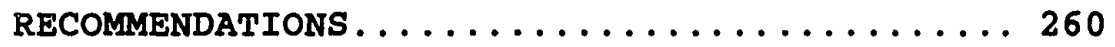

Synthesis of study Findings......... 262

Linking Observations To Proposed

Theory And Hypothesis............. 266

Frame of Reference For Evaluating

s/HMO Findings..................... 273

Policy Principles within which s/HMO

Findings Are Assessed............ 275

Ideas For Operationalizing S/HMO

On An Urban Scale................ 279

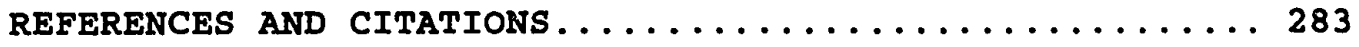

APPENDICIES

A FOR CHAPTER I................... 293

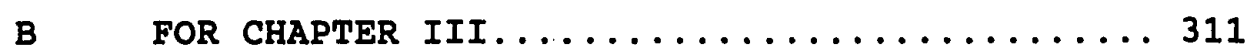

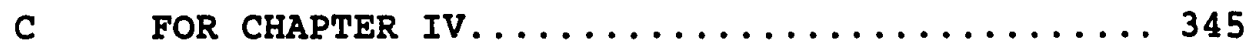

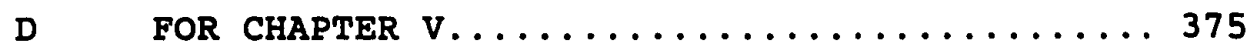

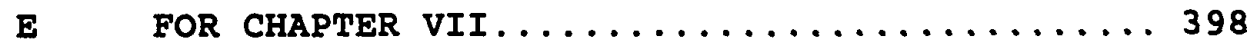

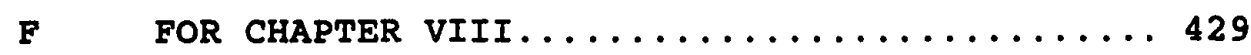


III Predicted Nursing Home Age Based Use Rates.. 24

IV Nursing Home Residents In SNF and/or ICF Facilities on At Least one Day of The study Period In The Overall Data Set.......... 73

Not Mutually Exclusive Count By Research Population Member Eligibility Groups.......

The Seven Combinations of Model B Clustered By Members Who Were "Never s/HMO" And "s/HMO sometime" ........................

VII Overall 1st Admission Rates Standardized BY Sex \& Age Cohort Unique Number of ICF Residents Admitted In The SP Per 1000 Cost or Risk or S/HMO Member Groups Which Are Not Mutually Exclusive (By Gender) .......... 107

VIII Overall Raw Rates for 1st Admission By Sex \& Age Cohort Unique Number of ICF Residents Admitted In The SP Per 1000 Cost or Risk or S/HMO Member Groups Which Are Not Mutually Exclusive (By Age Cohort)........ 109

IX

Sex By Age Cohort Standardized Rates For All Unique Number of Nursing Home Residents By Level of Care Admitted During The SP Per 1000 Cost or Risk or S/HMO Member Groups Which Are Not Mutually Exclusive (Male \& Female) ...................... 112

Non-Standardized Number of Nursing Home Residents Per 1000 Research Population Members, And Per 1000 Population NCHS 1985 National Sample, And Per 1000 Population In Multnomah County Age 65 And over Estimate overall \& By Gender.................. 117 
XI Overall Rates, Standardized By Age And Sex, For Days of Nursing Home Residence During The Study Period Per Member Year of Not Mutually Exclusive Eligibility status Cost, Risk, s/HMO In The study Period......... 119

XII Overall Rates, Standardized By Age And Sex, For ICF Days of Nursing Home Residence During The Study Period Per Member Year of Cost, Risk, s/HMO study Period Eligibility By Gender...................... 122

XIII Overall Raw Rates By Age \& Sex For ICF Days of Nursing Home Residence During The study Period Per Member Year of Cost, Risk, S/HMO Eligibility status In The study Period By Age Cohort................ 123

XIV Use Rates, Standardized By Sex And Age Cohort For ICF Days In SP Per HPM Year of Eligibility By Cost, Risk, s/HMO Eligibility status.................. 124

Raw Rate For ICF Days Per Member Year of Eligibility By Members In Cost, Risk, or S/HMO Eligibility Status By Age Cohort..... 127

XVI ICF Days Used Per Research Population Member Based on Raw Data For Subsets I And II And overall Data set of ICF Residents In Not Mutually Exclusive Cost, or Risk, Or S/HMO Eligibility Status........ 129

XVII Mean Length of Stay For All ICF Residents In Subsets $I, \operatorname{Ir} \ldots \ldots \ldots \ldots \ldots \ldots \ldots \ldots \ldots$

XVIII Overall Days of stay By Time Period Clusters During The study Period For ICF, SNF, SNF+ICF..................... 132

XIX Use Rates, Standardized By Sex And Age Cohort For SNF+ICF days In SP Per HPM Year of Eligibility By Members In Not Mutually Exclusive Cost, Risk, or s/HMO Eligibility status................. 139 
(Rates Are Standardized By Age Cohort Within Gender) Number of ICF \& SNF Residents Per 1000 Risk \& S/HMO Individual Members, Separated By "New" And "Conv" Status At Time of Enrollment In Risk or S/HMO Eligibility scatus................ 147

XXI Raw Rates By Age Cohort For ICF Residents Per 1000 HPM Subset For: New And Converted $\mathrm{S} / \mathrm{HMO}$ And Individual (Non-Group) Risk KP Members....................... 149

XXII (Rates Are Standardized By Age Cohort Within Gender) Number of ICF \& SNF Days For Individual Risk And S/HMO Residents According To New And Converted Status During Their Nursing Home stay.................. 152

XXIII (Rates Are Standardized By Sex And Age Cohort) ICF And SNF Residents Per 1000 Members For: A. New Risk Enrolled Before April, 1985; B. New Risk Enrolled After 1984; C. Cost Medicare Converted To Risk Before April, 1985; and D. After April 1985, As Individual Members.................. 154

XXIV (Rates Are Standardized By Sex And Age Cohort) ICF/SNF Days Per Member Year of Eligibility: A. New Risk Enrolled Before April, 1985; B. New Risk Enrolled After 1984; C. Cost Medicare Converted To Risk Before April, 1985; and D. After April

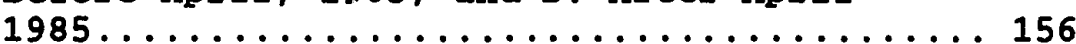

XXV Source Location From which ICF Residents Came At The Time of Their First Admission... 163

XXVI Source Location From which ICF Residents Came At The Time of Their First Admission other Than Home or ICF................. 164

XXVII Disposition Location To Which ICF Residents Went Following Their Last Known Discharge... 166

XXVIII Disposition Location To Which ICF Residents Went Following Their Last known Discharge Other Than Home or ICF............... 168 
XXIX Summary of Disposition For Last known ICF Discharge For All New And Converted S/HMO ICF Residents And (Only) Individual Risk Residents By Subsets (A) Pre-tefra and (B) Tefra Contract Risk Eligibility Status..... 169

XXX Summary of Disposition For Last ICF Discharge To Combined Home, RCF, AFC, ALF, ILF The Sum of Which Is presented As

"Community" other Than ICF........... 173

XXXI Proportion of Risk And S/HMO Residents Still present(SP) In An ICF At The Time Data Was Collected And Therefore Missing From Data In Discharge Disposition Tables...

XXXII Summary of Location Prior To First ICF Admission For New And Converted S/HMO ICF Residents And (Only) Individual Risk Residents By Subsets (A) Pre-TEFRA And (B) TEFRA Contract Risk Eligibility status.....

XXXIII Community Based Facilities other Than ICF To Which Individual Risk And S/HMO ICF Residents Went Following Discharge.

XXXrV (Rates Are Standardized By Age Cohort And Gender) Total Payments Received By Nursing Homes Within One Year After The Study Period For ICF Residents/Member Year of Eligibility In Exclusive Eligibility Clusters: N1 "Never S/HMO" (C, C+R, R); N2 - "S/HMO Sometime" (S, C+S, R+S. C+R+S) .......... 185

XXXV (Rates Are Standardized By Age Cohort and Gender) Total Payments Received By Nursing Homes Within One Year After The study Period For ICF Residents Per 1000 Members Mutually Exclusive Eligibility Clusters: N1 "Never S/HMO" (C, C+R, R); N2 - "S/HMO Sometime" (S, C+S, $R+S . C+R+S) \ldots \ldots \ldots \ldots 187$

XXXVI Absolute Dollars, As Payments Received For SNF And ICF Services During The study Period, Within one Year After The study Period................... 189 
XXXVII Total Payments Received By Nursing Homes Within one Year After The Study Period For ICF Residents Per 1000 Members Mutually Exclusive Eligibility Clusters: $N 1$ = "Never $S / H M O "$ (C, $C+R, R$ ) ; N2 = "S/HMO sometime" (s, $C+S, R+S . C+R+s) \ldots \ldots \ldots \ldots \ldots \ldots$

XXXVIII NPAR1WAY Nonparametric Analysis of Variance Tests For Differences In Payment Means Between Cluster A (Never $S / H M O$ ) And Cluster B (S/HMO Sometime) of Analysis

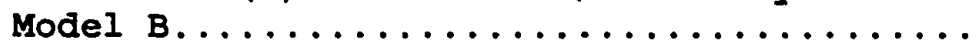

XXXIX (Rates Are Standardized BY Age Cohort and Gender) Total Payments Received By Nursing Homes within One Year After The study Period For ICF Residents Per 1000 Members in Mutually Exclusive Eligibility Groups: i.e., Cost, Cost+Risk, Risk, s/HMO, Cost+ s/HMO, Risk+S/HMO, Cost+Risk+S/HMO........ 195

XXXX (Rates Are Standardized By Age Cohort and Gender) Total Payments Received By Nursing Homes Within One Year After The study Period For SNF \& ICF Residents Per 1000 Members in Mutually Exclusive Eligibility Groups: i.e., Cost, Cost+Risk, Risk, s/HMO, COst+S/HMO, Risk+S/HMO，COst+Risk+S/HMO..... 198

XXXXI Per Diem Payments Including sNF+ICF stay By Each Category Presented For All Members Whose First Admission \& Last Discharge Was Within The study Period................ 202

XXXXII (Rates Standardized By Sex By Age Cohort) Rate of ICF First Admits In The study Period / 1000 Members (HPM) For Whom Nursing Homes Received Medicaid Funds For ICF or SNF stay Before 07/01/89..........206

XXXXII Percent of Total ICF Residents By Cost, Risk \& S/HMO During The study Period who Received Medicaid Assistance With Payment of SNF+ICF Bills During And Up To One Year Post study Period................... 207 
XXXXIV Percent of Total ICF Residents In "Never S/HMO \& "S/HMO Sometime" During The Study Period who Received Medicaid Assistance For Payment of Nursing Home Bills During And Up To one Year After The study Period...... 209

XXXXV (Rates Are Standardized By Age Cohort and Gender) Medicaid Payments Received For ICF Residents One Year After The Study Period For ICF Residents/Member Year of Eligibility In Mutually Exclusive clusters: N1 - "Never S/HMO" (C, C+R, R); N2 "S/HMO Sometime" (S, C+S, R+S. $C+R+S) \ldots \ldots 216$

XXXXVI (Rates Are Standardized By Sex And Age Cohort) Medicaid Payments Received For SNF and ICF Care Before, During and After the Study Period Through 06/30/1989 For Members Residing In ICPs During The Study Period /1000 Research Population Members In "s/HMO Sometime" or "Never S/HMO," (Model B, Format A) ....................... 220

XXXXVII Medicaid Payments As A Percent of Total Payments For ICF Residents In "Never S/HMO \& "S/HMO Sometime" Received Up TO One Year After The Study Period.......... 222

XXXXVIII (Rates Are Standardized By Sex And Age Cohort) Medicaid Payments Received For SNF and ICF Care Before, During and After the Study Period Through 06/30/1989 For ICFs or SiF Study Period Residents Per 1000 Members In "S/HMO Sometime" Or "Never S/HMO,"..... 222

XXXXIX (Rates Are Standardized By Sex And Age Cohort) Medicaid Payments Received For SNF and ICF Care Before, During and After the Study Period Through 06/30/1989 For Members Residing In ICFs During The study Period $/ 1000$ Research Population Members In "s/HMO Sometime" or "Never S/HMO," (Model B, Rows $3 \& 4$ of Format B) .................. 225

Mean of All Payments Received For S/HMO And Risk Nursing Home (SNF And ICF) Residents Who Became Welfare Dependent..... 
LI Rank Order of Top Ten DRGs From The Last Hospital Stay Prior To First ICF Admission

In The study Period................ 242

LII Rank order of Top Ten DRGs From The Last Hospital Stay Prior To First SNF Admission In The study Period................ 245

IIII Rank Order of Top Ten DRGs From Multnomah County Hospitals who Were Discharged To Nursing Homes (SNF-ICF).

IIV

Rank Order of Top Fifty Percent of ICD9 Codes From The Last Hospital stay Prior

To First SNF Admission In The study Period.. 248

LV

Rank Order of Top Fifty Percent of ICD9 Codes For Secondary Diagnosis From The Last Hospital Stay Prior To First ICF Admission

In the study period............... 250 
LIST OF FIGURES

1. Overall Nursing Home Resident Data Set Showing Combinations of Admissions Before, During And After The study Period And shows Which Combinations Comprise Subsets I \& II \& Overall (subset III)................ 90

2. Mutually Exclusive Model Used To Present Rates On Payments Received/1000 Research Population Members In Model For Analysis B...........

3. Model For Analysis And Format To Present Observations In Chapters IV Through X...... 98

4. Probability of Residing In A Nursing Home During The Study Period Per 1000 Research Population Members............. 113

5. Cumulative ICF Days Per Member Year of Eligibility During The study Period........ 125

6. All Days To Discharge By Time Periods....... 134

7. Percent of Distribution By Location To Which Last Discharged During The Study

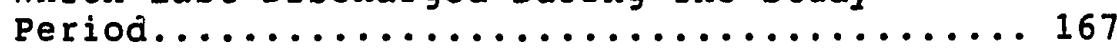

8. Total Dollars Received By Nursing Homes For SNF \& ICF Residents Within one Year After The study. Period Per 1000 Members...... 188

9. Percent of ICF Residents For Whom Nursing Homes Received Medicaid Payments During or Within One Year After The Study Period...... 208

10. Medicaid Dollars Received By Nursing Homes For SNF \& ICF Residents Within One Year After The Study Period Per 1000 Members..... 223 
CHAPTER I

INTRODUCTION, BACKGROUND INFORMATION, RESEARCH ENVIRONMENT

This country is in the midst of re-examining the role of public programs in carrying out intergenerational obligations. [1]

\section{INTRODUCTION}

The social problem addressed by this study is that many older Americans suffer catastrophic damage due to financial burdens resulting from use of nursing home services which are not paid for by entitlements or private insurance. Demand is growing for changes in national health policy which expand entitlements that protect the elderly, and other disabled persons, against the prospect of financial ruin resulting from their out-of-pocket payment for long-term care services. "One of the most serious gaps in our health care system is the failure on the part of both the public and private sectors to afford any of our generations protection against the devastating costs of long-term illness."[2]

However, resistance is extant among policy makers and in the private sector as well, because the commitment of resources needed to close this gap is potentially large. And, major changes may be required in our health care 
system in order to make it financially reasonable to adopt meaningful, entitlement-based, long-term care benefits, or some combination of entitlement services and private insurance or managed care programs involving long-term care. There are concerns about adopting such policy.

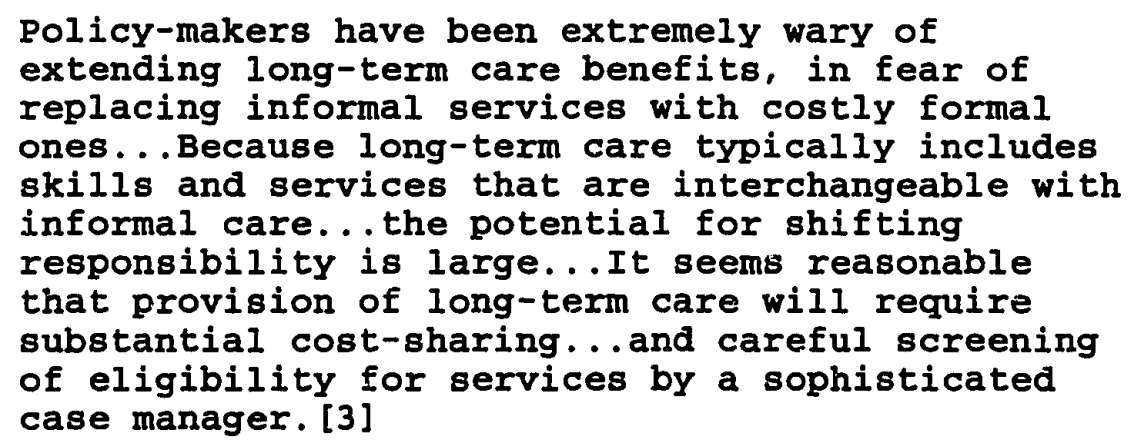

Another reason for being cautious in adopting policy which requires inter-generational transfer of resources, is that the future capacity for increasing this mandate is uncertain. Many technologically advanced nations are experiencing concurrent inflationary expenditures for health related services and escalating need for such expenditures, due to aging populations. Such trends are associated with changes in: social policy to provide for needs of aging cohorts in their population, the expanded infrastructure developed to attain goals of such policy, and ability of productive sectors within their populations to support public and private financing of such services.

Several cross-national studies have been done to document patterns of change in health care costs among the 
twenty-five nations participating in the organization for Economic Cooperation and Development (OECD). These studies are used to project the effect of escalating cost patterns over the next few decades. All 25 nations share a common concern about their ability to sustain the present level of inter-generational transfer of resources needed to support social and health policies already adopted beyond the next decade. Projected costs, related to adoption of such social policy in America, portend a very high burden for younger citizens who would pay for long-term care of their elders.

While social and health policy achievements are many, over the last three decades, most of these nations soon will be confronted by a marked decline in aged-dependency ratios. This ratio is that portion of the population contributing to the gross domestic product (GDP) divided by the population not contributing to the GDP. The changing aged-dependency ratio results from declining birth rates and increasing proportions of unemployed persons, of whom the aged are an increasing component. While no devastating change is projected regarding this socio-demographic condition prior to the year 2010; thereafter, the impact is catastrophic, given other entitlements and encumberments of government, and barriers to entrepreneurial growth which confront the private sector. Hard choices will exist for policy makers and individual citizens alike, in most of these 25 nations, regarding expansion of entitlements, 
especially present health benefits. Specifically,

Setting aside significant differences among countries, it appears likely...that by the year 2030 OECD countries will be faced on average with total health expenditures some 30 per cent higher, [than in 1986] and per capita health expenditures some 20 per cent higher as a result of population aging. At current levels of expenditure [1986], this represents an additional burden of 3 percent of the GDP. However, this presumes that all other potentially cost-inducing factors are held constant... it could require significant allocations from other competing goals and a political

willingness to provide the mechanisms which will

accommodate such a shift in priorities. [4]

What can the United States learn from other nations, whose declining aged-dependency ratio precedes ours and whose social policy is more expansive regarding long-term care benefits, regarding policy solutions?

In most Scandinavian countries, the United Kingdom, the Netherlands and in some Canadian provinces, where long-term care is almost exclusively delivered by the public sector, extensive screening programs exist to ensure use of resources which adhere to social policy goals, and which minimize poor public interest use of such resources.

specifically, complex issues are negotiated regarding family capacity for care-giving, use of personal finances, alternative methods of managing dependency, community options for placement, and differences of provider opinion regarding use of medical services. Social policy in Denmark establishes multidisciplinary assessment committees to 
negotiate such complex issues. However, OECD investigations suggest that policies for financing and delivery of longterm care services are fraught with conceptual as well as ethical difficulties; effective oversight is problematic without carefully devised incentives for cost-effective use of public and private sector funds.

Furthermore, such LTC and other health policies about chronic illness must be coordinated with those in the acute care area for the non-elderly population. Many OECD countries have pursued social policies regarding care of their aged which places the medical model of health service within the context of a broader gerontologic policy model.

In America, some scholars of geriatric care policy, such as Duncan Neuhauser, advocate that care of the elderly should not start with the medical model, but rather with a social support model under which medical care functions in a supportive but subsidiary role. It is his view that capitation reimbursement schemes and social/Health Maintenance organizations provide an opportunity to accomplish that, even if not fully realized.[5]

This notion has been espoused over the last decade by health care analysts, such as carroll Estes, who are concerned about the future role of academic health centers in America. She advises medical schools to pursue training of medical students in community-based settings where the orientation toward elderly persons and long-term care 
eschews the traditional medical model and shifts to a broader goal than medical treatment; one which encompasses socio-cultural, behavioral and environmental realities for chronically ill and dependent aged persons. [6]

Christine Cassel, Chief, Division of General Medicine, School of Medicine, University of Chicago, also advises a shift in teaching which emphasizes that treatment goals involve quality of life aspects which are as much or more relevant to the patient's welfare, than abnormal laboratory findings or any specific diagnosis. [7]

Adoption of such concepts by the community of formal, health care givers means that long-term institutional care providers will be expected to pro-actively coordinate traditional nursing home services with non-institutional community based care. Achievement of such concepts may require financial incentives which encourage discharge procedures that ensure continuity of social, psychological and health care needs of elderly residents. Such concepts may produce living arrangements for the elderly which have yet to emerge, and which may formally link providers of such living arrangements with the current nursing home model. [8] The challenge, then, is to find solutions to the current and impending demand for long-term care needs without enlarging the health care related public financing crisis which now besiege many nations, including America. What kind of policy changes are likely to move us in that 
direction? Theodore Marmor perceives the sweeping reforms, which he believes necessary for America's health care system, are unlikely because pluralist politics permit only incremental movement of social welfare policy here. But, he also believes that many key constituents of America's health care system accept that such change must come. [9] If other nations in OECD can manage to formulate, adopt, and internalize such national policy regarding long-term care of dependent elderly, so can the United States, even with its heterogeneous cultures, divergent state governments, and inharmonious public-private sector health care partnerships.

Indeed, a few trial projects exist in both public and private sectors regarding long-term care services which reflect social policy adopted in many other nations, and which incorporate some of the ideological changes, social policy objectives, multidisciplinary based managed care procedures, and financial goals discussed above.

These trial projects contribute information needed to guide policy formulation about social and health policy related to care of elderly persons. Policy adoption and ensuing implementation must also be guided by experiences of such trial projects. Mazmanian's view of incremental change, as the cornerstone of America's public policy adoption process, is embodied in these trial projects. This trial project process has moved the HMO concept 
into the mind-set of government and some politicians, as the solution of choice for containing growth in health costs at an acceptable level and widening the scope of benefits to better protect individuals and government pocket-books. That private organizations had successfully established todays HMO model 'well before the term "HMO" appeared in legislation, is also part of that incremental change concept. It is in this context that the research for this dissertation occurred.

A goal of this study is to contribute to incremental movement toward public and private domain adoption of operationally sound policy solutions which improve access to certifiably needed ITC services for this nation's elderly, at an affordable price.

The policy context within which this contribution is pursued, involves expanding Medicare entitlement linked Supplemental Benefit schemes for limited LTC insurance protection against early stage expenses for LTC, which are privately financed. Improved access to and coordination with most health and many social services is also part of that benefit scheme, which pursues creative home-care alternatives to institutionally provided services.

The hoped-for contribution of this study is that policy makers and private organizations will be better informed about the emerging outcome of one trial project with strong potential for nurturing policy changes which encompass LTC 
needs of beneficiaries without losing sight of looming inter-generational burdens to be borne by today's youth. That trial project is the Social/HMO as implemented by Kaiser Permanente (KP), Northwest Region, as one of four sites selected for this legislatively supported project. The S/HMO combines efforts of public and private sector organizations to shield the aged against shorterstay costs of long-term care institutions and formal care provided in the home as an alternatives to and preventive measure against needed long-term care services which are institutionally provided by Intermediate Care Facilities, known as ICFs. The S/HMO concept seeks to minimize personal expenditures for such services by maximizing authorized uses of S/HMO's limited Expanded Care Benefit through managed care concepts adopted by KP and the other three demonstration sites. While seeking better methods for accessing LTC services by the elderly, this trial also tests use of financial incentives designed to encourage cost effective cholces regarding LTC services. Such choices are negotiated between patient or patient's family, Center for Health Research case managers, attending physicians, and other providers within and outside of KP. This model conceptualizes financial outcomes which do not exacerbate inflationary trends in the health services industry.

The consolidated, prepaid $\mathrm{s} / \mathrm{HMO}$ model has been looked to by many policy-makers and providers as a 
rational way to deliver managed, integrated health and long-term care (LTC) services (Callahan and Wallack, 1981: Rivlin and Wiener, 1988)...The SHMO was designed to expand prepaid coverage of community and nursing home care in a controlled manner and to link these expanded LTC services with a complete acute care system. [10]

At the KP S/HMO Demonstration Project site, the HMO component of this project had been in operation for over 40 years. Therefore, the basic goal of the trial has been focused on designing and implementing LTC services under the Expanded Care Benefit package (ECB). Congressionally mandated waivers to Medicare and Medicaid laws/regulations were granted to allow and encourage integration of alternative LTC services with all Medicare entitlement benefits. The first trial project period was from 1985 through 1988. That is the time line within for which data was collected in support of research undertaken for this dissertation study. Two Congressional extensions have been granted since then, ending in 1995.

Studies are needed which inform policy makers with data about comparative differences in use of LTC services by persons having and not having various forms of fiscal protection against costs of LTC, of which the S/HMO ECB is one. Such information is not available from the $s /$ HMo sites at this time, although much is known about other outcomes from the $\mathrm{s} /$ HMO studies. No literature is available which makes this specific comparison between $S / H M O$ and Risk Medicare Beneficiary use of ICFs. 
Therefore, the research task of this study is to collect data about, and describe differences in, ICF services used by both trial project $s /$ Ho members and other KP Medicare beneficiaries enrolled in RP under HCFA contracts for fixed capitation reimbursement. Such Medicare beneficiaries are known as "Risk" members. S/HMO members are enrolled under such risk agreements, but HCFA capitation reimbursement for $\mathrm{s} / \mathrm{HMO}$ members who are certified as eligible for ICF (or SNF) level nursing home care is at 100 percent of the institutional rate cell rather than 100 percent of the average adjusted Medicare rate in the county of residence for Risk beneficiaries.

The purpose of identifying descriptive differences between S/HMO and baseline data for other Risk members, is to show how Risk beneficiaries with an ECB use ICF services compared to how other Risk beneficiaries without any ECB use ICF services, during a portion of the initial waiver trial period. Observed differences are presumed due to, at least partially, case managed ECB. Such observations could provide a base of knowledge sufficient to undertake studies which confirm that persons with S/HMO ECB choose and use institutional long-term care services in a way that differs from persons who do not have such benefit options. Research findings of that nature are central to policy information needed from the $\mathrm{s} / \mathrm{HMO}$ Demonstration Projects. It was not known whether such differences existed prior beginning this 
study. Views differed among $s /$ HMO investigators about how ICF use might differ between Risk and s/HMO members.

Another task of this study was to provide descriptive information about possible affects of the ECB on the need for and extent of Medicaid assistance with payment of $\mathrm{s} / \mathrm{HMO}$ member costs incurred from residing in nursing homes. Risk spend-down experience is compared with that of S/HMO to infer possible affects of the ECB on preventing or deferring welfare status.

Such descriptive observations are synthesized into policy recommendations conceptualized for use with s/HMO trial project outcomes, pending other confirmatory studies. These recommendations therefore are used to formulate recommendations for follow-up research needed to confirm descriptive findings of this study.

\section{BACRGROUND INFORMATION}

Background information is presented next which attempts to place the work of this study into an overall policy and operational context at the time of the study period (July 1 , 1986 through June 30,1988$)$, from which KP members were selected in order to identify and evaluate their nursing home utilization. This is followed by a description of the s/HMO program at this site. Chapter II presents conceptual issues related to the purposes of this study, noted above, from which six research questions are derived for guiding 
the research methodology pursued. Demand for nursing home care in America results from several situations which interact to produce inflationary growth in expenditures. Such inflatjonary trends may be partly related to the institutional bias of social policy programs legislated for health care. In America, the predominant public policy for financing nursing home services is Medicaid. The operational solution emphasized by Medicaid in most states is institutional care rather home or foster home care. For those elderly whose informal network of support is missing, alienated, or incapable of caring for them on a continuous basis, institutional care may be the only choice.

state policies on Medicaid and on Supplemental security Income for the aged vary greatly, which translates into significant differences between states regarding publicly funded access to LTC. Compression of state and local government budgets causes increased pressure for ways to reduce public financing of LTC; one method is to reduced the number of people who become eligible recipients of Medicaid. This is occurring at a time when the number of elderly in need of such support is increasing.

It is not a credit to the social policies of America that some elderly, most of whom have been financially self-sufficient over their life course, conclude it in abject poverty because LTC costs exceed their savings and other assets. This situation occurs because neither private 
insurance nor public policy has created a system of financing which spreads the risks of LTC costs across a large population base, as with most other health care costs. There are numerous issues associate with this lack of social and health policy for LTC services; some of these are presented, below. They include demographic trends, effects of inflation related to LTC services, the supply of nursing home beds, Spend-down trends and state policies on Medicaid, and trends in nursing home utilization. These issues provide the policy context in which this study commenced, as well as a basis for conceptualizing the circumstances from which research questions arise, in this study.

It is well known that people age 65 and older consume a highly disproportionate amount of health care services. They comprise about thirteen percent of the population, yet onethird of all national medical and hospital care expenditures are for the aged. As the proportion of young to old changes, so will the demand for allocation of limited resources shift to the elderly. Inter-generational transfer may become a serious burden on the nation's working population by the year 2010. [11]

As inflationary rates of institutional LTC continue to increase faster than the All Urban Consumers, All Items Indexes, Consumer Price Index (CPI), the issue of how to finance those services becomes more acute, and the matter of more effective management of LTC services more pressing. 
Nursing home expenditures continue to grow at a rate exceeding that of the CPI. In 1988, it is estimated that all long-term care costs for the elderly totaled $\$ 42$ billion including formal and informal care costs, which was about 9 percent of the total health care expenditures for the year. In 1987, nursing home care for the elderly totaled 32.8 billion which was over 20 percent of total health care expenditures for those age 65 and over for that year.

of this amount, 57 percent was paid privately (excluding private long-term care insurance), 36.3 percent by Medicaid, 3.4 percent by other government programs, 1.8 percent by Medicare, and 1 to 2 percent by private long-term care insurance. Nursing home costs have been raising steadily at an annual rate of about 10.5 percent ...From 1988 to 2018 , total nursing home cost are expected to grow from $\$ 33$ billion to 98.1

billion. [12]

Some nursing home residents become dependent on Medicaid after spending down their assets to a welfare eligible level. Over half of all nursing home occupants are reportedly not poor upon admission to a nursing home, but become so in less than one year.

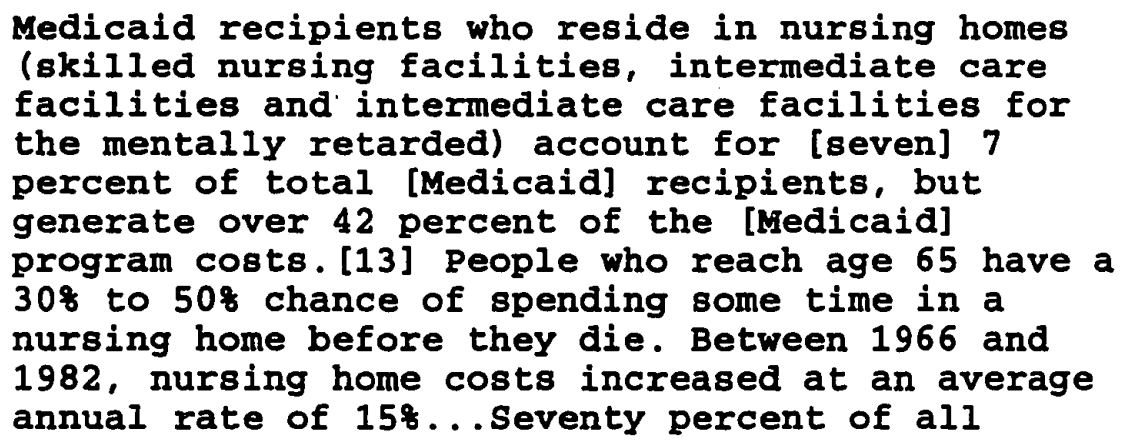


single people admitted to a nursing home go broke within three months: $50 \%$ of couples are impoverished within six months after one spouse is admitted. [14]

In 1990, Medicaid paid for 45.5 percent of all nursing home care and was the largest third party payer of long-term care. In 1985, Medicaid paid 50.4 percent of all nursing home costs. (Op. Cit. [12], p. 28) states are struggling to reduce expenditures for nursing home services and this decline in percent of total nursing home cost paid by states suggests they may be succeeding. There are many approaches to that process.

state governments often adopt a policy of restricting licensure for existing beds and prohibit construction of added beds as one means of suppressing utilization of nursing homes. Development of a national policy on availability of beds is complicated because demographic, cultural, social customs, geographic proximity of families, the local economy, and other trends cause great variability in choices for ITC between geographic regions in America.

The range in supply of nursing home beds/1000 population over age 64 among all states in 1985 was from 26.2 beds /1000 residents in Florida, age 65 and over, to 90 beds/1000, in Minnesota. [15]

In 1986, nationally, supply of nursing home beds, excluding those for the mentally retarded, was 1.5 million, averaging 51.7 beds/1000 population age 65 or more. Within 
this aggregate pool of LTC beds, there were 33.7 SNF beds/1000 population age 65 or more, 14.1 ICF beds /1000, and 13.8 uncertified nursing home beds /1000. In the western states, there were 42.3 nursing home beds/1000 age 65 or more, of which $34.3 / 1000$ were SNF and $3.8 / 1000$ were ICF; the remaining were uncertified.[16]

The proportion of ICF to SNF beds in oregon is unique among the western states in which SNF beds predominate. In oregon, there are far more ICF beds than SNF beds and, no doubt, this is related to the state and federal contract for pass-through of Medicaid funds.

In oregon an aggressive program has been in place since 1980 to reduce the number of nursing home beds per 1,000 population over age 65. The oregon State Health Plan established in 1980 an overall goal of 40 beds per 1,000 population age 65 and over, which was intended to reduce the supply from 50.3 beds per 1,000 (range by county was $25 / 1,000$ to $99 / 1,000)$.

The State Health Planning Council's policy objective, of 40/1,000 population, which also happened to be the goal proposed by the National Governors Conference 1980, was conditional in that the Council acknowledged that goal could "...only be reached if it is approached hand-in-hand with the development of alternative services." [17]

A 20 page chapter of that document was developed regarding "Alternatives to Institutional Care For The 
Elderly and Disabled." A primary solution to attainment of that goal was shifting Medicaid placements from nursing homes to other community facilities, such as Adult Foster Care or Residential Care Facilities.

By 1985, there were 16,068 nursing home beds in free standing facilities, having an average occupancy of 89.3 percent, in oregon. The statewide bed availability rate was then down to 45.1 beds /1000 population age 65 and over. Additionally, there were 293 hospital based nursing home beds in oregon. Of the 173 certified nursing homes in oregon, 109 were licensed to operate ICF beds.

oregon has pursued alternatives to nursing homes for Medicaid eligible persons during the last fifteen years, beginning with the Senior Health Improvement Project (SHIP) funded by a federal Models Project Grant (90-A-1606) from the Administration on Aging, Department of Health Education and Welfare. It developed Placement Information Base (PIB) criteria for assessment of Medicaid applicants in order to identify persons who could be placed in alternative care sites. This helped attain the 1980 ratio or 40 occupants 11000 persons.

Thus, in 1985, the state of Oregon represented an ideal environment in which to implement the s/HMO Expanded Care Benefit, since residents of the state were already conditioned to the idea that community based care was a reasonable alternative to nursing home care. 
Demographic changes are well documented about growth rates among age cohorts of 65 and over. The demand for nursing home care is expected to continue increasing, because the proportion of elders over age 75 is increasing faster than the general population. Age cohorts 65 to 100 have almost tripled since 1900; those over age 75 will comprise between seven percent of this nation's population by $2000 \mathrm{AD}$. and, "... in 2030 there will be as many people over age 75 as there are today over age 65."[18]

As a percent of the total population, those over age 75 are expected to increase from 5.0 percent in 1990 to 7.7 percent by 2030, while the total population over age 64 may increase 18.3 percent in 2030 .

The 1983 report of the U. S. Senate Special Committee on Aging senate projected the inter-generational transfer dependency ratio of non-aged working to non-working aged to increase from $18 \%$ in 1980 to 218 in 1990 and to 338 by 2025 . That is, under such forecasts, by the year 2025 there will be one, non-working elderly person for every 3 working persons in America. Some estimates suggest 2.5 to 1 is more realistic. [19]

Predictions vary regarding elderly citizen needs for chronic care services, at the point where aged-dependency ratios are a serious threat to the well being of this nation's economy. Researchers at the Urban Institute and Duke University "...project that in 2020, the disabled 
elderly population could be as low as 10 or as high as 14 million; in 2060, it could be as low as 14 or as high as 24 million." [20]

One demographer at the National Institute of Aging estimates that by the year 2080, the number of persons in America, age 85 and over, could be 72 million rather than the 18.7 million estimated by the Census Bureau; currently, there are 3.3 million. [21]

Furthermore, due to the relative increase in persons age 85 and the over, the proportion of disabled is projected to increase from 23.7 percent in 1985 to 28.6 percent in 2060, within the elderly population. Projections vary because of differences in how chronic disability is defined, and because of differences in assumptions about mortality rates. If disability rates decline as rapidly as mortality rates, there could be 20 percent fewer disabled in the year 2020. Table I represents one of many projections of age cohort distribution across the next fifty years.

A correlation between age and increasing use of nursing homes is clearly documented. As one study indicates, "...age is a very important factor. Among those 65 to 74 years old, the occupancy rate is less than 2 percent. It raises to about 7 percent for those age 75 to 84 , and then jumps to 20 percent for those 85 and older." (Op. Cit. [18])

Another study indicates 1.28 of those age 65 to 74 , and 5.9 percent of those age 75 to 84 , rising to 23.7 percent 


\begin{tabular}{cccc}
\hline \multicolumn{5}{c}{ TABLE I } \\
PEPULATION ESTIMATE THROUGH 2045 \\
1990 & $(000)$ & $(000)$ & $(000)$ \\
2000 & 18,035 & $75-84$ & $85-100$ \\
2025 & 33,188 & 10,349 & 3,313 \\
2045 & 31,202 & 12,318 & 4,926 \\
(Bureau of Census, Series & p-25, Nr. 952,1983, Table 6) \\
\hline
\end{tabular}

among those age 85 and over.[22]

There is agreement among demographers, in spite of differences over assumptions, that the absolute and relative number of disabled elderly will increase and corresponding demand for long-term care services will increase, dramatically. Brookings Institute has developed a sophisticated system for making and updating projections of the elderly population using long-term care services. The Brookings Long Term Care Financing Model includes many assumptions about personal income and other factors thought to affect demand and ability to pay. Findings suggests a stronger statistical relationship between: level of income (less or more than $\$ 10,000)$, disability, and long-term care services needed/used. [23]

This model makes a range of assumptions about induced 
demand resulting from a variety of private and public insurance programs. It considers the effects of relaxed financial eligibility for long-term care or expanding benefits, based on the Canadian experience and Channeling Demonstration Projects, which suggested that more community based services did not reduce demand for nursing home beds.

A central research question in this dissertation study is whether or not nursing home use changes when induced demand for community based services is introduced, under case managed conditions. Findings to this question are presented in Chapter v.

In the year 2018, just 25 year ahead, their 1990 prediction estimates the range of elderly persons using formal Medicare home health services as 5.88 million (low) to 7.88 high and baseline as 6.36 ; the low estimate for institutionalized nursing home residents is 3.03 to 5.02 and baseline is 4.02 . That represents an increase of over 75 percent from current nursing home use: "...the number of elderly using nursing homes during the course of a year is expected to increase from 2.3 million in 1988 to about 4 million in 2018." (op. cit. [20], pp. 8, 21)

The proportional increase of elderly in the total population is projected to raise by 61 percent. The Urban Institute baseline projection for nursing home use in 2020 $A D$ is 4.32 million, and about 20 million additional persons in the cormunity needing formal and informal care giver 
assistance.

of the $1,491,400$ residents living in 19,100 nursing homes, nationwide in 1985, 1,325,800 (88\%) were 65 year or older. The largest age group of nursing home residents was age 85 or more (45\%); the next largest was age 74 to 85 (398); in age cohort 65-74 only $16 \%$ of all residents. In a study by weissert, a method of predicting nursing home bed demand was developed, based on levels of chronic dependency among the elderly, as measured by Katz's activities of daily living (ADL) scale of dependency. Weissert projected in 1985, using 1977 and 1982 bureau of census population forecasts for age and sex, that the use of nursing home beds may double by the year 2000. this data reportedly is similar to that observed in the longitudinal Framingham study. [24]

The 1985 national aggregate ratio of residents per 1000 population age 65 is shown in Table II, below, as a reproduction of Weissert's estimated rates. These ratios provide a frame of reference for ratios produced in chapters $V$ through IX in this study. The above ratios also are consistent with those computed by the office of Actuary, U.S. DHHS based on 1977 NCHS data. [25] Nursing Home utilization rates also are a function of frequency of admission and average length of stay for each admission. Seventy-five percent of nursing home discharges are alive (although about eight percent go immediately to hospitals to 
die). Table III is derived from Weissert's projections for nursing home occupants per 1000 population age 65 and over.

TABLE II

NATIONAL RATIO OF RESIDENTS/1000 POPULATION

IN NURSING HOMES

AGE GROUP $\quad$ NURSING HOME OCCUPANCY
65-74
$12.5 / 1000$ (AGE 65+)
75-84
$57.7 / 1000$
$85+$
$219.4 / 1000$
$65+$ (AVERAGE)
$46.1 / 1000$
(MALES - 29.0/1000;
FEMALES - $57.7 / 1000)$

\begin{tabular}{ccc}
\hline & TABLE III \\
PREDICTED NURSING HOME AGE BASED USE RATES & Nursing Home/population \\
Age Cohort & & $\frac{10 / 1000}{65-69}$ \\
\hline $70-74$ & - & $21 / 1000$ \\
$75-79$ & - & $45 / 1000$ \\
$80-84$ & - & $98 / 1000$ \\
$85+$ & - & $217 / 1000$ \\
\hline
\end{tabular}

During the last three decades nursing homes have become increasingly the solution used for resolving problems of 
chronic dependency when volunteer home support is not available. "From the end of the 1960 s to the early 1980 s the number of residents in nursing homes more than doubled, from 790,000 to almost 1.4 million." [26]

The pool of LTC consumers could swell significantly if the capacity or willingness of the informal system to care for the very old is altered by changes in social, cultural and economic customs. Factors which reinforce this concern include: increased divorce rates, smaller families, lack of proximity to family members, age of siblings who may care for their elderly parents, and unavailability of females due to their joining the work-force. While there are theoretical counter arguments, many indicators exist which suggest that the proportion of older people requiring formal LTC support systems will expand continuously over the next several decades.

A primary cause of expanding demand for ICF services is due to the growing number of females over age 85 who live alone without access to family members or other informal groups who might support them.

As discussed above, policies of the $1970 \mathrm{~s}$ and $1980 \mathrm{~s}$, regarding suppression of nursing home beds, drove the ratio of SNF and ICF beds from 53.4 in 1978 to 52.5 in 1989, in an effort to cut costs. In oregon and washington, colorado and Wisconsin, the rate decreased by at least 17 percent, which may have serious consequences in the face of above 
projections. This diminished bed supply has led to a marked rise in other facilities providing services to the less severely disabled including board and care, assisted living facilities, congregate housing, and continuing care communities in these states. Such trends are following in other states.

The Brookings Institute projects that the number of elderly using paid home care services will raise by 60 percent in the next 25 years. Another study projects that by 2030,46 percent of all elderly will live alone, compared to 38 percent in 1990 (op. cit. [20], pp 10, 18) Given predicted increases in chronic disability and decreasing estimates of old persons with family or other support systems, it is apparent that demand will increase for paid personal care services which are provide in the home or otherwise in evolving community based facilities.

Managed care systems are leading the way in providing paid services in the home to this growing proportion of disabled population living in community, but private insurance programs are following this path of solutions to both bed shortages and cost containment for those elderly whose care can be managed in non-institutional settings. Cost projections, related to the above trends, are staggering. Between now and 2018, nursing home expenditures alone are expected to triple from over $\$ 42$ billion to $\$ 120$ billion in 1987 dollars, and triple again by 2048 to $\$ 350$ 
billion dollars. The range of assumptions for these 2018 projections, is $\$ 66$ billion to $\$ 145$ billion. Concurrently, the ratio of working age population to all estimated disabled elderly decreases; over the next 75 years it is estimated to drop from $21: 1$ to $9: 1$. (Op. Cit. [20], p. 14)

This above overview of socio-demographic issues related to LTC needs helps explain why governments and private business at all levels are seeking methods of reducing current and impending expenditures for LTC services. The prospective tax base for publicly financed support and care of the elderly is expected to diminish. Business does not wish to channel funds needed for capital growth and ownership earnings into expanded health care benefits, or increased taxes. Per capita expenditures for acute care can be expected to increase significantly in the foreseeable future, in addition to ITC due to the above changes in social characteristics of Americas population.

Lack of private insurance or entitlement benefits for financing LTC results in an increasing demand for Medicaid as the number of very elderly increases. "A major barrier to development [of LTC insurance] has been the lack of information on which to base utilization and cost estimation." [27]

Trial programs such as S/HMO represent potential methods for shifting some of the burden from government to 
the private community. The urgency of obtaining information from S/HMO, and other trial programs cannot be overstated. Many public programs have experimented with alternatives to institutional care in an effort minimize the public financing burden of nursing home care.

If private insurance carriers and direct service provider organizations are to pick up an increasing portion of nursing home expenditures, it is important for them to become knowledgeable about utilization rates.

Hopefully, data from this study will be useful to private organizations interested in developing and marketing ITC benefits. Exactly when, if ever, market products by private insurance carriers will become widely available is uncertain. However, meaningful efforts are being made by private organizations to understand, and prepare for that event. [28]

\begin{abstract}
By June, 1990, approximately 1.6 million Americans had purchased long-term care insurance... The market for long-term care insurance resembles the market of Medicare Supplemental or "Medigap" insurance in the 1970s, which varied greatly in value and coverage... [among the many issues complicating the marketing of such coverage, one is especially troublesome about having some uniform criteria about]... How insurers determine whether policyholders are impaired in their ADLs and thus eligible for benefits... another is how they link impairment to medical necessity. [29]
\end{abstract}

The Robert Wood Johnson Foundation is sponsoring trial projects which encourage public and private ventures in 
promoting LTC insurance which emphasize in-home care, case management and personal asset protection to policy holders.

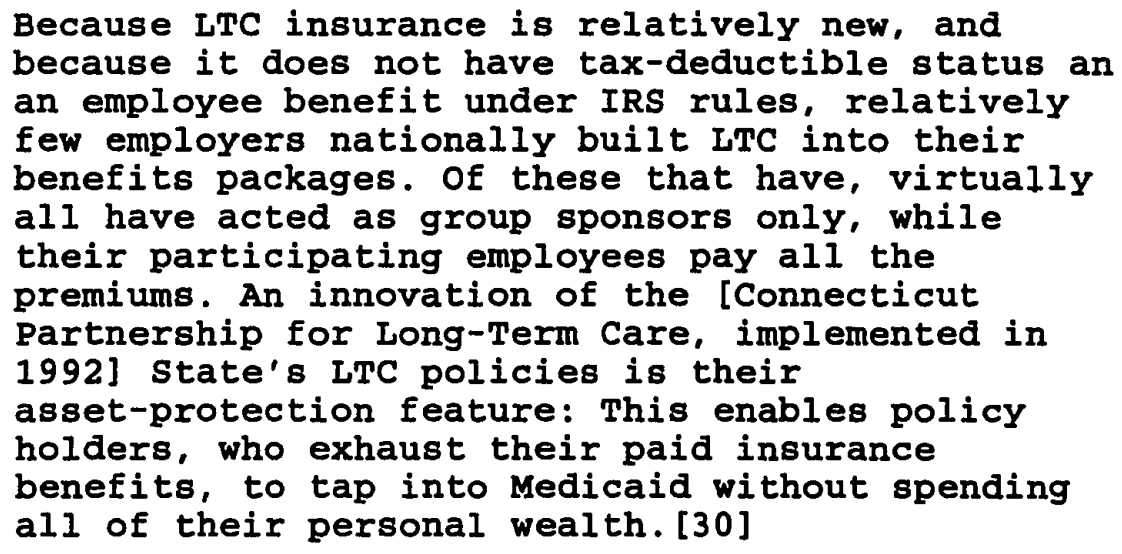

The demonstration project referenced above is a ten

year trial. Connecticut sees it as a way to contain Medicaid costs. Insurers see it as a way to promote LTC policies.

Employers see it as a way to decrease concerns of employees

over care of a disabled spouse, or parents.

Although pressure exists for Congress to require minimum policy benefits, guaranteed standards of access to benefits and non-cancelable terms, great variation exists.

Coverage for long-term disabilities, Alzheimer's disease and other dementias has grown briskly in the last five years, to an estimated $\$ 3$ billion in annual premiums. About 2.7 million people, most starting in their late $60^{\prime} \mathrm{s}$ and 70 's have bought policies. Depending on age and what is covered, the cost may be $\$ 1000$ to $\$ 4000$, a year. About 8 percent of large and medium sized employers sponsor long-term care [benefits], and 18 percent more intend to do so by 1995 ... Industry executives and Congressional health policy aids doubt that sweeping long-term care measures will be enacted soon. [31] 
As insurance carriers, and medical care provider organizations which operate under fixed payments or capitation arrangements, progress toward large scale marketing of prepaid, long term care services for chronically dependent older Americans, it is likely they will seek information about the effects of alternative services on the utilization of nursing home services.

While the literature on such utilization information is expanding generally, very little is available regarding a specific subset of the general Medicare population; specifically Medicare beneficiaries enrolled in HMOs under capitated "Risk" Contracts. The absence of such LTC information makes information from this study of potential interest to such health care organizations. Future research and actuarial studies seeking to create and market a ITC benefit may be interested in knowing effects of prepaid, Expanded Care Benefits on institutional care.

The foregoing summary of experience in nursing home use rates provides background information against which to compare findings in this study. A summary of factors likely to affect those rates over the next few decades, emphasizes the need for production of information presented by this study. This background information suggests the policy context for this study is complex and dynamic.

A summary is presented next of the specific research environment in which this study is conducted, i.e., at one 
of four sites (Raiser Permanente, Northwest Region), where a Social Health Maintenance Organization Demonstration Project is operationalized. Salient aspects of the S/HMO project are presented first.

\section{RESEARCH ENVIRONMENT}

The S/HMO Demonstration Project represents an incremental movement in social policy which embraces many of those notions about care of the aged practiced by OCED nations, and which are advocated by leadership at some of this nations academic medical centers with a history of forging change within the medical community.

Most health care research evaluate what exists. The S/HMO is an operationalized program of research to demonstrate what can be done to bring consumer, provider, government and private sector interests together to test the financial feasibility of adding a privately financed long-term component to entitlement benefits which risks shifting of informal services to formal care, yet which engages the patient's social sunbort svstem to facilitato

home based care when possible.

s/HMO is a complex program which is implemented in a sophisticated organizational structure servicing a fully diversified cross-section of the Medicare population in Multonomah county by a staff which is experienced in multidisciplinary team care. It is a formidable undertaking 
to do a comprehensive research analysis of all effects of the S/HMO Expanded Care Benefit (ECB). In part, the research process is complicated because it is difficult to separate possible effects of ECB from other contractual health care services provided to each Medicare beneficiary at this site. This study pursues one small step in that process. The specific policy issue addressed in this study is, will use of and expenditure for ICF services change, given this ECB.

The research process includes identifying and evaluating differences in ICF use rates, and related expenditures, between two Risk contract Medicare beneficiary groups, one of which, S/HMO, has a trial benefit (ECB) covering long term care services not covered by Medicare, and the other, Risk, does not. ECB allows limited home care services as a substitute for nursing home care, as well as nursing home services not covered by Medicare, as well as additional pharmacy services. A complete outline of $\mathrm{s} / \mathrm{HMO}$ benefits is presented in Appendix $A$.

The conceptual model presumes that beneficiary and HMO staff make rational choices about uses of the Social/HMO Expanded Care Benefit so that out of pocket payments to nursing homes by the beneficiary are minimized, without denying nursing home services when reasonable alternatives to nursing home admission do not exists.

Medicare beneficiaries enrolled in the S/HMO pay to RP 
a fixed monthly amount adjusted annually by KP, in addition to other charges for Medicare Supplemental Insurance Benefits. The Health Care Financing Administration makes monthly capitated payments to KP for entitlement services covered under Medicare, Parts $\mathrm{A}$ and $\mathrm{B}$.

In this study, the measure of difference in ICF use rates and expenditures is between S/HMO and non-S/HMO Risk beneficiaries enrolled in the same HMO during the same time period, cared for by the same providers. Non-s/HMO use rates are the baseline since they are the result of rational decisions by persons without LTC insurance, beyond Medicare entitlements and supplemental benefits, to use out-of-pocket assets for ICF care. The policy issues is, do persons with ECB use more ICF services, or less ICF services, than those who do not have ECB?

This study does not create a statistical model suitable for generalizability of specific quantitative findings regarding effects of ECB on ICF services. Study outcomes are based on the empirical experience of one s/HMO site. However, trends and overall observations may be transferable to other s/HMO sites. Findings or/and recommendations provided may be adapted to fit operational and research conditions unique to other current and future s/HMO locations.

A graphic and narrative summary of the overall sHMO experience at the $\mathrm{KP}$ site is presented in Appendix A. All 
such information was prepared by the S/HMO research staff and not by this investigator. Permission to use same is granted by the Project director.

The Center for Health Research (CHR) provides an environment especially amenable to research on issues related to utilization of health care services. At least a dozen reason are cited for conducting research which explains utilization outcomes observed; several models for doing so are identified. "In general, none of these models adequately predicts differences in or explains a great deal of the variation in medical care utilization."[32] ICF services are no exception to this view; they are much studied yet little agreement exists on models in the literature explaining why people with similar health conditions and similar socio-economic attributes have such varied long-term care utilization experiences. One area where findings are consistent is that there are two basic use patterns in nursing homes; those which are termed short stay and those termed long stay. However, even here definitions are inconsistent. Some see short stay as under 90 days, while others see it as six months or even less than one year. Most agreement exists about any stay in excess of one year as a long-stay resident. All studies advise that is is necessary to recognize and even treat these as distinct groups of nursing home users. In this study that is accomplished by creating an overall data set and two subsets 
in which long-stay residents are removed, successively. This was described earlier in Chapter III.

Considerable literature exists regarding the S/HMO ECB provider-consumer model and certain outcomes of the overall and site specific Demonstration Project. Some of these are summarized in Appendix A, in a list prepared by the CHR staff.

However, almost no studies report on differences in ICF use rates between the capitated members enrolled in the ECB model for S/HMO and other "Risk" members enrolled under capitated contracts between HCFA and HMOs.

Capitation was allowed for HMOs by Congressional policy when it passed prospective payment legislation in the Tax Equity and Fiscal Responsibility Act (TEFRA) of 1982 . Prior to 1982, an operationalized model for the TEFRA legislation was implemented in 1980 by the CHR as a Medicare Demonstration Project known as Medicare Plus. That project terminated in December 1984. The research sequel was S/HMO, called Medicare Plus II (PIus II), which began in April 1985 and it included the ECB, which had not been operationalized anywhere in the nation, before then. Plus II has had two extensions, currently running until 1995.

Kaiser Permanent (KP), Northwest Region, contracted with HCFA to enroll new Medicare and convert existing Medicare members, including those from the 1980-84 Demonstration Project, into TEFRA defined capitated 
contracts beginning in April, 1985. These KP enrollees are identified as Risk or Medicare Plus members in this study. Some aspects of the ECB are discussed next because they are central to understanding the differences between Risk and $s / H M O$ eligibility status of Medicare beneficiaries in this study. S/HMO member ICF use rates are compared to Risk group member baseline ICF use rate and expenditure data, in order to determine if differences exist. The conceptual notion in this study is that the ECB can be expected to correlate with differences in ICF use rates, if there is a difference in such use rates. The research task is to determine if there are differences and if so, to establish patterns of differences between the baseline and trial groups.

One essential component to the S/HMO ECB is case coordination, or case management, of s/HMO members at high risk of needing LTC services covered by the ECB. Several methods were/are used to identify such members.

Upon enrollment in S/HMO, and annually thereafter, questionnaire data was obtained from each s/HMO member asking for a self assessment of their health status, dependency requirements, and other socio-economic and demographic information. ADL criteria were used to screen s/HMO members into levels of need for LTC services, similar to those adopted by the state of oregon for categorizing Medicaid applicant's social and health dependency status. No 
means tests are administered, under any circumstances, to s/HMO members. If the initial screen qualified them for LTC, a second evaluation was done using a Comprehensive Assessment Form (CAF) to establish an initial member care plan.

That is, if a s/HMO member met the LTC high risk criteria, they were assigned to a case coordinator who managed the ECB thereafter, using a computer based record of their needs, ECB services arranged, and measure of changes in status under the care plan goals. Non-CHR staff members did hospital, HHA and SNF utilization review regarding s/HMO member entitlement services, just as they did with other KP Risk members. Oversight was the responsibility of s/HMO staff for services not covered by Medicare Parts A, B or basic supplemental benefits. Coordination of ECB and entitlement benefits was a joint responsibility of CHR and other RP staff.

$s /$ Ho members who met the qualifying criteria for receiving LTC were offered a range of benefit options and the choice was negotiated by the case coordinator. A monthly expenditure cap was established, although it could be exceeded, with senior management approval. Not all nursing home eligible clients chose to receive services at the time offered because they had and preferred to use their informal support networks. [33]

In addition to the screening system just described, 
other KP staff participated in identification of $\mathrm{s} / \mathrm{HMO}$ members who qualified for ECB services. Physicians or other licensed care givers notified the case coordinator when thee admitted a S/HMO member to the hospital, or to the KP Home Health Agency, or an SNF, ICF or other care location which they facilitated, even if that member had not be identified as qualifying for ECB services. Upon such notice the case coordinator initiated the initial screen and if appropriate a CAF assessment and care plan was implemented.

There is considerable integration of skilled medical system services with paraprofessional or long-term care types of services: $37 \%$ of persons eligible for ECB services had some care charged to their Medicare benefit. Medicare accounts for about one-fifth (218) of reported costs. The level of Medicare involvement in nursing home service packages indicates that even the limited short-term nursing home benefit available under SHMO's expanded care contributes significantly to the ability to serve this population. A study of data from Kaiser Permanente sHMO site found that $58 \%$ of nursing home admission under the expanded care benefit were fore convalescence or respite and designed to keep patients in the community or prepare them for return to the community after hospital admission... The case manager coordinates and utilizes the informal care giving that is available [by integrating medical and formal care services]. When a patient has no informal supports, more formal services in the home may be needed... the last resort is nursing home placement. SHMO data indicates that only 128 or the total [s/HMO] membership had no informal support system...To date, sHmo experience indicates that function- ally disabled and medically complex geriatric patients can be maintained for long periods in their own homes, even when their informal support systems are not strong. [34] 
strong motivation existed for early identification of, and follow-up intervention by case management for, s/HMO members meeting $\mathrm{ECB}$ criteria, because HCFA reimburses the HMO at $100 \%$ of the institutional capitation rate for all s/HMO members determined to be in high risk status. Otherwise, capitated rates are $95 \%$ of the Average Adjusted Per Capita Cost for the county of residence of the members, under the TEFRA HMO contracts.

What is missing from these reports, regarding ICF care used, is whether use rates and expenditures were different for $s / H M O$ members than for other Medicare beneficiaries and especially those under capitated TEFRA HMO contracts. That is the contribution of this dissertation study.

What is not included, however, is whether Risk members paid more, the same or less out-of-pocket costs than S/HMO members, for formal home-based care giver services and whether the combined ICF (and AFC, RCF or convalescent facility) and in-home formal care expenses were different for Risk members than for $S / H M O$ members. This latter consideration is recommended as a future research project.

The next chapter presents conceptual and operational issues relevant to the debate about whether and how to provide long-term care benefits to the elderly population. 
CHAPTER II

POLICY ISSUES ADDRESSED BY THIS STUDY

\begin{abstract}
As we brace ourselves societally for increased demand for health care due to increases in the elderly population, it becomes more important, in fact critically important, to search for better ways to provide hospital care, physician care, and long-term care. [35]
\end{abstract}

There are several policy issues which give rise to the research questions asked in this study. The overarching policy concept addressed by this study is, to what extent will increasing requirements for chronic care be met by private insurance, or joint public and private insurance programs, which expand Medicare linked supplemental benefits to include long-term services?

In recent years a variety of solutions to this policy problem have been proposed, such as that set forth by the 1990 Pepper Commission. But, uncertainty remains about many aspects of such solutions, such as whether or how to place the burden of initial costs for institutional long-term care (LTC) on the private domain or on public financing programs, and how should the burden of costs be shared for those unfortunate few who become very long-stay long term care nursing home residents.

Policy solution options include expanding entitlement 
benefits, or creating public programs which encourage intended to motivate and assist informal care givers with care-giver tasks necessary to maintain a chronically ill, severely dependent, frail elderly person at home. The complexities of policy decisions regarding this policy problem are extant.

Uncertainty about interaction between policy decisions regarding formal, and especially institutional long-term care and policy decisions regarding informal home-based care, is central to the debate about solving the overall policy problem of very limited entitlements for America's elderly regarding the continuum of services needed by chronically $i l l$ and severely disabled dependent persons, most of whom are at the end of their life cycle.

\begin{abstract}
The growing public support for a wide variety of alternatives to institutionalization indicates that the initial policy issue is not whether home health services are less costly, but how these services should be organized and financed for maximum efficiency and effectiveness. Whether a shift in medical care utilization patterns can be accomplished, thus reducing need for nursing home beds, remains to be seen. [36]
\end{abstract}

Several trial programs, such as the Channeling Demonstration Project and on Lok, have been conducted which experiment with a variety of options to determine whether community-based programs defer or prevent institutionalizing chronically ill and dependent elderly. Some have assessed the apparent cost effectiveness of case management to 
coordinate community services with other levels of care. Most have resulted in better care of elderly patients and better understanding of how to provide these services, but have not been encouraging from a cost effectiveness standpoint. [37]

One demonstration project, known as the Social Health Maintenance organization, or Social/HMO (S/HMO) has enlarged on the knowledge base from other demonstration projects and applied managed-care procedures to long-term care, which were developed for integrating acute care, home health agency, SNF and ambulatory services. Thus, integrating chronic care services into existing managed care practices for members enrolled under capitation contracts with HCFA, and other purchasers of HMO benefits, has brought something to the policy solution which others did not.

Specifically, the S/HMO is able to interact with all components of a comprehensive health care system to arrange services, covered by the member's benefits, in a cost efficient way while adapting those services to the member's needs. S/HMO addresses the overarching policy question by expanding existing capitated HMO benefits to encompass various forms of home and community-based services, allowing for short-stay (100 days) nursing home care, beyond Medicare SNF entitlements, including Intermediate Care Facility (ICF) stays. Long-term stay nursing home care is not covered. The S/HMO Expanded Care Benefit (ECB) is case managed 
and includes an on-going process of actively identifying frail elderly, from among the s/HMO membership, who may qualify for nursing home care, and therefore the ECB, as determined by standard, comprehensive assessments of their dependency based on dysfunction in activities of daily living. Members who qualify for nursing home care are further assessed for family or other network capacity to support the elderly member's needs and a plan is negotiated to arrange for supplementary services required, if any for home based maintenance and health services. [38]

Is the s/HMO Demonstration able to produce outcomes which differ from other projects? Most projects have led to increased use of formal services for community-based care without significantly decreasing institutional care, and at a significantly greater cost. Their use of a LTC benefit may have differed, somewhat from the way s/HMO conceptualized the use of an ECB.

Specifically, the targeted use of the S/HMO ECB is to help functionally impaired members remain in a home environment, who otherwise might not succeed at that without the availability of formal care givers to provide communitybased personal and domestic services essential to remaining at home, in addition to skilled provider care. Such services do not have to be tied to an episode of illness to qualify for them. of course, many many persons who qualify for the ECB also required hospital and other entitlement covered 
benefits. Decisions are made by case managers regarding allocation of Expanded Care Benefits between community-based services rendered in the home care and non-Medicare covered institutional chronic care services.

The [S/HMO] goal was to stimulate members to utilize their existing informal care network so that they could remain in their own homes and avoid nursing home placements as long as possible...this benefit was designed to serve only the more severely impaired portion of the population... Since this benefit is renewable as long as the patient continues to be eligible for services and remains at home... the combined annual maximum is $\$ 12,000$ [less $10 \%$ copayment] and the SNF/ICF coverage is not renewable in a calendar year unless the member has been out of an institution for 60 days... The community-based services often support the member's primary caregiver and provide needed respite... In this way, an important goal of supporting the informal care system rather than replacing it with formal services is achieved. (Op. Cit. [38], p. 12)

The applied research question, targeted by this dissertation study, asks whether s/HMO members who qualified for the ECB used more or less ICF services than might have been expected had they not had an ECB? It also acknowledged that there may not be any difference.

If the research outcome was that fewer ICF services were used by $s / H M O$ than Risk members, it could be inferred that S/HMO ECB was a force leading to diminished use of ICF services due, at least in part because formal, communitybased care deferred or prevented the need for ICF Care. If the research outcome was that more ICF services were used by $s / H m o$ than Risk members, it could be inferred that 
S/HMO ECB was a force contributing to increased use of ICF services while expanding home-based care options.

If no meaningful differences were observed in ICF use than it could be inferred that little interaction existed between community-based care programs and ICF care requirements.

Any of these findings would also serve as directional information for use in hypothesis-based tests needed for further confirmation of findings from descriptive research which this study is intended to accomplish.

Numerous applied research questions follow from this operational concept of integrating a chronic care benefit with other HMO services. Those of interest in this study were published in 1988 (Op. Cit. [36], pp. 62-63) as well as in more recent literature by referenced authors. They are rephrased into the following policy issues. If the ECB goal of stimulating members to use existing informal care-giver networks was attained, did it also reduce use of ICF services? If the goal of supporting informal care givers through ICF respite care was effective, did that affect ICF rates? If the goal to defer long-term nursing home stays was met, did it reduce expenditures for nursing home care? If increase use of community-based care was stimulated by ECB, did it reduce the likelihood of becoming a welfare dependent nursing home resident? Was it likely that a residual effect of the S/HMO ECB was to reduce Medicaid costs for S/HMO 
members?

Although data regarding ICF care was reported so long as it was covered by the ECB, such data was not maintained after a member consumed their ECB. Conceptually, there were dichotomous outcomes for ICF rates and little agreement existed among $\mathbf{S} /$ HMO staff about which outcome was most likely. If the incentives of the ECB were effective it could eliminate those enabled to remain at home, thus leaving those with no option as long-term permanent nursing home residents. That could push days-used rates higher. If the benefit worked ideally, few members would use ICFs other than as a respite for informal care givers.

The role of ICF services under the S/HMO chronic care benefit concept was not clear. This study provides some descriptive information intended to help clarify that role.

This study addresses a few policy issues raised in the literature about the likelihood that the S/HMO Expanded Care Benefit influences member use of ICFs in a way that would not occur in the absence of an ECB.

One of these issues is related to the S/HMO policy objective to keep disabled and dependent elderly at home. Another is related to possible effects of $\mathrm{s} / \mathrm{HMO}$ policy on short-stay and long-stay or permanent placements in ICFs. "It can be posited that one reason for including ICF coverage within a community-based services program is to provide an additional resource to help people stay in their 
own home." [39] Information was presented at that time

(1988) which supported that notion.

[However]...the proportion of expanded care benefit costs consumed by ICF care is not insignificant, even though most of the members served are served in their own homes... The observed pattern of use of institutional LTC contrasts sharply with the patterns observed from national data regarding individuals not in SHMOs. The pattern displays more frequent short-stay admissions to nursing homes, and probably less frequent permanent placements... About half of the institutional admissions were discharged to their homes and another 47 died while in the institution. Only 66 of the discharges were assessed as resulting in relatively permanent placement placement. What remains to be analyzed is the relative cost of care in those different groups: (op. Cit. [39], p. 20)

No data was available about non-s/HMO captitated Risk HMO member patterns of ICF use, against which to compare s/HMO patterns, reported above. This research issue is addressed by this dissertation study.

Although different admission patterns of long and short stay patients were reported, neither cumulative days of stay nor costs were reported. This study describes days of stay differences including proportions of long- and short-stay, and puts these observations into relative context by comparing s/HMO rates with a meaningful baseline (Risk), which until now has not been done. This pattern of long and short stay is reported elsewhere in the literature.

Nursing homes in the United states and Australia both served two different groups of persons and 
each group had a distinctive utilization pattern. The larger group consisted of persons who enter and leave within a short time (70\% within one year) using a smaller proportion of nursing home days. The other group was made up of persons who remained for an extended period (until they died or were near death) and used a larger proportion of nursing home days...short-stayers constituted $58 \%$ of nursing home admissions... long stayers constituted 428 ... the average length of stay for short stayers was 1.8 months, and they were generally discharged within a year. On the other hand, the average length of stay for long-stayers was 2.5 years. [The above stay patterns are for SNF and ICF residents, however] ...98\% of Medicare covered [SNF] persons left within one month... The two main characteristics of long-stay nursing home residents were mental deterioration and Medicaid coverage. The long-stay residents probably remained in a nursing home because they could no longer sustain themselves in the community. The Medicaid coverage could have been the reason for or the consequence of their long stay. [40]

This issue has important implications for policy formulation regarding long-term care benefits. Terms of debate about the most efficacious but affordable solution to spend-down, and reduction of Medicaid costs, center around this phenomenon of short-stay and long-stay nursing home patterns. The central policy notion is that the private domain cannot easily insure against long term stay costs, but who should insure against front-end costs, and for what objectives is at issue. If traditional insurance methods are used it obviates the role of government in holding down escalating prices covered by first-dollar and co-pay money. "In fact, in the absence of strong regulations, the incentives in a front-end benefit private market would 
encourage rather superficial home-care benefits and the diversion of expensive expensive home-care beneficiaries to nursing homes."[41]

Cost comparisons between Risk and S/HMO are limited to those for nursing home revenues. Data, needed to include comparison of community-based care for both long-and shortstay groups, was not obtained, nor was such data for Risk members. The original intention of this study was to partially fulfill that objective by comparing community-based care cost differences for Risk and s/HMO who became recipients of Medicaid funds following ICF stay, in addition to ICF and SNF care costs.

However, the policy issue regarding differences in Medicaid expenditures is partially addressed. That issue was raised but not answered in prior literature on S/HMO, at least in a relative sense, where $s / H M O$ is compared to a baseline of community experience. In this case, that baseline is the Risk group of KP members studied.

Presumably, capitated Risk members would provide the ideal baseline against which to measure $s /$ HMO rates if ICF use, since Risk members had nearly all benefits of $\mathrm{s} / \mathrm{HMO}$ members except ECB, and some enhanced drug benefits. To answer the above questions, use rates/expenditures for S/HMO members are compared with those for Risk HMO members. Rates are also used to answer research questions raised about whether the S/HMO concept offers new hope for ameliorating 
prospects of spend-down and dependency on Medicaid as the inale of one's lifetime experiences. 
CHAPTER III

RESEARCH DESIGN AND METHODOLOGY

\begin{abstract}
Designing a randomized experiment should never preclude the simultaneous design of fallback quasi-experiments which will use the same data base as the randomized experiment. Measures should be collected that will improve our inevitably partial understanding of any selection process which results because the random assignment has broken down. [42]
\end{abstract}

The research methodology experience of this study is, in many ways, as important as observations produced and conclusions drawn. This is so, because such large scale research is complex, opportunities for making costly errors abound, and project management concepts which have been tested and debugged may contribute as much to successes of future studies as use of statistical procedures which correctly infer findings.

Project management problems were encountered from start to finish in this project; the way in which these problems were addressed contributes to development of planning recommendations for future research projects which could build on data used for this study.

Lack of large project research experience by this investigator resulted in less than full understanding of these research methodology and project management problems. 
SETTING LIMITS ON THE RESEARCH PLAN FOR THIS STUDY

This study collects baseline data on nursing home use rates and patient revenues which are used to produce descriptive information needed for performing exploratory analysis of differences between Risk and s/HMO Medicare member use of ICF services, during the study period. Observed differences, if any, and related analysis are used to formulate tentative policy recommendations about advancing the S/HMO-ECB concept as an effective and widely affordable method for protecting elderly persons against asset depletion caused by front-end expenses for formal ITC services. Such recommendations require further, confirmatory research as justification for adopting legislation and/or committing private organization resources which embrace s/HMO as part of a new national policy on ITC. This is a descriptive study limited to hypotheses generating findings. Hypothesis testing with inferential statistics could result in more meaningful if not generalizable statements about possible causal relationships between ECBs and ICF use rates/expenditure rates. But, baseline data about Risk member use of ICF was unavailable for comparison with S/HMO member use of ICFs.

s/HMO nursing home data is juxtaposed with Risk member nursing home data because the latter represents how capitated HMO members resolve LTC needs in the absence of LTC insurance or an ECB, beyond what entitlements and 
Medicare Supplemental benefits provide. This points out two assumptions in this study. First, it is presumed that s/HMO member overall use of ICFs would be similar to that of Risk, if they were enrolled as Risk members. That presumes they come from the same population as Risk members, relative to descriptive parameters which might affect ICF use. Second, this study presumes that if $\mathrm{S} / \mathrm{HMO}$ member use of ICFs is substantially different from that of Risk, then having an ECB and associated managed care processes must influence member's decisions sufficiently to alter how ICF services are used.

These assumptions, in the context of policy issues discussed in Chapters I and II lead to a series of research questions, presented next. They also underscore the underlying reason why this study is exploratory in nature: there is no basis on which to hypothesize that s/HMO use rates are different from those of Risk members because there was no baseline data available for the latter. The research plan for this study did not assume that s/HMO rates were different than those for Risk. Such assumptions would have been required for hypothesis testing procedures. As two biostatisticians at Stanford University have recommended:

Researchers should be encouraged to realize that one does not go to trial until considerable preliminary evidence is in hand, much of it is quantitative in nature. Extensive exploratory data analysis and meta-analysis on related issues prior to going to trial are essential to plan effective strategy and to define a critical 
effect size. Statistical hypothesis testing is often premature, done at a stage when costeffective planning is not possible. [43]

Thus, production of such baseline data for use in developing such models is an important research task of this study. This chapter reviews the research methodology used to carry out the tasks of research in this study. The flow of this lengthy discussion is organized into steps (one though nine), which are underlined to denote successive stages in the research plan use to accomplish this study. An original objective of this descriptive study was to identify ways to organize and use the extensive information needed for this kind of research project.

The first step in the research plan of this study was to clarify the conceptual framework of policy issues, and the policy problem addressed by S/HMO, from which research questions in this study are derived.

The second step in the research plan was to establish exactly what research questions needed to be answered in order to expand on knowledge about $\mathrm{S} / \mathrm{HMO}$ as a suitable policy solution for the problem(s) identified. One established, those questions would guide development of the research plan and ensuing research methodology.

Six research questions were selected from policy issues discussed in Chapters I and II They are:

Research Question I: Are there differences between Cost, Risk, and s/HMO eligibility groups regarding the number of 
ICF residents per 1000 research population members during the study period?

Research Question II: Are there differences between S/HMO and Risk member ICF days in residence during the study period per member year of eligibility?

Research Question III: Are there differences between S/HMO and Risk members regarding the means of total payments received by all nursing homes in which each resident lived for all periods of stay before, during, and after the study period through June, 1989, per 1000 study period members?

Research Question IV: Are There Differences Between S/HMO and Risk Research Population Members Regarding the Probability of Receiving Medicaid Funds To Pay Nursing Home Bills?

Research Question V: Are There Differences In the Proportion of S/HMO and Risk Members Who Were ICF Residents During the Study Period, Who Also Were Medicaid Recipients within one Year Following The study Period?

Research Question VI: Are there differences in Medicaid payments received by nursing homes for members residing in ICFs during the study period per 1000 research population members?

In respective Research Questions I-III, the dependent variables are: number of ICF residents in the study period; number of ICF days in the study period; total payments (dollars) received by nursing homes before, during and after the study period, through June, 1988 for members in ICFs during the study period.

Step three of the Research Plan required determination of the study period because that would identify the research population data for whom historic data would be needed. The study period of nursing home utilization selected was July 1, 1986 through June 30, 1988. Medicare beneficiaries enrolled during one or more days of that time span, and who 
met other criteria defined below, constitute the universe for this study, hereafter called the research population. The decision to choosing a twenty-four month study period was made upon reviewing several organizational and operational issues discussed next. Organizational issues involved policy changes affecting membership size and use of the Expanded Care Benefit for ICF services.

If the study period started too soon after March, 1985, then neither Risk nor S/HMO members would have had time to develop patterns of nursing home use which might be influenced by their HMO eligibility status. Start-up began after March, 1985, for both TEFRA Medicare capitation Risk contracts and s/HMO Medicare Demonstration Project. By July, 1986, results of initial marketing efforts for new s/HMO members was mostly completed, as was initial conversion of Cost members to Risk or $\mathrm{S} / \mathrm{HMO}$ status. The research methodology problem, posed by this unstable period of eligibility status, was whether the high proportion of cost conversions to Risk status in some way biased use rates. A method of analysis was developed to consider that matter, discussed later.

One organizational decision affecting selection of the study period was that after July, 1988, s/HMO marketing was expanded beyond Multnomah County to include Washington and Clackamas Counties. This would have increased the number of potential nursing homes from which data must be gathered. It 
would have increased the size of the research population substantially without an immediate corresponding increase in S/HMO members use of ICFs, thus producing a confounding relationship between rate numerator and denominator.

Another organizational issue related to policy changes regarding use of chronic care benefit of 100 days of coverage beyond the Medicare Part $A$ and Supplemental Benefit Plan) for ICF and SNF care, which could influence use rates for s/HMO members. Beginning in July, 1988, a succession of limits were implemented by s/HMO, regarding the extent to which the S/HMO Expanded Care Benefit could be used for payment of nursing home expenses. These were needed to emphasize the $\mathrm{s} / \mathrm{HMO}$ objective regarding use of ECB funds, which was for home and community based LTC services principally and to avoid, if possible, reliance on ICF services to compensate for a member's 1088 of capacity to function independently. In January of 1989, use of ECB for SNF and ICF services was substantially restricted, compared to the uses of ECB during the study period. These issues led to selection of July 1, 1988 for a study period ending date. Such policy changes also reflected an important dimension of $\mathrm{KP}^{\prime} \mathrm{s}$ organizational objective for the S/HMO program.

Medicare Plus II made a conscious decision to adhere to strict eligibility criteria [regarding nursing home certification (NHC)], a decision that was guided by the demonstration site's principal focus, learning, to underwrite a long term care benefit... [Also] During the first two years of the demonstration, there was a loophole 
in Oregon's NHC criteria which qualified a person as NHC if he/she was incontinent, but was otherwise functionally independent and healthy. In January 1987, at HCFA's request, the NHC criteria were revised to be consistent with the state's (Oregon) new interpretation of the incontinence criterion. [44]

An operational issue influenced when to commence the study period. Some time was needed for the case manager process to become an established and effective component of the S/HMO program. At the same time, KP expanded its use of geriatric nurse practitioners to make nursing home site visits for level-of-care recertification on all members. By mid-1986, they performing routine patient assessment and updating orders for all ICF patient care in Multnomah County, sometimes on a monthly basis, or quarterly.

Although Risk patients were not case managed, the nurse practitioner program assured that both Risk and s/HMO members in ICFs and SNFs in Multnomah county were closely monitored for appropriateness of utilization. This suggests that utilization review differences could be ruled out as a likely cause of differences in use rates between Risk and S/HMO, if differences were found to exist.

Given the above considerations, the two year study period selected seemed to offer the best opportunity to measure whether differences occurred in the use of ICF services between $\mathrm{S} / \mathrm{HMO}$ and Risk members, because S/HMO members had a relatively high freedom of choice to select nursing homes as a location for satisfying their LTC service 
needs, during that time. Once the research population was defined, then sampling estimates could be undertaken. Thus, the fourth step in the research plan involved selection of residents needed to answer Research Questions I and II. The research proposal presumed that random, or stratified random sampling would be used to carry out that process. Estimating sample size required knowing or having a basis for estimating variation of the parameter values. Variance in days in nursing home, or mean lengths of stay, was not known for the Risk Group.

The adequacy of existing information to properly estimate variability in mean LOS for Risk members in ICFs, and therefore sample size, was in doubt. Data regarding national studies of nursing home use, which were published by 1989 , when this study was operationalized, did not seem to fit state of Oregon experience. State of oregon nursing home survey data was not based on information needed to establish reliable LOS parameters. Therefore, a combination of estimates were developed using models which were being produce and published for the first in 1988. A brief summary of method used and results is presented. A comprehensive discussion in available in Appendix B.

A sample size was estimated from information extracted from the literature. But, this involved much uncertainty about what variability in days of stay should be used to estimate sample size. Such variability also would determine 
the amount of difference between the mean LOS for the two groups that needed to be identified in order to determine that their difference was significant.

Based on national data, mean days of nursing home stay ranged from 1.5 to 2.5 years depending on the source and location. Considerable information is now available on the effects of relatively small numbers of long stay residents on the mean of shorter stay patients which, by far, comprise the largest portion of ICF residents. It was not available in 1989. Of course the maximum variability allowed was 730 days, due to censoring caused by the study period. In fact, a small proportion of ICF residents used close to 730 days. Assistance was obtained from Center for Health Research biostatisticians, in making some of the computations needed for sample size estimates but the variability used in that process was based on this investigators interpretation of the literature.

Computations about variability were performed based on the Ravlin and Weiner model (1988), shown in the Appendix B. These computations were done by age cohorts. The computed variability in days of stay in a nursing home was used to estimate sample size required in each age cohort cell for Risk (Medicare Plus) residents estimated to be in ICFs.

These estimates are for Research Question II.

At .90 power to detect a difference of 180 days between Mean LOS, and an alpha level of .05, for age cohort 65-74, the sample size estimate for Risk residents was 313; 
At .80 power to detect a difference of 180 days between Mean LOS, and an alpha level of .05, for age cohort 65-74, the sample size estimate for Risk residents was 176;

At .80 power to detect a difference of 180 days between Mean LOS, and an alpha level of .10, for age cohort 65-74, the sample size estimate for Risk residents was 138 ;

The above sample size estimates were done by age cohort, as indicated. When sample sized for age cohort 65-74 were compared to the estimated number of KP Risk Medicare ICF residents in Multnomah County, (see Appendix B). It was apparent that insufficient nursing home residents were available from which to select a random sample of the size needed. Even if taken collectively for the three years, $\{75+65+72-212\}$, it appeared that at .80 power and an alpha level of .10 that a 65 percent sample of residents was needed; if an alpha of .05 was used, an 83 percent sample was needed. At .90 power and an alpha of .05 , only $2 / 3$ the estimated sample residents needed were available.

One overall estimate indicated that 2,864 sample ICF residents was needed. Other overall sample estimates were done based on being able to detect a difference of 20 days between group mean LOS. For an asymmetrical distribution, a sample size of 3300 was required. For a normal distribution, a sample size of 2000 was needed; normality could not be assumed in this study. Thus, 3300 nursing home residents was even larger than the high estimate done using the Ravlin and Weiner based estimate model (Appendix B, high $=2438$, low 1625). It was so much larger than Appendix B that the notion 
of doing a study based on random sample design was abandoned. The decision to forego random sampling was solely that of this investigator, and not others at CHR.

Therefore, the research methodology in this study is not based on inferential statistics. Some $F$ tests are used to suggest where differences in means is statistically significant, in Chapters VII and VIII but the hypothetical population suggested by doing such tests is simply conceptualized as KP capitated Medicare members, generally. As it turned out, there were 395 Risk Medicare ICF residents in the overall data set. That was about what was required for the total Risk sample at .80 power at an alpha level of .10. There were 820 unique persons in ICFs during the study period. That was about one-half of the low Ravlin and Weiner estimate. The total SNF+ICF unique persons was 1160 , or about $2 / 3$ of the low $R \& W$ estimate. It was close to the estimate in Appendix B. It is worth noting that benchmark studies in this area by Liu and Manton, used samples of over 6500 nursing home residents.

In retrospect, the variance selected for use in estimating sample size, and factor (difference in days in an ICF between groups) of detectability between means ( 6 months) were both inappropriate. While it is true that potential variance was the maximum of the study period days (730), the problem would have been resolved by focusing on the shorter-stay ICF residents. This is recommended for 
future studies.

The research methodology problem is that to detect the small amount of difference in days used, between Risk and S/HMO, requires a large sample. Using only shorter stay members, for example only those with one year or fewer days, would facilitate answers to Research questions I, II and III. That is, since the S/HMO ECB benefit cannot cover a protracted time period, only those with one year of stay or less, could be done. This presumes having Los data in the data base used for sample selection. Persons selected in the random sample whose days of stay exceeded one year could be set aside and replacing by accepted replacement sampling techniques. This may require drawing a number larger than the sample estimate. Appendix $B$ includes estimates of ICF stays by proportional rates derived from a lifetime use formula published by Meiners and Trapnell, [45] and developed further by Rivlen and wiener. [46]

In reality, another sample size issue existed for the three Research Questions, IV-VI, in that the number of Risk members likely to spend-down to Medicaid status was not documented, and information about $\mathrm{s} / \mathrm{HMO}$ member expenditures known to spend down was unknown.

Here, variability of the response variable, time to spend- down, was noted in the literature as being one year or less. But, that included SNF care as well, and a sizable number of ICF residents in this study did not reside in 
SNFs. The measure of variability for days to spend-down was confused by a longer time span than the study period including dates beginning with the advent of $\mathrm{s} / \mathrm{HMO}$ and Risk (4/85) through June, 1989, when data was collected.

This study increased the level of appreciation about advice by one author on estimating variability for sampling in support of inferential testing:

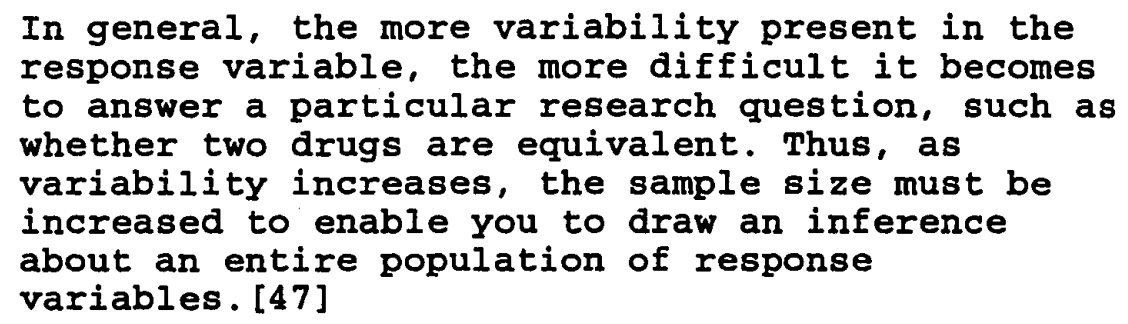

Since data collection, based on random sample estimates, was abandoned, a decision was made to pursue the study on the basis of obtaining and evaluating descriptively population data and true nursing home data for that research population. Thus, all utilization data was collected on all KP members residing in all SNF or ICF institutions in Multnomah County during the study period, provided they were age 65 or more by July, 1988 .

Later, an indirect test was made to determine the likelihood that all KP residents of Multnomah County, identified by zip code, who were in SNF or ICF locations during the study period, where in these 48 nursing homes. This was done by running data from the current RP "RARE" data base files on nursing home residents, implemented in 
1990 and loaded with all data by the end of that year, to determine how many of these residents were in nursing homes outside of Multnomah County. Approximately five percent were located in facilities in Washington, Clackamas or Clark counties. There were no operational changes to suggest that more than 5 percent of the residents identified in this dissertation study were located outside of the 48 facilities from which data was obtained in Multnomah County.

other studies, performed by experienced investigators, have found major research methodology problems when working with nursing home sample data. Such reports are recently published, as discussed next.

The 1985 National Nursing Home Survey data was flawed seriously by sampling problems. That study was done by the National Center For Health Statistics, but not published until March, 1990. In 1992 corrections were published because threats to the validity of sampling estimates confounded the published values. This was caused by survey questionaire misuse by nursing homes. Also, sampling problems were related to sample design which failed to relate correctly to the stated research objectives. Sample estimates of days used in the 1985 NNHS were erroneous.

Information was collected on discharged events
rather than discharged residents. [In addition,
This was compounded by repeated selection of
sampled residents due to failure to account for
multiple facility admissions and due to]...
variation across facilities as to how stays are 
defined. [The NCHS warns about nursing home survey samples as follows:] Construction of length of stay estimates is complicated in surveys such as the NNHS, which use facility based definitions of a nursing home stay as the sampling frame for current and discharged residents. It is necessary to consider a resident's entire pattern of nursing home usage, including multiple nursing home stays and intervening hospital utilization, in calculating length of stay. [48]

This NCHS warning surfaced problems similar to those identified during step five in the research plan, which was the data collection phase. After making the decision to collect data on all possible KP Medicare members residing in all SNF and ICFs in Multnomah County during the study period, work was began in improving the lists of names on hand from records used to reimburse KP physicians for such nursing home visits.

Three years of monthly lists were key punched and converted to a summary of unique members at each facility. Monthly lists were valuable because it demonstrated to those nursing home administrators who opted to have their staffs provide requested data, that the task was limited. That is, not all records had to be reviewed. This assumption was in error. As valuable as they were, it was discovered that as much as 10 percent of listings either listed person who were not KP members during the study period, or failed to list such persons. Some physicians made visits without claiming reimbursement.

over 75 percent of the data was collected personally by 
this investigator during initial site visits. Among those facilities preferring to assemble the data, it was necessary to review and redo much or their work, including a review of all records for all residents during the two year study period. The greatest problem with facility staff work involved definitional differences between facilities about discharges vs transfers to the hospital and back. Also, discharges between levels of care was often complicated by intervening hospital stays, and failure to correctly record discharges. The data collection forms and instructions were tested at two nursing homes in advance of use.

Great attention to validation of dates, in this study, assured high reliability of data concerning cumulative days in residence by level of care for all nursing homes used by each resident. Some residents were readmitted up to 10 times during the 24 months.

Another problem encountered which future research may consider is the complexity of finding historic business records of nursing homes. Frequent change of ownership is a problem commonly understood to exist among proprietary nursing homes. Owners are not required by law to leave business documents, other than registers of admission and discharge, after transfer of ownership. The law does require that medical records be left at a facility. As a result, it was necessary to track down several prior owners and obtain permission to go to off-site storage locations where such 
documents were kept, presumably for audit and tax purposes.

Another complexity of procuring business records relates to corporate ownership for non-profit and for-profit organizations. Some facilities do not store historic documents on-site but rely on, or are required by, central business offices to receive input, process and return it, as needed, and store it. Thus, in some cases, it was necessary for the facility to retrieve computerized historic records. In several instances, that cost either the facility or the investigator a not-so-nominal fee.

In those cases where business records could not be obtained for all dates of residence, generally for long stay residents, it was necessary to compute the amount of funds received by the nursing home. This was accomplished by use of files to which access was granted in 1991 by the Medicaid Audit Department, Senior and Disabled Services Division, State of oregon, for research purposes only. They contain data regarding operating costs by year as well as Medicaid reimbursement rates allowed by year. Days of stay were multiplied by operating cost and Medicaid rates. Such documents are filed at CHR where they can be treated by the confidentiality standards which apply to research involving human subjects research.

Documentation of admission and discharge dates was not always clear in business records of some nursing homes. It was necessary to obtain facility permission to extract such 
data from patient care records. In some cases that was done by facility personnel where confidentiality issues were a matter of concern.

Age eligible Medicare status need not have preceded the member's admission to a nursing home, since days of cost, Risk or S/HMO eligibility commenced with Medicare status, which allowed censoring of nursing home days which preceded or succeeded initial and terminal eligibility dates. Since nursing home days were censored by study period dates, days of stay for long term residents admitted before age 65 , and before the study period, were excluded from the analysis rates. However, such data was collected, because it was needed for identifying financial records.

If a suspected member resided in a nursing home during the study period, data was collected from the beginning of their first admission to the facility through the period of June, 1989. Such data was used for post-study period analysis of spend-down and Medicaid eligibility and expenditures ends with that June, 1989.

Some other data collection issues are listed. Admission and discharge dates were entered as identified by nursing home records. A problem requiring hundreds of data entry corrections resulted from both interfacility and intrafacility transfers in which the discharge data and readmission date were the same. This prevented separation of time periods by programming language subtraction. Systematic 
modifications were made to either discharge date or readmission date.

The preceding aspects of data collection took considerably longer than projected in the original research plan. It is essential to allow adequate time for data collection involving such complexities. All 48 facilities were prepared for this study by preliminary letters from nursing home associations, the Center for Health Research, and Portland state University. Data collection packets were provided and formally arranged meetings were conducted with facility management.

Much negotiations was required since facilities do not generally open records to investigation except when required to do so by law. Access was generally, good, and resistance was readily overcome when facility management learned that this investigator was concurrently completing a six month traineeship as a nursing home administrator, approved by the oregon Board of Examiners for Nursing Home Administrator licensing. In instances where resistance was encountered, some contacts were made by KP visiting nurse practitioners familiar to those facilities. Their presence made data collection easier.

on site data collection from a large number of LTC institutions under separate ownership or control involves considerable research time, expense, experience, knowledge and collaborative support. 
Survey collection forms were tested at two facilities in advance of June, 1989, though to be representative of the 48 sites. The first mistake this choice involved was to select one out-of-state site. Record keeping is conditioned by state Medicaid auditing, a fact not fully appreciated at the time. The second mistake was that two facilities were selected because they were known to have good record keeping practices from prior experience. The recommendation derived from this experiences is that test sites include facilities with the least developed business practices. Record keeping practices at some facilities were inadequate, including some under "chain" ownership.

Even though many issues arose in the data collection phase of this study, the reliability of data collected is good. It was not necessary to drop any residents from the data set because minimum data was lacking or because it was unacceptably incomplete.

Identification of KP members was assisted by a preliminary list extracted from 48 monthly lists of nursing home visits by $\mathrm{KP}$ recertification staff. In addition to looking for these persons, it was possible at most sites to scan admission and discharge registries for all persons admitted, including other KP names. Such registries usually include the location from which residents came or went, which helped with that identity.

The resulting data set of nursing home residents is 
summarized in Tables IV, V, VI, below. It is the residual of 1421 nursing home residents identified during data collection whose data collection forms were key punched into a VMS support system and down-loaded to $\$ 1032$ for initial testing. Another 200 names were discarded because of eligibility status, age, or zip code questions.

The fifth step in the research plan involves the complicated task of clarifying the research population. This activity was started at the same time as estimation of sample size but delayed when random sampling was abandoned as the basis for data collection. The final research population comprise members with Multnomah County zip codes who were age eligible Medicare beneficiaries during the study period. Model A represents eligibility data for three groups among which members moved during the study period, making these groups not mutually exclusive. The above summary of research population eligibility groups is presented because Models $A$ and $B$ are used in analysis of data are throughout the rest of this study. Model $B$ views eligibly grouping in a different way, by identifying each combination (seven) of the three eligibility groups in Model A. Model B is shown in Table VI. Days of eligibility are identified precisely for each of the 19,261 subjects. Note: Model $A$ and Model $B$ refer to methods of organizing the research population into separate datasets for purposes of analyzing the data. 
TABLE IV

NURSING HOME RESIDENTS IN SNF AND/OR ICF

FACILITIES ON AT LEAST ONE DAY

OF THE STUDY PERIOD

IN THE OVERALL

DATA SET

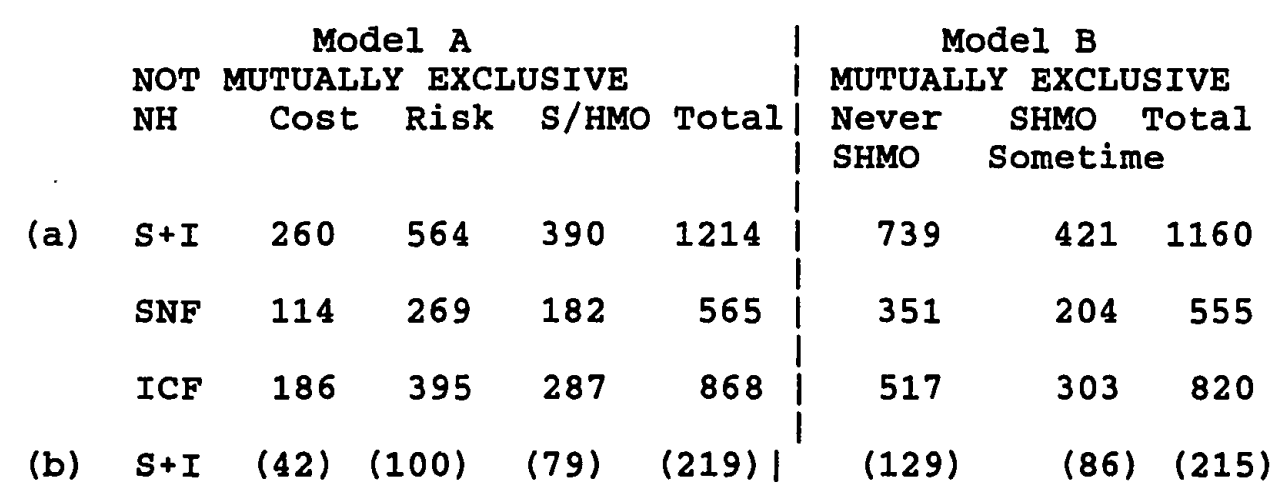

Research Population by eligibility groups above col.:

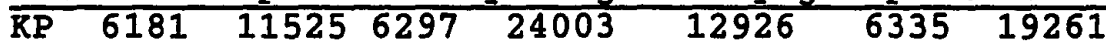

(a) $\mathrm{S}+\mathrm{I}$ is the unique count of residents

(b) ( ) is the number in both SNF and ICF

TABLE V

NOT MUTUALLY EXCLUSIVE COUNT BY RESEARCH POPULATION MEMBER ELIGIBILITY GROUPS

Cost members: 6,181

Risk members: 11,525

SHMO members: 6,297

24, 003 Model A

Unique count of members: 19,261 Model $B$

or more groups: 
TABLE VI

THE SEVEN COMBINATIONS OF MODEL B CLUSTERED BY MEMBERS WHO WERE WERE "NEVER S/HMO" AND "S/HMO SOMETIME"

$\begin{array}{lrr}\text { Cost only } & 2321 & \text { (7 Eligibility Groups } \\ \text { Cost+Risk } & 4608 & \text { of Model B, Format B) } \\ \text { Risk Only } & 5997 & \\ \text { S/HMO Only } & 2510 & \\ \text { Cost+S/HMO } & 2517 \\ \text { Risk+S/HMO } & 874 \\ \text { Cost+Risk+S/HMO } & \frac{434}{} \\ \text { (C, C+R, R) } & 19,261 \\ \text { (S, C+S, R+S, C+R+S) } & 12,926 \text { (2 Clusters of Model B, } \\ & \frac{6,335}{19,261} \text { Format A) }\end{array}$

Duration of eligibility in each status is important in this study since it represents the time of exposure during which nursing home admission may occur while enrolled in Cost or Risk of $\mathrm{S} / \mathrm{HMO}$.

Among Risk members, 398 of the 11,525 enrollees were eligible throughout the study Period (730 days), while 798 had 365 or more Risk enrollment days. Among s/HMO members, $55 \%$ of the 6,297 sHmo enrollees were eligible throughout the study Period, while $77 \%$ had 365 or more sHMo enrollment days. Cost days were affected by the involuntary conversion of Cost to Risk among Medicare members during the period $4 / 85$ through $12 / 86$. The result of conversion diminished cost enrollment to less than $20 \%$ of its December, 1984 count. Thus, 248 of the 6,181 Cost enrollees were eligible throughout the study Period while 36.68 had $365+$ days. 
Many problems regarding use of the above data needed resolution before using it in rate numerators. These are discussed because future research of this nature will encounter similar problems, unless they use the data base created for this study. Many of the solutions were very time consuming, technical in nature and required much expensive computer time.

Given the problems of securing research membership data, a decision was made to obtain research population data while gathering or working with collected nursing home data. To resolve the problem of identifying the full research population, the Center for Health Research committed resources to extract Medicare data from the membership file needed to meet requirements of Research Questions I and II. Determination of residence was established either by having a Multnomah county zip code for their personal residence, including that of a nursing home or other custodial facility, or that of a subscriber with whom they lived, such as a child, or sibling or other person in a role allowed by KP to act as a subscriber. The list of Zip codes finally used included those at the margin of county lines in 1988, rather than in 1985-87. Zip code areas are continually changing.

For some members, this criteria was complicated by having seasonal residence in other states, or other counties in oregon, while maintaining their primary residence in 
oregon. Some members were out of the area for large portions of the study period, and if such information was known, they were deleted. Such data was cross-checked with three sources including the overall membership data base, Medicare Durable Medical Equipment contract records, and KP Claims and Billing Department records, all of which were used in the early stages of this study to help identify probable nursing home users, and to confirm member eligibility status. Persons who retained their Multnomah County zip code to continue eligibility in KP, but who declared an out of (KP) service area residence, were excluded from the research population.

Enrollment in KP as a cost, Risk or $\mathrm{s} / \mathrm{HMO}$ member was determined by membership file first-Medicare eligibility date in each of these three status. Some members were age 65 and Medicare eligible except for being employed with health benefits which were primary to Medicare entitlements, so they were removed from the research population.

A similar problem existed in eligibility data except the problem was extant where overlapping coverages occurred. Generally, these were not data entry errors but due to dual status involving dual premiums or both spouses covered by joint policies of the other. All such overlapping dates had to be resolved before eligibility date profiles could be created and before cross-match of nursing home dates and eligibility periods could be done. 
The sixth step in the research plan, undertaken before June, 1989, involved acquiring data for analysis of ICF residents who became Medicaid dependent. Such data was needed to because it was the only source of data from which Risk ICF resident socio-economic and ADL health status indicators could be obtained. It would also provide a basis for comprehensive comparative analysis between Risk and S/HMO, regarding other factors that ECB which could account for any differences observed between Risk and S/HMO ICD use patterns.

The State of oregon had agreed to provided all data needed on KP members who became Medicaid from year beginning 1986 through 1989. Such information had to be selected from a large bank of month-end tape files by SSD staff using the mainframe at SSD. Arrangements were made for acquiring such data which was to be transferred to CHR on down-loaded EDP files.

Five months, out of 36 months, of preliminary data was received from Adult and Family Care Services files. This data was being cross-matched with KP Medicare member data in order to have SSA numbers and HIC numbers matched with the encoded SSD number which SSD used to ensure confidentiality of the 360 data base Medicaid Master files. Such information is subject to Human Subjects Protection research protocol and not in any way available for commercial use. The state of oregon would benefit by this research in that it needed 
information to determine the cost-effectiveness of purchasing the ECB for Medicaid members enrolled under S/HMO. Substantial time was invested in this effort by CHR and this investigator. But, Measure 5 resulted in termination of down-loading activities which only the state of oregon could do. As a result, an important element of this study was discontinued.

The seventh step in the research plan includes the very time consuming problem of data management procedures required for this study. Four basic data sets were created for the analysis of nursing home data. The source files from which supporting data was obtained includes the Membership Information database. Other subsidiary files used to supplement this data has been mentioned above. Collectively, KP membership information was loaded into the first data set and arranged into two files; one was a multiple record file and the other was a file on one comprehensive record per member.

The Nursing Home Resident file was created, from data collected as described. Financial data was separated from utilization data. No member names were included to assure confidentiality. This was the second basic data set.

Data was extracted from the RP Hospital Discharge Data Base for use in Chapter IX analysis of differences in ICD9 primary and secondary codes prior to the first study period ICF admission. This was the third basic data set. 
s/HMO member intake questionnaire and survey update data is stored at the CHR. A small amount of data was downloaded from it to enable analysis of members who were newly enrolled compared to those who converted from other KP status. Risk data on this matter was available from the membership data base. That was the fourth basic data set. This file was used to confirm that all members in the Nursing Home data set had been certified for ECB services at some point.

All of the source files are huge and the resulting four study files are large. Numerous skills had to learned to transfer and manipulate data extracted for use in this study. The software system used to transfer information from three of these data base files was Compuserve 1032 designed for handling large data sets in their initial form. It is not a system intended for use in data analysis. After information was assembled in 1032 , it was transferred to the SAS software systems which operates in the host VMS system used by the CHR.

Considerable energy was required to learn SAS programing at a sufficient skill level to array subsetted and nested data in mutually exclusive data sets. Attention to proper relationships between and within data sets was required to avoid computational errors which would undermine the validity of findings. Some understanding of set theory is important for a study of this nature. 
File structure required much attention in this study, as files were needed which stored data in multiple records for each member, and other files needed to have all data about each member in one record. Flow charts and file name systems were needed to facilitate return to files for correct data at different stages of its evolution.

Quality control procedures were essential to assure correct input when constructing a data set, as well as for output of programming commands. Consultation with seasoned programmers at CHR was essential with complex files but only the investigator can know if the output is good or bad, which only comes from an intimate knowledge of data under use. Much time was consumed in this study acquiring the skills and experience to progress with confidence in the results.

The eighth step in the research plan involved creating rates which would be used in comparing Risk and S/HMO utilization patterns, trends in nursing home revenue for Risk and S/HMO members, and Medicaid data.

In this study, rates for each eligibility group are compared to establish differences in utilization of and expenditures for ICF services. Rates for Risk eligibility group members serve as the baseline against which rates for s/HMO members are contrasted. If substantial differences are observed, and the pattern of differences is clear, then that pattern is interpreted as an indication that the S/HMO 
Expanded Care Benefit may be influencing those observations. Raw rates are determined for Research Questions I-IV, by dividing the dependent variable for each eligibility group by independent variable for each eligibility group data. Age differences were observed for each eligibility group by comparing the mean, median, first (Q1) and third interquartile (Q3) ages. These are summarized in Chapter IV. While mean ages are quite similar, considerable differences existed between median ages and $Q 3$ ages. Since age distribution within eligibility group could affect observed differences in use rates, an age-adjustment procedure is used to remove such potential affects. The distribution of members within each gender and age cohort of the total research population is considered to be a "standard population" which is used to perform that age-adjustment.

Specifically, the proportion of members in each age cohort within gender is determined for the the overall research population. The use rate, as determined for each age cohort within gender for each eligibility group, is multiplied by the proportion of members in each each age cohort by gender for the overall "standard" research population. Each of these computed, or "standardized" use rates are added to create a composite standardized use rate for each eligibility group. This age-adjustment procedure corrects for apparent differences between eligibility group 
specific rates which result from age distribution

differences.

This is called the direct standardization method of

performing an age-adjustment. The result of this

multiplication is a computed rate that can be expected in

the standard population if those age-specific rates had

prevailed. Apparent differences between actual rates for

each eligibility group may be eliminated by this process if

actual differences existed.

Direct standardization may be applied only when the schedule of specific rates for a given population is available... Consistent inequalities among [actual] specific rates, stratum by stratum, yield direct adjusted rates bearing the same inequalities... [but] bear in mind that an adjusted rate, no matter which method is used, has meaning only when compared with a similarly adjusted rate. Its magnitude means little in and of itself...The magnitude of the rate, however, is seen to depend strongly on the composition of the standard population. [49]

of course, examination of actual "crude" rates is an essential part of the analysis and must be done preliminary to comparison of standardized rates, since the latter can mask changes in rate differences between strata. According to J. Fleiss, a biostatistician, it is wise to use more than one index for summarizing age- and sex-specific incidence rates. is simply a value necessary (op. cit. [7]). After considering differences in actual rates, and examining patterns observed for computed age-adjusted rates which are the principal values used in tables presented in Chapters 
IV through IX, then rate differences are evaluated and findings are reported within the chapter in which such rates are presented.

Other factors than age differences are considered in the production of rates for each Research Question. One of those is whether the conversion of Cost Medicare members to Risk Medicare status, or the transfer of pre-TEFRA Risk members into TEFRA capitation contracts, affected Risk baseline rates differently than $\mathrm{S} /$ HMO rates? The specific concern was whether a disproportionate number of members with prior nursing home use ended up in Risk status due to both HCFA requirements regarding enrollment of existing KP members in s/HMO, and the criteria excluding nursing home residents from enrolling in S/HMO? Pre-TEFRA Risk members were not allowed to enroll in $\mathrm{s} / \mathrm{HMO}$ until after 1988 . If Cost members were in a nursing home when they applied for s/HMO, they could not be accepted, although some were enrolled who had previously resided in a nursing home.

The ninth step in the research plan was developed after looking initially at the overall rates. This involved creation of Analysis Models $A$ and $B$, and subsets $I$ and II of the overall model. These were the solution selected to deal with the problem described in the preceding paragraph. Specifically, two subsets were created from within the overall data set of nursing home residents; both excluded some or all members from each eligibility group who had been 
in a nursing home before the study period. These subsets are used to evaluate how the conversion process might have affected use rates based on the history of nursing home admissions before the study period. Neither are intended to replace the overall rates as the principal finding but only to surface the direction in which such rate patterns after effects of initial cost conversions and pre-TEFRA Risk transfers are considered.

Two basic eligibility status models are used for analysis. Model A identifies research population members by Cost, Risk or S/HMO status for use as a denominator; it additively counts each member with more than one eligibility status while in respective groups, when the denominator is per 1000 members.

There are 19,261 members by unique count, but 24,003 members when multiple status is counted, thus 4,742 members were enrolled in two or more eligibility status during the study period.

However, in Model $A$, the denominator allocates exact days of eligibility for multiple status members to respective Cost, Risk or S/HMO groups. Thus, eligibility status days are not over-counted and, Model A is a very good evaluation tool to use in answering research questions about cumulative days of stay per member year of eligibility. Not only is it a precise measure of such rates, and provides a basis for relative comparison of Risk and s/HMO, it also 
measures precisely the relative opportunity for each eligibility group member to be in a nursing home as a cost, Risk or SHMO beneficiary.

Model A also allocates ICF or SNF days to each eligibility status so that no days of nursing home stay are overlapping across two eligibility status.

Model $B$ addresses the issue of multiple eligibility status where the denominator is per 1000 members by isolating the 7 combinations of the three groups (Cost, Risk s/HMO) so that the denominator of each combination is a unique (or mutually exclusive) member count. These seven combinations are reduced to two clusters of members who were "Never-S/HMO" or who were "S/HMO-Sometime" when using Analysis of Variance $F$ tests for significance of difference between the means for the two groups, Risk and s/HMO.

Model $B$ is used for presenting financial data because the data collection methodology made it impossible to associate payments received with each stay, or level of care, in multiple level facilities. Therefore, financial data, as a numerator, could not be cross-matched with level of care eligibility status. Model B resolves that analytic issue.

The short-coming of Model $B$ is that it does not differentiate cost from Risk from $S /$ HMO ICF days within each mutually exclusive cluster, for multiple eligibility users; only that they were never in $\mathrm{s} / \mathrm{HMO}$ or in $\mathrm{s} / \mathrm{HMO}$ sometime. 
In addition to Models $A$ and $B$, it seemed necessary to create subsets I and II from the overall ICF resident data set. The methodological logic for Subset II was to clarify whether the conversion of Cost members to Risk status affected use rates differently than s/HMO rates, in the sense that cost members could be in a nursing home at the time they were transferred to Risk status, whereas that was a much less likely event for cost or Risk members who converted to s/HMO (not accepted if in a nursing home at time of $\mathrm{s} / \mathrm{HMO}$ application). It appears that conversion did influence rates, but the pattern that emerged did not reverse the overall direction.

Subset II is used as the primary tool to clarify this conversion issue. It removes 71 of the 1331 SNF and ICF residents who were in the nursing homes when the study period started. These 71 residents were either discharged and not readmitted, or never discharged.

The first subset is selected by the criteria of not having been in either an SNF or ICF nursing home prior to the first ICF admission during the study period. This is called subset I in the graph below.

Subset II includes all those in subset $I$, and in addition, includes all those who had an admission during the study period but had been in a nursing home prior to their first ICF admission during the study period; i.e. they may have been in either an ICF or SNF at the beginning of the 
study, or admitted and discharged before the study period. Up to this point, subset II is the same as that used for Research Question I. But, an additional 71 persons are removed who had long nursing home use records.

All ICF residents in subsets I and II are also in the overall data set used for output of overall rates for ICF days per year of member eligibility. The difference between overall rates and those for subset II is that seventy-one members are in the overall ICF resident data set who were not in Subset I or subset II.

A visual aid is presented below which shows how these two subsets, and the 71 others, are nested within the overall nursing home user data set. The justification for removing these 71 residents from subsets $I$ and II is that they had a history of nursing home use which, in many cases, preceded the implementation of $S / H M O$ and Risk TEFRA enrollment programs in April, 1985. Also many of these 71 residents remained throughout all or most of the study period, and none of those discharged were readmitted. Although they are a legitimate part of the overall data set for production of rates [nursing home days per member year of eligibility], it is also valuable to observe whether the S/HMO rate remains below that of Risk without their influence, and to clarify rates for members whose lifetime use of nursing homes began in proximity to or after implementation of $\mathrm{s} / \mathrm{HMO}$ and Risk. 
There were three methodological reasons for creating subset I. First, Subset I allows analysis of nursing home use rates for residents who were at the beginning of their lifetime use of nursing homes; therefore, it also eliminates the problem of left censoring of utilization data from before the study period. Utilization analysis of nursing home residents requires recognition that many residents have ongoing, although not necessarily continuous, residency status. In this study, about 758 of all nursing home residents had 3 or less admissions, but the other $25 \%$ trailed off to a maximum of 10 admissions. This recurrent admission process must be accounted for when developing conclusions or doing estimates of true use rates. Many studies have failed to do so, resulting in flawed findings. The 1985 National Nursing Home Survey (NNHS) produced seriously flawed data because it failed to ask for a correct history of prior nursing home admissions. Therefore, in 1992 a revision of findings was published.

The methodological issue is, how does the investigator manage left and right censoring of data, to correctly estimate true lifetime use rates? The answer is to be very clear about the descriptive data beyond censored study points. While the original research plan included survival estimates, but until descriptive data was fully understood, model building for that kind of analysis was not appropriate. 
Recent studies by Mark Meiners, including a meta-analysis of all spend-down research through 1991, and by Thomas Bice 1990, including a study of the 15 year Connecticut nursing home data base, showed how the 1977 and 1985 NNHS findings under-estimated lifetime nursing home use projections due to censoring without proper descriptive data about pre and post sample readmisisons. In this study, Subset $I$ is used with financial data up to one year after the study period, in an effort to capture a high proportion of lifetime use-rate data.

The second reason for creating subset $I$ was to facilitate a meaningful analysis of Medicaid dependency patterns for shorter-stay residents, by eliminating left censoring (prior nursing home) use as a reason for differences between Risk and s/HMO for becoming welfare dependent. Since spend-down occurs between 1-2 years after first admission as a private pay resident for 95 of Medicaid dependent persons, this study is able to identify a presumably reliable rate on that event, given that this study's data base includes data one year after the study period.

A third reason for subset $I$, was that a few long stay residents can badly distort descriptive parameters of financial data in this study. Several residents had well over $\$ 100,000$ in nursing home revenues, which strongly affected the mean of total payments received. 
Beginning and ending dates were cross-matched against beginning and ending dates of study period. The above graph shows which combinations of admissions within the study period (SP) were assigned to data subset II and data subset I within the overall dataset.

Figure 1, below, presents unique combinations of admissions to nursing homes during the study period.

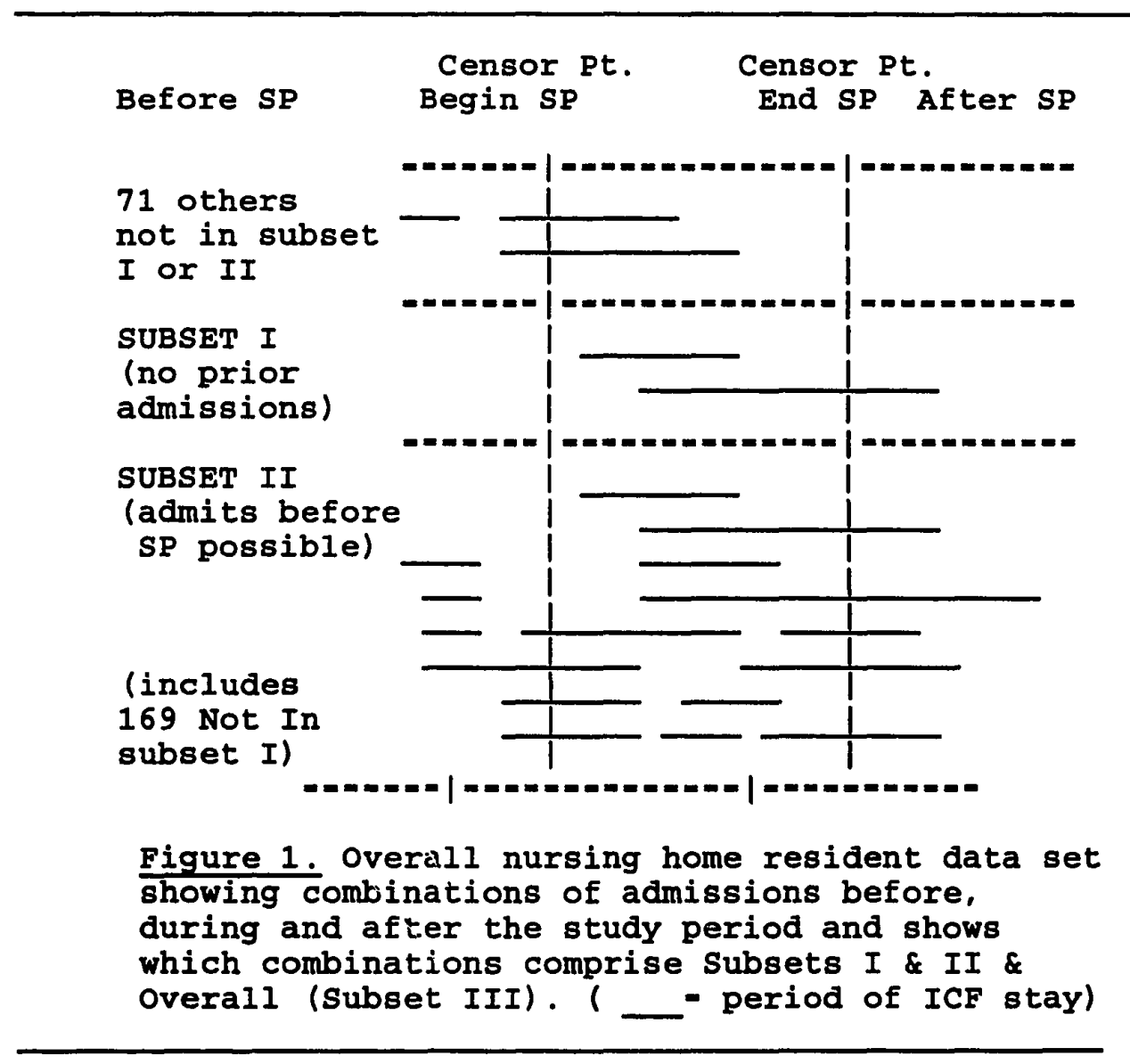

Both TEFRA Risk and S/HMO eligibility status commenced in April, 1985; this raises the question, why was the Risk 
use rate for ICF days in residence per year of eligibility so much higher than that for $s / H M O$ ?

of these 71 members, thirty-four were in Risk eligibility status, twenty-seven were in Cost status, and only four were in S/HMO status.

All of the 34 Risk members were cost conversions or roll-over members from the Medicare Plus Demonstration Project. This suggests that Cost and Risk member ICF user history was substantially more established than that of s/HMO when the study period began.

The question raised here is, does removal of these 71 residents account for some part of the rate differences in days of stay per year of eligibility between Risk and s/HMO?

Several macro-level reasons come to mind. Effects of managing the S/HMO Extended Care Benefit could produce the difference. S/HMO members in ICFs tended to stay for shorter periods but have more readmissions.

Could some characteristic of the Risk population bias their health and social status toward greater need for ICF services? Those in Medicare Plus might be older.

Could some characteristic of the S/HMO population bias their health and social status against need for ICF services such as the enrollment policy prohibiting acceptance of persons in a nursing home at the time they applied? Adverse or favorable selection bias were issues of concern to researchers in both Medicare Plus (PreTEFRA RISR) and 
Medicare Plus II (S/HMO).

Or, could the process of converting Cost to Risk members could have produced an administratively induced bias, based on the order in which conversions were implemented?

Regarding those seventy-one members in the overall data set, sixty-six were in ICFs, of whom thirty-three were in the Risk eligibility status. All thirty-three Risk members were in "group sponsored" status, rather than "individual payer" status. That means all such nursing home users were receiving a health benefit from a prior employer, union, or other collective sponsor who paid some portion of the Medicare Supplemental Insurance benefit premiums to KP for that member. Such payments did not include money for ITC benefits.

Another characteristic of these 33 Risk nursing home users is that their mean days (333.2 days in the study period) of stay was substantially greater than any other subset observed, including "individual" Risk members from the pre-TEFRA Demonstration Project. Median days for these thirty-three Risk members was 353 days; Q3 was 570 days, and Q1 was 86 days). Several were in ICF status across the entire study period. Furthermore, they had the highest mean age (88.2) and median age (87.1) of any subgroup from within the overall data set of nursing home residents; ( $Q 3$ was 93.1 and $Q 1$ was 84.8 ). In contrast, cost members within this 
subset of 66 ICF users, had a mean of 206.9 days of stay, a median of 137 days; ( $Q 3$ - 349 days, and $Q 1=25$ days).

Apparently, these 33 Risk ICF residents contributed heavily to the overall Risk rate. Why didn't s/HMO members have more ICF users who had transferred from Cost or Risk status? No doubt one of the answers is that one of the few barriers to converting into $s /$ HMO was that the member could not be in a nursing home at the time of application. Given this difference between Risk and S/HMO groups, it seems reasonable to remove at least those members from the Risk data set whose history of ICF utilization was known to load the rates with days of stay at the front-end, many of whom remained throughout all or most of the study period.

A third concern about factors potentially affecting use rates is related to the rate denominator in Research Question I. Specifically, some members were in more than one eligibility status, as noted earlier in this Chapter. However, that problem is eliminated in Research Question II, by using mutually exclusive days of eligibility per member per year, which absolutely eliminates overlapping counts.

Except for Research Question I, this problem is resolved either by using days of eligibility as the denominator, or creating mutually exclusive combinations and clusters of the three eligibility groups, which is called Analysis Model B. Mutually exclusive groups are for the denominator in Research Questions III, V, VI, in contrast to 
Analysis Model A, which is used in Research Question I. It should be noted that the most frequently used denominator in the literature on nursing home utilization appears to be a count of the population or "per 1000 population." That is a useful crude rate when not comparing population subsets between which population members move. Model B allows the use of that denominator (/1000 members) because it controls for, rather eliminates double counting.

Analysis Model $B$ uses the the three not-mutually exclusive eligibility groups, Cost, Risk, and s/HMO from analysis Model A, but they are organized into seven mutually exclusive combinations of eligibility which include: Cost, Cost and Risk, Risk, S/HMO, Cost and S/HMO, Risk and S/HMO, Cost and Risk and S/HMO. Additionally, these seven groups are divided into two clusters, the first of which includes groups one through three who were members that were never enrolled in s/HMO during the study period. The remaining four groups comprise the second cluster which includes members who were in $\mathrm{s} / \mathrm{HMO}$ sometime during the study period. The seven groups under Model B are called Format B; the two clusters are called Format A, of Model B. Each resident can only be in one of the seven groups in Format B, or one of the two clusters of Format A. Figure 2 lists Formats $A$ and B of Model B showing combination elements. Thus, Formats $A$ and $B$ of Analysis Model $B$ are used to present differences in the mean of payments received/ 1000 research population. 


\begin{tabular}{|c|c|c|}
\hline Format $\mathrm{A}$ & Format & B \\
\hline Never S/HMO\} & $\begin{array}{l}\text { Cost only }= \\
\text { \{Cost+Risk }= \\
\text { Risk only }=\end{array}$ & 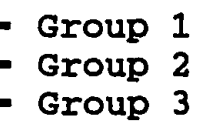 \\
\hline s/HMO sometime\} & $\left\{\begin{array}{r}\text { \{s/HMO only } \\
\text { \{s/HMO+Cost } \\
\text { \{S/HMO+Risk } \\
\text { \{s/HMO+Cost+Risk }\end{array}\right.$ & $\begin{array}{l}\text { - Group } 4 \\
\text { - Group } 5 \\
\text { - Group } 6 \\
\text { - Group } 7\end{array}$ \\
\hline
\end{tabular}

Figure 2. Mutually exclusive model used to present rates on payments received/1000 research population members in Model for Analysis B.

A mutually exclusive model (Model B) is used for three reasons. First, at some facilities data collection problems prevented reliable allocation of financial information according to ICF or SNF levels of care. Second, financial data cannot be allocated to periods of eligibility the way days of stay were in Chapter IV. Third, reliable allocation of financial data to the study period was not possible because financial records at some facilities did not specify periods of stay for which funds were received. Financial records for multiple admission residents on Medicaid were especially difficult to interpret, regarding periods of stay represented by the payments. These issues were discussed in greater detail in Chapter III.

Units of measurement for the rate used for financial information are as follows: total payments received for each 
member who resided in a nursing home during the study period are accumulated within each of the seven mutually exclusive groups (Format B) and two mutually exclusive clusters (Format A); the sum of such payments forms the numerator in this rate. The membership count allocated to each group or cluster forms the denominator. The numerator is first divided by the denominator to produce the non-standardized rate (payments/group members \{not just residents\}), which is multiplied by the standardizing ratio of age cohort distribution within gender to adjust for effects of differences in gender by age cohort between each group or cluster. The standardized rate is multiplied by 1000 , the result of which is presented in tables prepared for Chapters VII and VIII.

A mutually exclusive model is used for financial rates for three reasons, in addition to the issue of not-mutually exclusive denominators. First, at some facilities data collection problems prevented reliable allocation of financial information according to ICF or SNF levels of care. Second, financial data cannot be allocated to periods of eligibility the way days of stay were in Chapter IV. Third, reliable allocation of financial data to the study period was not possible because financial records at some facilities did not specify periods of stay for which funds were received.

Financial records for multiple admission residents on 
Medicaid were especially difficult to interpret, regarding periods of stay represented by the payments. These issues are discussed in greater detail in Chapter III.

Revenues received by nursing homes means total debited to accounts receivable for each member from all sources for SNF and ICF use prior to, during and up to one year after (before July, 1989) the study period.

subset II is used as a surrogate for the overall data set in Chapters VII and VIII, because nursing home use prior to the study period affects the amount of payments received rate used to measure differences between Risk and S/HMO. Hopefully, such organization will assist the reader(s) in moving through a great deal of numeric information without loosing track of the evaluation process use to summarize patterns or similarities and differences between Risk and S/HMO member use of ICFs during the study period. Figure 3, below, outlines the use of Models and $B$ in subsequent chapters.

\section{APPROACH TO EVALUATION OF FINDINGS}

A consistent format is used to present data and report findings as shown in Figure 3 , and the following subtitles: Pre-observation comments, Research Goals, Research Question, Rates for overall and/or subset II, and/or subset I data, Observations for each table, Summary of findings, Research Methodology Issues, Policy Research Recommendations. 


\begin{tabular}{|c|c|c|c|}
\hline & MODEL $\mathrm{A}$ & $\begin{array}{l}\text { MODEL B } \\
\text { Format a }\end{array}$ & $\begin{array}{l}\text { MODEL } B \\
\text { Format } b\end{array}$ \\
\hline $\begin{array}{l}\text { File } \\
2 \cdot\end{array}$ & PERSON BASED & GROUP BASED & CLUSTERS \\
\hline $\begin{array}{l}\text { MODEL } \\
\text { CONSTRUCT }\end{array}$ & $\begin{array}{l}\text { NOT MUTUALLY } \\
\text { EXCLUSIVE } \\
\text { BET'WN C_R_S } \\
\text { (COST_RISK_SHMO) }\end{array}$ & $\begin{array}{l}\text { MUTUALLY MUTU } \\
\text { EXCLUSIVE } \\
\text { ELIG. GROUPS } \\
\text { C, CR, R, S, } \\
\text { CS, CR, CRS }\end{array}$ & $\begin{array}{l}\text { ALLY } \\
\text { EXCLUSIVE } \\
\text { CLUSTERED } \\
\text { NEVER SHMO VS } \\
\text { SHMO SOMETIM }\end{array}$ \\
\hline $\begin{array}{l}\text { CRITERIA } \\
\text { FOR MATCH } \\
\text { OF RPM } \\
\text { ELIG \& } \\
\text { NH ADMIT } \\
4 .\end{array}$ & $\begin{array}{l}\text { ELIG.STATUS } \\
\text { IDENTIFIED } \\
\text { BY RPM FOR } \\
\text { EACH ADMIT }\end{array}$ & $\begin{array}{l}\text { ELIG. STATUS } \\
\text { IDENTIFIED BY } \\
\text { GROUPED ELIG. } \\
\text { STATUS FOR } \\
\text { ADMITTED RPM }\end{array}$ & $\begin{array}{r}\text { ELIG. STATUS } \\
\text { IDENTIFIED } \\
\text { BY CLUSTER } \\
\text { FOR ADMITS }\end{array}$ \\
\hline $\begin{array}{l}\text { Research Qu } \\
\text { CRITERIA } \\
\text { FOR COUNT } \\
\text { AS ADMIT }\end{array}$ & $\begin{array}{l}\text { estions I, II: } \\
\text { ADMIT DATE } \\
\text { >30JUL' } 86 \\
\text { <01JUL' } 88 \\
\text { AGE ELIG } \\
\text { MEDICARE }\end{array}$ & $\begin{array}{r}\text { ADMIT DATE } \\
>30 \text { JUL' } 86 \\
\text { <01JUL' } 88 \\
\text { AGE ELIG } \\
\text { MEDICARE }\end{array}$ & $\begin{array}{r}\text { ADMIT DATE } \\
>30 \text { JUL' } 86 \\
\text { <01JUL'88 } \\
\text { AGE ELIG } \\
\text { MEDICARE }\end{array}$ \\
\hline a. & $\begin{array}{l}\text { POSS ADMIT } \\
\text { B4 SP } \\
\text { NO NH ADMIT } \\
\text { B4 SP }\end{array}$ & $\begin{array}{l}\text { POSS ADMIT } \\
\text { B4 SP } \\
\text { NO NH ADMIT } \\
\text { B4 SP }\end{array}$ & $\begin{array}{l}\text { POSS ADMIT } \\
\text { B4 SP } \\
\text { NO NH ADMIT } \\
\text { B4 SP }\end{array}$ \\
\hline $\begin{array}{l}\text { R. Q.III-VI } \\
\text { CRITERIA } \\
\text { ADMISSION } \\
\text { TO COMPARE } \\
\text { DAYS USED }\end{array}$ & $\begin{array}{l}\text { a. } \\
\text { IN NH ON } \\
\text { 30JUL' } 86 \\
+ \text { ADMITS } \\
\text { DURING SP } \\
\text { b. } \\
\text { ADMIT AFTER } \\
\text { 30JUN' } 86 \\
\text { BUT PRIOR } \\
\text { NH USE POSS } \\
\text { C. } \\
\text { ADMIT AFTER } \\
\text { 30JUN' } 86 \\
\text { \& NO PRIOR } \\
\text { NH ADMITS }\end{array}$ & $\begin{array}{l}\text { a. } \\
\text { IN NH ON } \\
\text { 30JUL' } 86 \\
+ \text { ADMITS } \\
\text { DURING SP } \\
\text { b. } \\
\text { ADMIT AFTER } \\
\text { 30JUN' } 86 \\
\text { BUT PRIOR } \\
\text { NH USE POSS } \\
\text { C. } \\
\text { ADMIT AFTER } \\
\text { 30JUN' } 86 \\
\text { \& NO PRIOR } \\
\text { NH ADMITS }\end{array}$ & $\begin{array}{l}\text { IN NH ON } \\
\text { 30JUL ' } 86 \\
+ \text { ADMITS } \\
\text { DURING SP } \\
\text { b. } \\
\text { ADMIT AFTER } \\
\text { 30JUN' } 86 \\
\text { BUT PRIOR } \\
\text { NH USE POSS } \\
\text { c. } \\
\text { ADMIT AFTER } \\
\text { 30JUN' 86 } \\
\text { \& NO PRIOR } \\
\text { NH ADMITS }\end{array}$ \\
\hline
\end{tabular}

Figure 3. Model for Analysis and format for presenting observations in Chapters IV through $\mathrm{X}$. 
ICF VS SNF VS NURSING HOME

An issue is addressed next which was referenced in Chapter I regarding nursing home level of care. It is discussed because Federal regulations modified legislation and regulations pertaining to nursing homes which created or distinguished between that level of care in which skilled nursing care (RN) was required continuously, and that level of nursing home care in which skilled nurses were required intermittently for patient care.

By this action, HCFA decreed that SNF and ICF falsely differentiated patient needs and that each patient must be rated according to a score derived from a Minimum Data set of criteria prescribed by HCFA. This raises the question about the relevance of findings in this study regarding ICF care, given this policy change. Aside from the historic value, ICF level care was the term that the nursing home industry generally understood to differentiate chronic long term institutional care from post hospital convalescence for Medicare patients. Insurance carriers offering policies made and continue to make that distinction. It remains the terms of reference used within the nursing home industry outside of the Veterans Administration.

States pursued Medicaid pass-through funds based partly on formulas related to SNF and ICF level of care. Therefor, states differentiated patients in a way which followed that policy choice. To this extent, elimination of the terms SNF 
and ICF was a rational policy change.

In the state of Washington, about 908 of all Medicare and Medicaid admissions to nursing homes were SNF, and 108 ICF, during the 1980s. In Oregon, the converse existed where $90 \%$ of Medicare/Medicaid admissions were ICF and $10 \%$ SNF. Large variation existed among states many regarding such classification practices. For example, some states allowed use of ICF level care for a patient being fed via nasogastric or gastic tube, while others required they be classified as SNF, for purposes of Medicaid reimbursement. [per 9/15/92 phone conversation with Elizabeth Cornelius, Ph.D., Office of Demonstrations and Evaluations, HCFA., regarding HCFAs plan to use the MDS as a basis for reimbursing nursing homes].

It is important to note that published research about nursing home utilization focuses mostly on SNF Medicare services and ICF services by individuals who are welfare recipients. Prior to 1991 and implementation of OBRA '87, Medicare files contained only SNF reimbursement related information. State and federal Medicaid data regarding ICF services included only welfare recipients. Nursing home utilization rates and financial data on SNF and ICF services not covered by Medicare or Medicaid has low visibility among journal articles.

Even reported findings on the s/HMO participant's use of ICFs includes only that portion within the benefit limit 
as authorized by "the sHMO and expenditures paid for by Medicare." [50]

In this study, nursing home utilization and expenditures data, regarding Subject's use of SNF and/or ICF services, includes non-Medicaid and non-sHMO ICF data, in addition to SNF Medicare, and SNF data beyond that covered by Medicare but before Medicaid eligibility, and data while Medicaid eligible.

ISSUES RELATED TO GENERALIZATION OF STUDY FINDINGS

Caution is required about any generalization of any studies regarding nursing home utilization. That caveat applies to this study, for reasons other than not using random sampling and inferential statistics as the basis for analysis.

Large variation exists between states and within states regarding several important variables commonly used to describe urban nursing home utilization. These variables include: age distribution of the elderly; differences in health conditions of residents, as defined by DRG/ICDM hospital codes and by $\mathrm{ADL}$ defined functional disabilities; methods used by states to classify nursing home residents as SNF or ICF [during the time period of this study]; availability of SNF and ICF beds; availability and state policy on the use of other levels of long-term care facilities. 
variance in age distribution is considerable between states and counties among Americans over age 64. The age cohort for those 85 and over is a target group. regarding nursing home use rates, because the proportion admitted to a nursing home is high. Census data for 1990 indicate that Midwestern states have very high distributions of such elderly. In MSA wichita, Kansas, 13.6 per cent of those over age 64 were 85 years old or more, while in the Multnomah County, only $6 . x \%$ were 85 years or more [1990 census]. State of Kansas - 12.38 vs state of oregon 9.98. [51] The final chapter is used to synthesize findings from the next six chapters in this study. Some hypothesis testing recommendations are made related to such findings. 
OVERALL USE RATES FOR RESEARCH QUESTIONS I AND II

There is growing evidence that suggests that management control practices may be associated with lower costs in health care facilities. [52]

The purpose of this chapter is to present use rates which respond to policy issues of interest in this study. Research Questions I and II are presented in this chapter which respond to two of those policy issues.

A population based policy concern is addressed first. Specifically, does the s/HMO Expanded Care Benefit influence the rate at which ICF services are accessed? This is not an issue of frequency of access, rather one of initial access. There are several issues related to this policy concern. Does this ECB increase the use of ICF services in addition to providing formal home care services? Does the ECB benefit appear to improve access to ICF services for members whose needs are certifiable at that level of care? Baseline Risk rates provide the comparison against which conclusions are descriptively inferred in this study, regarding such policy concerns. Research Question I responds descriptively to that policy concern.

Improved access is an important goal so long as resulting residency patterns don't become excessive or 
inflationary. In a risk-based HMO, that is a critical operational issue. It is also a matter of substantial interest on the part of health care policy makers who are pondering whether long-term care entitlements are an affordable national goal, and looking to s/HMO for some answers.

Thus, the second policy concern addressed in this chapter is whether the operational principles of managed care, as practiced in this HMO, contain utilization of ICF services by $\mathrm{S} / \mathrm{HMO}$ members certified for use of their $\mathrm{ECB}$ to cover costs of ICF services? Research Question II responds descriptively to that policy concern. The baseline for comparison is Risk member ICF use rates.

Under Research Question II, values from the Overall data set are used to produce baseline use rates for Risk and S/HMO nursing home residents. These rates form the basis of evaluation of differences in this study. Descriptive data from two subsets (I, II), are extracted from the overall data set; they are used to identify patterns of change from overall utilization differences when residents with nursing home admits prior to the study period are removed.

Subset II was used for Research question I. That is, members who were in a nursing home on day one of the study period are removed, if they did not have a subsequent readmission during the study period. Such persons were almost exclusively Risk and Cost members; most of the Cost 
members involved had began their use of nursing homes before the Risk TEFRA and S/HMO commenced. That was the case for some of the Risk members because they had been participants in the Medicare Plus Demonstration project.

Subset I provides a view of use rate patterns for first time ICF residents. Thus, nursing home residents are removed from subset I who did not any prior admission to an ICF before the study period. Such persons are just beginning their lifetime use of nursing homes, compared to the overall data set which includes many persons part way into their life cycle of nursing home use.

Patterns are summarized regarding differences in use of ICF services associated with three age cohorts based on non-standardized rates.

Raw data is used to display the percent of distribution of cumulative days used by nursing home residents within five length of stay (LOS) time ranges during the study period. This information is provided in response to the policy research concern about what proportion of $\mathrm{s} / \mathrm{HMO}$ nursing home users are reasonably protected against nursing costs by front-end, shorter-stay, LTC benefits? In contrast, what proportion of s/HMO nursing home users are likely to go through the ECB financial shield and begin a period during which they must rely on personal assets to cover nursing home costs. That circumstance places them in peril of catastrophic financial harm. The policy issue is what 
proportion of nursing home users are served by the ECB at this S/HMO Demonstration site.

Some Overall SNF and combined SNF+ICF rates are

presented where they help clarify use rate patterns and trends for ICF residents.

Research Question I in this study asks whether differences exist between three eligibility groups regarding respective probabilities of becoming an ICF resident during the study period. The units of measurement in this rate are the unique number of members admitted to an ICF during the study period while enrolled in Raiser Permanente as Cost, or Risk, or S/HMO Medicare beneficiaries, per 1000 members of Cost, Risk, or s/HMO eligibility groups. By definition, this research question excludes persons in a nursing home at the beginning of the study period.

Research Question I: Are There Differences Between Cost, Risk, and S/HMO Regarding The Number of ICF Residents Per 1000 Research Population "Members" During The Study Period?

Members may have become an ICF resident while in more than one eligibility status. This could occur under two circumstances: first, if a member's eligibility status changed while they were in a nursing home they are credited as having an admission under each eligibility status. Or, if a member's first ICF stay was all under one eligibility status, but a subsequent admission was under another status, then one admission per eligibility group was counted. 
Specifically, twelve percent (nineteen of the one-hundred fifty-four cost ICF residents) subsequently were in Risk status as ICF residents. Four percent (eleven of the two-hundred eighty-two S/HMO ICF residents) also had ICF admissions during the study period while in Cost or Risk: two were in Cost and nine were in Risk. Model $A$ is used to present probability of admissions, in Table VII, below.

TABLE VII

OVERALL 1ST ADMISSION RATES STANDARDIZED BY SEX \& AGE COHORT UNIQUE NUMBER OF ICF RESIDENTS ADMITTED IN

THE SP PER 1000 COST OR RISK OR S/HMO MEMBER GROUPS WHICH ARE NOT MUTUALLY EXCLUSIVE

(BY GENDER)

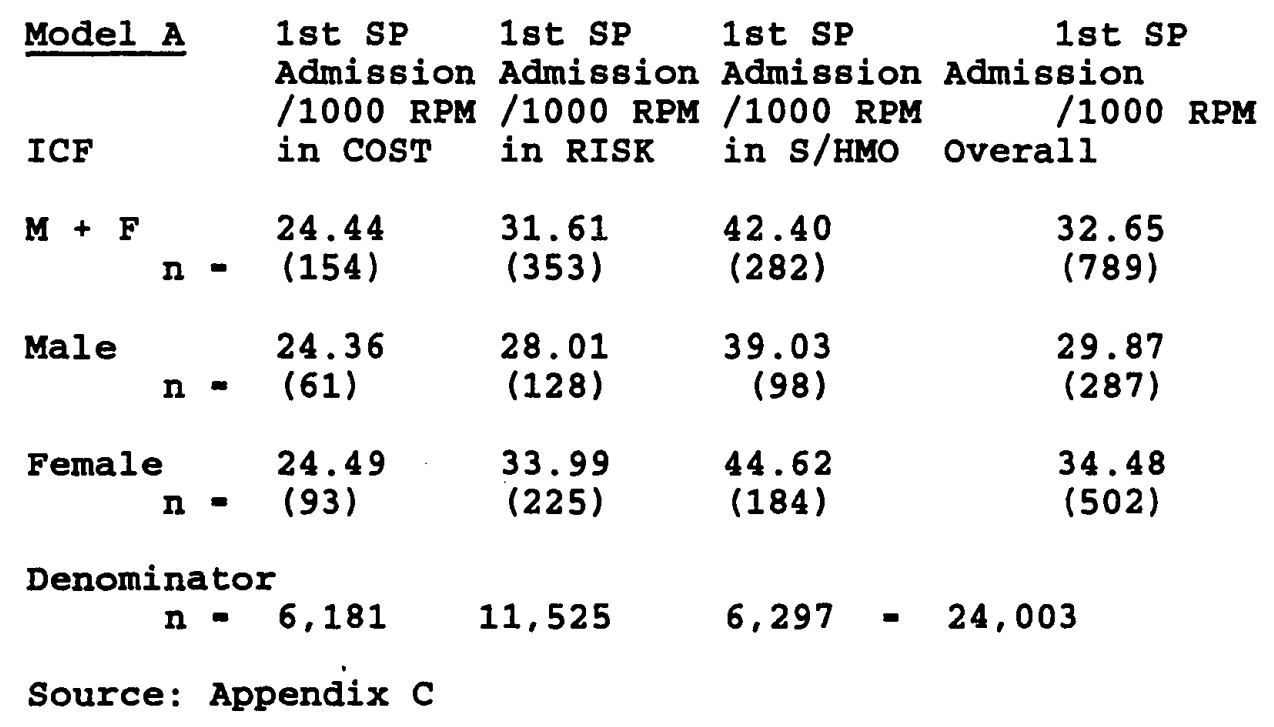

The standardized rate per $1000 \mathrm{~s} / \mathrm{HMO}$ members who became ICF residents during the study period was thirty-four percent greater than that rate for Risk members, and 
seventy-three percent greater than the rate for cost members. This overall rate represents the probability of becoming an ICF resident while in the research population as Cost, Risk or s/HMO Medicare status during the study period. The above observations are important because they suggest that access to ICF services is facilitated by the ECB for persons certified as needing that level of care. Within eligibility groups, females in S/HMO status were fifteen percent more likely to reside in an ICF than S/HMO males; females in Risk were twenty-one percent more likely than Risk males to reside in an ICF, while almost no rate difference by gender occurred for members while in Cost status.

Between eligibility groups, the probability of being in an ICF was thirty-nine percent greater for $s /$ HMO males than Risk males, and was thirty-one percent higher for s/HMO females than Risk females. Clearly, the probability of becoming an ICF resident was greatest for s/HMO members, based on differences in age adjusted use rates.

The literature on nursing home use leaves little doubt about a strong association between age and probability of becoming a nursing home resident. This is because older persons tend to differ from younger persons regarding the type and effects of chronic illness. Chronic illness patterns are evaluated in Chapter IX in an attempt to determine is hospital discharge information suggests that 
s/HMO members health status differs from Risk in a way which explains use rate differences. That evaluation suggests as much similarity in health status as dissimilarity. Some studies estimate that twenty percent of the population over age eighty-five reside in nursing homes. That suggests the probability of becoming a resident must be high which is examined in the next table. Rates presented in Table VIII are based on raw data and are not the result of any adjustment for differences in age cohort distribution of the Cost, Risk or s/HMO members.

\section{TABLE VIII}

OVERALL RAW RATES FOR 1ST ADMISSION BY SEX \& AGE COHORT UNIQUE NUMBER OF ICF RESIDENTS ADMITTED IN THE SP PER 1000 COST OR RISR OR S/HMO MEMBER GROUPS WHICH ARE NOT MUTUALLY EXCLUSIVE

(BY AGE COHORT)

\begin{tabular}{|c|c|c|c|c|}
\hline RAW Rates & $\begin{array}{l}\text { 1st SP } \\
\text { Admission } \\
/ 1000 \mathrm{HPM} \\
\text { in COST }\end{array}$ & $\begin{array}{l}\text { 1st SP } \\
\text { Admission } \\
\text { /1000 HPM } \\
\text { in RISR }\end{array}$ & $\begin{array}{l}\text { 1st SP } \\
\text { Admission } \\
/ 1000 \mathrm{HPM} \\
\text { in s/HMO }\end{array}$ & $\begin{array}{l}\text { 1st SP } \\
\text { Admission } \\
\text { /1000 HPM } \\
\text { Average }\end{array}$ \\
\hline $\begin{array}{l}\text { ICF } \\
\text { Age Cohort } \\
65-74\end{array}$ & in $\cos T$ & $\begin{array}{c}\text { in RISR } \\
6.82\end{array}$ & in $\mathrm{S} / \mathrm{HMO}$ & Average \\
\hline $75 \_84$ & 25.22 & 32.90 & 52.61 & 36.54 \\
\hline $85-105$ & 109.09 & 136.74 & 139.65 & 130.58 \\
\hline
\end{tabular}

Source: Appendix C

Based on Raw Rate Differences, within S/HMO, members in age cohort $85+$ were 1.7 times more more likely to reside in ICFs than S/HMO age 75-84. Within Risk members in age cohort 
85+ were 3.2 times more more likely to reside in ICFs than Risk age 75-84.

Comparison between $\mathrm{S} / \mathrm{HMO}$ and Risk shows that in age cohort 65-74, the rate of first admission during the study period was 1048 greater for $S / H M O$ than Risk members; and in age cohort 75-84 the rate of first admission during the study period was $60 \%$ greater for S/HMO than Risk members; and in cohort $85+$, the rate of first admission during the study period was 2.18 greater for $\mathrm{S} / \mathrm{HMO}$ than Risk members. Why is this pattern of differences occurring? Is age distribution different among $\mathrm{S} / \mathrm{HMO}$ than Risk members?

Mean and median ages of research population members within each eligibility status do not suggest that overall, first-admisson ICF rates would be much different for s/HMO than for Risk or Cost, as summarized next.

Specifically, age parameters of research population members by eligibility group are summarized. Mean age for: Cost - 76.1, Risk - 75.2, S/HMO - 76.3; Median ages are: Cost - 74.6, Risk - 74.1, S/HMO - 75.2; Interquartile ages: Cost $=70.9-80.2$, Risk $-68.8-79.9$, S/HMO $=70.8-80.8$. These descriptive data show that $\mathrm{S} / \mathrm{HMO}$ members are approximately one year older, on average, than Risk members, which raises doubts about age as an explanatory variable for the above rates/1000 research population eligibility group members. If mean age of research population members does not explain differences between $\mathrm{s} / \mathrm{HMO}$ and Risk probability rates 
for ICF use, are there differences in SNF rates which could suggest reasons for rate differences? This is explored in Table IX which compares Overall rates for the probability of admission to an ICF and to an SNF, as well as comprehensive nursing home age-adjusted use rates.

The above observations reinforce the first use rate findings that ECB help facilitate access to ICFs. Mean age is very similar between Risk and S/HMO research population members .

Many members who became ICF residents were admitted to an SNF before or after an ICF stay. For certain chronically ill patients, ICF care is a multidirectional extension of either post-hospital recuperation or deteriorating health. Some patients go into an ICF and after a time need skilled care continuously, as provided by SNFs: Table IX shows both, followed by Figure 4, showing ICF, SNF, and SNF+ICF rate The probability of S/HMO members becoming an SNF resident was fifteen percent greater than that for Risk members during the study period.

The probability of $\mathrm{s} / \mathrm{HMO}$ members becoming a nursing home resident, using either ICF or SNF care, or both, was twenty-seven percent greater than that for Risk members during the study period.

Within S/HMO, the rate per 1000 members of having one or more admissions to an SNF during the study period was fifty-eight percent less than the rate for having one or 
more admissions to an ICF. Within Risk, that rate difference was only thirty-six percent.

TABLE IX

SEX BY AGE COHORT STANDARDIZED RATES FOR ALL UNIQUE NUMBER OF NURSING HOME RESIDENTS BY LEVEL OF CARE ADMITTED DURING THE SP PER 1000 COST OR RISK OR S/HMO MEMBER GROUPS WHICH ARE NOT MUTUALLY EXCLUSIVE

(MALE + FEMALE)

Model A

1st SP

1st SP

Admit

1st SP 1st SP

Admit Admit Admit $/ 1000$ HPM /1000 HPM /1000 HPM /1000 HPM in $\cos T$ in RISK in $S / H M O$ Overall

ICF 24.44

31.61

(353)

42.40 n $=(154)$

$(282)$

32.65

SNF 16.73

23.27

n $=(105)$

(261)

26.78

(176)

22.45

SNF+ICF $\quad 41.16$

54.60

$n=$ (259)

(614)

69.18

(458)

54.96

Denominator
$n-6,181$
11,525
6,297
- 24,003

Source: Appendix C

The focus of this study is on ICF level care, and not SNF level care, because entitlement benefits and Medicare Supplemental benefi.ts do not cover ICF care expenses. But, SNF utilization rates are very interactive with ICF rates.

The above rate is important because it emphasizes that entitlement benefits for SNF care are being used in similar ways by Risk and s/HMO members. 


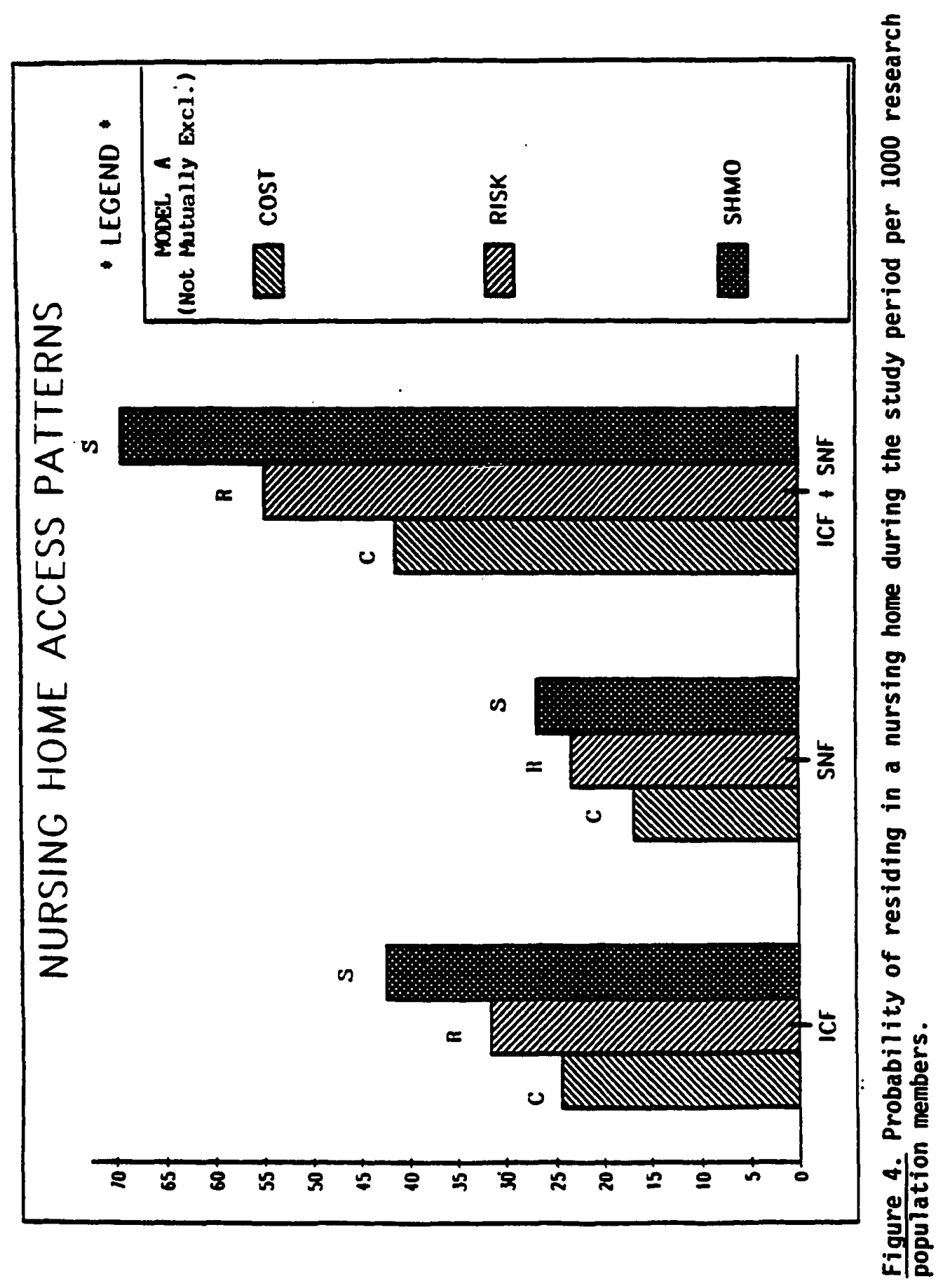


It also suggests that access for both Risk and S/HMO members is based on Medicare entitlement and basic Supplemental Plan coverage, rather than on ECB. The slightly higher S/HMO rate is not surprising because the ECB allowed S/HMO member to use up to $\$ 10,500$ for SNF care, during the study period, if approved by the case manager. The lower Cost member use rate not surprising since cost members were not required to carry the Medicare Supplemental benefit until after they were offered Risk conversion, or were exempted from conversion.

A use rate which measures the probability of becoming an ICF resident is much different that a rate which measures frequency of admission, which is not presented in this study although produced in the course of data analysis. Some members were admitted ten times to nursing homes during the study period, but that is the product of interfacility transfers, short term use for family care-giver respite, or other services best provided by an institution. However, about seventy five percent of all nursing home stays were accounted for by members with three admissions or less.

Frequency of admission may be an indicator of nursing home policy on managing patients with a change of health status, or of the appropriateness of physician admitting practices to a given level of care, or of the nature of services provided at a given facility. For example, some facilities expect their turnover rate to be higher if they 
receive many patients with short life expectancy, or with post-fracture rehabilitation needs, or for respite care. The probability of becoming a nursing home resident is affected by many factors, also. If one population subset is comprised of persons with more disabling chronic illness than another, is the variance in rates affected? If one population subset is comprised of persons with insurance benefits, unlike another population subset, do those who have insurance use the service more than those who do not? In this study, an overarching policy question is, did the Expanded Care Benefit affect nursing home use; more specifically, did it affect ICF use?

Measurement of utilization often involves several tests of empirical information in order to adequately clarify such questions. Generally, literature which reports on nursing home utilization includes at least two, and often three, rates including: [number of residents per 1000 population, or/and number of admissions]; [days in residence per year, and/or mean length of stay]. Rates used by HMOs are often expressed as annualized member months of eligibility, i.e., year of eligibility per member. That convention is used here.

In 1985, the non-standardized, national sample of the number of residents age 65 and greater in nursing and personal care homes was 46.2 per 1000 population. This is somewhat less than that for Risk and $s /$ HMO and somewhat 
above that of Cost members. National sample data was based on a count of residents for calendar year, 1984 and includes some residents from a level of care in licensed or certified institutions whose admitting criteria were less restrictive than for intermediate care facilities in oregon. Facilities providing only mental health services are excluded. [53] [54]

The rate presented for Multnomah County in Table $x$, below, is based on a one day annual survey of all nursing home residents in all nursing homes. [55] The average of two years is used for the rate of 42.4 per 1000 population age 65 and over. Population data for Multnomah County is from Portland State University. [56]

Caution is urged in comparing KP research population rates with national nursing home use rates, because survey and sample definitions may be inconsistent with those in this study regarding criteria for admission to ICFs.

The data is presented here as a flag for possible future research comparing HMO member use of nursing homes with non-HMO members. Table $X$ is not presented as baseline findings The overall $\mathrm{KP}$ rate of nursing home residents per 1000 research population members is thirty percent higher than the estimated rate for Multnomah County. Given the caveats and conditions for use, above, observations are limited to the following points: sample based estimates for the national rate is twenty percent lower than overall rates for KP research population members. 
TABLE X

NON-STANDARDIZED NUMBER OF NURSING HOME RESIDENTS PER 1000 RESEARCH POPULATION MEMBERS, AND PER 1000 POPULATION NCHS 1985 NATIONAL SAMPLE, AND PER 1000 POPULATION IN MULTNOMAH COUNTY AGE 65 AND OVER ESTIMATE OVERALI \& BY GENDER

\begin{tabular}{|c|c|c|c|}
\hline Model A & $\begin{array}{l}1985 \\
\text { National } \\
\text { SNF+ICF+? } \\
\text { Nurs. Home } \\
\text { /1000 Pop } \\
\text { (estimate) }\end{array}$ & $\begin{array}{l}1986-87 \\
\text { Mult. Cnty } \\
\text { SNF+ICF } \\
\text { Resident } \\
\text { /1000 Pop } \\
\text { (estimate) }\end{array}$ & $\begin{array}{l}\text { 1986-88 } \\
\text { Res. POp. } \\
\text { SNF+ICF } \\
\text { Resident } \\
/ 1000 \text { HPM } \\
(C+R+S)\end{array}$ \\
\hline $\begin{array}{l}M+F \\
\text { Male } \\
\text { Female } \\
65-74 \\
75-84 \\
85+\end{array}$ & $\begin{array}{r}46.2 \\
29.0 \\
57.9 \\
12.5 \\
57.7 \\
220.3\end{array}$ & $\begin{array}{l}42.4 \\
x \star \star \\
x \\
x \\
x \\
x\end{array}$ & $\begin{array}{r}55.3^{\star} \\
50.9 \\
58.2 \\
17.4 \\
64.5 \\
192.6\end{array}$ \\
\hline
\end{tabular}

The National rate is nine percent higher than that for Multnomah County. Policy analysis which attempts to compare non-HMO data with HMO data is outside the context of this study, but could be valuable information for policy makers interested in generalizing findings regarding the Expanded Care Benefit portion of the s/HMO Demonstration Project.

In order to obtain a comprehensive view of overall utilization rates, it is necessary to evaluate other use rates than that presented for Research Question I. The rate of choice for assessing nursing home utilization is days in 
S/HMO.

Research Question II asks whether differences exist between three eligibility groups regarding respective days of stay as an ICF resident during the study period. Units of measurement are cumulative days attributed to all members while residing in an ICF during the study period as Cost, or Risk, or S/HMO Medicare beneficiaries per member year of eligibility in Cost, Risk, or S/HMO KP eligibility groups.

Research Question II: Are There Differences Between S/HMO and Risk Member ICF Days in Residence During study Period/ Member Year of Eligibility?

Days of ICF residence are apportioned according to their overlay on periods of eligibility in Cost or Risk or S/HMO RP membership. If the resident's eligibility changed while in an ICF (or SNF), their days in residence are censored by such eligibility dates. No member was in an ICF residence across all three eligibility status even though some members were enrolled as Cost, and Risk, and s/HMO during the study period.

The unit of measure in tables presented under Research Question II is: [days of ICF stay per days of eligibility within each Medicare beneficiary group during the two year study period]. Conversion of that rate denominator is needed to create relative values for comparing Cost, Risk and s/HMO rates and because denominator values are so large. Thus [days of eligibility during the study period], become [per 
member year of eligibility].

Table XI presents overall rates for all members residing in an ICF or and SNF, or both, including: those in a nursing home at the start of the study period, those admitted and discharged during the study period, and those in a nursing home at the end of the study period. Periods of stay which crossed the beginning and ending dates of the study period were censored, accordingly.

TABLE XI

OVERALL RATES, STANDARDIZED BY AGE AND SEX, FOR DAYS OF NURSING HOME RESIDENCE DURING THE STUDY PERIOD PER MEMBER YEAR OF NOT MUTUALLY EXCLUSIVE ELIGIBILITY STATUS COST, RISR, S/HMO IN THE STUDY PERIOD.

Model A Male + Female Days
/Yr EIig
COST ICF SNF
Days /Yr Elig RISR

4.77

1.00

$$
0.92
$$$$
3.45
$$

0.88

0.94

Source: Appendix C

Based on the above rates, the answer to Research Question II is that overall age-sex standardized relative rates are significantly less for s/HMO than for Risk or Cost ICF residents, regarding days of stay per member year of group eligibility.

This is a very meaningful finding because it suggests that, while access to ICF care may have been enhanced by the 
S/HMO ECB program, S/HMO members were able to find other options more readily than Risk members, or that S/HMO members were able to leave sooner, or dependency on ICF services could be minimized. Chapter VI information suggests that a much higher proportion of $s /$ HMO members went home following ICF admission than Risk members.

In empirical terms, the ICF rate for cost residents was forty-three percent more than the ICF rate for $S / H M O$ residents; the ICF rate for Risk residents was thirtyeight percent more than the S/HMO ICF rate.

Little variation existed between SNF rates. The Risk rate was four and one-half percent above the s/HMO rate. These observations suggest that even though the probability of residing in an ICF was much higher for s/HMO members than other Medicare members, the time spent in an ICF by S/HMO nursing home residents was less than that of other Medicare members. This observation is also made regarding overall nursing home use. This finding has not been reported in the Iiterature on S/HMO site studies.

At least one report has identified the higher probability of ICF admissions for $s /$ HMO than non-s/HMO.

The observed pattern of use of institutional LTC contrasts sharply with patterns observed in national data regarding individuals not in sHMOs. The pattern displays more frequent short-stay admission to nursing homes and probably less frequent placements. [57]

One masters thesis (1992) identified this trend based 
on data limited to $\mathrm{S} / \mathrm{HMO}$ members in the last year of life who used nursing homes. "There was no significant difference in ICF utilization between the study populations in the last year of life, although the Plus II population showed a tendency for a higher mean ICF admissions (p-0.14) [than Plus I Risk] [58]. One study in a non-S/HMO setting reported a similar finding in a 1988 study of 3,316 residents of six Continuing Care Retirement Communities, (CCRC) which provided for nursing home care as a part of the financial investment of their members.

\begin{abstract}
A CCRC combines the finance and delivery of longterm care services within a single organizational context and insures against long-term care costs. [One of the findings reported in this study was that]...across all age categories, the lifetime risk of nursing home entry was greater among CCRC residents than among persons in the community... and across communities, the risk of entry was found to vary dramatically, suggesting that management decisions are critically important for controlling the use of nursing home services. The same study found that the length of stay per admission is shorter in a CCRC than in the general community...CCRC nursing home entrants were found to enter nursing homes twice as often as their counterparts in the general community. [59]
\end{abstract}

In the overall data set, ICF use rate patterns by gender are consistent with the expected lower rate for males and higher rate for females, as summarized below, in Table XII. The rate was thirty-six percent higher for Risk males than s/HMO males, and forty percent higher for Risk females than S/HMO Females.

The literature reports a greater likelihood of home 
support systems for males and females, suggesting that the female pattern is especially important.

TABLE XII

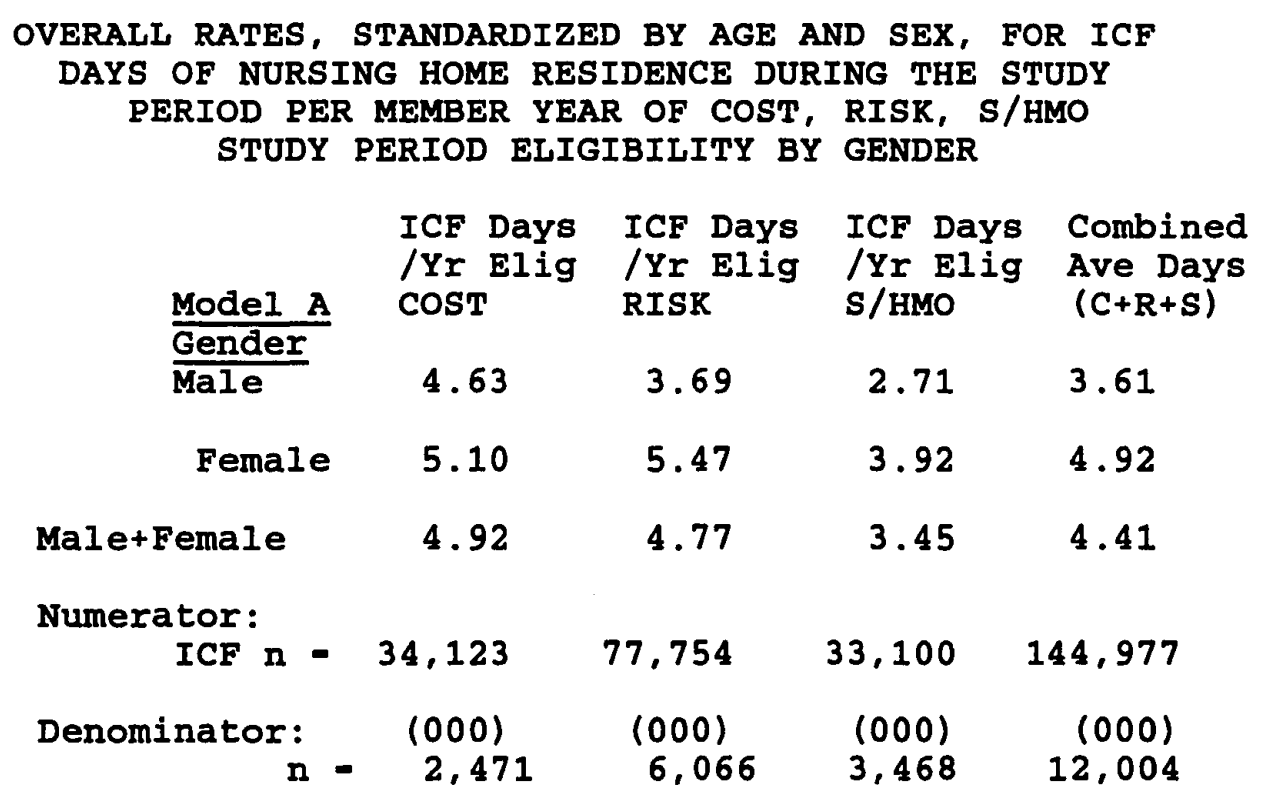

Source: Appendix C

Before examining age cohort patterns of nursing home use, a summary is provided of parameters for age within each eligibility group of ICF residents during the study period. Mean ages for ICF residents in the study period are:

Cost $=85.0$, Risk $=84.5, \mathrm{~S} / \mathrm{HMO}=82.9$. Median ages are: Cost -86.0, Risk -85.2, S/HMO $-83.4 . Q 3$ ages are: Cost = 80.6-90.4, Risk - 79.2-90.1, s/HMO = 77.7-87.8.

Among ICF users, the first and third interquartile mean value $(Q 1, Q 3)$ of ages for $S / H M O$ members were 1.5 years younger than Risk at $Q 1$, and 2.3 years less than Risk ICF 
users at Q3. Median age is 1.8 year less, and mean age is 1.6 years less for S/HMO than for Risk ICF residents. An examination of non-standardized (Raw) ICF rates by age cohort is presented in Table XIII, below.

\section{TABLE XIII}

OVERALL RAW RATES BY AGE \& SEX FOR ICF DAYS OF NURSING HOME RESIDENCE DURING THE STUDY PERIOD PER MEMBER YEAR OF COST, RISK, S/HMO ELIGIBILITY STATUS IN THE STUDY PERIOD BY AGE COHORT

Model A (RAW) Age Cohort 65-74 $75-84$ $85-105$

\begin{tabular}{|c|c|c|c|}
\hline $\begin{array}{l}\text { ICF Days } \\
\text { /Yr Elig } \\
\text { COST }\end{array}$ & $\begin{array}{l}\text { ICF Days } \\
\text { /Yr Elig } \\
\text { RISR }\end{array}$ & $\begin{array}{l}\text { ICF days } \\
\text { /Yr Elig } \\
\text { S/HMO }\end{array}$ & $\begin{array}{l}\text { Combined } \\
\text { Ave Days } \\
(C+R+S)\end{array}$ \\
\hline 0.66 & 0.66 & 0.81 & 0.70 \\
\hline 5.69 & 5.57 & 3.84 & 4.58 \\
\hline 20.71 & 22.81 & 13.62 & 19.73 \\
\hline
\end{tabular}

Source: Appendix C

The S/HMO raw rate for age $65-74$ is twenty-three percent greater than that for Risk. The Risk rate is fortyfive percent greater than that of $\mathrm{s} / \mathrm{HMO}$ in age cohort 75-84 and sixty-seven percent greater than that of $s /$ HMO in age cohort 85-105. Thus, in the overall data set, s/HMo rates are consistently less than Risk rate in each age cohort and gender cell.

As noted at the beginning of this chapter and as discussed in Chapter III, overall rates are contrasted with Subsets I and II, presented in Table XIV, below, in order to see how patterns change when residents with prior nursing 
home admissions are removed. Figure 5 presents data from Tables XI and XIV, following the latter table.

In Subset II, the s/HMO rate of days in residence per year of eligibility is ten percent less than that of Risk and eight percent less than the cost rate for ICF members admitted during the study period. Thus, use rate differences between Risk and $\mathrm{S} / \mathrm{HMO}$ are not as disparate after removal of those 71 residents ( 34 Cost, 33 Risk, 5 s/HMO) in an ICF at the beginning of the study period who were not readmitted during the study period. It appears that Risk and $S / H M O$ rates were the same under these conditions of comparison.

TABLE XIV

USE RATES, STANDARDIZED BY SEX AND AGE COHORT FOR ICF DAYS IN SP PER HPM YEAR OF ELIGIBILITY BY COST, RISK, S/HMO ELIGIBILITY STATUS

$\begin{array}{llll}\text { Model A } & \text { ICF Days } & \text { ICF Days } & \text { ICF Days } \\ \text { Male }+ \text { Female } & \text { /Yr Elig } & \text { /Yr Elig } & \text { /Yr Elig Ave } \\ \text { COST } & \text { RISR } & \text { S/HMO Combined }\end{array}$

$\begin{array}{lllll}\text { Subset } I & 2.26 & 2.58 & 2.67 & 2.56\end{array}$

$\begin{array}{lllll}\text { Subset II } & 3.45 & 3.51 & 3.19 & 3.41\end{array}$

Source: Appendix C

Subset II stili included other residents who had admissions before the study period. In subset $I$, the S/HMO rate was three percent above that for Risk. In subset I all members with prior nursing home experience are removed. 


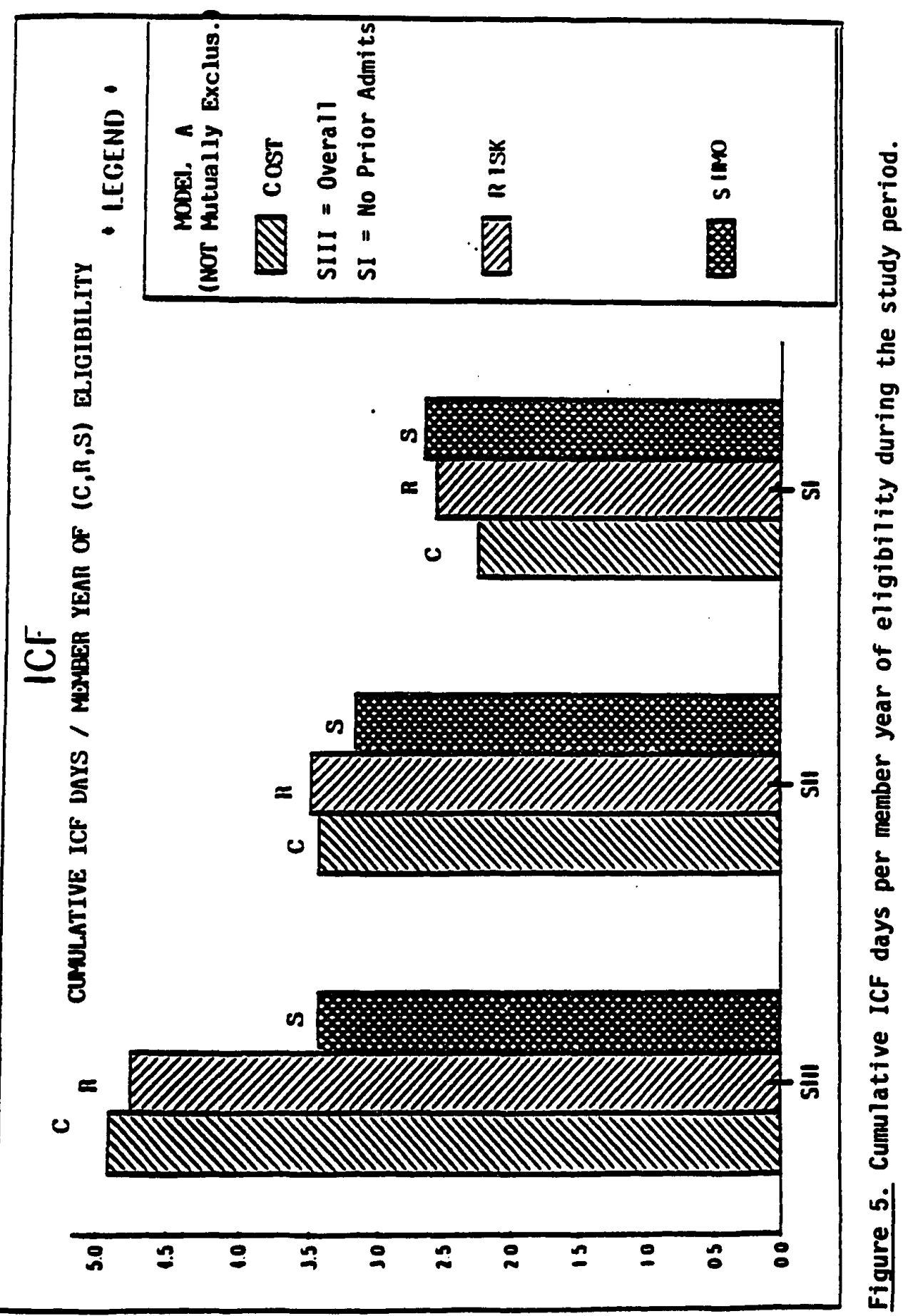


Because information in Chapter VII suggests that s/HMO days may be less than Risk when the year following the study period is considered.

This underscores an important policy research issue when doing policy analysis about nursing homes; lifetime use patterns are likely to differ significantly from those observed in shorter term patterns. Caution is emphasized about formulating policy on one time rates such as those in the National Nursing Home Surveys or annual state surveys. Differences within Risk and $\mathrm{s} / \mathrm{HMO}$ are important observations in the table below. Specifically, the magnitude of change in use rates between subset II and subset I shows that the S/HMO rate reduced by only half that of $\mathrm{Risk}$ in Table XV, below. The change in rates between subset II and Subset I, below, was nineteen percent for S/HMO, while the change in rate between subset II and subset I was thirty-six percent for Risk.

A similar pattern is observed for raw rates by age cohorts. Raw annualized use rates within age cohort display two patterns of special interest regarding differences between subset I and subset II for Risk and S/HMO days used during the study period per member year of eligibility. The first pattern shows that $\mathrm{s} /$ HMO rates are greater in the two younger age cohorts but less in the oldest age cohort in both subsets I and II, as seen in Table XV, below. Within age cohort $85+$, s/HMO is twenty-eight percent below Risk. 
TABLE XV

RAW RATE FOR ICF DAYS PER MEMBER YEAR OF ELIGIBILITY BY MEMBERS IN COST, RISK, OR S/HMO ELIGIBILITY STATUS BY AGE COHORT

Model A

$\frac{\text { Age } 65-74}{\text { Subset I }}$

subset II

Age 75-84

Subset I

Subset II

Age $85+$

Subset I

Subset II

Source: Appendix C
ICF Days ICF Days ICF Days

(RAW)/Yr Elig/Yr Elig/Yr Elig $\cos T$

RISR S/HMO

Average

$\begin{array}{llll}0.26 & 0.56 & 0.56 & 0.50\end{array}$

0.34

0.61

0.77

0.60

$$
2.32
$$

2.50

3.52

2.77

3.74

3.33

3.76

3.54

10.67

11.56

9.01

10.64

15.77

16.41

11.83

14.96

Within Subset II, this rate for $\mathrm{S} / \mathrm{HMO}$ age 85 and over is thirty-nine percent less than Risk. The oldest $\mathrm{s} / \mathrm{HMO}$ members use less ICF services, collectively, than their counterparts in Risk. This pattern is recurrent in all other rate comparisons. Some variable(s) is/are affecting ICF use rate patterns for "old old" $s /$ HMO members which is not affecting "old old" Risk ICF residents, or at least not so it is visible in measurement rates used here.

The opposite trend occurs between subsets I and II in age cohort 75-84 and this pattern also prevails under all tests of difference. Specifically, this rate is forty-one 
percent greater for S/HMO than for Risk ICF users in Subset I, and thirteen percent greater for subset II.

Within S/HMO age cohort $75-84$, the rate decreases thirty-one percent between subset II and subset I; within Risk the rate decreases forty-two percent between subsets I and II.

These two patterns. and trends form a recurrent observation throughout all remaining analysis in this study, including analysis of Medicaid rates and analysis of expenditures for nursing home care. The above table suggests that the ECB program could be managing different age cohorts differently. Or, different needs may exist in each age cohort for Risk members than for s/HMO members.

Several other views of this changing pattern are presented. First, raw rates for days of stay are compared by days per research population member. Rates, for ICF days used per research population member in Table XVI, follow the same trends as the pattern observed for the preceding rate, days used per member year of eligibility. However, there is an important difference. The magnitude of difference between Overall data set values and those of subsets I and II is much less. Days of eligibility were selected over members as the denominator because the latter should give a better indicator of the opportunity of having days in a nursing home relative to the days in each eligibility over which that event was occurring. At least the above table confirms 
that the pattern prevails under both denominators of members in the eligibility group and days of eligibility.

TABLE XVI

ICF DAYS USED PER RESEARCH POPULATION MEMBER

BASED ON RAW DATA FOR SUBSETS I AND II

AND OVERALL DATA SET OF ICF RESIDENTS

IN NOT MUTUALLY EXCLUSIVE

COST, OR RISR, OR S/HMO

ELIGIBILITY STATUS

Model A $\underline{\text { Male +Female }}$ ICF Days /member $\cos T$

ICF Days /member RISK

ICF Days /member Ave s/HMO Combined

overall

5.52

6.74

5.25

6.04

subset II

3.88

4.97

4.84

4.66

Subset I

2.56

3.68

4.05

3.49

Source: Appendix C

Raw data is used to give another view of utilization differences for Risk and S/HMO ICF residents by subsets I. II. Descriptive parameters of mean, median and third interquartile range days of stay are shown in Table XVII, below for Cost, Risk and S/HMo groups in Model A. In fact, the values shown in the third interquartile range of days of stay may be an indication of what is taking place among among $s /$ HMO members that accounts for the trends observed in the preceding two tables.

The length of stay (LOS) variance is substantially less among $\mathrm{s} / \mathrm{HMO}$ members than among Risk or cost members. But, more important, is the pattern of descriptive information 
about S/HMO members; its central tendency is closely formed at 100 days, similar to that allowed by the ECB, then.

TABLE XVII

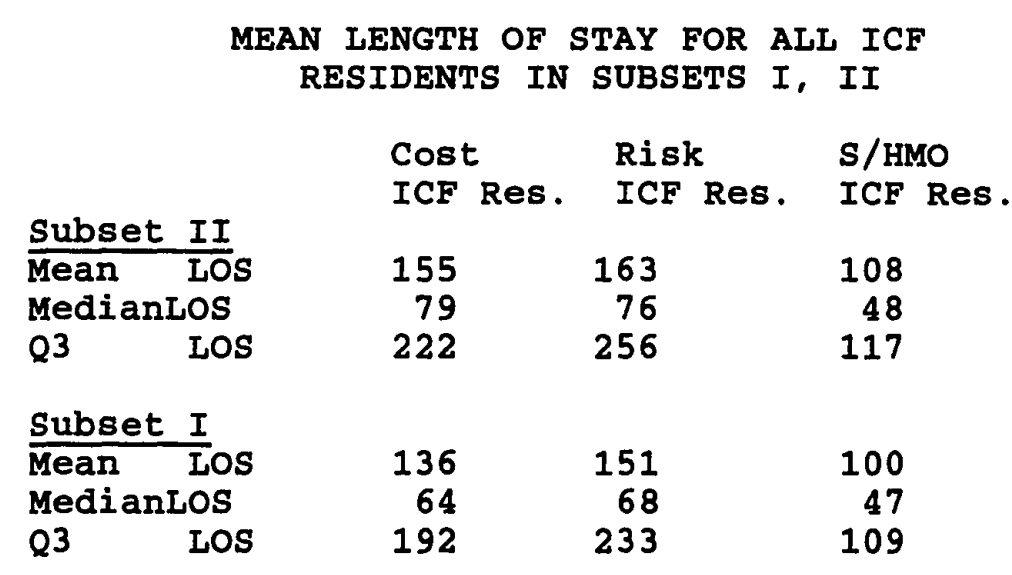

Note: numbers are rounded to nearest whole number.

Substantially less LOS variance about the S/HMO mean, than about the Risk mean LOS, reinforces the notion that use rates for S/HMO were influenced by the ECB benefit level of 100 days maximum. Since this is a two year study period, the question needs pursuing about whether the mean LOS of 100 days is a lifetime pattern for that subset of s/HMO users or simply coincidence which would change over a longer period of time. In policy issue terms, is this rate mainly a product of balancing managed care practices with health care needs or is it a chance observation? If not the latter, this is an encouraging finding about the policy concern for the manageability of insured LTC. The 100 day limit was a non- 
renewable benefit from one year to the next unless out of an ICF and in a home while on ECB benefits for 60 days or more. These LOS data observations lead to another important evaluation process of differences in use rates. That is, how do time periods of stay differ when cumulative days are clustered according to notions of shorter-stay and longer stay. A policy issue of some interest is the basis for this evaluation step. There is some debate about whether the ECB days of nursing home coverage (100 days) is sufficient? The policy issues is, how long should such a benefit provide for in order reasonably protect most shorter-stay nursing home residents against using all their personal assets for expensive nursing home costs? One test of this question is what proportion of days in ICF residence would be covered, on average, by 100 days?

As seen in the above table of mean and median lengths of stay, mean and $Q 3$ LOS are very close to the 100 day limit of non-renewable ECB for nursing home use. Seventy percent of S/HMO ICF residents had 100 or fewer ICF cumulative days of stay during the two year study period. Ninety-one percent of S/HMO ICF residents use 200 days or less during the study period. Table XVIII, below, provides a better understanding of the variance in LOS within and between S/HMO and Risk nursing home users. Fifty-six percent of Risk ICF residents had 100 or fewer ICF cumulative days of stay during the two year study period. 
TABLE XVIII

OVERALL DAYS OF STAY BY TIME PERIOD CLUSTERS DURING THE STUDY PERIOD FOR ICF, SNF, SNF+ICF

\begin{tabular}{|c|c|c|c|c|c|c|}
\hline \multirow[b]{2}{*}{ Days } & \multicolumn{2}{|c|}{$\begin{array}{l}z \text { of } \\
\text { Total } \\
\text { ICF Days }\end{array}$} & \multicolumn{2}{|c|}{$\begin{array}{l}\& \text { of } \\
\text { Total } \\
\text { SNF Days }\end{array}$} & \multicolumn{2}{|c|}{$\begin{array}{l}8 \text { of } \\
\text { Total } \\
\text { SNF+ICF }\end{array}$} \\
\hline & Risk & SHMO & Risk & SHMO & Risk & SHMO \\
\hline $1-30$ & 31.28 & 37.98 & 54.08 & 52.38 & $36.4 \%$ & 38.68 \\
\hline $31-100$ & 24.98 & 32.38 & $36.8 \%$ & 38.08 & 29.18 & 32.18 \\
\hline $101-200$ & 13.08 & 12.48 & 4.28 & 6.88 & 10.18 & 14.18 \\
\hline $201-365$ & 13.98 & 8.518 & $3.8 \%$ & $1.7 \%$ & $11.4 \%$ & $7.8 \%$ \\
\hline $366-730$ & 17.08 & 8.98 & 1.28 & 1.28 & 12.78 & 7.38 \\
\hline
\end{tabular}

That is, over fourteen percent fewer Risk ICF residents would have been within the 100 day limit. A policy issue is, would a higher proportion of Risk ICF residents have used fewer total ICF days had they had ECB benefits?

Among those S/HMO residents further analysis is needed to determine if they were concurrently using there ECB, or if they used it all, for community based home care? Among Risk and S/HMO the analysis of ICD hospital discharge codes used in Chapter IX needs to be applied to this subset, together with ADLs, which need to be obtained for the Risk Residents.

An analysis of total days of ECB eligibility is needed for the $\mathrm{s} / \mathrm{HMO}$ subset of under 100 days. This would require a cross match of nursing home admission and discharge dates 
with dates of recertification for ECB.

The subset of $\mathrm{S} / \mathrm{HMO}$ members who also were Risk member needs to be cross-matched with $s /$ HyO users remaining under 100 days to determine how many used their $\mathrm{ECB}$ benefits and reverted to Risk; in the study period that appears to have occurred rarely; confirmation is needed. Winety-one percent of S/HMO ICF residents used 200 days or less during the study period; eighty-three percent of Risk members required 200 days or less of ICF stay, as seen in Figure 6, next.

SNF rates are nearly identical when grouped. Ninety percent of both Risk and S/HMO members stayed 100 days or less. This suggests that utilization control was good. The real question for policy purposes is, what proportion of SNF patients needed to use the full 100 days of SNF care? These values are too close to the Medicare benefit limits to not suspect a correlation between benefits and discharge.

Eight-seven percent of Risk nursing home users remained, or used fewer than 366 days out of the 730 potential SNF and ICF days during the study period. A high proportion of elderly research population members were eligible during that entire time. Ninety-three percent of S/HMO nursing home residents used less than the equivalent of one year of nursing home days. Since a high proportion of longer stay patients had multiple admissions, these Los data do not necessarily reflect continuous stay. 


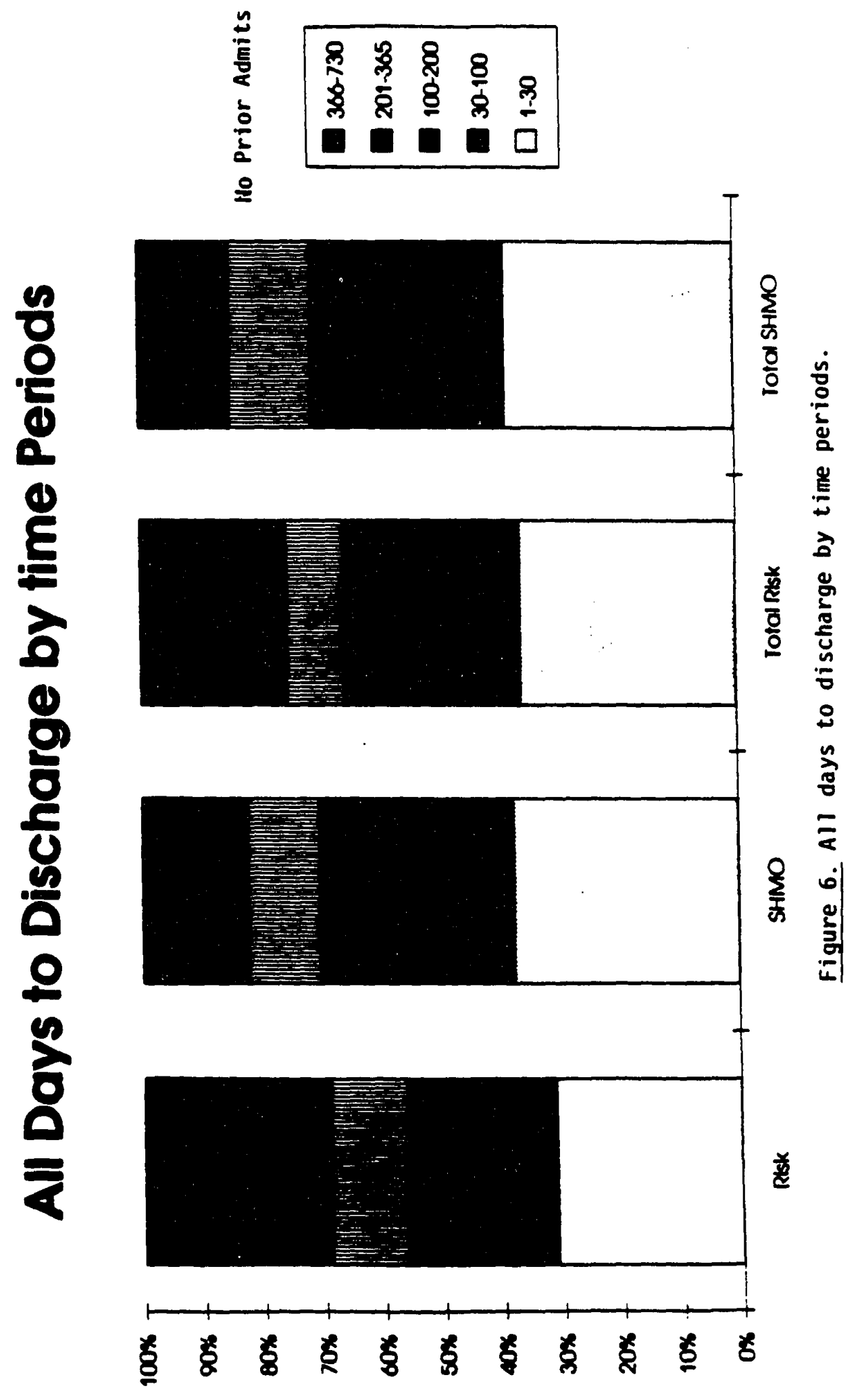


In September, 1990, the first definitive study of

estimates regarding cumulative days of nursing home stay, based on sample histories of multiple facility and multiple admissions data. The findings presented in the above tables, based on absolute rather than sample data, show lower patterns of nursing home utilization than has been estimated for the nation. "Nearly two-thirds of nursing home stays, however, have been for 6 months of less and only about 16 percent of persons stayed for longer than two years." (op. Cit. GAO [20] p. 12). Another view of LOS patterns is available from the Urban Institute.

Until 1985, the NNHS length of stay information consisted only of one isolated stay per nursing home resident. Consequently, if an individual had more than one nursing home stay, those days could not be linked to analyze patterns of multiple admissions... Our second aim is to demonstrate the importance of accounting for multiple admission when estimating the number of people who would be affected by front-end policies such as those proposed by the former Commissioner Ball and the Pepper Commission.. The median sample stay of the 65-74 age group (74 days) is 22 days shorter than that of age group $85+$ (96 days), the difference increases to 51 days after adjusting for multiple stays... of the short stay group with no prior days, 33 percent were nursing home deaths [we included persons who were known to have died in a hospital after discharge from a nursing home], and 35 percent were discharged alive to a private residents...individuals with minimum stays of one year have a negligible chance $\{5 \%\}$ of returning to a private residences noted, limitations of the NNHS do not allow us to fully capture the incidence of multiple admissions, either from the perspective of lifetime nursing home use or episode of illness surrounding the sample... [a simulated model was developed to estimate true comprehensive nursing home use patterns by 
adjustment for multiple admissions indicates that 25 percent fewer persons would be fully covered by the three-month front-end policy [recommended by the Pepper Commission] ...paraphrase...12 $\%$ stayed 90-180 days and 180-365 days each in the simulated model."[60]

Using the Liu and Perozek simulated model of estimated cumulative days of nursing home stay as the baseline of comparison for Risk and S/HMO SNF+ICF data, suggests that 15 percent more Risk residents and 20 percent more S/HMO residents accumulated less than 100 days of nursing home stay that the model suggests. It also suggests about 28 more more of the sample model remained 90-180 and 180-365 days, than was observed in this dissertation study of the true population.

The Connecticut Nursing Home Data System study, by Liu and Manton did an analysis of the complete length of stay distribution on an admission cohort of nursing home patients in that state. Even that data base, which is perhaps the most complete state wide data base on nursing home use in America, required estimates for missing information related to left (entry) and right (truncated tail of the cohort distribution) censoring. Their model estimates the cumulative discharge rate to be 34 percent at 90 days, and 43 percent at 180 days and 52 percent at one year. [61]

Several factors could affect the difference between the Liu and Manton model of Connecticut data and this study. It may be that the two year study period falsely censored many 
Risk and $\mathrm{s} / \mathrm{HMO}$ members whose true cumulative days of stay would be longer when examined. Such examination is entirely feasible since all utilization and financial data was collected on all nursing home members prior to the study and up to one year after.

Another nursing home care model-building issue exists which is unique to the S/HMo research population, at least at the KP S/HMO Demonstration site. This issue relates to some proportion of members being eligibility for ECB services on an intermittent basis. That does not necessarily mean they were not eligible for $\mathrm{ECB}$ while in a nursing home, but it could be a consideration in differentiating possible effects of S/HMO ECB on ICF use. In this $\mathrm{s} /$ HMO research environment any model which is created to estimate comprehensive lifetime use rates must also consider eligibility status for both the benefit and eligibility groups. That adds some serious complications to the model building effort. [62]

The S/HMO ECB allowed up to 100 days beyond what Medicare covered in either an SNF or ICF during the study period. That suggests the likelihood of some interaction between SNF and ICF use rates, beyond what exists for persons not having sHMO benefits. One policy issue arising from this situation is whether the comprehensive use of nursing home services is different for S/HMO that Risk? The research question pursued next is whether the ICF 
pattern observed above differs from use rates for combined SNF and ICF stay? In the next analysis, Table XIX, that question is examined on a group basis; that is persons with only SNF care are included in the rates as well as persons with both SNF and ICF stays.

Among shorter-stay residents, it is not always easy to differentiate between persons qualifying for SNF care and those belonging in ICF care. Making that differential determination sometimes requires a lengthy period during which a patients condition is stabilizing. For this reason it is well to begin this evaluation from an overall use rate perspective.

overall, the s/HMO rate, above, is thirty-two percent less that that for Risk, and is seven percent less than Risk in subset II. In subset I Risk is nine percent below that of S/HMO for combined SNF+ICF nursing home use.

once again, the reversal of the use rate patterns in Subset I suggests the need for analysis of utilization data following the study period. Analysis of payments receives suggests that the $S$ /HMO rate in subset $I$ should also be less than that for Risk.

The observation inferred by the above rates is that the trend of effects of S/HMO ECB on Overall nursing home use rates is similar to that for ICF only, but the magnitude of differences are less. Based on the earlier comparison of SNF rates this is not surprising. 
TABLE XIX
USE RATES, STANDARDIZED BY SEX AND AGE COHORT FOR SNF+ICF DAYS IN SP PER HPM YEAR OF ELIGIBILITY BY MEMBERS IN NOT MUTUALLY EXCLUSIVE COST, RISK, OR S/HMO ELIGIBILITY STATUS

$\begin{array}{lllll}\text { Model A } & \begin{array}{l}\text { SNF+ICF } \\ \text { NH Days } \\ \text { /Yr Elig } \\ \text { COST }\end{array} & \begin{array}{l}\text { SNF+ICF } \\ \text { NH Days } \\ \text { /Yr Elig } \\ \text { RISK }\end{array} & \begin{array}{l}\text { SNF+ICF } \\ \text { NH Days } \\ \text { S/HElig } \\ \text { S/HMO }\end{array} & \begin{array}{l}\text { SNF+ICF } \\ \text { /Yr Dlig } \\ \text { Combined }\end{array} \\ \text { Overall } & 5.95 & 5.69 & 4.32 & 5.35 \\ \text { Subset II } & 4.22 & 4.34 & 4.07 & 4.23 \\ \text { Subset I } & 2.84 & 3.17 & 3.45 & 3.19 \\ \text { Source: Appendix C } & & & \end{array}$

\section{SUMMARY OF FINDINGS}

Two Research Questions were addressed in this chapter. The findings for both show that ICF utilization was different between $\mathrm{S} / \mathrm{HMO}$ and Risk members during the study period. Specifically, Overall rates in Research Question $I$, indicates that $\mathrm{s} / \mathrm{HMO}$ members were much more likely to become ICF residents, but the overall rate in Research Question II, shows that S/HMO used substantially fewer ICF days than Risk members. Thus, the inference is that the ECB facilitates access to ICF services that they may not have enjoyed, but also facilitates both effective management of that access by case management which can offer a home based alternative to institutional care as a means of meeting the elderly members LTC needs. 
Subset I has the effect of removing residents whose days of stay spanned the study period and may have included many months or years on either side of the twenty-four month window of analysis. Subset I also has the effect of removing one of the study period censoring factors, wherein length of stay is potentially terminated, unnaturally, on one end. However, it also reduces the use rate pattern more likely to surface from a lifetime of nursing home needs. Subsets $I$ is introduced as a tool with which to surface questions about the overall rate patterns.

In age cohort $85+$, the $\mathrm{s} / \mathrm{HMO}$ rate is consistently less than Risk under all conditions. This suggests that S/HMO ECB may be affecting the oldest age cohort differently than younger s/HMO members.

Mean lengths of stay were less for S/HMO than for Risk members and variance about the mean is considerably less, suggesting that the 100 day ECB limit was an influence in ICF use rates.

Patterns of stay demonstrate that more $\mathrm{s} / \mathrm{HMO}$ residents remained in nursing homes (SNF+ICF) for fewer cumulative days than Risk members. Given the time-span of this study that is only a tentative pattern, but the trend is important because it reinforces the observations from use rates that $s /$ Ho member's use of nursing homes is influenced in ways not observed for Risk members, which infers that the s/HMO $E C B$ must be considered as a a strong influence in member 
decisions about home based vs. institutional based care. No doubt, there were other organizational influences which affected utilization of S/HMO ICF residents, as well as socioeconomic and health factors.

DISCUSSION: EXTERNAL ISSUES POTENTIALLY AFFECTING USE RATES

Why aren't differences in use rates constant between the three criteria for which rates are produced, i.e., (1) all residents in study period; (2) those admitted during the study period, who had a history of SNF/ICF admissions before the study period; (3) residents whose initial ICF admission was during the study period? Is it due to magnitude of rate changes in S/HMO, or Risk or both?

The likely answer to higher S/HMO rates in subset I is that as case management skills improved, members certified for nursing home care who were less dependent, were placed at home, leaving a higher proportion of longer-stay placement. Eliminating $\mathrm{S} / \mathrm{HMO}$ members with nursing home stay prior to the study period probably would have eliminated the shorter-stay, multiple admission residents.

At the same time, there were other organizational and environmental events taking place which were more likely to affect the $R$ isk ICF rates than $s /$ HO rates. It was a dynamic period, regarding nursing home utilization.

In fact, at least three other policy events were taking place, which deserve noting, that could have affected use 
rates, especially those for Risk members. They were not measured or otherwise studied as a part of this research project. Their affect on Risk and s/HMO use rates would have been strongest in the latter half of the study period. That was also a time when SHMO member use of nursing homes was increasing. Tighter controls over nursing home use would have a greater influence on subset I than the overall data set because

First, during the study period, HCFA regulations were clarified, regarding capitation payments, to include higher monthly reimbursement for Risk Medicare members in nursing homes, including those in ICF and residential or foster level care facilities. This higher capitation payment recognized greater expenses incurred by HMOs from caring for members in nursing homes. KP also recognized it needed to do a better job of managing nursing home expenses.

Although KP was a model for hospital utilization review, it had not applied that model to $\mathrm{KP}$ members in nursing homes. Beginning in 1985, such a program was pilot tested using one geriatric nurse practitioner to do on-site review for level of care needs. This supplemented Medicare's requirement of initial and quarterly physician review for SNFs, and annual review for ICFs. By late 1987 and early 1988, that program was expanded to include three nurse practitioners to do ICF review; it was not uncommon that many of the $600+$ ICF patients were reviewed quarterly, and 
in some cases monthly.

Second, KP hospital emergency rooms developed lists of elderly persons who frequented ERs for problems which could be stabilized or resolved quickly, including short term use of in home services by community based care givers. Such persons might otherwise have been placed in an ICF, or other suitable institutions.

Third, by the end of the first year in S/HMO, SNF costs were escalating rapidly. This may have led to more intensive utilization review of S/HMO members in SNFs, resulting in an increasing substitution of ICF care for SNF care, when ever needs of such S/HMO members could be safely and adequately met at the lower level of licensed staffing required of ICFs.

How these utilization activities contributed to the pattern of changes in rates observed for the overall data set of ICF users, is not known, but they were part of the changing process of managing long term care needs of KP members during the study period.

If these events are as described, it only strengthens the Overall data set findings for Research Question II. That is, even under an environment of increased use of ICFs, and under the most conservative measure of ICF use rates, no difference in days used per member year of eligibility was observed between S/HMO and Risk eligibility groups. And, under the overall use rates, s/HMO nursing home residents 
clearly used fewer ICF days per year of member eligibility than did Risk members who resided in ICFs.

POLICY RESEARCH OBSERVATIONS REGARDING ICF USE RATES

Answers to Research Questions I and II raise some key issues about operational implementation of a s/HMO model. one issue is, under what conditions is it operationally rational to expand a Medicare Supplemental health care benefit so it includes some reasonable amount of ICF level long-term care services, without leading to an economically untenable affect on the financial well being of the HMO? Another issue is that an Expanded Care Benefit does not, a priori, lead to inflationary use of ICF services, given the conditions under which use rate differences were measured in this study. The fact that $\mathrm{s} / \mathrm{HMO}$ members were more likely to become ICF residents did not materialize into more ICF days in residence. This suggests the possibility that a managed care system can successfully manage ICF use rates, probably even more rigorously than initially managed by $\mathrm{KP}$ and $\mathrm{S} / \mathrm{HMO}$ case coordinators.

From a national policy perspective, the outcome of rates for Research Questions I and II should offer encouragement about enlarging s/HMO Expanded Care Benefit trial programs wherein a rigorous test of difference between trial and control groups is conducted including comparison of the community based component of an ECB. 
The next chapter examines rate differences in Risk members who pay Medicare Supplemental Benefit dues out of pocket compared to those whose dues (premium) are paid via a group sponsor. It also examines differences in use rates between Risk and $\mathrm{S} / \mathrm{HMO}$ members who are enrolled from the community in contrast to those who converted from within KP. And, differences within Risk membership are viewed regarding those who rolled over into TEFRA Risk status from Medicare Plus Demonstration Risk status. Did these factors influence Risk or S/HMO rates in a way which warrants caution about findings regarding overall ICF use rates, presented above? 
CHAPTER V

USE RATE DIFFERENCES BASED ON SOURCE OF ENROLLMENT

The [Medicare Plus Prospective Payment] project stimulated a new planning process for geriatric care and fostered the development of the Social HMO demonstration (Medicare Plus II) that began serving serving Medicare beneficiaries with expanded in-home support service benefits in March 1985. [63]

Within the Risk research population group there were Medicare members $(2 / 3)$ who paid the Supplemental Benefit directly to KP, and there were others $(1 / 3)$ whose supplement Benefit premium was paid by a group retirement program sponsor. This chapter compares S/HMO members, all of whom paid their premium directly to RP, with those Risk members who also paid RP directly, as individual subscribers.

Within S/HMO and individual Risk eligibility groups, there were two basic categories of enrollees; those who converted from Cost status within KP and those who enrolled directly from the community and were new to KP. This chapter compares new and converted rates for $\mathrm{Risk}$ and $\mathrm{S} / \mathrm{HMO}$ members.

Also, a substantial portion of the direct pay Risk membership was comprised of transfers for the Medicare Plus Demonstration project. They were not allowed to become s/HMO members during the study period, or until 1989. Rates for individual Risk and $S / H M O$ members are compared next, in 
order to answer Research Questions Ia and IIa.

Research Question $I_{1}$ : what Differences Existed Between Individual Risk Members and S/HMO Members Regarding the Probability of Becoming An ICF Resident Per 1000 Members?

Table Xx, below, shows standardized use rates for this Research Question. SNF rates are also presented.

TABLE XX

(RATES ARE STANDARDIZED BY AGE COHORT WITHIN GENDER) NUMBER OF ICF \& SNF RESIDENTS PER 1000 RISK \& S/HMO INDIVIDUAL MEMBERS, SEPARATED BY "NEW" AND "CONV" STATUS AT TIME OF ENROLLMENT IN RISK OR S/HMO ELIGIBILITY STATUS

\begin{tabular}{lllll} 
Model A & $\begin{array}{l}\text { Risk } \\
\text { New }\end{array}$ & $\begin{array}{l}\text { Risk } \\
\text { Conv }\end{array}$ & $\begin{array}{l}\text { S/HMO } \\
\text { New }\end{array}$ & $\begin{array}{l}\text { S/HMO } \\
\text { Conv }\end{array}$ \\
Male + Female & $/ 1000$ & $/ 1000$ & $/ 1000$ & $/ 1000$ \\
\hline Individual ICF & 37.34 & 30.26 & 43.94 & 46.37
\end{tabular}

Overall [Total Risk] $\quad 31.61$ [Total s/HMO] 42.40

$\begin{array}{lllll}\text { Individual SNF } & 32.36 & 16.35 & 28.49 & 26.85\end{array}$

Overal1 [Total Risk] 23.27 [Total s/HMO]

26.78

(Individual)

ICF numerator denominator $168 \quad 118$ 4194

131 2913

Source: Appendix D

No residents are included in the rates who were in a nursing home at the beginning of the study period. Risk rates presented in this chapter exclude numerator and 
denominator values for "group" members, and include only "individual" Risk member values.

s/HMO members were about thirty percent more likely to become ICF residents than individual Risk members. Both new S/HMO and converted S/HMO were more likely to reside in an ICF than either individual new or converted Risk members. This is consistent with previous findings in which group and individual Risk members are compared with s/HMO, regarding risk of ICF admission.

Individual new Risk and new $\mathrm{s} / \mathrm{HMO}$ members were twenty-three percent more likely to become ICF residents during the study period than were converted Risk members. New S/HMO members were six percent less likely to become ICF residents during the study period than were converted $\mathrm{s} /$ HMO members. This is important because a finding of little difference suggests that s/HMO sampling procedures used to control against adverse selection bias resulted in a wide cross section of the community, rather than attracting many persons expecting to access and use ICF services. "If subscribers representing higher than average risk choose the plan, then that plan is said to have experienced adverse selection from the group in question. "[64]

Similarly, new Risk members were ninty-eight percent more likely to become SNF residents during the study period than were converted Risk members. New S/HMO members were 6.18 less likely to become SNF residents during the study 
period than were converted Risk members.

The broad policy concern addressed in this chapter is about the uncertainty of effects on an ECB program which enrolls new Medicare members directly into a capitation program on the basis of receiving a LTC benefit.

Table XXI shows that removal of group Risk members from the data set affects $\mathrm{Risk}$ rates in age cohort 75-84 by raising the Risk rate to that of S/HMO. Apparently, more group Risk members were younger than non-group Risk members.

TABLE XXI

RAW RATES BY AGE COHORT FOR ICF RESIDENTS PER

1000 HPM SUBSET FOR: NEW AND CONVERTED

$S / H M O$ AND INDIVIDUAL (NON-GROUP)

RISR KP MEMBERS

\begin{tabular}{lllll} 
& \multicolumn{2}{c}{ Individual } & \\
Risk & Risk & S/HMO & S/HMO \\
Model A & New & Conv & New & Conv \\
Male + Female & $/ 1000$ & $/ 1000$ & $/ 1000$ & $/ 1000$
\end{tabular}

age 65-74

RAW (ICF)

17.98

3.61

14.13

14.48

age $75-84$

$\overline{\mathrm{RAW}}$ (ICF)

42.20

28.04

55.51

51.92

age $85+$

$\frac{\text { RAW }}{\text { (ICF) }}$

$114.80 \quad 163.00$

123.63

152.02

Source: Appendix D

Specifically, comparison of rates for probability of admission of individual Risk and $\mathrm{S} / \mathrm{HMO}$ members shows the same pattern of reduced use of ICF and SNF services for age cohort 85 new members, compared to both converted members 
and to new members in age cohort 75-84. This pattern was not observed when Risk Group members were included. S/HMO rates exceed those of individual Risk members in age cohorts 65-74 and 74-85.

Overall differences between age cohorts 75-84 and 85+ may simply represent differences in nursing home placement efforts by families, providers, or welfare case managers. or, it may be that those who survive age cohort 75-84 simply have different states of disability and dysfunction, or/and their dependencies can be satisfactorily managed in non-nursing home surroundings.

The similarity of rates for new Risk and $s /$ HMO in age cohort $85+$, and higher rate for $s / H M O$ than Risk in age 75-84 was observed in the overall rates presented in Chapter IV. Within s/HMO there is little rate difference that suggests new members needed more ICF services than converted s/HMO members. In fact, among the group most likely to use ICF services, age $85+$, converted $\mathrm{S} / \mathrm{HMO}$ members were 238 more likely to reside in an ICF during the study period than were new $\mathrm{S} /$ kMO. Interestingly, that same observation is made about their respective SNF use rates.

A more focused policy question arises about the above patterns regarding the need for research about the effects of early screening on ICF access. This is really an issue of how these two groups were accessing care If converted members with established physician relations were ending up 
in nursing homes and then notifying S/HMO, that was circumventing the screening and case management process. If new members, without established physician relationships were identified by early screening and intervention was occurring before nursing home entry occurred, then the s/HMO system was working better for new members than converted members .

Within Risk there is a notable difference in rates between new and converted Risk members which suggests that age cohort 65-84 new Risk members needed ICF services more than converted members, while new Risk members age $85+$ had less need for ICF services converted "old-old" Risk members. That is not observed for SNF level care.

Research Question IIa: Are There Difference In ICF Days Used During The Study Period Per Member Year of Eligibility Between S/HMO and Individual Risk Members?

In the Table XXII, below, Cost rates are removed. Overall, s/HMO members resided in ICFs significantly fewer days per year of eligibility than overall individual Risk members.

The overall new $\mathrm{S} / \mathrm{HMO}$ rate is twenty-nine percent less than that rate for individual new Risk members, and the overall converted $\mathrm{S} / \mathrm{HMO}$ rate is forty-seven percent less than that for individual converted Risk members. Rates for "group" Risk residents must be lower than rates for individual Risk members, based on observations 
from the above table. Why group Risk rates may be lower than individual Risk rates is a matter of some policy interest.

TABLE XXII

(RATES ARE STANDARDIZED BY AGE COHORT WITHIN GENDER) NUMBER OF ICF \& SNF DAYS FOR INDIVIDUAL RISK AND S/HMO RESIDENTS ACCORDING TO NEW AND CONVERTED STATUS DURING THEIR NURSING HOME STAY

Individual

Risk New Risk Conv s/HMO New S/HMO Conv
ICF Days/ ICF Days/ ICF Days/ ICF Days/
Yr Elig Yr Elig Yr Elig Yr Elig

Model A Yr Elig YrElig Yr Elig Yr Elig

Male+Female

$\begin{array}{lllll}\text { Overall } & 5.41 & 4.69 & 3.99 & 3.19\end{array}$

[Total Risk] $\quad 4.77$

[Total SHMO]

3.45

Source: Appendix D

If a S/HMO ECB was marketed to sponsors of group retirement plans, benefit rates might have to be adjusted if the difference was great. Some follow-up studies on this policy issue is needed.

One additional issue is addressed before leaving this question of rate differences between converted and new members in Risk and S/HMO. Specifically, are use rates different for Risk members during the study period who transferred from the Medicare Plus Demonstration Project, than use rates for Risk members who enrolled after April, 1985, following TEFRA-authorized HMO capitation contracts? 
If so, is there some apparent reason for such differences which could influence interpretation of composite rate differences between $S / H M O$ and Risk during the study period? Use rates and age differences for each of the four Risk subsets are compared, as a method of clarifying this research question.

HCFA required an enrollment ratio of three ("new") community residents to one ("converted") KP Cost Medicare member during the Medicare Plus Demonstration Project. Since that project did not offer an Expanded Care Benefit, nothing was known about differences between new and converted pre-TEFRA Risk members regarding their need ICF services. However, a great deal is known about other characteristics which could affect ICF use rates.

The entire Spring 1984 edition, volume 5, Number 1, of The Group Health Journal is devoted to an analysis of enrollment differences between new and converted Medicare Plus Demonstration Project members. For example, it was reported that Risk "conversion members generally are sicker than the new members, especially with regard to heart disease and hypertension." (Op. Cit. [63])

Research Question $I$ is revisited in Table XXIII, next, with Risk data only (S/HMO data is omitted). Model A is used to present the following table, which separates individual Risk members into four subgroups in order to observe what proportion of ICF residents per 1000 Risk members were 
transfers from the Medicare Plus Demonstration Project $(1980-84)$.

TABLE XXIII

(RATES ARE STANDARDIZED BY SEX AND AGE COHORT) ICF AND SNF RESIDENTS PER 1000 MEMBERS FOR:

A. NEW RISK ENROLLED BEFORE APRIL, 1985;

B. NEW RISK ENROLLED AFTER AFTER 1984;

C. COST MEDICARE CONVERTED TO RISK BEFORE APRIL, 1985; AND

D. AFTER APRIL 1985, AS INDIVIDUAL MEMBERS

\begin{tabular}{lllll} 
Model A & Risk & Risk & Risk & Risk \\
Male + Female & New $<85$ & New $>85$ & Conv $<85$ & Conv $>85$ \\
\cline { 1 - 4 } & $/ 1000$ & $/ 1000$ & $/ 1000$ & $/ 1000$ \\
& (a) & (b) & (c) & (d)
\end{tabular}

Subset II

$\begin{array}{lllll}\text { (ICF) } & 43.09 & 24.94 & 46.41 & 28.60 \\ \text { (SNF) } & 36.43 & 22.58 & 26.39 & 15.08\end{array}$

Combined Risk Rate: ICF - 31.61; SNF - 23.27/1000 HPM Source: Appendix D

The probability rate for becoming an ICF resident was over seventy percent greater for Medicare Plus Demonstration Project Risk members than that rate for Risk members whose Risk eligibility status commenced after April, 1985. Also, this SNF rate was higher for pre-TEFRA Risk members. Age differences among research population members in each subset follow the pattern of differences in this use rate.

As will be seen later in this chapter, Risk members who converted from Cost into the Medicare Plus Demonstration 
Project (1980-1984) had the highest probability rate of becoming an ICF resident during the study period, of all eligibility subsets examined, including S/HMO. However, their days in an ICF were slightly lower than other subsets, but it appears that their death rate was high. Among Risk members, they were most likely to be admitted to an ICF from home, and most likely to be discharged to a community-based convalescent center, as presented in Chapter VII.

Table XXIV summarizes differences in the rate for nursing home days by the four Risk subsets. New pre-TEFRA Risk ICF residents used more days than converted pre-TEFRA Risk members. New individual TEFRA Risk ICF residents used fewer ICF days than converted individual TEFRA Risk members. Overall, new pre-TEFRA Risk nursing home residents used more combined days per year of eligibility than residents in any other Risk subset. This is not surprising, given their mean age. In contrast, converted TEFRA Risk members admitted to an ICF had the highest rate, but the lowest mean age.

The rate of ICF days per member year of eligibility for new Risk members enrolled from 1980-1984, Column a, was twenty-three percent greater than that rate for Risk members who converted from Cost to Medicare Plus during 1980-84, as shown in Table XXIV, below.

For new Risk members whose eligibility status was established after April, 1984 (Column b), this rate was forty-three percent less than the rate for converted Risk 


\section{TABLE XXIV}

(RATES ARE STANDARDIZED BY SEX AND AGE COHORT)

ICF \& SNF DAYS PER MEMBER YEAR OF ELIGIBILITY:

A. NEW RISK ENROLLED BEFORE APRIL, 1985;

B. NEW RISK ENROLLED AFTER AFTER 1984;

C. COST MEDICARE CONVERTED TO RISK

BEFORE APRIL, 1985; AND

D. AFTER APRIL 1985

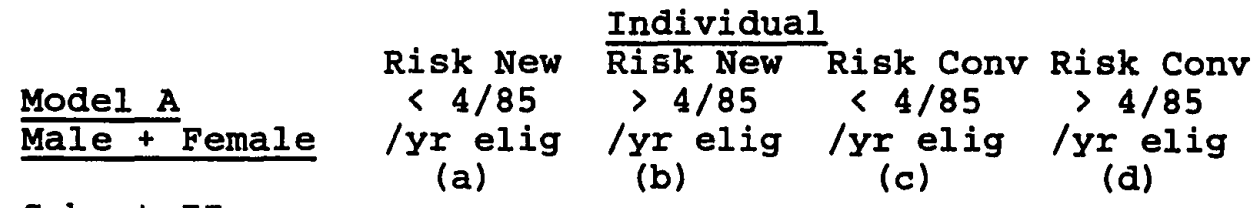

Subset II

$\begin{array}{lllll}\text { (ICF) } & 4.37 & 3.27 & 3.56 & 4.69 \\ (\mathrm{SNF}) & 1.25 & 0.72 & 0.68 & 0.65\end{array}$

Source: Appendix D

members whose eligibility status succeeded April, 1984, (Column d). New Risk members from pre-TEFRA used about 80 percent more SNF days than members who converted to Risk enrollment after the TEFRA contract was implemented.

SUMMARY OF OBSERVATIONS ABOUT RATES IN THIS CHAPTER AND THEIR IMPLICATIONS REGARDING PRIOR OBSERVATIONS

- New and converted $\mathbf{S} /$ HMO members had a greater probability of entering an ICF during the study period than either new and converted Risk members. This rate difference is probably not due to selection bias and not age related.

- The probability of becoming an ICF resident was slightly greater for Medicare Plus Demonstration Project (pre-TEFRA) Risk members than for $\mathrm{S} /$ HMO members. Since rates are age adjusted, the fact that the mean age of Risk Medicare Plus Demonstration Project members was four years older than that for $\mathrm{s} / \mathrm{HMO}$ does not explain the finding. If 
this is related to the phenomenon of pre-TEFRA Risk members having had more time to develop a physician relationship and admission to an ICF was more easily authorize

- The probability of becoming an ICF resident during the study period was nearly two times greater for Risk members who had been in the Medicare Plus Demonstration Project (1980-1984), than for Risk members who enrolled after the TEFRA capitation contract was implemented (4/85). The mean age of Risk Medicare PIus Demonstration Project members was six years older than Risk for members enrolled after April, 1985. No age adjustment was made within Risk, so age is a possible factor regarding this difference.

- Individual Risk rates are higher than group Risk rates; the policy issue is whether persons with employer sponsored retirement benefits use less institutional LTC that individual payers, after age and gender adjustments are made. Such differences were not clarified in this study.

- The converted TEFRA Risk rate was higher than rates for new TEFRA Risk subsets during the study period (1986-1988). A similar finding was reported about such conversions under the Medicare Plus Demonstration Project (pre-TEFRA) during the period 1980-1984.

- However, pre-TEFRA new Risk Rates were higher than pre-TEFRA converted rates during the study period, after the long term care ICF Risk residents were removed from the data set. This shift emphasizes the need for lifetime use rates.

- Within age cohort $85+$, the rate for TEFRA Risk residents was substantially greater than the rate for Medicare Plus Demonstration Project Risk members during the study period. This was explained by the days used rates for converted (Cost to Risk) TEFRA Risk members.

- The overall $s / H M O$ rate was clearly less than the rate for either new or converted individual Risk ICF residents.

- In age cohort 85 , new $S / H M O$ and converted $\mathrm{S} / \mathrm{HMO}$ rates for ICF days are lower than individual new Risk and individual converted Risk rates.

- The similarity between S/HMO and individual Risk research population members, regarding age and gender distribution as well as similarity in numbers makes a valuable contribution to descriptive data produced in this study. Specifically, it confirms that between the most ideally matched Risk and S/HMO groups, the pattern observed in Chapter IV is upheld. The S/HMO member probability of 
but for S/HMO ICF residents, days of stay are substantially less for S/HMO than Risk residents. This strengthens the notion that the ECB and related case management process improves access but that does not lead to inflationary use. Instead, it suggests that such $\mathrm{S} / \mathrm{HMO}$ access patterns can be managed effectively with financial incentives and formal care alternatives. The ECB allows dependent elderly to remain in a home environment who otherwise might have continued in an ICF. Confirmation testing of this concept is needed because it is has significant policy implications.

RESEARCH METHODOLOGY ISSUES RELATED TO THIS CHAPTER

In this study, the separation of group from individual enrollees is slightly artificial, in that $\mathrm{KP}$ members may have been enrolled in groups prior to converting to individual Risk or S/HMO status. In the process of evaluating s/HMO and Risk data for creation of variables and files to be used in analysis, some absolute numbers were produced. It is clear from some evaluation of historic membership files that the transition from non-Medicare employment status with group health benefits to Medicare status, greatly alters the proportion of HMO members covered by group benefits compared to Medicare members who pay for Medicare benefits out of pocket. In order to affect widespread acquisition of a Medicare based Expanded Care Benefit, it is probably necessary to market it to employers, in the absence of a national policy for long-term care. If marketed to persons below age 65, an important policy issue would be that group rates be continued after age 65 . 
POLICY RESEARCH ISSUES

Future studies should consider random sampling which is designed to select individual Risk members for the control group so that the size of the Risk group is balanced with that of $s / H M O$, making it easier to perform statistical analysis which require balanced cell sizes. In order to do an analysis of difference in Risk group and Risk individual payers it would be appropriate to identify all individual members who had previous group status and remove them from the individual Risk sample.

The research question of interest between group and individual members is whether having a retirement benefit, which pays all or part of the basic Medicare Supplemental Benefit, is associated with differences in nursing home use? That is, do retirees who have a work related retirement benefit which makes capitated payments to the HMO for entitlement based Supplemental Benefits, have different ICF use rates than those who pay for these benefits out of pocket.

A follow-up analysis of differences between rates for retirement benefit groups and individual payer groups is recommended. In this study about 900 Risk members were removed from the individual Risk data set who also were carried in the Risk Group data set. There were other instances of duplicate status. Validity of differences between individual and group rates was in question and 
direct comparison of those rates was avoided. That is why conclusions about effects of group rates were inductively arrived at by showing differences between individual Risk rates and overall $\mathrm{Risk}$ rates.

Thus, it is almost certain that group rates were lower than individual Risk rates, but to what extent is uncertain. This discussion is presented here because it has policy implications regarding prospective marketing of an Extended Care Benefit to Medicare (on non-Medicare) members enrolled in the HMO via sponsored retirement benefit plans. For one thing, employers may wish to know the extent to which they feel they might be subsidizing and adjusted community rate which included individual Medicare Supplemental Benefit premiums which included an ECB.

Employers may want to know that kind of information if they are considering the purchase of an Expanded Care Benefit for future retirees as part of their retirement benefits package. This is a policy research marketing issue about whether group members are less likely to represent adverse selection bias than individuals members who want to buy an Expanded Care Benefit. This question was not fully or successfully addressed by this study. But, hypothesis can be tested based on descriptive data presented.

In future studies, confirmation of the potential selection bias identified in this chapter may be justified for HMO rate setting purposes. Public policy for widely 
available LTC benefits needs to be informed about such actuarial issues because of the implications for Medicaid expenditures. 


\title{
DIFFERENCES BETWEEN INDIVIDUAL RISK AND S/HMO RESIDENTS REGARDING ADMISSION AND DISCHARGE PATTERNS
}

\begin{abstract}
Numerous studies suggest that one-fifth to one-third of those in institutions are receiving an inappropriate level of care. For example, of the million or so institutionalized elderly, 17 to 25 percent are there only because of no alternative social support system. [65]
\end{abstract}

Admission and discharge patterns are another important component in the analysis of nursing home utilization. Thus, ICF admission and discharge patterns are evaluated to determine if there are differences between four individual Risk subsets, and two subsets in s/HMO, which differentiate new members from converted members.

The objective of this analysis is to clarify whether differences in location prior to the initial ICF admission and after the final ICF discharge, during the study period, provide macro level clues to characteristics about each subset of ICF users which may help explain differences in use rates. Findings may also suggest other areas of analysis which may explain differences in use rates. This also may further clarify whether some use rates are indeed affected by some form of selection bias, as tentatively observed in Chapter V. 
The analysis presented in Table XxV, below, examines differences in source location prior to first ICF admission. only data from subset $I$ is used because it captures the true source location from which members came at the time of first ICF admission. Subset $I$ is used for last known disposition of members not residing in an ICF when data was collected.

TABLE XXV

SOURCE LOCATION FROM WHICH ICF RESIDENTS CAME

AT THE TIME OF THEIR FIRST ADMISSION

Source Location

Percent of AlI S/HMO ICF Residents

12.28

3.18

4.48

$13.7 \%$

$66.7 \%$

ICF

other Com. *

Home

Hospital/ER
Percent of

Al1 Risk

ICF Residents

Nothing remarkable is presented in the above table, regarding differences between Risk and S/HMO for source location prior to first ICF admission.

"Other" community facilities is a greater source of admissions in Risk than S/HMO, but the difference is offset by a higher percent of admission from home in s/HMO than Risk. That is an expected difference given the emphasis on home placement by Extended Care Benefit case coordinators. 
Similarity of hospital and SNFs as a source location is an important observation because it suggests that the progressive care of Medicare patients in capitated status was managed in a similar way, and that discharge utilization review practices were similar.

This similarity between source locations does not follow through into ICF discharge disposition location, which is a very important observation, given differences in observations about rates presented in earlier chapters.

This similarity is the basic reason for presenting the above table. Comparison of source locations and disposition locations leads to some important policy implications and is the basis for recommending some research hypotheses in the final chapter. Table XXVI shows admitting source locations other than ICF and Home to clarify dissimilarities between admission and disposition sites.

TABLE XXVI

SOURCE LOCATION FROM WHICH ICF RESIDENTS CAME AT THE TIME OF THEIR FIRST ADMISSION OTHER THAN HOME OR ICF

\begin{tabular}{|c|c|c|}
\hline $\begin{array}{l}\text { Source } \\
\text { Location }\end{array}$ & $\begin{array}{l}\text { Percent of } \\
\text { All s/HMO } \\
\text { ICF Residents }\end{array}$ & $\begin{array}{l}\text { Percent of } \\
\text { All Risk } \\
\text { ICF Residents }\end{array}$ \\
\hline $\begin{array}{c}\text { RCF } \\
\text { AFC } \\
\text { ALF } \\
\text { ILF } \\
\text { * Other Community } \\
\text { Ootal Community }\end{array}$ & $\begin{array}{r}0.48 \\
2.48 \\
0.08 \\
1.6 \\
\frac{4.48}{13.78} \\
18.18\end{array}$ & $\begin{array}{r}1.18 \\
3.28 \\
1.48 \\
1.88 \\
7.58 \\
10.58 \\
18.38\end{array}$ \\
\hline
\end{tabular}


* "OTHER COM." means community based facilities other than ICFs, or Home, including: (RCF) Residential Care Facilities, (AFC) Adult Foster Care facilities, (ALF) Assisted Living Facilities, and (ILF) Independent Living Facilities.

Probably, the higher level of AFC as a source of admission is related to the high proportion of Medicaid recipients who are placed in AFCs as an alternative to ICF, although the substitutability of AFC for ICF placement is not pervasive.

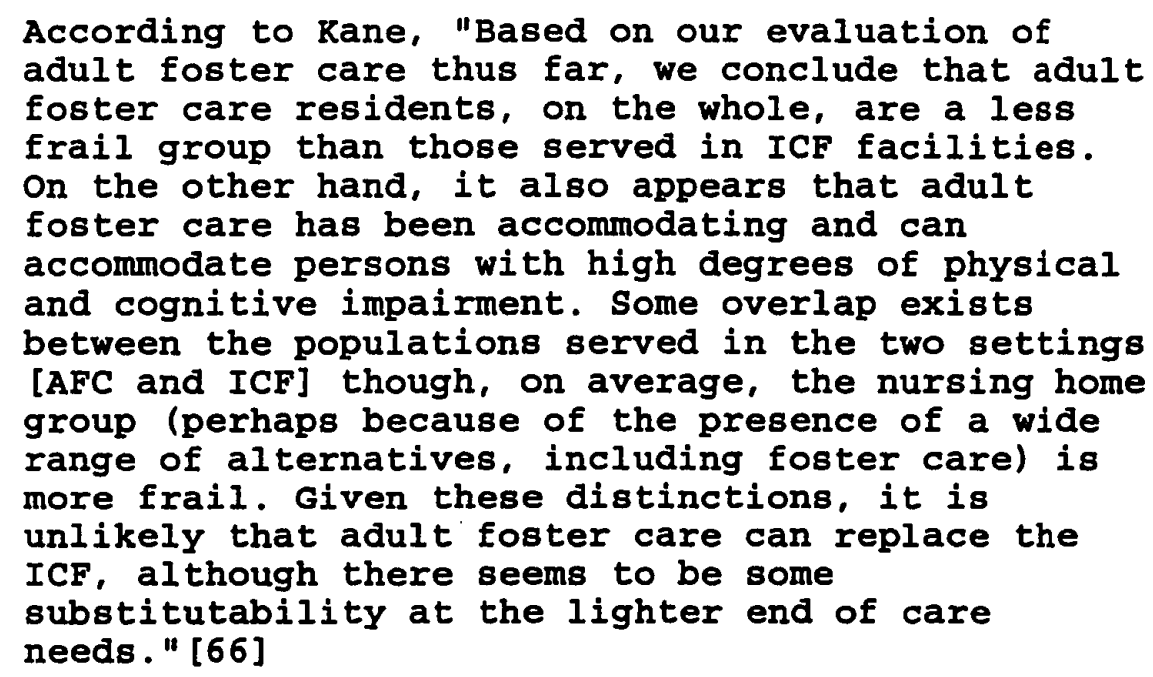

Discharge to an AFC or other community based location is not represented as substitutability of services in Table XXVII. While that may be the case, it is very likely that numerous circumstance precipitate discharge to ACFs from ICFs, including conditions of financing, social support networks, functional status, medical conditions, needs assessment and satisfaction with environmental conditions. 
TABLE XXVII

DISPOSITION LOCATION TO WHICH ICF RESIDENTS WENT FOLLOWING THEIR LAST RNOWN DISCHARGE

\begin{tabular}{|c|c|c|c|}
\hline $\begin{array}{l}\text { Disposition } \\
\text { Location }\end{array}$ & $\begin{array}{l}\text { Perc } \\
\text { All } \\
\text { ICF }\end{array}$ & $\begin{array}{l}\text { cent of } \\
\text { S/HMO } \\
\text { Residents }\end{array}$ & $\begin{array}{l}\text { Percent of } \\
\text { All Risk } \\
\text { ICF Residents }\end{array}$ \\
\hline SNF & & $0.8 \%$ & 0.78 \\
\hline ICF & & $6.7 \%$ & 9.78 \\
\hline Other Com. * & & 12.48 & 10.98 \\
\hline Home & & 32.28 & 17.28 \\
\hline Hospital/EF & & 11.08 & 16.28 \\
\hline Death at IC & CF & 30.68 & $35.5 \%$ \\
\hline
\end{tabular}

Figure 7 presents some interesting observations and surfaces information not previously reported in the literature, regarding comparison of two capitated groups, one having and ECB and the other not having and ECB.

Last S/HMO ICF discharges were much more likely to be sent home than Risk residents at last discharge. Given the goals of the S/HMO Expanded Care Benefit, this is an encouraging observation.

S/HMO ICF days of stay are similar to those of Risk in the data set used for these tables (Subset I), so that longer convalescence is not a likely explanation. Age of ICF users was similar also, as was gender mix. The proportion of deaths in ICFs was sixteen percent higher for Risk than S/HMO, which may reflect that S/HMO members had more options 


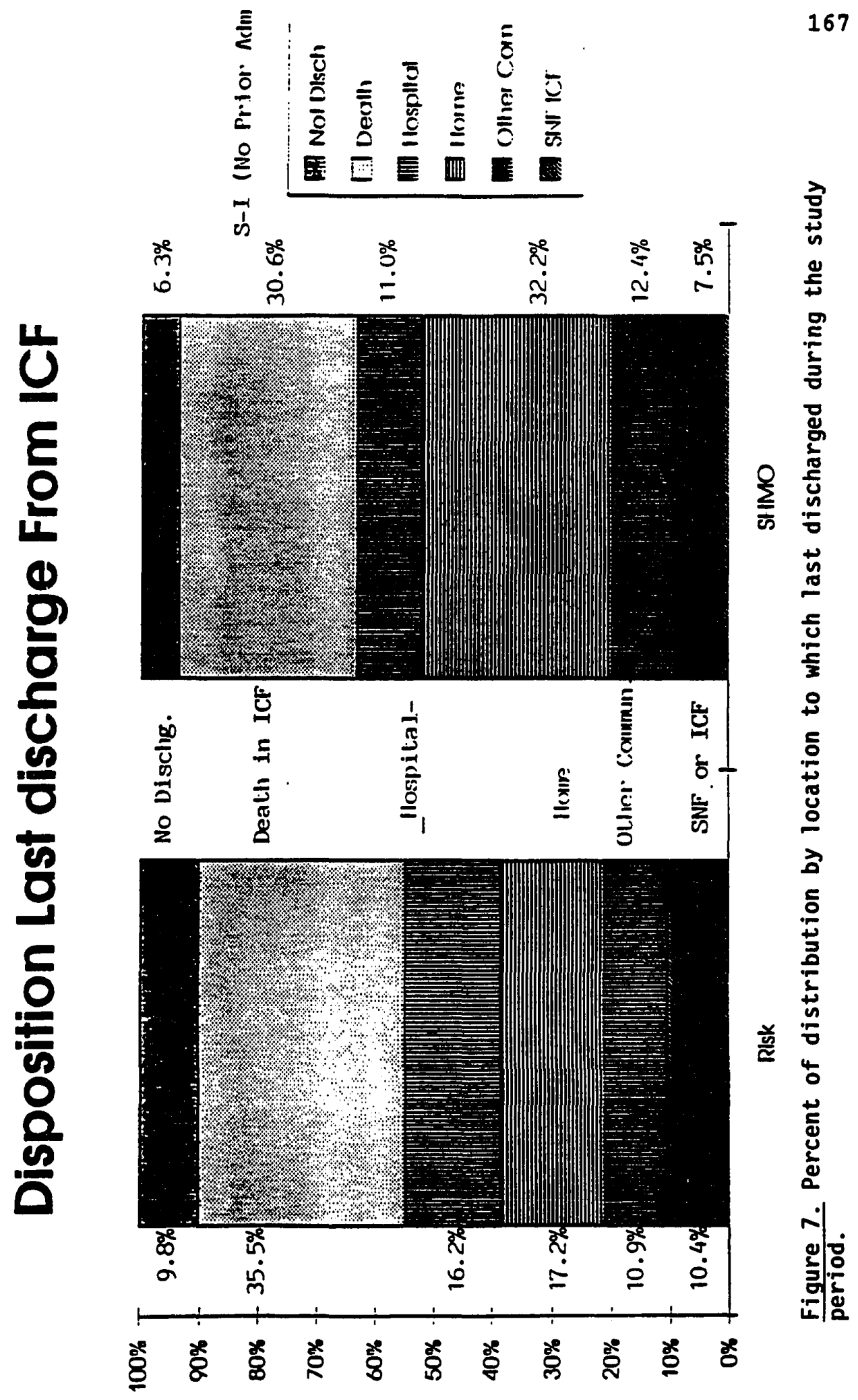


to choose from for their final period of life.

Without data on differences in ADLs or IADLs, which give categorical values to differences in dependency, it is difficult to say whether chronic disability levels were a cause for difference in home discharge rates. That data is needed for residents in the data set. It is possible that such information could be obtained from nursing home records on these persons, at a reasonable cost.

A companion observation is the difference in percent of discharges between Risk and s/HMO to other community facilities. Although $\mathrm{s} / \mathrm{HMO}$ is only fourteen percent greater than Risk, when taken together, the difference between s/HMO and Risk is impressive, as seen in Table XXVIII, below.

TABLE XXVIII

DISPOSITION LOCATION TO WHICH ICF RESIDENTS WENT
FOLLOWING THEIR LAST KNOWN DISCHARGE
OTHER THAN HOME OR ICF

\begin{tabular}{|c|c|c|}
\hline $\begin{array}{l}\text { Source } \\
\text { Location }\end{array}$ & $\begin{array}{l}\text { Percent of } \\
\text { All S/HMO } \\
\text { ICF Residents }\end{array}$ & $\begin{array}{l}\text { Percent of } \\
\text { AIl Risk } \\
\text { ICF Residents }\end{array}$ \\
\hline $\begin{array}{c}\text { RCF } \\
\text { AFC } \\
\text { ALF } \\
\text { ILF } \\
\text { * Other Community } \\
\text { Otome } \\
\text { Total Community } \\
\text { (excluding ICF) }\end{array}$ & $\begin{array}{r}0.88 \\
9.08 \\
0.88 \\
1.68 \\
12.48 \\
32.28 \\
44.68\end{array}$ & $\begin{array}{r}2.2 \% \\
6.5 \% \\
1.1 \% \\
1.1 \% \\
10.98 \\
17.28 \\
28.1 \%\end{array}$ \\
\hline
\end{tabular}

Forty-five percent of all S/HMO ICF residents in subset I were discharged to a community location other than another 
ICF, at the time of their last discharge, compared to twenty-eight percent of all Risk last known discharge placements. That is, discharge planners were able to place fifty-nine percent more last ICF discharges in community based facilities than they arranged for Risk members in Subset I.

Again, the percent of ICF discharges sent to AFC sites is of interest because it is different than source admissions data; the percent of $\mathrm{S} / \mathrm{HMO}$ members is higher than that for Risk.

Table XXIX shows use rate differences between S/HMO and individual Risk ICF residents.

TABLE XXIX

SUMMARY OF DISPOSITION FOR LAST KNOWN ICF DISCHARGE FOR ALL NEW AND CONVERTED S/HMO ICF RESIDENTS AND (ONLY) INDIVIDUAL RISK RESIDENTS BY SUBSETS

(A) PRE-TEFRA AND (B) TEFRA CONTRACT RISK ELIGIBILITY STATUS

\begin{tabular}{llllllll} 
Disp. & S/HMO & S/HMO & Risk & \multicolumn{2}{l}{ Individual } \\
Loc. & New & Conv & New & New & Conv & Risk \\
& & & $<4 / 85$ & $>4 / 85$ & $<4 / 85$ & $>4 / 85$ \\
& \& Colf & Col & \& Col & \& Col & \& Col & \& Col
\end{tabular}

$\begin{array}{rrrrrrr}\text { SNF } & 00.0 & 03.1 & 02.2 & 00.0 & 00.0 & 00.0 \\ \text { ICF } & 16.0 & 18.9 & 13.7 & 14.3 & 11.4 & 19.4 \\ \text { OTHER COM. } & 11.7 & 05.5 & 12.1 & 07.1 & 15.7 & 08.4 \\ \text { HOME } & 36.2 & 37.8 & 14.4 & 21.4 & 25.7 & 13.9 \\ \text { DEATH } & 19.1 & 15.0 & 20.1 & 14.3 & 17.2 & 19.4 \\ \text { HOSP/ER } & 17.0 & 19.7 & 37.5 & 42.9 & 30.1 & 38.9 \\ \text { total } & 100.0 & 100.0 & 100.0 & 100.0 & 100.0 & 100.0\end{array}$

Percentages for each of the five location variables 
differ somewhat for individual Risk members. Differences between new and converted $\mathrm{s} / \mathrm{HMO}$ and Risk members contribute to information about their use rates. They also suggest the importance of obtaining socioeconomic variables which characterize differences in member groups that may facilitate community based discharges.

The substantially lower percentage of $\mathrm{s} / \mathrm{HMO}$ discharges to the hospital, relative to any of the four individual Risk subsets, offers some clues about differences in rates for ICF days in Chapter $v$.

ICF death ratios parallel their days used rates. That is new pre-TEFRA and converted TEFRA use rates were higher and so are their ICF death rates. The same is observed about new $\mathrm{s} / \mathrm{HMO}$. This suggests those subsets may have included more permanent residents who remained until death.

The lower percent of discharges to hospitals for both s/HMO subgroups is an observation for which no answers are surfaced in this study. It is interesting that both TEFRA Risk subgroups had higher ratios of discharge to the hospital and for death than the two older Risk pre-TEFRA.

The proportion of discharges to community facilities, other than ICFs, is presented below in summary form followed by a table giving supportive detail.

S/HMO members receiving ECB services at home, whose only use of ICFs was for respite, could be expected to have fewer days in residence. 
Differences in discharge patterns to other ICFs is relevant to differences in ICF use rates because of several reasons. Discharges directly from one ICF to another occur for a limited number of reasons, some of which include: (a) resident is in a facility not accepting Medicaid patients, which was a factor for both Risk and S/HMO members whose initial nursing home stay was in a nursing home with which RP contracted for SNF and ICF services, that discontinued participation in the Medicaid program; (b) patient became Medicaid eligible in a facility that did not have Medicaid beds which were available, i.e., all licensed Medicaid beds were filled; (c) S/HMO Case coordinator requested that member be moved to an ICF facility with which S/HMO contracted; (c) patient or visiting RP nurse practitioner was not satisfied with care given by a nursing home; (d) patient had needs better provided for at another nursing home, such as those with advanced dementia; other patient/family dissatisfaction.

These reasons are listed because they suggest that operational issues need to be considered as an explanatory variable for determining why the percent of discharge to other ICFs might be higher for S/HMO; and indeed both S/HMO subsets are higher than three of the four Risk subsets.

Equally important, they suggest the need to examine differences in rate of spend-down to Medicaid eligibility as a reason for differences in length of stay. If Risk rates 
are higher than S/HMO, which Chapter VIII suggests is true, then more Risk members could reside longer in ICFs, or AFCs, because that is where the state of oregon requires them to be, or/and that is where families want them to be, in order to continue to be recipients of Medicaid support. one operational reason for rate differences is that $\mathrm{s} /$ HMO members may have been located at nursing home sites where closer monitoring of continuing need for ICF care could be conducted by KP utilization review staff. This was done by both s/HMO case coordinators and geriatric nurse practitioners whose responsibility was to frequently assess changes in patient condition, oversee quality of nursing home care, communicate with patient, physician and family and about patient needs, and advise s/HMO coordinators as needed. Such patient oversight was also conducted on Risk and cost members, but without s/HMO case coordinator participation. Indirectly, therefore, ICF transfers imply possible reasons for shorter stay, even for those s/HMO members who were considered permanent placement, but whose condition might eventually permit use of other community based institutions.

When the overail pattern of ICF discharges to both HOME and community facilities other than ICFs is examined, the trend is clear: both "new" and "conv" s/HMO members were transferring from ICF to community based living arrangements at significantly greater rates than any of the four Risk 
subsets presented above. Of the four Risk subsets, only those who converted to Medicare Plus Demonstration Project in 1980-1984 had a lower but similar pattern of discharges. S/HMO ICF residents also were discharged to reside in their home or someone's home at a much greater rate than Risk members.

Analysis of ICF discharges to other community based locations, besides ICFs, is presented in Table XXX, below.

TABLE XXX

SUMMARY OF DISPOSITION FOR LAST ICF DISCHARGE

TO COMBINED HOME, RCF, AFC, ALF, ILF

THE SUM OF WHICH IS PRESENTED AS

"COMMUNITY" OTHER THAN ICF

\begin{tabular}{|c|c|c|c|c|c|c|}
\hline $\begin{array}{l}\text { Disp. } \\
\text { Loc. }\end{array}$ & $\begin{array}{l}\text { S/HMO } \\
\text { New } \\
\text { After } \\
\text { \& Col }\end{array}$ & $\begin{array}{l}\text { S/HMO } \\
\text { Conv } \\
\text { After } \\
\text { \& Col }\end{array}$ & $\begin{array}{l}\text { Indiv } \\
\text { Risk } \\
\text { New } \\
\text { Before } \\
\text { \& Col }\end{array}$ & $\begin{array}{l}\text { Indiv } \\
\text { Risk } \\
\text { New } \\
\text { After } \\
\text { \& Col }\end{array}$ & $\begin{array}{l}\text { Indiv } \\
\text { Risk } \\
\text { Conv } \\
\text { Before } \\
\text { \& Col }\end{array}$ & $\begin{array}{l}\text { Indiv } \\
\text { Risk } \\
\text { Conv } \\
\text { After } \\
\text { \% Col }\end{array}$ \\
\hline $\begin{array}{l}\text { \#OTHER COM. } \\
\text { HOME } \\
\text { COMMUNITY }\end{array}$ & $\begin{array}{l}11.7 \\
36.2 \\
47.9\end{array}$ & $\begin{array}{l}05.5 \\
37.8 \\
43.3\end{array}$ & $\begin{array}{l}12.1 \\
14.4 \\
26.5\end{array}$ & $\begin{array}{l}07.1 \\
21.4 \\
28.5\end{array}$ & $\begin{array}{l}15.7 \\
25.7 \\
41.4\end{array}$ & $\begin{array}{l}08.4 \\
13.9 \\
22.3\end{array}$ \\
\hline $\begin{array}{l}\text { * RCF } \\
\text { *AFC } \\
\text { *ALF } \\
\text { *ILF }\end{array}$ & $\begin{array}{l}3.2 \\
5.3 \\
1.1 \\
2.1\end{array}$ & $\begin{array}{l}0.0 \\
5.5 \\
0.0 \\
0.0\end{array}$ & $\begin{array}{l}1.4 \\
5.0 \\
1.4 \\
4.3\end{array}$ & $\begin{array}{l}0.0 \\
0.0 \\
7.1 \\
0.0\end{array}$ & $\begin{array}{l}7.1 \\
5.7 \\
2.9 \\
0.0\end{array}$ & $\begin{array}{l}2.8 \\
5.6 \\
0.0 \\
0.0\end{array}$ \\
\hline
\end{tabular}

* OTHER COM. includes community based facilities other than ICFs for care of disabled and dysfunctional dependent persons, including: (RCF) Residential Care Facilities, (AFC) Adult Foster Care facilities, (ALF) Assisted Living Facilities, (ILF) Independent Living Facilities.

The percent of discharges to Adult Foster Care, shown 
in the table below, is similar across five of the six subsets, which probably is related to members who became Medicaid recipients, since the state of oregon vigorously pursued that solution an alternative to ICF level care. It is not clear why no Risk members who enrolled after April, 1985, were discharged to AFC facilities But the combination Assisted Living Facility and Adult Foster Care suggests that new Risk members enrolled after April, 1985 may have had sufficient personal assets available to them to afford ALF and avoid the need for Medicaid funds, or were not as disabled or dependent as other Risk subset members. There were only 15 such members and their use rates were lower than S/HMO or the other three Risk subsets, especially among females, as may be observed in use rate tables presented above. They also were slightly younger which may not have affected use rates but may correlate with having more personal assets.

Table XXXI shows the proportion of residents still present in nursing homes at the time data was collected; they are omitted from the tables in this chapter. Not surprisingly, fewer Medicare Plus Demonstration Project members remained in an ICF than Risk TEFRA members or S/HMO members. Why such a disproportionate percent of converted Risk TEFRA residents remained in nursing homes is not known, but it suggests a higher proportion of long term residents existed in that subset than among new Risk TEFRA residents. 


\section{TABLE XXXI}

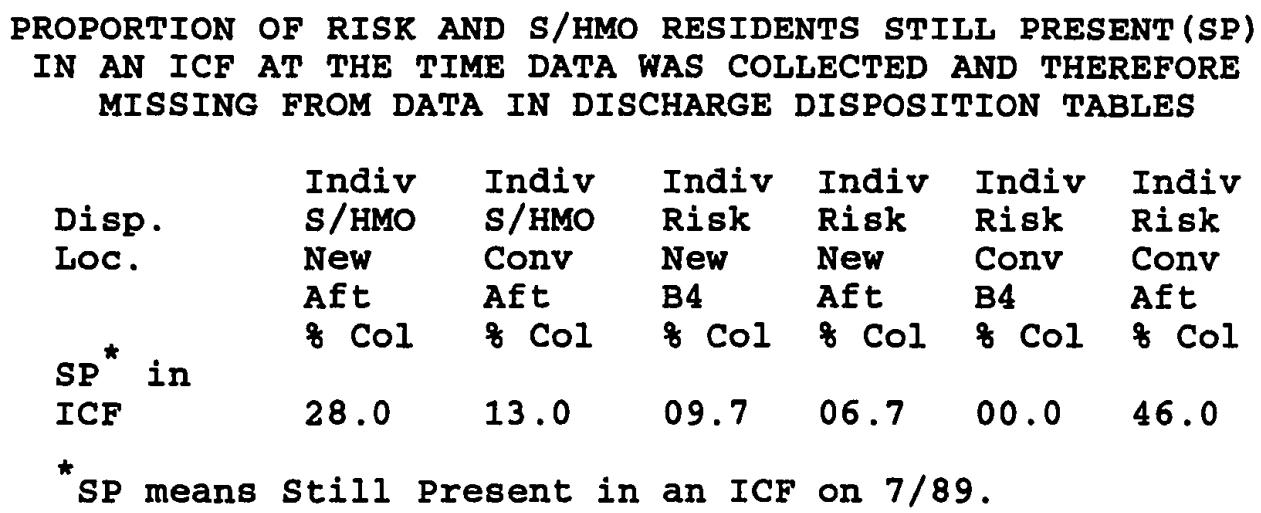

No doubt, the very high proportion of Risk members who converted from cost to Risk during or before the study period affected the Risk rate. Risk conversions after April, 1985 had the highest days in age cohort $85+$ per member year of eligibility, by far, of all subsets examined. (See Appendix B). Apparently, that conversion factor also resulted in more long term permanent placements than in other Risk subsets. Unless those Risk members were in a nursing home before having an opportunity to apply for s/HMO, why they did not do so is an interesting research question. Indeed, some of them were among the 71 ICF users identified as "outliers" in Chapter IV.

The higher proportion of new $s /$ HMO members who were still present suggests that recruitment of new members from the community included a higher proportion of persons who would become permanently placed in ICFs than occurred from 
higher proportion of new members were older, so that the cohort survival phenomenon may have been a factor.

Is there anything instructive about patterns of locations from which first ICF admission come, regarding the above subsets? The following table points to the need for clarifying possible differences in medical status of patients admitted to ICFs, given the very high proportion of admissions from hospitals.

With the exception of Risk members whose eligibility data in that status was established after April 1985, no remarkable differences exist between subsets. clearly, Cost who converted to Risk after April, 1985, had a need for more intense nursing and personal care after being in community facilities than other Risk or S/HMO members. If nothing else this observation signals the need for HMOs to monitor care rendered to $\mathrm{KP}$ members in non-nursing home community based facilities.

An interesting use rate observation is made about "HOSPITAL/ER as a source of first ICF admission. This rate appears to be substantially higher than that for national data. Since this data is from a period of time after DRGs had their first impact on nursing home admission rates, it is doubtful that this observation is associated with that phenomenon. Location prior to first ICF stay is shown next in Table XXXII. 


\section{TABLE XXXII}

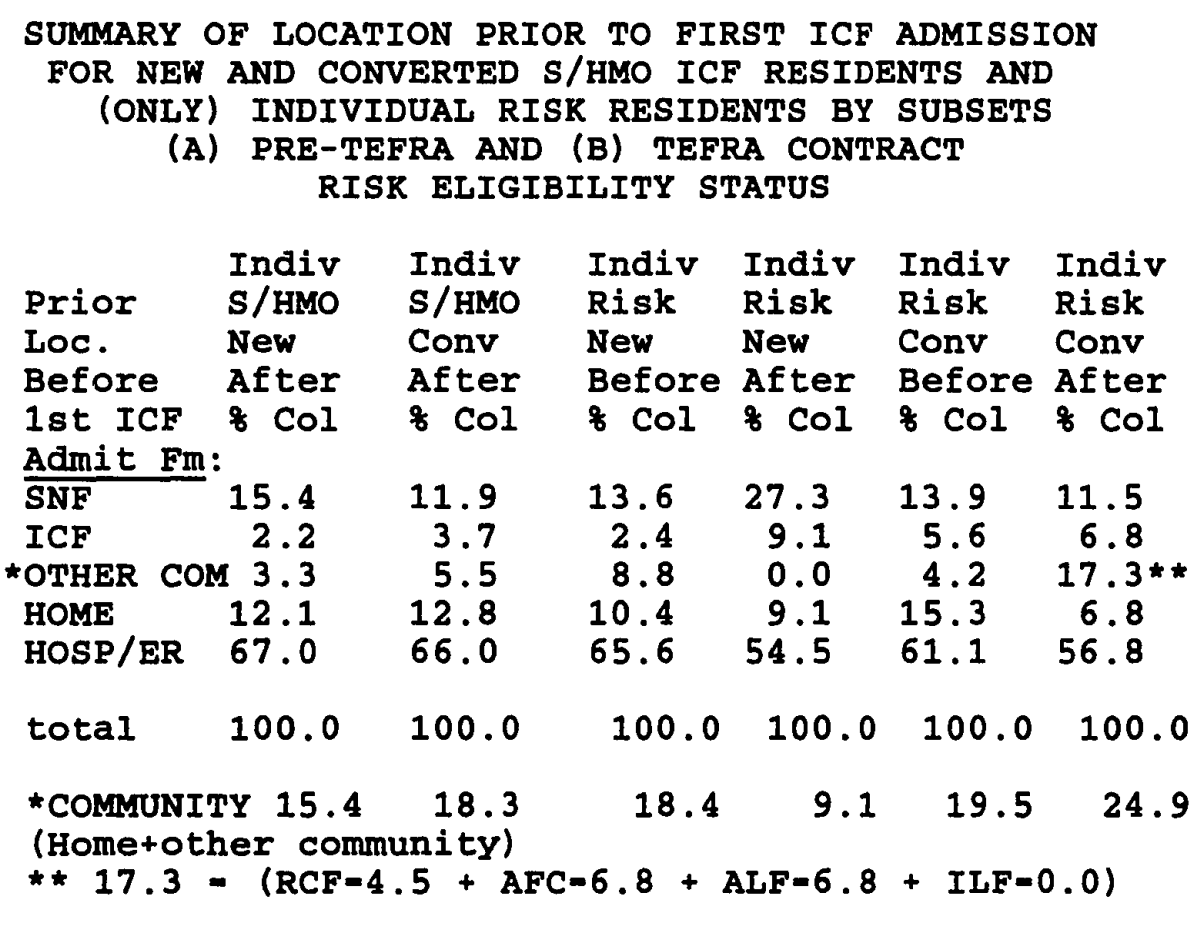

This study shows a trend similar to but slightly higher than the national pattern in the same time period, especially for S/HMO members. Why is that so? This pattern is one of several good reasons to examine differences in hospital discharge diagnosis from 1985 through the study period among Risk and s/HMO members admitted to ICFs within the study period. That is accomplished in the next chapter.

Analysis of mortality patterns among ICF users includes admission and discharge analysis, but also requires death certificate data and hopefully information regarding location of following discharge. In this study, the RP 
membership information data base includes a variable regarding reason for termination, including death. That data is used for the following table. Termination data is generally entered as the last day of the month in which enrollment ended. Since nursing home data collection commenced in June, 1989, termination data is used for that month regarding members in respective eligibility group subsets, who were also in ICFs.

Table XXXIII is provided in response to Research Question II, regarding how differences in days used per year of eligibility might have been affected by differences in death rates. One possibility is that higher death rates resulted in earlier censoring of days in residence.

TABLE XXXIII

COMMUNITY BASED FACILITIES OTHER THAN ICF TO WHICH INDIVIDUAL RISK AND S/HMO ICF RESIDENTS WENT FOLLOWING DISCHARGE

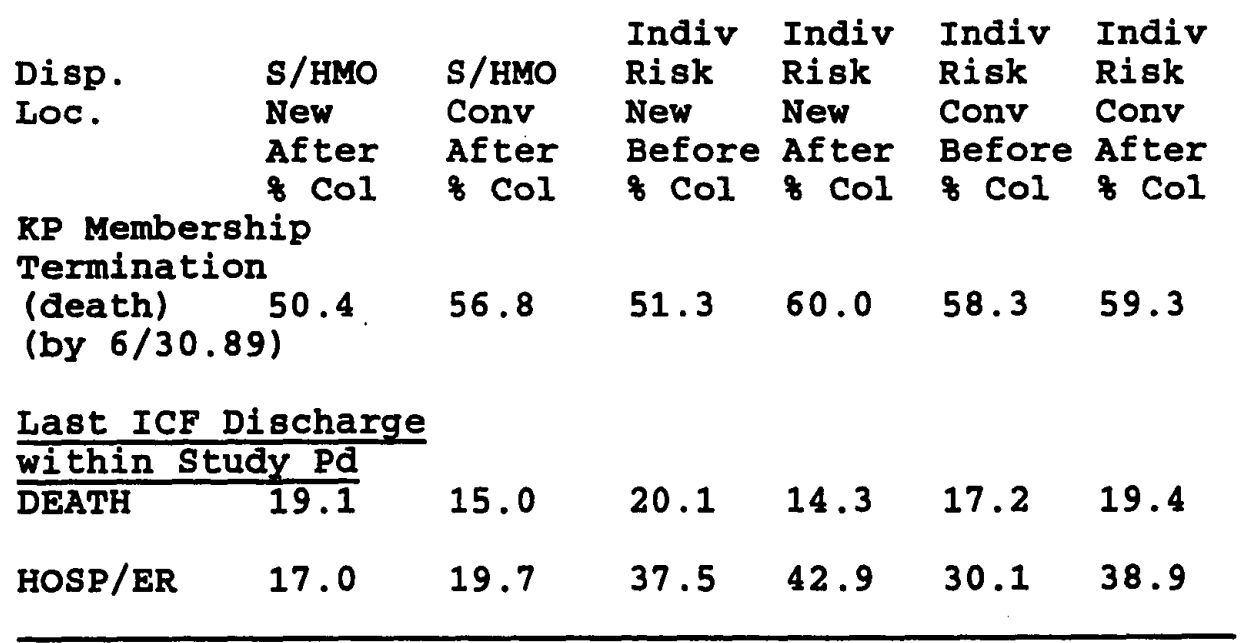


The combination of hospital discharges and death while in an ICF is not a precise measurement of death rates because not all last ICF discharges to the hospital were followed by death. However, it is a good indicator. When compared to percentages of ICF residents whose membership was terminated due to death either during the study period or within one year after the study period, the combining of variables "DEATH" and "HOSP/ER" looks like a reasonably good estimator of mortality rates for last ICF discharges.

Risk ICF residents may have had a higher incidence of mortality during the study period than s/HMO ICF users, based on this crude measure of death. If that is correct, that only adds to the reasons for looking to variables other than age and gender, to explain lower $S / H M O$ rates than Risk rates for cumulative days of stay in ICFs during the study period.

It is likely that ICF days in residence were affected somewhat by the higher death rates, observed from $\mathrm{KP}$ membership data. If taken together, DEATH and HOSP rows suggest somewhat higher rates of mortality for Risk ICF users who had enrolled as new Risk members after April, 1985 and Cost conversions to Medicare Plus (Risk) Demonstration Project.

Risk ICF residents who enrolled after April, 1985 had the lowest percent of last ICF discharge to Home and highest rate of last discharges to the Hospital; combined discharges 
to hospital and percent of members dead by July, 1989, suggests that health status of Risk members enrolled after April, 1985 may have been a contributing factor to nursing home utilization rates.

\section{POLICY RESEARCH ISSUES}

This chapter suggests that ECB is an incentive for limiting ICF level services, when alternative home care is a accessible, and that this incentive is likely to affect both new and converted HMO, in a similar way. The observed data also suggests that individual payers are likely to respond to incentives positively. Such observations have not been reported previously comparing ICF discharge patters of two HMO capitation groups.

This chapter provides baseline data about nursing home use rates regarding sources of admission to and discharge from ICFs. The pattern of discharges from ICFs to community based institutions, other than ICFs and to Home, merits further research to identify whether closer monitoring and early intervention could improve such rates.

Policy and policy based protocol for such monitoring and early intervention care is an important utilization issue relevant to any effort to extend Expanded Care Services to an entire HMO population. Of course, it also is fundamental to good patient care of dependent elderly persons. Use rates may have been affected by differences in 
such monitoring practices.

Values for variables examined in this chapter suggest they could serve as useful explanatory data, together with ADLs, health status (ICD codes) and mortality data, to clarify whether length of ICF (or total nursing home) stay differences between $\mathrm{S} / \mathrm{HMO}$ and Risk are influenced by the existence of an Extended Care Benefit and related case managers, or more likely due to differences in those covariates. To do so would require some form of analysis which clarified interactions between independent variables and length of stay, as well as interaction between independent variables. No doubt, multicolinearity would be extant between them, in any regression procedure used for such analysis.

Based on descriptive information which these tables contain, SNF appears to the only variable of doubtful value for use in building a multivariate model with which to help clarify differences in ICF use rates between $S / H M O$ and Risk residents. 
CHAPTER VII

REVENUES RECEIVED BY NURSING HOMES FOR RESIDENT CARE

"Econometric cost functions cannot yet provide
ratesetters with predictions about the cost of the
efficient provision of nursing home care appro-
priate to patient needs. In any case, the design
of reimbursement systems must be founded not only
on technical information but also on public policy
goals for long-term care." [67]

The policy issue addressed in this chapter is whether the combined effects of ECB incentives for improved access to ICF services and S/HMO managed care policies, operational practices and related LTC goals result in s/HMO eligibility group ICF costs which are different from Risk eligibility group ICF baseline costs.

As observed in Chapter IV, overall data set rates for S/HMO member access to ICFs was much greater then that of Risk members, however, Days of ICF stay were substantially less for $\mathrm{S} / \mathrm{HMO}$ than for Risk ICF residents. The combined effects of access and cumulative stay comprise overall ICF utilization and cost outcomes. It was not know whether rates for total nursing home revenues for s/HMO ICF residents differed from those for Risk ICF residents before the study. This chapter presents an analysis of differences between rates of nursing home revenues for $\mathrm{s} / \mathrm{HMO}$ and Risk ICF residents per $1000 \mathrm{~s} / \mathrm{HMO}$ and Risk members. Another view 
of relative payment differences is also presented by comparing differences in mean of revenues per s/HMO resident and mean of revenues per Risk resident; tests are used to indicate whether differences are statistically significant.

The numerator used for computation of rates comprises all payments received (revenues) by nursing homes for care they provided to research population members who became ICF and/or SNF residents during the study period. The denominator used in this rate is members; specifically, per 1000 members.

Nursing home revenues, for such residents, include all moneys received during the period mid-1985 through June 1989 (before, during, and after the study period), by all nursing homes in which any resident resided during the study period (July, 1986 through June, 1988).

In this chapter, revenues are not reported for all residents in the overall dataset. Instead, only subset II and Subset I residents are used. Subset II revenues are reported because residents in that data set did not reside in nursing home before 1985, thus their utilization history meets the time limitation, mid-1985 through mid- 1989. Subset I revenues are reported because residents in that data set had not used nursing home services before the study period. Thus, the rate comparison reported below for subset I has the effect of limiting numerator revenues to a three year period (July, 1986 through June, 1989). 
Rates (for nursing home revenues for ICF residents /1000 \{Risk $>$ \} (SHMO\} members) include composite payments recorded on nursing home ledgers for SNF and ICF services during that four year period. Such rates are also presented for combined SNF and ICF residents.

In this chapter, only Analysis Model B is used for analysis of differences in both rates of payments received: (a) per 1000 research population members; (b) per member year of eligibility; and mean of revenue for each resident. A graphic illustration of the two Model B formats is shown in Chapter III, indicating combinations of group status used to create denominators for mutually exclusive membership status tables presented in this chapter.

The advantage of using a denominator of per member year of eligibility is that it measures relative time of study period eligibly by enrollment status during which the event (numerator) may occur. However, in the case of payments received, the numerator used is not left and right censored, thus reflecting more of a lifetime use than a study period use. In this case the rate which is least distorted by factors affecting numerator values is that derived for Subset I. However, rates for all subsets are shown in Table XXXIV, below, which responds to Research Question III.

Research Question III, asks: Are There Differences Between S/HMO and Risk Members Regarding The Means of Total Payments Received By All Nursing Homes In which Each Resident Lived For All Periods of Stay Before, During, And After The Study Period Through June, 1989? 
TABLE XXXIV

(RATES ARE STANDARDIZED BY AGE COHORT AND GENDER)

TOTAL PAYMENTS RECEIVED BY NURSING HOMES WITHIN ONE YEAR AFTER THE STUDY PERIOD FOR ICF RESIDENTS/MEMBER YEAR OF ELIGIBILITY IN EXCLUSIVE ELIGIBILITY CLUSTERS: N1 - "NEVER S/HMO" (C, C+R, R); N2 - "S/HMO SOMETIME" ( $S, C+S$, $R+S$. $C+R+S)$

Model B, Format A. Male + Female (dollars are rounded)

(See graph, s1-s111)

ICF Residents Subset I (graph-s1)

Subset II (graph-S11)

overall (graph-s111) \$623
(N2)

stdidz

payment (SNF+ICF) /HPM Yr. SP Elig. for ICF Residents

Col. (b) $\$ 272$

$\$ 251$

$\$ 278$

The difference in standardized rates for subset I nursing home users is 14 percent. This rate is probably not affected in any way by the conversion process of transferring Cost members to Risk or Risk members to s/HMO. Since a smaller proportion of $\mathrm{s} / \mathrm{HMO}$ members were Medicaid, the value of total payments for $\mathrm{s} / \mathrm{HMO}$ members is closer to private pay billed charges and less suppressed by Medicaid payment. Total payments for males were $\$ 280$ per member year of eligibility for Risk and $\$ 227$ for S/HMO; a 23 percent difference, but approximately 20 Percent less total payments for males than for females. 
In Table XXXV, below, the standardized rate of money received by nursing homes, per 1000 members, was twelve percent less for "S/HMO Sometime" ICF residents (column a), than that for "Never S/HMO" ICF residents (column b) under Subset I (no nursing home use prior to the study period by ICF residents). Figure 8 shows total ICF payments.

The standardized rate of money received by nursing homes, per 1000 members, was sixty-three percent less for "S/HMO Sometime" ICF residents (column a), than that for "Never S/HMO" ICF residents (column b) under subset II (ICF residents may have had nursing home admissions prior to the study period).

Unlike for the Subset I rate, of ICF days per member year of eligibility presented in Chapter IV, the "S/HMO Sometime" rate for money received is substantially less than "Never S/HMO" under subset I, as well as in subset II. This finding is important for both policy issue and study outcome reasons.

The above finding suggests that the ECB as administered by the $\mathrm{s} / \mathrm{HMO}$ influenced outcome differences. This finding provides a basis for the formulation of specifichypotheses testing statements with which to accept the likelihood of that observation in a repeated, sample based study from another time period. Such research is recommended to confirm the findings of this study. Regarding SNF+ICF residents, rates for money received 
are nine percent less for "S/HMO Sometime" than for "Never

S/HMO" in subset I. This is a true assessment of "nursing

home" revenues under current Medicare Regulations which

does not differentiate SNF and ICF as levels of care.

"The SNF-ICF distinction is clearly not a stable product definition appropriate for use across the nation: the estimated SNF-ICF cost differentials vary among state studies, and direct studies of production methods and state regulations have shown that the SNF and ICF designations mean different things in different states." (Op. Cit. [67], p. 61)

TABLE XXXV

(RATES ARE STANDARDIZED BY AGE COHORT AND GENDER) TOTAL PAYMENTS RECEIVED BY NURSING HOMES WITHIN ONE YEAR AFTER THE STUDY PERIOD FOR ICF RESIDENTS PER 1000 MEMBERS MUTUALLY EXCLUSIVE ELIGIBILITY CLUSTERS: N1 - "NEVER S/HMO" (C, C+R, R); N2 = "S/HMO SOMETIME" (,$C+S$, $R+S$. $(+R+S)$

(N1) Stdizd

Model B, Format A. Male + Female (dollars are rounded) (See graph, s1-S111) I $\overline{\mathrm{CF}}$

Subset I (graph-s1)

subset II (graph-s11)

Overal1

SNF+ICF

Subset I

(graph-s111)

$\$ 1,069$

$\$ 530$

$\$ 861$
(N2)

stdidz

Paymt Rates

11000

members

Col. (b)

$\$(000)$

$\$ 397$

$\$ 446$

$\$ 495$

$\$ 485$

$\$ 543$

Source: Appendix E 


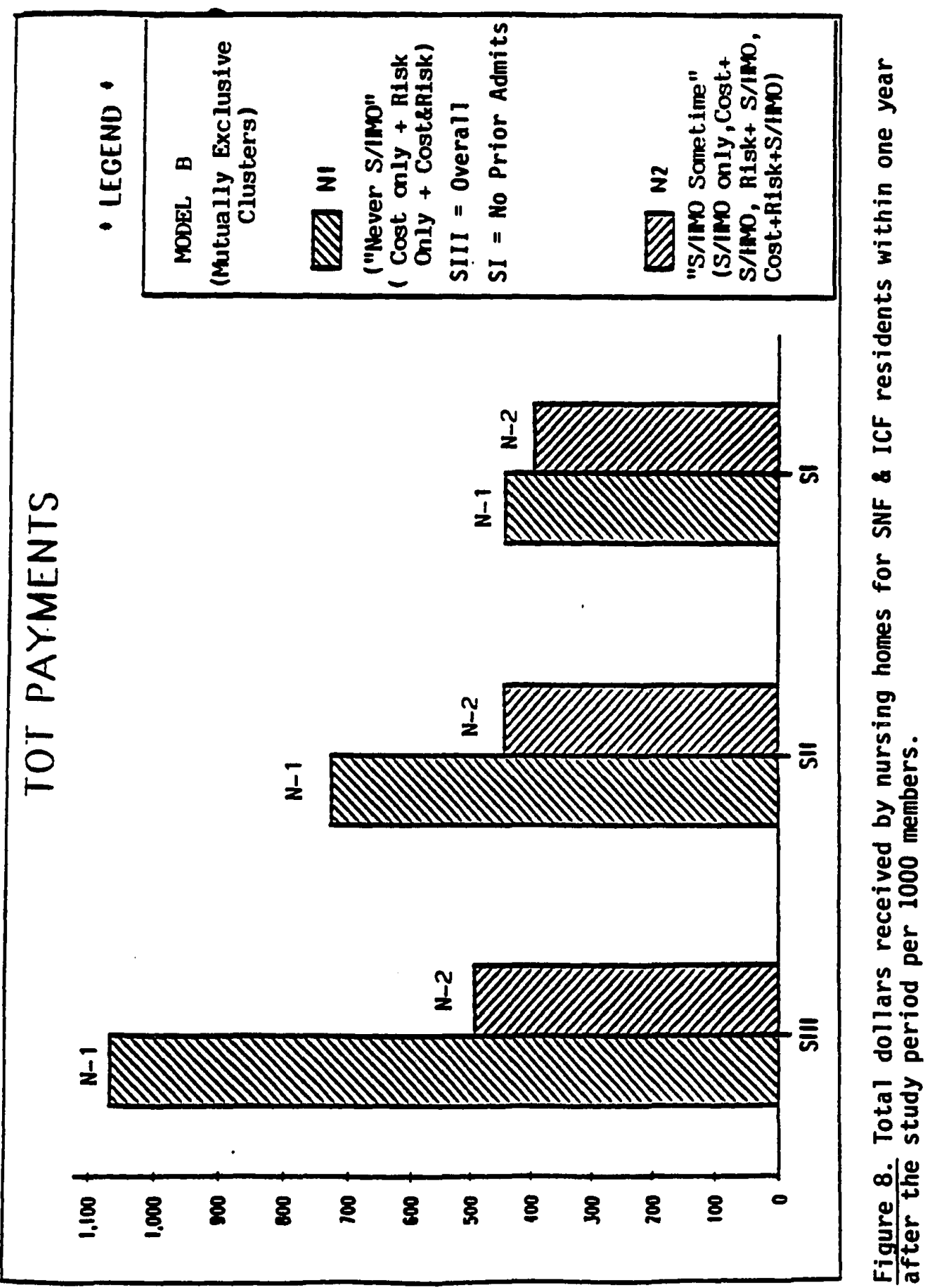


Absolute dollars for rates are given in Table XXXVI, below. A note about these data on absolute dollars is that in the Overall data set, not shown, about $\$ 400,000.00$ in payments had been made for $s / H M O$ members prior to the beginning of the study period, whereas about four million $(\$ 4,000,000$.$) had been received by nursing homes for$ services used by "Never S/HMO" members before the study period. Subset I helps to confirm the credibility of rate differences observed in subset II which include payments received prior to the study period. Table XXXVI, below, is a longitudinal measure of expenditures for residents in a nursing home during the study period.

TABLE XXXVI

ABSOLUTE DOLLARS, AS PAYMENTS RECEIVED FOR SNF AND ICF SERVICES DURING THE STUDY PERIOD, WITHIN ONE YEAR AFTER THE STUDY PERIOD

$\begin{array}{lllr}\text { Subset I } & \text { Never S/HMO } & \text { s/HMO Sometime } & \text { Total } \\ \frac{(\text { SNF+ICF) }: \$ 6,653,168 .}{\$ 3,265,816 .} & \$ 9,918,984 . \\ \frac{\text { Subset II }}{(\text { SNF+ICF) }: \$ 10,787,054 .} & \$ 3,651,839 . & \$ 14,438,893 .\end{array}$

Rate include payments received up to one year after the study period. Data used in this study is reliable and fully documented from 1985 through 1989. Observations in the above tables are presented with considerable confidence.

In the following table, non-standardized rates are 
presented by age cohort so the magnitude of difference between age cohorts is clarified, and so that true total dollars can be presented in a meaningful way. Second, rates for the overall dataset are presented so that the effect of those 71 members removed to create subset II can be conveyed.

Model B is used for analysis in this chapter, which prevents a direct comparison with Model $A$ rates used in Chapter IV. The trends and patterns from both models suggest that objectives of the S/HMO ECB program are being achieved. Table XXXVII, below, shows unadjusted rates for total nuring home payments received for research population members residing in ICFs during the study period per 1000 members. A statistically significant difference exists between the mean of such payments for Risk and S/HMO members. In subset I and age cohort 75-84, the "Never s/HMO" rate is twenty-six percent less than for "S/HMO sometime." It is the only instance where that is observed.

In Subset I and age cohort $85+$, the "S/HMO Sometime" rate is forty-nine percent less than that for "Never S/HMO." These rate differences are consistent with rate differences observed in Chapter IV under the not-mutually exclusive model for days of residence per member year of eligibility. The "S/HMO Sometime" rate in subset I under age cohort 65-74 is thirty-nine percent less than that for "never S/HMO." This is notable because the SNF+ICF "S/HMO sometime" 
rate of days in residence per member year of eligibility in Subset I, Chapter IV, Table XVI, was nine percent greater than that for "Never S/HMO."

TABLE XXXVII

TOTAL PAYMENTS RECEIVED BY NURSING HOMES WITHIN ONE YEAR AFTER THE STUDY PERIOD FOR ICF RESIDENTS PER 1000 MEMBERS MUTUALLY EXCLUSIVE ELIGIBILITY CLUSTERS: N1 - "NEVER S/HMO" (C, C+R, R); N2 - "S/HMO SOMETIME" $(S, C+S$, $R+S . C+R+S)$

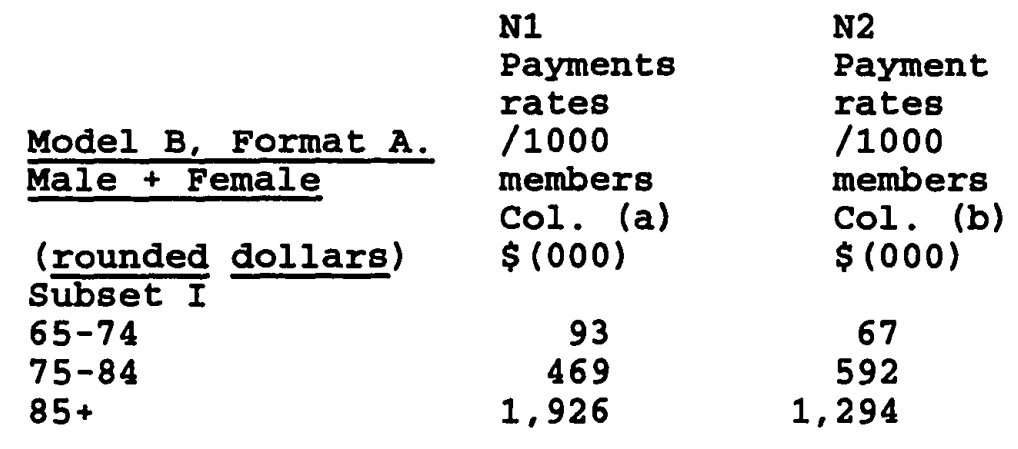

Subset II

65-74

75-84

119

776

$85+$

3,274

99

607

Overall

65-74

75-84

144

1,172

$85+$

4,821

116

625

1,815

Source Appendix E

Based on the above observations about differences in rates, it is possible to state a null hypothesis that, Differences Are Not Statistically Significant Between the Mean of Payments For "Never S/HMO" and "S/HMO Sometime" 
Nursing Home Residents: a. Overall, b. by Sex, c. by Age Cohort.

In the following three tables, a trial run test of the above hypothesis is are presented using nonparametric Analysis of Variance procedures to measure statistical significance of difference in means of payments received for each of the two clusters of ICF residents, "Never S/HMO" and "s/HMO Sometime."

The raw mean is the quotient of the sum of payments received for all ICF residents divided by the total number of ICF residents in each group under Analysis Model B. A nonparametric test of ranked revenues per member is used to compute a mean for use in this test of differences because distribution of variance about mean revenues is not normal. since statistical tests are intended for use with random samples to determine the probability that differences observed are probably real and not the result of chance sampling, it may be seem superfluous to use an Analysis of Variance test here. That is, rates from this study are presented on the basis of being derived from an entire research population, where differences are real and not the product of chance sampling.

A critical assumption of the analysis of variance procedure is that differences in values are distributed in a statistically normal way. The SAS procedure known as NPARIWAY provides a nonparametric option for an analysis of 
variance test; it does not compute an $F$ value based on the assumption of normal distributional of (payments) values about the means for each group compared. Rather, it ranks all the observations and computes an $F$ value based on difference in rankings.

This analysis of variance $F$ tests procedures is used here in the context that data used were hypothetically representative of all KP Medicare members during the period 1985 - 1990, and a random drawing from that membership would have a good chance of looking like the two years actually used in the research population.

A relaxed condition for rejecting the null hypothesis is selected because it is a trial process. Determining statistical significance is based on an alpha level of .10 . That is, a probability (p) value is computed to determine if the mean score (ANOVA Mean Score F) produced by the statistical procedure is likely to be the result of chance sampling or if difference in means is likely to be real. Thus, in Tables XXXVIII and XXXIX, $p$ values of .10 or more require that the null hypothesis, above, be accepted. values of less than .10 allow rejection of the null hypothesis.

The probability value of $F$ for mean scores under columns $A$ and $B$, below, suggest that the difference is statistically significant in every instance of comparison, except in age cohort 75-84. Thus, parts $a$ and $b$ of the null 
hypothesis, above, are rejected; part $c$ of the above null hypotheses statement is not rejected because the $p$ value of $F$ is .10 . Although the acceptance or rejection premise is based on the $p$ value being greater than .10, rejection is hard to defend for a rounded value of exactly .10. Indeed, throughout this study, a pattern of higher rates for s/HMO than Risk residents was consistent in age cohort 75-84.

TABLE XXXVIII

NPAR1WAY NONPARAMETRIC ANALYSIS OF VARIANCE TESTS FOR DIFFERENCES IN PAYMENT MEANS BETWEEN CLUSTER A (NEVER S/HMO) AND CLUSTER B (S/HMO SOMETIME) OF ANALYSIS MODEL B

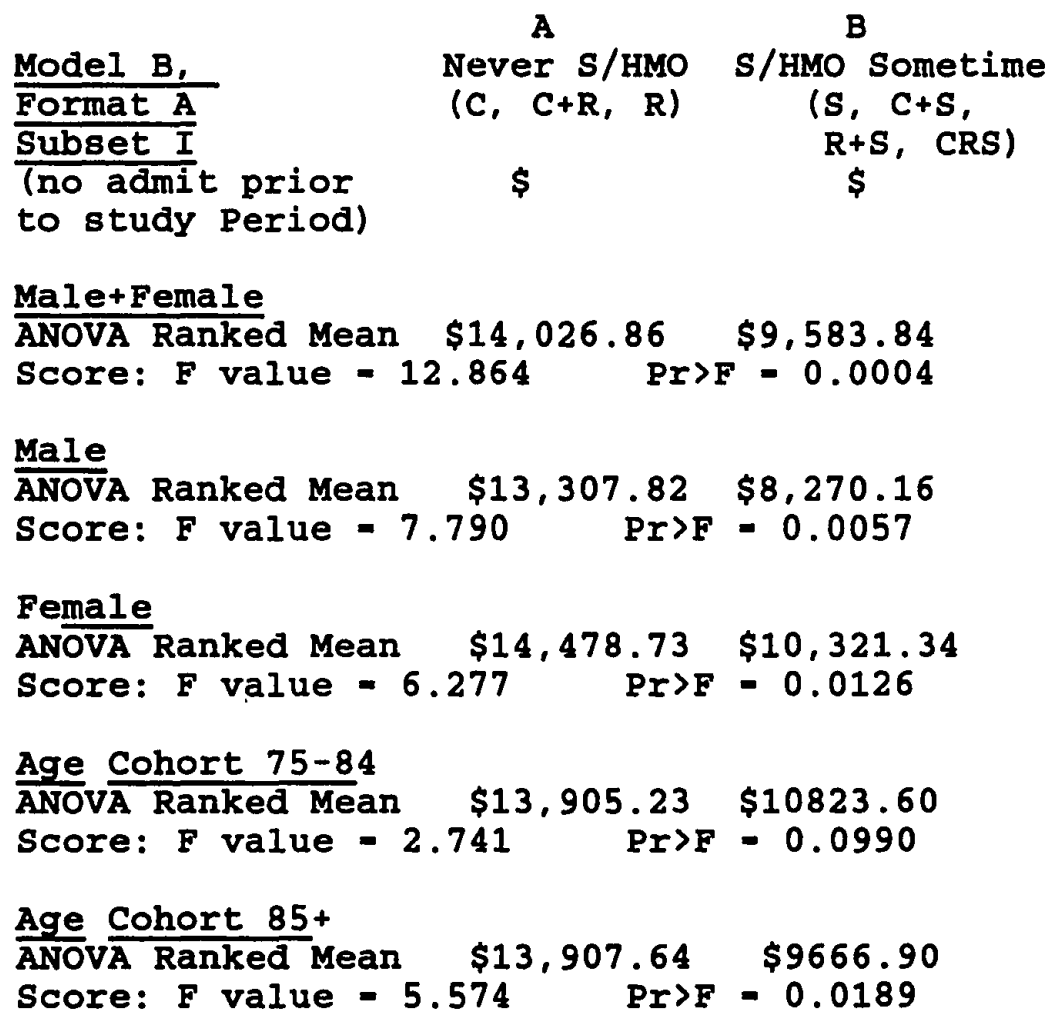


Next, payment rates per 1000 members are examined again under Model B, in Table XXXIX, below, but using each combination of member eligibility. In Format $B$, each eligibility group is listed vertically on the left side of the following tables, where as clustered groups were listed horizontally along the top of the previous two tables.

TABLE XXXIX

(RATES ARE STANDARDIZED BY AGE COHORT AND GENDER)

TOTAL PAYMENTS RECEIVED BY NURSING HOMES WITHIN ONE YEAR AFTER THE STUDY PERIOD FOR ICF RESIDENTS PER 1000 MEMBERS IN MUTUALLY EXCLUSIVE ELIGIBILITY GROUPS: I.E., COST, COST+RISK, RISK, S/HMO, COST+S/HMO，RISK+S/HMO, COST+RISK+S/HMO

$\begin{array}{llc}\text { Model B. Format B. } & \begin{array}{c}\text { Stdizd Pmts } \\ \text { for ICF Res }\end{array} & \text { for Idiz Pmts } \\ \text { Male }+ \text { Female } & / 1000 \text { member } & / 1000 \text { member } \\ \text { Subset I } & \text { Subset II } \\ \text { Col (a) } & \text { Col. (b) } \\ & \$(000) & \$(000)\end{array}$

\begin{tabular}{|c|c|c|c|}
\hline$\frac{\text { Row }}{1 .}$ & $\frac{\text { Group }}{\text { cost only }}$ & 444 & $\begin{array}{c}\text { (rounded dollars) } \\
1,093\end{array}$ \\
\hline 2 . & Cost+Risk & 597 & 743 \\
\hline 3. & "new" Risk & 378 & 611 \\
\hline 4. & "new" s/HMO & 409 & 517 \\
\hline 5. & Cost+SHMO & 338 & 479 \\
\hline 6 . & Risk+SHMO & 304 & 241 \\
\hline 7. & Cost+Risk+SHMO & 129 & 173 \\
\hline
\end{tabular}

Overall Mean payments $\$ 430$ $\$ 631$

Per 1000 member for ICF residents Source: Appendix E 
In Subset I (column a), rows 3 and 4 show that the "new" S/HMO rate is eight percent greater than "new" Risk for payments received per 1000 members. "S/HMO Only" ICF days per year of member eligibility were fifteen percent less than that for Risk. S/HMO only and Risk Only members were either enrolled directly from the community, rather than converting from within $\mathrm{KP}$, or at age sixty-five (65) they enrolled directly into Risk or S/HMO.

Since rates for payments received by nursing homes included SNF and ICF payments, the observation of this reversal may be related to the higher use of SNF by S/HMO than by Risk members. This observation is repeated when SNF and ICF days are combined, as seen in the following table. Observations regarding rows 3 and 4 (new Risk vs. new S/HMO) of Format $B$ do not alter the overall findings of Format $A$ in Analysis Model B.

It does confirm similar observations about "new" s/HMO members using more days in the study period than "new" Risk members. In fact the rate for payments received suggests that the difference in days used prevailed for study period residents who continued use of nursing homes over the year succeeding the study period.

It can be stated that "new" $s /$ Ho members would have been a greater liability than other groups of $\mathrm{s} / \mathrm{HMO}$ members as well as "new" Risk members, relative to total nursing home expenditures for those who resided in ICFs during the 
study period, under subset I. That was not true when residents were included who had began their use of nursing homes prior to the study period, in Subset II.

In Table XXXIX, above, rates confirm what was observed in Table xxxVIII, regarding substantial savings in nursing home expenditures for s/HMO member in ICFs.

What explains the greater expenditures for nursing home care by Cost members who converted to s/HMO than occurred for Risk members who converted to $\mathrm{s} / \mathrm{HMO}$ (rows 4 and 5 , column (a), in Table XXXx, below? Prior use is not an explanatory variable in subset $I$, column (a); very few s/HMo members became nursing home residents between the time of their application and effective date in s/HMO. Fewer yet had been in a nursing home but discharged prior to the time of application for S/HMO, although those who were may have had significant nursing home experience. S/HMO applicants were rejected if in a nursing home at that time.

In the preceding table of ICF residents, new S/HMO residents in subset I was the single group rate, within the cluster of group rates for "S/HMO sometime," which exceeded any of the group rates for "Never s/HMO."

This was true for both SNF plus ICF and ICF rates in Subset I. It was not observed in subset II. It is possible that nursing home use prior to becoming a s/HMO member affected rates in row 5 resulting in a higher relative payment level than for "Never s/HMO." 
TABLE XXXX

(RATES ARE STANDARDIZED BY AGE COHORT AND GENDER) TOTAL PAYMENTS RECEIVED BY NURSING HOMES WITHIN ONE YEAR AFTER THE STUDY PERIOD FOR SNF \& ICF RESIDENTS PER 1000 MEMBERS IN MUTUALLY EXCLUSIVE ELIGIBILITY GROUPS: I.E., COST, COST+RISK, RISR, S/HMO, COST+S/HMO, RISR+S/HMO, COST+RISK+S/HMO

Model B, Format B. Male + Female

Row Group

1. Cost only

2. Cost $+R i s k$

3. "new" Risk

4. "new" s/HMO

5. Cos $t+$ SHMO

6. Risk+SHMO

7. Cos $t+$ Risk+SHMO

Mean Payments

Per 1000 members

for SNF+ICF Days-SP

Source: Appendix E
Stdizd

Payments

for ICF

\& SNF Res. $/ 1000$ members Subset I

(a)

$\$(0.00)$
Stdidz

Payments

for ICF

\& SNF Res.

$/ 1000$ members

Subset II

(b)

$\$(000)$ (rounded dollars)

$524 \quad 1,206$

656

823

478

785

548

670

502

549

329

266

184

221

$\$ 515$

$\$ 750$ 


\section{SUMMARY OF OBSERVATIONS}

- Less revenue was received for ICF residents who were in the cluster $\mathrm{S} / \mathrm{HMO}$ Sometime then for nursing home residents in the cluster Never In S/HMO, under both subset $I$ and Subset II of Format A, Model B.

- Less revenue was received for every age cohort of ICF residents who were in the cluster S/HMO Sometime then for nursing home residents in the cluster Never In S/HMO, under both Subset I and Subset II of Format A, Model B.

- Less revenue was received for nursing home residents (SNF plus ICF) who were in the cluster S/HMO sometime then for nursing home residents in the cluster Never In S/HMO, under both subset I and Subset II of Format A, Model B.

- A statistically significant difference in means was observed, confirming that cluster rates for $s /$ Ho sometime were less than cluster rates for Never S/HMO, with one exception. This was observed for each test of gender, as well as for all residents in subset $I$, and for age cohorts 65-74 and 85+, but not for age cohort 75-84. The analysis of variance test for difference in means represents the sum of nursing home payments received for all ICF residents in each cluster divided by the number of residents in that cluster. This exception is consistent with rates for ICF days per member year of eligibility. This exception did not appear in Subset II.

- More revenue was received for nursing home residents who were enrolled directly into s/HMo as new members during the study period, i.e.. in the cluster s/HMO sometime, then for nursing home residents who were enrolled directly into Risk as new members during the study period, i.e., in the cluster Never In S/HMO, under Subset $I$, but not under subset II of Format B, Model B.

\section{POLICY RESEARCH ISSUES}

It is difficult to find data in the literature which is comparable to that presented in this chapter. The generic problem is finding nursing home payments for ICF services; much data is available for SNF revenues or computed costs, but little is reported on ICF revenues. Even less is 
reported on total nursing home revenues (SNF+ICF) for those who become ICF residents. While Medicaid and Medicare data is often reported, private pay revenue, is rarely reported.

The 1985 National Nursing Home Survey presents the most recent characteristics of nursing homes, nation-wide. In 1985 there were 19,100 nursing homes with 1,624,200 beds of which 69 percent were under proprietary ownership. Nursing homes are certified for SNF, ICF, both SNF and ICF, or not certified by Medicare and or Medicaid. IN 1985, 758 of nursing homes had some beds certified as meeting SNF and/or ICF level criteria by either Medicare, Medicaid or both, accounting for 89 percent of total nursing home beds. Of certified nursing homes $(14,400), 40$ percent were state or Federally certified for both SNF and ICF beds, accounting for 50 percent of all certified beds. Occupancy levels were in excess of 90 percent. The average daily rates for private pay was $\$ 61$ for SNF, $\$ 48$ for ICF $\$ 31$ for RCF level services. In the west, these per diem rates were $\$ 58.22-\mathrm{SNF}$, $\$ 47.44-I C F$ and $\$ 28.52$ for RCF. Medicaid ICF was 43.02. [68] The use rates per 1000 population age 65 and over are closer to those in this study than to Multnomah County. They are as follows: age 65-74 - 12.5/1000; age 75-84 $57.7 / 1000$; age $85+219.4 / 1000$ and overall ages $65+46.1$; all Males - 29.0/1000; all females 57.7/1000.[69]

Another study presents a slightly different estimate of per diem costs from the 1985 NNHS. "Calculated at the sample 
means, marginal costs in SNF facilities in 1986 were $\$ 130$ for a Medicare day, $\$ 74$ dollars for a private day and $\$ 56$ for a Medicaid day."[70]

similar, but not comparable per diem payments are presented below for nursing home users in this study. Data includes SNF+ICF payments received for every category; thus per diem rates are higher than those presented above. Data in the following table is for nursing home residents whose first admission occurred prior to the study period, and whose last discharge data was before the end of the study period. Thus, none of the financial data is for days of stay after the study period, as is the case in all previous tables. This subset of users is the only way that revenues can be restricted to study period nursing home use in this study.

Since SNF+ICF payment information is combined, the proportion of days (denominator) in SNF may be greater for S/HMO than Risk, thus lowering the rate. No weighting is done to adjust for such differences. These are raw rates. However, the pattern is too consistent across level of care and Models $A$ and $B$ to discount the apparent lower mean daily revenue for $s / H M O$.

Table XXXXI, below, presents average daily revenues per research population member as a measure of differences between Risk and S/HMO ICF residents whose entire history of nursing home use occurred within the study period. By 
definition, these are not very long-stay residents.

\section{TABLE XXXXI}

PER DIEM PAYMENTS INCLUDING SNF+ICF STAY BY EACH CATEGORY PRESENTED FOR ALL MEMBERS WHOSE FIRST ADMISSION \& LAST DISCHARGE WAS WITHIN THE STUDY PERIOD

\section{Category}

Model A SNF ICF

$$
\begin{array}{lll}
\text { Per Diem } & \text { Payments } & \text { For SNF+ICF } \\
\text { Cost } & \text { Risk } & \text { SHMO } \\
\$ 229.86 & \$ 257.75 & \$ 200.35 \\
\$ 153.11 & \$ 129.73 & \$ 99.08
\end{array}
$$

Model B

SNF+ICF *
SNF
ICF

\begin{tabular}{cr} 
Never SHMO & SHMO Sometime \\
\cline { 2 - 2 }$\$ 111.92$ & $\$ 82.53$ \\
$\$ 249.38$ & $\$ 196.26$ \\
$\$ 122.33$ & $\$ 97.62$
\end{tabular}

*Comprehensive Nursing Home Average Revenue/Day SNF overlaps with ICF; SNF+ICF - unique count 
CHAPTER VIII

DIFFERENCES IN MEDICAID STATUS BETWEEN ELIGIBILITY GROUPS

[An]...indicator of the spend-down phenomenon is found in the recent paper of Liu and Manton (1989), based on the National Long Term Care follow-up survey...over a two year period in a community sample of disabled elderly who were initially non-Medicaid, the risk of becoming Medicaid eligible was $31 \%$ for those who experienced a nursing home stay and $7 \%$ for those who did not....it is relevant to know that spend-down outside the nursing home among the disabled is not negligible... Because the [disabled] group not using nursing homes was about 7 times the number admitted to these facilities, they accounted for about threefifths of the individuals who spent down in the two year period...the missing piece is the number of dollars involved for each group. For example, spend-down for community based care may affect elderly near the cutting edge of eligibility for Medicaid and it may not require high expenditures over a long period to make them eligible. The situation could be quite different for spend-down due to nursing home stays. More frequently this may affect individuals whose financial resources are depleted after meeting large costs for appreciable periods of time... In the 1985

NNHS, $36 \%$ of patients discharged within three months, and $58 \%$ of those with stays of at least a year.... [were on Medicaid when discharged] [71]

Two policy issues are addressed by this chapter. The

first issue is how to reduce the incidence of persons who enter a nursing home and thereafter become dependent on Medicaid for payment of charges. This study asks what effect 
is the S/HMO concept likely to have on that problem. The second issue is how to reduce the extent of public financing required for nursing home residents who spend down. This study asks what solution does the s/HMO concept offer regarding that problem.

The S/HMO Demonstration project has not tracked ICF use rates of Risk members for purposes of developing control group data with which to compare S/HMO member use of ICFs, nor did HCFA evaluators. Therefore, data has not been available regarding comparison of Medicaid expenditures for $\mathrm{s} / \mathrm{HMO}$ and Risk members.

specifically, this chapter makes several comparisons of $\mathrm{S} / \mathrm{HMO}$ and Risk information collected during this study. Two rates are used, the probability of ICF and SNF residents becoming Medicaid eligible up to one year after the study period and Medicaid payments received by nursing homes during and up to one year following the study period per 1000 members.

This study does not present other information about the Medicaid experience of research population members. That goal was part of the original research proposed to the Dissertation Committee in 1988. The colloquium proposal included undertaking multivariate analysis of effects which various independent variables or covariates might have on use rates. In fact, preliminary data required for that research procedure, was partially obtained from the senior 
Services Division, State of Oregon, Medicaid data base files in 1990 and 1991. But the SSD Data Processing Department stopped down-loading records of KP members on Medicaid, for use at the Center for Health Research, following passage of Measure 5 in 1991. Data in this chapter is limited to only that collected from nursing homes in 1989 and 1990. Two Research Questions guide production of rates in this chapter. The first policy question addressed is, in what way does S/HMO ECB appear to reduce the incidence of spend-down related to use of ICF services?

Research Question IV: Are There Differences Between S/HMO and Risk Research Population Members Regarding the Probability of Receiving Medicaid Funds To Pay Nursing Home Bills?

Table XXXXII, below, presents the number of ICF members, admitted during the study period, per 1000 Cost, Risk, S/HMO eligibility group members for whom nursing homes received Medicaid payments in one year post study period.

In subset II, the probability rate of becoming a Medicaid Recipient, during or within one year after the study period, was forty-four percent less for s/HMo members who were admitted to an ICF during the study period, than that rate for Risk members.

In Subset I of Table XXXXII, the probability rate of becoming a Medicaid Recipient, during or within one year after the study period, was twenty-three percent less for s/HMO members who were admitted to an ICF during the study 
period, than that rate for Risk members.

TABLE XXXXII

(RATES STANDARDIZED BY SEX BY AGE COHORT)

RATE OF ICF FIRST ADMITS IN THE STUDY

PERIOD /1000 MEMBERS (HPM) FOR WHOM

NURSING HOMES RECEIVED MEDICAID

FUNDS FOR ICF OR SNF STAY

BEFORE 07/01/89

Model A Cost Admts Risk Admts S/HMO Admts $/ 1000$ HPM $/ 1000$ HPM $/ 1000$ HPM

$\begin{array}{llll}\text { Subset II } & 9.04 & 9.23 & 6.40 \\ \text { Subset I } & 6.68 & 6.79 & 5.53\end{array}$

Source: Appendix F

Subset I and II rates, above, include the 124 AFC welfare recipients enrolled in s/HMO during the study period, 9 of whom were admitted to an ICF after study period began. Data for AFC nursing home residents are removed from other information presented in this chapter. Only 10 of these 124 were in a nursing home during the study period; one had been in a nursing home prior to the study period.

Research Question V: Are There Differences In the Proportion of S/HMO and Risk Members who Were ICF Residents During the Study Period, Who Also Were Medicaid Recipients within one Year Following The study Period?

Table XxxxII, above, describes the probability of research population members becoming Medicaid dependent. Another way of describing differences between cost, Risk and S/HMO member's Medicaid Assistance patterns is to present 
the percent of nursing home residents from each group for whom nursing homes received Medicaid payments at some time during the study period and up to one year thereafter. Raw data is used for these proportions, as shown below in Table XXXIII.

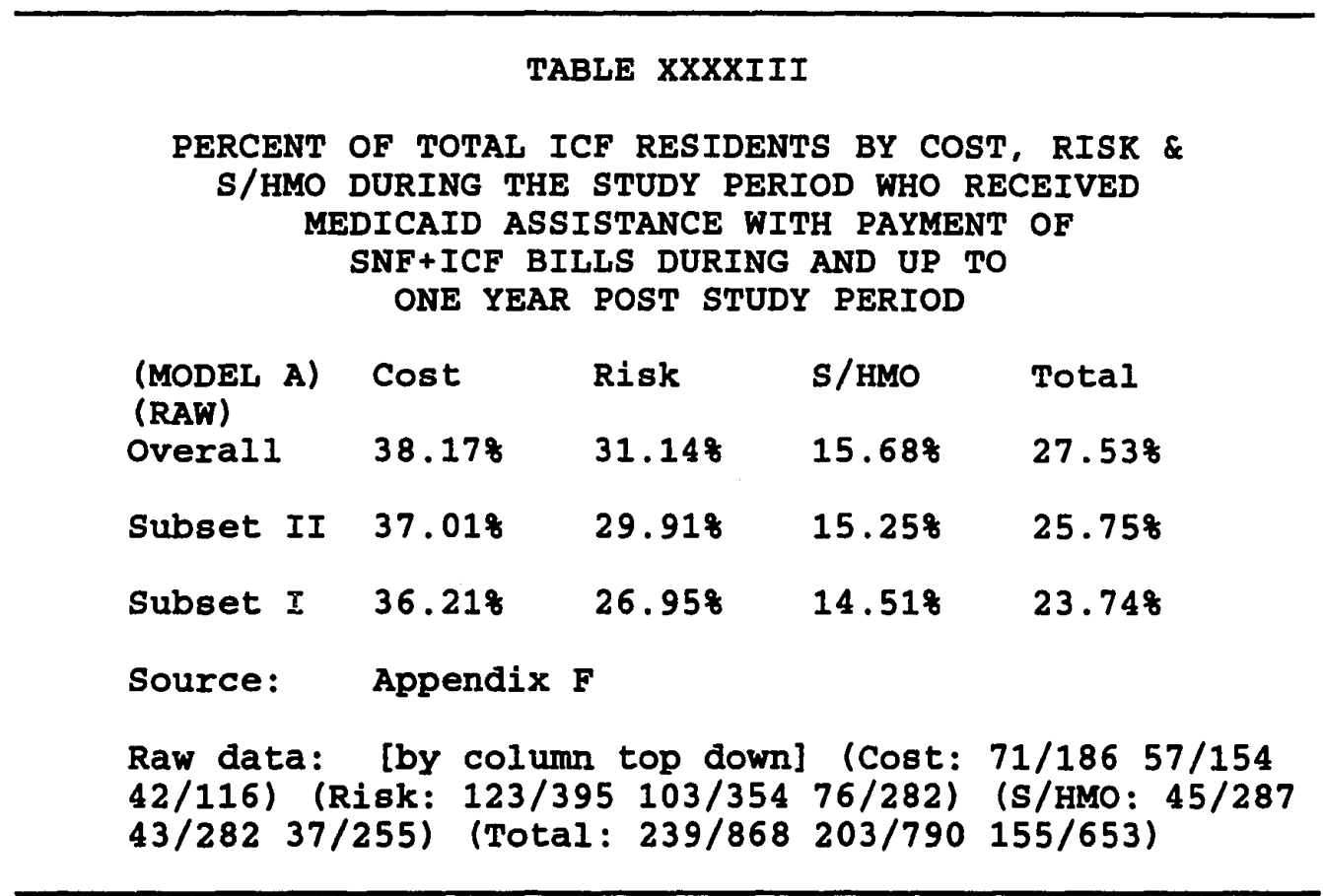

The pattern of differences in welfare dependency is clear and the resulting conclusion is inferred with some confidence that ECB contributed strongly to deferring or avoiding the need for Medicaid assistance by S/HMO ICF residents, relative to such need by Risk and Cost members who were ICF residents during the study period. Figure 8, next, summarizes these differences. 


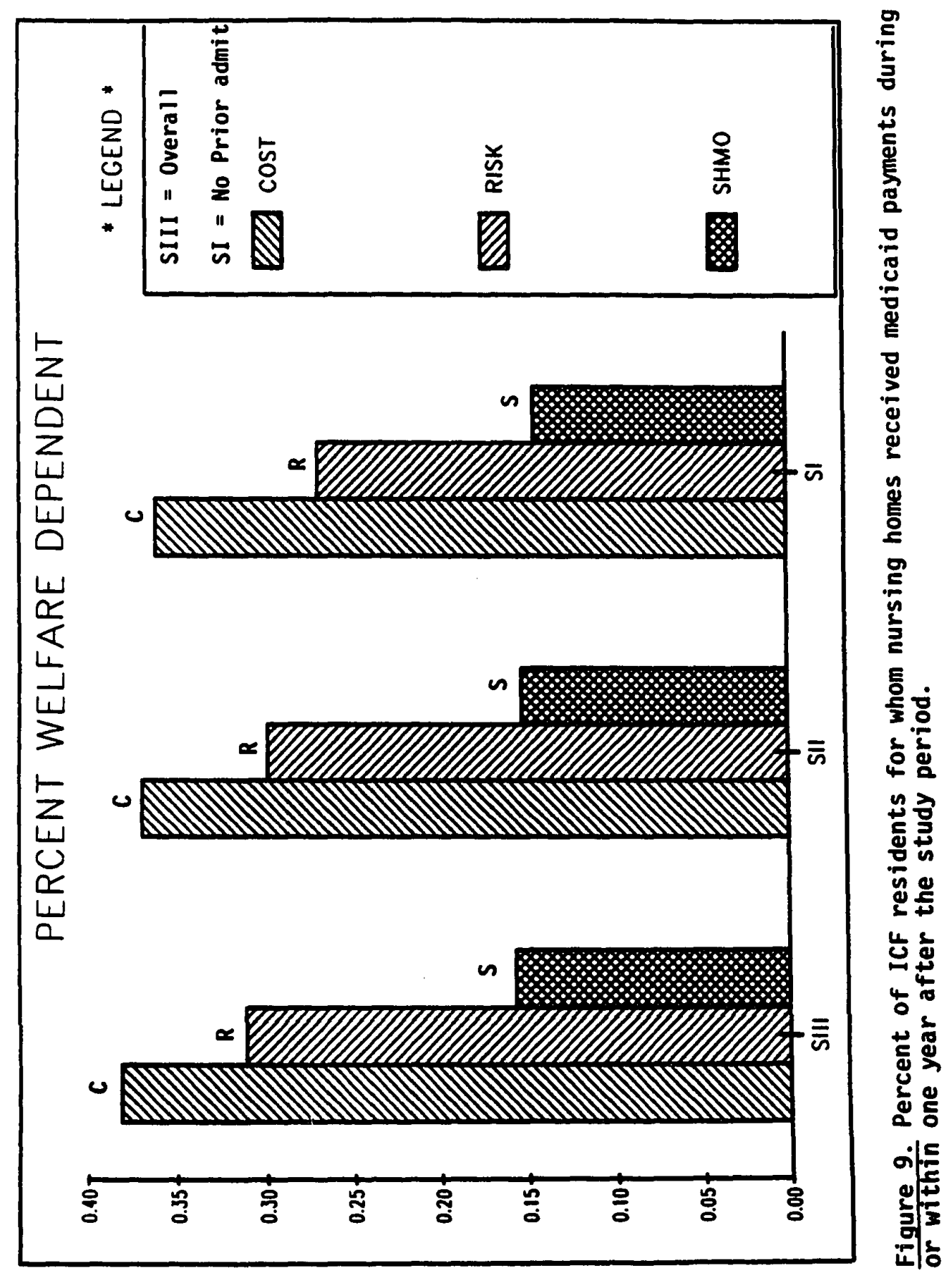


Another view of data on total payments is presented next in Table XXXXIV, regarding Research Question IV.

TABLE XXXXIV

PERCENT OF TOTAL ICF RESIDENTS IN "NEVER S/HMO \&

"S/HMO SOMETIME" DURING THE STUDY PERIOD WHO RECEIVED MEDICAID ASSISTANCE FOR PAYMENT

OF NURSING HOME BILLS DURING AND

UP TO ONE YEAR AFTER

THE STUDY PERIOD

(MODEL B)

Never S/HMO S/HMO Sometime Combined

$\begin{array}{llll}\text { Subset I } & 29.868 & 14.078 & 23.15 \%\end{array}$

The pattern is the same in the two preceding tables. They suggest that the likelihood of spend-down by s/HMO members, as viewed under subset $I$, is about half that of Risk members.

However, some cautions are needed about interpreting the values in the above three tables.

Spend down, as related to nursing home use, by definition means member assets did not qualify him/her for Medicaid assistance with payment of nursing home bills before first being admitted to an ICF or SNF.

In reality, Medicaid spend-down is a function of all medical services which reduce a person's assets to a level qualifying them for Medicaid funds to pay such bills. Thus, a person could be in a nursing home, and not be eligible for assistance, be discharged to a hospital SNF and readmitted 
to an ICF after having become eligible for Medicaid

assistance. This is an important issue to address in clarifying effects of any insurance or service based LTC program on that need for Medicaid help. Without clarifying total health costs, it is hard to predict a cause-effect relationship, or even correlate any one aspect of health services, with the moment of spend-down.

In a prepaid, group practice, health center based, capitated HMO, where the continuum of each patient's care is not interrupted by competing groups of providers, care needs are efficiently coordinated with the full range of health service providers. Spend-down occurs as a part of this process. However, among capitated HMO members, nearly all health costs are covered; seldom to Risk and S/HMO Medicare beneficiaries use services not substantially covered by their benefits.

Therefore, aside from persons on welfare prior to admission to a nursing home, it is unlikely that persons would have spent down before entering a nursing home as a $\mathrm{KP}$ member. Risk or $\mathrm{S} / \mathrm{HMO}$ members newly enrolled directly from the community could be an exception to that general condition. This does not mean that converted Risk members in ICFs during the study were not Welfare recipients. It is very likely, indeed almost certain, that some Risk ICF residents in Subset II were Medicaid dependent when the study period began, because many of them had begun their 
nursing home experience before the study period.

For that reason, findings from subset I are emphasized as the most meaningful data regarding possible influence of S/HMO regarding answers the policy issues and Research Questions asked in this chapter. It is unlikely that many Risk ICF residents, whose initial nursing home admission occurred during the study period, were on welfare at that time. Since that information is not known, caution is needed about the interpretation of rates presented. The important issue is the pattern and trend of differences between Risk and $s /$ Ho they suggest existed.

While the term spend-down is used above, it is used with less precision than may be appropriate for some analysis.

\begin{abstract}
A recent study found that 58 percent of all nursing home residents remained non-Medicaid patients during their stays and only 7 percent spent down during their stay to become Medicaid eligible. The remaining 35 percent were Medicaid eligible when they entered. Successfully targeting 7 percent of the population who spend down is the key to attaining the [RWJF] project cost containment goals. [72]
\end{abstract}

"RWJF" refers to Robert Wood Johnson Foundation planning grants awarded to eight states, including oregon. The purpose of RWJF research money is to promote use of and study the effects of various insurance policies, and mechanisms for marketing long-term care insurance, on the extent to which it protects elderly persons against 
impoverishment resulting from long-term care needs, and the extent to which it reduces Medicaid costs.

The above citation noted that only 7 percent spent down, from the 65 percent who were not Medicaid eligible at the time of nursing home admission; 35 percent were already on Medicaid upon nursing home admission.

It is uncertain how observations in the above table relate to the above citation without knowing how many Risk members were on Medicaid at the time of admission. Such information could strengthen the importance of observations offered above.

Given the above observations and caveats, it is interesting to note that one study of Medicare nursing home residents (SNF+ICF), who became Medicaid dependent in the States of Michigan, California and New York during the same time period as this dissertation study, showed outcomes somewhat similar to this study for Californians. Specifically within 90 days of nursing home admission, 40.8 percent of admissions were Medicaid dependent and within 180 days 51 percent were Medicaid dependent. Rates were substantially lower in Michigan and New York, than in California. In all three states, "... not only is the prevalence of Medicaid covered nursing home residents highest for the very old who are female...but the annual rate of entry to the nursing home is highest for this group as well. Females who are very old also had the lowest rates 
of both nursing home discharge of any kind and discharge to the community."[73]

Age cohort $85+\mathrm{s} / \mathrm{HMO}$ females were substantially lower users of nursing homes than Risk members, and their rate of Medicaid dependency and raw rates of Medicaid payments received per member year of eligibility was much lower (Subset I, raw rate for female Risk - $\$ 209 /$ member year of eligibility; female s/HMO - $\$ 51 /$ member year of eligibility. Differences in Medicaid payments are discussed next. The policy issue from which the next Research Question arises is what cost containment practices can be invoked which slows or reverses the inflationary trends of Medicaid payments for nursing homes? Numerous insurance schemes and trial service plan projects have been and are being tested by many States. The policy question is what prospect does the S/HMO ECB concept offer as a policy solution?

The analysis of changes in Medicaid spending on acute-care and long-term care services revealed that growth in long-term care spending continue to outstrip growth in acute-care spending... Medicaid spending in constant dollars increased much faster for the aged and disabled than for adults and children [during 1984-87]...outlays for nursing home care increased by 3.98 per year in constant dollars, reflecting a 1.7 percent per year increase in recipients, as well as a 2.1 percent per year increase in real expenditures per recipient. [74]

ICF services have become the second largest single contributor to total Medicaid payments (inpatient hospital services are the largest single contributor). In 1989, [non ICF-MR] ICF services accounted for 16.3 percent of all Medicaid payments... If we combing ICF and SNF 


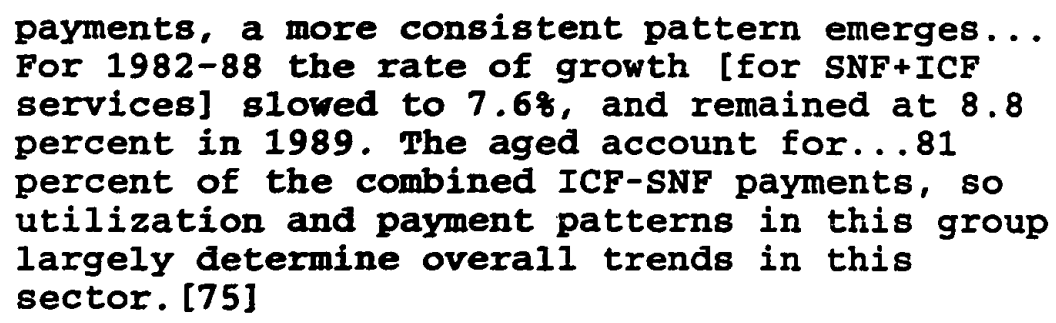

The policy issue of increasing Medicaid cost for

nursing home services is essentially one of cost containment given the proportion of state welfare budgets consumed by nursing home payments for welfare recipients.

Research Question VI: Are There Differences In Medicaid Payments Received BY Nursing Homes For Members Residing In ICFs During The Study Period Per 1000 Research Population Members?

Nursing home business records differentiated Medicaid payments from other sources of revenue on a consistent basis which allowed data collection to proceed as planned for this variable. Rate information on payments received by nursing home for residents is presented as a consolidated amount, not distinguishing between payments received for SNF level care and ICF level care.

The following tables present rates based on payments received by nursing homes for ICF residents per 1000 members by eligibility group. Payments received include all money recorded on nursing home ledgers as received; amounts owed or billed but not collected are excluded. If the nursing home was licensed for both SNF and ICF services, and both levels of care were provided then payments received for both 
levels of care are included in rates under this research question even though the resident status is presented in following tables as ICF level care.

Payments received by all facilities for all admissions are accumulated into a single value for each resident. Payments include those received from before the study period beginning with 1985 (in Subset II), during, and after the study period through June of 1989 .

Subset II approximates a longitudinal study of four years of nursing home use, $4 / 85$ through $6 / 89$, for all s/HMO and Risk members who were in a nursing home during the study period. Persons in a nursing home before or after the study period, but not during the study period, are not included in that four year span of information. This span of time is important to both Subsets I and II for different reasons. It assures that Subset I includes only persons without prior nursing home use, and therefore subset I does not represent a cross-section of all users the way most nursing home surveyors do. In Subset II, it assures that Medicaid costs for those residents with prior nursing home stays are not misrepresented, due to left censoring.

Medicaid spend-down rates can be very misleading without knowing what proportion of persons in a sample have had previous bills to pay for nursing home care. Only since the 1985 National Nursing Home Survey have researchers worked to clarify the effects of prior admission on 
spend-down estimates based on national survey data.

Likewise, without collecting all data from all nursing homes used by any given resident, Medicaid cost estimates per resident can be very misleading.

In effect, Table XXXXV, below is a longitudinal study of three years duration.

\section{TABLE XXXXV}

(RATES ARE STANDARDIZED BY AGE COHORT AND GENDER) MEDICAID PAYMENTS RECEIVED BY FOR ICF RESIDENTS ONE YEAR AFTER THE STUDY PERIOD FOR ICF RESIDENTS/MEMBER YEAR OF ELIGIBILITY IN MUTUALLY EXCLUSIVE CLUSTERS : N1 - "NEVER S/HMO" (C, C+R, R) : N2 - "S/HMO SOMETIME" (,$C+S$, $R+S .(+R+S)$

Model B, Format A.

Male + Female (dollars are rounded)

ICF Residents ONLY Subset I

Subset II

Overall
(N1) Standardized

Medicaid

Payments

(SNF+ ICF)

/HPM Yr. of /HPM Yr. of SP Elig.

for ICF

Residents

Col. (a)

$\$ 53$

$\$ 103$

$\$ 40$

$\$ 160$

$\$ 51$

SNF+ICF RESIDENTS Overall stdiz rate

$\$ 505$

Within Study Period ICF RAW Rate 
The above table includes all nursing home residents admitted during the study period with no prior admissions. Subset I represents a "clean catch" specimen for analysis of rate differences.

As a control or confirmation of this comparative standardized measurement, the comparable raw rate is presented as the last line in the above table for ICF residents who were Medicaid recipients and whose first nursing home admission occurred in the study period and whose last discharge date occurred in the study period, and for whom no subsequent admission occurred within the following year.

The difference (61 percent) in Medicaid payments per member year of study period eligibility is substantially greater, between $N 1$ and $N 2$ for ICF residents in subset $I$, than the difference (14 percent) in total payments for $N 1$ and N2 ICF residents in Subset I (see Table XXXIV).

The above data show that the Medicaid Payments for such Never S/HMO residents was $\$ 8.07$ per member year of study period eligibility $(\$ 2,814,939 / 348,825)$, while the Medicaid Payments for S/HMO Sometime Medicaid ICF residents was $\$ 3.15$ year of study period eligibility $(\$ 889,438 / 282,317$ study period eligibility days).

Although Medicaid payments comprise slightly less than half of nursing home revenues, little information is known about some important aspects of residents who enter a 
nursing home under private pay reimbursement term and subsequently spend their assets down to levels of welfare eligibility and therefore become Medicaid Dependent. Among such persons, it is not well understood how effective private or public risk pooling schemes might be with respect to averting nursing home related spend-down; varying opinions exist on this matter.

Liu and his associates show that nationally in the early $1980 \mathrm{~s}$ most spending down of assets occurred among those residing outside of nursing homes. (27) [Liu \& Manton., "The Effect of Nursing Home Use in Medicaid Eligibility," The Gerontologist, 30 (February, 1990), 12]... on the other hand, the large portion of Medicaid beneficiaries who became eligible due to spend down, and the larger portions of all spending devoted to their care, could be seen as targets for potential reductions in Medicaid outlays for nursing home care. The interest in private insurance for long-term care or mixed publicprivate programs springs partially from this possibility. (28) [Meiners, "Reforming Long-Term Care Financing Through Insurance, "Health Care Financing Review (Annual Supplement, 1988), p. 109-121]

Our findings regarding the timing of spending down suggest, however, that public policies and private insurance schemes aimed at averting spending down among nursing home residents may have only small effects. The majority of people who enter nursing homes stay only brief periods, and many of these appear to be using nursing homes as adjuncts to acute hospital care or as substitutes for hospice services, both of which result in relatively short stays. These people are unlikely to spend down, for substantial portions of their nursing home charges undoubtedly are paid by the Medicare program. Others who stay longer and eventually spend down convert to Medicaid on average rather early in their stays and remain institutionalized as Medicaid beneficiaries for relatively long period. This leaves little time for public or 
private programs to affect the course of spending down while perhaps committing them to lengthy benefit flows.

Finally, one might note that, regardless of the path to dependence, the Medicaid program may not be the appropriate public instrument for providing assistance. Rooted as it is in traditions of welfare policy, with its accompanying means-tested administrative regulations, the program invites abuse, perpetuates unfairness, and becomes with each new safeguard administratively more complex. (29) [Moses, "The Fallacy of Impoverishment," The Gerontologist, 30 (February, 1990), p. 21-25] . Unfairness emerges from two considerations. First, some individuals and families transfer costs to others by being inclined to and adept at skirting asset depletion requirements. Additionally, the Medicaid program's status as a direct transfer program deprives it of a rationale for intergenerational equity such as that which underlies the Social security program. (30) [Aaron, et.al.. Can America Afford to Grow 0ld: Paying for Social Security, The Brookings Institution, 1989]. This absence, perhaps more than other considerations, argues for abandoning current arrangements in favor of either publicly enforcing savings or public incentives that encourage voluntary saving. Delay in instituting such programs prolongs unfairness that can only grow when today's middle-aged population retires shortly after the turn of the century. [76]

Among research population members in this study, an important but missing component of information is the Medicaid status of residents at the time of nursing home admission. Although observations in the preceding and following tables show that $s /$ HM members were less likely to be or become Medicaid dependent than Risk members, it is uncertain what proportion of Risk members were already on welfare at the time of first nursing home admission. While 
it is unlikely that the availability of such data would substantially alter the observed differences between Risk and $S$ /HMO in subset $I$, the certainty of this finding remains open to interpretation.

Table xxxxvI, below, provides another view of the same numerator data used in the table above; the denominator below is per 1000 members over the two year study period.

TABLE XXXXVI

(RATES ARE STANDARDIZED BY SEX AND AGE COHORT) MEDICAID PAYMENTS RECEIVED FOR SNF AND ICF CARE BEFORE, DURING AND AFTER THE STUDY PERIOD THROUGH 06/30/1989 FOR MEMBERS RESIDING IN ICFS DURING THE STUDY PERIOD/1000 RESEARCH POPULATION MEMBERS IN "S/HMO SOMETIME" OR "NEVER S/HMO," (MODEL B, FORMAT A)

N1 Never S/HMO

Model B Format A

Overall

subset II

Subset I (C, $C+R, R$ ) Public Pmts Rec'vd $/ 1000$ members for ICF Res. rounded $(000)$ $\$$ 275 176 91
N2 S/HMO Sometime $(S, C+S, R+S, C+R+S)$ Public Pmts Rec'vd $/ 1000$ members for ICF Res.

(000)

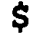

91

80

59

Source: Appendix F

The overall standardized rate of Medicaid payments for ICF residents, per 1000 "Never S/HMO" members is three times 
that rate for "S/HMO Sometime" cluster. Under Subset II, the "Never S/HMO" rate of payments for ICF residents, per 1000 members is twice that of the rate for "s/HMO sometime" cluster. Two important observations are made about the difference in rates between the "S/HMO Sometime" and "Never S/HMO" clusters under subset I.

In previous chapters rates of utilization under subset I have shown either no difference between Risk and S/HMO or a trend of $\mathrm{s} / \mathrm{HMO}$ slightly exceeding Risk. But, that is not the case with rates for payments received.

Second, the magnitude of difference in rates on payments between "Never S/HMO" and "S/HMO" sometime in Subset $I$ is great enough to suggest that while days in residence were about the same for Risk and S/HMO, (Chapter IV), the $\$ 12,000$ front-end ECB payments for ICF services notably reduced the level of payments needed by Medicaid to cover nursing home debts of residents who spent down.

A different view of the answer to this Research Question is presented next. Total payments received includes Medicaid payments. Table XXXXVII, below, presents that proportion of total payments received during and up to one year after the study period for ICF residents, which were publicly financed by Medicaid. Table XXXXVIII and Figure 9, show total Medicaid payments received by nursing homes as a percent of total payments within one year after the study period for SNF and ICF residents, many of whom were in both 
TABLE XXXXVII

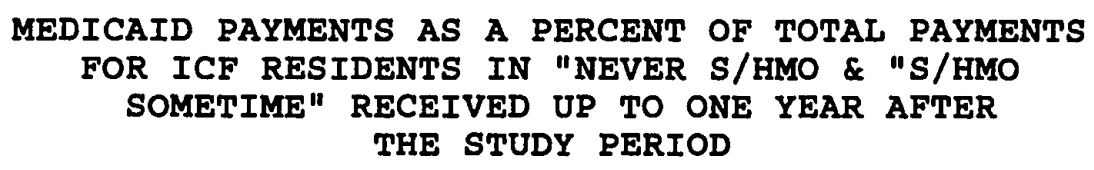

Model B

(N1)

(N2)

(RAW) Never S/HMO S/HMO Sometime Combined

$\begin{array}{llll}\text { Subset I } & 20.24 \% & 14.82 \% & 18.48 \%\end{array}$

In the above table, Raw data for each value listed are: $\mathrm{N} 1=\$ 1,132,843 / \$ 5,597,881 ; \mathrm{N} 2=\$ 398,719 / \$ 2,690,737$.

TABLE XXXXVIII

(RATES ARE STANDARDIZED BY SEX AND AGE COHORT) MEDICAID PAYMENTS RECEIVED FOR SNF AND ICF

CARE BEFORE, DURING AND AFTER THE STUDY

PERIOD THROUGH 06/30/1989 FOR ICFS OR

SIIF STUDY PERIOD RESIDENTS PER 1000

MEMBERS IN "S/HMO SOMETIME"

OR "NEVER S/HMO,"

N1

Never $S /$ HMO

Model B

Format A
(C, $C+R, R)$

Public Pmts

Rec'vd for

SIF and/or ICF

residents

$/ 1000$ members
N2

s/HMO Sometime $(S, C+S, R+S, C+R+S)$ Public Pmts Received for SIT and/or ICF residents $/ 1000$ members

rounded

S:F and/or ICP

\begin{tabular}{llc}
\multicolumn{1}{c}{ rounded } & $(000)$ & $(000)$ \\
& $\$$ & $\$$ \\
Overall & 583 & 328 \\
Subset II & 212 & 80 \\
Subset I & 109 & 67 \\
Source: Appendix F & &
\end{tabular}

Source: Appendix F 


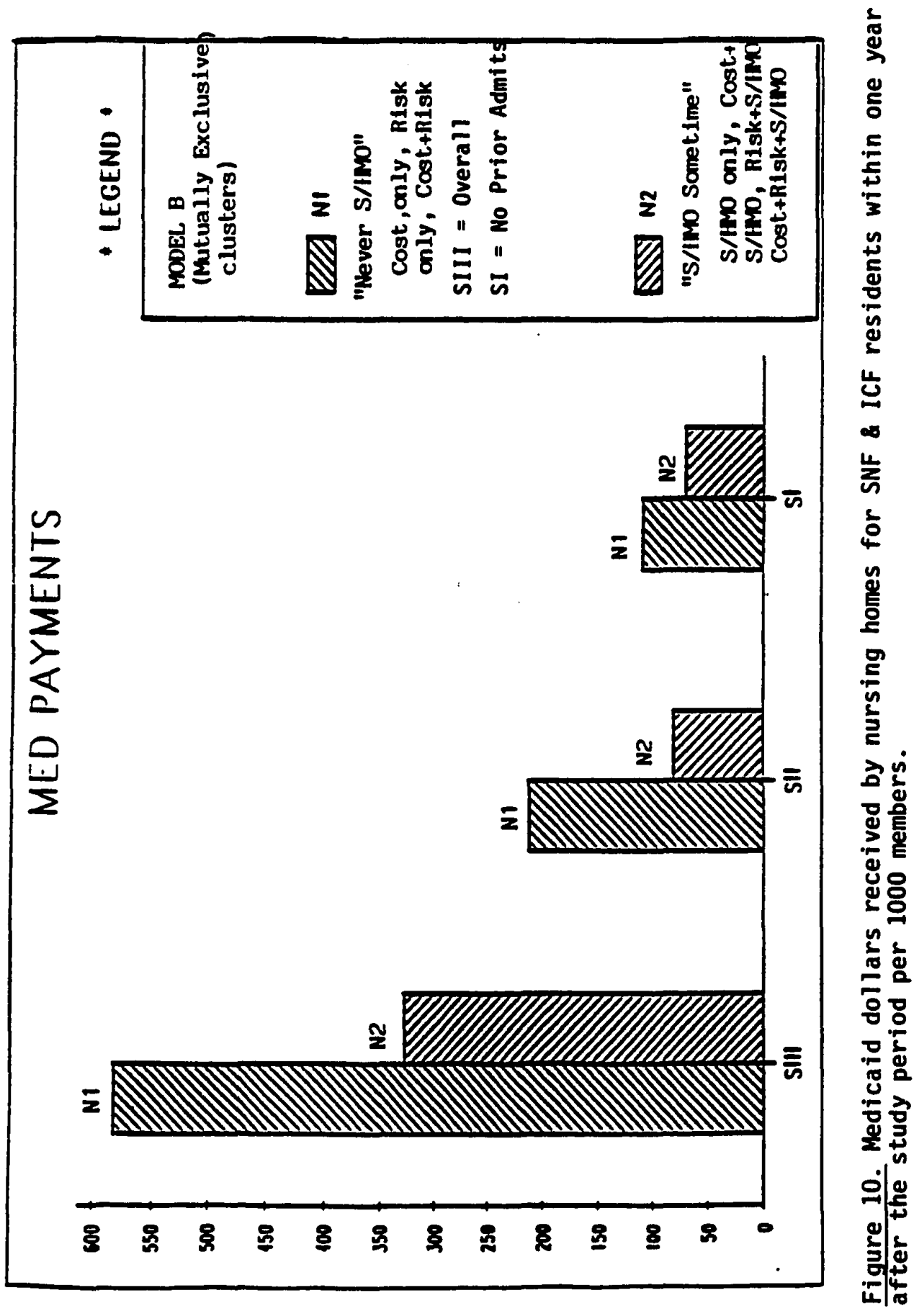


SNF and ICF status. Again, these rates measure nursing home Medicaid payments for the Never $s /$ Ho and $s /$ HMO Sometime clusters (N1, N2 respectively).

The pattern is consistent with that for ICF residents only. This further supports the notion that the ECB reduces the requirement of Medicaid assistance for S/HMO members who become nursing home residents.

Under Model A, when an adjustment was made for residents in both $\mathrm{Risk}$ and $\mathrm{S} / \mathrm{HMO}$ status, the raw data showed that Medicaid payments for SNF and ICF residents, as a percent of total nursing home payments received, was greater for Risk residents than for S/HMO residents.

Specifically, under subset $I$, in the adjusted Model $A$ for SNF plus ICF residents, nineteen percent (18.6\%) of total payments received within one year after the study period for Risk residents were from Medicaid sources $(\$ 845,245 / \$ 4,544,872)$. The adjustment allocates duplicated payment equally between Cost and Risk or S/HMO and Risk. Under Subset I, in the adjusted Model A for SNF plus ICF residents, fourteen percent of total payments received within one year after the study period for s/HMO residents were from Medicaid sources $(\$ 428,768 / \$ 3,023,555)$. The adjustment allocates duplicated payment equally between cost and $\mathrm{S} / \mathrm{HMO}$ or $\mathrm{S} / \mathrm{HMO}$ and Risk. Also, all AFC money is removed. One of the research issues examined in Chapter $v$ was whether differences in use rates existed between Risk and 
S/HMO members enrolled directly from the community. Table XXXXIX compares individual $\mathrm{s} / \mathrm{HMO}$ and Risk members.

\section{TABLE XXXXIX}

(RATES ARE STANDARDIZED BY SEX AND AGE COHORT) MEDICAID PAYMENTS RECEIVED FOR SNF AND ICF

CARE BEFORE, DURING AND AFTER THE STUDY PERIOD THROUGH 06/30/1989 FOR MEMBERS RESIDING IN ICF DURING THE STUDY PERIOD / 1000 RESEARCH POPULATION MEMBERS IN "S/HMO SOMETIME" OR "NEVER S/HMO," (MODEL B, ROWS $3 \& 4$ OF FORMAT B)

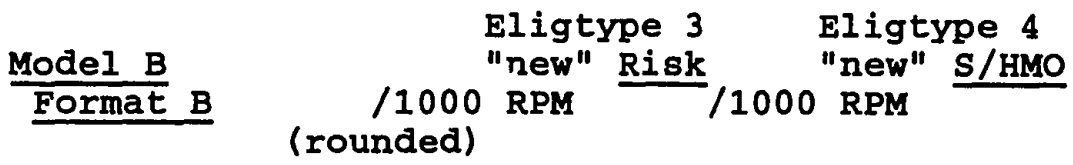

Male + Female Subset II

Subset I

Male

subset II

Subset I

Female

subset II

Subset I
138 $\$(000)$ (598)

8 Diff 87
83

102

65

161

96
$\$(000)$

68

〈68 >

68

99

(73\%) 55

Source: Appendix F

Model B, Format B allows an examination of such members. Thus, only two of the seven mutually exclusive groups under Model B, Format B, are presented next. Such members are called "new" in this study because they were not enrolled in KP as Medicare members at the time they became 
capitated Risk enrollees or S/HMO enrollees. However, "new" also includes members who were enrolled in KP as non-Medicare members but, became Risk or $s /$ Ho upon reaching age 65 .

Comparison of direct "New" members is of special interest because of the uncertainty about whether either introduced some kind of bias which might affect rates in a way which the other did not. That is, do "new" Risk members who became ICF residents show different trends in spending down, and in Medicaid expenditures, than "new" s/HMO members? Is there adverse selection, likely to disfavor the HMO or carrier financially, or is there some positive selection, likely to favor the HMO or carrier financially, which surfaces in Medicaid rate analysis? Rate differences for "new" Risk and "new" $\mathrm{S} /$ HMO are examined next. The overall rate is dropped because it badly distorts this rate for "Never S/HMO" residents.

Except for "new" S/HMO males in subset $I$, rates of Medicaid payment per 1000 members are substantially lower for "new" $S$ /HMO than for "new" Risk. In Subset II, the "new" s/HMO public assistance standardized rate is fifty-nine percent less than that for "new" Risk ICF users, regarding payments per 1000 mutually exclusive members who became ICF residents during the study period.

In Subset I, the "new" $S /$ HMO public assistance standardized rate is thirty-eight percent less than that for 
"new" Risk ICF users, regarding payments per 1000 mutually exclusive members who became ICF residents during the study period.

Why is this rate for "new" $s /$ HMO females seventy-three percent less than that for "new" Risk Females? Why is this rate of Medicaid payments per 1000 members for "new" s/HMO females twenty-four less than that for "new" s/HMo males? It appears from this table and from rates in previous chapters that there is a group of "new" $\mathrm{s} /$ HMO males who had a significant requirement for nursing home services and possibly represent a condition of adverse selection at the time of enrollment.

Whether "new" s/HMO females represent favorable selection is less clear than that for $\mathrm{S} / \mathrm{HMO}$ males, because the female rate does not hold across SNF level care. Could it be that case management processing of $\mathrm{S} / \mathrm{HMO}$ members was more successful in placing or keeping females in community settings upon determining their need for ICF care, than males?

Under the Overall data set for Model A, there were 420 SNF and/or ICF S/HMO nursing home residents, ten(10) of whom were AFC members, (persons enrolled in $\mathrm{s} / \mathrm{HMO}$ as Adult and Family Care welfare recipients by the state of oregon), nine (9) of whom were not admitted to nursing homes until after the study period. One hundred thousand dollars $(\$ 106,435.00)$ were received by nursing homes for these ten AFC members, of 
which twenty-eight thousand dollars $(\$ 27,876)$ were paid by the state of oregon. Only 124 or the 6,306 s/HMO members were recipients of AFC assistance during the 24 month study period. In the following analysis, they are removed from nursing home data to facilitate a summary of spend-down by members who were not already on welfare.

of the remaining 411 S/HMO residents who were not AFC welfare members, fifty-five, or thirteen percent spent down to become dependent on public financing for part of their nursing home charges. State of oregon Medicaid payments for those fifty-five residents totaled six hundred thirty-five thousand dollars $(\$ 635,043.00)$.

of the 421 overall S/HMO nursing home residents, 73 were in either Cost or Risk before, during or after the study period; thus, 347 were not in nursing homes while in non-s/HMO status. Over three million dollars $(\$ 3,129,648.00)$ were received by nursing homes before June 30, 1989, for care rendered to these 347 SNF or ICF S/HMO residents who were not in Cost or Risk status while in a nursing home. of the 347 s/HMO "only" eligible nursing home residents, forty-five or thirteen percent $(45 / 347-12.97 \%)$ became Medicaid recipients by one year after the study period, for whom four hundred fifty-six thousand dollars $(\$ 456,644.00)$ of Medicaid payments were received by nursing homes .

If Medicaid payments $(\$ 27,876)$ for the four AFC are 
removed $(\$ 456,644-\$ 27,876=428,768)$, fourteen percent $(\$ 428,768 / \$ 3,129,648=13.7 \%)$ of total payments for non-AFC S/HMO residents who were not also in Cost or Risk came from public funds (fifteen percent including AFC funds $\$ 456,644 / \$ 3,129,648-14.598)$ during the four year period (4/85-6/89) of nursing home experience for $s /$ HMO residents who were in a nursing home during the study period.

The difference in Medicaid payments between non-AFC Overall s/HMO in all eligibility status, and Overall s/HMO who did not reside in nursing homes under Cost or Risk status is $\$ 206,000$ for ten residents $(\$ 635,043-\$ 428,768$ $\$ 206,275)$ with Cost or Risk status.

Table Xxxxx gives another view of Medicaid patterns is derived from the mean of all payments received for $\mathrm{s} / \mathrm{HMO}$ and Risk nursing home (SNF and ICF) residents who became welfare dependent.

TABLE L

MEAN OF ALL PAYMENTS RECEIVED FOR S/HMO AND RISK NURSING HOME (SNF AND ICF) RESIDENTS WHO BECAME WELFARE DEPENDENT.

Model A, Subset I - Payments Per Resident (7/86-6/89) In SNF and/or ICFs in study Pd. Risk S/HMO

$\begin{array}{lll}\text { Mean }-\$ 10,044 & \$ 10,069 \\ \text { Median }-\$ 5,533 & \$ 6,168 \\ \begin{array}{l}\text { Third } \\ \text { Quartile }\end{array} & \$ 17,050 & \$ 9,511\end{array}$


This above data are discredited some by not having a good way to remove the proportion of payments received from either Risk or $\mathrm{s} / \mathrm{HMO}$ which Cost, Risk and $\mathrm{s} / \mathrm{HMO}$ residents incurred while in all eligibility status. The higher payment per resident at $Q 3(\$ 17,050)$ suggests that there were more Risk members under subset I who stayed longer and that probably accounts for the greater proportion of Risk members who spent down and the higher rates per 1000 members in tables above.

\section{DISCUSSION ABOUT FINDINGS}

Differences in total Medicaid payments are large, depending on the criteria of prior nursing home admission. In the data set containing overall cost, Risk and s/HMO information the total of Medicaid payments received was $\$ 9,508,935$. In subset II, for those admitted during who may have had prior nursing home use, the total of Medicaid payments received was $\$ 3,196,436$. In subset $I$, for those admitted during the SP with no prior nursing home admissions, the total amount of Medicaid payments received was $\$ 1,816,313$.

Medicaid payments lag behind dates of service by months, while determination of eligibility, assets, and assessment for level of care allowed by Medicare is done. A percent may be withheld until adjustments from the prior year are determined. Therefore, studies involving Medicaid 
payments must allow a substantial period following dates of ICF residence for reasonable completion of payments. In this study one year was allowed, after the study period. Thus, payment data for residents admitted earlier in the study will be more complete than for those admitted later. However, the data collection process causes difficulties in making reliable comparison between eligibility groups in all but subset I.

Many factors could influence financial data leading to the slightly higher amount of Medicaid payments for S/HMO members who spent down to welfare, (only) under subset I. Did a greater proportion of $\mathrm{S} / \mathrm{HMO}$ members in nursing homes die sooner than Risk members, thus reducing the time during which they remained on Medicaid? Apparently not, based on life-status data presented in Chapter VI.

Or, were they discharged from the nursing home by $s / H M$ case managers to continue their care in a community based setting, unlike Risk members? Were S/HMO members otherwise managed differently than Risk members regarding solutions for their long-term care needs? Further studies are recommended and needed to clarify that question. Such research could use the data base created for this study and build on it buy collecting and adding needed information. Did those who remained in nursing homes through the period during which Expanded Care Benefits paid for SNF services beyond those covered by Medicare, or beyond the 
period of ICF stay covered by ECB, leave S/HMO and become Risk or Cost members as they became Medicaid dependent? OnIy two instances of that occurred during the study period.

Did S/HMO members have more liquidible assets, than Risk members, with which to delay the time to spend-down? Or, did the combination of $\mathrm{ECB}$ and personal assets result in the reduction of need for public assistance? This is unknown without having state of oregon SSD data from the Medicaid files in Salem, Oregon.

Did S/HMO ICF members have more support group options than Risk allowing them to avoid spend-down? If so, was it because they were there to begin with or because case managers in S/HMO used ECB funds to create surrogate support groups which afforded them the option of non-institutional long-care, not similarly available to Risk members?

What is different about users or/and use of SNF services by S/HMO members from Risk members that results in less reliance on public assistance for S/HMO than for Risk? This issue is especially perplexing, considering that s/HMO overall use of SNFs is greater than that of Risk members.

There is much work to be done with the data sets in this study regarding explanatory analysis which might clarify why findings are as observed, in addition to the premise that $S / H M O$ ECB influences the results of nursing home spend down. The need exists for greater understanding in general regarding factors affecting spend down, in order 
to formulate policy which effectively shifts from means

tested eligibility to risk-sharing and intergenerational

financing of protection against catastrophic effects of

long-term care for the elderly.

The incidence of Medicaid dependency in this study more closely approximates the recent findings of studies from the Connecticut nursing home data base, which is much lower than most other studies.

The Connecticut data have been used to derive alternative spend-down estimates that provide some idea of the magnitude of the biases introduced by some of the methodological issues raised earlier. As noted earlier, Connecticut data clearly illustrated the effect of measuring spend-down over multiple versus singular admission; measures of SP1 and SP2 were virtually doubled when multiple admissions were taken into account.

In the more recent (1991) study of Connecticut data, the authors [Gruenber et. al.] noted the effect of using a resident versus an admission cohort on the measure of SP1. Using a 1978-79 admission cohort in the connecticut data, the SP1 estimate was found to be 21\%; using the one-day 1985 resident view, the authors measured SP1 as almost 408! As they argue, this illustrates effects of considerably greater lengths of stay represented in the resident sample in connecticut. [77]

In this dissertation study, 30 percent of subset I ICF residents in Never S/HMO status, whose initial nursing home stay occurred during the study period, were welfare dependent one year after the two year study period; 14 percent of such S/HMO sometime members were welfare dependent. This was similar to that for Subset I Risk 
residents (27 percent) and s/HMO residents (14 percent); overall, including cost residents (36 percent), the rate of welfare dependency, used as a proxy for spend down, was 24 percent, under terms of evaluation established for subset I.

\section{SUMMARY OF OBSERVATIONS}

- Fewer S/HMO ICF residents per 1000 members, admitted during the SP, became Medicaid dependent within one year after the study period, then did Risk ICF users.

- At least 628 fewer S/HMO ICF residents were receiving Medicaid than Risk ICF residents, during or within one year after the study period. Among ICF residents with no nursing home admissions prior to the study period (subset I), at least 438 fewer S/HMO residents were receiving Medicaid assistance one year after the study period.

o. S/HMO ICF residents admitted during the study period, who were Medicaid dependent within one year after the study period, required substantially less public financial assistance per 1000 members than did Risk members, based on Medicaid payments received by nursing homes within one year after the study period.

- The state of oregon apparently paid substantially less for $S / H M O$ ICF residents who became Medicaid dependent than for Risk ICF residents who became Medicaid.

\section{POLICY RESEARCH RECOMMENDATIONS}

Data from the State of Oregon's Data Base on Medicaid case worker files is needed, as originally planned for this study. Such information would provide variables for explaining whether Risk and S/HMO ICF residents who became welfare were different, as measured by ADLs, personal income socio-demographically, by available family or other support groups, by prior Medicaid status. 
And, this study could be extended to compare differences in community based services. This level of research could definitely help test a predictive hypothesis for causal relationships between S/HMO and reduced dependency on Medicaid assistance. 
CHAPTER IX

MEDICAL STATUS OF NURSING HOME RESIDENTS

The non-Medicare nursing home population is quite distinct from the Medicare Population.. In our Medicare analysis, the first pure type was characterized by a cancer diagnosis. In this [non-Medicare] analysis, our first pure type involves some hip fracture, in addition to cancer, as primary diagnosis. This [nonMedicare] group is distinctly older than the Medicare group, but is still predominantly female, unmarried, incontinent and generally bedfast. In contrast to the Medicare population, senility, chronic brain syndrome, circulatory, and other chronic conditions are prevalent... The second pure group [among non-Medicare nursing home residents] is associated with a primary diagnosis of stroke [being] male, married, incontinent and with a high prevalence of persons who are chairfast...and a wide range of medical problems (diabetes, bedsores, kidney failure, circulatory diseases). [78]

This chapter investigates the discharge diagnosis patterns for S/HMO and Risk ICF residents who had hospital stays preceding their ICF stay. This focuses on health status indicated by medical conditions associated with secondary diagnosis at the time of hospitalization preceding the first ICF admission of Risk and $\mathrm{s} /$ HMO members during the study period. This asks whether S/HMO members who were hospitalized and ended up in ICFs had different illness patterns from Risk residents.

The following discussion provides a frame of reference regarding use of hospital discharge diagnosis as indicators of health status for ICF residents. 
Hospital discharge diagnosis are not equivalent substitutes for basic measures of cognitive or physical disability which cause member's dependency and therefore need for ICF care. Those measures, such as activities of daily living measures (Katz's ADL scales), are not part of the hospital discharge diagnosis coding system, even though they may be included in a hospital chart. ADLs were not obtained for Risk members and therefore not available for comparison with those known for s/HMO members, in this study.

Hospital discharge diagnosis do specify the chronic illnesses which give rise to ADL dysfunction. Related conditions are otherwise defined by the International Classification of Diseases, ICD9 codes, and Diagnostic Related Groups, DRGs.

Discharge diagnosis used in this study are from the last hospital stay following April, 1985 which was prior to the first ICF admission after June, 1986. In some instances a lapse of over one year occurred between hospital discharge and ICF admission. Not all ICF residents were hospitalized before their first ICF stay, although a very high proportion were hospitalized during the course of nursing home care, often preceding their death.

Residents already in an ICF at the time of admission are also excluded because most were hospitalized prior to the start-up of S/HMO and TEFRA Risk programs. Therefore, 
fewer nursing home residents are evaluated in this chapter than in prior chapters. Hospital discharge may have been to an SNF as an intermediate step to ICF admission; SNF stay is considered later in this chapter.

The TEFRA Act allowed some waivers from Cost Medicare regulations for Risk and S/HMO members. One wavier removed the requirement of three days hospitalization prior to an SNF admission. Thus, S/HMO and Risk members could be evaluated and admitted directly to an SNF, as well as an ICF, if their health condition permitted. Therefore, SNF or ICF admissions might be ordered following a visit to the physician's medical office or the hospital emergency room. Often, patients were held in an Emergency Room (ER) Holding bed for up to 24 hours to permit evaluation and observation for changes in health status. No doubt, such waivers affected hospitalization patterns and therefore availability of discharge diagnosis preceding SNF stay if not ICF stay.

One study observed that, in 1985, the most frequent location from which residents were admitted [to a nursing home] was a general or short-stay hospital (378)...elderly residents were more likely to be admitted from short-stay hospitals (398) [than any other location and]...38 percent of nursing home residents had previous nursing home stays... Several studies found that prior nursing home residence for hospitalized patients was associated with a very high probability of continued institutionalization. Lewis, Cretin, and Kane found that transfers between nursing homes and hospitals (in both directions) occurred in 54 percent of first-time admissions to nursing homes in the 2 -year period following admission. [79] 
S/HMO and Risk ICF users were no exception. Sixty-five percent of S/HMO, and sixty percent of Risk first ICF admissions came from a hospital. During the two year SP, the largest number of readmissions to ICFs and SNFs was nine, although approximately seventy-five percent of all nursing home users had three or less nursing home admissions. Published studies, which employ DRG or ICD codes as a tool for analysis, are not always explicit about how many and which hospital stays are used for ICD source data.

Hospital discharge diagnosis may not reflect chronic conditions leading to a nursing home stay. For example, a high proportion of last nursing home discharges to the hospital precede a patient's demise. The primary diagnosis might be pneumonia as a complication of emphysema. The patient may have been in a nursing home because of advanced dementia. Many readmissions are for a procedure, such as stabolizing a fractured femur, and related secondary diagnosis that reflect basic chronic conditions which contributed to the incident, such as diabetes or orthostatic hypotension, may not be recorded.

One the other hand, ADLs may not be the reason for admission to an ICF. One common cause of first ICF admission is that a care-giver spouse/relative dies or becomes disabled, and no alternative care givers are available to continue home care of the elderly dependent person. DRGs and ICD codes are products of a hospital 
discharge. Medical conditions observed during an emergency room visit are not coded unless the patient is hospitalized. Nursing homes record the location prior to admission, and it is common practice to note "hospital" even if the patient was only seen in the ER but not admitted to hospital. Thus, tables presented in chapter VI on location before and after ICF admission may not coincide with numbers in this chapter.

Primary diagnosis is the dominate factor in selecting a Diagnostic Related Group (DRG) which best summarizes the overall reasons for hospitalization. Computer programs are used to weight how all associated ICD9 codes contribute to the selection of DRGs. [80]

DRGs are selected via an algorithm which considers primary and secondary diagnosis, age, severity of conditions, presence of systemic problems or procedural complications, invasive procedures performed while hospitalized, and a few other conditional factors such as length of stay exceeding the average length of stay for a primary diagnosis. Numerous primary diagnosis are clustered by DRG. Currently, there are 487 DRGS, which are clustered into 28 Major Diagnostic Categories (MDC). [81] Up to eight secondary diagnosis are also recorded upon discharge. Such information is extracted from documentation in the medical record which was entered during each period of hospitalization by all providers. Secondary diagnosis 
identify other conditions diagnosed and either treated or considered as co-morbidities influencing medical or/and social decisions regarding the primary condition under treatment. For each of these nine medical conditions, a code is selected which best fits the patient's condition during each hospitalization. Codes used in this study are those listed in the International Classification of Diseases, 9 th Revision, or "ICD9". These codes and related DRGs are stored in the KP Hospital Discharge data base used for this study. Secondary ICD9 diagnostic codes have been used in some post-acute care studies to predict and describe utilization trends. One study, done at the Rand Corporation, tests the power of secondary diagnosis to predict which patients receive care in particular post-acute settings [rehab., SNF, HHA] . This RAND study used all secondary diagnosis available (4) from hospital billing records which were listed for each of five DRGs (DRG 14: stroke, DRG 88: COPD, DRG 127: Heart Failure, DRG 209: Major Joint Procedures, DRG 210: Hip \& Femur Procedures). These DRGs were selected at Rand because their were association strongly with SNF and HHA.

The overall findings of that study, regarding secondary diagnosis is noted. Secondary diagnoses are quite important in determining how likely a patient is to use post-acute care in a particular setting. With the exception of DRG 209 (major joint procedures), secondary diagnosis appear to be more important than are different primary diagnoses within the DRG. Moreover, the secondary diagnoses that are associated with high utilization of postacute care are very often apparently unrelated to the primary diagnosis. An important determinant of 
whether a stroke patient used SNF care, for example, seems to be whether he or she had a secondary diagnosis of pneumonia. [82]

In this study, five DRGS are used as measures of health status among nursing home users because they are known to be reliable predictors of nursing home admission, based on the RAND study. However, they did not account for a majority of discharge DRGs for nursing home users studied.

Therefore, a ranking of the top twenty DRGs is

shown for Model $A$ by Cost, Risk and s/HMO members. If DRGs were ranked from all hospitalizations associated with each ICF user, the order of frequency is different than that presented in Table LI for the last hospital stay before first ICF study period admission.

TABLE LI

RANR ORDER OF TOP TEN DRGS FROM THE LAST HOSPITAL STAY PRIOR TO FIRST ICF ADMISSION IN THE STUDY PERIOD

$\begin{array}{cc}\text { Rank of } \\ \text { Risk } & \text { of } \\ \text { DRGs } & \text { total } \\ \# & \text { DRGs }\end{array}$

Rank of S/HMO \& of DRGs total \# DRGs

$\begin{array}{lr}1 & \frac{127}{14} \\ 3 & 140 \\ 4 & \frac{147}{187} \\ 5 & 1 \frac{89}{38} \\ 6 & 395 \\ 7 & 141 \\ 8 & 209 \\ 9 & 280 \\ 10 & \end{array}$
\begin{tabular}{r}
$\frac{127}{14}$ \\
140 \\
\hline 187 \\
89 \\
138 \\
395 \\
141 \\
209 \\
280
\end{tabular} 5.88 5.28 5.28 4.08 3.58 2.98 2.98 2.38 2. 38 $2.3 \%$ $140 \quad 6.58$ $\overline{127} \quad 5.88$ $\overline{121} \quad 3.98$ $210 \quad 3.98$ $182 \quad 3.28$ 193.28 $39 \quad 2.68$ $174 \quad 2.68$ $277 \quad 2.68$ $\frac{1.98}{36.28}$ 
DRG: 14-stroke, 19-cranial/peripheral nerve disorder, 39-Removal of eye lens, 89-pneumonia, 121-heart related circulatory disorder(MI/arrest/hypertension). 127-heart failure shock/hypertension, 138-cardiac arrhythmia fibrillation tachycardia, 140-cardiac arrhythmia ischemia ro/MI angina, 141-orthostatic hypotension \& syncope(unconscious due to circulation, 174 -gastrointestinal hemorrhage/ulcer, 182-digestive disorder gastrointestinal illness, 187-dental extraction or restoration, 209 -major joint repair or replacement, 210-hip and femur procedure, 277-injury infection or wound to skin, 280 open wound or trauma to skin (decubitus ulcer), 395-red blood cell disorder, anemia, transfusion reaction.

Before summarizing rank order differences in DRGs, it should be noted that six of these DRGs related to heart and circulation problems, two related to digestive tract, two related to skin, two related to joints. While exact match-up of DRGs shows a 308 common listing between Risk and s/HMO only two in each eligibility are different diseases than found in the other. Similarity of diseases predominates between the top 10 hospital discharge DRGs for Risk and S/HMO ICF residents, in spite of the following Ranking summary.

Within the ten most frequent DRGs for the hospital discharge prior to first ICF admission in the study period, three were common to S/HMO and Risk $(89,127,140)$. Although not shown, three were common between s/HMO and cost 189,127 182), and two DRGs were common to all three (pneumonia and heart failure).

The top ten DRGs accounted for 468 of all cost, 478 of all Risk, and $36 \%$ of all S/HMO. Over $60 \%$ of Risk DRGs were 
in the top twenty of all secondary diagnosis; about half in S/HMO.

Three of the top 10 Risk DRGs, and two in S/HMO, were common to the top five Rand DRGs selected on the basis of most frequent DRG-linked secondary diagnosis. A case could be made that the similarity was higher, due to marginal distinctions between DRGs and related secondary diagnosis. These observations suggest that a somewhat different health status existed among Risk and S/HMO members who became ICF residents, the most obvious condition missing from S/HMO, that is in Risk, is DRG 14 (stroke). Whether something different was taking place regarding ICF admission practices for stroke S/HMO members could only be determined by a careful analysis of stroke patients in both groups. But, it can be said that the most important difference in Risk and S/HMO hospital discharge diagnosis is that stroke is high in the rank order of DRGs for Risk but is missing from the top 10 for S/HMO hospital discharges.

DRG 88 (COPD) did not appear in the top twenty of any RP hospital discharge DRG lists except those for both Risk and S/HMO SNF DRG rankings for age 65-74. In the Rand study it was a predominate diagnosis. In contrast, pneumonia was in the top 10 of every DRG/ICD ranking list.

When the above table (only half of the top twenty DRGs identified) is compared to the composite of DRGs for all hospitalizations (before, during and after the study period 
through June, 1989) for ICF residents in the study period, the sum is: Risk $-1,282$ and $\mathrm{S} /$ HMO $=976$. There were 183 DRGs from the last hospital discharge before the first study period admission.

The composite rank order of all DRGs is not similar to that in the above table. Only two of the top ten in Risk and S/HMO (127 and 14) were common in both lists.

In contrast to the last hospital DRG rank-order profile before first ICF admission in study period, great similarity existed between last DRG rank-order and first SNF admissions, as shown in Table LII, below.

TABLE LII

\begin{tabular}{|c|c|c|}
\hline \multicolumn{3}{|c|}{$\begin{array}{c}\text { RANK ORDER OF TOP TEN DRGS FROM THE LAST } \\
\text { HOSPITAL STAY PRIOR TO FIRST SNF } \\
\text { ADMISSION IN THE STUDY PERIOD }\end{array}$} \\
\hline $\begin{array}{c}\text { Rank } \\
\text { Risk } \\
\text { DRGs } \\
\text { \# }\end{array}$ & $\begin{array}{l}\text { der } \\
\text { xx } \\
\text { common } \\
\text { DRGs }\end{array}$ & $\begin{array}{l}\text { Rank order } \\
\text { S/HMO } \\
\text { DRGs } \\
\quad \#\end{array}$ \\
\hline $\begin{array}{r}14 \\
209 \\
210 \\
127 \\
89 \\
320 \\
296 \\
39 \\
140 \\
79\end{array}$ & $\begin{array}{l}\mathbf{x x} \\
\mathbf{x x} \\
\mathbf{x x} \\
\mathbf{x x} \\
\mathbf{x x}\end{array}$ & $\begin{array}{r}14 \\
209 \\
210 \\
127 \\
296 \\
89 \\
174 \\
416 \\
96 \\
79\end{array}$ \\
\hline $48 . \overline{38 \text { tot }}$ & & Tot. DRG \\
\hline
\end{tabular}

As noted, seven of the top ten (and 13 or the top 20) last hospital DRGs before first SNF admission in the study 
period are common to S/HMO and Risk (Cost included 8 of the top 10 DRGS for Risk and S/HMO combined). As expected, the DRG rank order is quite different for those age $85+$ and those age $65-74$.

Hospital discharge practices had to be similar between Risk and $\mathrm{S} / \mathrm{HMO}$ members to produce a ranking of DRGs that is so similar. That is expected because s/HMO did not intervene or interact with the KP managed care process for Medicare covered services. At least the basis for post-acute care management was similar for Risk and S/HMO. AT the point when $\mathrm{s} / \mathrm{HMO}$ case managers intervene in long-term nursing home care, there was a reasonably similar health status among SNF level residents.

The five Rand DRGs included code 88 which is not an important health condition for hospitalization in either Multnomah County, or in KP ICF member care patterns. Pneumonia is (89) a key illness.

To study differences in discharge diagnosis between KP members and all of Multnomah County (including KP members), data was purchased from the state of oregon, Office of Health Policy, SAS data base for hospital discharges. The DRGs were ranked, together with number of patients and total hospital days. The result is reported in Table LIII. The similarity in the top 20 DRGs between Multnomah County and KP suggest KP members are not likely to require different nursing home services, overall, that other 
residents of the county in which they reside. of course, other factors than medical condition must be considered before any conclusions could be drawn.

\section{TABLE LIII}

\section{RANR ORDER OF TOP TEN DRGS FROM MULTNOMAH COUNTY HOSPITALS WHO WERE DISCHARGED TO NURSING HOMES (SNF-ICF)}

$\begin{array}{ll}\text { Rank Order } & \text { Rank order } \\ 1986 \text { DRG } & 1987 \text { DRG } \\ \text { Mult. Cnty. } & \text { Mult. Cnty. }\end{array}$

\begin{tabular}{lrr}
1 & 14 & 14 \\
2 & 210 & 210 \\
3 & 89 & 89 \\
4 & 127 & 295 \\
5 & 296 & 127 \\
6 & 209 & 209 \\
7 & 79 & 79 \\
8 & 429 & 320 \\
9 & 320 & 182 \\
10 & 416 & 174 \\
\hline
\end{tabular}

ICD codes are examined next for patterns in Risk and S/HMO member use of SNFs. Given the large number of ICD9 secondary codes available to select from and the fact that up to eight secondary codes were reported for each hospital discharge, dissimilarity is expected in comparing Ranked ICD9 secondary diagnosis. That was not the finding, as Table LIV shows. Similarities would be expected for primary diagnosis, since that is the major determinate of DRG codes. Secondary ICD9 codes are more likely to reflect chronic conditions. 
TABLE IIV

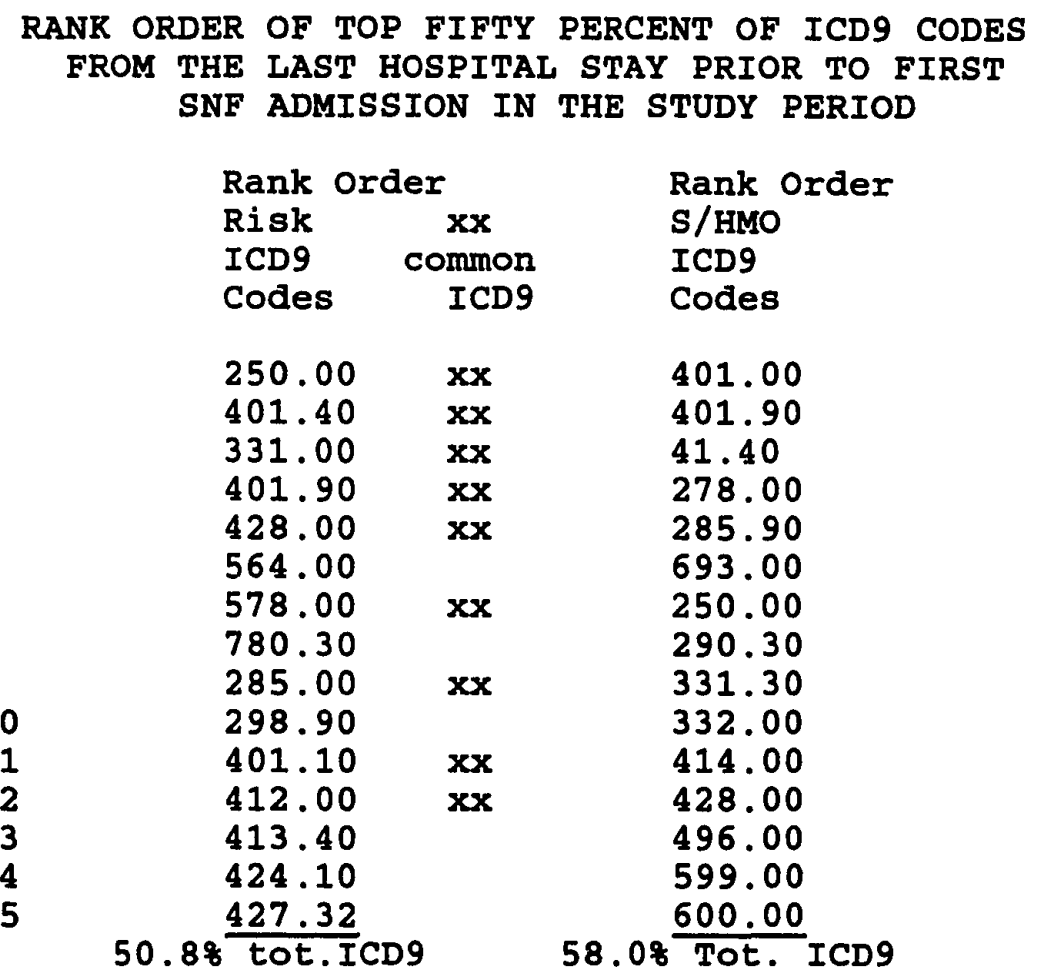

Seven of the top 15 secondary diagnosis (ICD9) codes were common between Risk and S/HMO members; the top 15 codes encompassed over 50 percent of all secondary diagnoses during the last hospital discharge prior to the time of their first SNF admission during the study period.

This reinforces DRG observations about SNF care, and further confirms that Risk and s/HMO members who entered SNFs, and who subsequently became ICF residents, had similar health characteristics.

That S/HMO members spent substantially fewer days in 
ICFs than Risk members, suggests events other than health status influence those rates. This provides some additional basis for recommending hypothesis-based tests of statistical inference to test the correlation between fewer days of stay and $\mathrm{S} / \mathrm{HMO} \mathrm{ECB}$.

In Table LV eleven of the top 20 secondary diagnosis for last hospital stay before first ICF admission were common to Risk and S/HMO ICF residents; the top 20 codes encompassed over 50 percent of all secondary diagnoses (2-9) during the last hospital discharge prior to the time of their first SNF admission during the study period. The are listed below:

Diagnostic name of ICD9 Codes listed: 41.40-E.Coli Bacterial Infection; 185.00-Malignant Neoplasm-Prostate; 198.50-Neoplasm-Bone, Bone Marrow; 244.90-Hypothyroidism, Unspecified Cause; 250.00-Diabetes Mellitus; 278.00 Obesity and Hyperalimentation; 285.90-Anemia; 290.00-Senile Dementia/Senile Organic Psychotic Condition; 290.30-Senile Dementia w/Deliriums; 298.90-Psychosis, Unspecified; 331.00-Alzheimers; 332.00-Parkinson's Disease; 365.90-Glaucoma; 401.10-Hypertensive Disease; 401.90-Hypertensive Disease; 412.00-Healed Myocardial Infarction; 414.10-Aneurysm-Heart Wall; 413.90-Unspecified Angina; 424.10-Aortic Valve Disorder; 427.00-Paroxysmal Tachycardia; 427.31-Atrial Fibrillation; 427.69-Premature Ventricular Contractions; 428.00-Cardiac Heart Failure; 438.00-Late effects of Cardiovascular Accident (CVA) : Aphasia Dysphasia, other paralysis; 440.90-Arteriosclerotic vascular Disease; 443.90-Peripheral Vascular Disease; 492.8-Emphysema 496.00-Chronic Obstructive Pulmonary Disease; 553.30-paraesophogeal/Hiatial Hernia; 599.00-Urinary Tract Infection; 715.90-0steoarthrosis unspecified;

Over 258 of all (736) secondary diagnosis from the last hospital stay before first ICF admission in the study period were common to Risk and S/HMO, under Model A in this study. 
TABLE LV

RANK ORDER OF TOP FIFTY PERCENT OF ICD9 CODES FOR SECONDARY DIAGNOSIS FROM THE LAST HOSPITAL STAY PRIOR TO FIRST ICF ADMISSION IN THE STUDY PERIOD

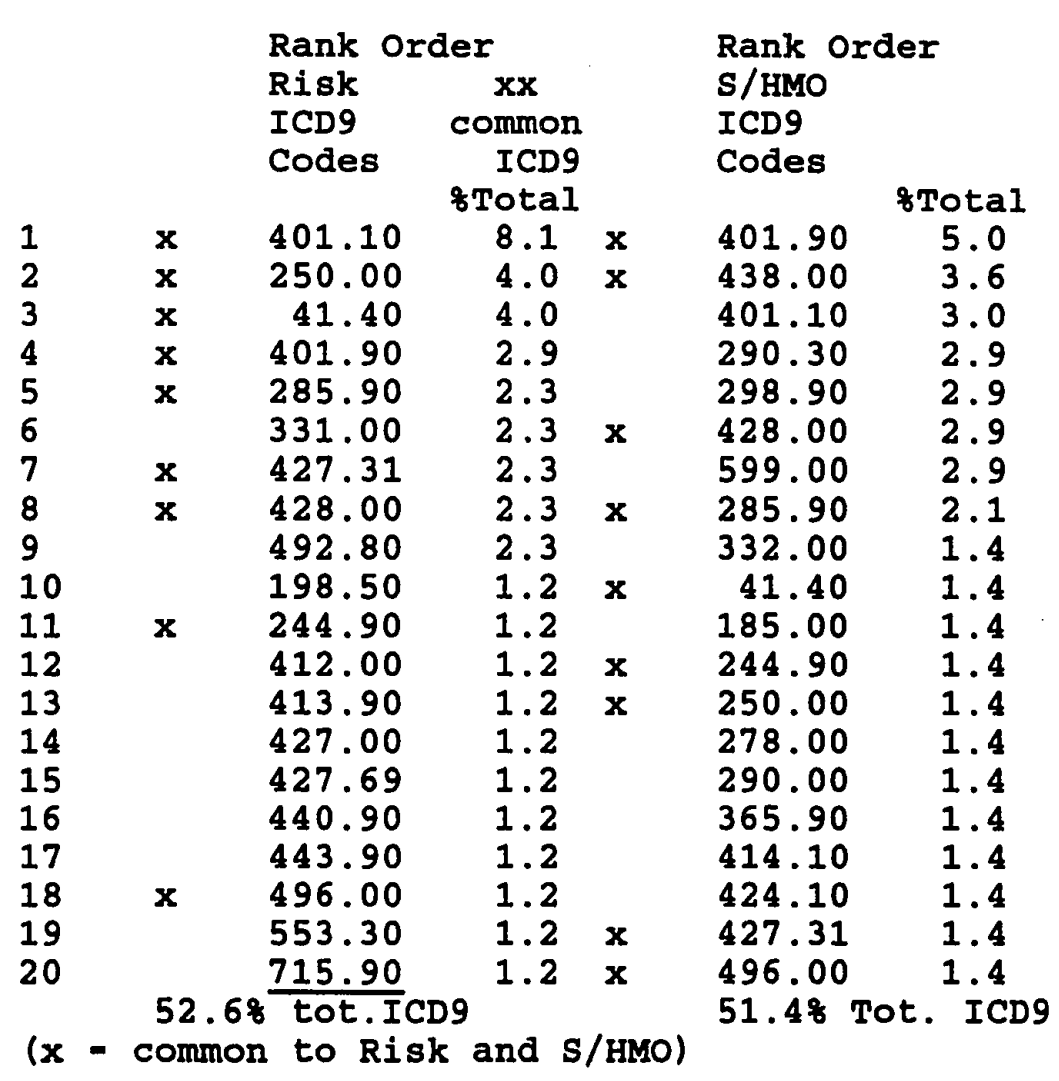

Of the total ICD9 secondary diagnoses for S/HMO, 6.28 were associated with psychiatric disorders, compared to 1.28 among Risk. Late effects (over 1 year post-stroke) of CVAs, such as paralysis, aphasia, dysphasia account for 3.6 of S/HMO residents while the same ICD9 code is not listed for Risk among the top $50 \%$ of secondary diagnosis in Risk ICF residents. Similarly, parkinsons disease accounts for 2.18 
of S/HMO and code 332 is not listed in the top $50 \%$ of Risk. Such patients require considerable personal care and medical attention.

To the extent that such differences may exist, it suggests that some S/HMO members who were in ICFs were very dependent, requiring continuous assistance. Such patients commonly become Medicaid dependent.

In 1988-89, the state of Oregon contracted with the School of Public Health, University of Minnesota, to evaluate differences between Medicaid and private residents in Adult Foster Care (AFC) homes in oregon, compared to Medicaid and private residents in ICFs. One measurement of difference included medical problems based on specific diagnosis identified by the providers. The proportion of ICF residents with dementia, heart disease, hip fractures, and bowel and bladder dysfunction was consistently higher than for AFC residents, but lower for mental illness, and similar in most other areas of chronic illness dysfunction. Kane, et. al., found the following.

Although some very impaired people live in foster care homes, foster care residents are, on average, less impaired than ICF residents. They also show that, within the foster care sector, private-pay foster care residents are more impaired than Medicaid foster care residents... In nursing homes a larger proportion (37\% Medicaid and 398 private pay residents) needed complete help with 5 or all $6 \mathrm{ADI}$ activities, whereas in foster homes 58 of Medicaid and $13 \%$ of private pay residents need 
complete help with 5 or 6 of those activities. Note, however, that the data show an extreme level of ADL impairments for private pay foster home residents. (This is consistent with the large numbers of private pay foster care residents with dementia as a medical problem)... on average, ICF residents had more cognitive impairment than foster care residents, and that private-pay foster care residents had more cognitive impairment than Medicaid residents. [83]

The above tables present some macro level patterns of chronic illness that suggest $\mathrm{S} / \mathrm{HMO}$ members may be more akin to the private-pay group and Risk more akin to the Medicaid group in the referenced study. This suggests that S/HMO may indeed be facilitating home based care for persons who otherwise would be found in ICFs.

The proportion of Risk ICF residents was greater than S/HMO ICF residents who had cardiovascular illness as an underlying chronic medical problem to there functional dependency, and chronic diseases associated with cognitive dysfunction was more prevalent among $s /$ HMO than among Risk ICF residents.

While these categories measure gross differences, they offer some basis for formulating a model which might be used to evaluate differences between Risk and S/HMO members in ICFs and Risk and S/HMO members in home and other community based settings.

Such comparison is recommended for measuring overall differences in use of ECB covered services between Risk and s/HMO. The analysis could include tests for correlation 
between ADL-Cognitive dysfunction scores and hospital secondary discharge diagnosis, as a method of evaluating the relationship between chronic disease and location of care.

one study recommendation is to examine diagnostic characteristics of $\mathrm{s} / \mathrm{HMO}$ and Risk members who spend down in order to determine the extent to which ECBs deferred spenddown for such persons. The issue is to develop models of financing solutions for long-term disabled persons, as well as shorter term disability.

The model for analysis in this study is based on use of secondary hospital discharge diagnosis to predict ICF use rates for Risk and $\mathrm{s} / \mathrm{HMO}$ members. The model includes 32 independent medical status variables which were selected for use in predicting nursing home use. They are regressed on the dependent variable, "days of ICF stay in the SP." This model is evaluated first by stepwise regression and then introduction of age, sex, and SNF status. A linear fit is not good. The fit is not improved significantly by use of a squared or log value for the dependent variable. The model is described below.

ICD9 hospital discharge codes were used to create thirty-two clusters of ICD9 codes which describe medical status based during hospitalization. Twenty five of these clusters describe chronic health conditions identified in a study undertaken by Manton, Liu and Cornelius in 1985 (op.cit. [79]). Those twenty-five conditions were 
statistically associated with nursing home residents as derived from the National Nursing Home Survey of 1977 . The authors were called and asked how they selected ICD codes to define clusters for such chronic conditions. Korbin Liu and Elizabeth Cornelius confirmed ICD9 codes were not used either to formulate the questionnaire or translate answers, and that no reference data set of ICD codes existed. Thus, all ICD9 codes were assigned to each variable based on ICD9 code definitions. Additionally, seven Major Diagnostic Categories from the DRG system were used to create clusters of other ICD9 codes not considered by the 25 other clusters. These 25 chronic conditions and 7 MDCs, together with age, sex, SNF status and eligibility groups (Risk and S/HMO only) comprise the independent variables in this regression model. ICF days comprise the dependent variable. A linear regression model is used to predict which, if any, of these thirty-two independent variables might be useful in explaining the variance between groups I, II (S/HMO, Risk) days of residence in ICFs during the study period. The number of ICF users available for this procedure is reduced by the criteria of prior hospitalization. Specifically, their were 789 members who were admitted one or more times to an ICF during the study period. of these users, only 373 , or 478 overall, had prior inpatient stay, ( 398 of Cost, 498 of Risk and $50 \%$ of S/HMO). These percentages are approximately $15 \%$ below those given in the 
previous chapter for Risk and S/HMO, based on nursing home records regarding source of first ICF admission.

Results of the model, based on the eight ICD9 clusters selected by the SAS stepwise regression option, include an adjusted $\mathrm{R}$-Square of 0.1139 for the linear regression. This is improved only slightly by squaring the value for the dependent variable, resulting in an $R$-Square value of 0.1472 , when age and sex and SNF are added to the eight ICD clusters.

Either the Regression model did not include ICD codes (clusters) or other variables needed to predict ICF days of stay, or there is little relationship between those selected and days of stay. This could also be interpreted as an indication that the S/HMO ECB was influencing days of stay and the model did not account for that variable.

Additional experimenting is needed to determine how to use ICD9 code information more effectively, with other independent variables, in order to help formulate hypothesis testing models which confirm or refute that the ECB and related case-management is affecting use rates of ICF, and other community-based long-term care services.

clarification of differences in health status between Risk and s/HMO members remains an important need to be considered in follow-up studies. The extent to which ECB arrangements for home-based formal care facilitates transfer of ICF level elder care to the community is a matter of 
great policy interest. Conventional understanding about this interchangeability suggests overlap only at the margins.

Although chronic disease is common in most elderly, the impact of disease and resulting impairments distinguish the nursing home resident from the community-dwelling elderly. Brody and Foley (1985) report that one-fourth of nursing home residents are dependent in all six activities of daily living (feeding, dressing, bathing, continence, using the toilet, and mobility), and the degree of dependency increases with age. Compared with 9 percent of noninstitutionalized elderly, 93 percent of nursing home residents require assistance in at least one activity of daily living. Cognitive impairment affects over half of nursing home residents. Behavioral problems are often the most burdensome aspect in caring for resident with cognitive impairment... [However, the] environment provides a context in which behavior can be adaptive or maladaptive. [84]

The Rane study of the oregon AFC-ICF trade-off

reinforces the notion for Medicaid residents that the interchangeability is limited, but reopens the debate based on findings about private-pay, non-Medicaid residents. Prior studies may need to be revisited in order to re-think and re-observe what can be done where for the elderly.

$$
\text { POLICY ISSUES }
$$

There are several caveats attached to comparison of Risk and S/HMO secondary discharge diagnosis from the last hospital stay prior to first ICF admission. On a Macrolevel,

The limitations of a disease-specific orientation are well recognized; assessment of disease-specific treatment and outcomes may indicate relatively 
little about the individual's overall health and well-being...the interrelationships of generic and disease specific approaches are depicted, with needs for care being determined both by disease and nondisease aspects of health. The nondisease aspects may include signs and symptoms that do not meet criteria for classification as diagnosis (ICD9 -CM) or limitations in function that create needs for care...Although relatively little has been done to introduce health status measurement into the policy debate, there are areas in which this has been accomplished and appears to have been influential...the three areas of policy application for health status information around which this discussion is organized are: 1) identification of high-risk and high-need populations, 2) assessing alternative financing methods; 3 ) evaluating alternative approaches for organizing health care. [85]

This reference surfaces two points regarding use of discharge diagnosis in this chapter. First, used alone, they are known to be weak predictors of nursing home and other long-term care requirements. There is increasing evidence that used jointly with other health and social status variables, ICD-9 codes may strengthen methods of classifying LTC users into user groups, including those around whose needs ECB support systems may be developed to enhance the prospects of satisfying LTC needs in community based settings, who otherwise would be in nursing homes. Second, policy regarding LTC programs must have some reliable outcome measures of LTC decisions. That involves preliminary clarification of health status related to 1088 of capacity for independent functioning and resultant dependency. Assessment of appropriateness of LTC care, therefore, must include clarification of underlying multiple 
chronic illness which affects functional well being. Enhancement of function as an outcome goal within a range of environmental locations, rather than alleviation of symptoms, means clarification of severity of disease as well as clarification of dysfunction. Improvement in the reliability and construct validity of instruments which measure enhancement of function against some baseline condition requires increased use of chronic illness diagnostic codes to clarify physical and psychosocial health status. [86]

Measures of functional enhancement should be considered as one of the outcomes for evaluating ECB incentive based, managed LTC programs, compared to such outcomes for Risk members. The policy research question is whether ECB, which reduces use of ICFs and substitutes home based care, leads to desirable differences in functional enhancements, as well as patient/resident and family satisfaction.

There is an increasing body of evidence that LTC needs of the elderly increasingly involve significant deficits in mental status as well as physical functional status. (op. Cit. [84], p. S17). S/HMO members in this study appear to have a substantially higher level of cognitive impairment than Risk members. This raises a question about whether the S/HMO program was especially attractive to families of persons concerned about the complications of caring for 
cognitively impaired elderly, and therefore a selection

bias. Some research follow-up is recommended to draw this issue to a more visible level. It has implications for policy benefit formulation and policy care solutions which may be different than for elderly with physiological dysfunction. on the other hand, it may not be an issue of enrollment selection but simply that the cognitively impaired person is often difficult to manage in a home setting resulting in institutional care as the solution of choice.

It is certain that incentives which influence provider and consumer decisions regarding use of LTC benefits will be of great interest as national policy on LTC evolves, given the projected demand for ITC services in the next 50 years.

The chronically disabled community resident elderly population was projected to increase from 5.6 to 15.4 million between 1985 and 2060. The comparable population 85 and over is projected to increase from 1.1 to 5.6 million... Defining health outcomes for the oldest old is difficult because of the high prevalence of c-omorbidity and functional

impairment ... [yet] Clinical studies...suggest that disability is reversible for a significant number of elderly persons-even at advanced ages... It is possible, however, to reduce the impact [of increased resources required to meet increased LTC needs] by intervening in what had been viewed as "immutable;" the age rate of physical and functional decline for elderly individuals. This has implications, not only for reducing the aggregate level of LTC demand, but also for improving social autonomy at the personal level. It raises the question of whether society is doing all that is possible to maximize the potential of individuals at later ages. [87] 
CHAPTER $\mathrm{X}$

\title{
SUMMARY OF FINDINGS AND POLICY RECOMMENDATIONS
}

\begin{abstract}
Ideological argument is an important and inevitable part of social and policy inquiry. However, such philosophical argument and interpretation would be most suitable for policy inquiry when it is somehow connected to and complemented by systematic empirical study... [but] objectivity in social inquiry....is less a matter of hypothesis testing or quantitative measurement than perceptiveness and open-mindedness; an ability to see how other agents organize their social world. [88]
\end{abstract}

This study attempts to inform researchers, analysts, policy makers and the public about one outcome of the s/HMO Demonstration project. Specifically, empirical observations are described regarding differences in ICF use rates and expenditures between TEFRA capitated HMO members and S/HMO capitated members whose Supplemental benefit package is expanded to include limited coverage of nursing home and formal home-based LTC costs.

Composite observations suggest that in the managed care setting studied, members who had S/HMo benefits and who were certified as eligible for ICF care, accessed nursing homes more readily but limited nursing home stays such that costs were substantially less than for capitated members who did not have s/HMO benefits.

These composite observations suggest that nursing home 
use rates can be meaningfully modified by use of home-based formal services, available through privately financed Medicare Supplemental Insurance benefits. It also demonstrates that such modification can be cost effective. Based on observations in this study, it is postulated that the operational effects of the s/HMO ECB significantly reduce overall nursing home use rates and costs. Therefore, it is likely that increased costs of formal home-based care are a rational and cost-effective LTC policy option in group practice based HMOs.

However, this study does not directly examine the outcomes of S/HMO policy and underlying operational theory regarding community-based LTC services, except as inferred by observed differences in ICF use rates and nursing home costs between Risk and S/HMO members. A comprehensive follow-up companion study is strongly recommended to assess that element of the $s /$ HMO concept.

An equally significant observation of this study is that Medicaid payments to nursing homes for $s / H M O$ members, as a proportion of total payments, were substantially less than for Risk members. If non-nursing home community-based spend-down and Medicaid dependency were found to be significantly less for s/HMO than Risk members, that would further support the s/HMO concept as an important building block for national LTC policy. Such analysis is needed. Findings of this study may help formulate answers to 
questions raised by Grannemann in 1989 [89] about whether capitated payment service delivery methods may be applied to LTC in the same manner as applied to Medicare and Medicare Supplemental benefits. This study suggests that to be true. This study also suggests that while demand for nursing home level care may be inelastic, [op. Cit. [89\}] methods of satisfying that demand may be altered given the price and conditions (contract terms) for use of LTC services which are embedded in the ECB of S/HMO. Observations which lead to that supposition need validation by confirmatory evaluation of variables not examined in this study.

\section{SYNTHESIS OF STUDY FINDINGS}

The overall argument, which evolves from collective observations in this study, is that use of and expenditures for ICF services by $S / H M O$ members appear to be strongly influenced by HMO practices and ECB incentives. Conclusions, about the apparent influence of the $s /$ HMO program on use of ICFs, are based on empirical comparisons between s/HMO members and other capitated Ho Medicare beneficiaries who do not have an ECB.

Collectively, measures used in this comparison suggest that the S/HMO concept is associated with:

- greater access to ICF services, based on observations that $S /$ HMO members had a much higher probability of being in a nursing home (SNF/ICF) than Risk members; 
- effective ICF utilization outcomes based on observations that S/HMO residents used substantially fewer cumulative days of ICF stay across a two year span of time, than Risk members, including days not covered by ECB. This was also true of $\mathrm{s} /$ HMO members whose nursing home stay included SNF and ICF services, and under conditions where only direct-pay (individual) Risk member rates were compared with $\mathrm{S} / \mathrm{HMO}$ rates. Risk group-sponsored member use rates were lower than individual Risk member use rates;

effective use of the ECB, based on the observation that s/HMO residents had a higher proportion of ICF days below the benefit limit (of 100 cumulative days), as well as a higher proportion of ICF days below one year (the point beyond which a majority of nursing home occupants, generally, become welfare dependent);

- adherence to the s/HMO goal regarding use of the ECB for home-based care when possible, based on the observation that S/HMO residents had a substantially higher proportion of last ICF discharges to home, than Risk Members;

- cost-effective outcomes based on the observation that $S /$ mo members, as an entire eligibility group, required fewer financial resources per 1000 members for ICF services, than Risk members. This was also true for combined SNF and ICF services, although differences for SNF only services were marginal;

- lower incidence of spend-down, based on the observation that S/HMO ICF residents had a lower rate of spend-down within one year after the study period, than Risk members ;

- potentially important outcomes regarding governmental goals for containment of escalating welfare payments, based on the observation that Medicaid payments were a smaller proportion of total nursing home revenues for $S / H M O$ ICF residents, than for Risk ICF residents.

- socially important outcomes regarding options to nursing home care for very elderly frail females in that use rates for such $s /$ HMO members were significantly lower than those for such Risk females.

Findings listed above are derived from the overall data set. When nursing home users are removed from the overall 
data set who had admissions prior to the study period, e.g., (Subset I), the second finding noted above (cumulative days used) is shifted to a finding of no statistically significant difference. However, the elimination of left censoring effects did not alter the fifth or sixth finding, above, i.e., payments received, and Medicaid payments as a proportion of total payments were less for S/HMO nursing home users than for Risk nursing home users.

Since payments include nursing home stays up to one year after the study period, this observation suggests that resident use patterns in subset I data had not sufficient time to develop into those observed in the overall data set. However, a basic change in use of ECB for nursing home care became effective in January, 1989, which complicates interpretation of findings for subset I financial data.

In this study, numerous measures of utilization were applied including rates whose denominators made numerator values relative, and central tendency values which observed effects of long-term residents on the length of stay means for the dominant group of nursing home users whose total stay was short. Even after the long-stay group was removed from the overall data set, cumulative days used within the study period by $S /$ HMO members were substantially less than those for Risk members.

The elimination of a statistically significant difference in cumulative days used, resulting from 
elimination of all Risk and S/HMO members with prior admissions (Subset I), emphasizes the importance of identifying rates over a long time period, if not over a lifetime of use rates. This is especially important when investigating patterns of spend-down, total nursing home payments, and likely effects of renewable LTC benefits on nursing home use rate patterns.

While this study did not encompass the time span needed to comprehensively examine life-time nursing home use rates, it did encompass a four year period (three for those with no prior admissions) for purposes of comparing relative rates of nursing home payment. And, it encompassed a two year period for comparing probability of admission and cumulative days used from all admissions. Other methods of evaluation are yet to be applied to data in this study, such as odds ratio survival predictions, as a method of resolving effects of left and right censoring of use rate data.

However, findings in this study do a reasonable job of informing policy makers about patterns of nursing home use for shorter-stay residents (under two years), most of whom will not re-enter nursing homes.

This study suggests that over two-thirds of $\mathrm{s} / \mathrm{HMO}$ member cumulative days of ICF stay totaled less than 100 days, and over four-fifths totaled less than one year. Risk member ICF users used more ICF resources than S/HMO members. This suggests that, during the time period studied, the ECB 
was providing a reasonable level of protection against asset depletion and against catastrophic loss of assets, for a large proportion of research population members needing ICF and overall nursing home services.

Based on observations summarized above, it appears that this study establishes part of the baseline needed to undertake comfirmatory hypothesis testing of differences between $\mathrm{S} / \mathrm{HMO}$ and Risk members enrolled during the study period, regarding comprehensive use of all formal ITC services available under the ECB. Such studies could strengthen policy-maker and public confidence in the s/HMO concept as a basic component of emerging national policy on LTC.

\section{LINKING OBSERVATIONS TO PROPOSED THEORY AND HYPOTHESIS}

"Theory implies considerable evidence in support of a formulated general principle explaining the operation of certain phenomena."[90]

The consistency of differences between Risk and s/HMO member ICF use patterns provides a basis for conceptualizing the existence of a strong relationship between the s/HMO program and ICF utilization outcomes. This conceptual conclusion leads this investigator to the following (two) tentative theories and related working hypotheses regarding differences between $\mathrm{S} / \mathrm{HMO}$ and Risk member use of and expenditures for ICF services, and spend-down patterns. 
General Theory I: Elderly people and/or family actively use benefits covering formal in-home care to reduce use of ICFs below the level used by people without home care benefits because, if at all possible, because it is financially rational to do so.

Program theory, derived from General Theory I, is that chronically ill, functionally impaired, certifiably dependent elderly s/HMO members readily access but limit use of ICF services by adopting home based care as an alternative through case manager negotiated use of formal care givers who assist informal care-givers committed to supporting such members at home, when financial incentives favor that option and when it is medically feasible.

Use rate patterns observed in this study suggest that continued pursuit of the s/HMO concept is justified as a widely affordable means of privately financing, limited coverage, front-end, LTC benefits. If ECB are managed carefully, in concert with entitlement and Medicare Supplemental Insurance benefits, to help disabled elderly persons either remain in a home setting longer than they otherwise might, or as a means of minimizing nursing home stays to respite use, then inflationary effects of such a benefit may be minimized or even avoided.

This theory and study observations lead to a hypothesis statement about $S / H M O$ as a policy choice for offering ITC benefits to HMO members on a widespread basis.

Working Hypothesis I: Combined non-entitlement LTC costs resulting from SNF+ICF plus formal in-home services used by study period $\mathrm{S} / \mathrm{HMO}$ members certified as needing ICF level 
assistance, do not exceed total SNF and ICF costs of study period Risk members.

An example of the potential for this, as seen in Chapter VII, is that savings of $\$ 238$ per Research Population member were attributable to S/HMO use of SNF and ICF total expenditures, over expenditures for Risk members. Assuming that 238 dollars represented an average savings for lifetime nursing home expenditures by members in this study, then over six million dollars (\$861-\$543-\$318 x 19261 pop. $\$ 6,125,000$ [rounded]) would be available for use by that population to spend on alternative LTC services, without increasing expenditures among those 19,261 persons. That is:

Actual Cost+Risk+S/HMO Total SNF+ICF revenues $=\$ 14,439,000$

* SNF+ICF Revenues Never S/HMO: \$861/HPM - \$16,584,000 SNF+ICF Revenues S/HMO Sometime $\$ 543 / \mathrm{HPM}=\$ 10,459,000$

* (HPM means $\$ /$ health plan member $x 19,261$ )

Furthermore, instead of spreading the ECB Supplemental Premium costs over 6317 s/HMO members, conceptually, all 19,261 members in the Research Population would have borne the price of such premiums.

There is considerable reason to believe that it is possible to successfully market broader coverages of Medicare Supplemental Insurance which include LTC benefits. One recent survey indicated that the "second most important [demand among LTC policy holders and non-policy holders] was establishment of a governmental long-term insurance program 
for which they [private policy holders] would pay

premiums.... [the author's considered opinion about this

finding was]...Unless and until the government clearly

defines its own role regarding long-term care, consumers may

be reluctant to purchase private insurance."[91]

A second theory also is surfaced by baseline

observations in this study, regarding apparent ECB effects

on Medicaid dependency patterns.

General Theory II: Maintaining elderly dependent persons at home with formal assistance, who otherwise would be in an ICF, is a cost-effect solution to containing welfare dependency because it reduces the probability of nursing home induced spend-down.

Program theory for the above General Theory II is that an HMO managed care benefit which provides consumer incentives for use of home-based care in lieu of ICF services when medically feasible, but which provides limited coverage of ICF and non-entitlement SNF costs, defers spend-down related to nursing home costs and reduces the level of public financing of nursing home costs.

Working Hypothesis II: Medicaid payments, as a proportion of total life-time costs for both nursing home and formal community based care, are less for $s /$ HMO members than for Risk members.

If, as seen in Chapter VIII, fourteen percent fewer S/HMO ICF users spent down, than did Risk users, and if over 5 percent less Medicaid funds were used to pay for $\mathrm{s} / \mathrm{HMO}$ than for Risk nursing home costs, then some kind of 
front-end, LTC policy, prior to Medicaid, may be an

affordable program in the public domain.

This notion may be postulated even more strongly if a secondary study observation about Risk-s/HMO spend down is true. It appears that spend-down rates for both Risk and S/HMO may be substantially less than is widely reported for elderly nursing home residents. That is, Risk and S/HMO apparent overall spend-down rates of thirty-one percent and sixteen percent, respectively, were less than the thirtyeight percent reported for nursing home users whose stay is three months, and fifty-eight percent for those whose stay is one year.

How meaningful is it that only sixteen percent of $\mathrm{s} / \mathrm{HMO}$ members were welfare dependent? Placed in the context of a recently (1992) released study by DHHS, regarding spend-down by elderly, it is an important outcome of the s/HMO Demonstration. DHHS said:

- Our review of these studies and methodological issues...lead us to believe the following are fairly safe conclusions:

- Approximately 1 in 4 persons admitted [to a nursing home] as private pay stay long enough to deplete assets to Medicaid levels;

- Approximately 1 in 3 persons eventually covered by Medicaid were not eligible when admitted; and

- Around 30-408 of Medicaid expenditures for nursing home care can be attributed to these asset spend-downers. [92] 
If a $s / H M O$ program were implemented which resulted in 158 spend down of all residents who were private pay persons at the time of admission, rather than a $25 \%$ spend- down rate, that could be a meaningful public policy change, relative to current Medicaid payments for true spend down patients.

Further, a S/HMO program could have an important affect on the proportion of persons admitted to a nursing home who already have become welfare dependent due to community-based ITC costs ( $7 \%$ of such persons are estimated to spend-down). Deferring spend-down may be a better target than preventing spend-down for that portion of the aged population at high risk of permanent or long-term institutional placement. This is another reason why data is needed from the state of Oregon SDSD 360 Medicaid files; it would allow identification of Risk vs. S/HMO community-based spend-down.

Literature on spend-down data must be viewed with caution because the basis on which estimates are made may be at issue and because censoring is very likely to confound spend-down observations. For example, a recent study on the Connecticut data base of nursing home users explored why variation is so great among studies which estimate the probability of spend-down, concluding that:

Most of the variation between spend down probabilities reported here, and those found in other studies, probably reflect the greater degree of censoring in others' data. [in Connecticut]... of the 41,845 people who first 


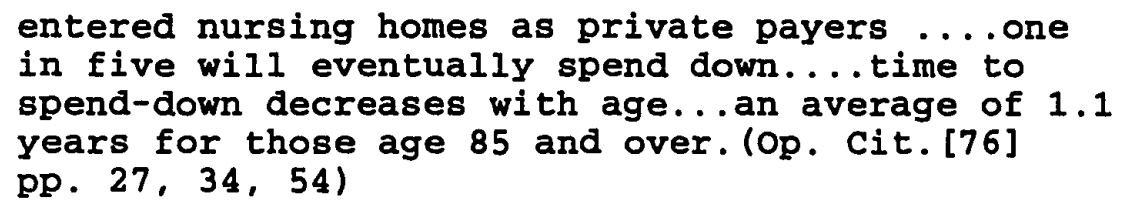

In this dissertation study, under subset $I$, all nursing home residents had at least one year, and some had three years, in which that spend-down could occur, following first ICF admission. Since most studies agree that about 50 percent who spend down do so within one year, it is likely that this dissertation study identifies a high proportion of those who would spend down. Thus, spend down rates reported in this study should not be seriously understated due to study period censoring.

However, spend-down does not translate directly to savings, since a large proportion of Medicaid recipients pay part of their bill from SSI or other private sources, such as probated estates

Hypotheses I and II are recommend for use in research that is broader in scope than was addressed by this study. Additional information is needed about use of and payments for formal LTC services in the homes and at other community-based locations of Risk members during the study period, as well as for $\mathrm{S} / \mathrm{HMO}$ members.

If findings from these hypotheses confirm observations in this study and expand findings to include difference in formal community care between Risk and s/HMO members, then a comprehensive, hypothesis derived, policy statement may be 
presented regarding effects of S/HMO ECB on overall LTC services and expendatures of HMO members.

There are other considerations for formulation of ITC policy than those discussed so far in this study. They are presented next, followed by some observations about how S/HMO, as LTC policy, holds up under the social criteria for evaluating ITC policy solutions.

FRAME OF REFERENCE FOR EVALUATING S/HMO FINDINGS

Findings of this study may be evaluated within the context of how they validate or modify above theory and related concepts of S/HMO policy.

During the period of time examined by this study, s/HMO policy allowed use of the total ECB value for nursing home services not covered by Medicare or RP Supplemental benefit package. This study concentrates on the outcome of that specific element of S/HMO policy.

A cluster of strategic theories was advanced by Leutz and Capitman in 1992 for meeting the needs of highly dependent frail and/or ill elderly persons in America. (Op. Cit. [41], pp. 217) These strategic theories and related operational concepts, paraphrased below, assert, that:

- a substantial portion of initial, formal, LTC costs can be met by widespread private sector risk pooling of fixed payments by Medicare beneficiaries.

- concomitant adoption of national policy on the role of government financing of LTC is needed to 
facilitate private sector involvement in LTC.

- managed care programs can do more to influence efficient use of alternative services at early stages of dependency than after the use of nursing home care has either commenced or been adopted as the solution of choice by families of elderly dependent members.

- while indemnity and non-HMo service based insurance policies for LTC are predominately for nursing home services, formal home based LTC services are manageable in an environment where incentives for cost efficient care exist, and where all levels of care-givers are continuously available to ensure proper management of home based care.

- early access to formal LTC services facilitates the opportunity to assist and teach family networks to maintain members at home;

- properly coordinated medical and social intervention at the earlier stages of dependency may either delay the need for ICF level services or even prevent it;

- most elderly who need institutional LTC will not remain on a prolonged basis, therefore targeting persons needing shorter term institutional care and ongoing home based care makes better policy sense in the private insurance market place, as a deterrent to spend-down.

- persons are less likely to become welfare dependent if maintained in a community based setting where informal care givers are supported by formal care giver services.

Certain operational concepts are derived from the above postulates/theories and adapted for use in the s/HMO

Demonstration setting. These concepts include:

- merging private financing of widely affordable but limited LTC insurance with the existing system of social insurance (Medicare);

- targeting use of home based formal services to encourage and support the role of informal care 
givers in maintaining ADL dependent elderly at home, as a first line of protection against nursing home costs, and allowing limited benefits or front-end institutional LTC costs which include incentives for moving to home based care;

- balancing protection of personal assets with early access to needed ICF level services, while protecting the pool of funds for LTC services so that many members benefit some, rather than a few benefiting greatly;

- using private sector LTC benefits to defer or prevent spend-down for many, rather than insure against catastrophic costs of those who exceed their LTC benefits and personal assets.

How well do observations in this study uphold theories and operational criteria presented above? The four concepts are the conceptual underpinnings of the S/HMO program. And, there is empirical support in this study for all of the above theories, including the second listed reference to government action supporting the S/HMO theory. Title 18 waivers were granted by congress enabling $S / H M O$ to be operationally integrated with other Medicare entitlements, and with the Medicaid program.

POLICY PRINCIPLES WITHIN WHICH S/HMO FINDINGS ARE ASSESSED

Two sources are referenced regarding principles for adopting national LTC policy. They also serve as a frame of reference for evaluating findings in this study. The first source is from a working paper to advance "strategies for strengthening Long-Term Care in the U.S.," funded by the John A. Hartford Foundation, 1990 by Leutz and Capitman. 
six dominant goals surfaced in that paper, among those advanced by the advisory body assembled for clarifying the "issues and options for reform." They are to:

Keep public costs low, Ensure equity in access to service, Assure efficient and high quality care, Protect assets of beneficiaries, Meet a range of long-term care needs, Provide consumer choices. [93]

Observations and findings in this study suggest that s/HMO outcomes, regarding use of ECB for nursing home care, were consistent with or/and supportive of all but the second goal. No quantitative evaluation of quality of care was done in this study.

The second source was published in 1988, by Rivlin and Wiener who brought into focus much of the technical information and concepts about policy options needed to overcome the nationwide problem, noted in Chapter II, of this study, especially that of not having any risk pooling system which reduces the impact of long-term care related catastrophic costs on individuals and governments.

They recommended six principles intended to guide the development of policy options into a collective solution for this overaxching LTC policy problem.

Those six principles are paraphrased below because, in addition to guiding national policy formulation, they provide a social, if not moral, frame of reference for summarizing how findings in this study might add to knowledge, if not understanding, about the role of $\mathrm{s} / \mathrm{HMO}$ as 
a viable LTC policy option.

These six principles propose that public and private

LTC policy must strive to:

- achieve scientific breakthrough and lifestyle changes which help reduce the incidence of chronic illness that results in profound disability at older ages;

- treat long-term care as a normal risk of growing old, and therefore use risk-pooling as one approach to paying for long-term care;

- require that all financing systems for LTC respect desires of most elderly to remain at home as long as possible and to reinforce the efforts of family and friends to provide informal care in a home environment;

- encourage the design of new payment mechanisms which can improve the quality, flexibility, and efficiency of the delivery system as well as access to it;

- ensure that both public and private sectors have major roles in the organized financing of long-term care;

- encourage the design of payment mechanisms which organize care in a way that increases patient satisfaction and minimizes institutionalization, such as social/health maintenance organizations, continuing care retirement communities, and other alternative living arrangements. (Op. Cit. [46], pp. 238-239).

only the first principle, above, is not addressed by the s/HMO concept, whether or not they are operationalized at all sites; obtaining scientific breakthrough is not a relevent criteria for use in evaluating s/HMO. Empirical observations in this study, or the economic concept of $\mathrm{s} / \mathrm{HMO}$ support the other five principles.

By design, S/HMO facilitates expansion of the HMO managed care process to include coordination of LTC services covered by a range of ITC benefit options. Also, by design, 
s/HMO Expanded Care Benefits provide social and economic incentives for members, who are certified as needing ICF level services, to use formal home care services as a means of deferring or precluding admission to an ICF.

Certain operational concepts guide managed care practices of those HMOs which function as a private domain, capitation financed, "closed panel" group practice health care organization. Such concepts include: a cooperative contractual relationship between medical group providers and the health care organization which charges the member or sponsor at fixed rates for a fixed period of time and minimizes copayments or other charges; full exchange of information about the benefit related service commitments to a known membership, full sharing of medical information among providers, division of labor among care givers which facilitates cost-effective delivery of care, comprehensive benefits at prices competitive within the market place, financial incentives for members to access care in a timely way, financial incentives for providers to treat member's health problems in a timely way.

The social/HMo is a geriatric health policy model which applies the above concepts to use of selected LTC services allowed under an expanded Medicare supplemental benefit. Such benefits are designed to stimulate use of home-based services, enhance access to nursing homes as an adjunct procedure for supporting home-based care. Therefore the 
operating concepts of $\mathrm{S} / \mathrm{HMO}$ are added to the above HMO concepts :

- provider guided selection of least skill intensive care suitable for the member's medical needs available under Medicare and Medicare Supplemental benefits;

- coordination of Medicare and Supplemental Insurance benefits with those available under the S/HMO ECB, emphasizing community services needed by elderly persons certified as physically and/or cognatively dependent due to chronic illness and/or degenerative health conditions;

- case-managed monitoring of appropriateness of level of care; negotiated use of $\mathrm{s} /$ HMO benefits which substitute formal home based care for nursing home services when feasible;

- financial incentives to reside in a home setting rather than in an ICF for those certified as eligible for admission to an ICF;

- LTC benefit limitations on institutional LTC services, in order to seek a balance between premium prices which a large cross-section of elderly can afford, yet which meaningfully shield a large proportion of members from front-end expenses of LTC services;

- an enrollment case-mix similar to a broad cross-section of the age eligible Medicare population regarding need for LTC services, so that an enroliment policy is needed, to ensure provision of LTC services within the benefit price structure, which guards against serious adverse selection. (Op Cit. [41] p.217)

IDEAS FOR OPERATIONALIZING S/HMO ON AN URBAN SCALE

Assuming that findings in this study were confirmed by follow-up research, proposed above, some kind of interim step is needed as a preparatory phase for adopting a s/HMO model as national LTC policy. That interim step could be an 
adaptation of the Urban Development Assistant Grants (UDAG) in which federal funds were used to stimulate private venture capital to up-grade the quality of life in blighted urban centers.

A few large scale demonstration projects could be undertaken within selected metropolitan statistical areas (MSA) to stimulate new arrangements between insurance carriers, large health benefit service organizations, providers, employers, state governments, and Federal agencies. Such arrangements would ensure that all Medicare Beneficiaries were covered by a comprehensive, Medicare linked, Supplemental benefit which included an Expanded Care Benefit. That benefit could encompass alternative care concepts in addition to home-based care.

Federal funds would not be awarded until trial MSA participants merged their interests into organized networks which comported with Rivlin and wiener type guidelines.

A few competing organizations could be formed in each trial MSA which could have variation in their delivery systems, as long as a floor of service and economic standards were met. Implementation would require some one-time solutions for Medicare persons already in nursing homes, hospitals, Medicare Respite and End Stage Renal Disease status.

start-up costs could include some form of financial incentives to both providers and Medicare beneficiaries. 
Beyond initial enrollment, limitations would be required to prevent adverse selection resulting from people moving into trial MSAs because they knew of their need for long-term care. Medicaid, or some other pooling concept would be used to reinsuring long-stay, long-term care persons who used up their ECB and personal assets. Aggressive, community-based, case managed control of such benefits would be required. Entitlement benefits under Medicare would tied to privately paid LTC Supplemental Benefits under capitation contract which linked defined organizations of physicians, hospitals and other providers into a cost-effective system. If the trial MSA happened to be Portland, oregon, it might be anticipated that an initial surge of nursing home use could occur. There is some indication that nursing home rates (SNF+ICF) for KP members collectively are higher than in the community from which the Research population came in this study. Risk members were 30 percent more likely than Multnomah County residents to be in an ICF, and S/HMO members were 67 percent more likely than the computed use rates of overall Multnomah County, including KP members. This information is presented to indicate that both Risk and S/HMO members probably represent a somewhat different population of Medicare Beneficiaries in the sense that all Risk and s/HMO members are covered by Medicare Parts $A$ and $B$ and have a comprehensive Medicare Supplemental Insurance Benefit as well. Many aged do not have such 
coverage, which places them at risk of spend down for costs that would be covered for KP members. Moving an entire community to that level of coverage, risks a change in LTC admission rates. But, if use rate patterns in this study were replicated in an urban model elderly health services concept, as proposed, then increased access would not lead to greater institutional expenses.

Demonstration projects having an entire urban population as its membership may be required to establish the S/HMO concept as a basic component of national ITC policy. This also could activate new behaviors in the private sector which would respond to LTC problems defined by this study.

A private sector response within such trial MSAs is unlikely until state and Federal governments coordinated their policy position to provide trial umbrella coverage for catastrophic LTC costs.

Given that environment, private insurance/service organizations could coordinate their marketing of LTC risk products with some financial confidence. Linking such products to the rest of the health care system then becomes the challenge. S/HMO is the logical model for making that connection. With carefully structured incentives, it may be possible to integrate a social model of LTC services with existing entitlement benefits at an affordable cost. 
REFERENCES AND CITATIONS

\section{Chapter I}

[1] Lave J.R., \& Silverman H.A., "Financing the Health Care of the Aged," The Annals of the American Academy of Political and Social Science: Health Care Policy In America; Vol 468, July 1983; p 163.

[2] Fleming, A.S., "Health Care: An Intergenerational Issue, " Intergenerational Programs: Imperatives, Strategies, Impacts, Trends, Ed. Newman S., Brummel, M.A.; The Haworth Press, Inc. Binghamton, NY 1989, p. 236 .

[3] Mechanic. D., "Health Care and the Elderly," The Annals of the American Academy of Political and Social Science: The Quality of Aging: Strategies for Interventions. Vol 503, May 1989, pp 96-97.

[4] Organisation for Economic Co-operation and Development, "Technology, Demographic Change and Long-term Care"; Financing and Delivering Health Care: A Comparative Analysis of OECD Countries, Social Policy studies No. 4, 1987, Publications Service, OECD, Paris, France; p. 91 .

[5] Neuhauser D., "The Future of Health Care Research In Cost Containment," The Medical Cost-Containment Crisis: Fears, Opinions, and Facts.; Ed. by McCue, J.D.; Health Adminsitration Press Prespectives, Ann Arbor, Michigan, 1989, pp 286-287.

[6] Estes C.I., Wiler P.G., "Health Professions Education For Care of the Elderly," Health Care for an Aging Society, Ed. by Andreopoulos, S., Hogness J.R., Churchill Livingstone, NY, 1989, pp 108-109.

[7] Cassel C.I., "Aging Society, Chronic Illness, And Future of clinical Training," Health Care for an Aging Society, Ed. by Andreopoulos, S., Hogness J.R., Churchill Livingstone, NY, 1989, pp 85-86.

[8] Osgood N.J., "Theory and Research In Social Gerontology," The science \& Practive of Gerontology: A Multidisciplinary Guide, Ed. by Osgood N.J., \& Sontz A.H.L., Greenwood Press NY, 1989. 
[9] Marmor T.R., Mashaw J.L., Harvey P.L., Americas

Misunderstood Welfare State: Persistent Myths, Enduring Realities, Basic Books, USA, 1990, pp 197, 230.

[10] Leutz W., Malone J., Kistner M., O'Bar T., Ripley J.M., Sandhaus M., "Financial Performance In The Social Health Maintenance Organization, 1985-1988, "Health Care Financing Review; Vol 12, Nr 1, Fall 1990, U.S. Department of Health and Human Services, Health Care Financing Administration, office of Research and Demonstrations, Baltimore, Ma. November 1990, p. 9.

[11] Harrington C., "Crisis In Long-Term Care: Part I, The Problem; Part II, Policy Options," Nursing Economics, January-February, 1985, Vol 3, pp 15-20, March-April, 1985, Vol 3, pp 109-114.

[12] Polich C., Parker M., Chase D., Hattinger M., Managing Health Care for the Elderly; John Wiley \& Sons, Inc. NY, 1992, pp 22-23.

[13] Oberg R., Polich C., "Medicaid: Entering the Third Decade, "Health Affairs, Vol. 7, Nr. 4, 1988 Fall Issue, p. 89 .

[14] Ruffenach G.I., "Nursing Home Care as a Work Benefit: More Firms offering Employees Insurance for long-term Costs," The Wall Street Journal, June 30, 1988, Section 2, p. 23 .

[15] Sirrocco A., "Nursing and Related Care Homes As Reported From the 1986 Inventory of Long-term Care Places," Advance Data, Nr. 147, National Center for Health statistics, DHHS, January 22, 1988, pp. 1-12, and states supplements.

[16] Sirrocco A., "Nursing Home Characteristics: 1986 Inventory of Long-term Care Places," Vital and Health Care Statistics, Series 14, No 33, National Center for Health Statistics, DHHS, March, 1989, pp. 1-32

[17] State Health Planning Department, Oregon State Health Plan, 1980, Section B, state of oregon, pp. 48, 50).

[18] Rane R., Ouslander J., Abrass I., Essentials of Clinical Geriatrics; McGraw Hill Book Co., N.Y., 1984, pp 17-31.

[19] Kane R., Kane R.A., A Will and a Way: What the U.S. Can Learn From Canada About Caring for the Elderly; Columbia University Press, N.Y., 1985, pp 13-14.). 
[20] United states General Accounting office, Long-term Care: Projecting Needs of the Aging Baby Boom Generation; GAO/HRD-91-86, B-243726 Human Resources Division, June 1991, pp 5-14.

[21] Kolata G., "New Views on Life Spans Alter Forecasts on Elderly," The New York Times, Monday, November 16, 1992, front page.

[22] Johnson C.L., Grant L.A., The Nursing Home In American Society The Johns Hopkins University Press, BAltimore, Md., pp.37-51.

[23] Lewin-ICF, Inc., Brookings Institute, Inc., Brookings/ICF Long Term Care Financing Model, Office of the Assistant Secretary for Planning and Evaluation, Department of Health and Human Services, February 1992.

[24] Weissert W.G., "Estimating the Long-Term Care Population: Prevalence Rates and selected Characteristics," Health Care Financial Review, Vol 7 , Summer 1985, pp. 84-85.

[25] Arnett R.H., McKussick D.R., Sonnenfeld S.T., Cowell C.S.. "Projections of Health Care Spending to 1990," Health Care Finnacing Review, Spring 1986, Vol 7, Nr 3, Fig. 5, p. 26 .

[26] Anderson R., Aday L., Chen M., "Data Watch," Health Affairs, Spring 1986, Vol. 5, Nr. 1, p 166.

[27] Meiners M.R., Trapnell G.R., "Long-term Care Insurance: Premium Estimates for Prototype Policies," Medical Care, October 1984, Vol. 22, No. 10, p. 903)

[28] Greenberg J., Westwater D., Leutz W., "Long-term Care Insurance: How Will It Sell?" Business and Health, Washington Business Group on Health, Nov., 1986, p. 20.

[29] U.S. General Accounting office, Testimony before the subcommittee on Health, Committee on Ways and Means, House of Representatives; Long-term Care Insurance: Risks to Consumers should Be Reduced, April, 1991, GAO/T-HRD-91-14, pp 1-22.

[30] Tenser J., "Long-term Care Insurance: The Rx for a graying America," Business and Health, Mid-March, 1992, pp53-57. 
[31] Freudenheim M., "Business and Health, U.S. May Impose Care- Policy Rules," The New York Times, Tuesday, November 101992 , C2.

[32] Greenlick M., Freeborn D., and Pope C., Health Care Research in an HMO: Two Decades of Discovery; The Johns Hopkins University Press, 1988, pp47, 48]

[33] Abrahams R., Nonnenkamp L., Dunn S., Mehta S., Woodward P. , "Case Management In the Social/Health Maintenance Organization," Generations, Fall, 1988 pp. 39-41.

[34] Abrahams R., Sternberg T., Zeps D., Dunn S., Macko P., "Integrating Care for the Geriatric Patient," HMO Practice, Vol 6., Nr. 4, December, 1992, pp. 16-27

\section{Chapter II}

[35] Shaugnessy P.W., Shaping Policy For Long-Term Care: Learning from the Effectiveness of Hospital Swing Beds; Health Administration Press, Ann Arbor, Michigan, 1991, p. 5 .

[36] Harrington C., Newcomer R., Estes C., Long Term Care of the Elderly, Sage Publications, Beverly Hills, Ca. 1985, pp. $62-63$.

[37] Weissert W.G., Cready C.M., Pawelak J.E., "Home and Community Care: Three Decades of Findings," in Peterson M.D., White D.L. (Eds.); Health Care of the Elderly: An Information source Book, Sage Publications, Newbury Park, Ca., 1989, pp. 39-126.

[38] Luetz W.N., Abrahams R., Greenlick M., Rane R., Prottas J., "Targeting expanded care to the aged: early SHMO experience" The Gernotologist, 1988, v28 pp. 4-17.

[39] Greenlick M., Nonnenkamp L., Gruenberg L., Leutz W., Lamb S, "The S/HMO Demonstration: policy implications for long term care in HMOs," Pride Institute Journal, 1988,7 , p. 16 .

[40] Shapiro E., Roos N.P., "Predictors and Patterns of Nursing Home and Home Care," in Peterson M.D., White D.L. (Eds.); Health Care of the Elderly: An Information source Book, Sage Publications, Newbury Park, Ca., 1989, p. 142.

[41] Leutz, W.N., Capitman J.A., MacAdam M., Abrahams R., Care For Frail Elders: Developing Community Solutions; Auburn House, Westport, Conn. 1992, p220. 
Chapter III

[42] Cook T.D., Campbell D.T., Quasi-Experimentation: Design \& Analysis Issues for Field Settings, Rand McNally, Chicago, 1979, p. 386

[43] Kraemer H.C, Thiemann S., How many Subjects? Statistical Power Analysis in Research, Sage Publications, Beverly Hills 1988, p. 96 .

[44] Office of Research and Demonstrations, Health Care Finincing Administration, U.S. Department of Health \& Human Services; Report to Congress: Evaluation of the Social/Health Maintenance Organization Demonstration, HCFA Pub. No. 03283, 1989, Section VI, p. 5.

[45] Meiners M., Trapnell G., "Long-Term Care Insurance: Premium Estimates for Prototype Policies, " Medical Care, vol 22, October, 1984, pp.901-11.

[46] Rivlin A.M., Wiener J.M., Caring for the Disabled Elderly: Who will pay? Donnelley and Sons, Co. Harrisonburg, va. pp. 262-264.

[47] Marks R.G., Designing a Research Project: The Basics of BioMedical Research Methodology; Van Nostrand Reinhold C., NY, 1982, p.121.

[48] Jonas, Madans, Rothwell, Bush, Feldman-Division of Analysis; "A Method to Redefine Stays on the 1985 National Nursing Home Survey," Vital and Health Statistics, Series 2: Data Evaluation and Methods Research, No 115, U.S. Department of Health and Human Services, Public Health Service, Centers for Disease Control, National Center for Health Statistics, DHHS Pulbication No. (PHS) 92-1389, March, 1992 pp.2-4.

[49] Fleiss J.I., Statistical Methods for Rates and Proportions; John Wiley \& Sons, NY, 1981, pp. 244-246.

[50] Harrington C, Newcomer R.J., "Social health maintenance organizations' service use and costs, 1985," Health Care Financing Review, Vol. 12, Nr. 3, U.S. Dept of Health and Human Services, HCFA, Office of Research and Demonstrations, Baltimore, Maryland, June 1991 pp. 43.

[51] Statistical Abstract of the United States 1991, U.S. Dept of Commerce, Bureau of the Census, 111 th ed. p. 23 
Chapter IV

[52] Smith H.L.. Fottler M.D., Prospective Payment: Managing for Operational Effectiveness; Aspen Systems Co., Rockville, Md, 1985, p. 121 .

[53] Public Health Service, Centers for Disease Control, National Center for Health Statistics; Health United States 1990; U.S. Department of Health and Human Services, DHHS Pub. No. (PHS) 91-1232, 1991; Hyattsville, Md.; p. 154 .; p. 23.

[54] Public Health Service, Centers for Disease Control, National Center for Health Statistics; Vital \& Health Statistics; "The National Nursing Home Survey-1985, Series 13, No. 97"; U.S. Department of Health and Human Services, DHHS Pub. No. (PHS) 89-1758, January 1989, Hyattsville, Md.; p. 154.

[55] The Multnomah County rate of residents per 1000 population is from the state of oregon, office of Health Policy, annual nursing home survey for 1986 and 1987.

[56] Center for Population Research and Census,"1985 Estimate of Population by Five Year Age Groups and by Sex For Oregon and It's cities," School of Urban and Public Affairs, Portland state University, Portland, Oregon, May 1986, pp1-3.

[57] Greenlick M.R., Nonnenkamp I.L., Greunberg I., Luetz W, Lamb S., "The S/HMO Demonstration: Policy Implications for Long Term Care In HMOs," Pride Institute, JI of Long Term Care Home Health Care, Summer 1988; $7(3): 15-24$, p21.

[58] Peterson K.N., Comparison of Service Utilization in the Last Year of Life between Two Medicare Populations, Masters Thesis, University of Washington, School of Public Health.

[59] Cohen M.A., Tell E.J., Wallack S.S., "The Risk Factors of Nursing Home Entry Among Residents of Six Continuing Care Retirement Communities," Journal of Gernotology: Social Sciences, 1988 , Vol 43 , No 1 p 515.

[60] Liu K., Perozek M., Effects of Multiple Admissions on Nursing Home Use: Implications for "Front-end" Policies; The Urban Institute, Wash. D.C., Sept. 1990, DHHS Contract No. HHS-100-90-0015, pp 1-17 
[61] Liu K., Manton K., Nursing Home Length of Stay and Spend- Down: Connecticut, 1977-1985; Health Care Financing and Administration, Cooperative Agreement No. 19-C-99020 and the Department of Health and Human Services Contract No. HHS-100-90-0015; August, 1990, Table 2 .

[62] HMO/CMP Manual-HCFA-75; Health Care Financing Administration Section 6008 "Accretion and Deletion" 04-85, p. 6-1-28 Rev.1

\section{Chapter V}

[63] Greenlick M.R., Lamb S.J., A Demonstration of Alternative Models for Prepaid Capitation of Health Care Services for Medicare Recipients: Final Report; Center for Health Research, Raiser Permanente, Northwest Region, August, 1985, p. 12.

[64] Wrightson C.W., HMO Rate Setting \& Financial strategy; Health Administration Press Perspectives, Ann Arbor, Michigan, 1990, p. 247.

\section{Chapter VI}

[65] Johnson C.I., Grant L.A., The Nursing Home In American Society; The Johns Hopkins University Press, 1985, p. 45 .

[66] Kane R.A., Hixon L., Kane R.L., Nyman J.A., "Executive Summary," Adult Foster Care In Oregon: Evaluation; Division of Health Services Research and Policy, School of Public Health, University of Minnesota;

\section{Chapter VII}

[67] Bishop C.E., "Nursing Home Cost studies and Reimbursement Issues," Health Care Financing Review; office of Research, Demonstrations, and statistics, Health Care Financing Administration, Spring 1980, Volume 1, Number 4, p. 47.

[68] Strathan G., "Nursing Home Characteristics: Preliminary Data From the 1985 National Nursing Home Survey, " NCHS Advance Data from Vital and Health Statistics. No. 131, DHHS Pub. NO. (PHS) 87-1250 Public Health Service, Hyattsville, Md., Mar. 27, 1987, pp.2-5. 
[69] Hing E, "Use of Nursing Homes by the Elderly: Preliminary Data From the 1985 National Nursing Home Survey," NCHS Advance Data from Vital and Health Statistics. No. 135, DHHS Pub. No. (PHS) 87-1250 Public Health Service, Hyattsville, Md., May 14, 1987, p.2

[70] Dor, A, "The Costs of Medicare Patients In Nursing Homes in The United States: A Multiple output Analysis," Jl of Health Economics 8 (1989), [The Urban Institute] May 1989, p. 254.

\section{Chapter VIII}

[71] Shapiro S., "An Overview of the Aged and Long Term Care Issues," from page 13 of the Manuscript Draft of a Commissioned paper presented at the conference on the Economics and Politics of Long-Term Care, sponsored by the University of California, Irvine, Graduate School of Management, and The FHP Foundation, Long Beach, California, held october 11-13, 1989.

[72] Human Resources Division, U.S. General Accounting Office; "Report to the Chairman, Subcommittee on Health and Long-Term Care, select Committee on Aging, House of Representatives," Long-Term Care Insurance: Proposals to Link Private Insurance and Medicaid Need Close Scrutiny; Sept., 1990, GAO/HRD-90-154, p 12 .

[73] Ray W.A., Federspiel C.F.. Baugh D.K., Dodds S.. "Experience of a Medicaid Nursing Home Entry Cohort," Health Care Financing Review; Office of Research and Demonstrations, Health Care Financing Administration, US DHHS, Summer 1989, Vol. $10 \mathrm{Nr}$. 4. Table 4, p. 58 .

[74] Chang D., Holahan J., Medicaid Spending In The 1980s: The Access-cost Containment Trade-off Revisited; Urban Institue Report 90-2, The Urban Institute Press, Washington D.C. 1990, pp. 73-74.

[75] Reilly T.W., Clauser S.B., Baugh D.R.. "Trends in Medicaid Payments and Utilization, 1975-89," Health Care Financing Review, 1990 Annual Supplement, Medicaid: Innovations and Opportunities. Office of Research and Demonstrations, Health Care Financing Administration, DHHS, December, 1990, p.31.

[76] Bice T.W., Nursing Home stays and Spend Down in the State of Connecticut: 1978-1983; Office of Family, Community and Long-Term Care Policy, Assistant Secretary for Planning and Evaluation, U.S. Department of Health and Human Services, Sept. 20, 1990, pp 59-60. 
[77] Adams E.K., Meiners M.R., Burwell B.O., A Synthesis and Critique of Studies on Medicaid Asset Spend Down; office of Family, Community and Long-Term Care Policy, office of the Assistant secretary for Planning and Evaluation, Department of Health and Human Services, January, 1992, p. 16.

\section{Chapter IX}

[78] Manton, K.G., Liu, R., Cornelius, E.S., "An Analysis of the Heterogeneity of $U$. S. Nursing Home Patients," Journal of Gerontology, vol 40, No 1, p. 39 .

[79] National Center for Health Statistics (NCHS), Vital \& Health Statistics, "Nursing Home Utilization By Current Residents: United states, 1985," Series 13, No. 102, October 1989, USDH\&HS, PHS CDC, pp. 18-20.

[80] Lorenz, E.W., Jones, M.K., The Physician's DRG Working Guide, 1991, st Anthony Publishing, Inc., USA)

[81] Fetter, R.B., Brand, D.A., Gamache, D., DRGs, Their Design and Development; Health Administration Press, Ann Arbor, Michigan, 1991, p. 41)

[82] Neu C.R, Harrison S.C, Heilbrun J.z., Medicare Patients and Postacute Care: Who Goes where? The Rand Publication Series, R-3780-MN, Santa Monica, Ca, 1989. pp. $29-48$.

[83] Kane R.A., Illston L.H., Kane R.L., Nyman J.A., Adult Foster Care In Oregon: Evaluation; Division of Health Services Research and Policy, School of Public Health, University of Minnesota, under contract with oregon Senior Services Division, Department of Human Resources, Saslow, M.G, Porject officer, with additional support from Administration on Aging and The Southmark Foundation on Gernotology; May, 1989, pp. 67, 70, 77 .

[84] Lekan-Rutledge D., "Chapter 25, Gerontological Nursing In Long-Term Care Facilities;" Matteson MA, Ed. Gerontological Nursing, Concepts and Practice, W.B. Saunders Company, Philadelphia, 1988, pp. 795-799.

[85] Lohr K.N.. "Advances in Health Status Assessment: Overview of the Institute of Medicine's Second Conference on Advances in Health Status Assessment ", Medical Care, Supplement, March, 1989, Vol 27 No. 3, p. S14. [funded by H. J. Raiser Family Foundation Grant] 
[86] Rothman M.L., Hedrick S., Inui T., "The Sickness Impact Profile as a Measure of the Health Status of Noncognitively Impaired Nursing Home Residents," Medical Care, Supplement, March, 1989, Vol 27 No. 3, p. S157-S167.

[87] Manton K.G., "The Dynamics of Population Aging: Demography and Policy Analysis," The Milbank Quarterly, Vol. 69 No 2, 1991, Milbank Memorial Fund, pp. 309-339.

\section{Chapter X}

[88] Paris D.C., Reynolds J.F., The Logic of Policy Inquiry, Longman, Inc. NY.NY., 1983, pp. 178-179.

[89] Grannemann T.W.. "Should Risk-Based Payments Be Used For Long-Term Care?" Ed. Scheffler R.M., Rossiter L.F.; Advances In Health Economics and Health Services Research, Volume 10, 1989: Risk-Based Payments Under Public Programs, JAI Press Inc., Greenwich, Conn, pp. 345-348.

[90] Webster's New World Dictionary of the American Language, Second Edition, The World Publishing Co, NY, NY. 1970, p. 1475 .

[91] Cohen M.A, Kumar N., Wallack S.S., "Who Buys Long-Term Care Insurance?" Health Affairs, Volume 11, Number 1, Spring, 1992, pp. 222.

[92] Adams R.E, Meiners M.R., Burwell B.O., A Synthesis And Critique of Studies on Medicaid Asset spenddown; Office of the Assistant secretary for Planning \& Evaluation, office of Family, Community and Long-term Care Policy, Department of Health \& Human Services, January, 1992, p. 29;

[93] Leutz W., Capitman J., Stratagies for strengthening Long-Term Care in the U.S.: Issues and Options for Reform, Working Paper \#1, February, 1990, Florence Heller Graduate School, Brandeis University, Waltham, MA, pp. 16-17. 
APPENDIX A

FOR CHAPTER I 


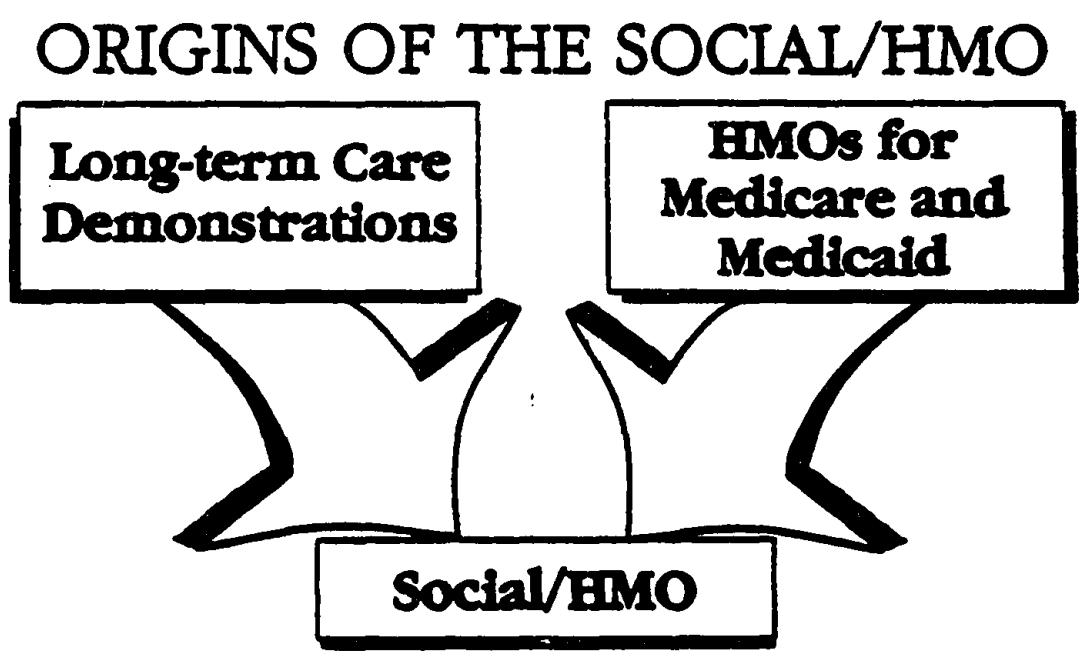




\section{Medicare Plus II}

Wi cu nase more cill credyecthan Alclikiser Plus provide you llety uans to pin Micliesor Pluw th.

Nissindre Plus to is a

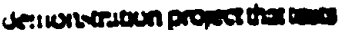
biller nulys of expund"se and

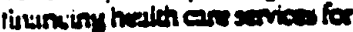

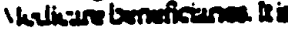
yenooned by Mudtean and liain Prmention.

\section{Idditional coverge}

fix s high maxtly proming

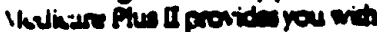

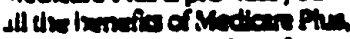

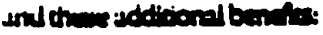

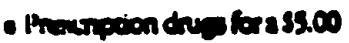
orreximas

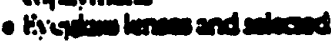
reamen an woros

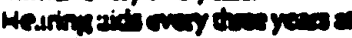
sonert theses

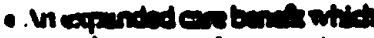

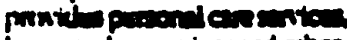

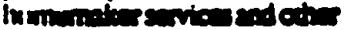

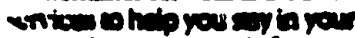

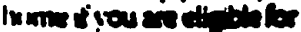

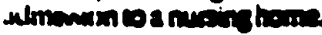

\section{How is worte}

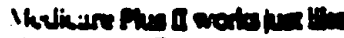

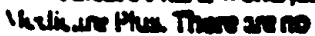

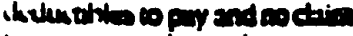

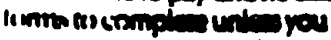

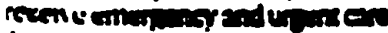
iroul a nontinies Prive

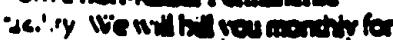

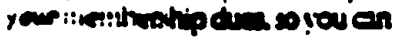

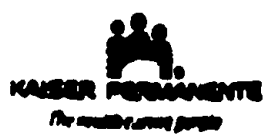
more phan your haids aipan Aly acesumen vou per whin you nawer astient in the bue

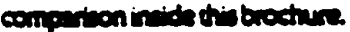

\section{An bi.npentin regutromer}

Dras you idin Kaim

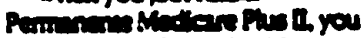

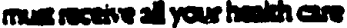

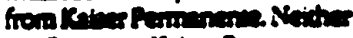

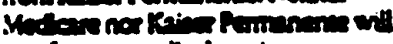

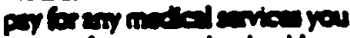

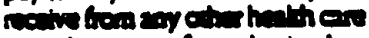

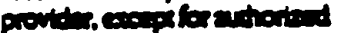

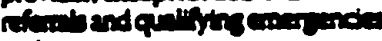
andur were

\section{Bow oging}

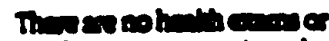

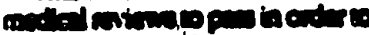

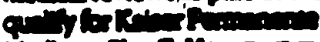

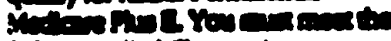

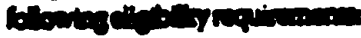

- Youmaberede.

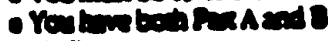
Nosoming

- Youm lyariaces of the

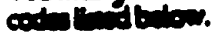

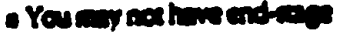

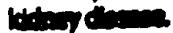

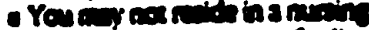

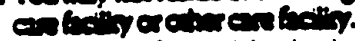

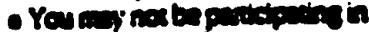

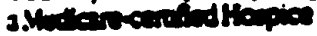
Pricine

\section{Rentur...}

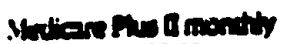

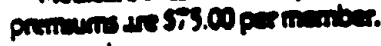
permorith
Service Ares 24p Codes

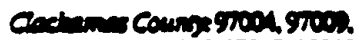
97011. $97013.97013 .97017 .5 \% 0$.

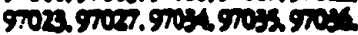

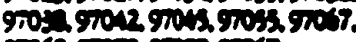
970. 970709702975

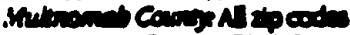

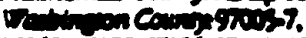

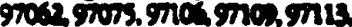

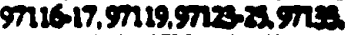

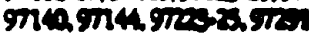

\section{Eom to eqp's}

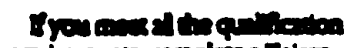

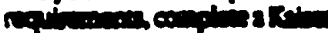

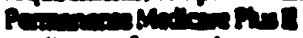

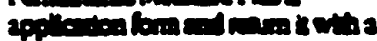
eppefyor manede Renming ThM Rin entor on

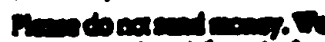

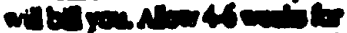

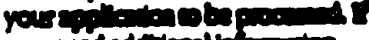

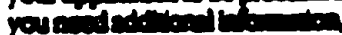

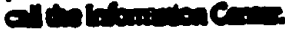

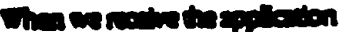

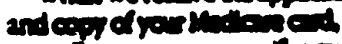
wem ace a goven

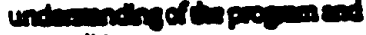
ines.res

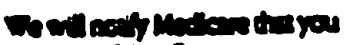

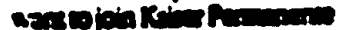

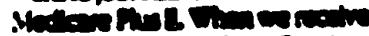

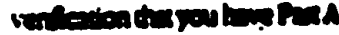

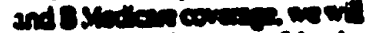

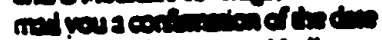

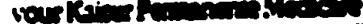

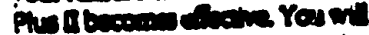

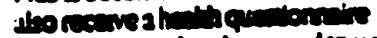

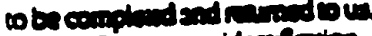

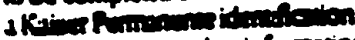

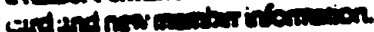




\section{The Medicare Phus II Expanded Care Benefit}

If you become eliomble for nursing home onre, this bernet. may help you say in your own home whte rectiving eare.

It provides sevice to you in

your home as well as antirences io fanily members who are for you. It may also inctude shorterm nursing home ore if you need is These sevices are in addition to whatever is covered under Mediare

\section{Inimy}

You mus be eligible for nursing home cerifiedion or cerofication is bered on the sria criterie eorblisterd by the sore of Oretion. The services you receive undes this bencix mus be awhorised by your Kaies Permanerie helth care teanses pare of your expanded are pien.

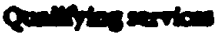 \\ ansom tom

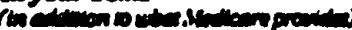

- Nid with pexomel are

- Homeroleservices

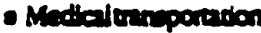

- Adulzerverecer

- Addivarel nusirzor

- Pitypial and cosupiciond therapy

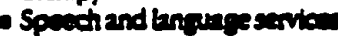
- Madicel social sevica

- Suppor and erinions for yoor arviver in your hom

- Refuris aron d with comsuring suvices such as metison-whendnon. medicelemeporetion

Inenom

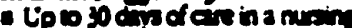

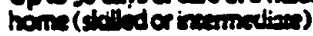

\section{Conmen}

in sour bom

Each monch Kaiser Permanente provides $90 \%$ coverege of up 10

31000 of expanded cere services in your home You pay the ermining 10\%. You are alo teponable for $100 \%$ of any expanded care expenses over the stoon per monch

Raiver Permanente will pey soiv of the con of preatibed expandad care services in rusing home for up $1030 \mathrm{dy}$. You poy the remining $10 n$ You are aloo repontele for $100 \mathrm{n}$ of any cons for mursing hom sevices beyond the 30 dy lix Thisbefefin reners onty frou formlen 60dys.

\section{0}

Thesesssoseroy

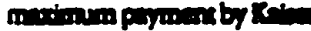
Peswenenie th appliasoto conblesion of e.pudeder

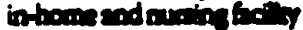
seviou You por forseven

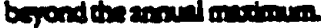

\section{Informetion Certes}

Porind (503)721.2000

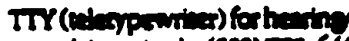
specthimpind ..(503) 7eteres

TTY wil fies fornarea cod (503) and (200)....(800) 2 a-7957

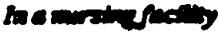
have bees or of a ming bom
Geperal exchusions for Medicare Phus and Medieare Phus II

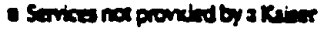
Permenasophyican

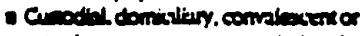

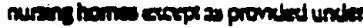

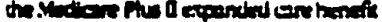
- Durieles

- Doun foxer

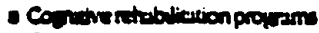

- Condisar ar.

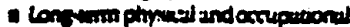

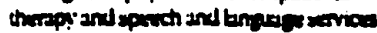

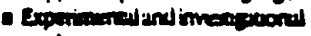
proouto

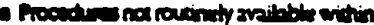
tocomonity

- Mapran

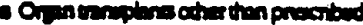

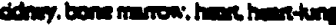
adeond troweter

- Nexilar

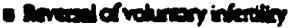

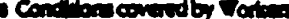

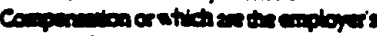

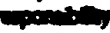

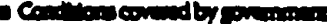

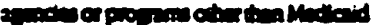

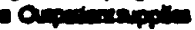

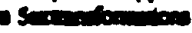

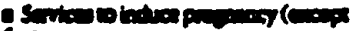

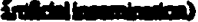

- Concidenar man

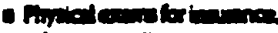

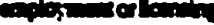

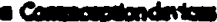

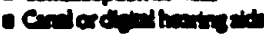

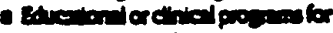

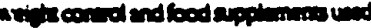

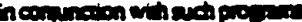

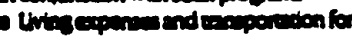

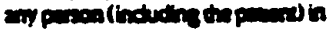

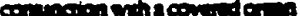

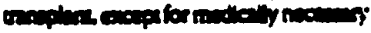

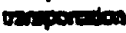

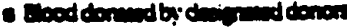

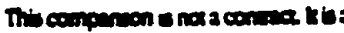

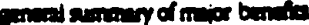

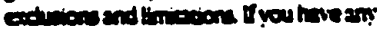

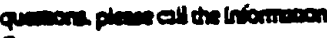
C.1. 


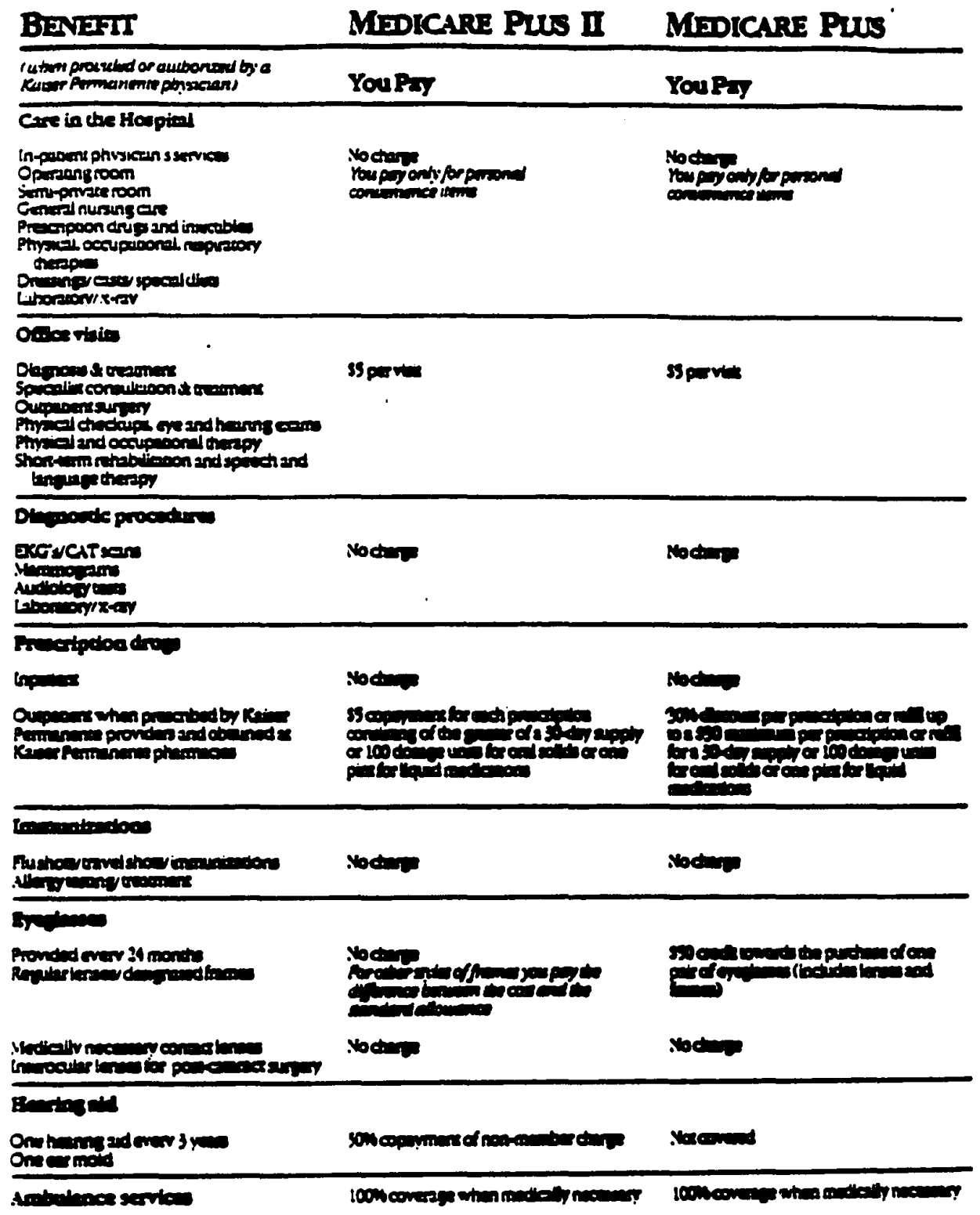




\begin{tabular}{|c|c|c|}
\hline BENDFI & Midicare Puts II & Modcara Pus \\
\hline 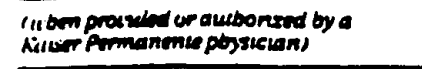 & Youfay & You Puy \\
\hline $\begin{array}{l}\text { Everifery and urent are } \\
\text { within the service ares }\end{array}$ & 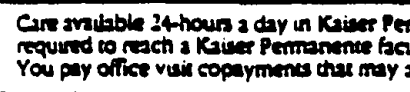 & 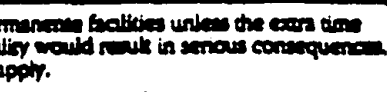 \\
\hline 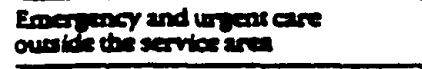 & 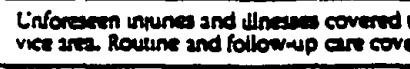 & 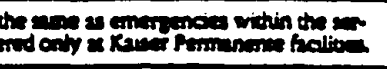 \\
\hline \multicolumn{3}{|l|}{ Dureble medlal equipoent } \\
\hline 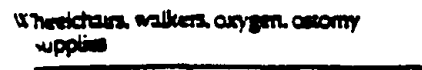 & Socture & Socter \\
\hline \multicolumn{3}{|l|}{ Henchedodon } \\
\hline 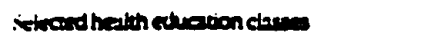 & Oarevery per dere & Ougresporten \\
\hline Specen e etweriond forums & Voctare & Nocture \\
\hline \multicolumn{3}{|l|}{ Mesalbonth } \\
\hline 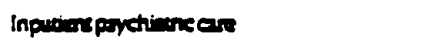 & 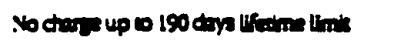 & 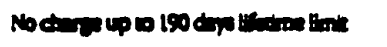 \\
\hline Promberstherept & $39 p=x$ & ssprowe \\
\hline \multicolumn{3}{|l|}{ 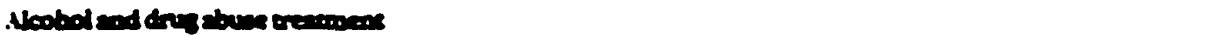 } \\
\hline 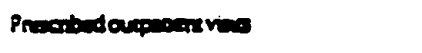 & ssperente & Mpant \\
\hline 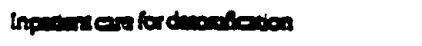 & Noder & 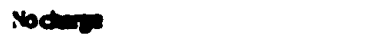 \\
\hline 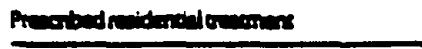 & Nodure & Soderes \\
\hline \multicolumn{3}{|l|}{ Beontrom } \\
\hline 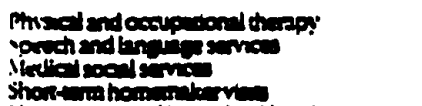 & 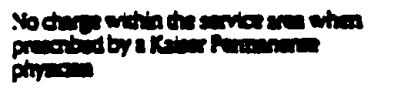 & 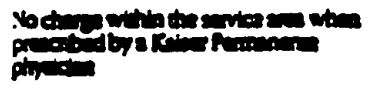 \\
\hline \multicolumn{3}{|l|}{ 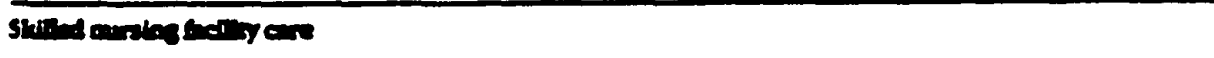 } \\
\hline 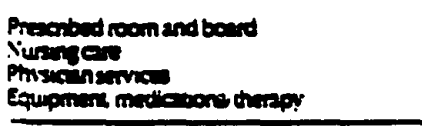 & 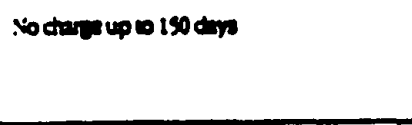 & קים \\
\hline \multicolumn{3}{|l|}{ Expodedere } \\
\hline Cirsinta ham & 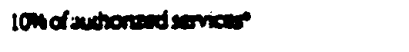 & Nacomant \\
\hline Car na numong folly & 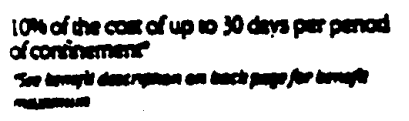 & Vorconed \\
\hline \multicolumn{3}{|l|}{ 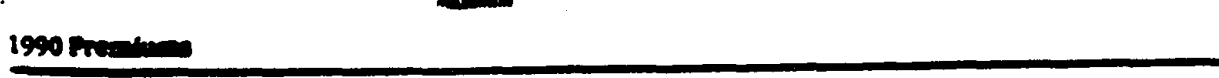 } \\
\hline 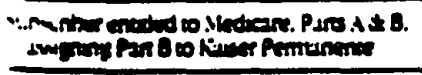 & 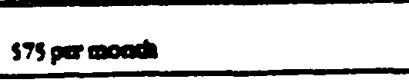 & unperect \\
\hline 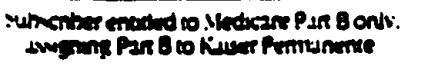 & $\cdot \mathbf{N} / \mathbf{A}$ & stesse preach \\
\hline
\end{tabular}


Kaiser Permanente Northwest Region

Center for Health Reserich

FACT SHEET

MEDICARE PLUS II DEMONSTRATION PROJECT

(Social Health Maintenance Organization)

1. The purpose of the demonstration is to expand long-term comunity-based support options avaliable to Medicare beneficieries under a prospective capitation payment systen, and to integrate these support services within managed medical care system.

2. Kaiser Permanente Northwest Region is one of four national demonstration sites:

- Minneapolis - Ebenezer/Group Health "Seniors Plus"

- New York - Metropolitan Jewish Geriatric Center "riderplan"

- Long Beach - Senjor Care Action Network "Scan Health Plan"

- Portland - KPMCP "Medicare Plus II"

3. A consortium composed of the four sttes and a tean of researchers at Brandeis University directs the project at a natlonal level. The Health Care Financing Administration (BCFA) provided evaluation funds and avarded the contract to the University of Callfornia San Francisco.

4. Medicare pays Kaiser Permanente for each enrollee at the rate of 100 percent of the MPCC (1.e., the average per capita Medicare cost in the county where the mamer lives, adjusted for age, sex and other factors). Medicare will not pay any other providers for services to these demonstration enrollees.

5. Per Member Per Month Payment (estimnted):

MEDICARE

$\$ 356$ (2008 APCC)

$\$ 146$ (from nsmber)

$\$ 500$ coesl $\mathrm{PH} / \mathrm{EM}$
MEICAIE/HDICAID

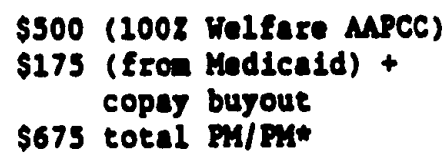

$\$ 675$ total PM/ Fit

- (Includes copareant buyout and comminity-besed ceres

6. As of January, 1993, the Medicare Plus II progran has over 4,600 members.

7. Enrollment Ellgibility requirements:

must be 65 or older;

must have Part $A$ and Part $B$ kedicare;

must reside in Oregon Count les of Multacmah. Bashington or Clackemes. 
SOCIAL HMO SITES:

BENEFIT AND CASE MIX

CHARACTERISTICS: 1991 UPDATE

\begin{tabular}{|c|c|c|c|c|}
\hline & $\begin{array}{l}\text { Kaiver } \\
\text { Personnerue } \\
\text { Pordind } \\
\text { Oregon }\end{array}$ & 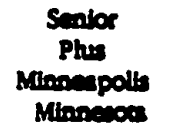 & $\begin{array}{l}\text { SCAN Fienth } \\
\text { Phan } \\
\text { Lore Bench } \\
\text { Callfornin }\end{array}$ & 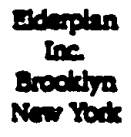 \\
\hline \multicolumn{5}{|l|}{1991} \\
\hline \multicolumn{5}{|l|}{ ITC Bendit } \\
\hline 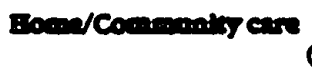 & $\begin{array}{l}s 12,000 / 5 r . \\
(\$ 1000 / 00 .)\end{array}$ & $20,400 / x$ & $\begin{array}{l}57,500 / 4 \pi \\
(3625 / m a)\end{array}$ & $26,500 / 2$ \\
\hline 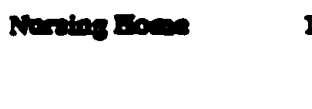 & $\begin{array}{l}14 \text { diphapell } \\
\text { of lingen }\end{array}$ & 21 dysispeil & 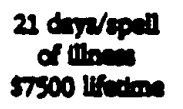 & $26,500 / 2$. \\
\hline Oonenthen & $312,000 / 21$. & $28,4004 \pi$ & $57300 / 51$. & $26,500 / y$. \\
\hline 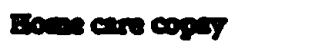 & 200 & 2001 & 57.sorvie: & suaverete \\
\hline 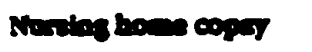 & 200 & 200 & 0 & $\mathbf{2 0 n}$ \\
\hline 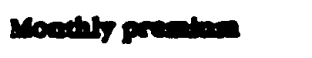 & s13s & 35273 & 0 & 936.47 \\
\hline 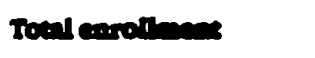 & 3413 & 308 & 290 & $4 i p$ \\
\hline 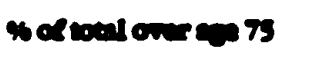 & $628 \%$ & 40.5\% & 4hen & 46m \\
\hline \multirow[t]{2}{*}{ 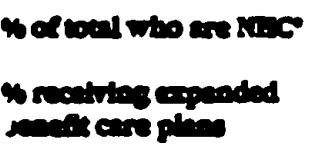 } & $126 n$ & $12 x$ & $5.4 \%$ & $5.5 \%$ \\
\hline & $7 x$ & $124 \%$ & 3.45 & 4.96 \\
\hline
\end{tabular}

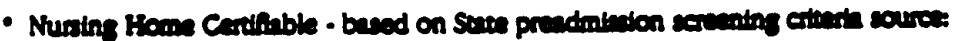




\begin{tabular}{|c|c|c|}
\hline \multicolumn{3}{|c|}{ Medleare Phus II Member Premiums } \\
\hline Yer & Monthly Premium & Enrollment County \\
\hline $1985-87$ & $\$ 49$ & Mulonomh \\
\hline $19: 8$ & 57 & Mulinocanh \\
\hline 1989 & 57.85 & Muliocomb/Washingtoe \\
\hline 1990 & 75 & Mulie/Wash/Chadenrms \\
\hline 1991 & 125 & Mule/Wash/Chadomas \\
\hline 1992 & 135 & Muir/Wash/Caclonmes \\
\hline
\end{tabular}


Kaiser Permanente Northwest Region Medicare Plus II

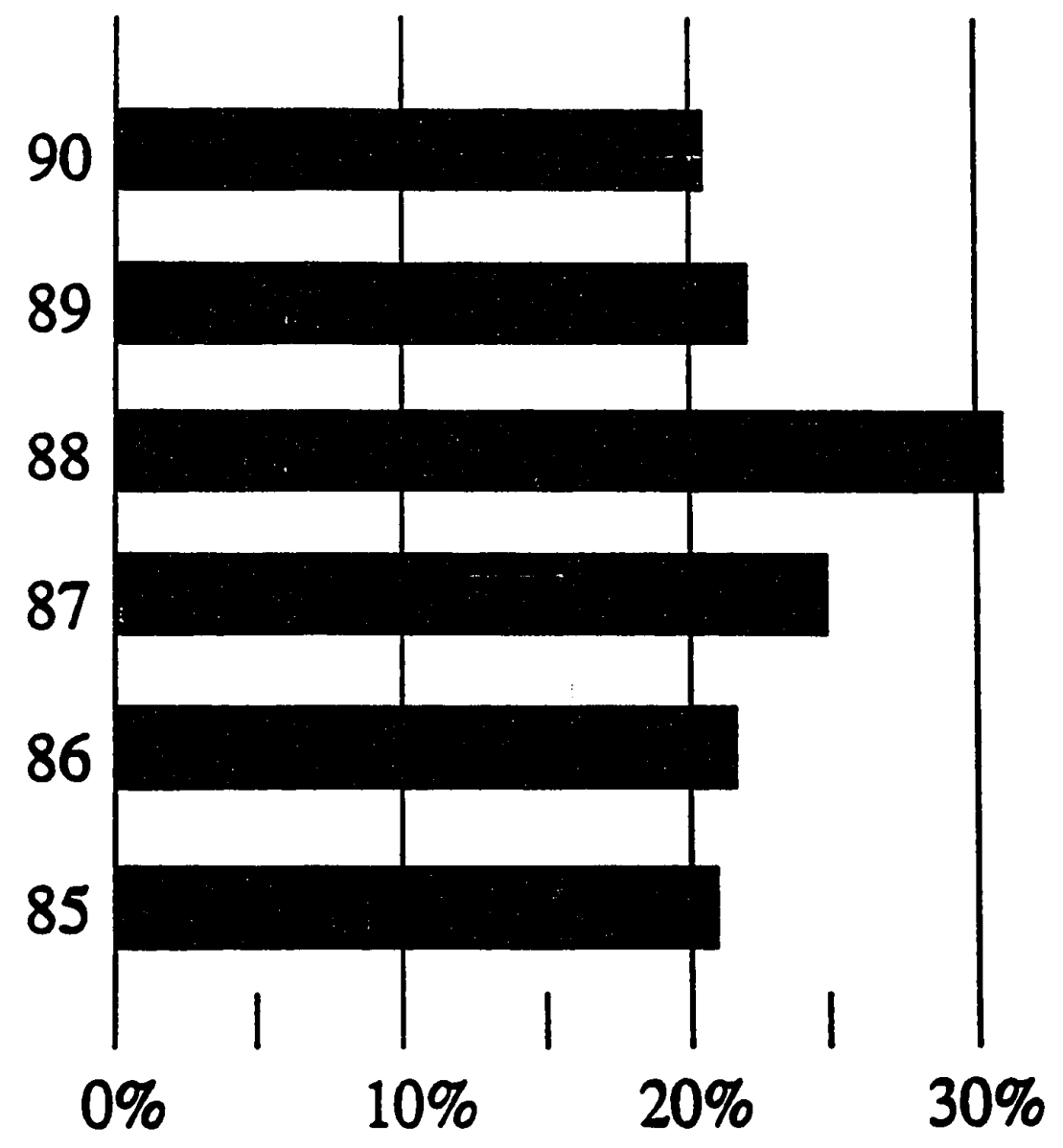

Percent of new members 80 years and older 


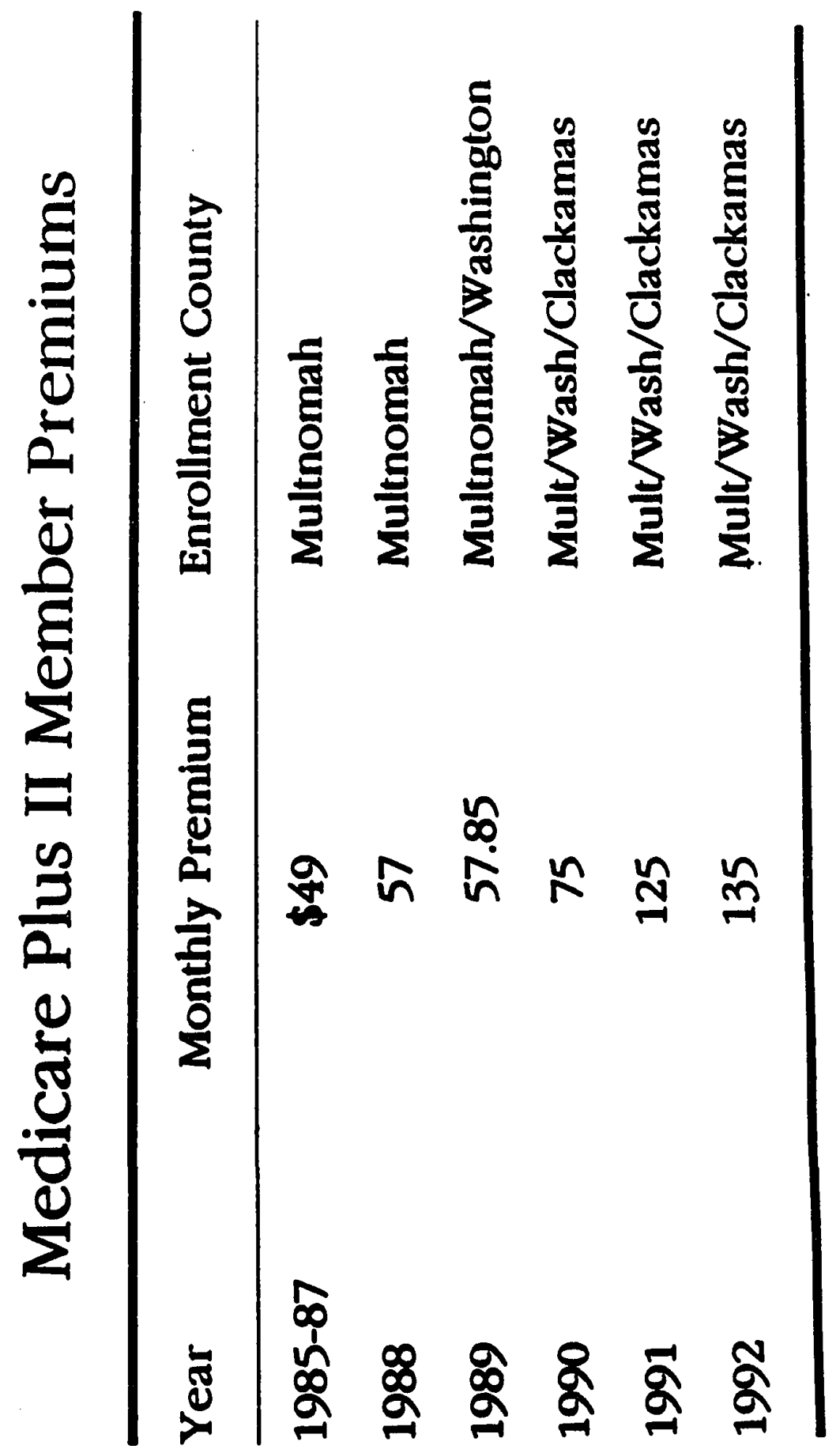




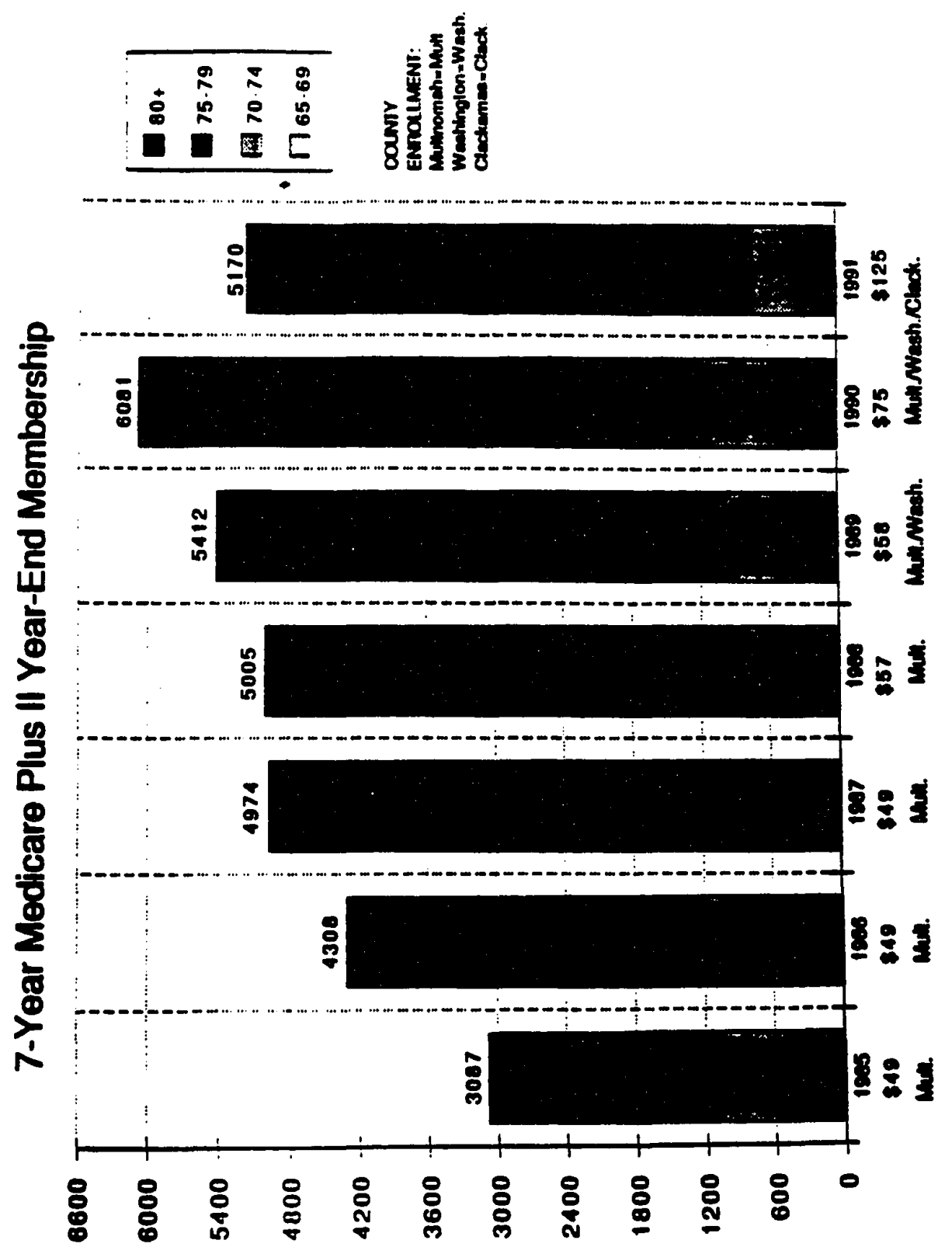




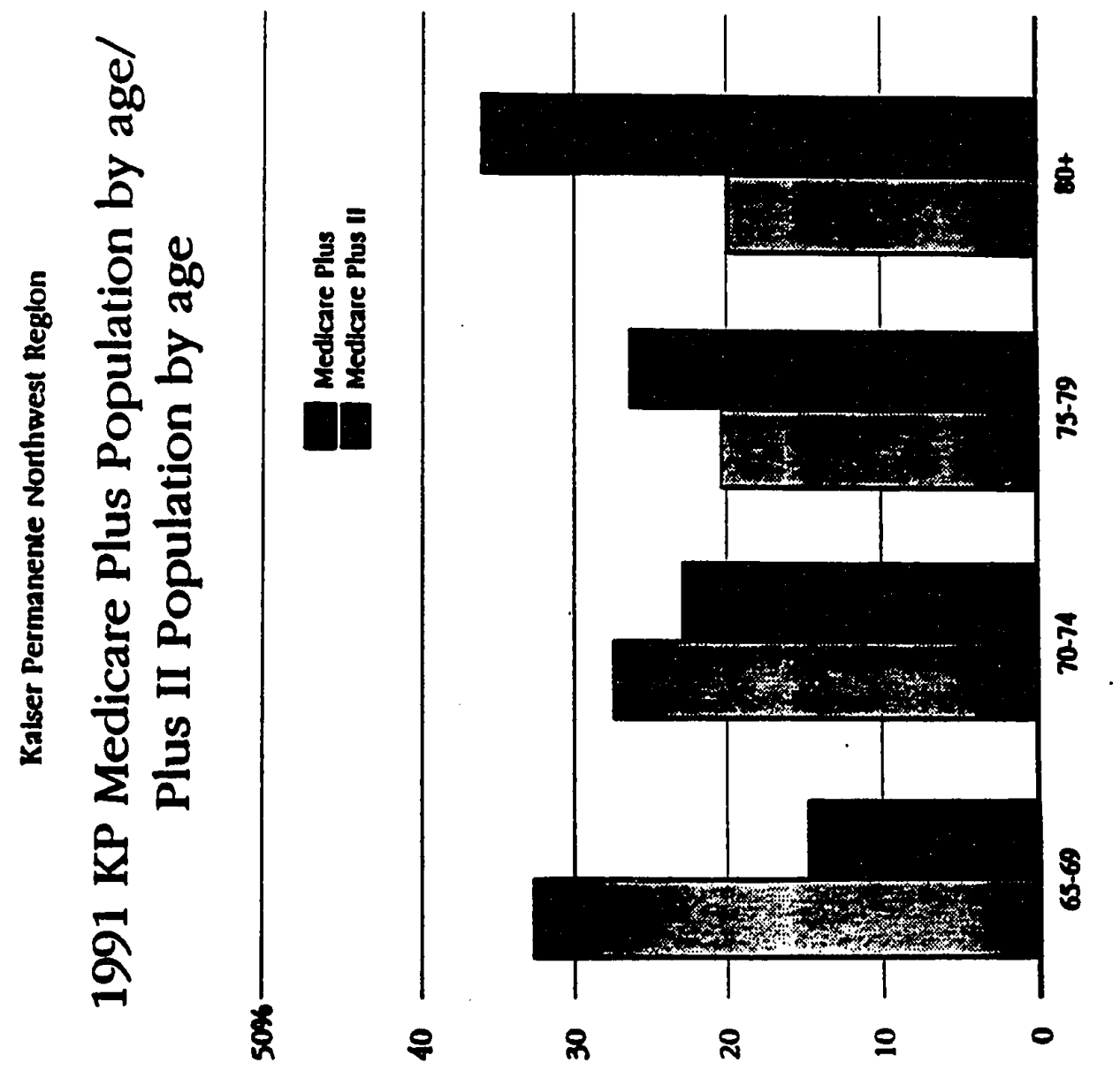




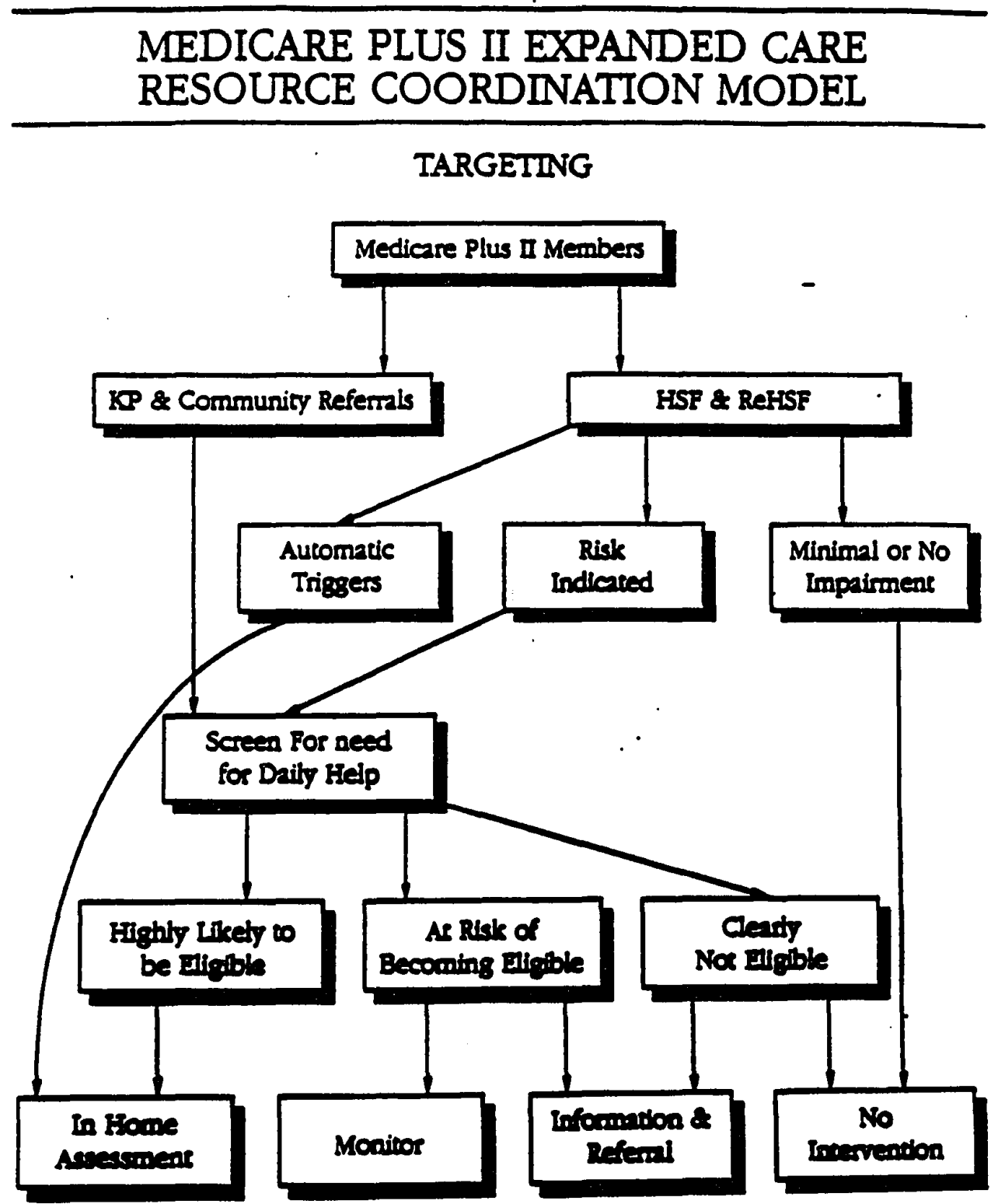




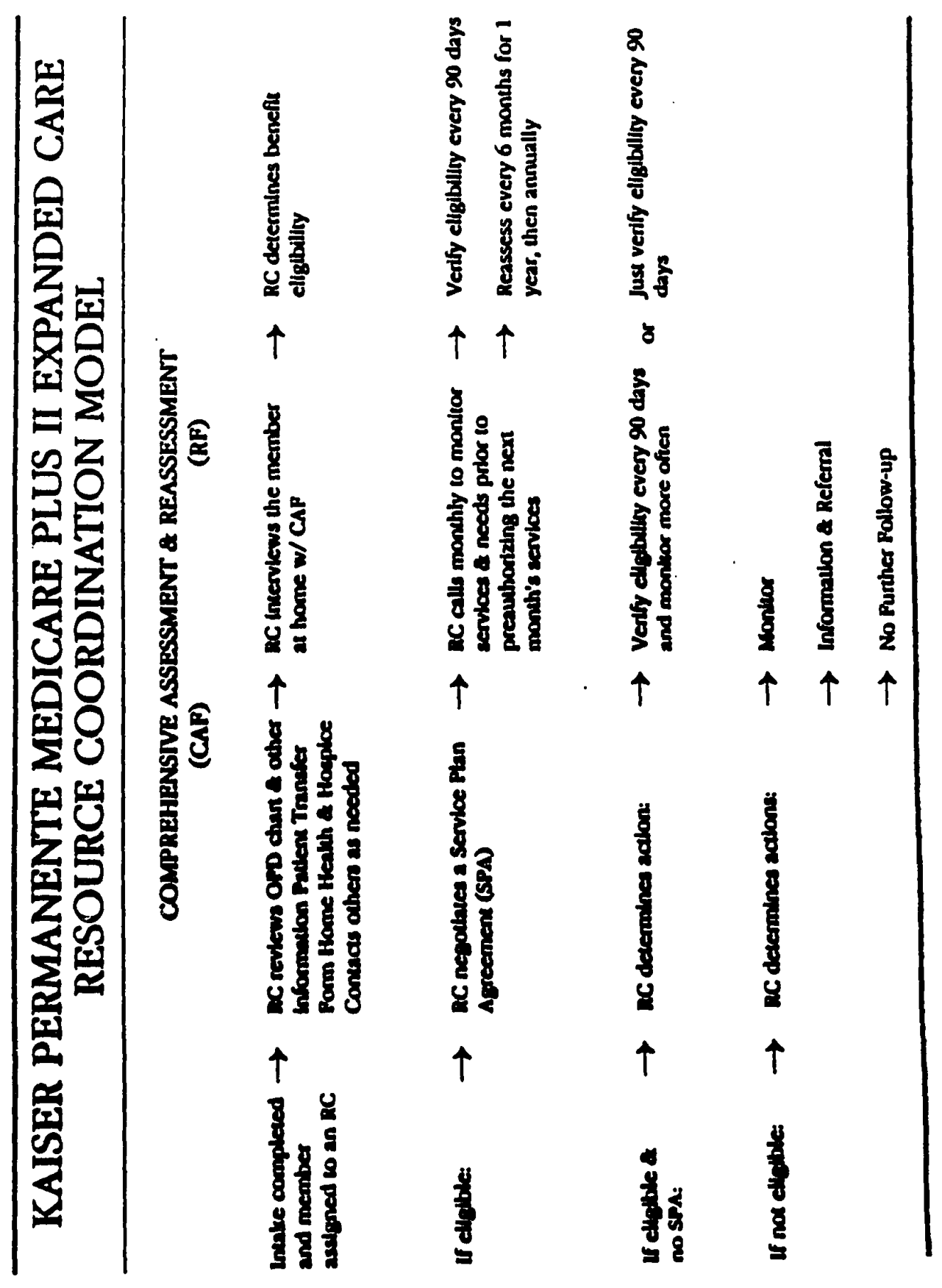




\section{MEDICARE PLUS II EXPANDED CARE RESOURCE COORDINATION MODEL}

\section{SERVICE PLAN AGREEMENT WITH ELIGIBLE MEMBERS}

(SPA)

RC negotiates the maximum service package and copayment, SPA.

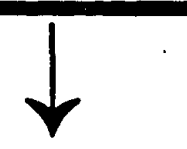

RC uses information from monthly pre-authorization calls and RF 10 renew or negotiate a new SPA at least every 6 months.

- The member must sign the SPA within 30 days of the star of services.

- The member musx sign a SPA to agsee to all new services and highes copayments.

- The member receives a copy and the original is kept in the members project chart

- The expanded care darabase connins an electronic todeler to treck the reurn of SPAs, authotizations and ascestronten. 


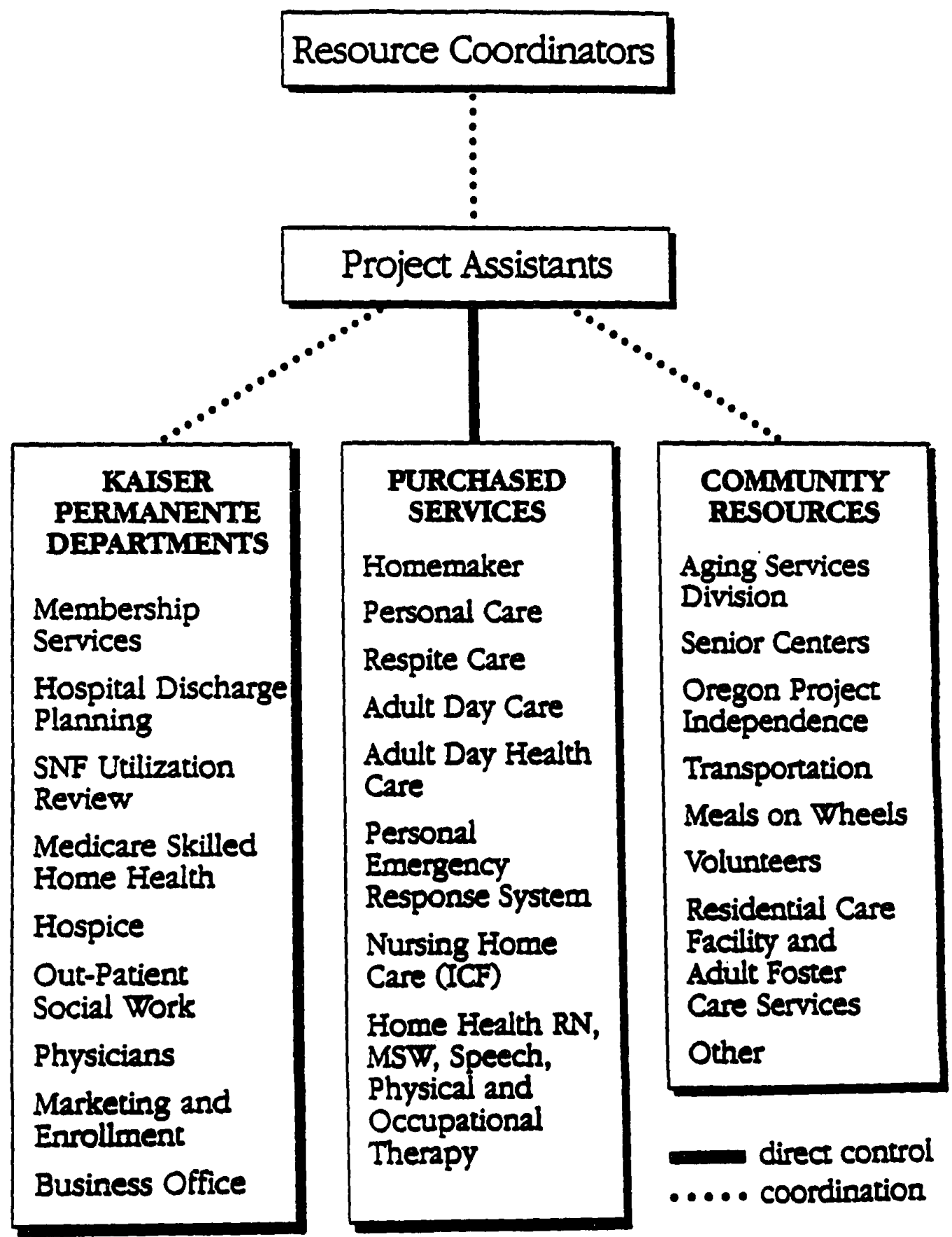




\section{SOCIALTMO BLBLIOGRAPHY}

\section{Articles}

R. Abrahams, J. Capitmen, W. Leuta, and P. Macko, "Variations in Care Planning Practice in the Sodal/FMO. An Exploratory Study," Cerontoloedit, December 1989, pp. $725-736$.

R. Abrahams, S. Dunn, P. Mecko, T. VonStemberg, and D. Zeps, "Integrating Care for the GeriatricPatient" EnMo Prection December 1992, vol. 6, no. 4, pp. 12-19.

R. Abrahams, J. N. Greenberg, L. Gruenbers, and S. J. Lamb, Reliable Ascessment Data in Muld-Site Programu: The Sodal HMO Example, "Eynluntion Reviate April 1983, pp. 153-169.

R. Abraharme and S. J. Lamb, Developing a Reliable Asceanent Technigue in Case-managed, Ceriatric Long-Tem Care Progenms," Ounliby Beview Bulletin June 1988, pp.179-186.

R. Abrahare and W. N. Leutr, The Consolldated Model of Can Manegement and Service Provision to the

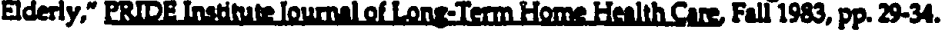

R Abrahars, L Nonnentenmp, S. Dunn, et al., "Cave Marugement in the Socin/Health Maintenance Organiza tion," Genarnitione, Fall 1983, Pp. $39-13$.

5. L Erwin S. Mehte, and B. L. Showe, "Social HMO. and Employers: A Budding Relationship for Retire Health Care," Helth Con! Mnnverment, July-Auguat 1987, pp. 11-20.

J. N. Grunberg and W. N. Leutz, "A Basic Stratwy for Finanding Long-Term Care," Hoaplal Properas February 198, Pp. 16-49, 72 .

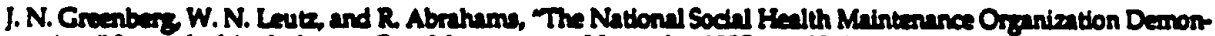

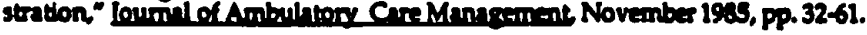

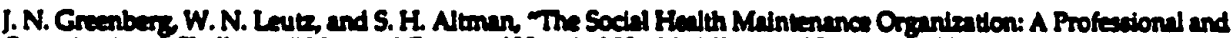

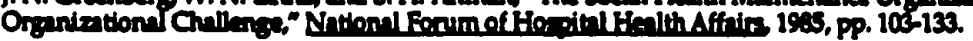

J. N. Guanbers W. N. Leut, S. Evirn et al., S/HMO: The Soda//Health Mainienance Organization and

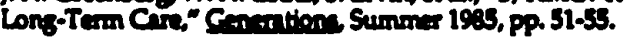

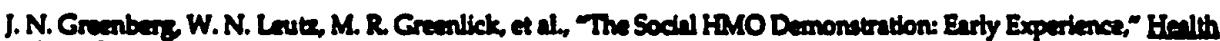
Affitio Summer 1988, pp.66-79.

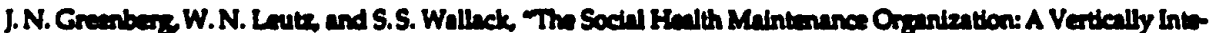

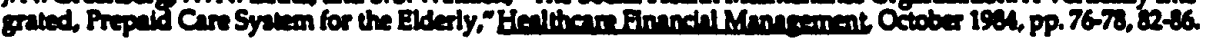

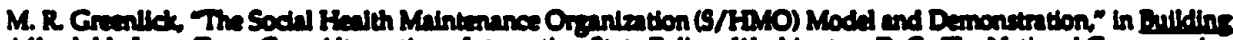

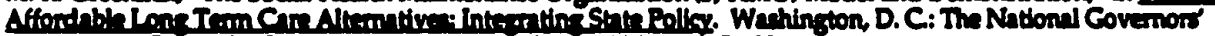

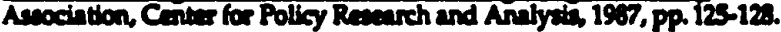

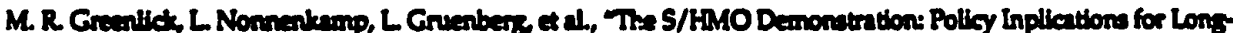

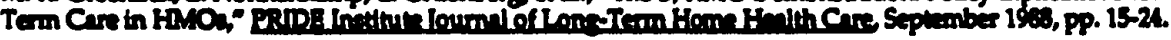

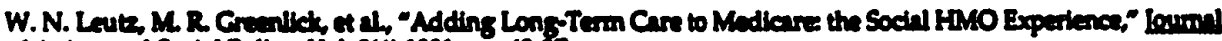
of Atingend Sodil Poli ar Vol 3(1) 1991, pp.698.

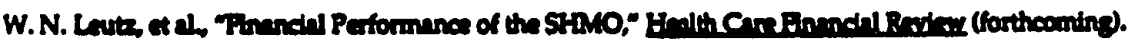

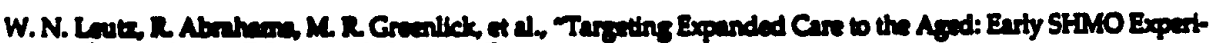

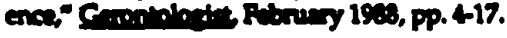

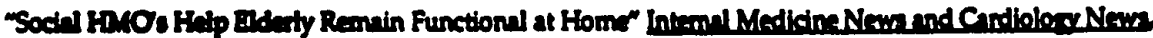
October 15-31 19.9, p.3

Bocks and Iropare

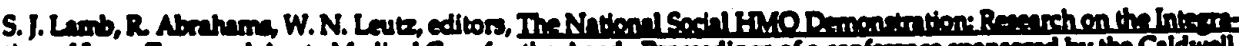

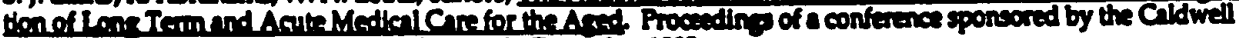
B. Enedosy Foundation, Claveract, New Yort, December 1906.

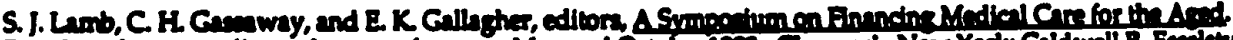
Band on the procendinge of two conferences, May and Oetober is.3. Civanct, New Yorte Caldwell B. Enelstyn Foundation isas

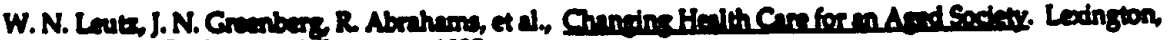
Miryland: D. C. Finth and Compeny, 1965. 
APPENDIX B

FOR CHAPTER III 


\section{APPENDIX FOR CHAPTER THREE}

The following is a complete presentation of the research plan originally proposed for this study in 1987.

\section{SAMPLE SIZE ESTIMATES, CALCULATIONS \& DISCUSSION}

\section{BASIC INFORMATION NEEDED FOR SAMPLE}

\section{Sampling}

The major technical issue affecting study period date selection had to do with the question of whether to gather data based on a stratified random sample, or collect data on all SNF and ICF nursing home residents, who were KP Medicare members in the study period which fit a predetermined set of criteria. If sampling was to be used, there were many issues to consider in determining sample size. These are critical issues to consider in sample planning for future studies similar in research design and composition to this one. These are listed briefly and then some issues are discussed in detail. A random sample was the preferred approach and sample size computations were developed after considerable work was done to estimate variabilty in length of stay. A summary of that work is included in Appendix $x-x$.

First, Research Questions I and II prospectively required different samples. Much more is understood about this as a result of evaluating the data, than prior to its collection.

Under Research Question I, a sample of the research 
population was required for which data on nursing home admissions was needed to compute the probablity of becoming an ICF resident during the study period. Here, the sampling question of how many population members was conditioned by not having good historic data on KP member use of ICFs which could predict the unique number of members likely to become an ICF resident in one year. A list of the total number of ICF residents from KP was available, but proved to be somewhat inaccurate.

Also, at the time this study was operationalized in June, 1989 there was little data outside of KP to clarify this question. State of oregon survey information is based on a one day annual determination of Census. The 1977 National Nursing Home Survey overstated the likely number because ICF was not clearly differentiated from other lower levels and the 1985 NNHS was not published. Longitudinal data discussed number of admissions or days per 1000 but residents per 1000 was vague. The count of members approved for ICF admission was known for $s / H M O$ members, but not Risk or Cost members.

In retrospect a minimum of 20 percent of the $s / H M O$ membership would have produced a total of about $25 \mathrm{~S} / \mathrm{HMO}$ ICF residents in one year, had the distribution of those using an ICF been normally distributed over the s/HMO membership, which it was not. of course stratification by gender and age cohort was required since the greatest proportion come from 
those over age 75 and the ratio of females to males who enter an ICF is at least 4 to 1 . Stratification also would have been needed by new vs converted proportions of the membership, since their use rates differed.

To do a random sample of the research population ment knowing who they all were, i.e., having knowledge of the cumulative enrollment and disenrollement across the 24 months. As discussed below, this investigator's access to that data was restricted to use of year-end files, December only. Measuring attrition between three successive year-end files seemed a plausable solution to estimating the total population numbers, but it did not solve the problem of drawing randomly from all members. Besides, it was shown after nine months into the study, that a substantial number of Medicare members enrolled and terminated between January and Novemeber each year. This was a serious problem of internal validity regarding sample selection bias: those likely to be missing were those who became members and died or left often because of spend-down in a nursing home. Precise identification of the entire membership was needed and that required waiting until needed information could be acquired from the comprehensive active and historic KP Health Plan membership mainframe data base.

Furthermore, Research Question II was going to required a complete eligibilty history on each sampled member in a nursing home to determine days of ICF stay 
attributable to each eligiblity status (Cost, Risk or s/HMO). Using year end-files assured project failure. In retrospect, several issues regarding sample procedures that were not considered in the sample estimate whihc could have confounded the relationship between dependent and independent variables.

First, there was a disproportionately high number of Cost members members who converted to Risk status, who had commenced their lifetime use of nursing homes before the study period and therefore were in nursing homes at the beginning of the study period. There were very few $\mathrm{s} / \mathrm{HMO}$ members in nursing homes before the study period. This difference affected the number of nursing home days for the overall data set of ICF residents. Validity of the proposed sample was doubtful for two related reasons.

First, it did not consider the overall or changing distribution of the three eligibility groups which should have been the basis for stratification.

Second, actual variance in cummulative ICF days of stay was quite different from that used to estimate minimum sample size. National data from tables in the 1977 National Survey on Nursing Homes was used in sample size estimates. Tables in chapter one initially were built from that data in 1988. National Nursing Home Survey data is complicated use and some uncertainty exists about length of stay values in these studies. Regionalized data may not reflect Multnomah 
County experience for many reasons. One reason is the extraordianry difference in definitions of nursing home as including or not including SNF, ICF and other custodial facilities.

In oregon, patients are discharged from ICFs to other alternative care facilities, such as Adult Foster Care and Residential Care facilities, in much greater proportions than in many states, some of which do not have such services developed to the extent of the oregon experience. This fact appears to reduce the length of stay in oregon for ICFs, which the sample estimate originally prepared did not consider. Actual cummulative lengths of stay for cost, Risk and $\mathrm{s} / \mathrm{HMO}$ members were much shorter than national data suggested. Variance of length of stay was great in this study, however, upon removal of the small proportion of very long stay patients, variance was much less.

As noted above, it is recommended by this investigator that sampling of nursing home residents be stratified by estimates about the proportion of short stay residents and long stay residents. And, it is recommended that a decision be made about minimum cell size required for tests of difference between rates. If balanced cell sizes are needed, and if cell sizes need to be a certain minimum size, the sample size must be enlarged to meet those research terms. Even with over 1100 total nursing home residents, some cell sizes in the rate matricies were too small for large sample 
inference.

In order to determine the sample size needed for this study several preliminary computations were done in the absence of true population data and true length of stay information. Specifically, estimates of the population, the number of members in ICFs, the mean LOS and the variance in LOS are needed for estimating sample size for the two key measures of utilization in this study: use rates per 1000 members in each population subset and LOS of ICF users in each population subset. The following tables provide the calculations used to develop estimates for each.

Estimates of Population Subsets

The total number of members enrolled during the period of this study for trial and control groups includes those who were active members at the end of the study period plus those who were members by terminated.

since the time frame of this study is over two years, composition of HMO population is dynamic. Enrollment changes in the Medicare subpopulation were especially significant during the period of time investigated by this study. They must be taken into account if research of the data at issue is to avoid criticism on management of risks to validity.

In 1985 Kaiser Permanente, Northwest Region, elected to become an HMO Medicare Risk contractor, under the newly passed TEFRA legislation. This set into motion, a process of converting Medicare Cost Contract Enrollees to Risk 
enrollment status which as inverted the relationship between Risk and Cost which existed then.

Prior to that time there were approximately 7500 Medicare members enrolled under the experimental capitation model (outlined in RFP HCFA-78-OPPR-22/PHG) named Medicare Plus. "The goal of the demonstration project, named Medicare Plus, was to increase HMo participation in the Medicare program .... that would allow Medicare members of an HMO to have prepaid benefits similar to the HMO's younger members."(Greenlick and Lamb, Final Report, Contract No HCFA 500-78-0078, p10.) These members did not have LTC Benefits.

In March, 1985, a program commenced, known as Medicare Plus II, which extended the concepts of its precedent, Medicare Plus, but added the Extended Care benefits. Thus, concurrently, new members were entering Medicare status under both SHMO and Risk categories, prior Medicare members were shifting from cost to Risk, in time both cost and Risk members were shifting to sHMO.

Because each contract category represented some selective admission practices and because the dynamics of patient care management are conceptually different in cost than Risk, making comparison of observations about effects of the extended care benefit difficult to translate. Also, because members in the cost group no longer represent a good cross section of the HMO population, this study excludes the Cost contract group. 
Additionally, Cost membership includes some members with benefit restrictions; namely Part $A$ only or $B$ only or not purchasing the supplemental benefit, all of which are required to be in the Risk Category.

Tables describing this HMO's Medicare composition during the period of this study follow. 
Table 1

PORTLAND AREA KAISER PERMANENTE MEMBERSHIP

DISTRIBUTION AMONG MEDICARE S/HMO

CONTRACT CATEGORIES DURING THE

SOCIAL/HMO DEMONSTRATION

PROJECT

YEAR/MONTH

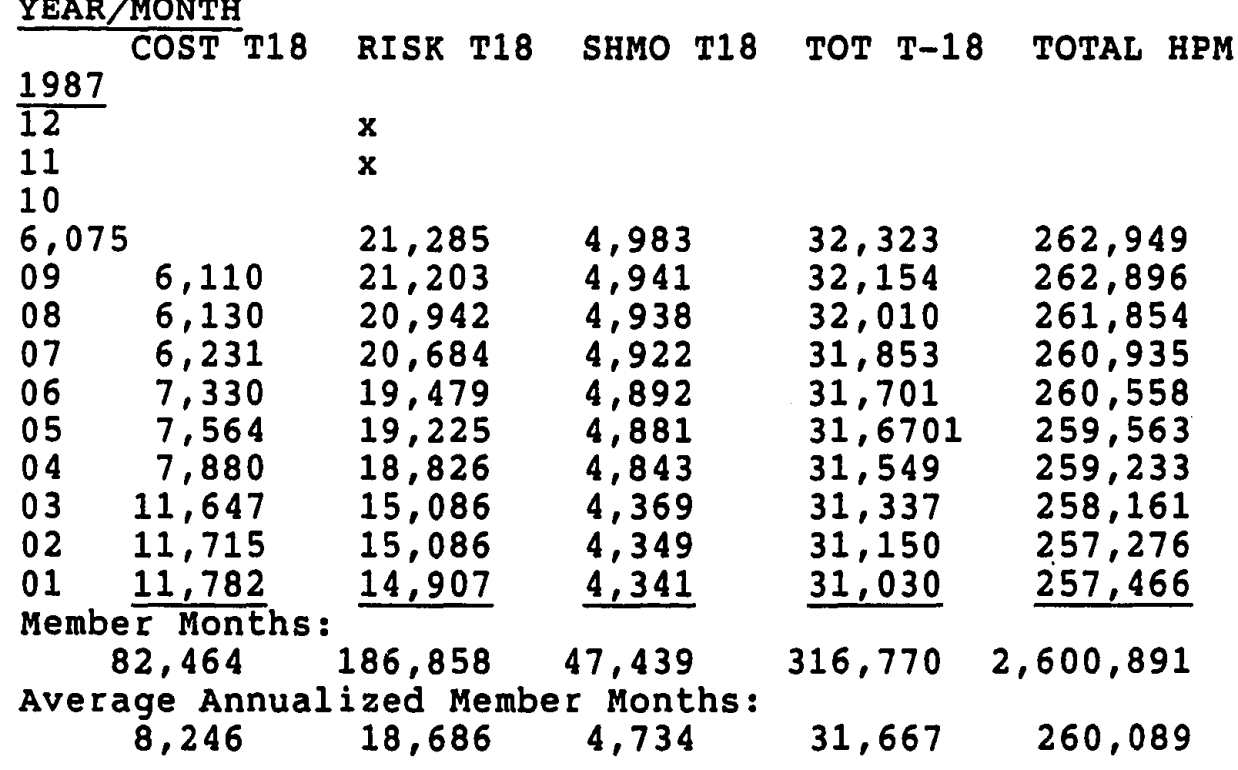


(continuation of Table 1)

\begin{tabular}{|c|c|c|c|c|c|}
\hline $\begin{array}{l}12 \\
11 \\
10 \\
09 \\
08 \\
07 \\
06 \\
05 \\
04 \\
03 \\
02 \\
01\end{array}$ & $\begin{array}{l}12,872 \\
12,892 \\
12,950 \\
13,105 \\
13,340 \\
13,387 \\
14,209 \\
14,486 \\
14,739 \\
15,433 \\
15,422 \\
15,968 \\
\end{array}$ & $\begin{array}{l}13,485 \\
13,238 \\
13,038 \\
12,721 \\
12,622 \\
12,331 \\
11,613 \\
11,283 \\
11,013 \\
10,860 \\
10,371 \\
10,063 \\
\end{array}$ & $\begin{array}{l}4,436 \\
4,272 \\
4,261 \\
4,257 \\
4,228 \\
4,119 \\
4,146 \\
4,144 \\
3,951 \\
3,852 \\
3,315 \\
3,205 \\
\end{array}$ & $\begin{array}{l}30,793 \\
30,402 \\
30,249 \\
30,083 \\
30,190 \\
29,837 \\
29,968 \\
29,913 \\
29,703 \\
30,145 \\
29,288 \\
29,236 \\
\end{array}$ & $\begin{array}{l}253,974 \\
253,345 \\
253,686 \\
252,230 \\
252,773 \\
253,354 \\
253,878 \\
254,245 \\
254,293 \\
254,259 \\
255,588 \\
256,634 \\
\end{array}$ \\
\hline ve & $\begin{array}{c}168,803 \\
\text { age Annual } \\
14,067\end{array}$ & $\begin{array}{l}142,638 \\
\text { Lized Membe } \\
11,865\end{array}$ & $\begin{array}{l}48,286 \\
\text { er Months: } \\
4,024\end{array}$ & $\begin{array}{r}359,807 \\
29,984\end{array}$ & $\begin{array}{r}3,248,259 \\
270,688\end{array}$ \\
\hline $\begin{array}{l}1985 \\
12 \\
11 \\
10 \\
01 \\
08 \\
07 \\
06 \\
05 \\
04 \\
03 \\
02 \\
01\end{array}$ & $\begin{array}{l}17,123 \\
17,123 \\
17,053 \\
17,249 \\
17,323 \\
17,614 \\
17,975 \\
18,431 \\
18,628 \\
18,199 \\
18,713 \\
18,690 \\
\end{array}$ & $\begin{array}{l}9,040 \\
8,846 \\
8,646 \\
8,284 \\
8,058 \\
7,836 \\
7,500 \\
7,446 \\
7,434 \\
7,486 \\
7,502 \\
7,504 \\
\end{array}$ & $\begin{array}{c}3,173 \\
3,096 \\
2,952 \\
2,815 \\
2,655 \\
2,306 \\
1,785 \\
770 \\
461 \\
173 \\
0 \\
0\end{array}$ & $\begin{array}{l}29,336 \\
29,065 \\
28,291 \\
28,393 \\
28,036 \\
27,756 \\
26,260 \\
26,647 \\
26,523 \\
25,858 \\
26,215 \\
26,199 \\
\end{array}$ & $\begin{array}{l}257,474 \\
257,724 \\
256,477 \\
255,690 \\
256,280 \\
256,284 \\
255,872 \\
254,637 \\
254,003 \\
249,009 \\
252,907 \\
252,441 \\
\end{array}$ \\
\hline & $\begin{array}{l}\text { Annu } \\
.847\end{array}$ & $\begin{array}{c}88,849 \\
\text { ized Mem } \\
7,404\end{array}$ & $\begin{array}{l}19,826 \\
\text { er Months } \\
1,652\end{array}$ & 301,288 & $3,067,637$ \\
\hline
\end{tabular}

Data in this table contrasts SHMO with all Medicare members enrolled in Kaiser Permanente (including SHMO). To develop a model of comparison, in the absence prior data, occupancy/1000 over-all for the population over age 60 in the state of Oregon is assumed to equal that of Kaiser Members. This is developed for use in sample size estimates 
given in the following table.

Table 3

RESOURCE DATA FOR ESTIMATING VARIANCE BETWEEN SHMO AND OTHER MEDICARE REGARDING EXPECTED VARIANCE

IN NURSING HOME OCCUPANTS PER $1000 \mathrm{KP}$

MEDICARE ENROLLMENT POPULATION

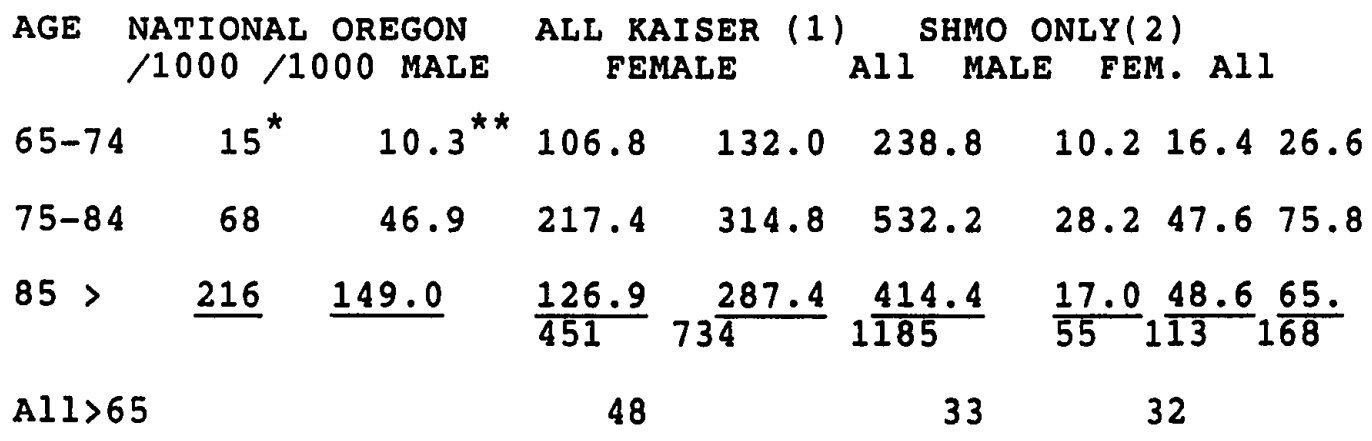

* Source of per 1000 use rate information are: (1)-National;

J. Ouslander, J. Beck; "Defining The Health Problems of The Elderly," Annual Review Public Health, 1982, 3:55-83, p.74.

(2) [data for 1980]. "Data Watch," Health Affairs, Spring 1987, p.178.

** Per 1000 data source-state of oregon

Executive Summary, Oregon Systems Development Project For Long Term Care, State of Oregon, March 1981, p. 4. Idata for 1980]

(1) For this estimate, use rate $/ 1000$ by all Medicare Kaiser Permanente Members age 65> is assumed to equal the State of Oregon. Age specific data the for state of oregon is derived by a constant multiplier of $.69[33 / 48=.69] \times$ the national use rate by 10 year age cohorts].

(2) For the estimate of sHMo a multiplier of $.957 \times$ the derived use rates for age cohorts. [31.6/33.0=.957 composite use rate $/ 1000$ population.] 31.6 is the true use rate per 1000 YTD 1987.

Linear application of these multipliers unquestionably compound other errors but, in the absence of any other data, and for sample estimate purposes only, these determinations are use to guide the sample size calculations. Table 4

\section{RESOURCE DATA FOR ESTIMATING VARIANCE BETWEEN MEDICARE AGE COHORTS}

Age Cohorts Male $:$ Female $\&$ Total $q$ 


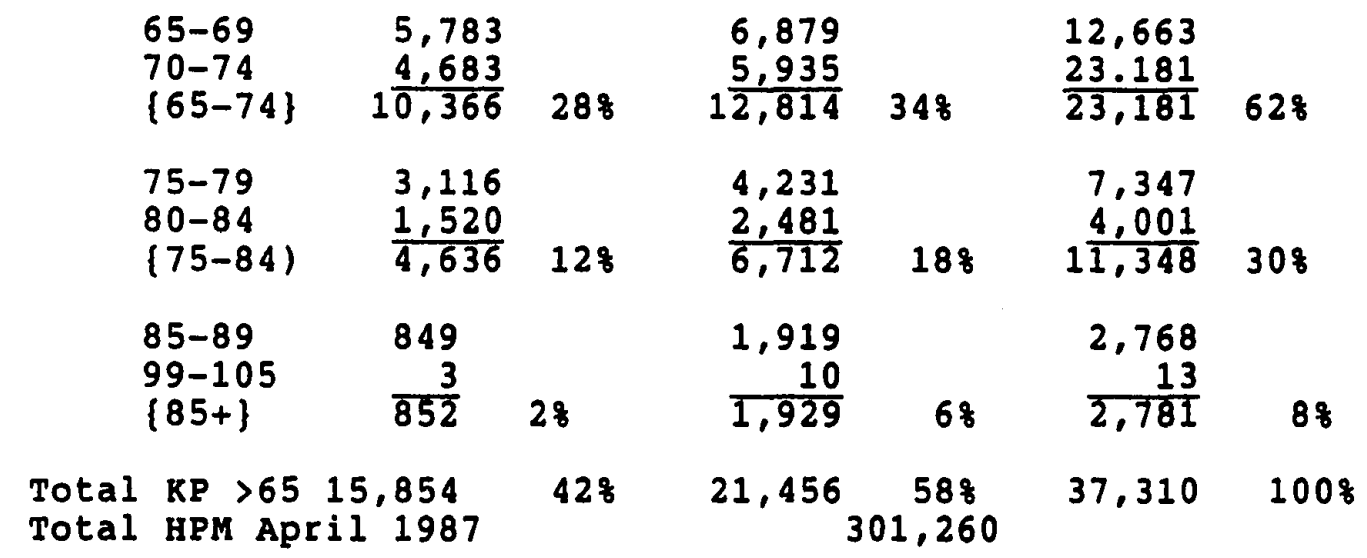

$(q=q$ Medicare $)$

April 1987 membership data is assumed to represent the YTD membership of all members age 65 and over including sHMO

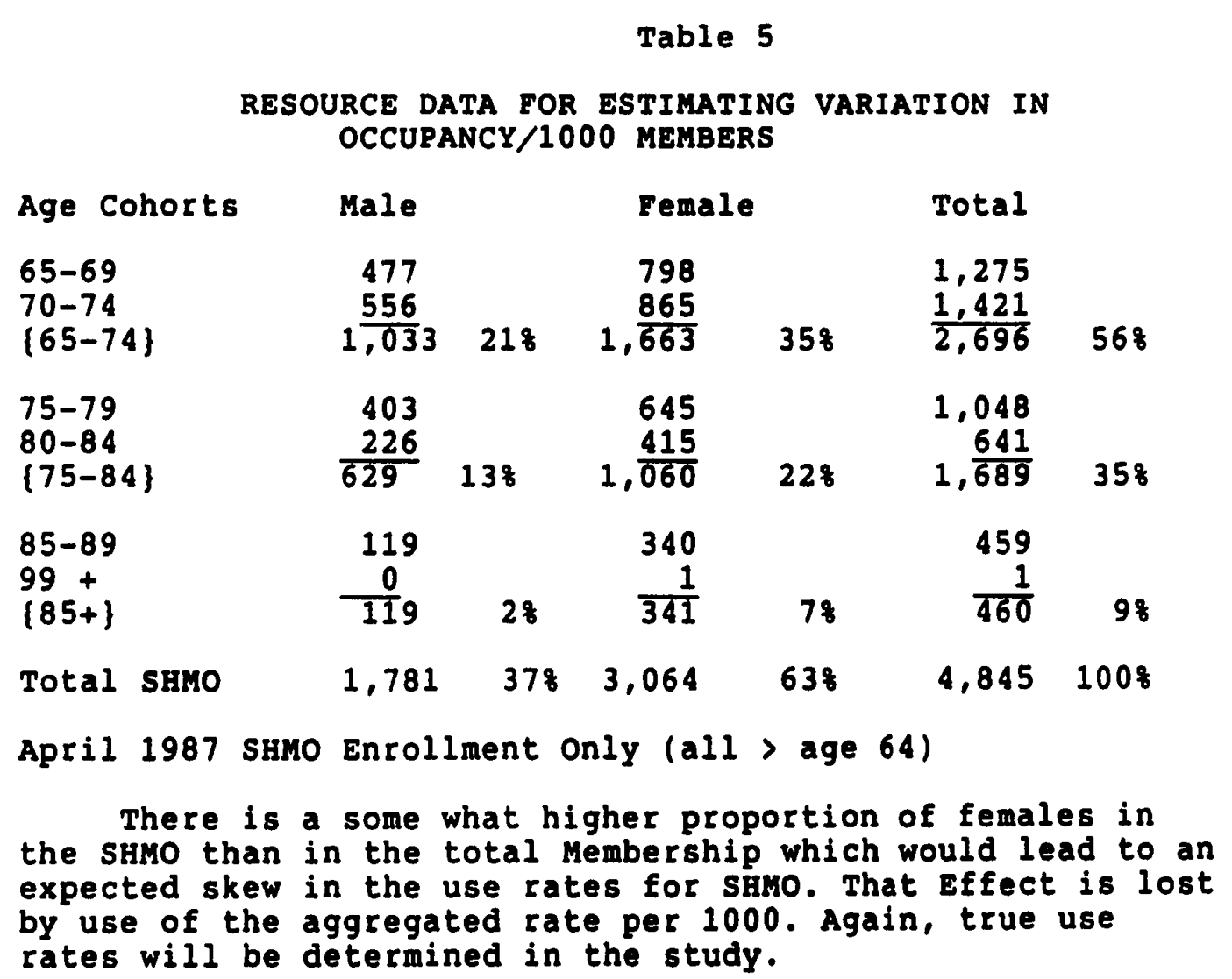


1987 YTD SNF Risk ${ }^{*}$ days used $=\underline{1785}$

Total Nursing Home days SHMO $=9562$ (This is an understatement by the number of ICF days used by sHMO emrollees who passed through the ceiling of benefit days. That number appears to be:

$9,562 / 301,742=31.6$ people $/ 1000$ total HPM in ICF IT IS NOTED THAT IN 198637 DAYS/1000 OCCURRED

It is appropriate to estimate the number who terminated and reenrolled to avoid double counting. This number is quite small; in 1986 a manual, limited effort was made to isolate reasons for termination indicating a regional 12 month total of 160 terminations who reenrolled. This number is too small to merit consideration for computing the estimated total. Kaiser Foundation Health Plan did not develop a method for determining the count of active and terminated members until July 1986 The following data relys on such information. Medicare Plus (control group) Estimates 
Reported Total Northwest Region Medicare Plus Membership:

RATIO OF ACTIVE TO TERMINATED

IN TOTAL RISK MEMBERSHIP

Date Active Active + Difference Ratio

$\begin{array}{lllll}7 / 85 & 15941 & 18500 & 2549 & 1.1605\end{array}$

$\begin{array}{lllll}6 / 87 & 24238 & 30190 & 5952 & 1.2456\end{array}$

$\begin{array}{lllll}6 / 88 & 27855 & 35474 & 7619 & 1.2735\end{array}$

Estimated Multnomah County Medicare Plus Membership is:

Date Active Term Ratio Region Risk HPM

$7 / 855578 \times$ (a) $1.1605 \quad 6473$

$6 / 878010 \times \quad$ (b) $1.2456 \quad 9977$

$6 / 888688 \times \quad$ (c) $1.2735 \quad 11064$

The above table is the result of an effort to estimate the true number of members in the Risk control group. This was necessitated by lack of access to the KP membership files at the time such information was needed to prepare estimated sample size. The above table was need to project the $\mathrm{N}$ (denominator) for the sample estimate formula. As it was determined in the first quarter of 1992, from data regarding the true research population first obtained in October, 1992, the cummulative number of Risk Medicare members which met the study criteria were 11,252 . The mean of the above three annualized estimates would have seriously underestimated the size of the Risk population. The above data underscores the problem of using one month-end file out of 12 month end files per year, to estimate research 
population data. Four months of analysis of month end files demonstrated the probable proportion of missing Medicare members was unacceptably high to use for this study.

Table 6

ESTIMATED RISK MEDICARE POPULATION IN MULTNOMAH COUNTY ICFS ON AVERAGE DURING EACH OF YEARS

OF THE TWO YEAR STUDY PERIOD

$7 / 86-6 / 87$

$\begin{array}{lccc}\text { Age } & \text { Female } & \text { Male } & \text { Total } \\ & & & \\ 65-74 & 24 & 17 & 41 \\ 75-84 & 57 & 33 & 90 \\ 85+ & 102 & 16 & 118 \\ \text { Total } & 183 & 66 & 249\end{array}$

$\underline{7 / 87-6 / 88}$

$\begin{array}{llll}65-74 & 35 & 23 & 58 \\ 75-84 & 69 & 42 & 111 \\ 85+ & 123 & 19 & 142 \\ \text { Total } & 227 & 84 & 311\end{array}$

Note: Actual total number of Risk ICF residents during study period was 562 which is about the same as combined number from above two years. [d] MULTONOMAH COUNTY TOTAL 1987 POPULATION (CENTER FOR POPULATION RESEARCH - PSU) DIVIDED INTO THE NURSING HOME (NH) POPULATION (SNF+ICE) AS DETERMINED BY SAMPLE FOR SEPTEMBER 30,1986 , AGE 65+ BY THE OFFICE OF HEALTH POLICY, STATE OF OREGON = NH USE RATE TIMES THE RATIO $(5 / 6=k)$ OF SNE TO ICF IN NH FOR RAISER PERMANENTE.

$A G E=65-74:$

FEMALE

(d1)

(k) $303 / 24706=.0102$

(d2)

$A G E=75-84:$

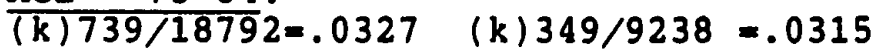

(d3)

$A G E=85+$ (e3)

TOTAL

(E1)

(k) $202 / 18711=.0090$ (k) $505 / 43417=.0097$

(e2)

(£2)

(k) $1088 / 28030=.0323$

(f3)

$\begin{array}{llll}\text { (k) } 1298 / 6264=.1726 & \text { (k) } 252 / 3079=.0681 & \text { (k) } 1550 / 9343=.1382\end{array}$ 
(d4)

Age 65-105

(k)2340/49726=.0393 (k) $803 / 31028=.0216 \quad(k) 3143 / 80790=.0324$

[e] ESTIMATED AVERAGE NUMBER OF MULTNOMAH COUNTY MEDICARE RISK HPM IN ICFS AT THE BEGINNING, MIDPOINT AND END OF THE STUDY PERIOD BASED ON THE USE RATE OF TOTAL MULTNOMAH COUNTY POPULATION:

$(a-c)$

(HPMxActive+Terminated ratio = True HPMxMultC Use Rate=ICF\#)

$7 / 86$ AGE HPM FEMALE $=T$ MALE $=T$ TOTAL $=T$

65-74

$1717 \times a=1993 \times d 1=12 \quad 1265 \times b=1468 \times$ e $1=133461 \times f 1=25$

$\begin{array}{lllllll}75-84 & 1263 & 48 & 735 & 27 & 2319 & 75\end{array}$

$\begin{array}{lllllll}85+ & 395 & 79 & 172 & 14 & 658 & 93\end{array}$

$\begin{array}{lllllll}\text { Total } & 3375 & 139 & 2203 & 54 & 5578 & 193\end{array}$

$6 / 87$

$\frac{6787}{65-74}$

2633

$33 \quad 1959$

$22 \quad 5720$

55

75-84

1653

$67 \quad 1024$

$\begin{array}{lll}40 & 3334 & 107\end{array}$

85+

583

$125 \quad 214$

$18 \quad 993$

143

Total

4824

$225 \quad 3197$

$80 \quad 8021$

305

$6 / 88$

65-74

2896

$38 \quad 1959$

$24 \quad 5720$

62

75-84

1716

$71 \quad 1115$

$45 \quad 3605$

116

85+

551

$121 \quad 244$

$21 \quad 1012$

142

Total

5163

$227 \quad 3525$

$84 \quad 8688$

311

The average for the three time periods are used to produce table [c]. 11064 
[f] COMPUTATIONS FOR THE ESTIMATE OF THE TRUE AVERAGE NUMBER OF $K-P$ MEDICARE MEMBERS IN ICFS IN EACH OF THE TWO YEARS STUDIED. PROBABILITY OF LOS IS DERIVED FROM REVALIN \& WEINER (MIENERS) MULTIPLIED TIMES DATA IN TABLE [C] FOR EACH YEAR. SEX IS COMBINED BECAUSE PROPABILITY DATA IS COMBINED. Estimates are reduced by factors of (2)\& (3) representing the average number of ICF readmissions/year (SHM=3) not accounted by Meiner's model.* $\begin{array}{lllll}\text { AGE MONTHS } & 1986-87 & 1987-88 & 1986-87 & 1987-88\end{array}$

(AVERAGE) HPM PROP. HPM PROP. EST \# ICF EST \# ICF $\frac{65-74}{0.5}$

$\begin{array}{lrrrrrrr}\frac{65}{0.5} & .274 & 11.2 & 24 \mathrm{mo} & 15.9 & 24 \mathrm{mo} & 268 & 381 \\ 1.5 & .109 & 4.5 & 8 \mathrm{mo} & 6.3 & 8 \mathrm{mo} & 36 & 50 \\ 2.5 & .079 & 3.2 & 5 \mathrm{mo} & 6.3 & 5 \mathrm{mo} & 16 & 23 \\ 4.5 & .105 & 4.3 & 3 \mathrm{mo} & 6.1 & 3 \mathrm{mo} & 12 & 18 \\ 9.0 & .098 & 4.0 & 1.3 \mathrm{mo} & 5.7 & 1.3 \mathrm{mo} & 5 & 7 \\ 18.0 & .106 & 4.3 & 6.1 & 4 & 6 & & \\ 30.0 & .067 & 2.7 & 3.9 & 3 & 4 & & \\ 42.0 & .027 & 1.1 & 1.6 & 1 & 2 & & \\ 54.0 & .027 & 1.1 & 1.6 & 1 & 2 & & \\ 66.0 & .021 & 0.9 & 1.2 & 1 & 1 & & \\ 73.0 & .084 & \frac{3.4}{40} & \frac{4.9}{57.9} & 3 \frac{3}{50} & 4 \frac{5}{99} & & \end{array}$

$*[1986-7 @ .50=175, e .33=117][1987-8 @ .50=250, e .33=166]$ $74-85$

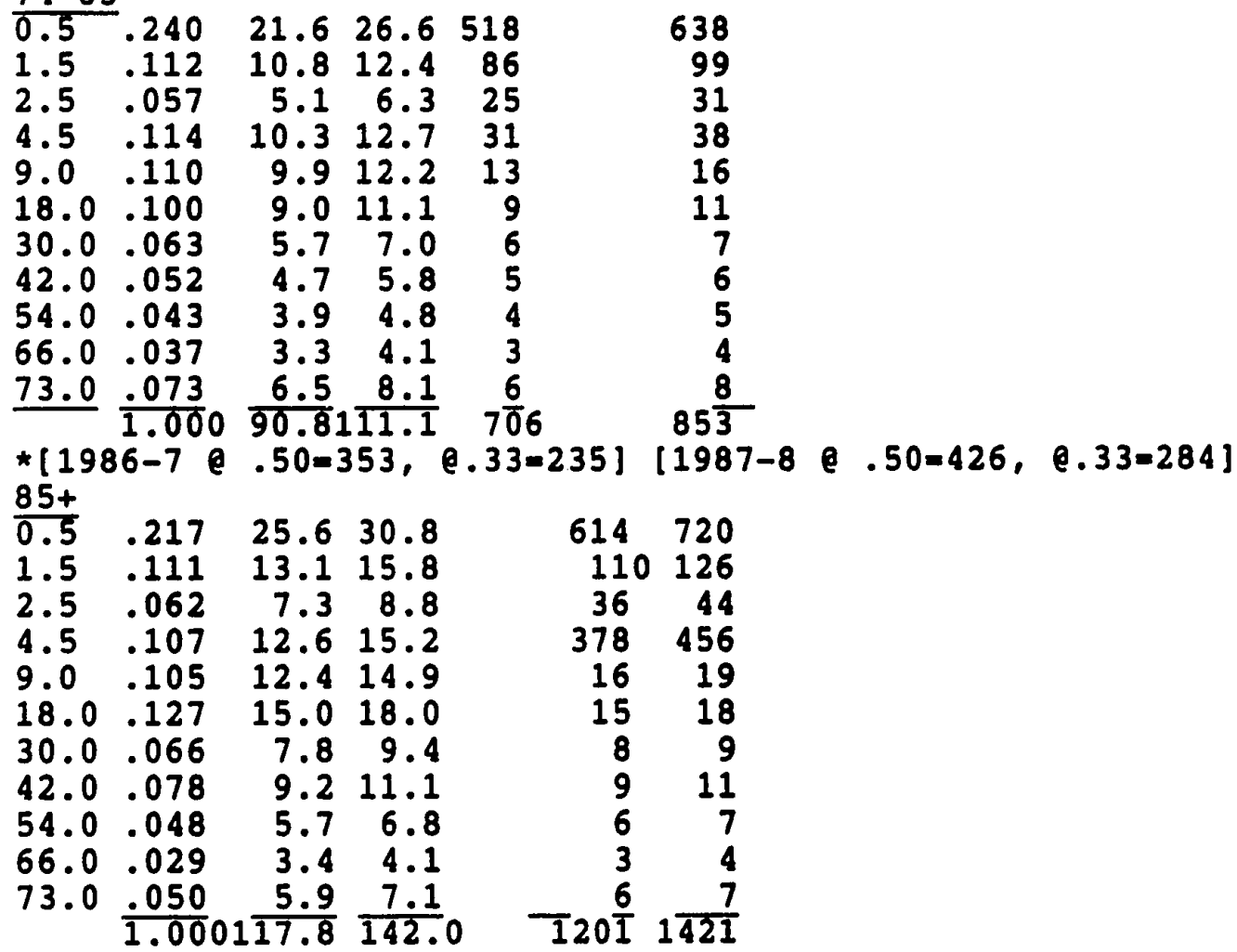


Copied Fron Earing for the Jisabled Elderly, Wino illi Paj? by h. hivion a j. Niener, Brookings institute, 1000

262 Caring for the Disabled Elderly

TA B LE A-s. Probability of Numing Home Length of Stay, by Age ar Entry and Mortaliy Status at Discharge

Perrent

\begin{tabular}{|c|c|c|c|c|c|c|c|}
\hline \multirow{3}{*}{$\begin{array}{l}\text { Length of } 2 x y \\
\text { Imoaths! }\end{array}$} & \multicolumn{6}{|c|}{ Apt at Enten: } & \\
\hline & \multicolumn{2}{|c|}{$65-74$} & \multicolumn{2}{|c|}{$75-24$} & \multicolumn{2}{|c|}{85 and over } & \\
\hline & Live & Dend & Live & Dend & Live & Dead & \\
\hline $\begin{array}{l}\text { Under } 1 \\
1-2 \\
2-3 \\
3-6 \\
6-12\end{array}$ & $\begin{array}{r}17.72 \\
5.68 \\
4.80 \\
5.88 \\
3.86\end{array}$ & $\begin{array}{l}9.68 \\
5.27 \\
3.13 \\
4.63 \\
5.97\end{array}$ & $\begin{array}{r}14.29 \\
6.50 \\
2.97 \\
5.07 \\
4.70\end{array}$ & 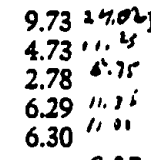 & $\begin{array}{r}11.66 \\
4.79 \\
2.41 \\
3.99 \\
3.12\end{array}$ & $\begin{array}{r}10.03 \\
6.32 \\
3.76 \\
6.74 \\
7.36\end{array}$ & $\begin{array}{l}21 \cdot i \\
-6 \\
12:-\end{array}$ \\
\hline $\begin{array}{l}12-24 \\
24-36 \\
36-48 \\
48-60 \\
60-72\end{array}$ & $\begin{array}{l}4.80 \\
2.19 \\
0.99 \\
1.04 \\
0.84\end{array}$ & $\begin{array}{l}5.84 \\
4.56 \\
1.72 \\
1.68 \\
1.29\end{array}$ & $\begin{array}{l}3.15 \\
1.43 \\
1.19 \\
1.09 \\
0.64\end{array}$ & 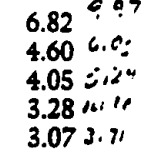 & $\begin{array}{l}2.97 \\
1.26 \\
1.17 \\
0.66 \\
0.18\end{array}$ & $\begin{array}{l}9.70 \\
5.29 \\
6.68 \\
4.15 \\
2.73\end{array}$ & 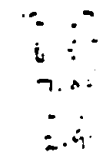 \\
\hline .93 and over & 1.91 & 6.52 & 0.74 & 6.577 .31 & 0.43 & 4.59 & $\therefore \cdots$ \\
\hline TOTAL & 49.70 & 50.30 & 41.77 & 58.23 & 32.65 & 67.35 & \\
\hline
\end{tabular}

Both the length of stay assigned by the model to nursing home entranes and the mortality stans of residents at time of discharge are based on estimates developed by Meiners and Trapnell from the 1977 National Nursing Home Survey and vary by an entrant's age at admission (able A-s). The Meiners and Trapnell lengths-of-stay probabilinies aggregated multiple admissions for parients readmitted to a nursing home soon after being discharged and were further modified to reflect increasing numbers of nursing home residents by age from 1969 to 1977.

All nursing home lengths of stay are assigned the midpoint of the extimate. Nursing home assigned lengths of stay are based on age and remain constant over the simulation period. Previous_nursing home residents reenter nursing homes at the same rate as people who have to never been instirutionalized.

\section{Home Care Use}

Noninstirutional services in the model include home health services, chore and homemaker services, personal care, and meal preparation services. Using data from the 1982 National Long-Term Care Survey of noninstirutionalized ehronically disabled elderly, the model places disabled people still living at home into one of four groups: those 
MEAN, STANDARD DEVIATION \& VARIANCE OF ICF LOS, BASED ON MEINER'S PROBABILITY MODEL FOR DISTRIBUTION OF LOS BY AGE FOR ICF ADMISSIONS

(RAVLIN AND WEINER PG 262) Male and female are combined

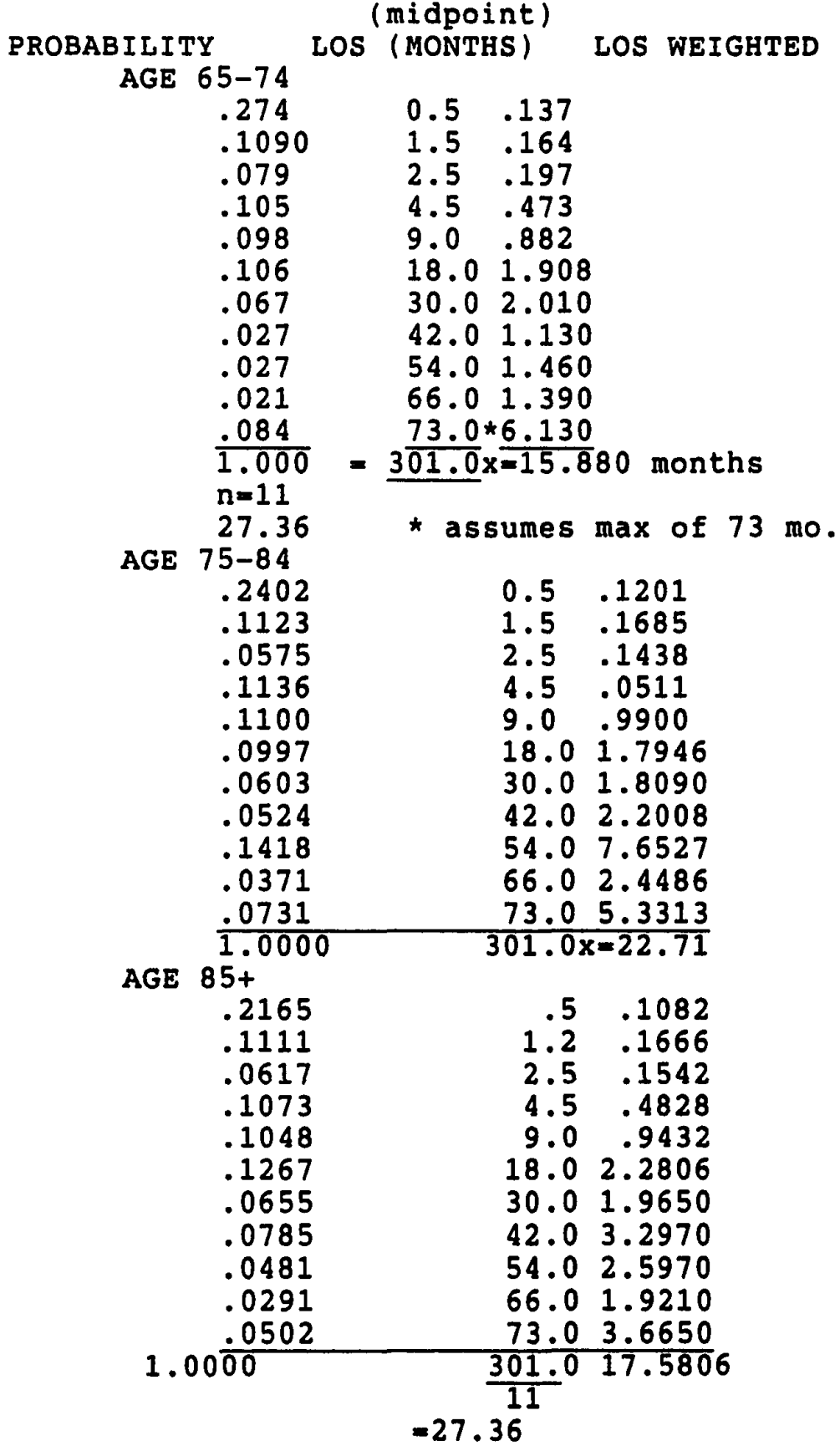


COMPUTIING SAMPLE SIZE FOR ESTIMATING THE AVERAGE DIFFERENCE IN RESPONSE VARIABLES BETWEEN TWO FACTOR LEVELS WHEN THE SAMPLE IS NOT REPEATED. SAMPLE SIZE ESTIMATES: (1). Determining Sample size for ICF Users

statistical tests of significance (measure of the probability of incorrectly rejecting the null hypothesis in favor of the alternative hypothesis) require that the researcher select a level of power (the probability of being able to properly reject the null hypothesis when it is false) appropriate to the study. Since this study is exploratory in nature, the criteria for avoiding error in hypothesis rejection is relaxed. A power of $80 \%$ is used. Smaller sample sizes of nursing home users are thereby permitted. Ability to detect a six month difference in mean LOS between Risk and $s / H M O$ was a criteria initially used fro the computation. Detection of smaller differences required a larger sample size for Risk and s/HMO groups

Based on the assumptions that distribution of ICF residents within respective control and trial groups is asymentrical (not normally distributed under a two tailed normal curve), and increments of measurement are not measured in continuous intervals, the sample size suggested for the data sets concerned with ICF utilization is shown: (To be used for determining the probability of becoming and ICF resident during the study period.

Computations about variability were performed based on the Ravlin and weiner model, shown in the above table. These computations were done by age cohorts. The computed 
variability in mean LOS is used to estimate sample size required in each cell.

At .90 power to detect a difference of 180 days between Mean LOS, and an alpha level of .05, for age cohort 65-74, the sample size eatimate for Risk ICF residents was 313;

At .80 power to detect a difference of 180 days between Mean LOS, and an alpha level of .05, for age cohort 65-74, the sample size estimate for Risk ICF residents was 175.6;

At .80 power to detect a difference of 180 days between Mean LOS, and an alpha level of .10, for age cohort 65-74, the sample size estimate for Risk ICF residents was 138;

When the above sample size estimates for age cohort 65-74 were compared to the estimated number of KP Risk Medicare ICF residents in Multnomah County, (see tables [c] and [e]; in [e] even taken collectively for the three years, $\{75+65+72=212\}$ it appeared that at .80 power and an alpha level of .10 that a 65 percent sample of residents was needed; at an alpha of .05 and 83 percent sample was needed. At .90 power and alpha of .05 only $2 / 3$ the required resdients were available. The overall sample size estimate was 2864 under one calculation. This was even larger than the high estimate 
done using the Ravlin and Weiner based estimate [table f] $($ high $=2438$, low $=1625)$ was so much larger than table [e] that they were of questionable use. A decision was made to collect data on every one, since the estimates seemed about the same size as the sample needed.

As it turned out, there was a total of 395 Risk Medicare ICF residents in the overall data set. That was about what was required for the total Risk sample. There were 820 unique persons in ICFs during the study period. That was about one-half of the low Ravlin and Weiner estimate. The total SNF+ICF unique persons was 1160, or about $2 / 3$ of the low $R$ \& estimate. It was close to the estimate in table [e]. It is worth noting that benchmark studies in this area by Liu and Manton, used Samples of over 6500 nursing home residents.

In retrospect, the variance selected for use in estimating sample size, and factor (difference in days in an ICF between groups) of detectability between means ( 6 months) were both inappropriate. While it is true that potential variance was the maximum of the study period days (730), the problem would have been resolved by focusing on the shorter-stay ICF residents. This is recommended for future studies. The ability to detect a difference of 6 months between means was far to large. The problem is that to detect the small amount of difference in days used between $\mathrm{Risk}$ and $\mathrm{S} / \mathrm{HMO}$, that was actually observed, requires 
334

a sample bigger than that estimated. Determining sample size based on the ability to detect differences in mean Los.

$2 \operatorname{THE} 9010.22$ Tiv e

$A$

$f$

- 274

.109

.079

-... . .

.106

...... . 067

.027

.027

. of y $.105^{\circ}$

- 021

$B$

$\because \because \operatorname{med} \beta$

$\therefore \cos (x)$

$.5^{\circ}=0.1370$

$1,5^{-}$

$2.5^{-}$

4,5

9.0

18.0

30.0

42.0

54.0

66.0

... . . . ..

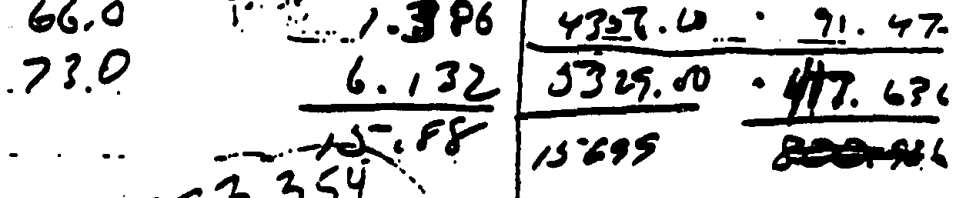

Vurimice

$\sigma^{2}=$

$3=3,354:$

$.80-2.9$

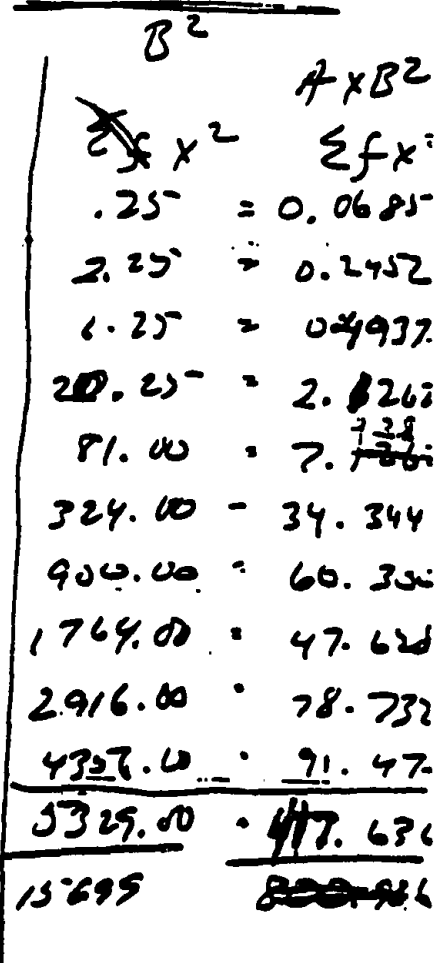

sample dive.

$\sum_{f} x^{2}-\left(\varepsilon_{f x}\right)^{2}$

$n=\frac{2 \sigma^{2}\left(z_{f}+z_{p}\right)^{n}}{\rho^{2}}$

$800.796-(15-58)^{2}$

$n=2(548.61)(10.2)^{3}$

$8002786-252,17$

$\therefore \quad 5.48 .64$

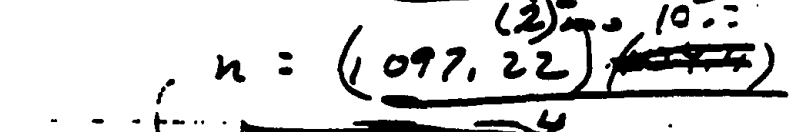

6

To ti $n=2854$

-...

Si],

$s^{2}=2 i 54 \% \therefore \quad(10 ; 0)$ 
335

MPUTI'NG SAMPLE SIZE FOR ESTIMATING THE AVERAGE DIFFERENCE IN RESPONSE RIABLES BETWEEN TWO FACTOR LEVELS WEEN THE SAMPLE IS NOT REPEATED

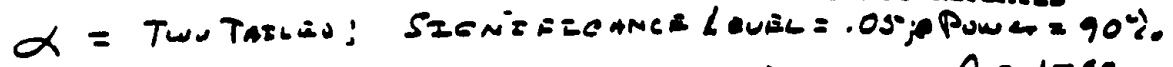

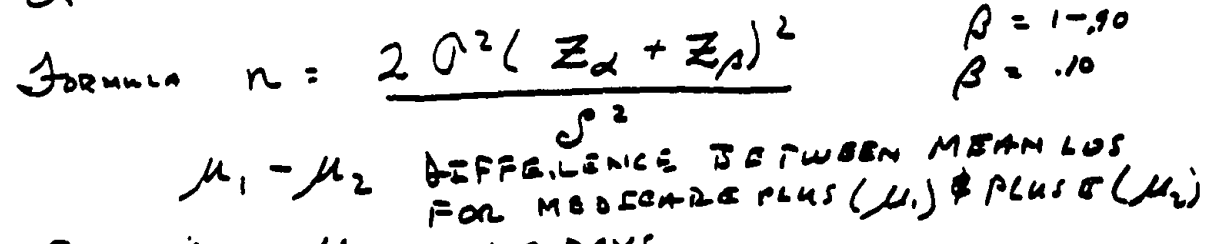

อง2.

$\left(z_{\alpha}+z_{\mu}\right)^{2}=10.2$

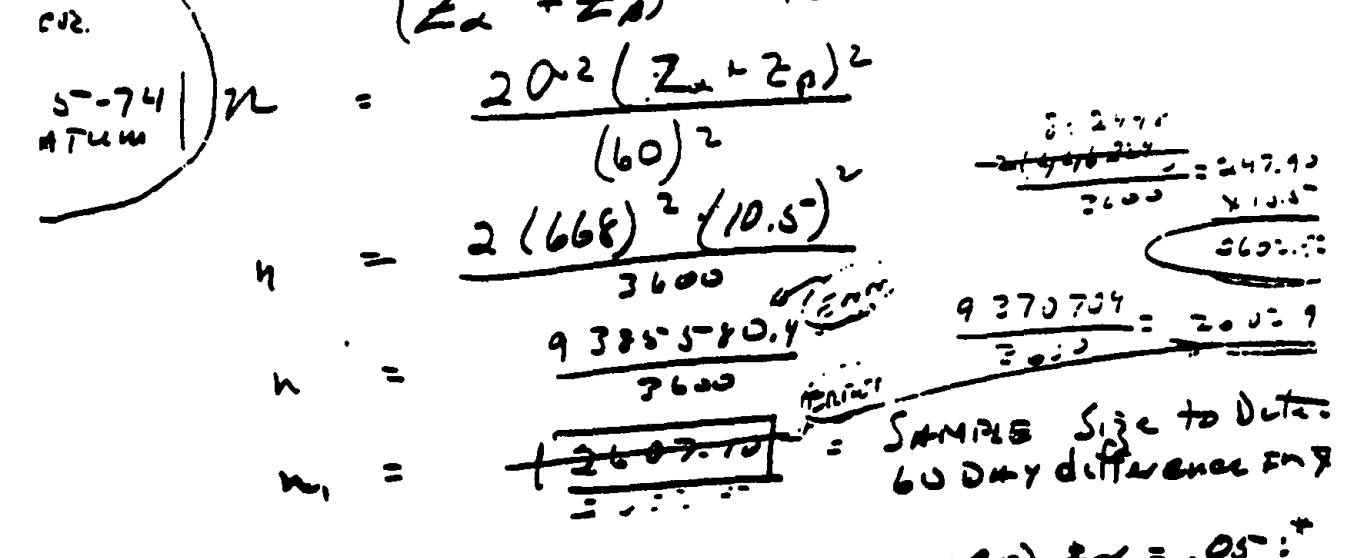

$\operatorname{lnm}=66 r$
$\min i 7=40^{\circ}$
ton $=-64$
$=\quad 5-84=600$

sumpter :

wen a carina + bu says

$$
\begin{aligned}
& \text { J } 1: \beta=20(\beta \text { ow } n=.80)+\alpha=.05: *
\end{aligned}
$$

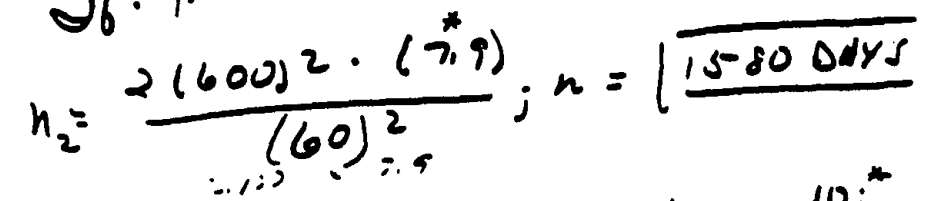

$$
\text { gl: } \beta=20(\text { power }=.80) \& \alpha=.100^{*}
$$


Formation of utilization comparisons by age stratification will require some weighting adjustment due to the disproportionate distribution. While all Medicare enrollees in risk and sHMO categories will serve as the sample, analysis will be done by use of sampling fractions. If distributions are also disproportionate between age distribution and sex further weighting fractions will be developed. Information regarding age and sex distributions are being developed and were not available for this document.

Time from enrollment status to time of ICF admission: It will also be necessary to determine the proper method of aggregating admissions and therefore this part of the study must consider time to readmission(s) and the times to discharge. Thus, total as well as increments of care must be considered.

The beginning point of this study may need to be staggered so that the true start of Risk Group is that when Cost to risk began.

Some testing of the need to delete all prior risk group enrollees will be undertaken. That is, of the 7500 Medicare Plus enrollees in risk already, is there a marked difference in the variables describing them in comparison to the sHMo group?

The objective is to create comparable states of stability and change. A measure of this stability will be 
to compare expense variances within the first two years with those of the third year to see if there is any trend or change suggest regarding the effectiveness of what sHMO is doing to attain cost effective LTC services.

In considering that hypothesis analysis concerned with time of enrollment eligibility to time of ICF admission, a further threat to validity exists. To help ensure comparability of the two groups, it is necessary to withdraw those members in the Risk category who were in a nursing home (or other facility) at the beginning of this study. This is necessary because none of the sHMo enrollees could be in a nursing home (or other facility) at the time of their application.

The concern is that they could constitute a large number of days which would skew the results, indefensible confounding the findings. Since the objective is to understand effects of the sHmo program (creating networks and alternative support groups to be used in lieu of ICF admission) on ICF use in contrast to no program this population adjustment is needed. It is feasible that some members will have been in ICF facilities from prior to the beginning of sHMO to present.

However, that group of individuals will be examined independently by use of a survival analysis. A survival curve will be developed for this subset which will also consider their admit time elapsed to discharge. Without this 
separation of ICF residents at the start of the study it may lead to a serious overstatement of what the sHMo program is doing to reduce utilization.

True data on ICF use rates are not available, currently. Obtaining it is one of the required functions of this study. It will be accomplished by going to each facility with member names and obtaining correct information.

Data, regarding the above characteristics of hospitalized members in each contract category, will be derived from the automated discharge abstract system for all hospitalized patients. All of the variables noted above, in the survey questionnaire, are available from this inpatient information system data base.

Since some Medicare members included in the study will have been hospitalized elsewhere, a search of all referrals during the study period will be done. Likewise, a review of all sHMo new enrollment forms will be undertaken. Where information has not been entered into the Kaiser Permanente Inpatient Information System from Discharge Summaries sent by those non- Kaiser Permanente hospitals, that will be undertaken. In addition, the Part $A$ intermediary may be asked for such information if needed. Consent will be obtained as needed.

Likewise, analysis of utilization information for all Medicare enrollees during the study period, who were 
admitted to SNFs is feasible by use of referral billing data for all sHMO and Risk members.

\section{Expense Data}

The time and effort required to collect expense information greatly exceeds that of utilization data. This is due to the protocol of providing many services via parties not employed by the ICF site. There will be multiple vendors and providers for each person confined in the ICF unit. For this reason a sample of the population will be drawn to accomplish an analysis of expenses. Otherwise the research expense, and logistics of data collection, become unmanageable and exceed any reasonable funding request.

Therefore, a stratified random sample of all members admitted to ICFs will be developed from admission and discharge lists by facility. These lists have been created and entered into a software program at Raiser Permanente but have not yet been tested and recompiled into software program files. They require validation by on-site verification at each ICF location. Utilization data will be confirmed in the process.

The size of the sample in each contract category, as well as that required for cells within in these category blocks is described below.

a. Variables which comprise sample cells include the following. 
(1) Age - ten year increments will be used. (65-74, $75-84,85-+$.

(2) Sex.

b. Weighting of the numbers in each will be needed because of disproportionate distributions under Age and sex, and because of cross-over expenses resulting when a member transfers from one of the categories to another.

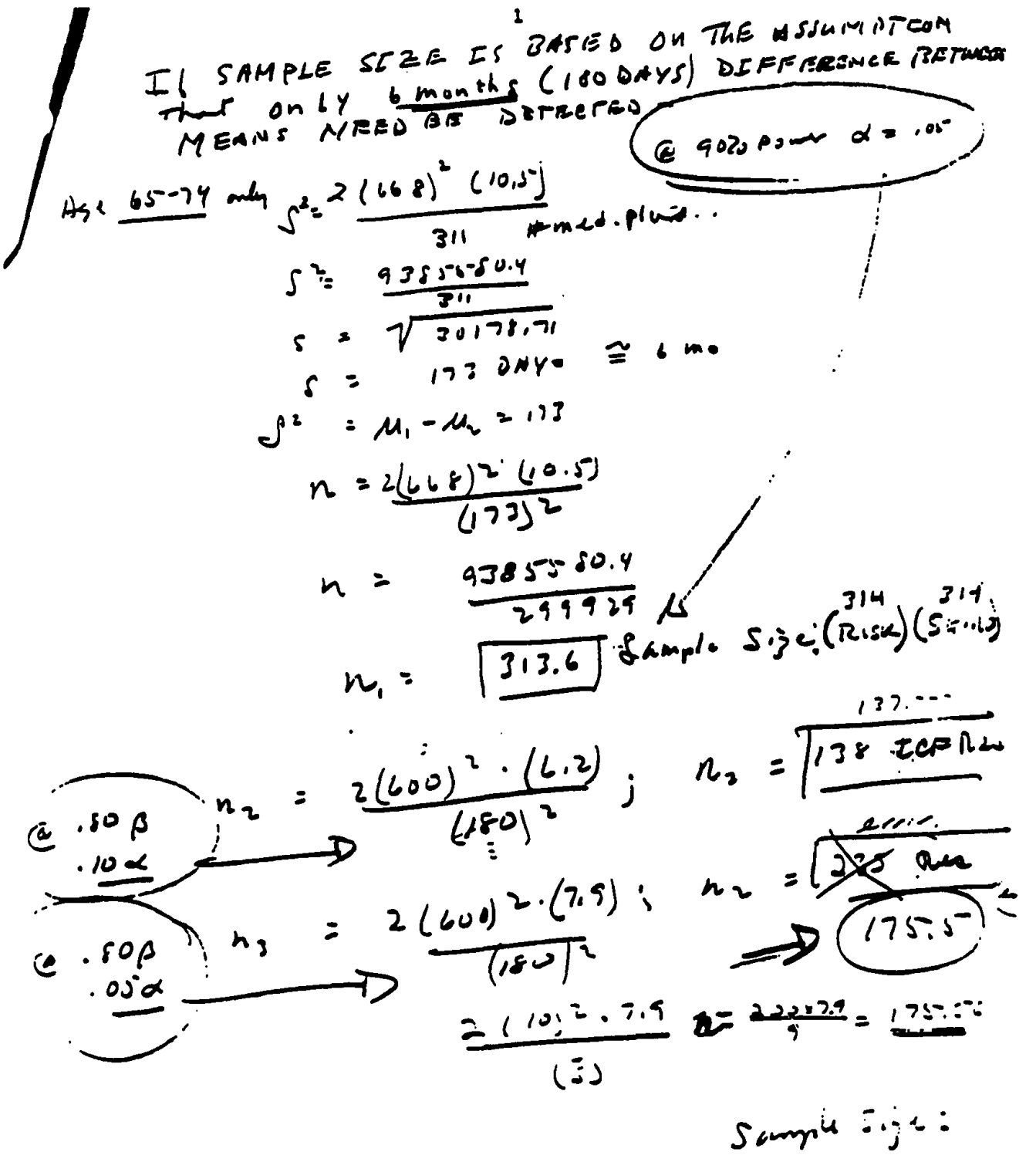


(2) Determining Sample size for Population subsets

\section{Method One--Normal Distribution}

Sample size assuming a normal, two sided distributed outcome, using a .05 level test sample size, $n$, per group, is given by the formula:

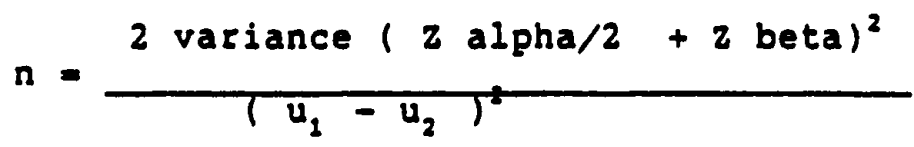

where:
(a) = (presumed constant) variability,
(b) $u_{1}, u_{2}$ are group means
(c) $2 / 2=100(1-/ 2)$ percentile for $N(0,1)$
ie, $\quad-.05, \mathrm{z} / 2=.025=97.5 \mathrm{th}$ percentile $=1.96$
alpha (type I error).-
beta $(1)$ type II error, (not rejecting when $u_{1} / u_{2}$ )
- 1 - power; at 908 power, beta $=.10$
$=z .10=$ goth percentile or 1.282
Lamda 1 - rate per 1000 for group one
Lamda $2=$ rate per 1000 for group two
$n=\frac{(2 / 2+2)^{2}(1+2)}{(2-2)^{2}}$
Power $=1-2 / 2-\frac{(1-2) n}{1-2}$
$\mathbf{n}=\mathbf{2}$
$2(3 / 2+3)^{2}$
๑.g. 0.1
$=0.5$
$-.10$
$=2(10)^{2}(1.96+1.282)^{2}=2102$ 
For example, if the Los data is normally distributed with a standard deviation of 200 days than we would have 90 percent power to detect a variance of 20 days per year for the sHMO group.

Method two--Non-Normal Distribution

An alternate Approach To Estimating Required Sample size is to use a poisson distribution which assumes a non-normal or asymmetrical distribution of variation in use rates.

$$
\begin{aligned}
\text { Power } & =1-3 / 2-\frac{n}{2} \\
(x) & =\begin{aligned}
\text { Probability } n(0,1)<x \\
\text { ie a cumulative distribution function }
\end{aligned} \\
n & =3000,=.1 \\
\text { Power } & =1-(-1.91),=978
\end{aligned}
$$

The asymmetrical distribution is a known characteristic of utilization in ICFs making the latter approach the better choice. Therefore, a sample size of 3,000 per population subset, i.e., (3,000 Risk $)+(3,000$ smyo $)$, will provide 97 percent power, (assuming a two-tailed .05 level test) for use in detect differences between the skmo and Risk study groups on the order of 0.1 standard deviation.

More formal justifications of sample size, because of the limited information on variability, is not considered to be fruitful for this study. Indeed, an important consequence of this study will be the generation of good data on the distribution and variation of utilization for SNF and ICF members of HMOs. 
Research Population Estimate One, (normal distribution)

When estimating the average difference in a response variable between two groups, and the size of the target population is not known, using the formula:

$\left[n=z^{2}\left(\operatorname{variance}_{1}{ }^{2}+\operatorname{variance}_{2}\right)^{2} / B^{2}\right] ;$ and,

Assuming normal distribution of Los data with a standard deviation of $+/-200$ days (two tailed) at an $.05 \%$ alpha level, and using $90 \%$ power to detect a variance between trial and control groups of 20 days, then a sample of 2200 subjects is required. Since it is almost certain that the distribution of days/1000 will be asymetrical, an even larger sample is desirable. The number of ICF residents among Medicare Plus members is not much larger than this number suggests is needed. The sHmo population is smaller .

Research Population Estimate Two, (non-normal) suggests:

\section{1 sigma}

$N=$ delta to get a $90 \%$ power = sample size of 3,000

To predict the variance on .1SD and an alpha level of .05 (Type I error) is only correct relative to the perturbation of data in the sample.

Using the above guestimate on size of sample (to be drawn from the total population of each subset for Risk and sHMO groups), for the period $6 / 85$ through $12 / 87$ (see chart two for range of enrollees) requiresd about 3000 per group to cover all the cells of age and sex in each group of cell. 
With a standard error of .2 and .3 for the estimate, at a $95 \%$ Confidence Interval, and at $a+/-6 q$, the probability of being admitted to a ICF over the life of the study is between 2 to 38 . 
APPENDIX C

FOR CHAPTER IV 


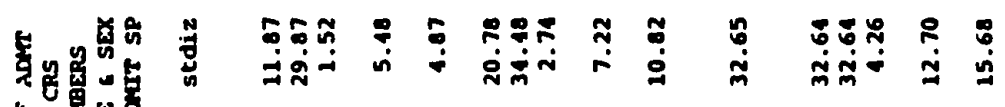

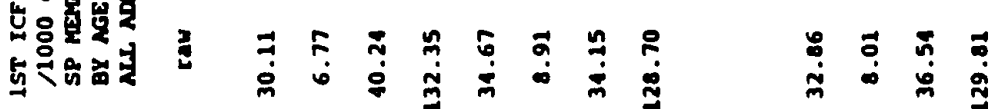

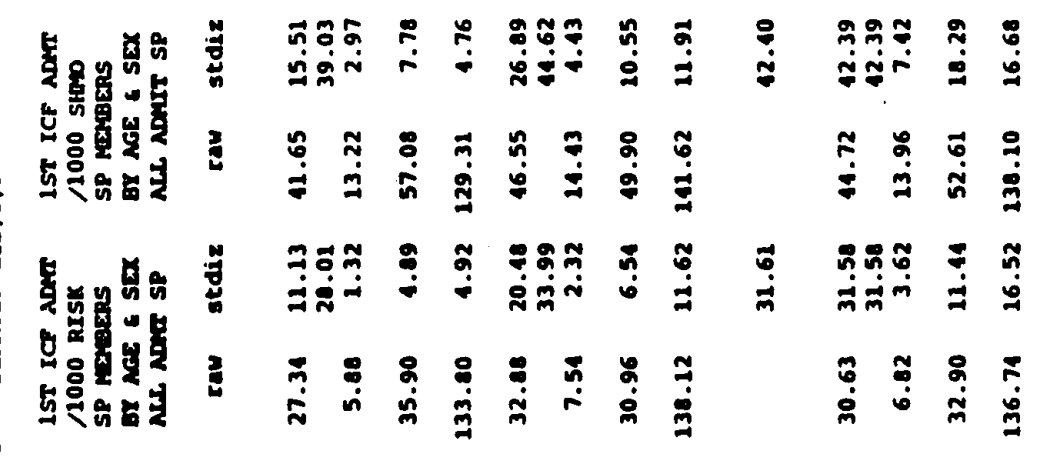

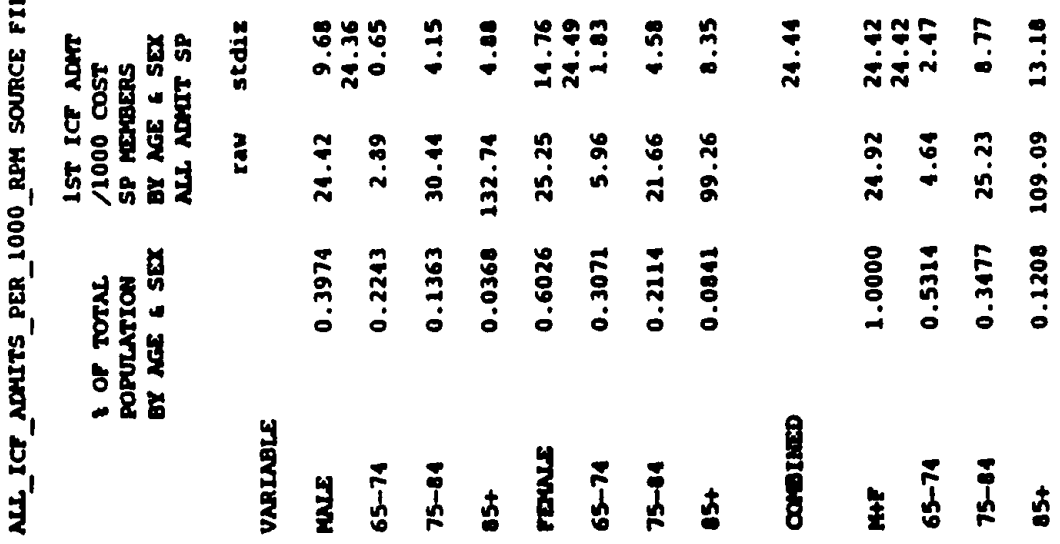




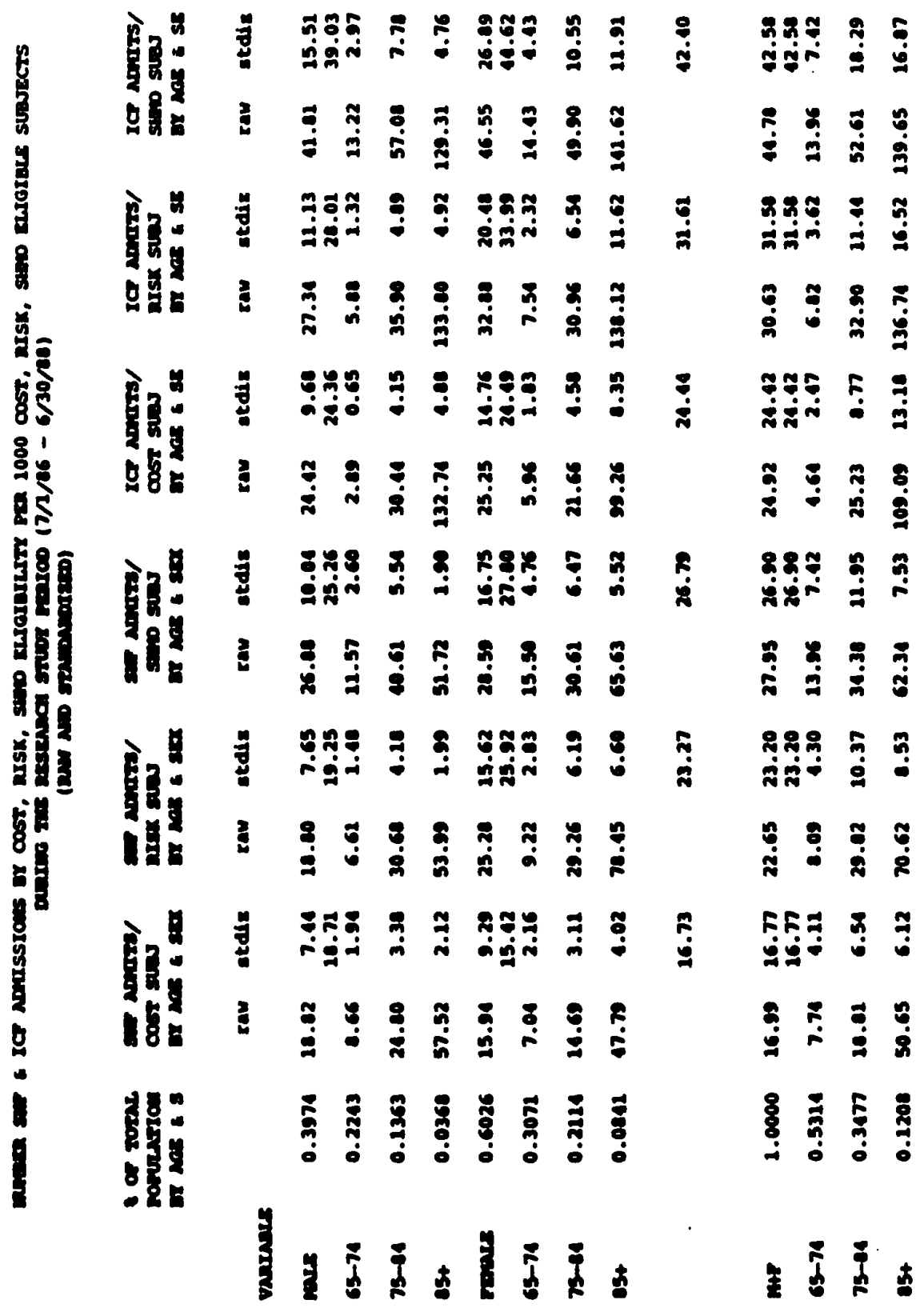




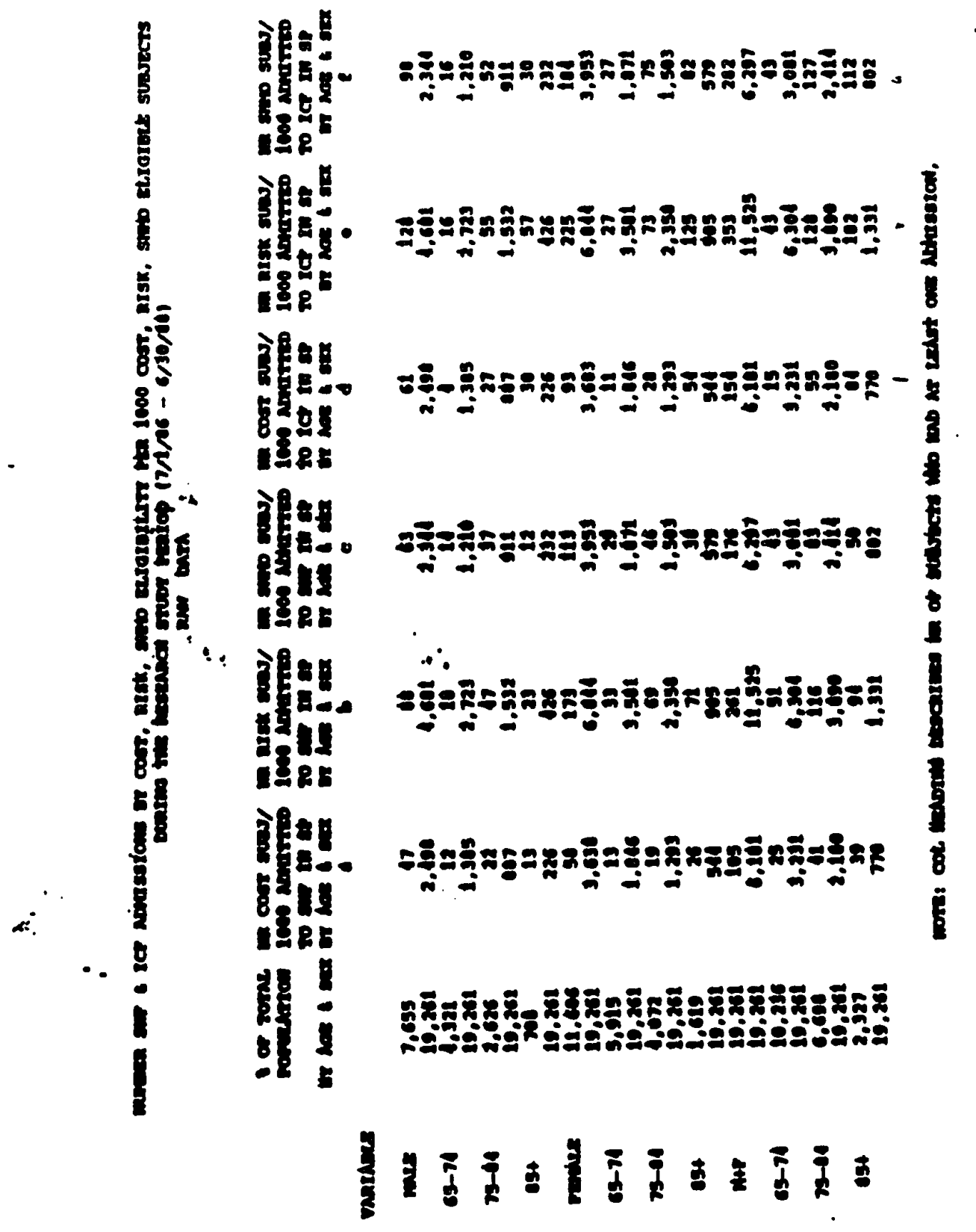




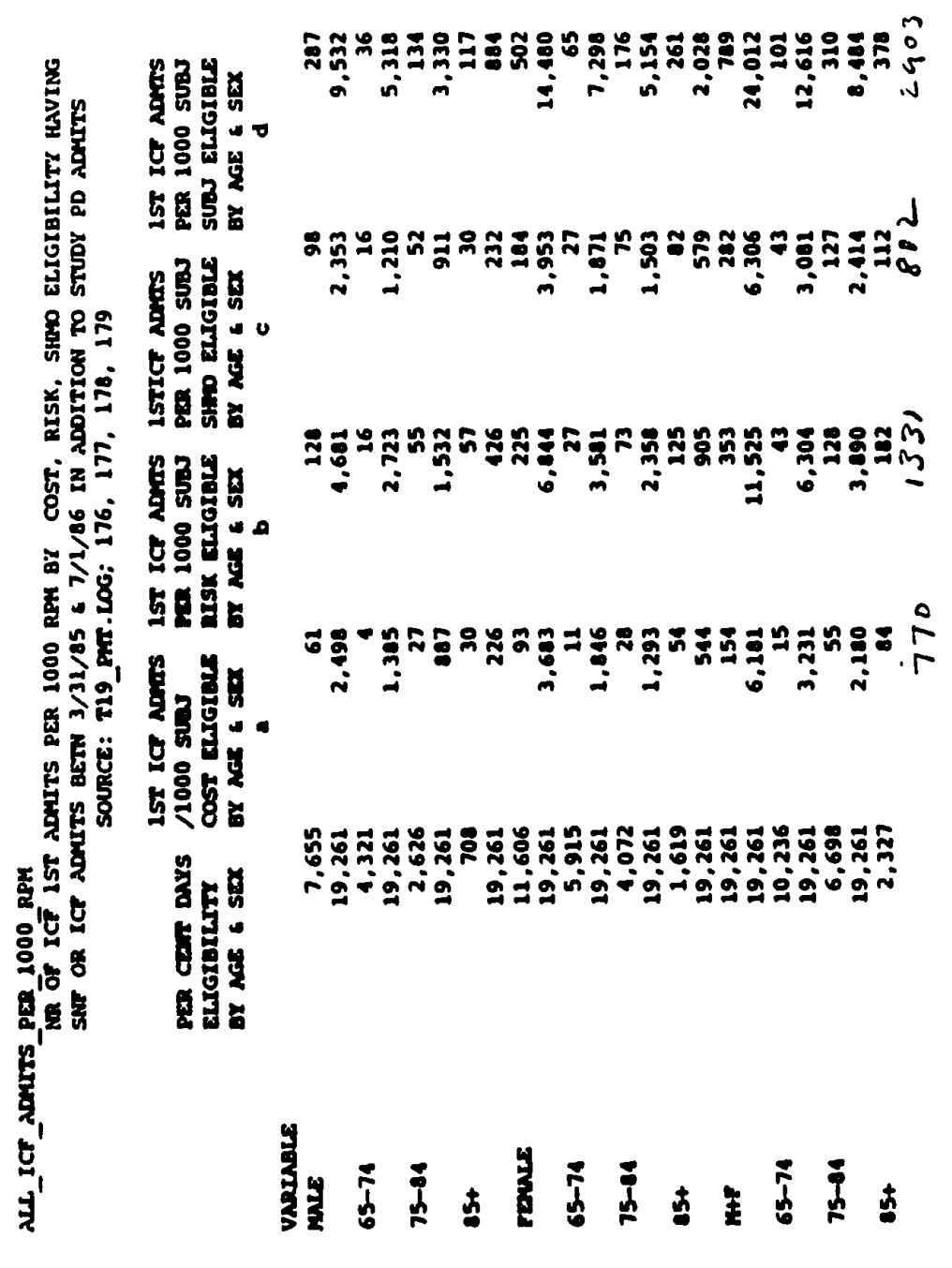




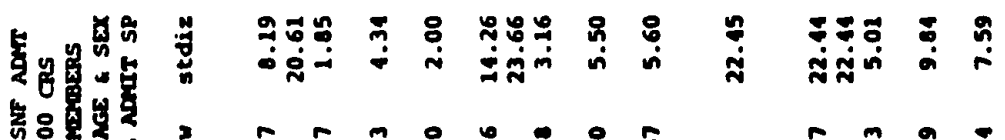

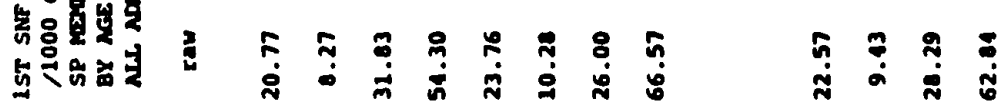

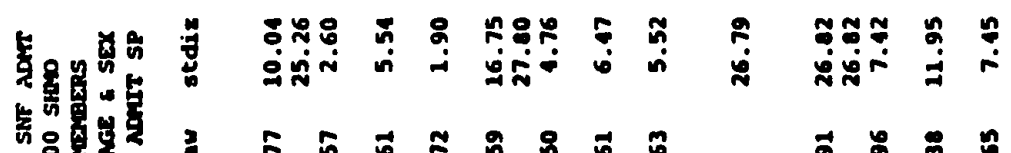

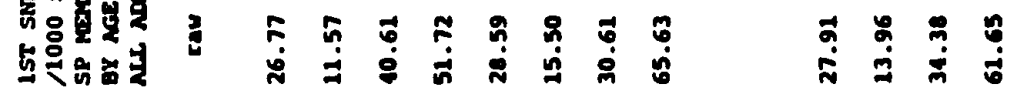

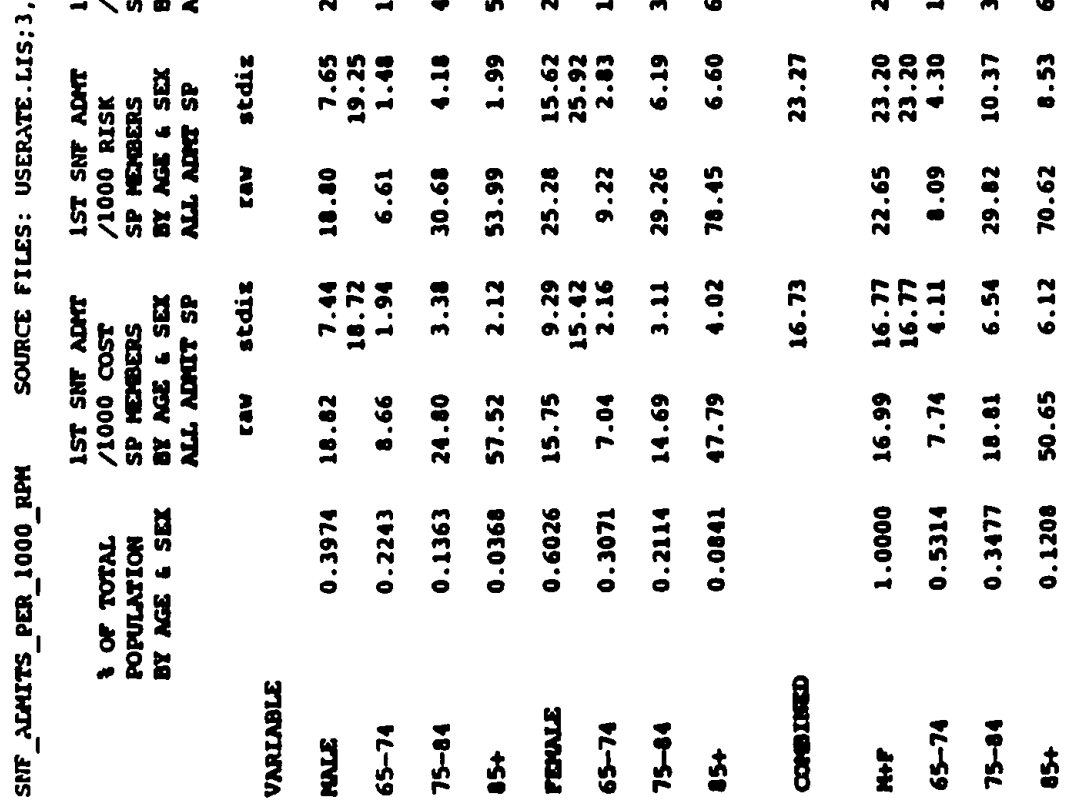




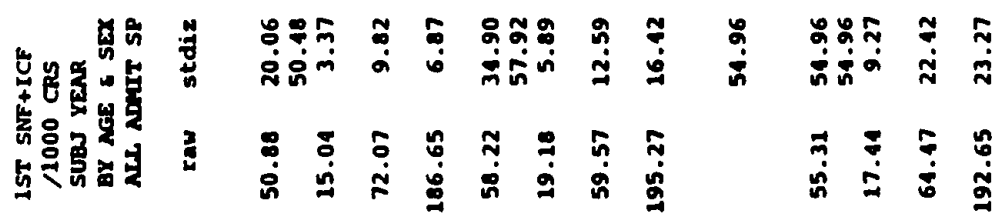

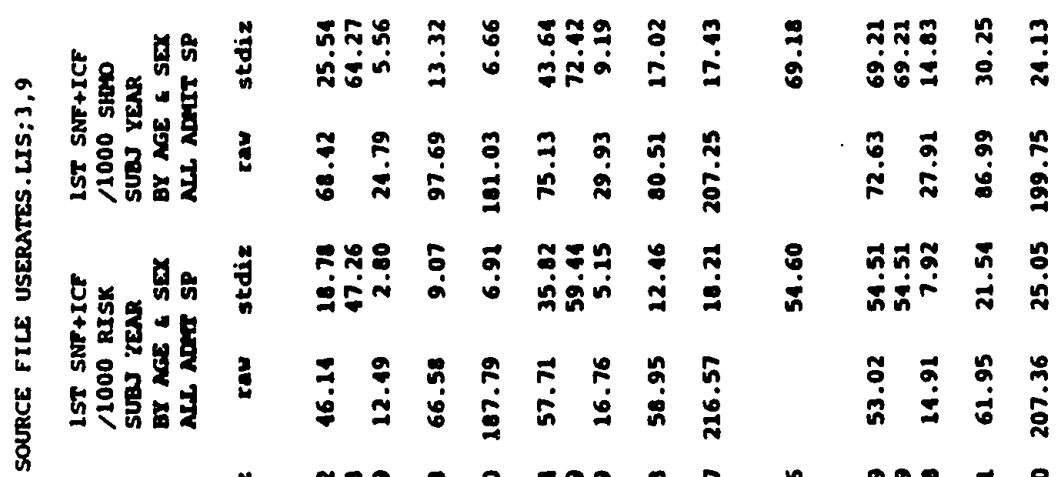

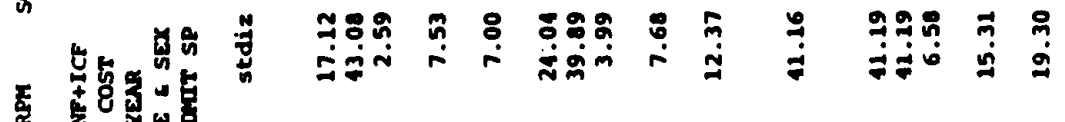

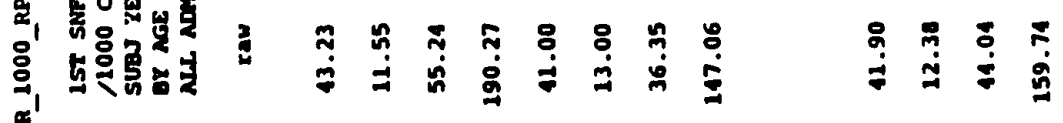

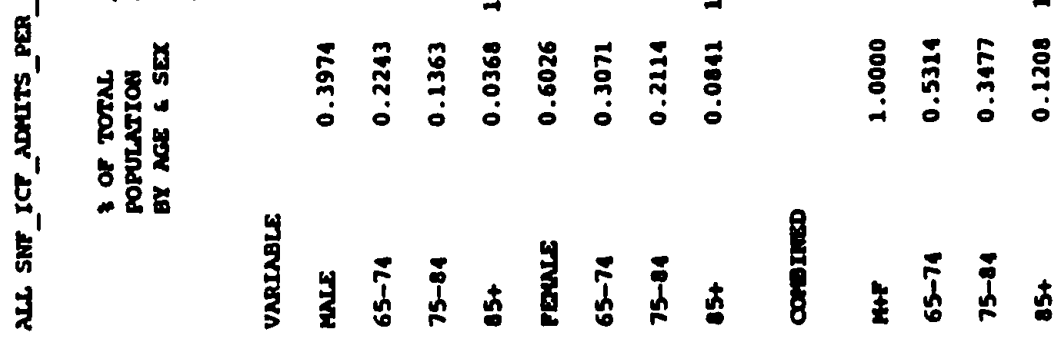




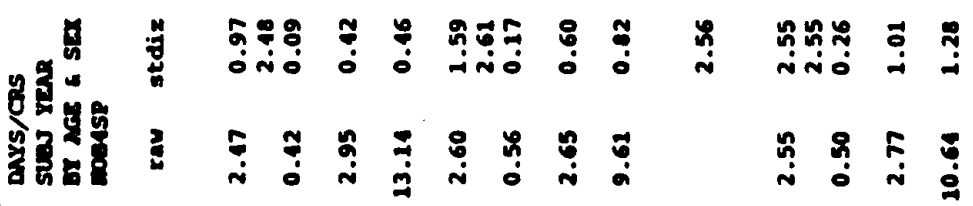

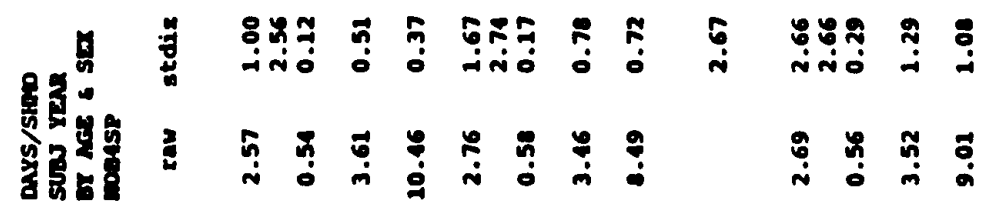

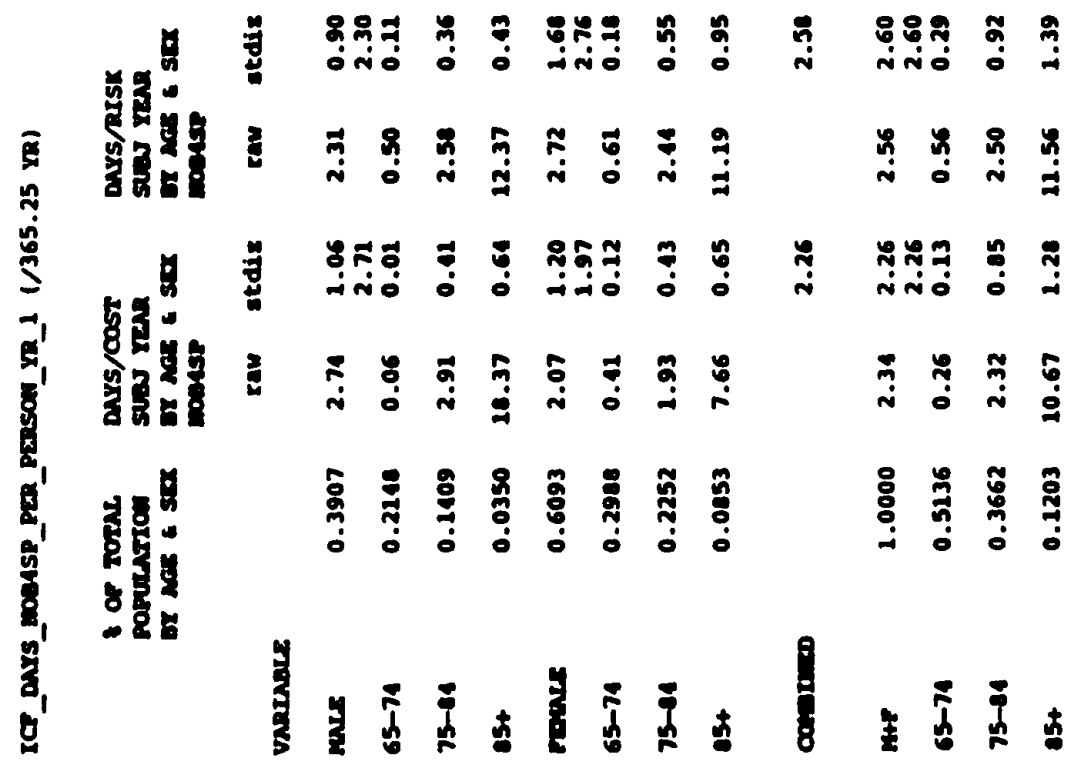




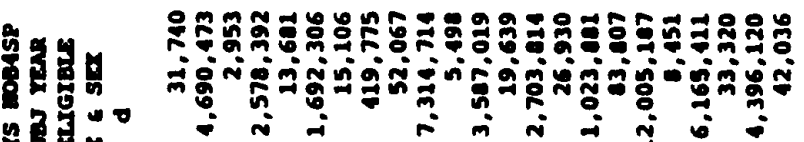

के

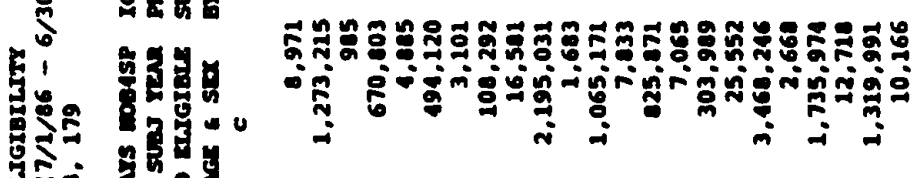

8.

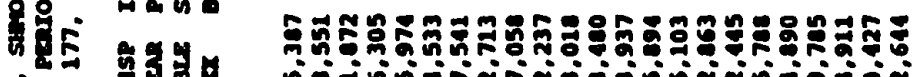

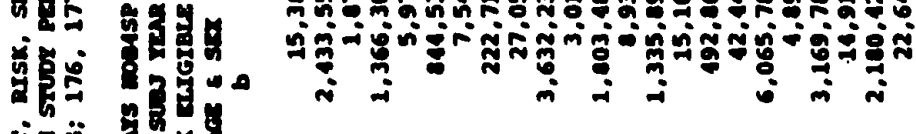

8\%

o

त्र

85

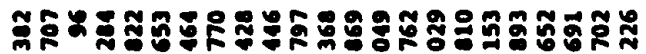

告

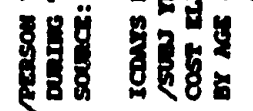

造哲

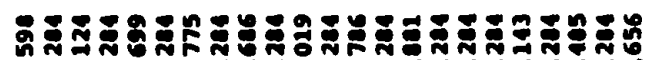

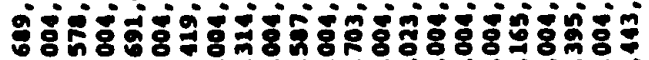

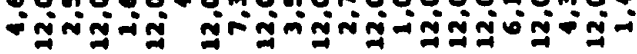

n

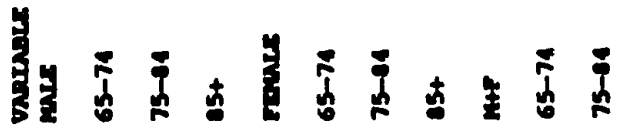




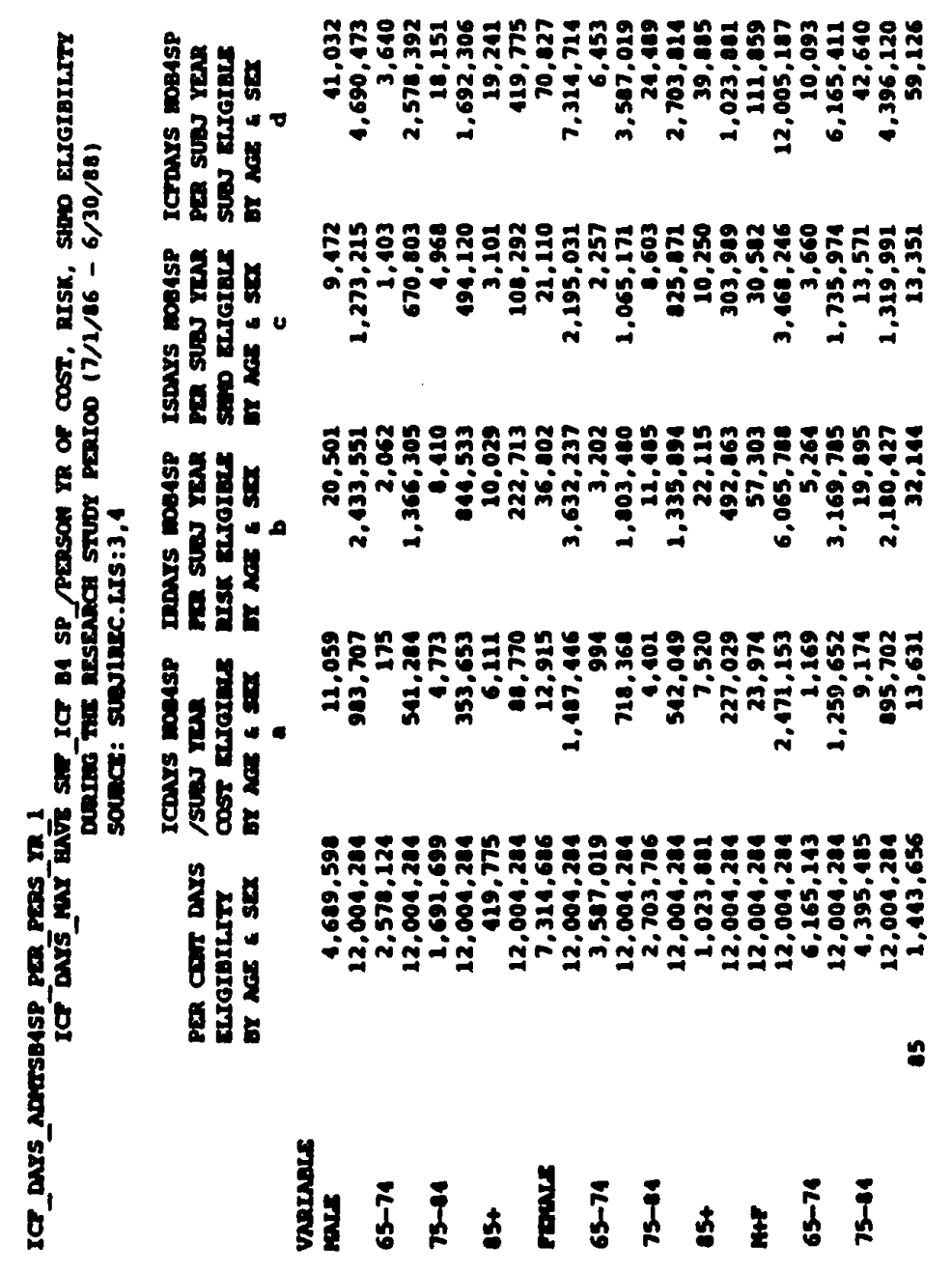




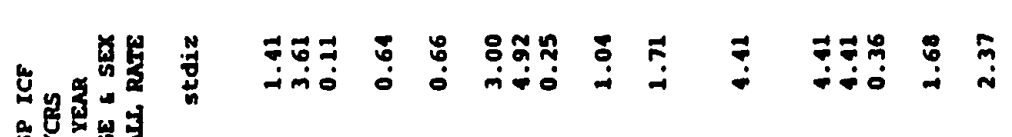

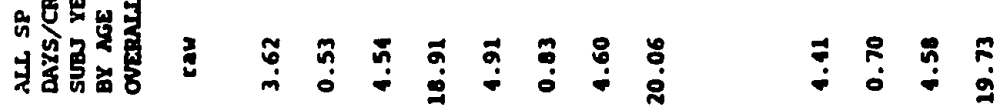

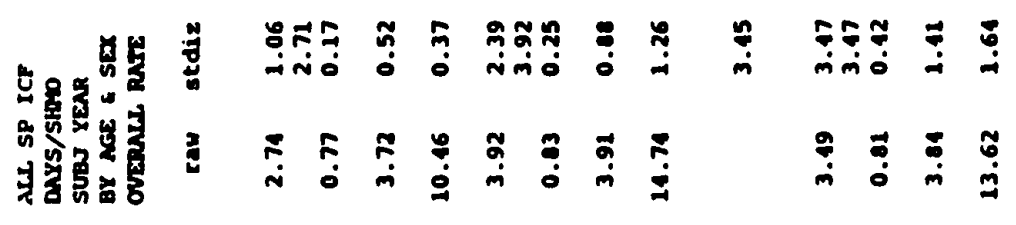

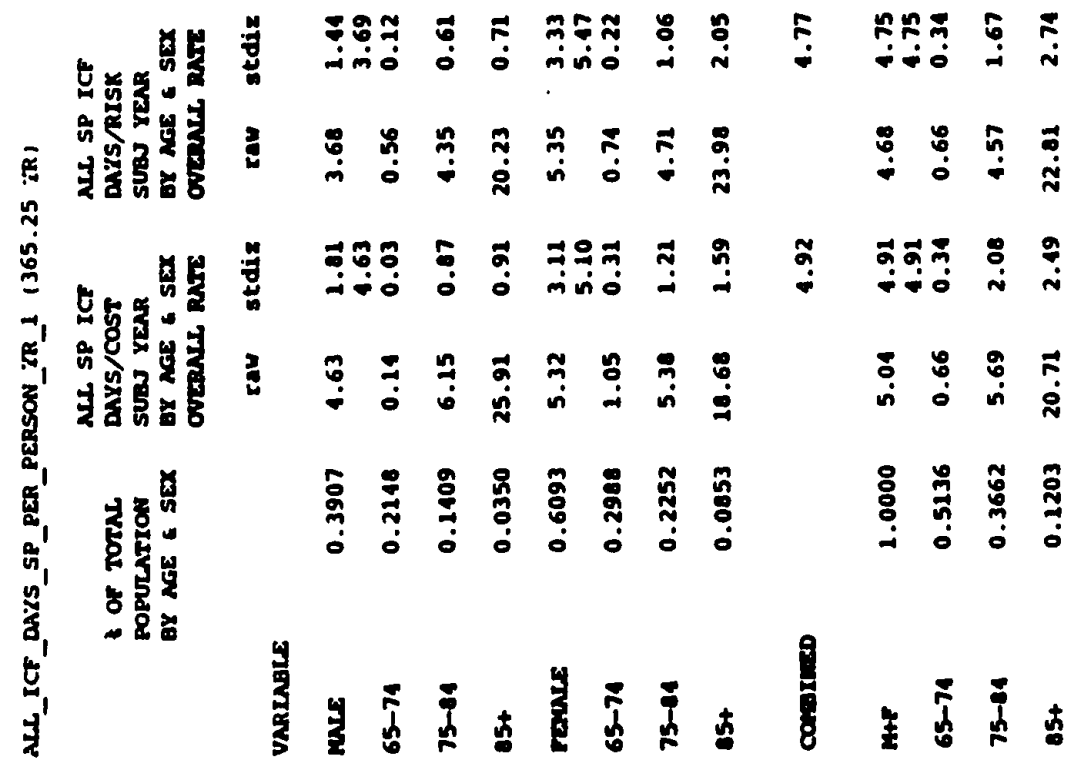




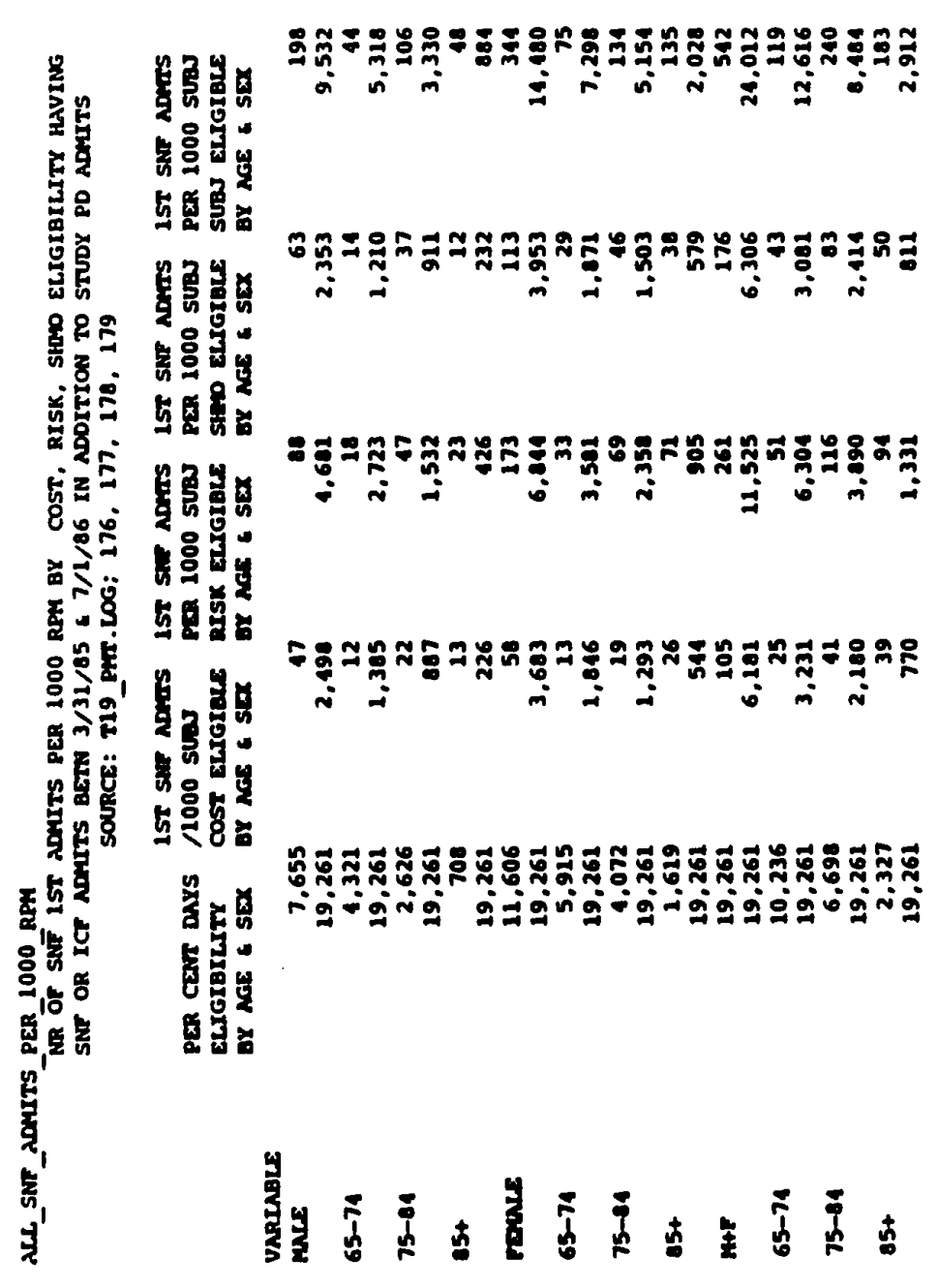




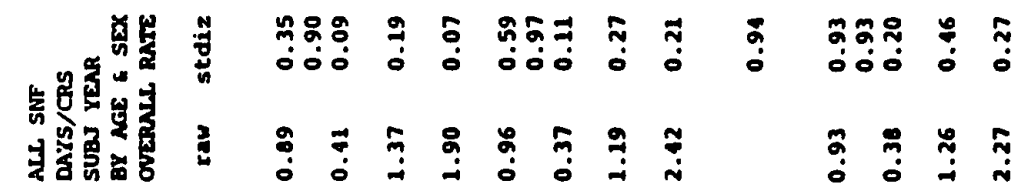

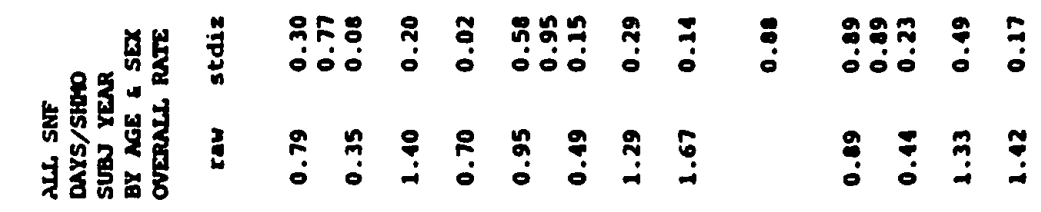

$$
\begin{aligned}
& \begin{array}{llll} 
& \\
4 \\
4
\end{array}
\end{aligned}
$$




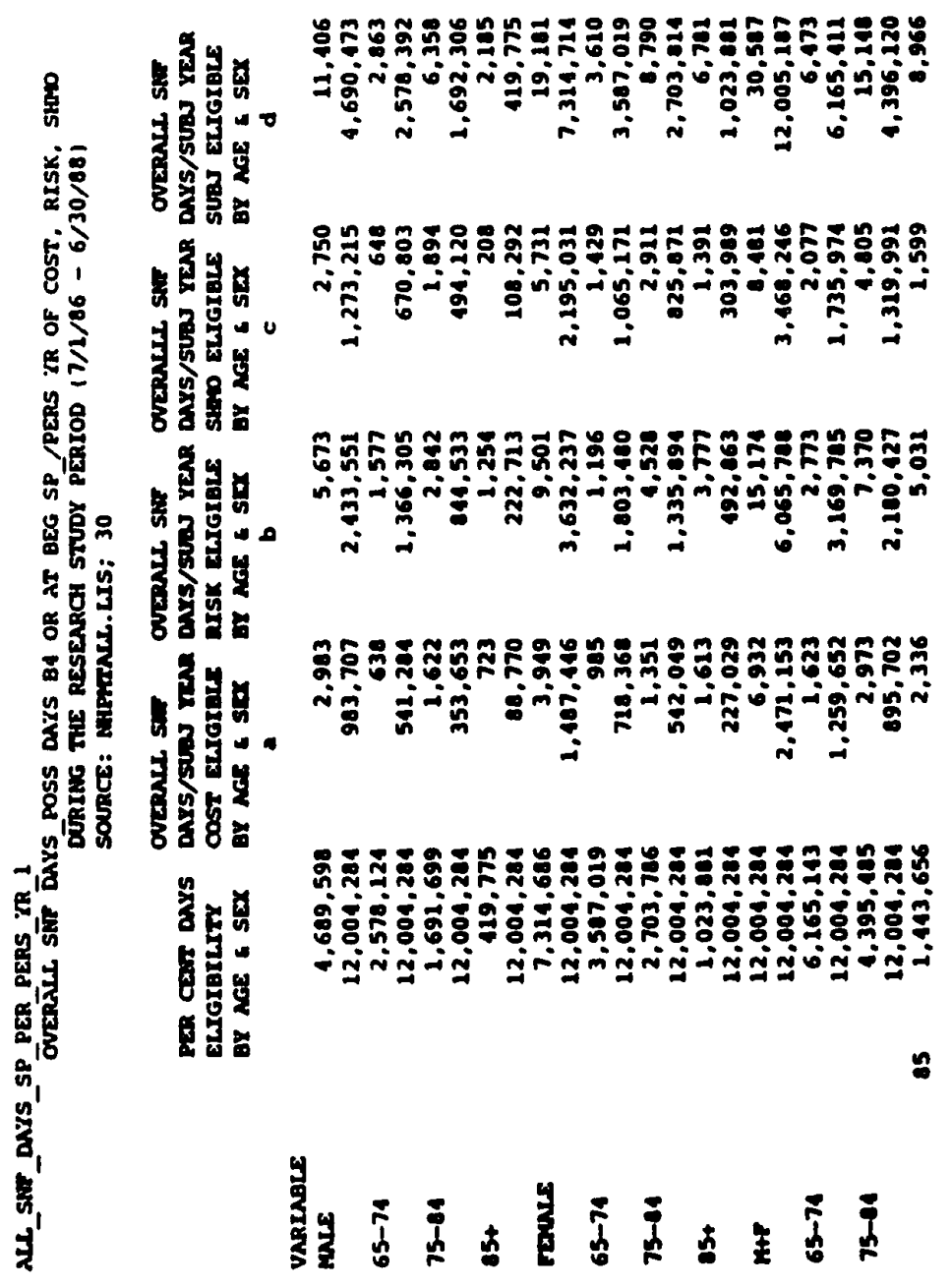




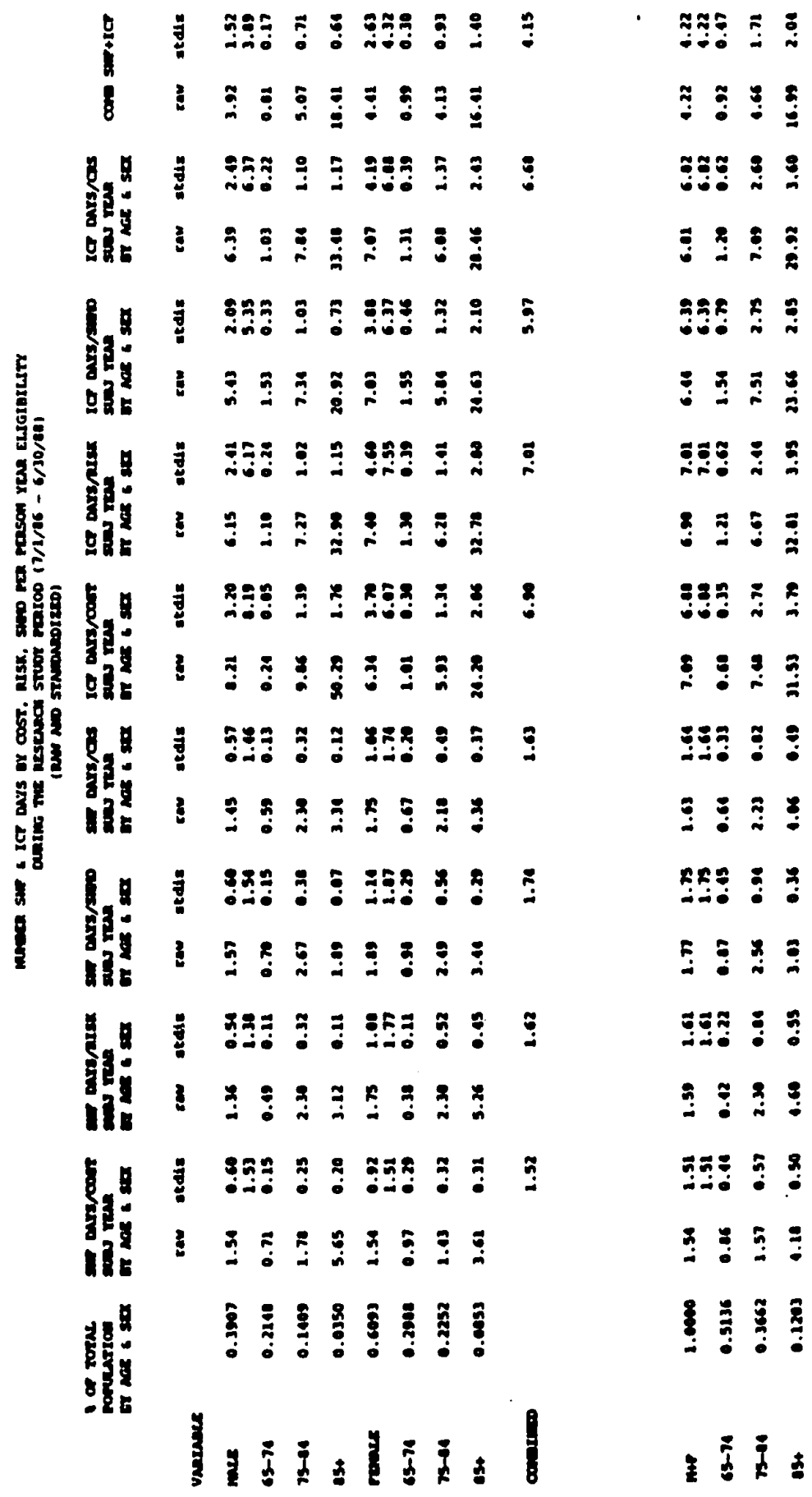




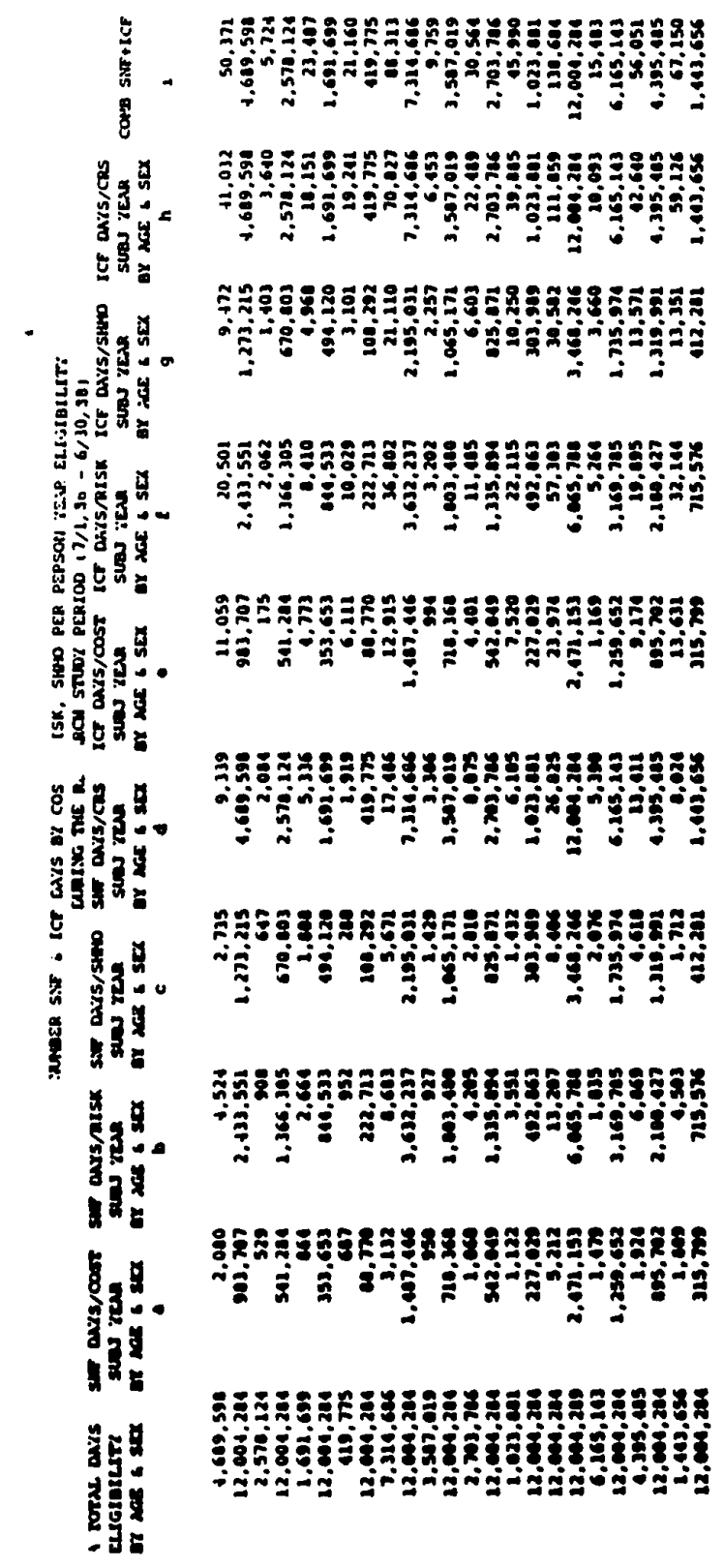

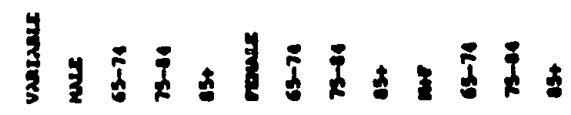




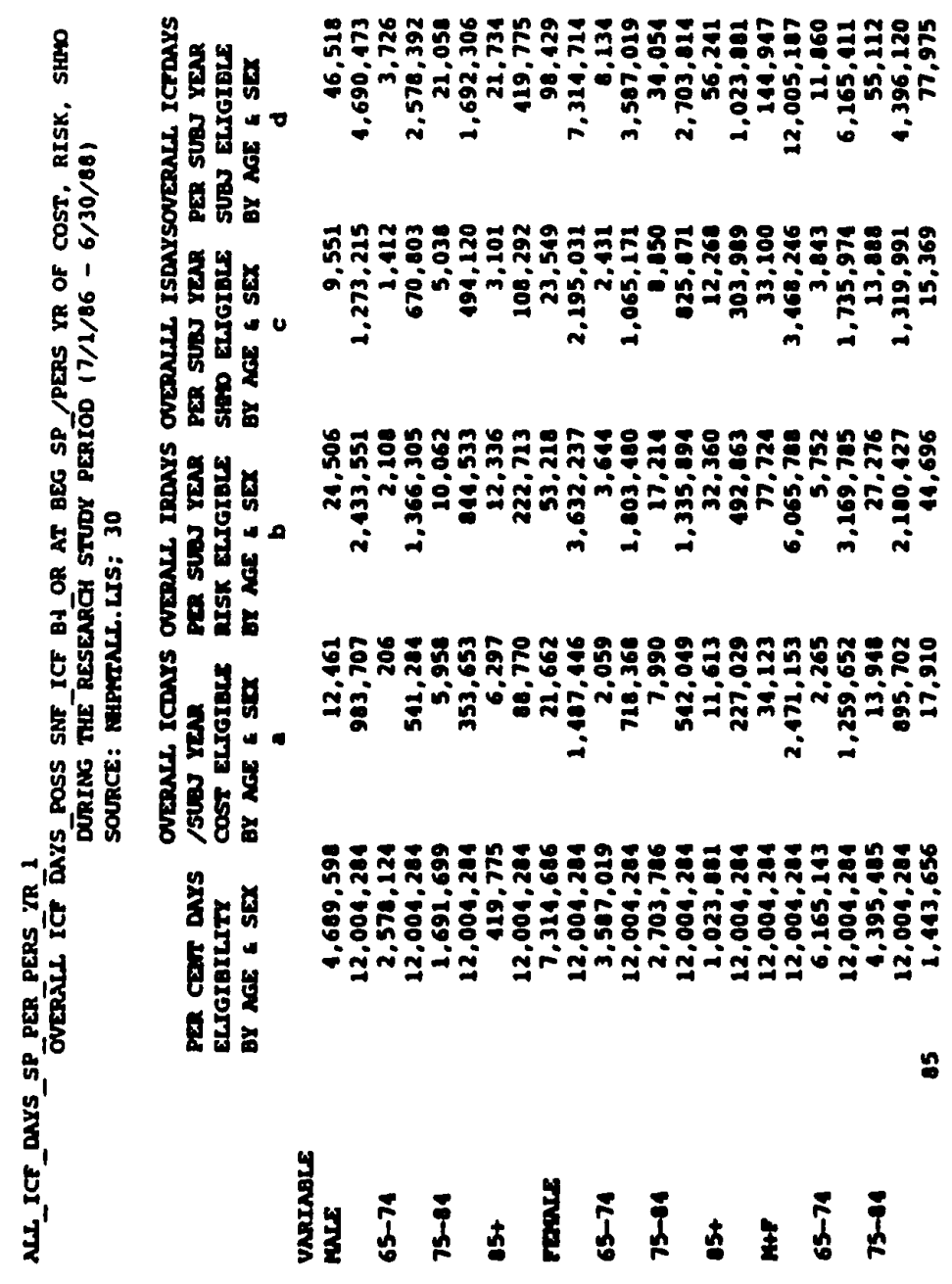




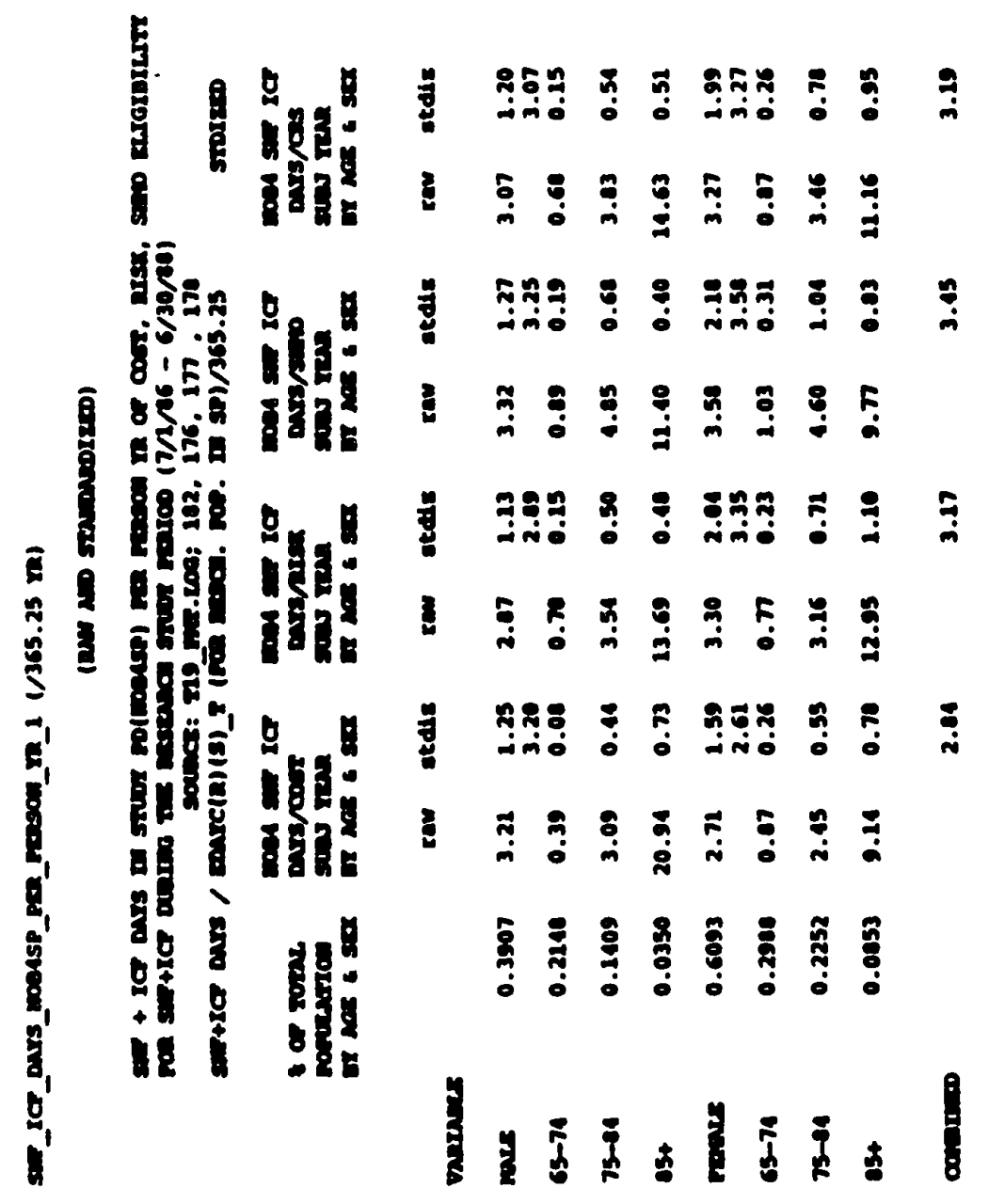




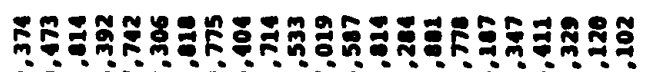

$\sum{ }^{\circ}$

照最

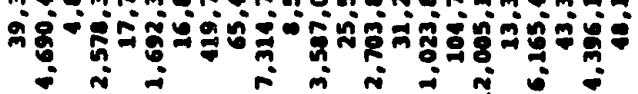

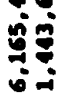

密量。

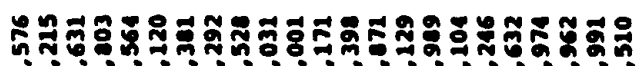

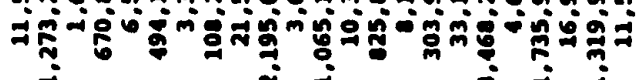

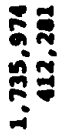

总最

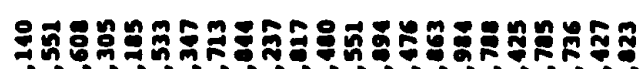

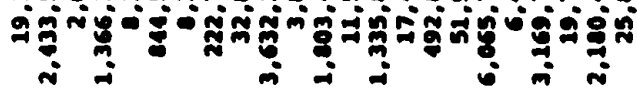

s.

㩆

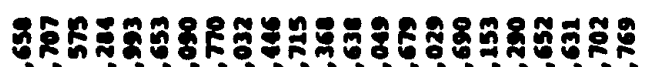

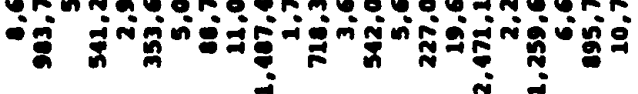

กิธู์

में

㩆易

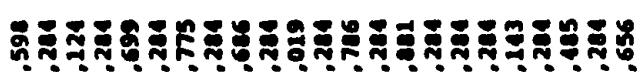

ơ

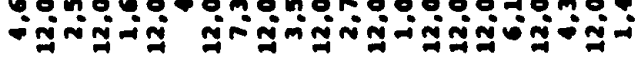

용

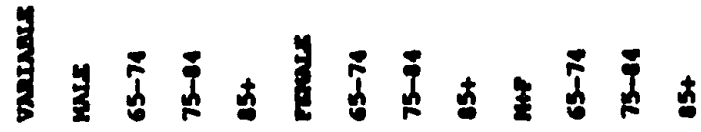




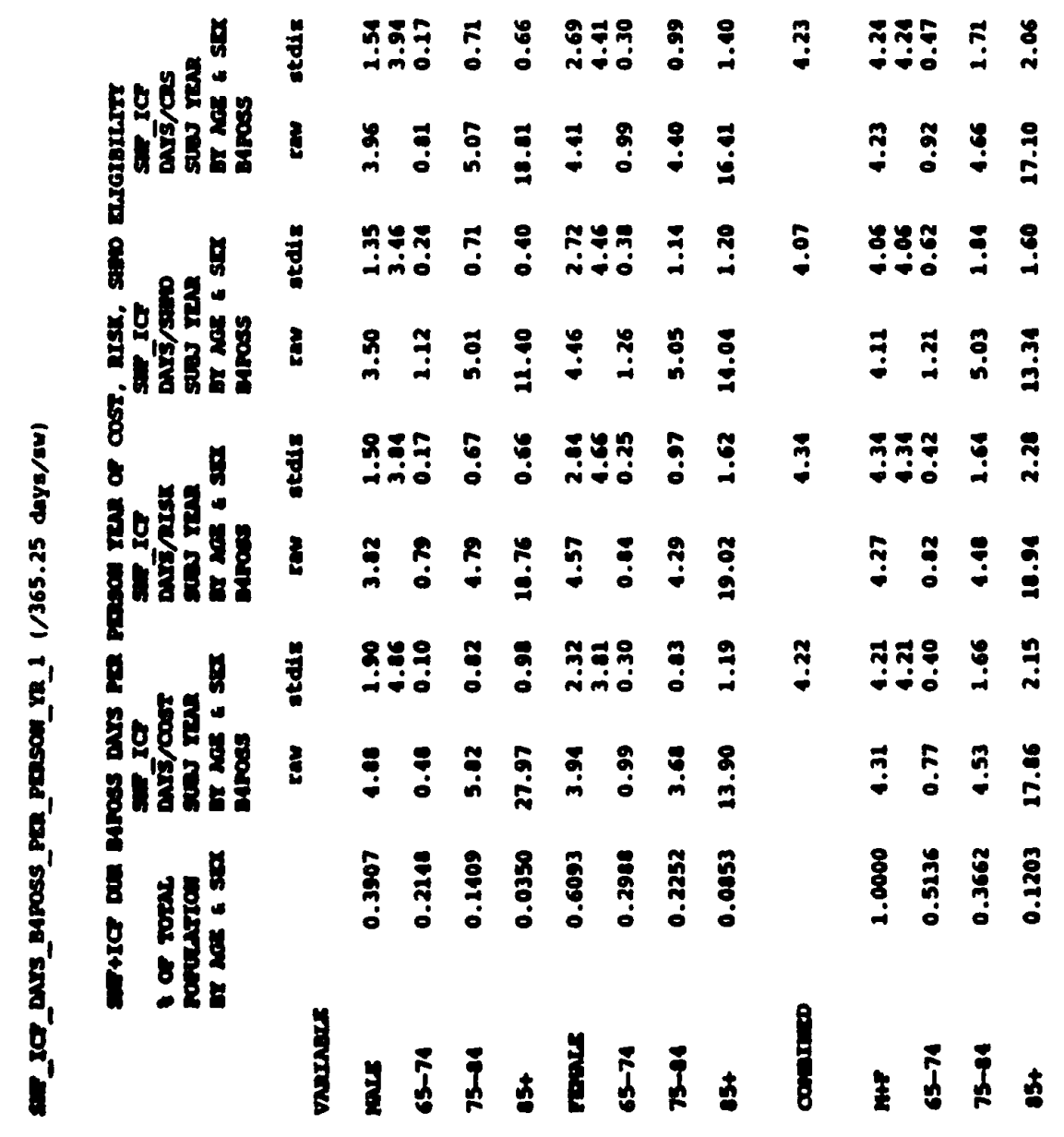




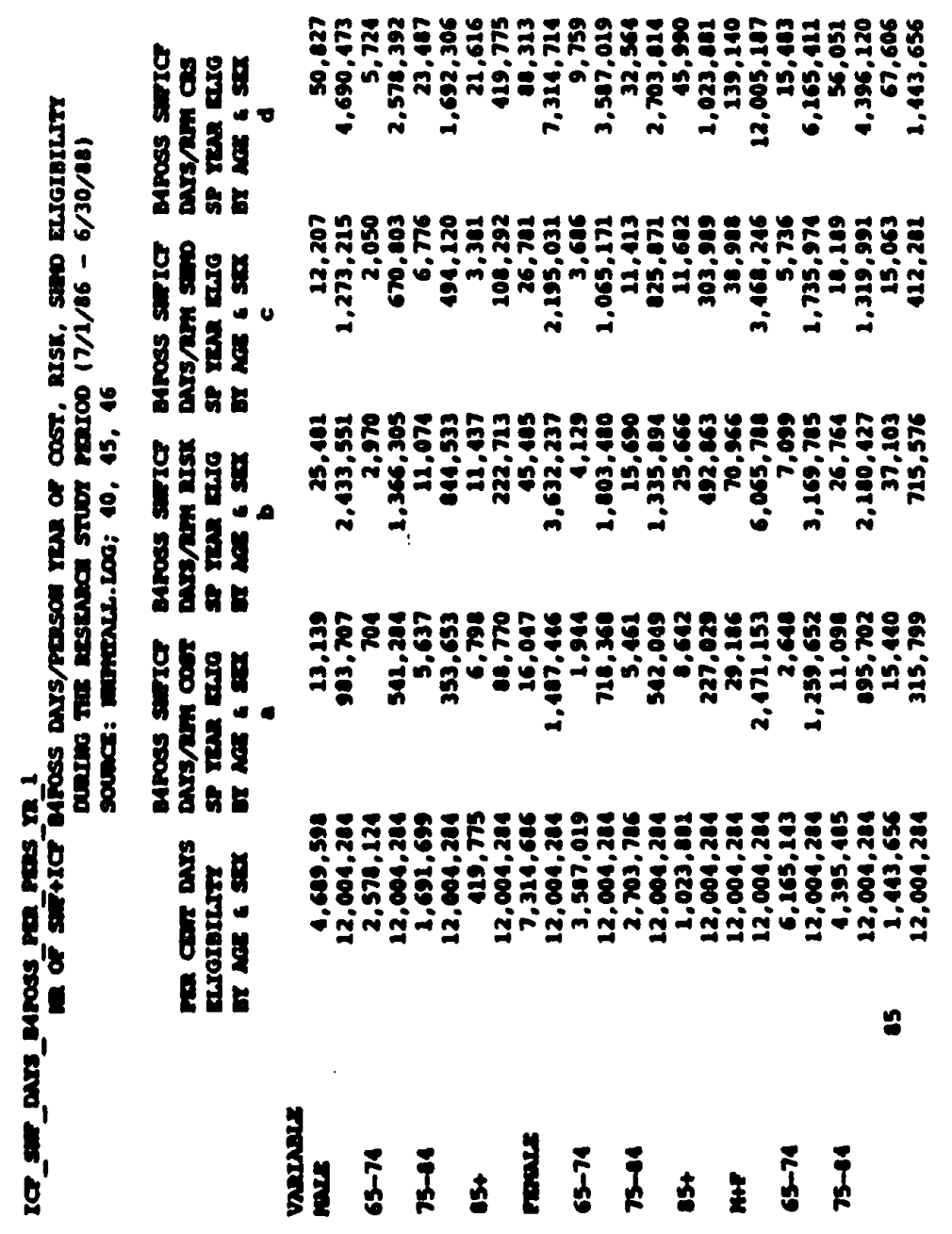




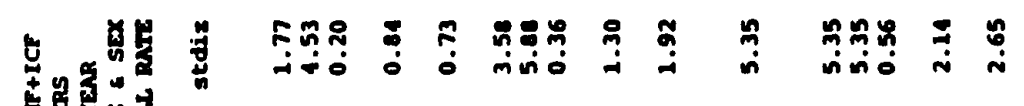

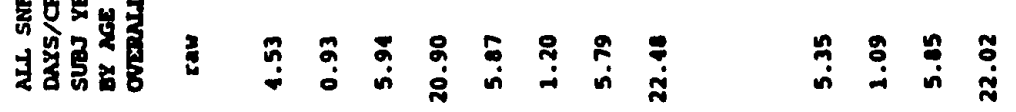

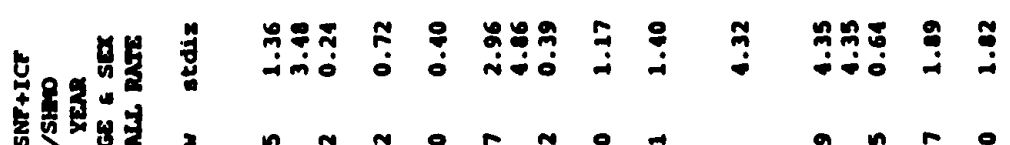

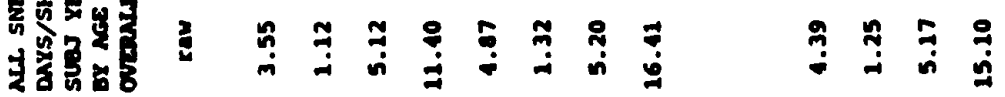

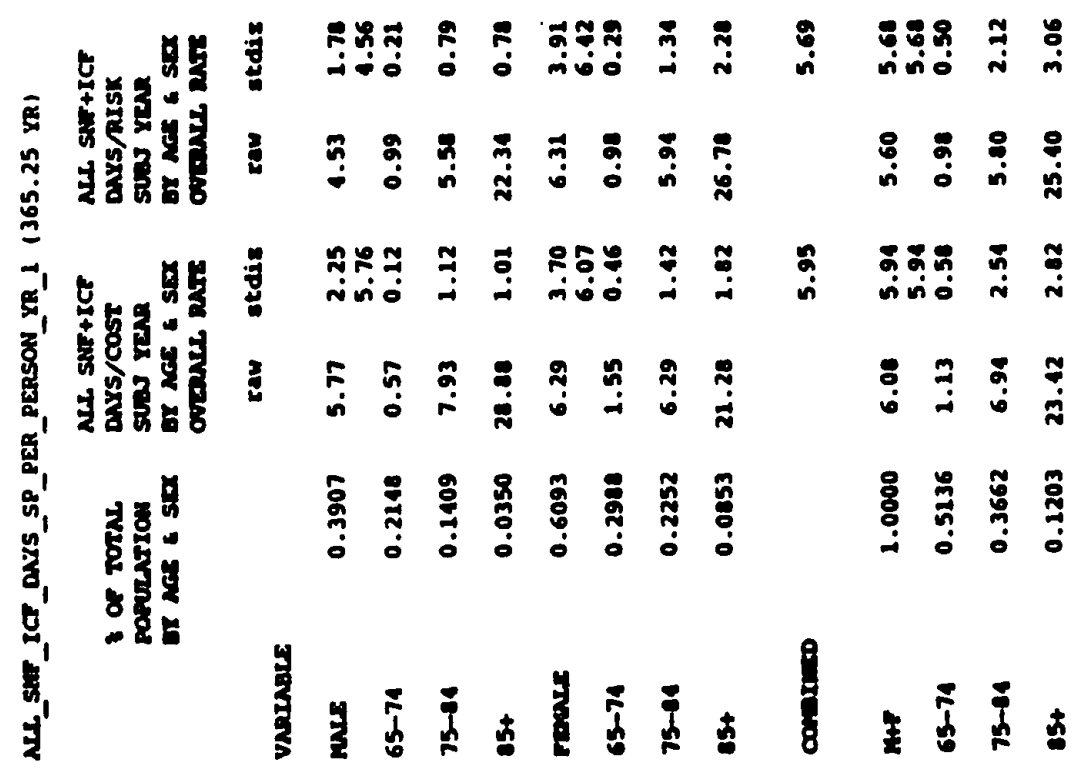




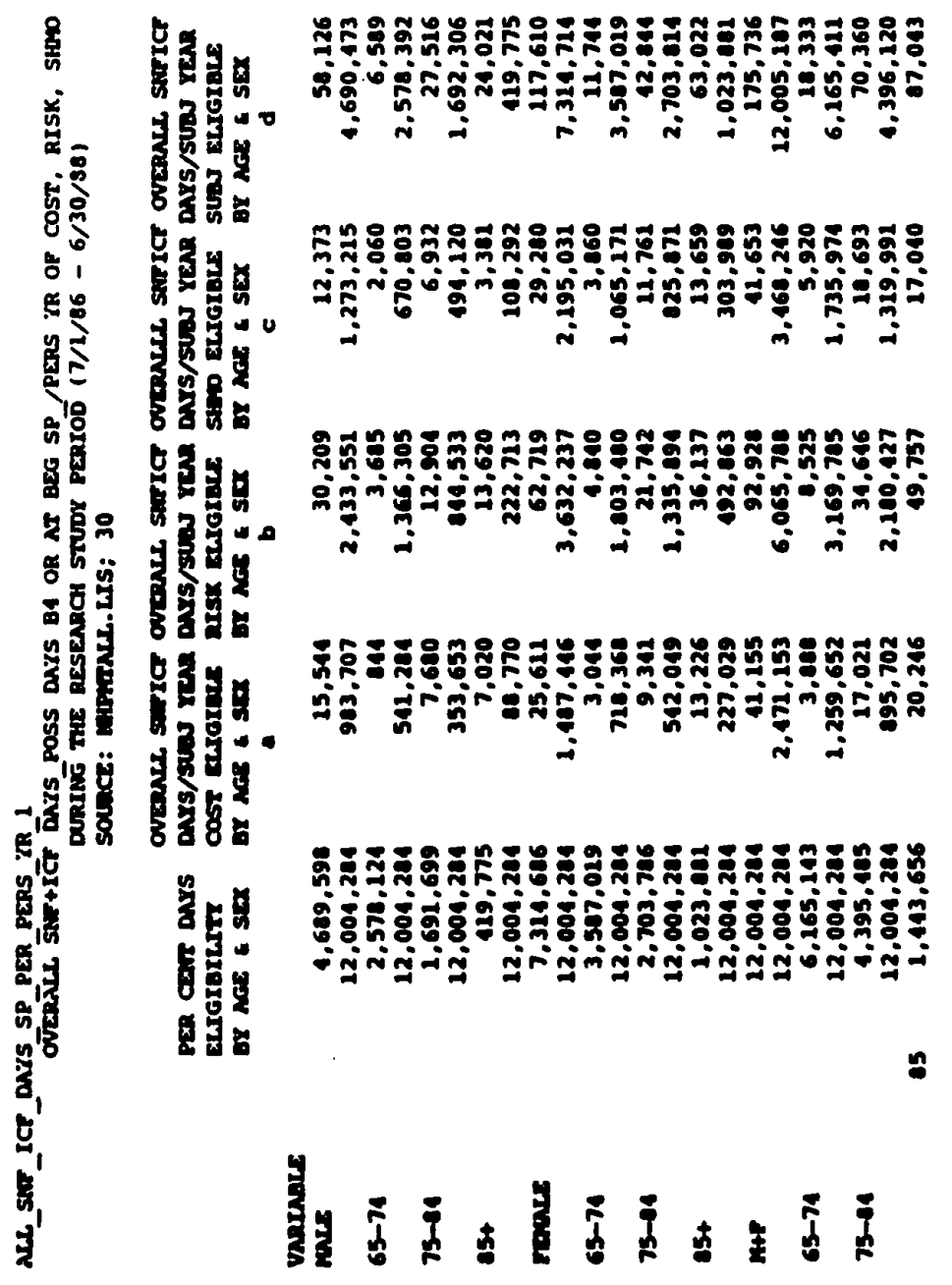




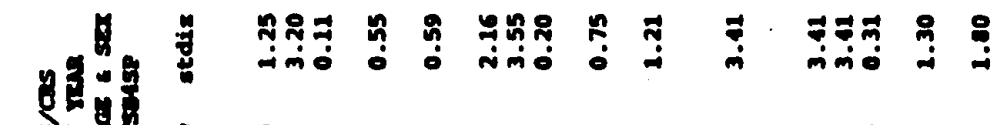

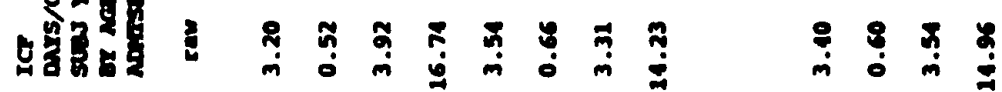

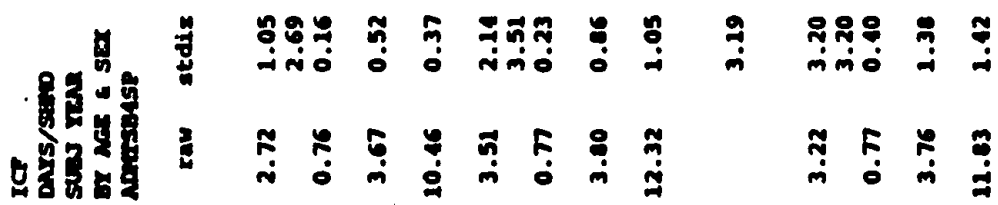

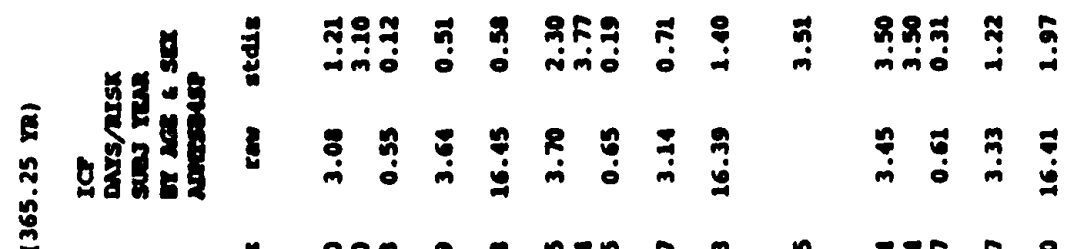

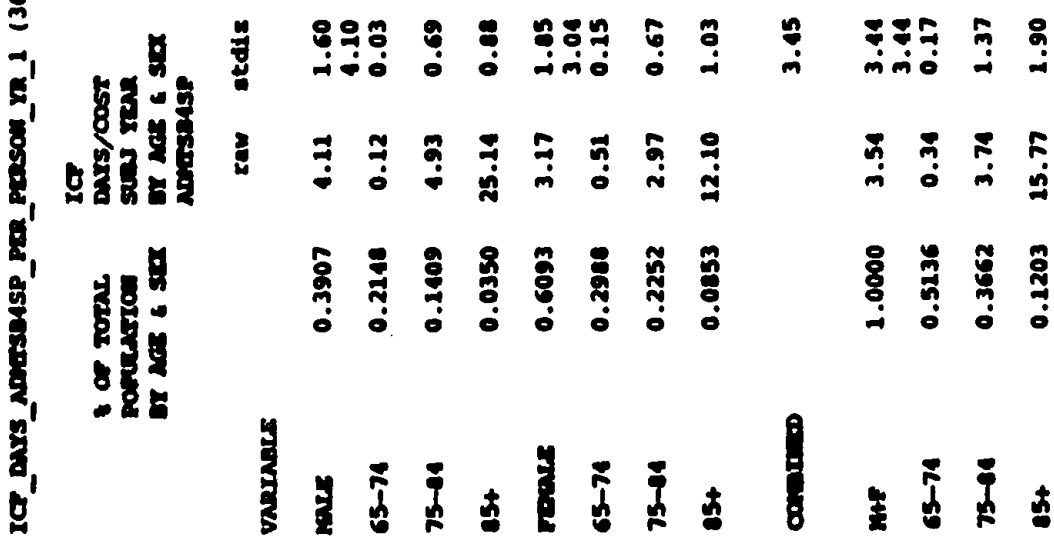




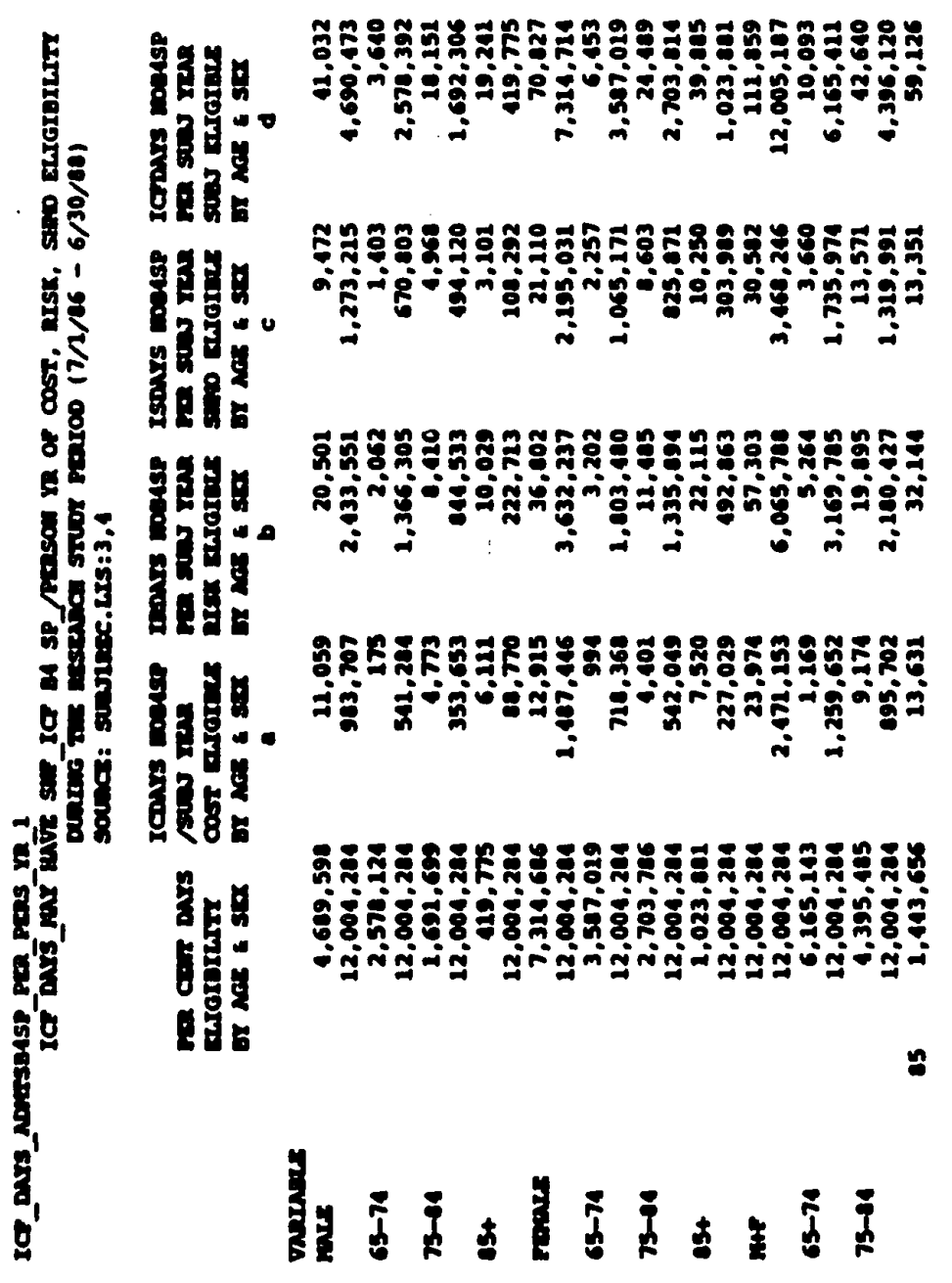




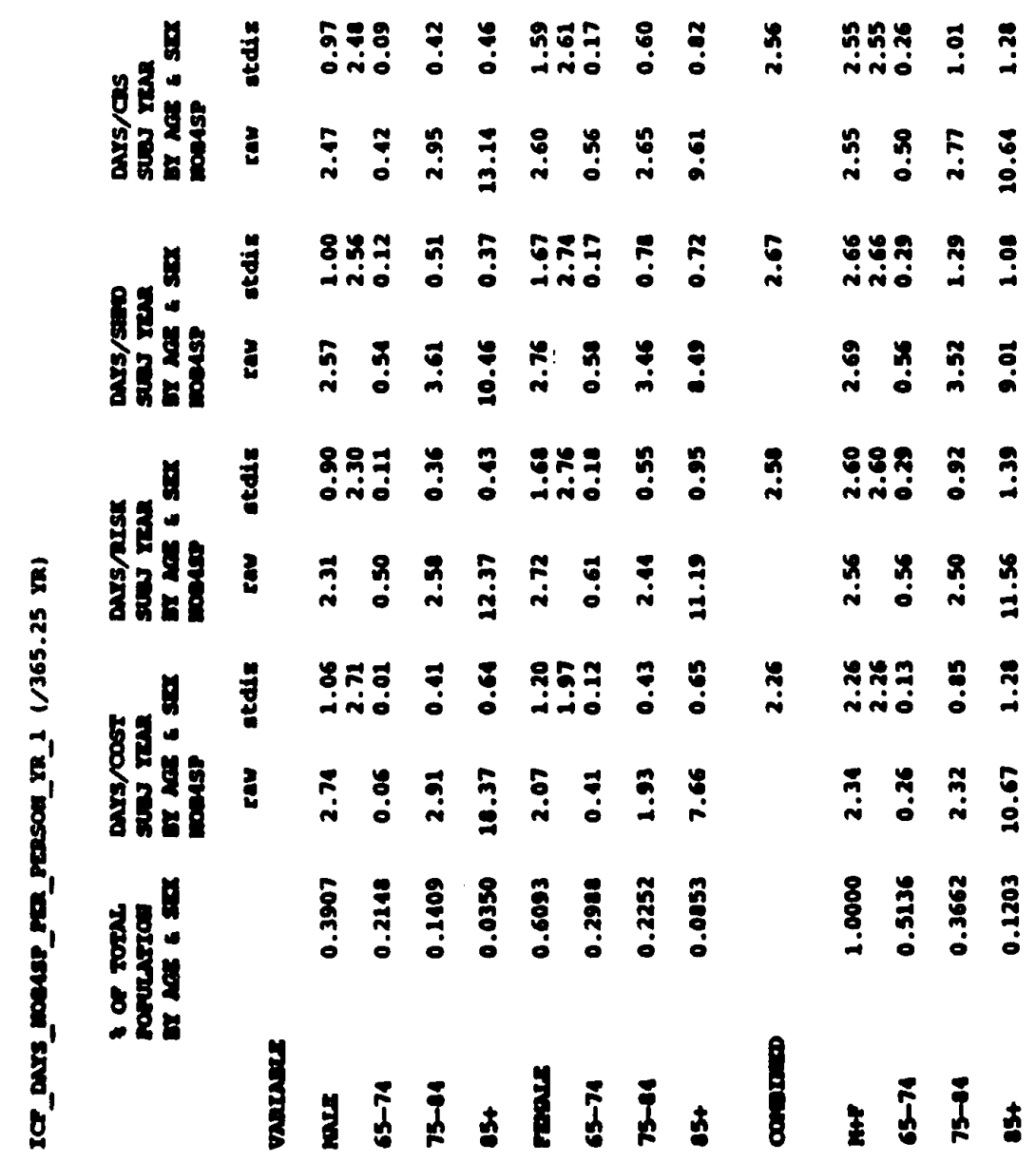




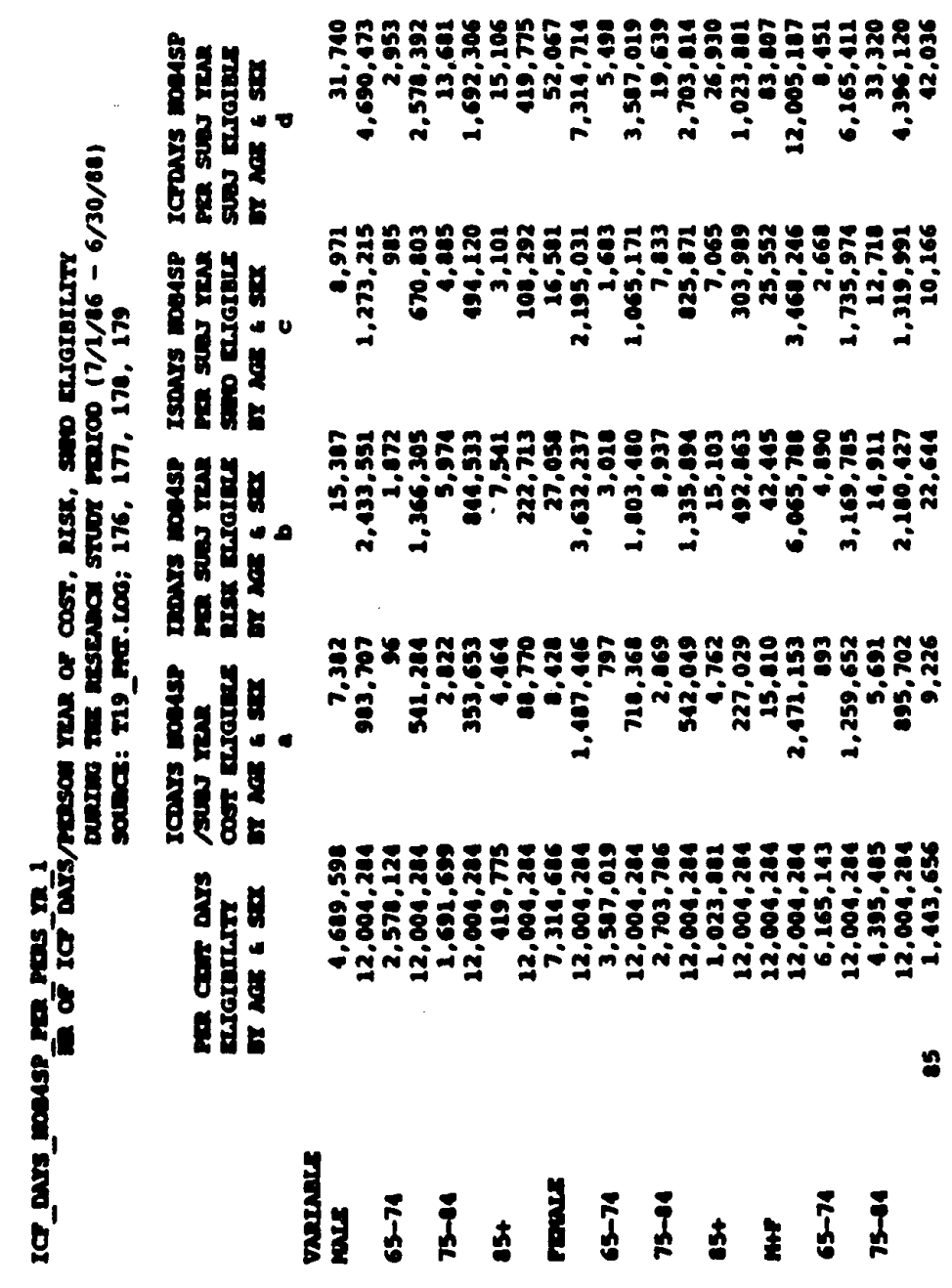




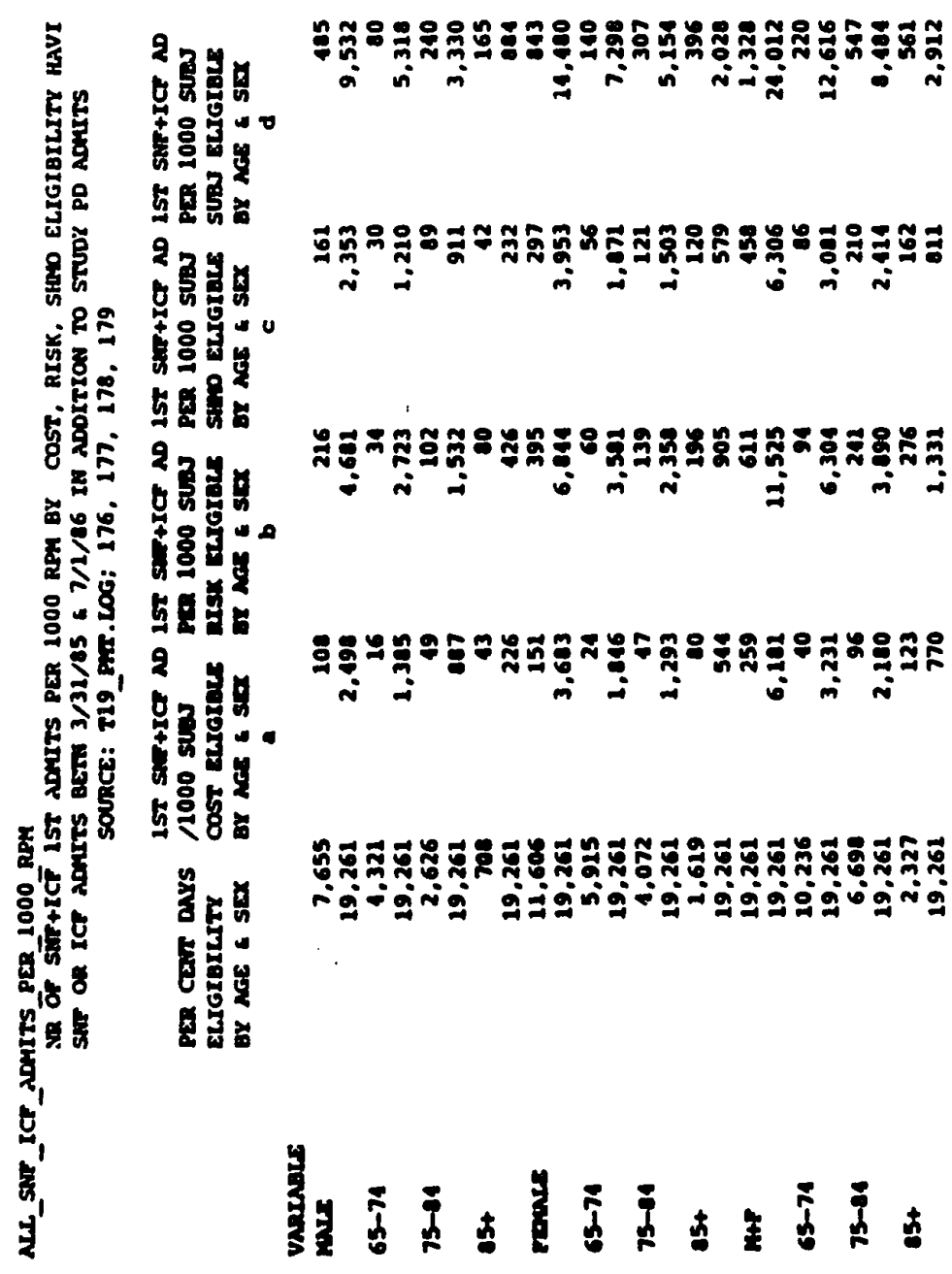




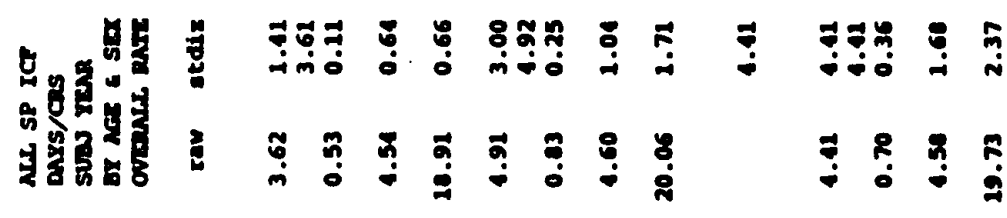

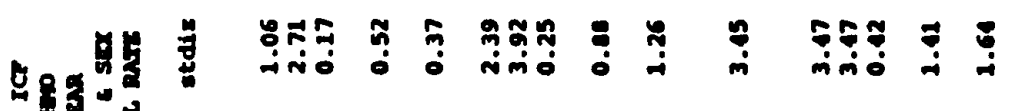

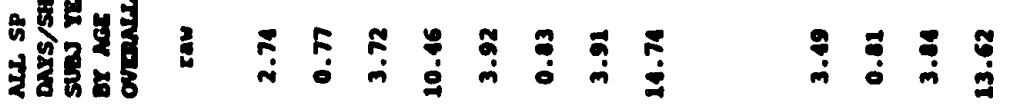

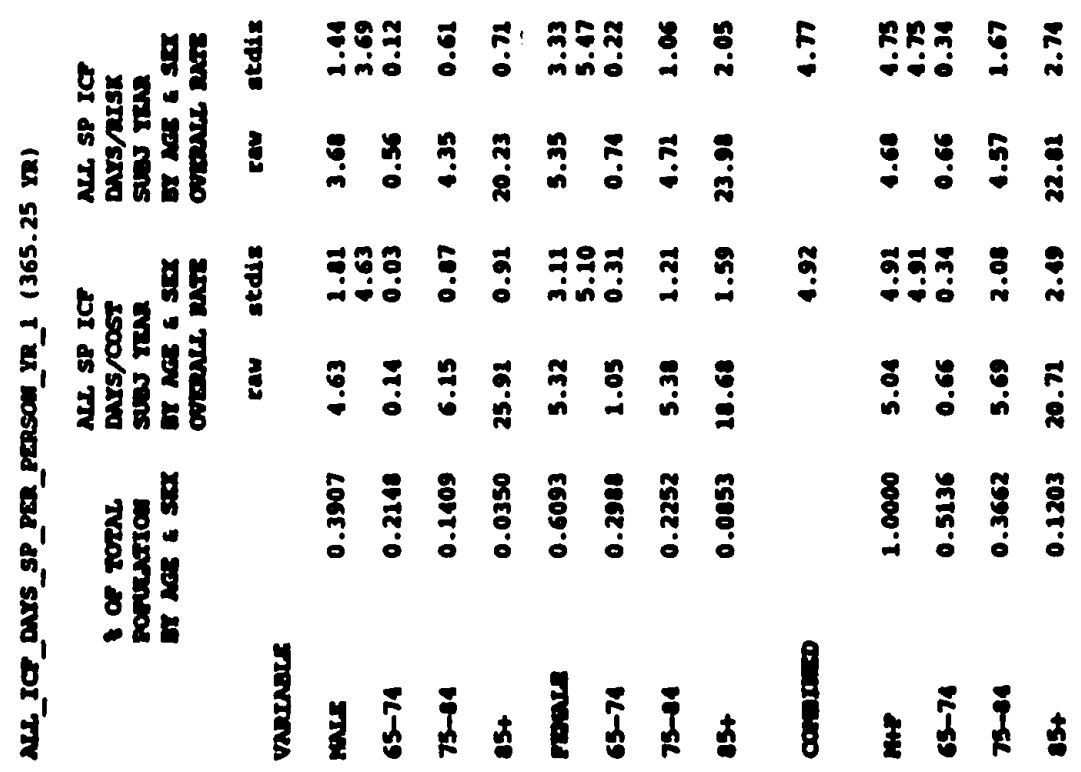




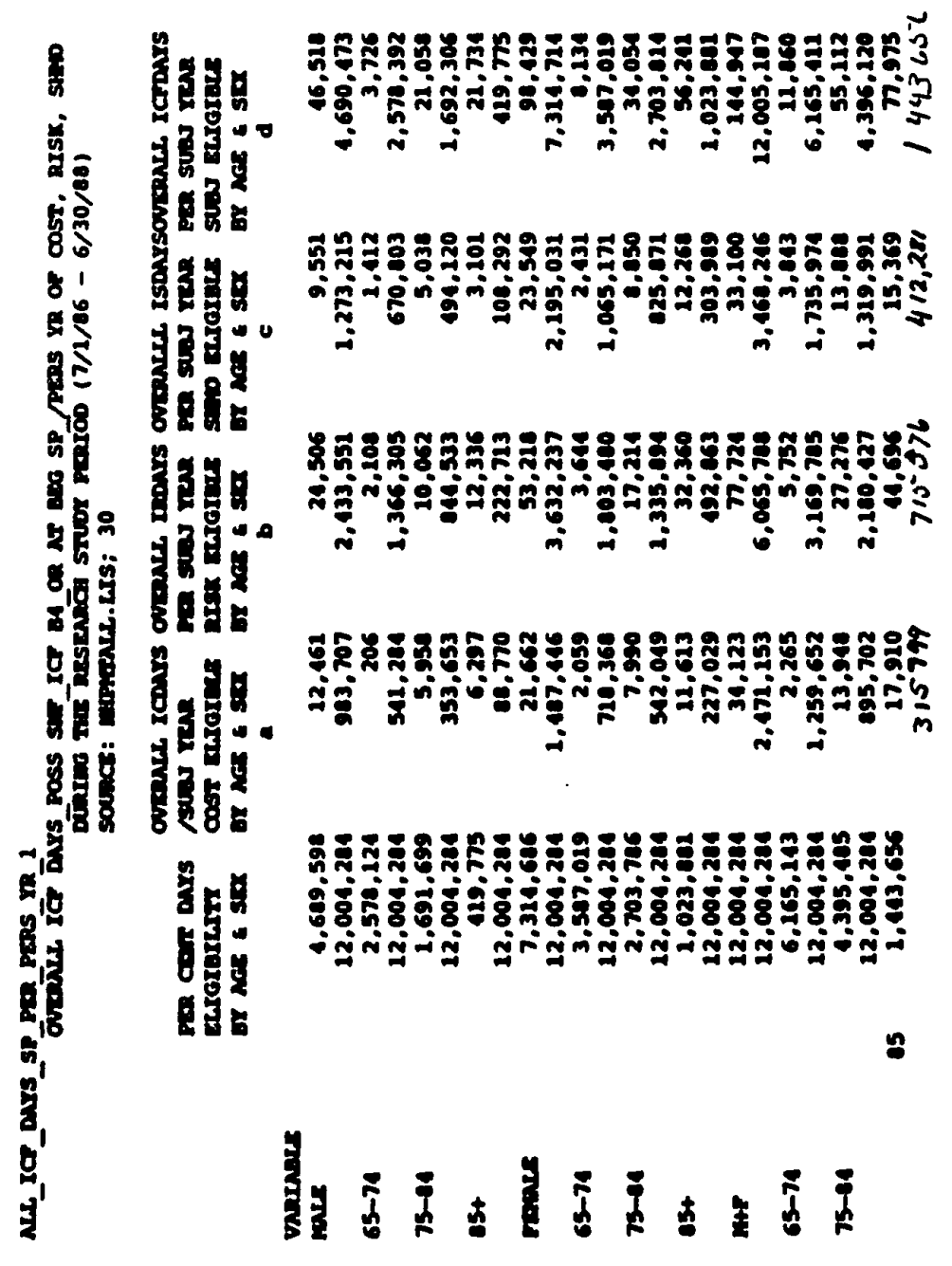


APPENDIX D

FOR CHAPTER V 


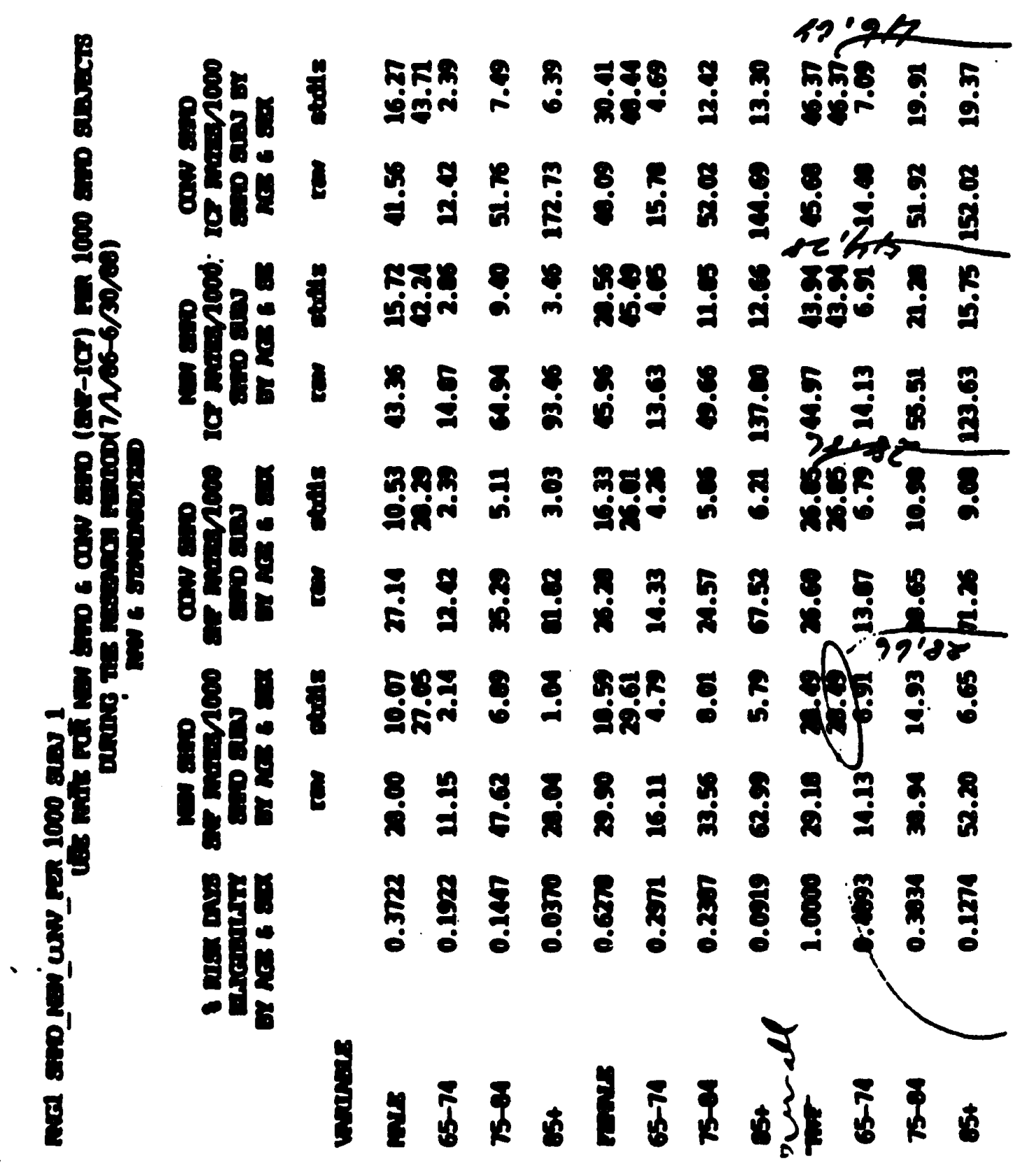




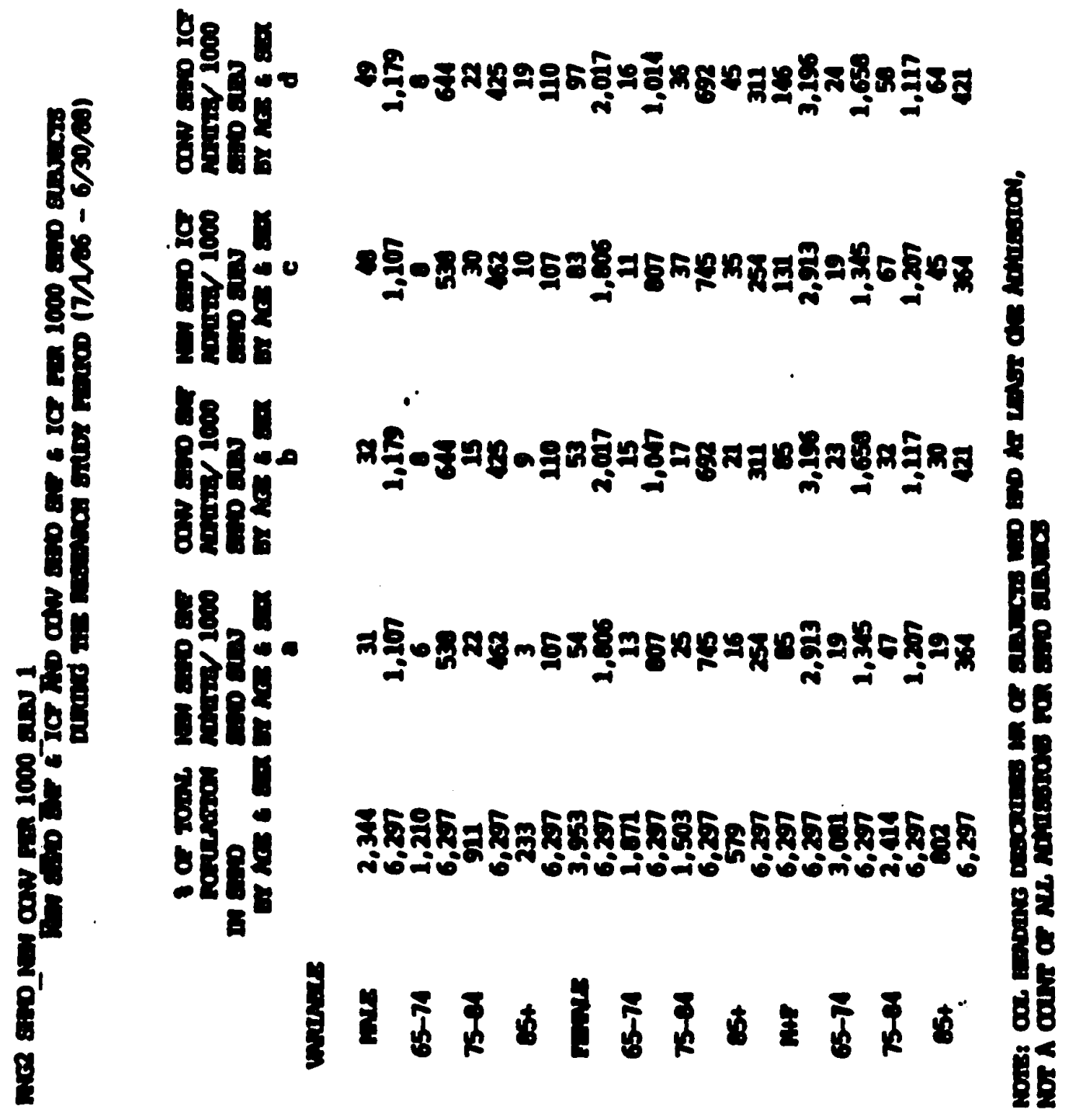




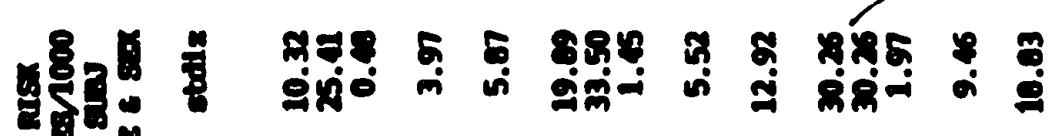
$8 e_{0}^{2}$

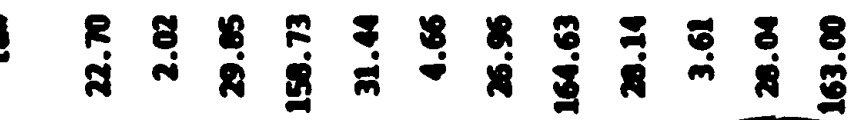

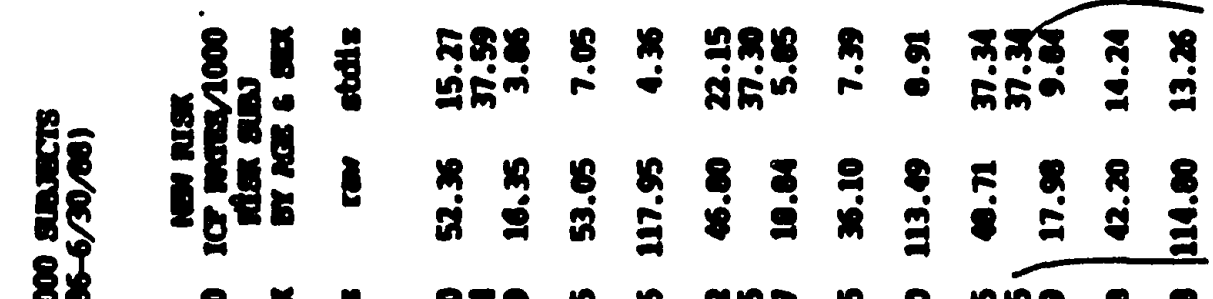

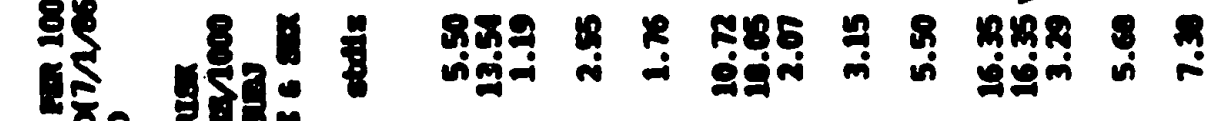

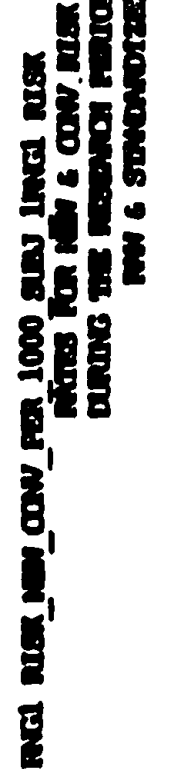
$8 \sin ^{2} \mathrm{c}$

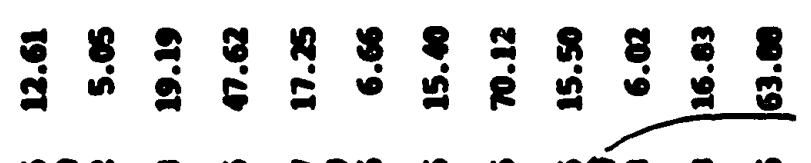

8ิ $\cos ^{2} \mathrm{~g}$

R. 影量

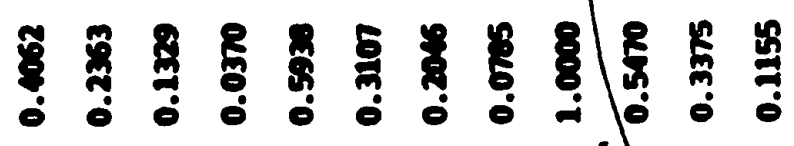
3. 


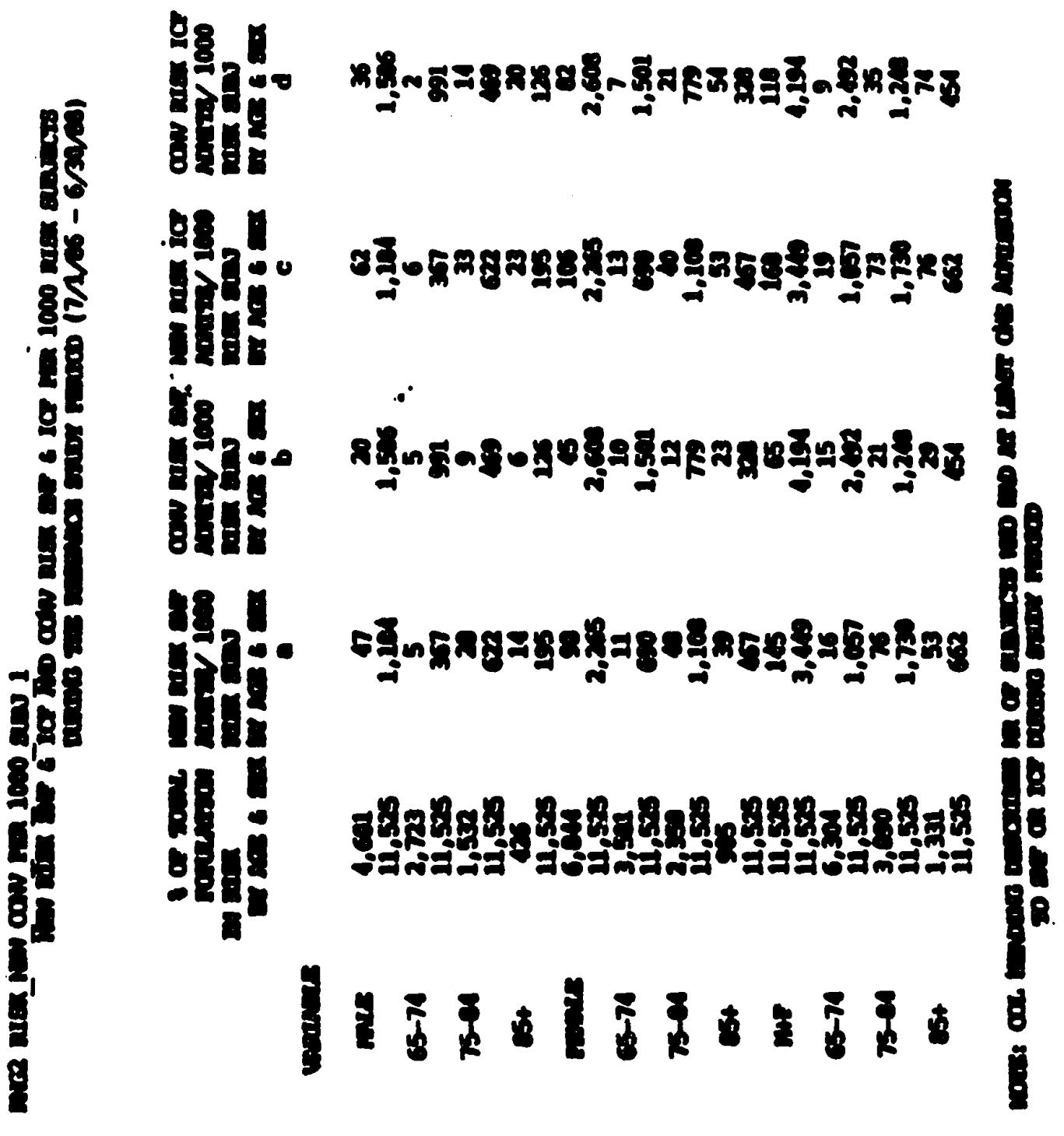




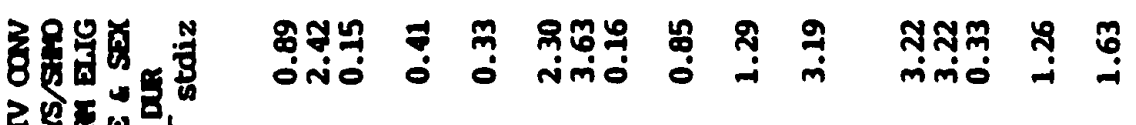

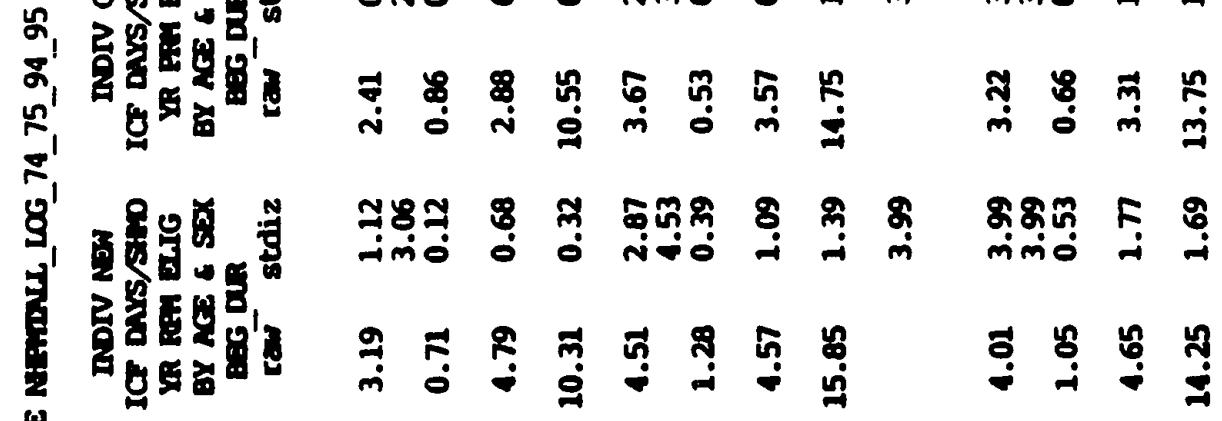

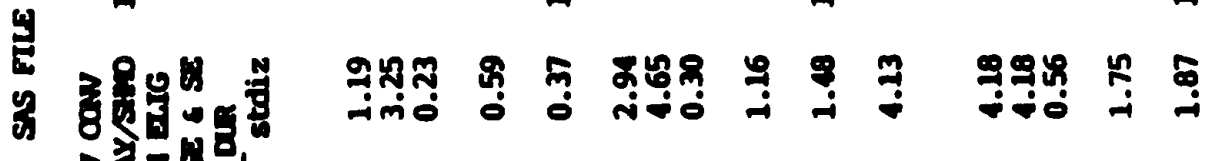

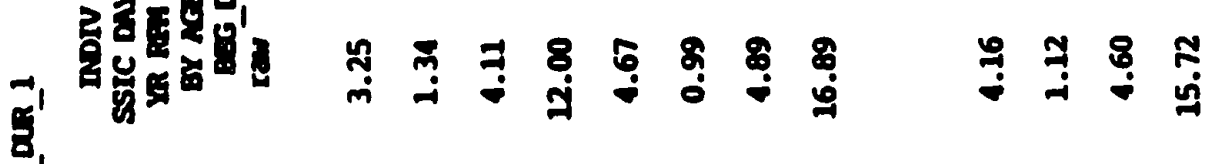
ه

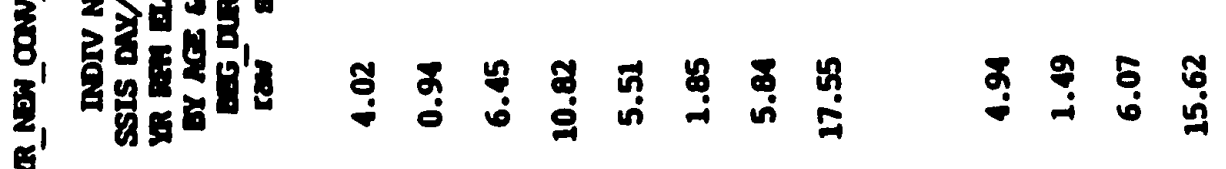

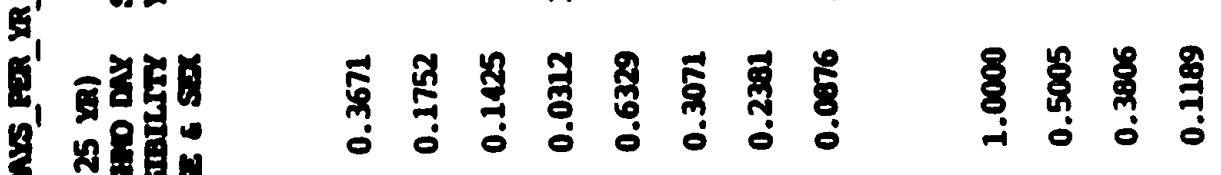

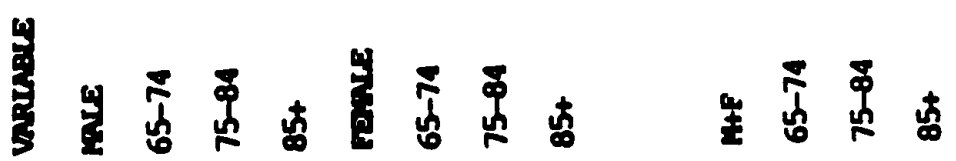




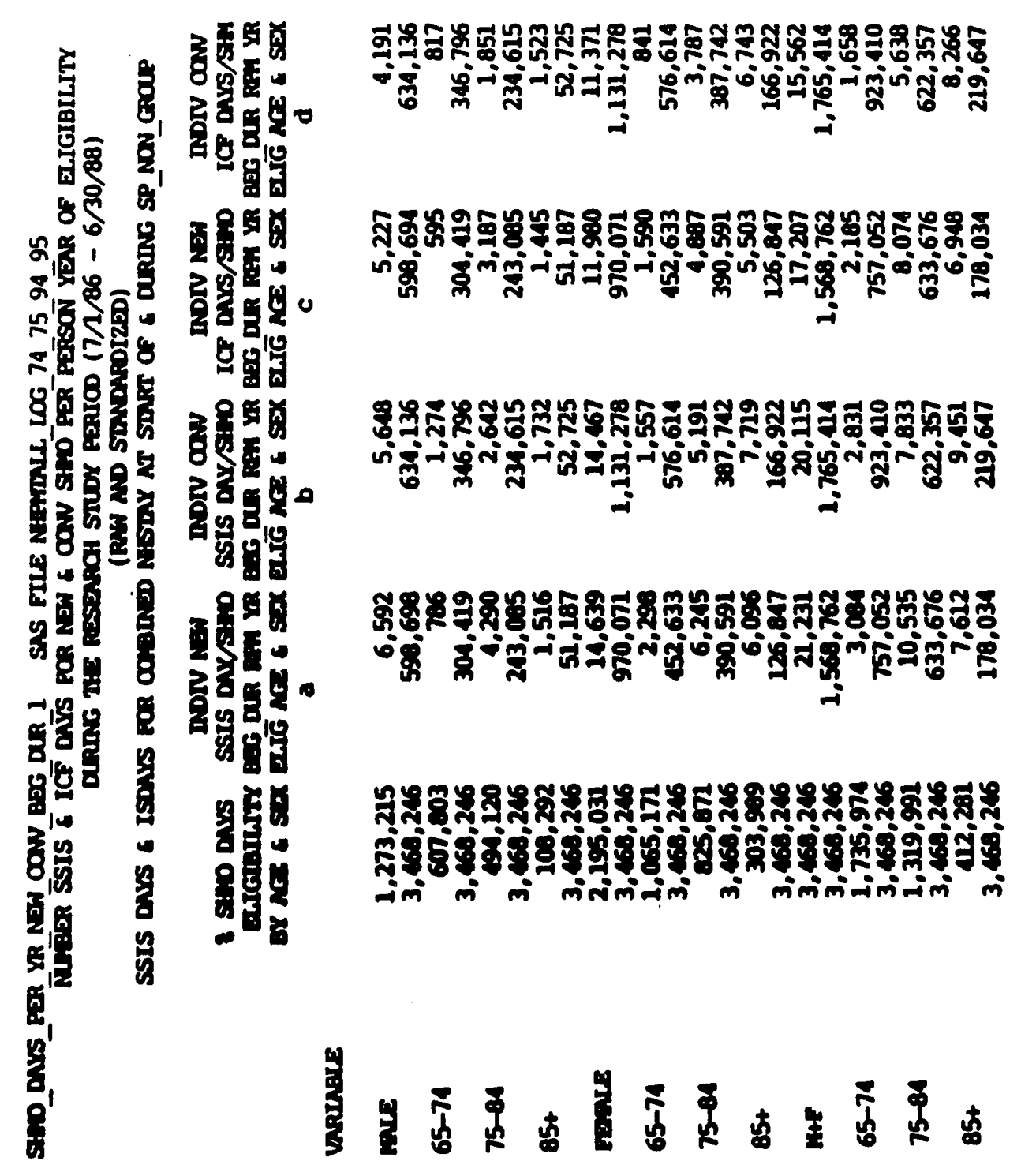




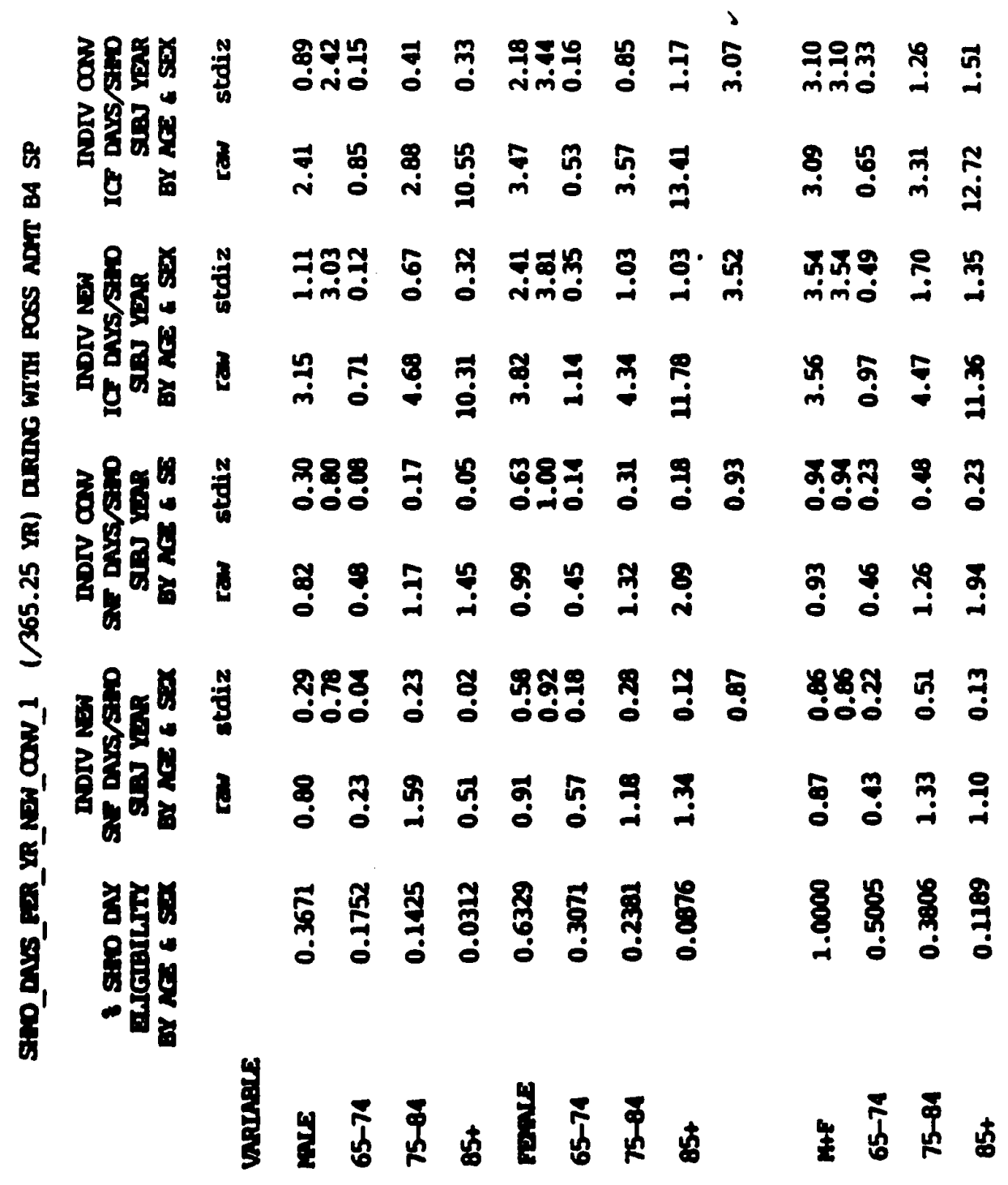




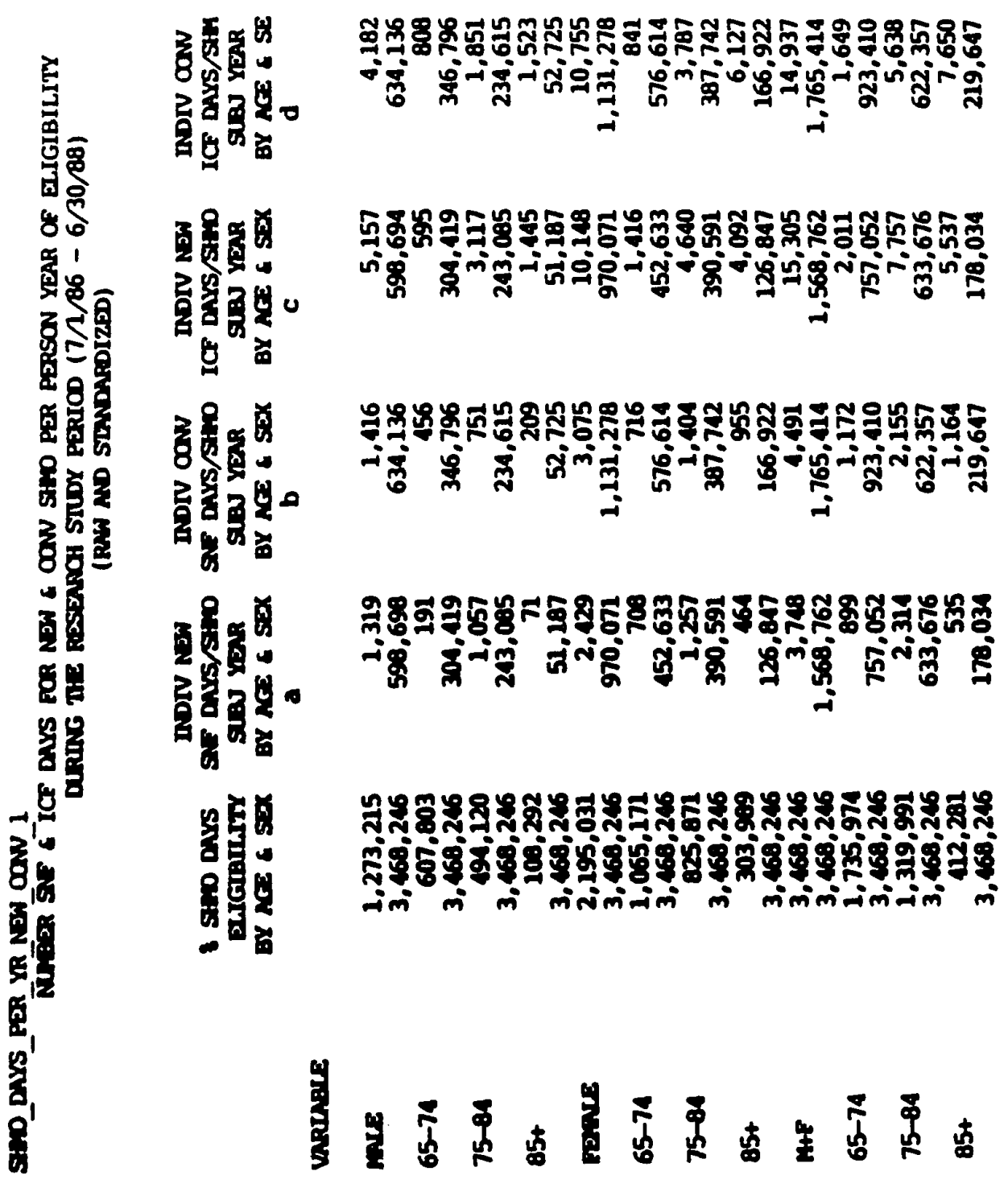




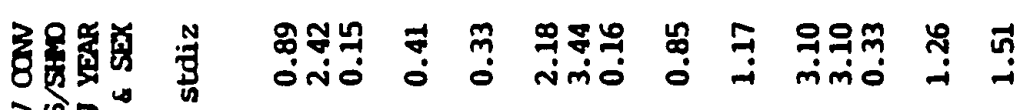

的阳

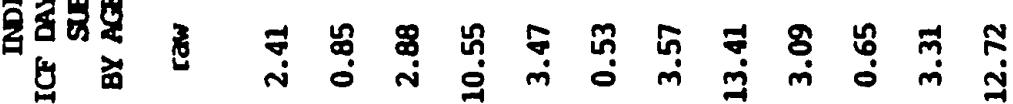

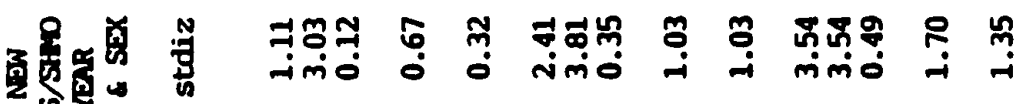

政

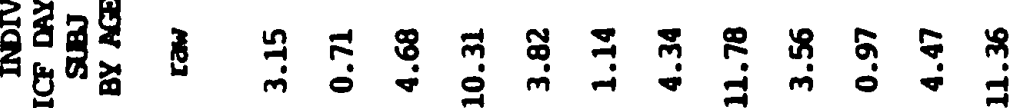

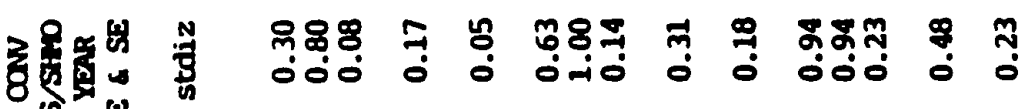

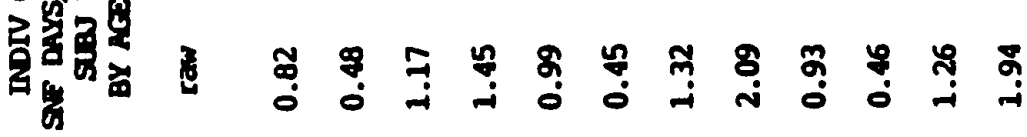

ณึ่

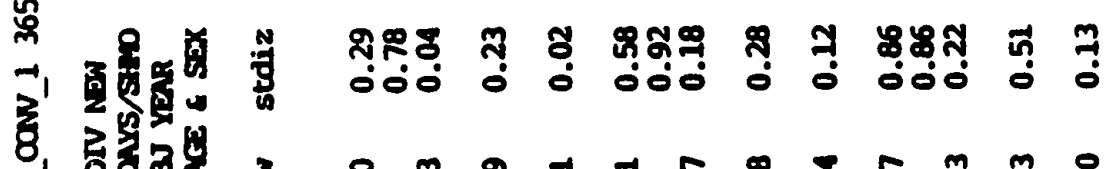

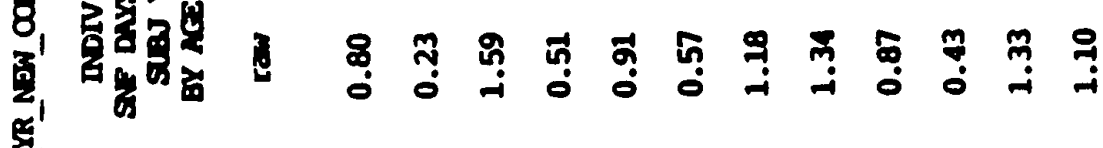

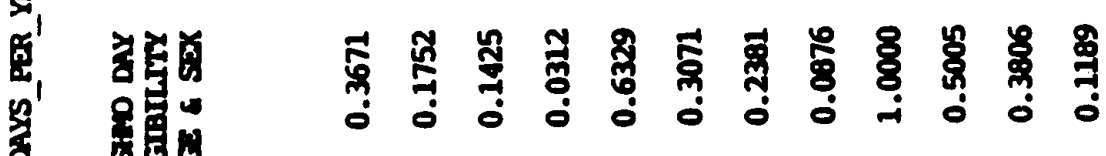

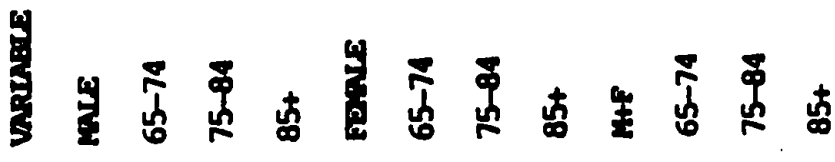




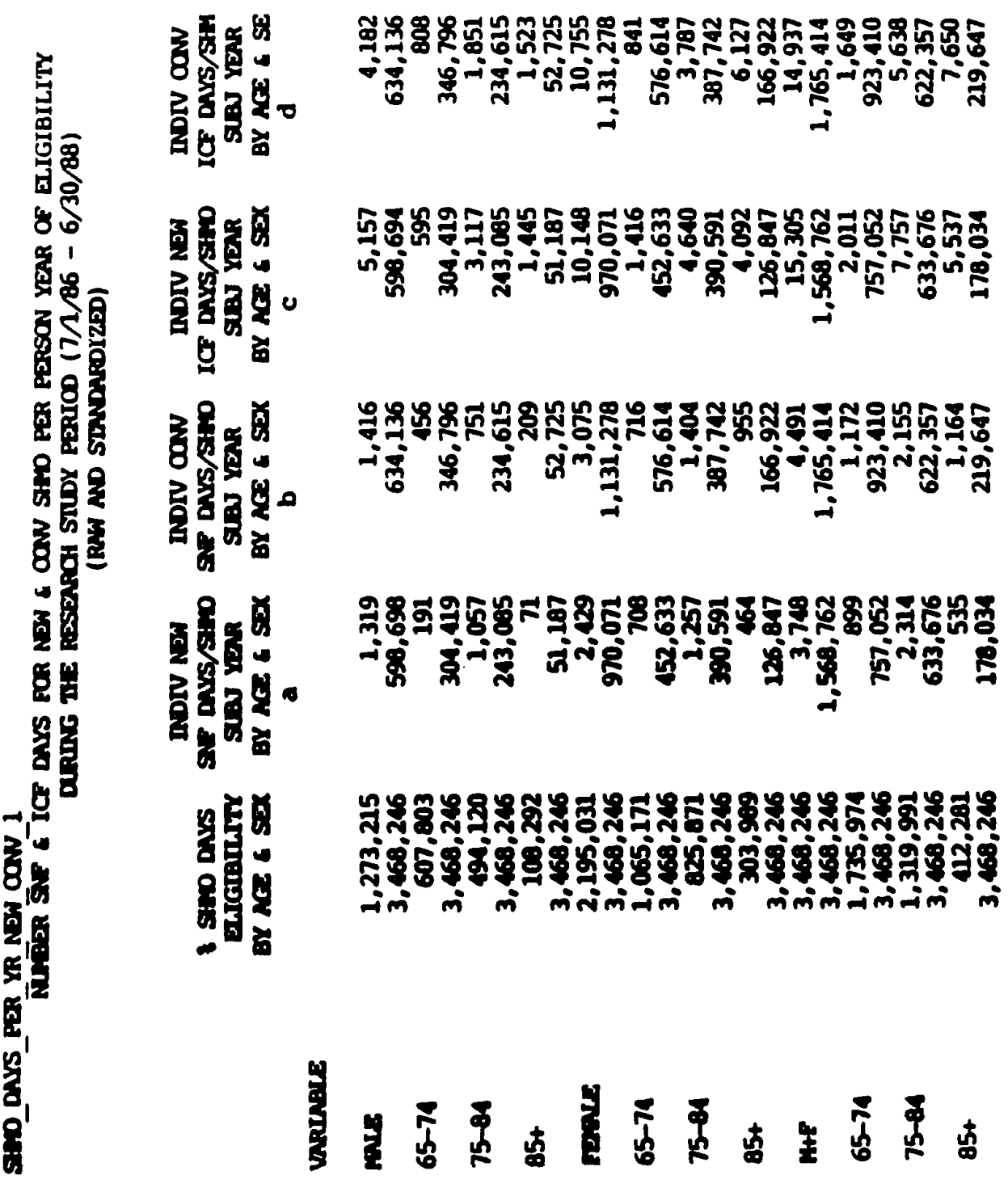




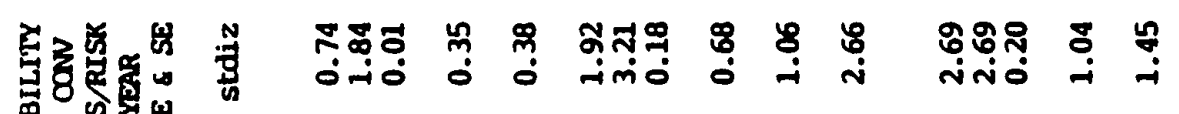

密实

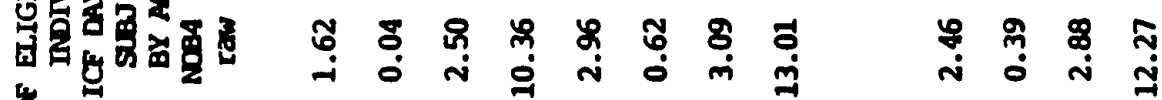

5

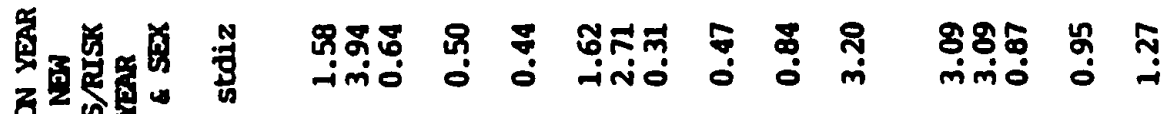

잉

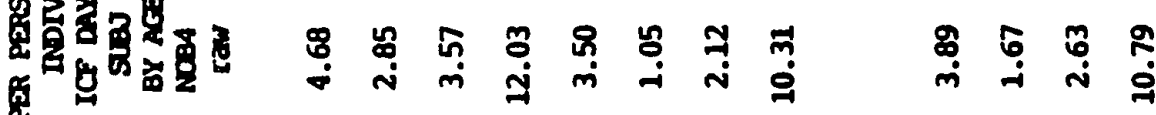

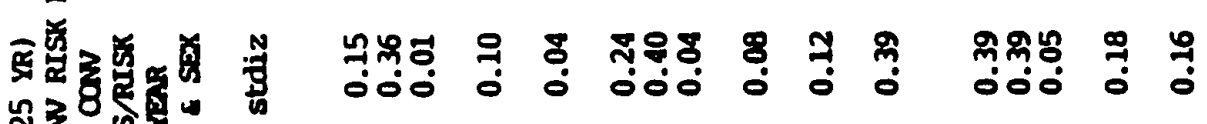

年层

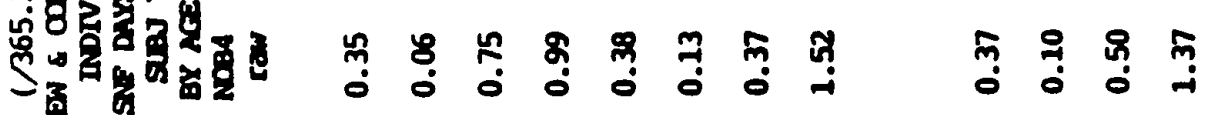

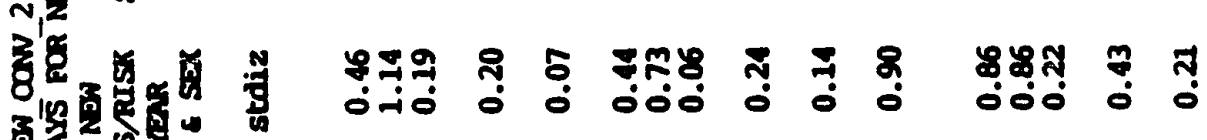

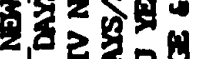

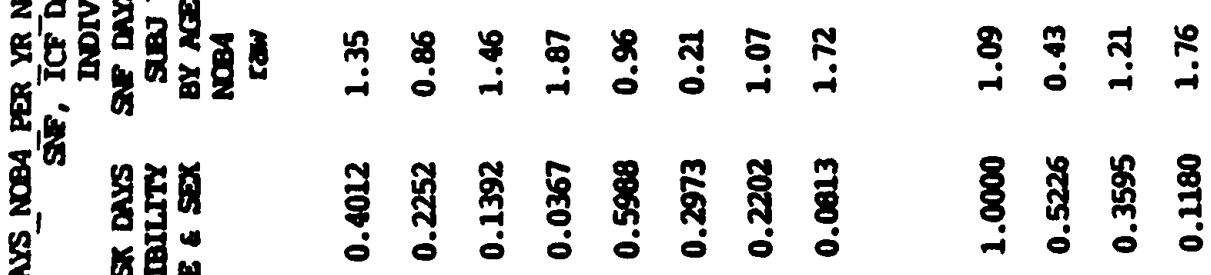

告

部面

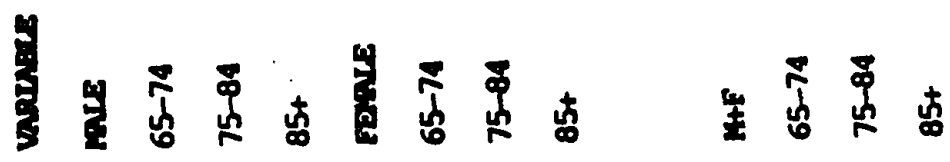




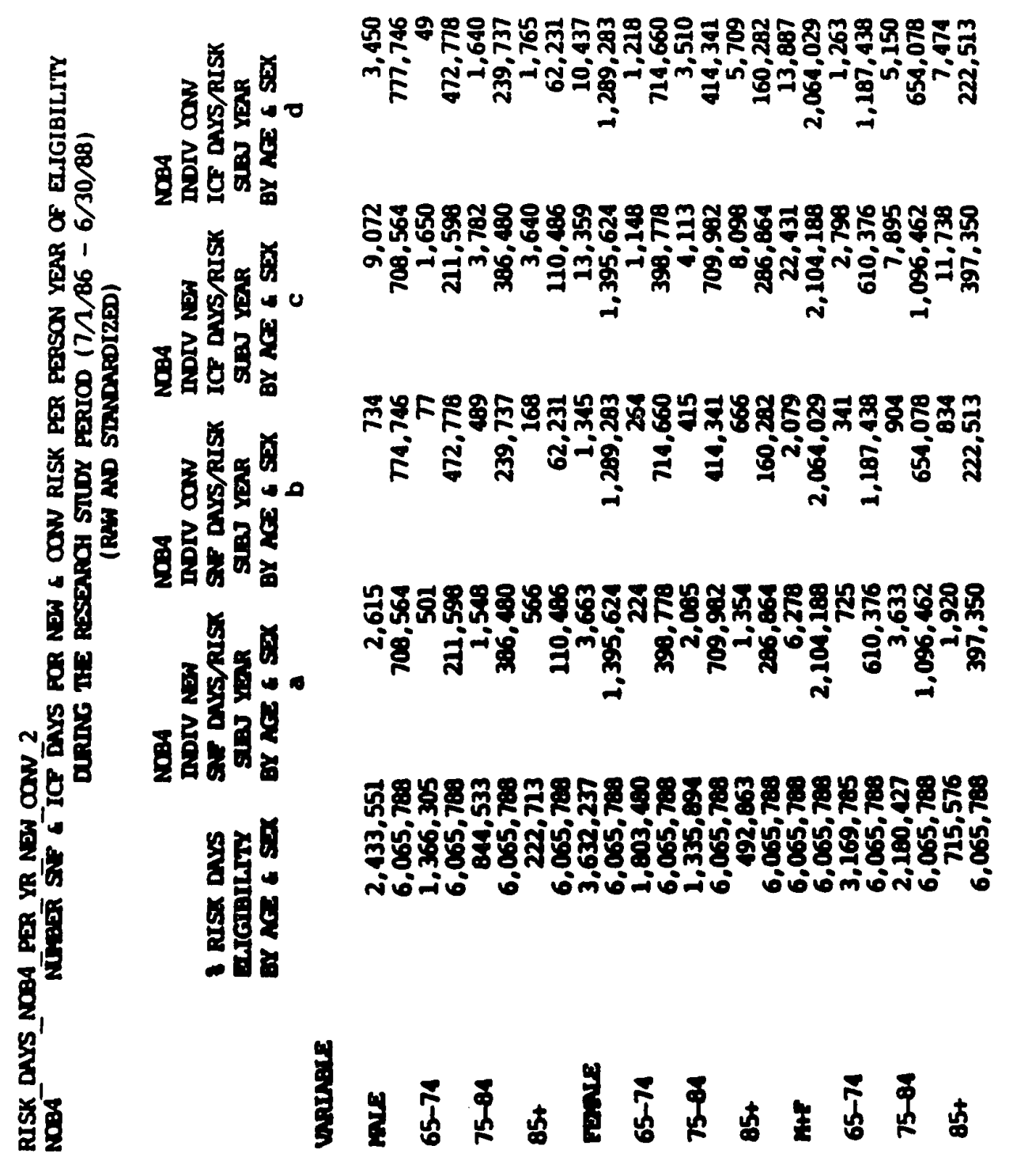




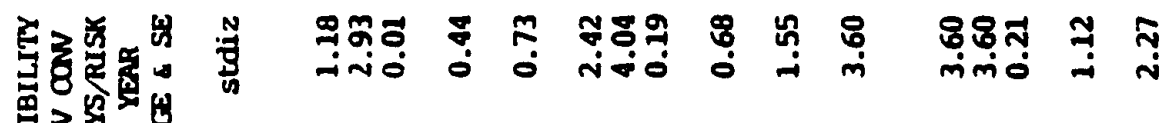

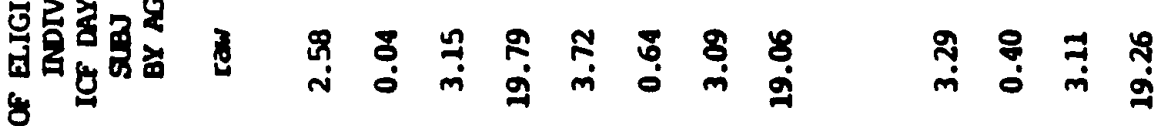

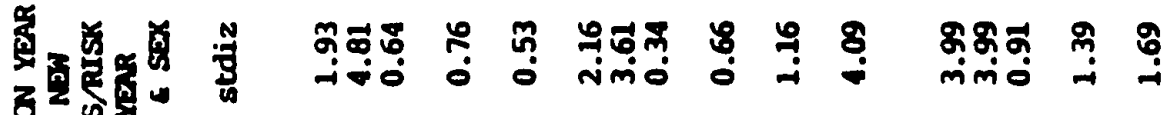

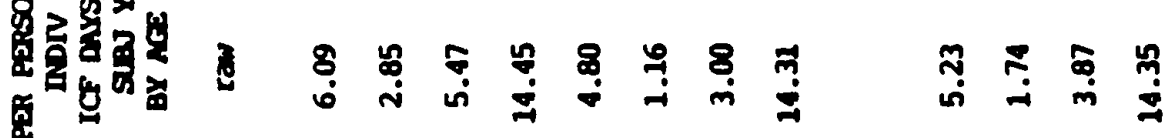

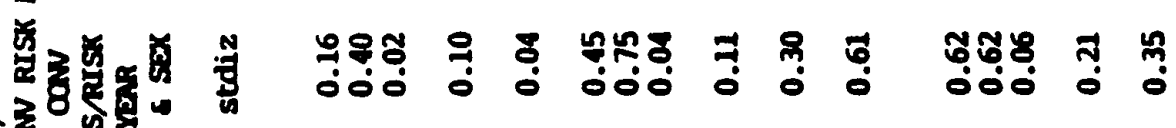

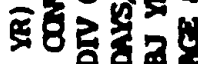

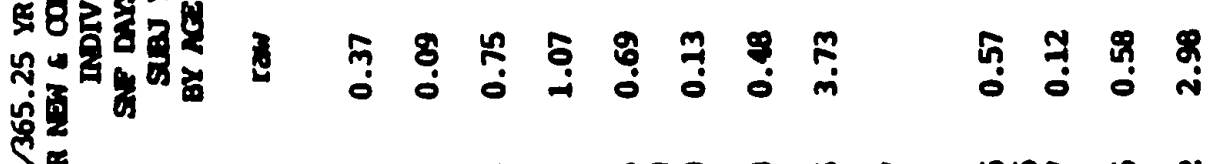

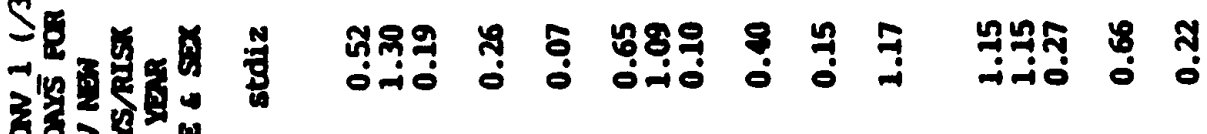

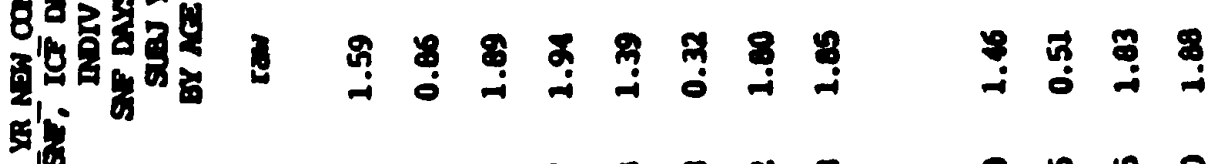

憵

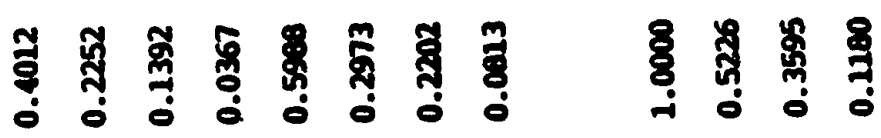

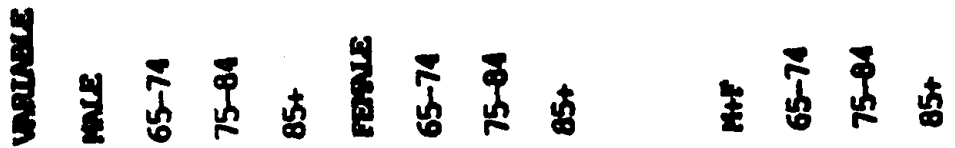




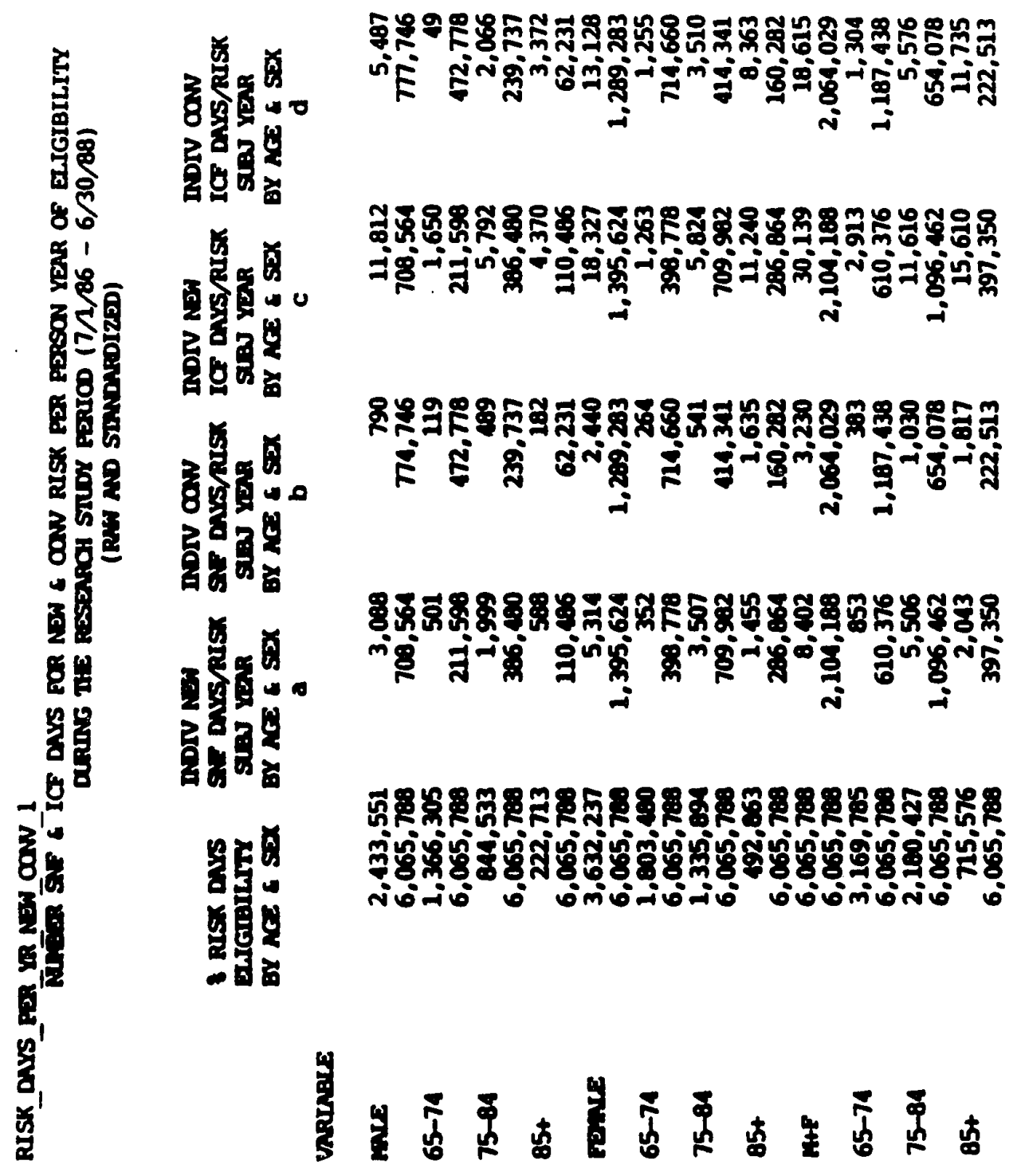




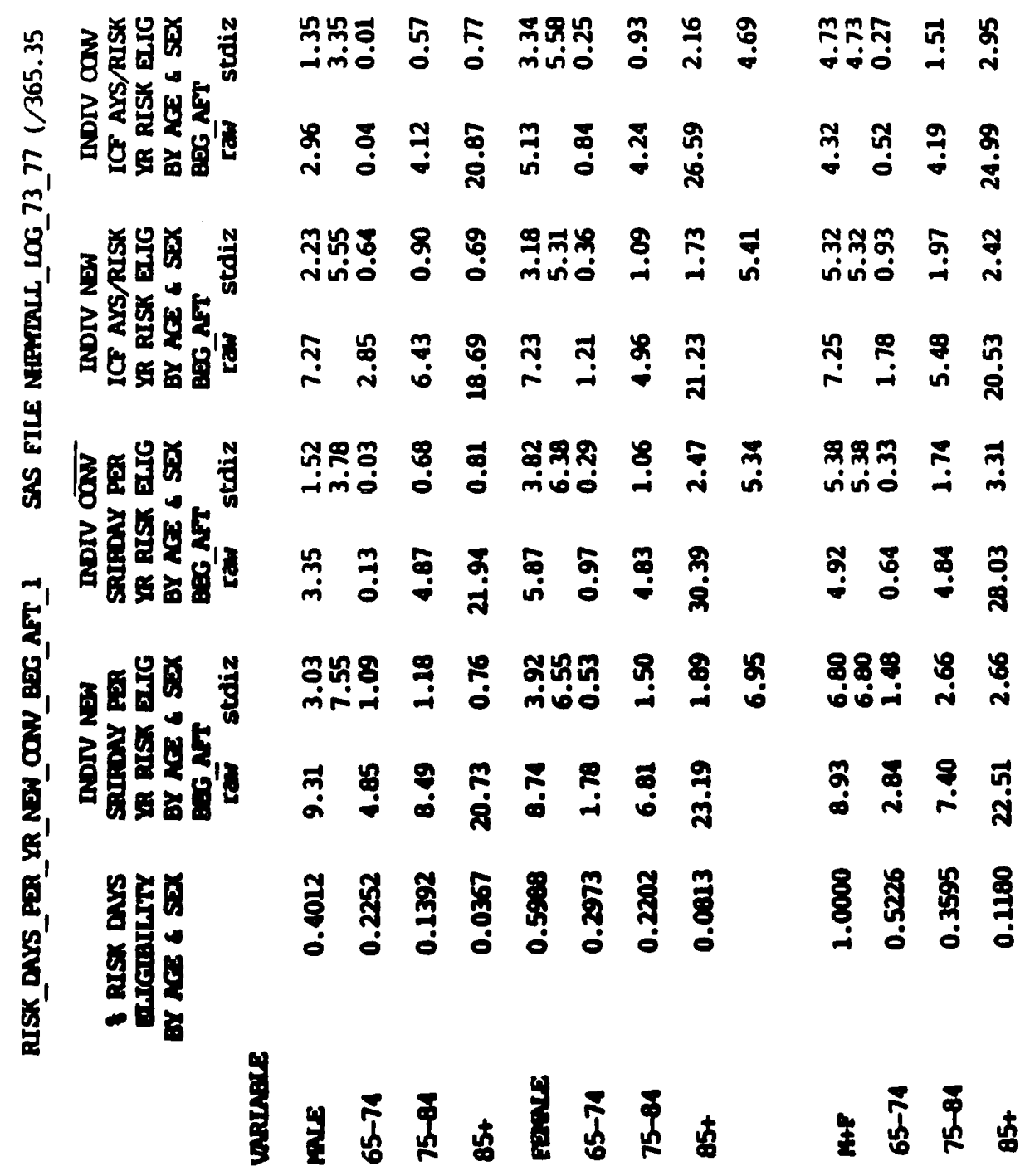




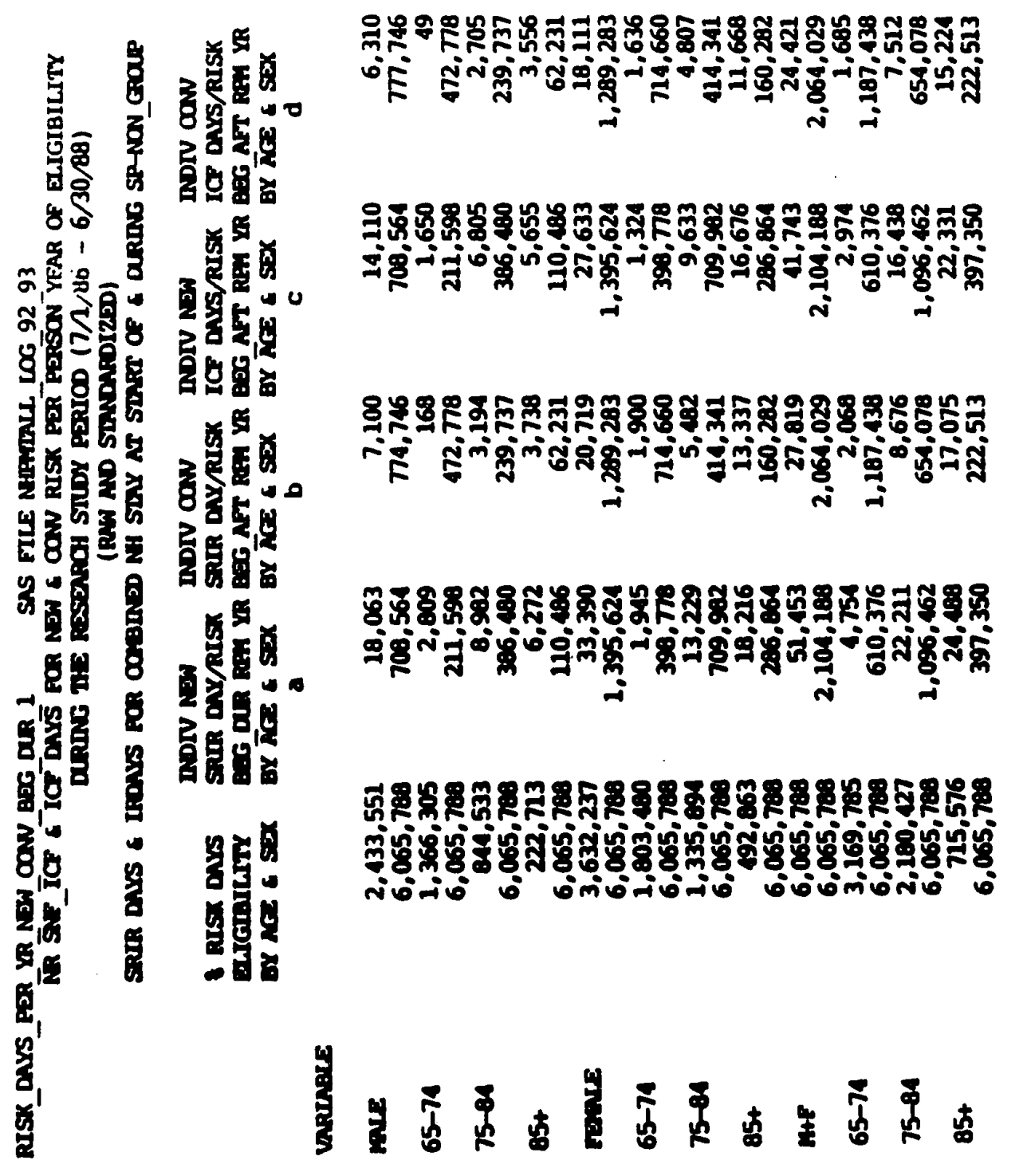




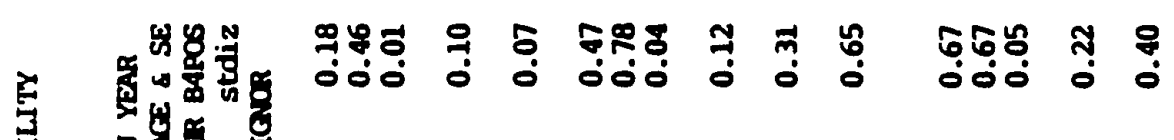

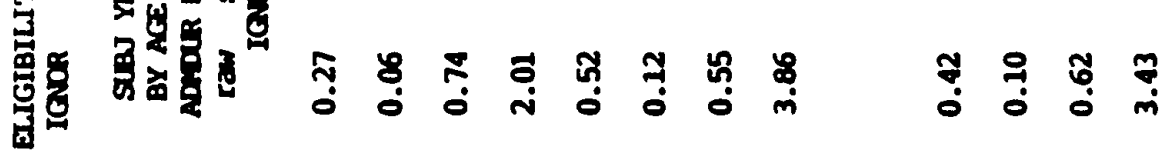

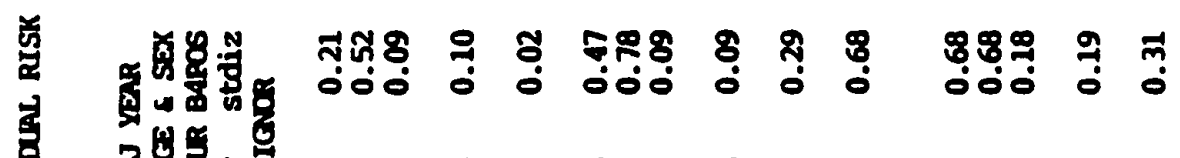

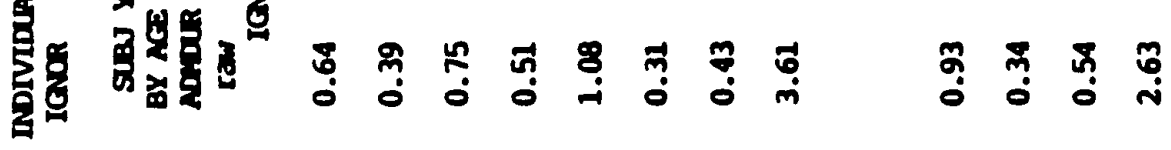

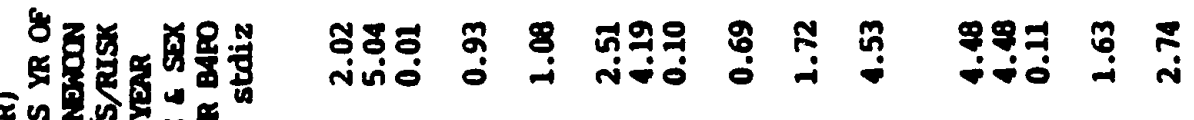
ang

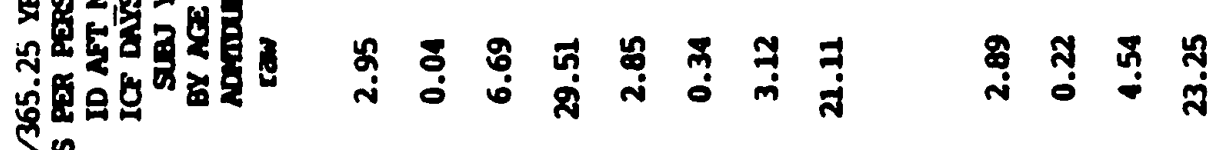

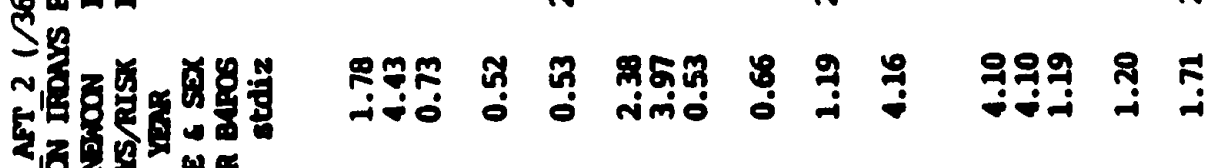

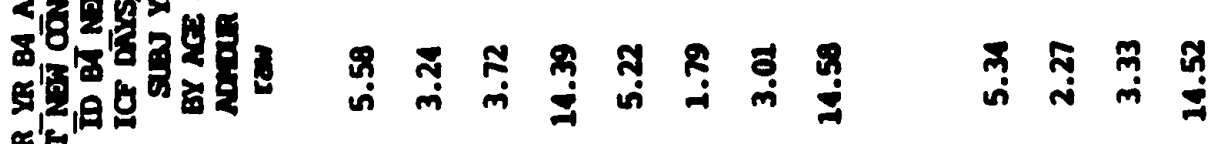

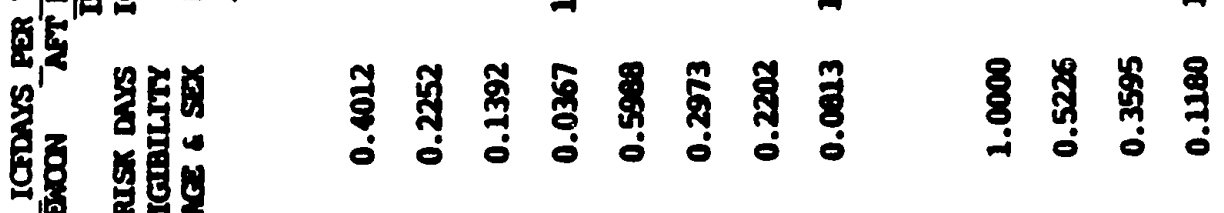
譬

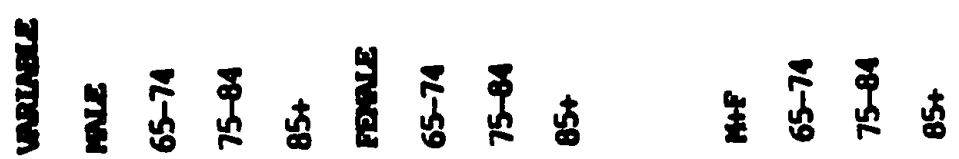




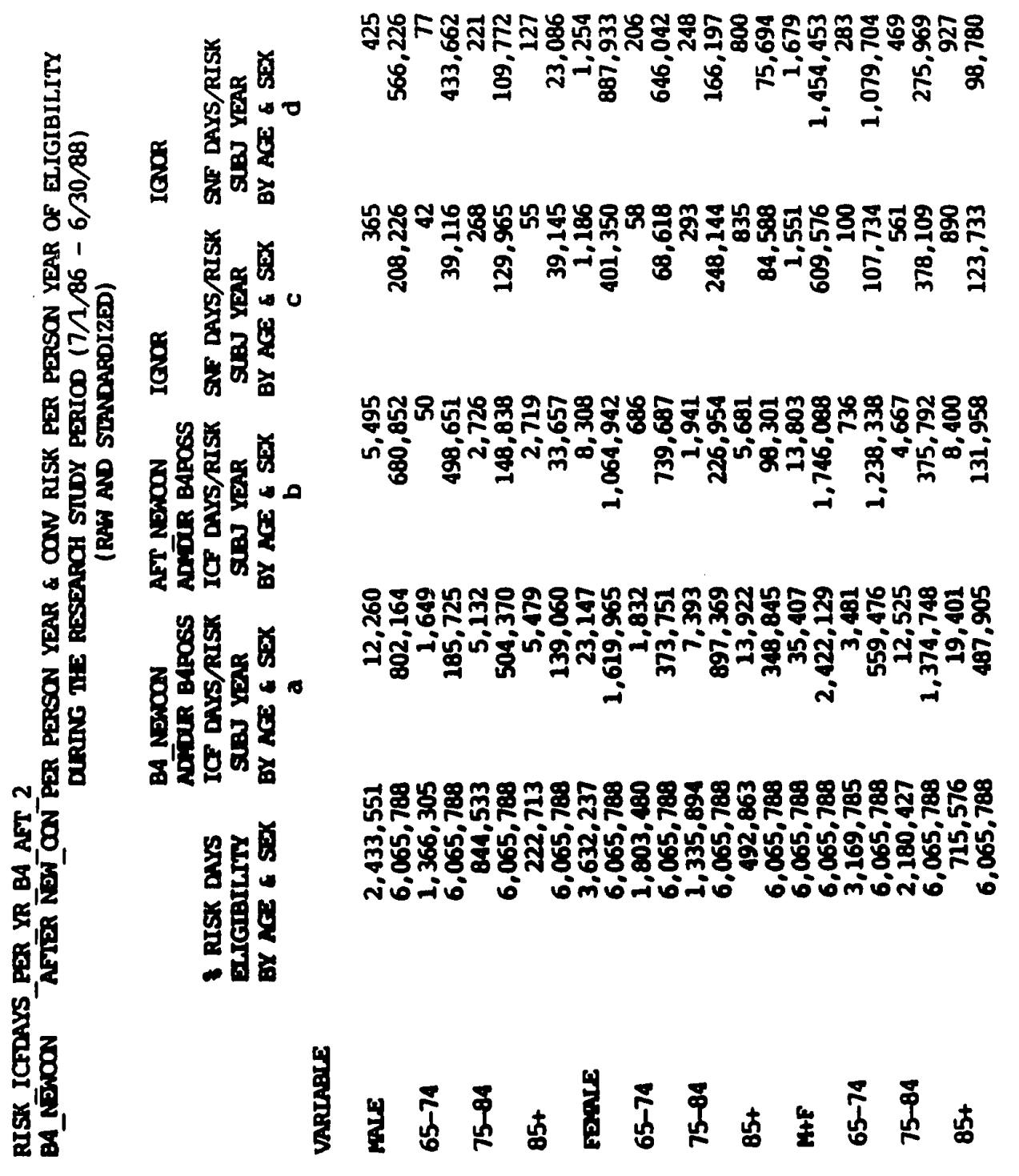




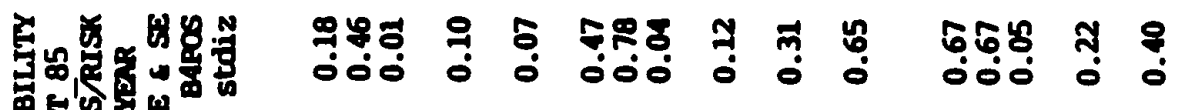
(1)

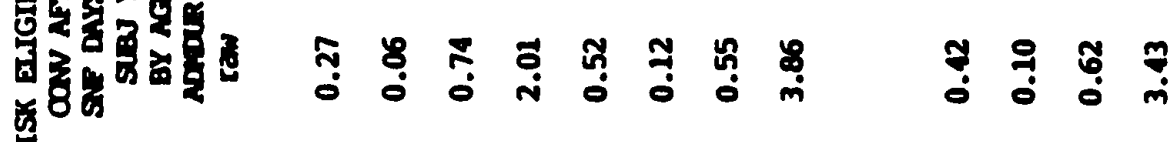

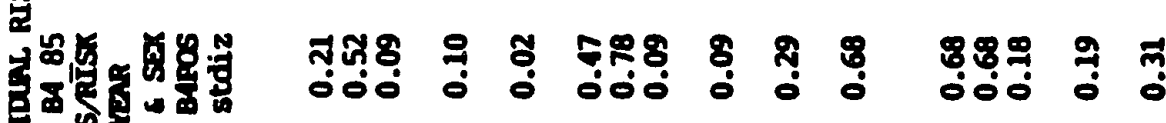

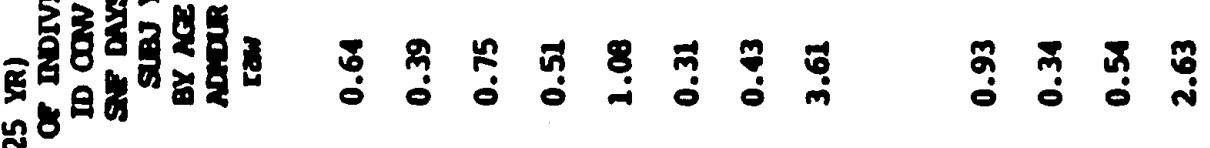

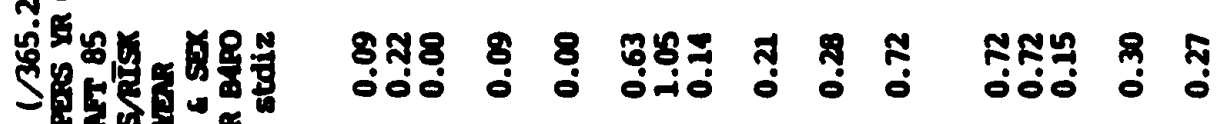

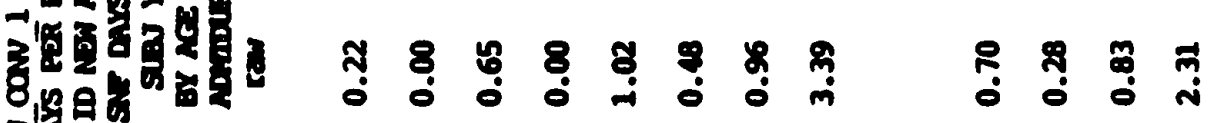
解

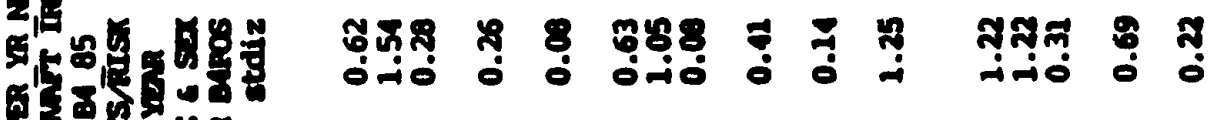

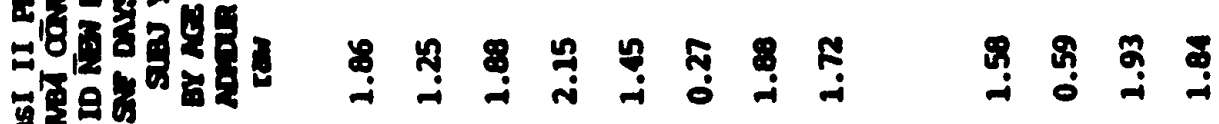
琶醇

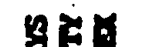

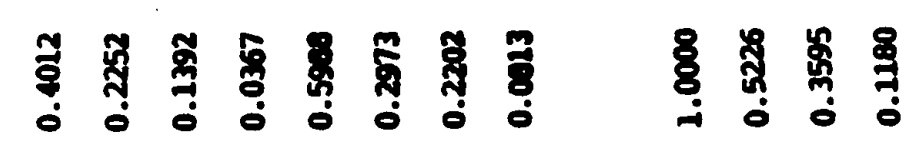

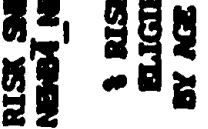

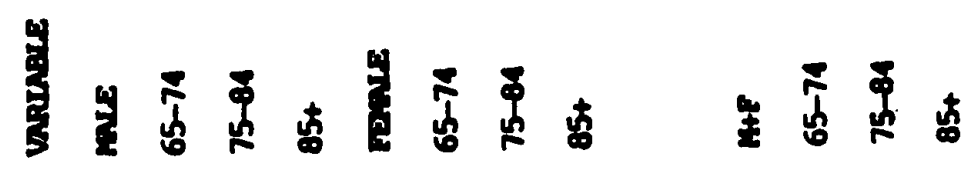




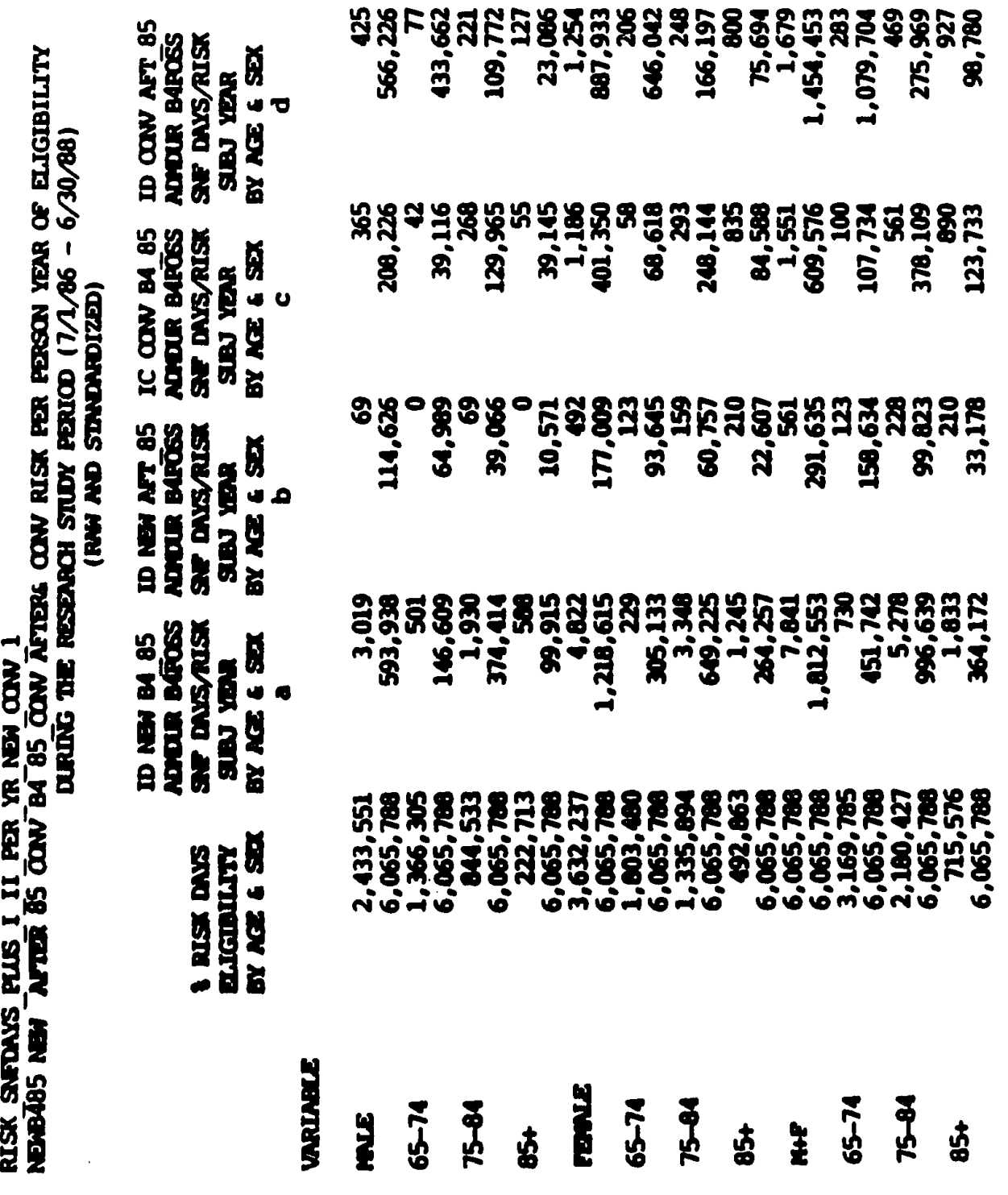




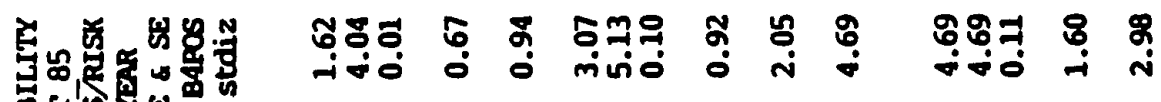

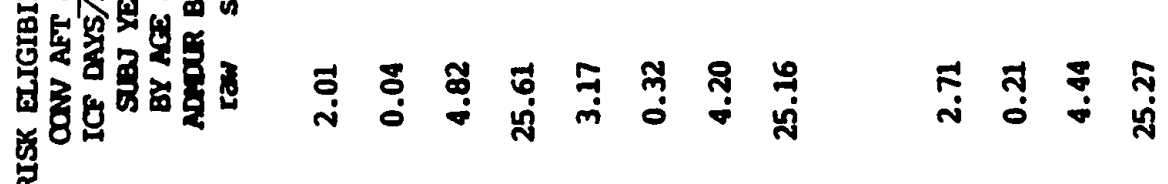

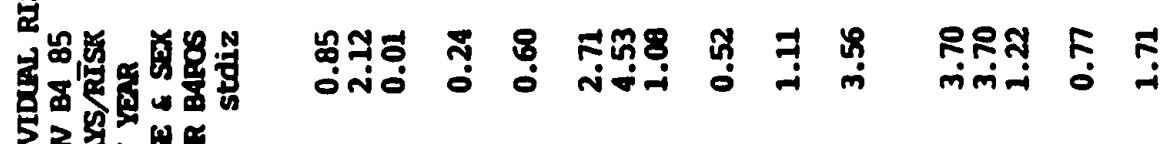

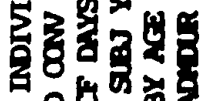

ริง

ว

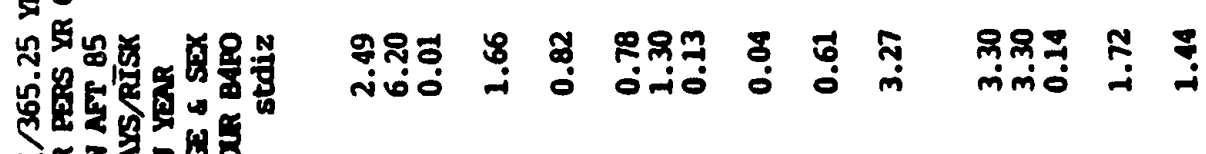

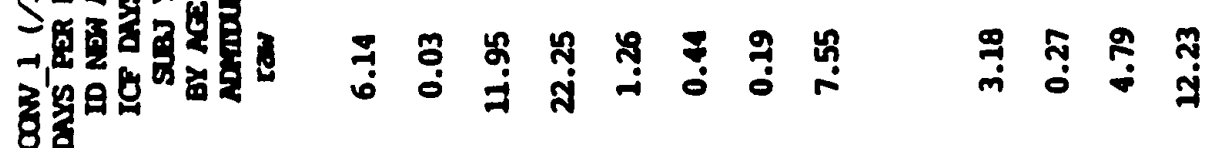

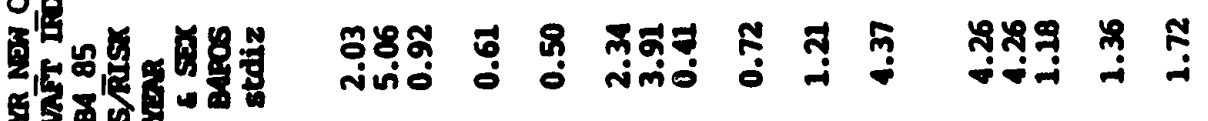
S?

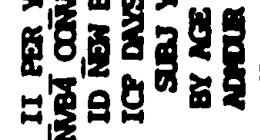

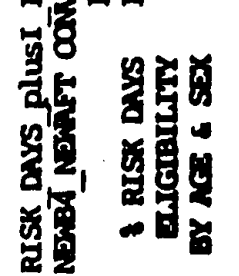

\&

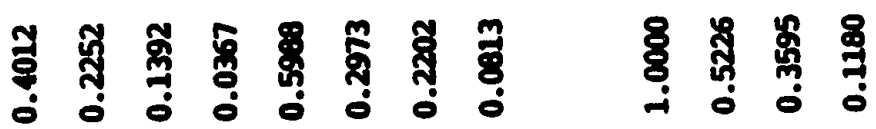

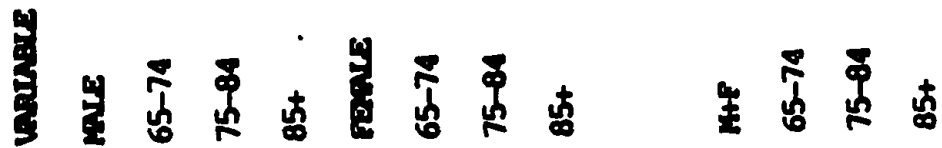




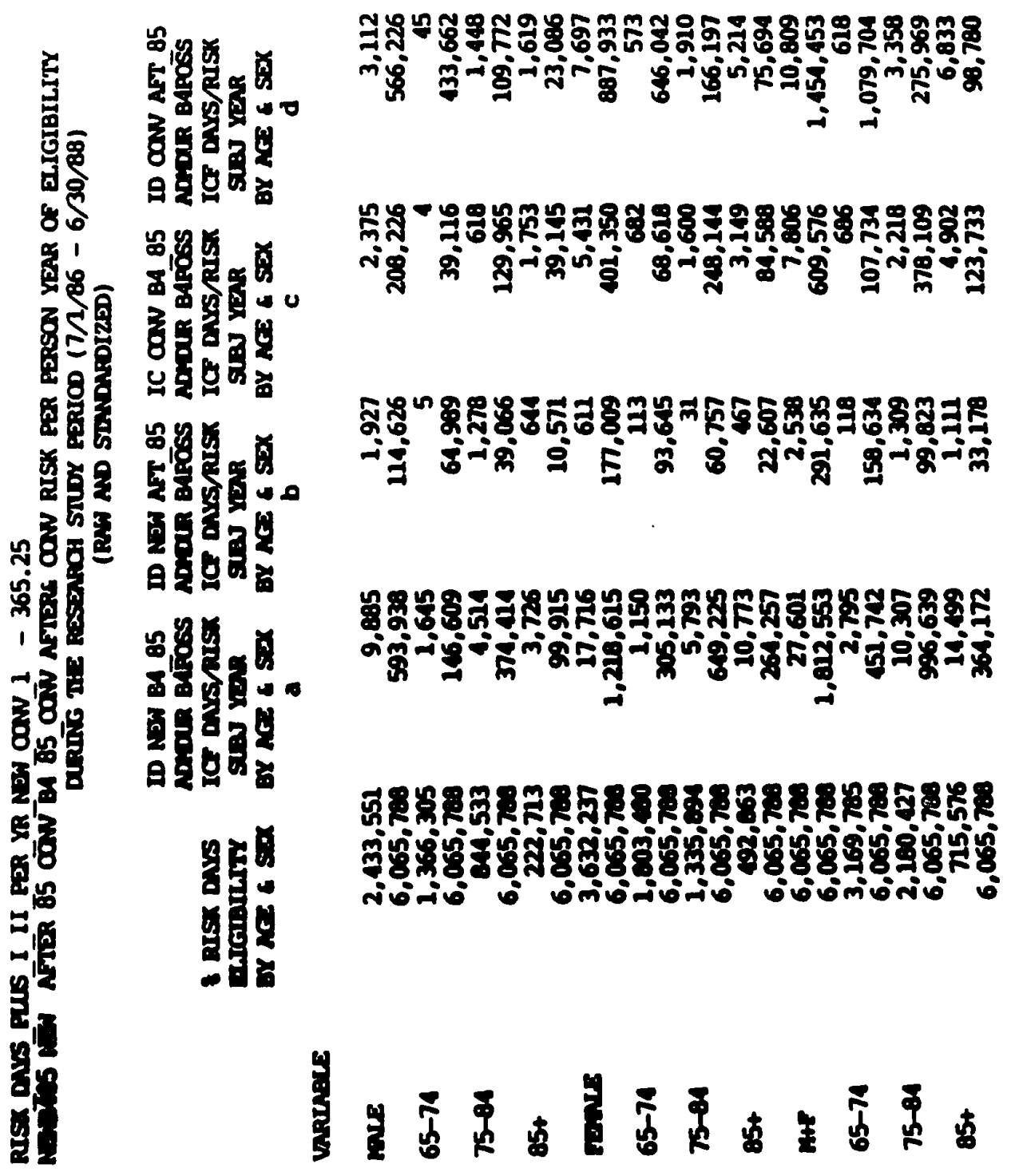


APPENDIX E

FOR CHAPTER VII 


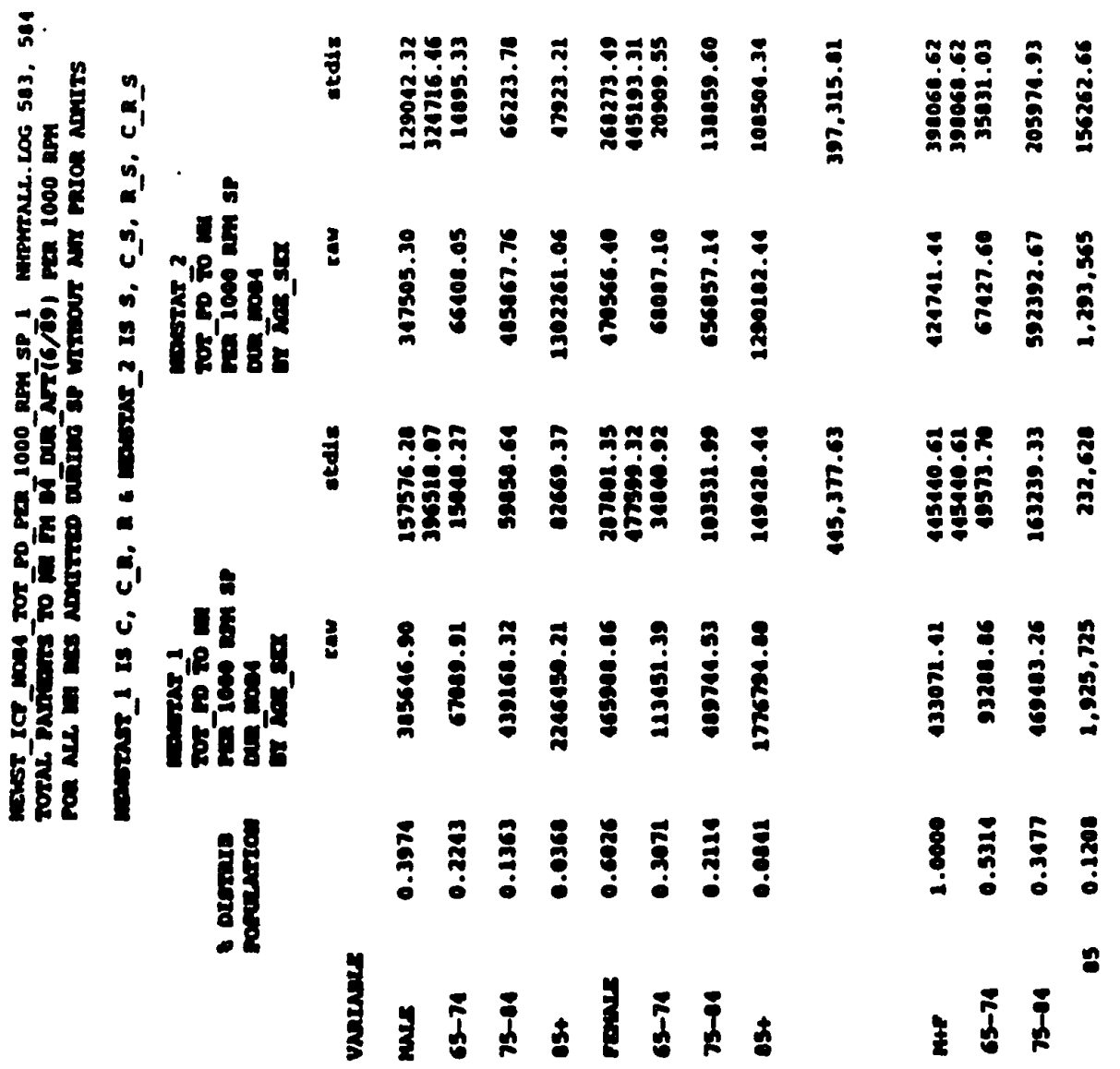




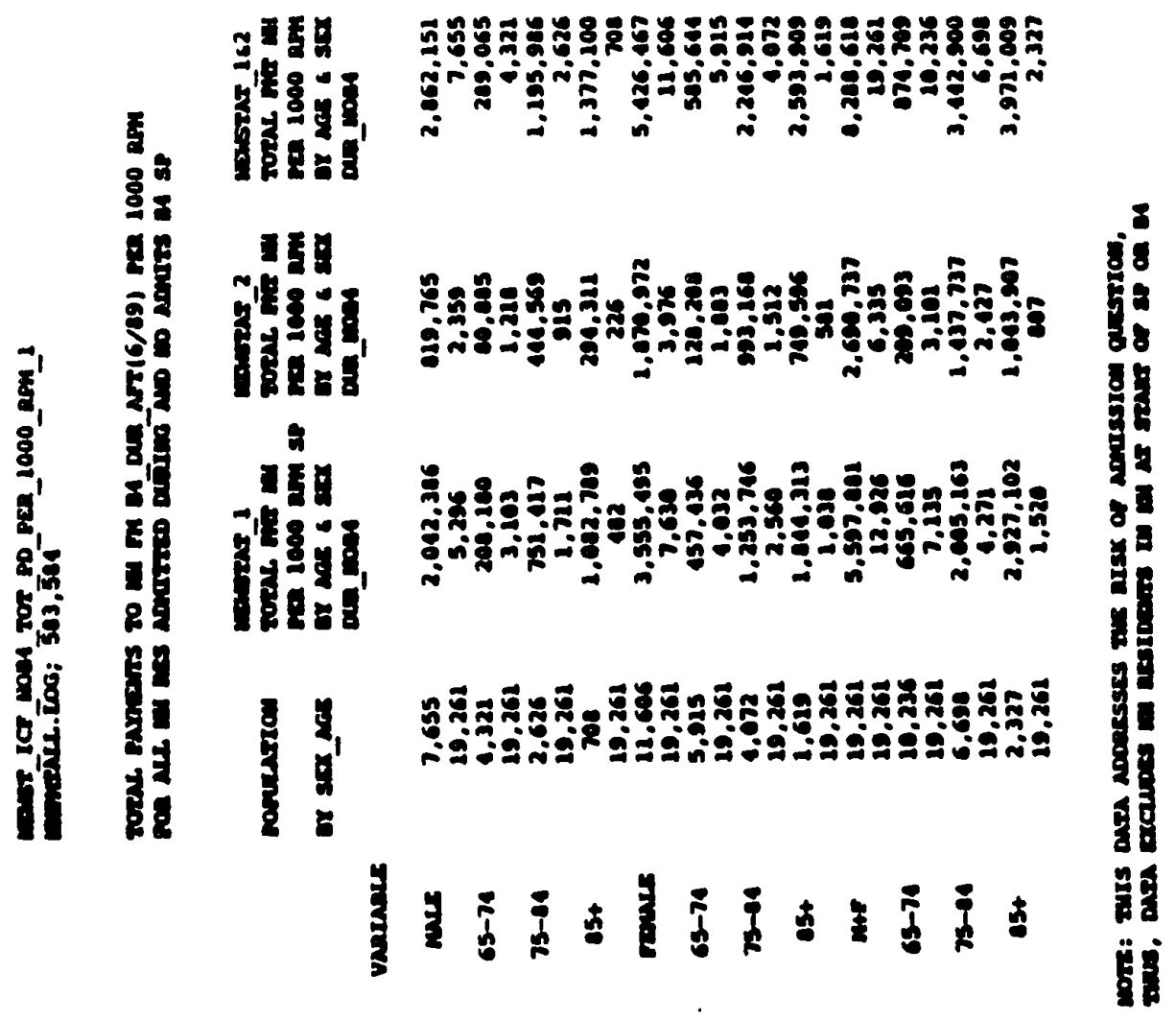




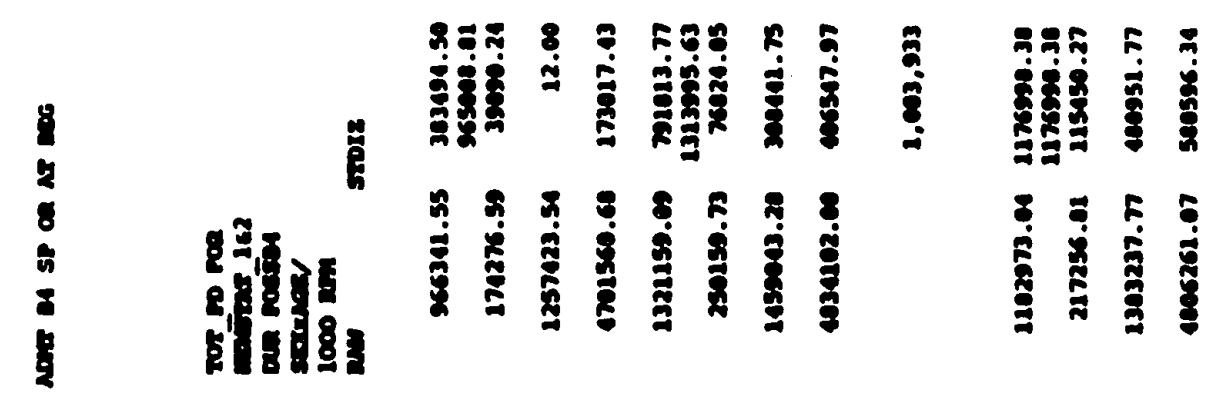

\section{ติ}

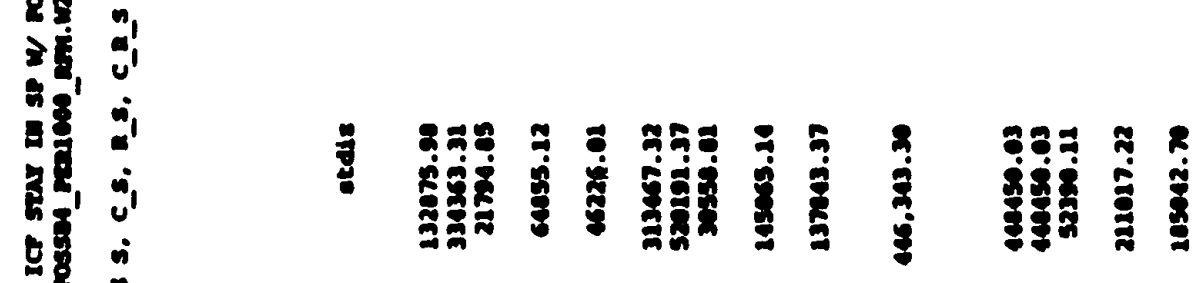

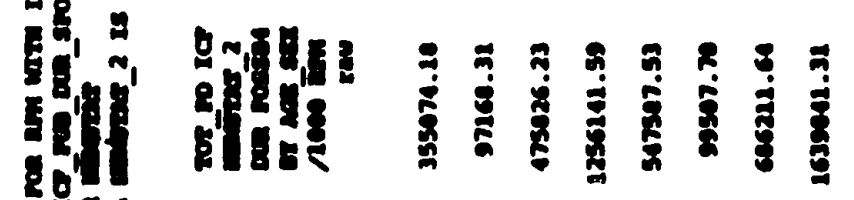

III

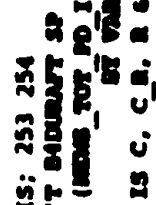

है

$8 \rightarrow x^{5}$

ปै

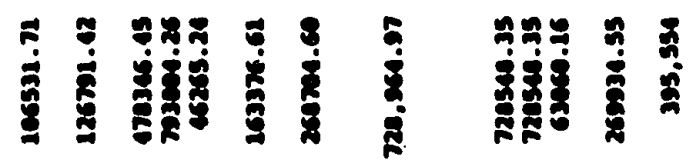
है8

5

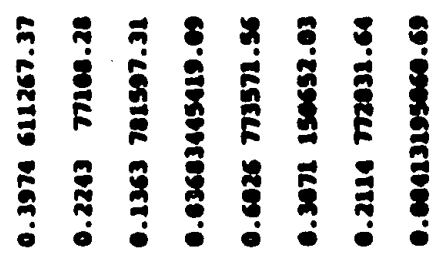

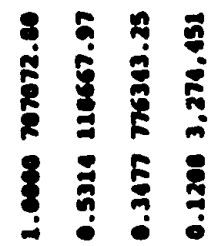

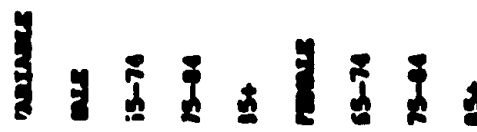

i 


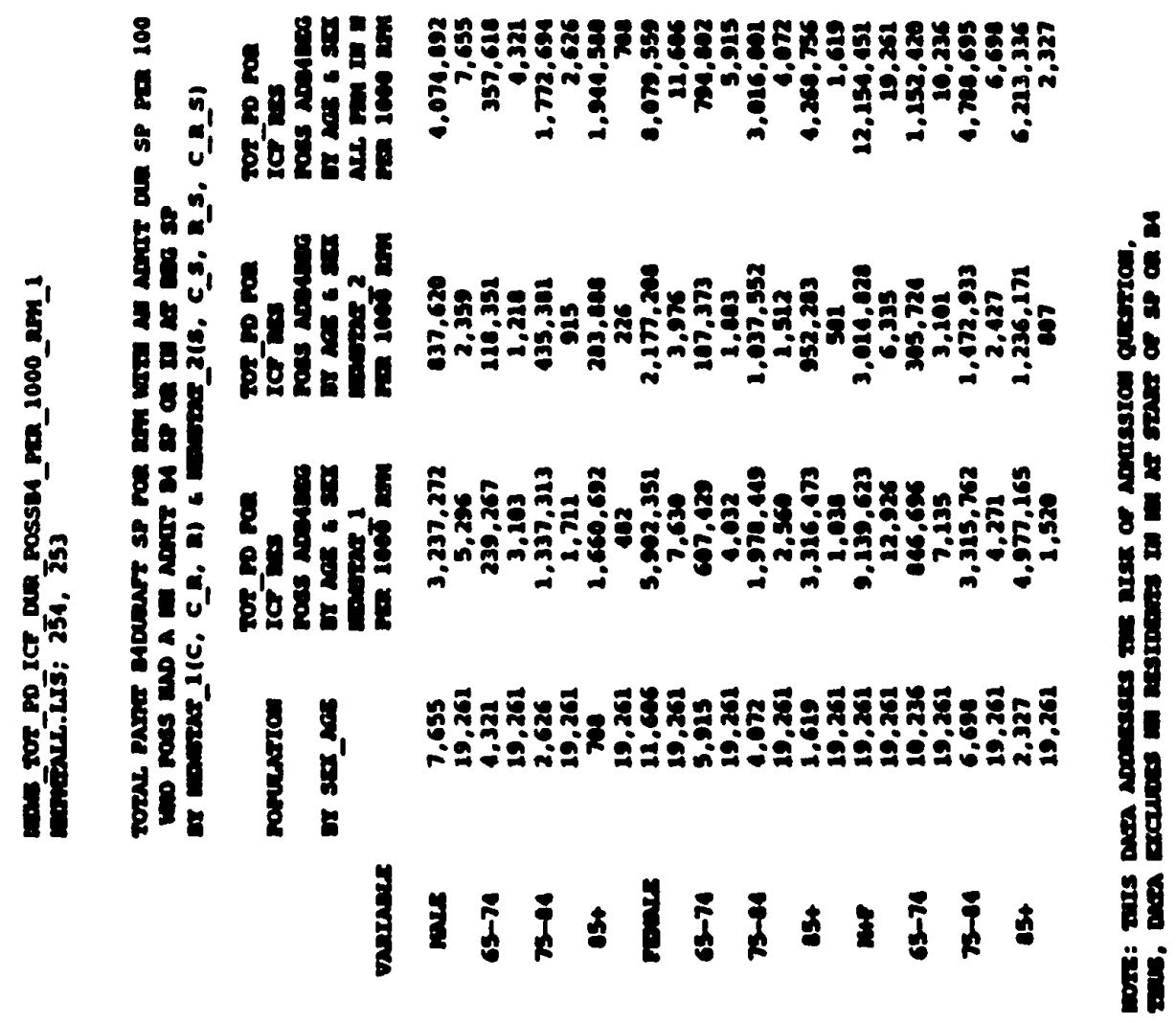


403

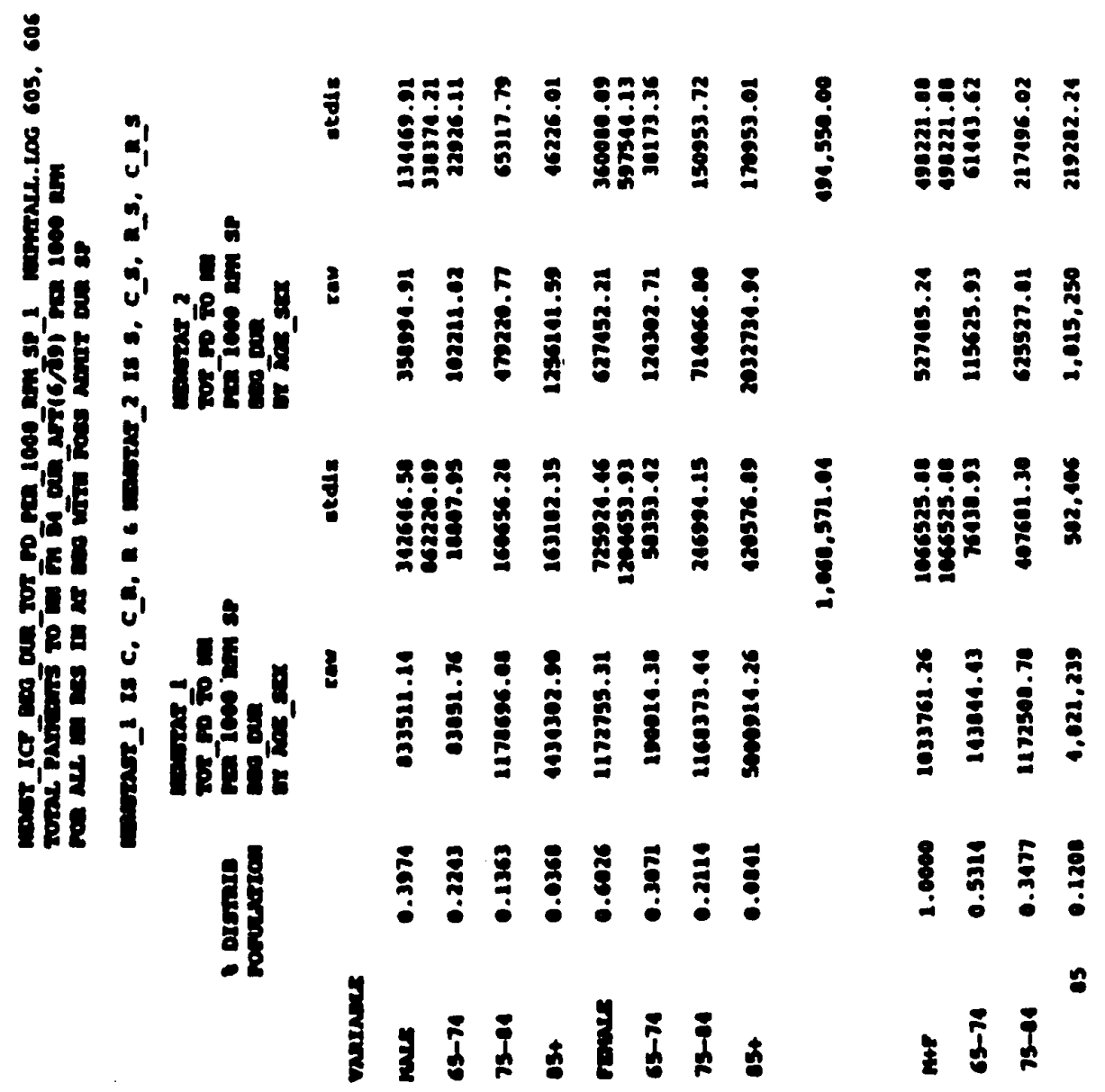




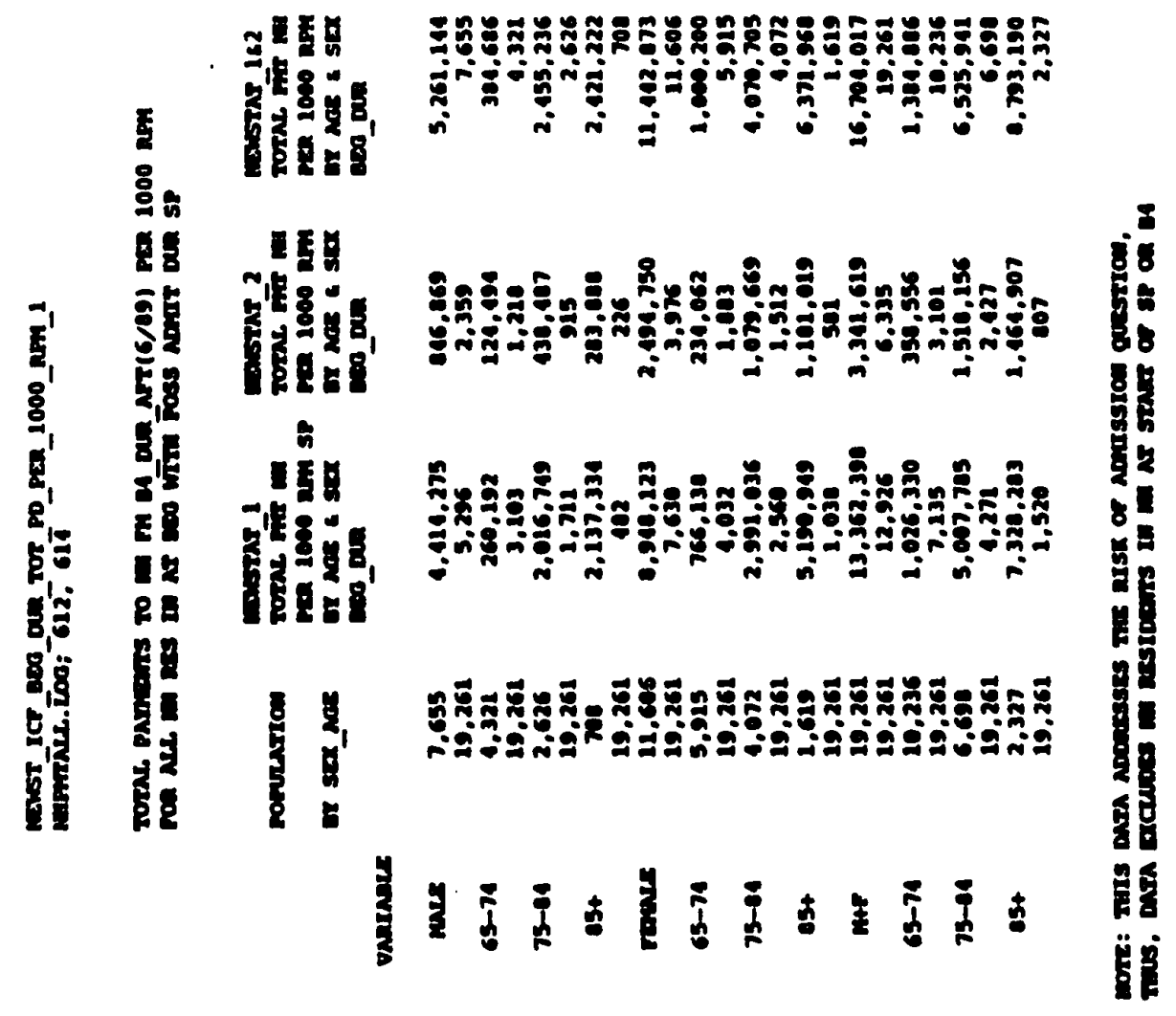




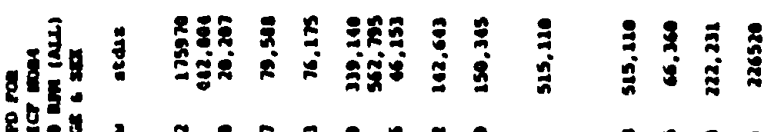

躍!

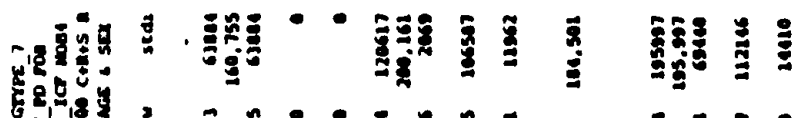

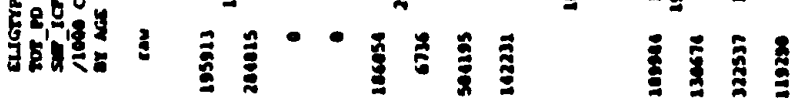

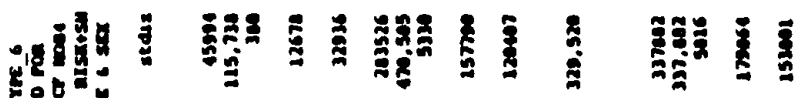

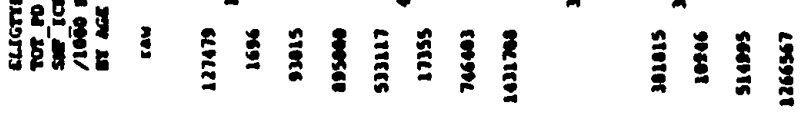

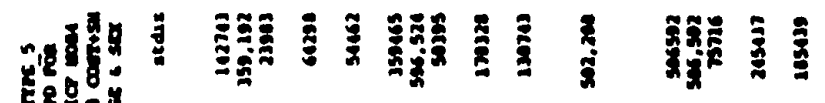

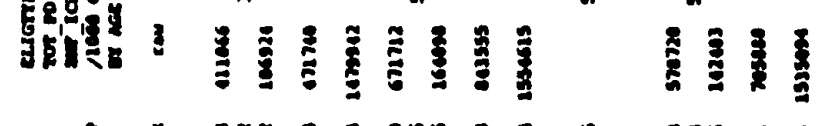

ว

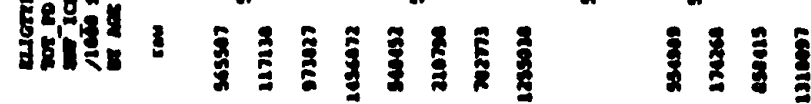

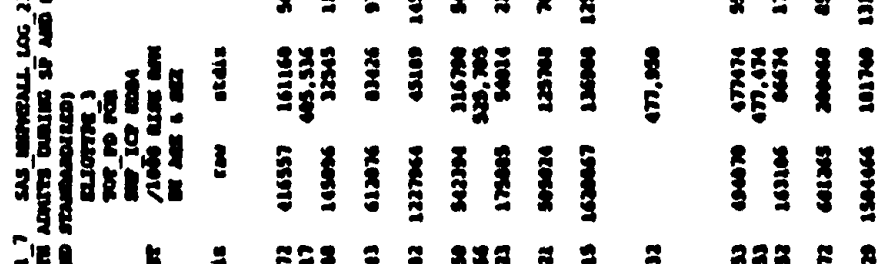

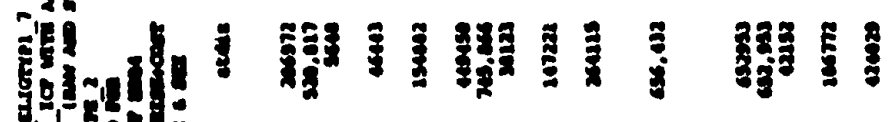
MIn:

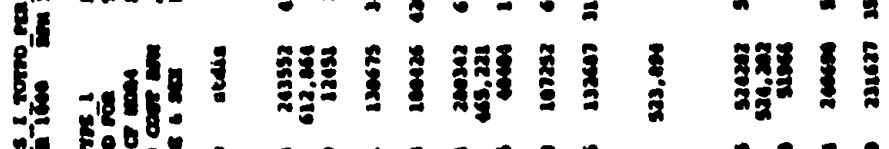

ami

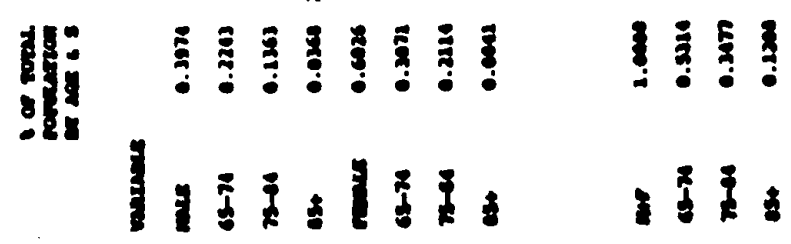




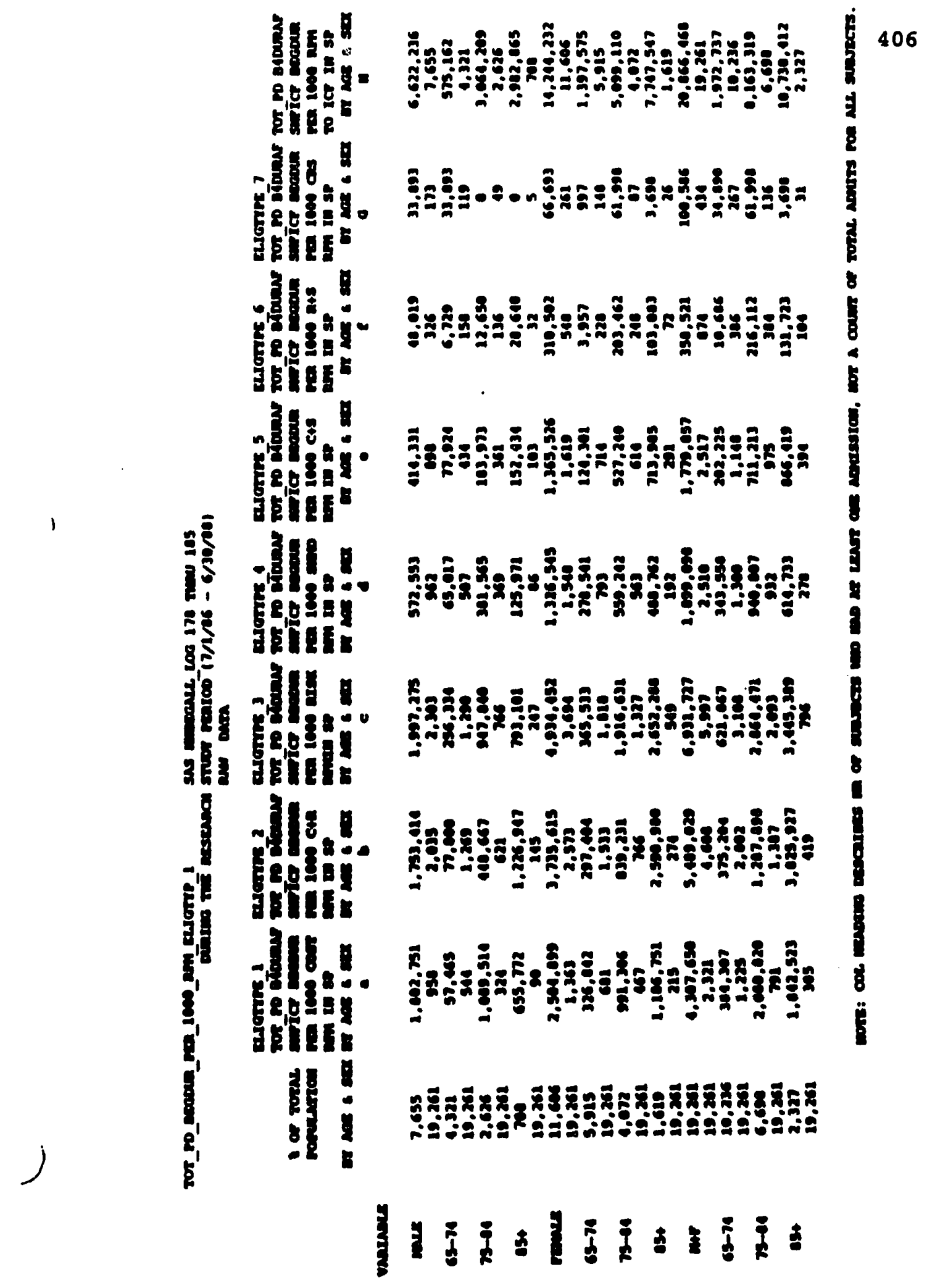




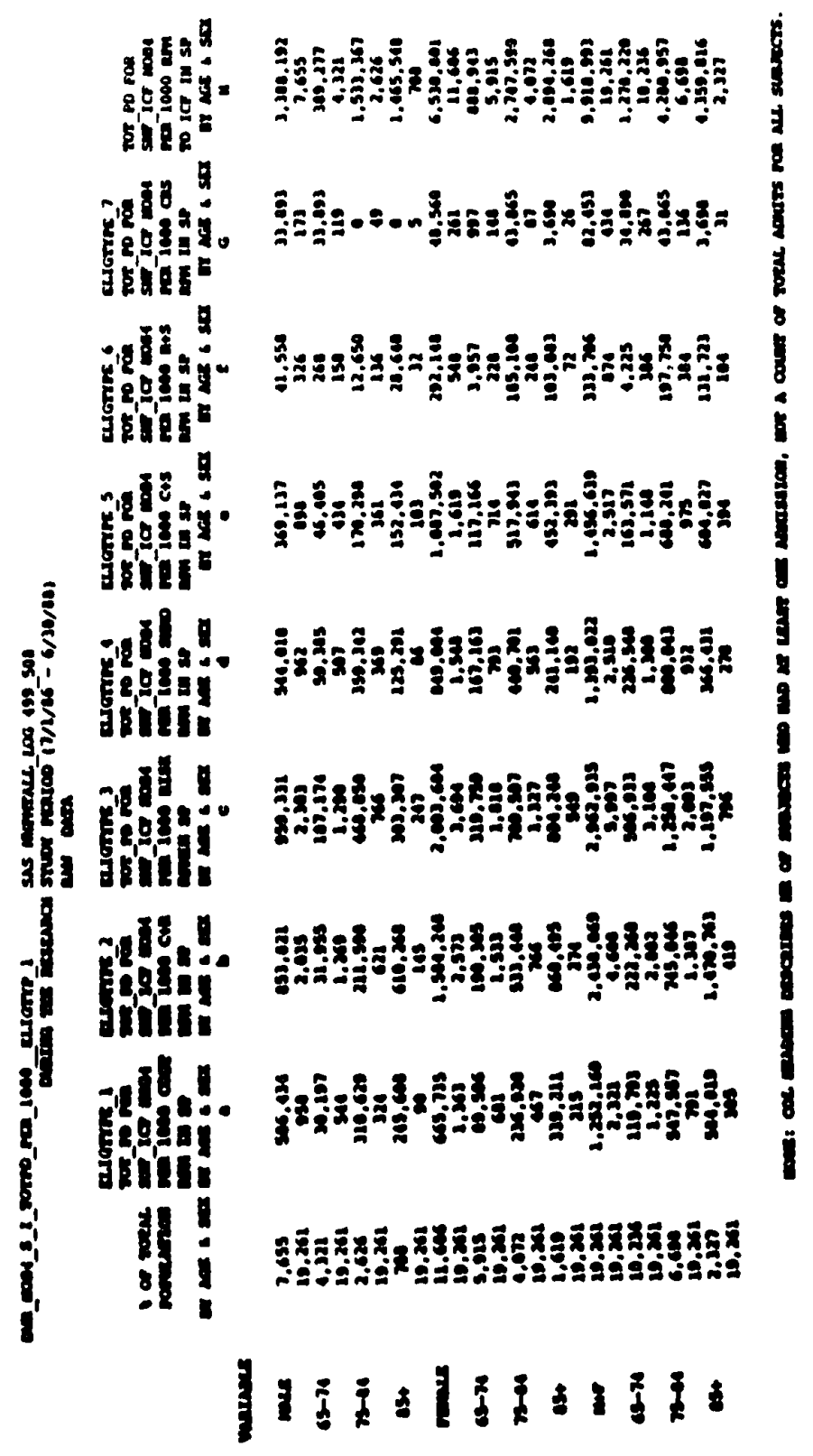




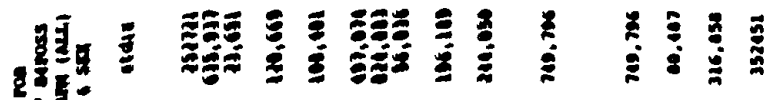

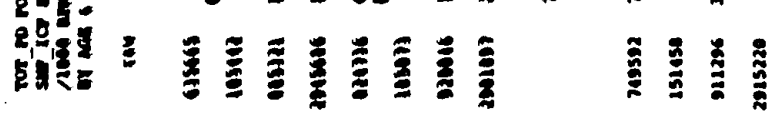

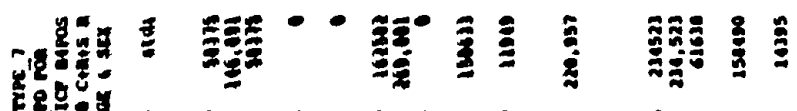

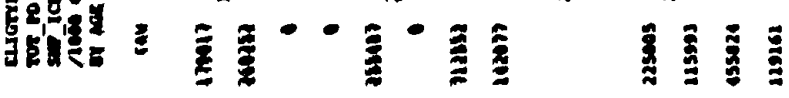

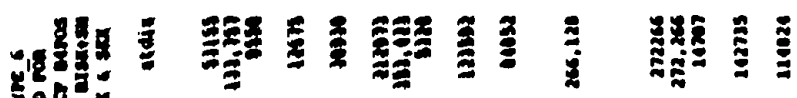

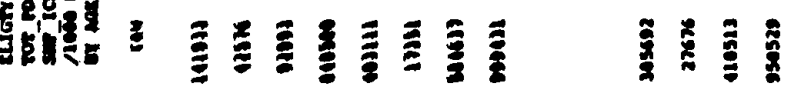

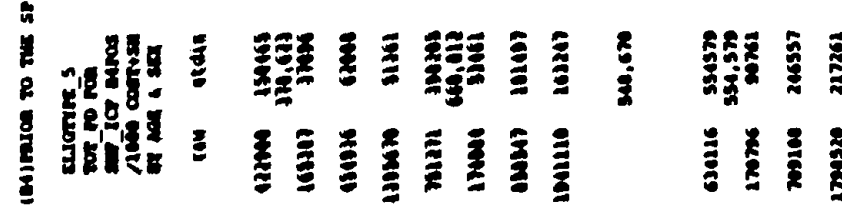

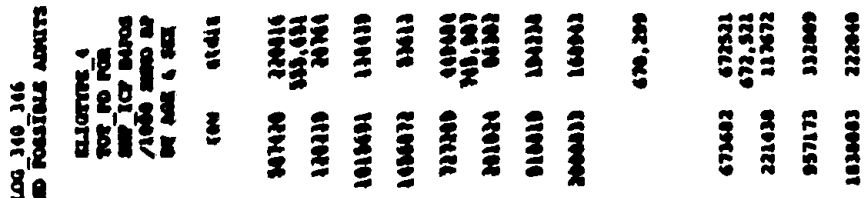

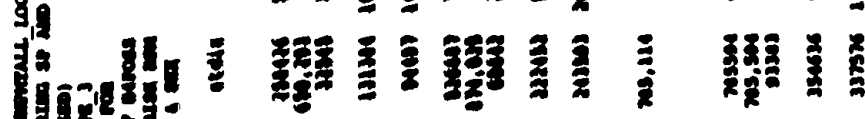
1.5

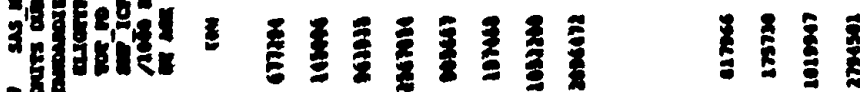

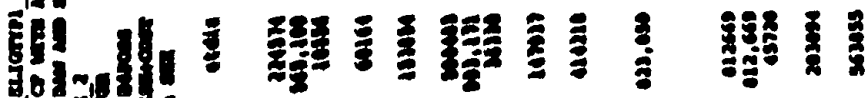

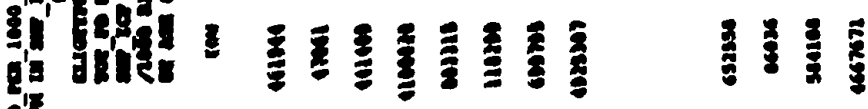

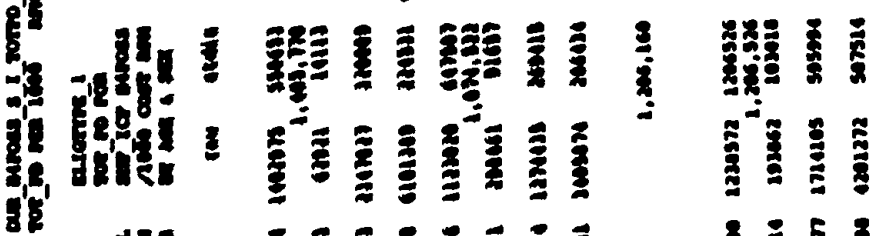

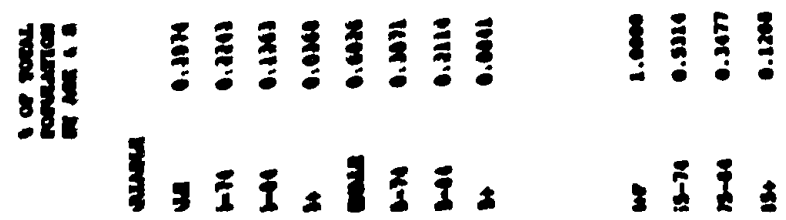




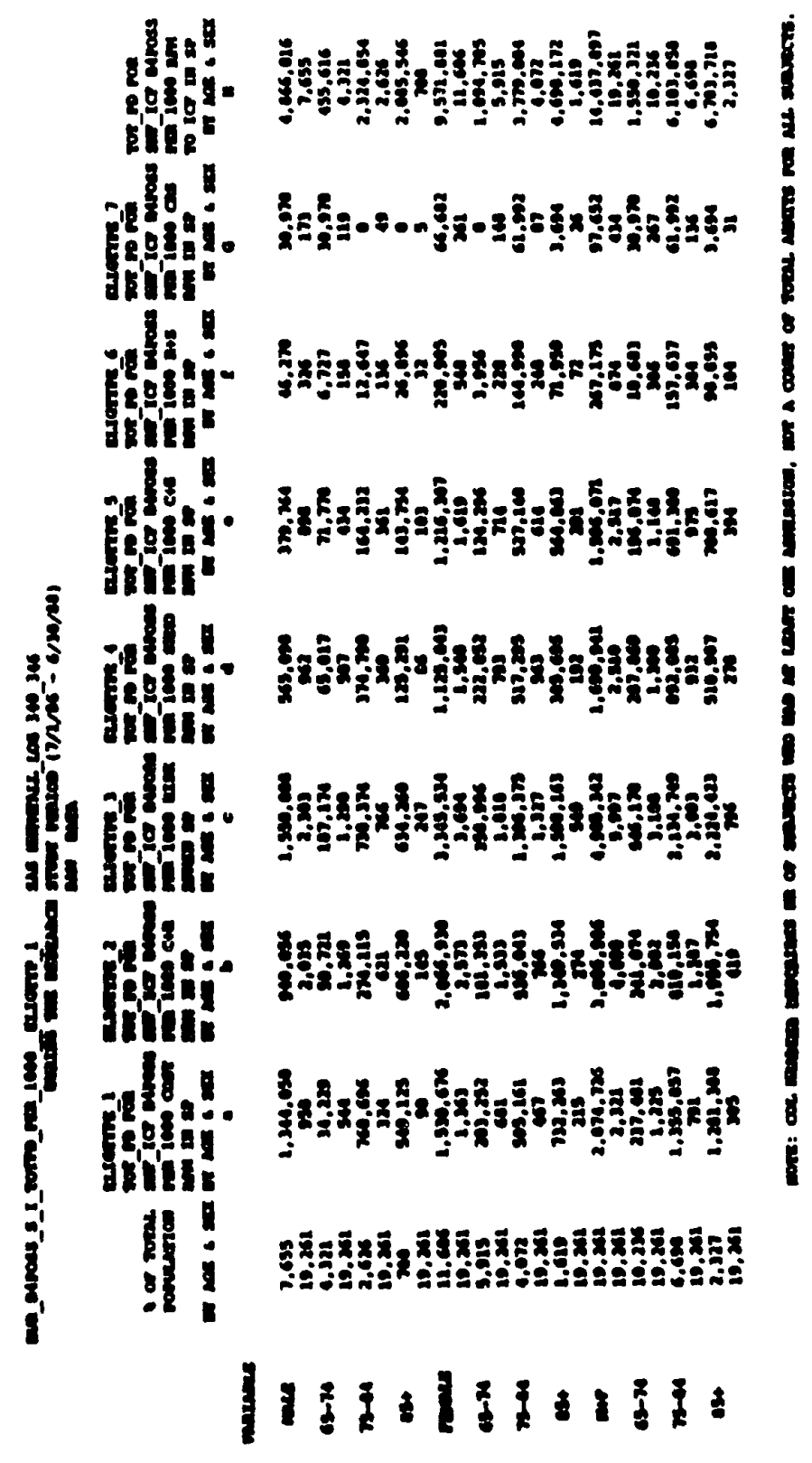




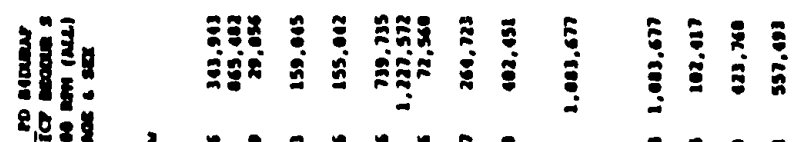

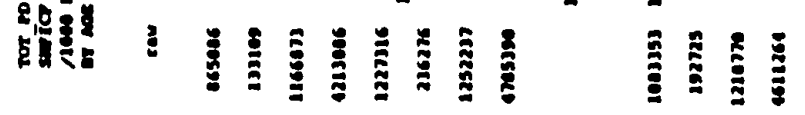

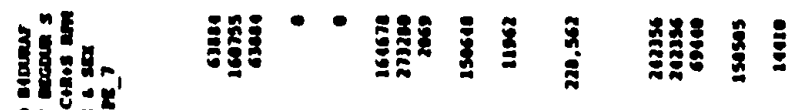

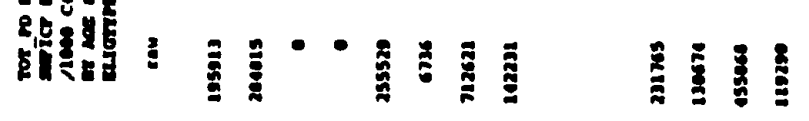

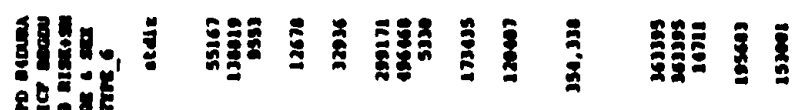

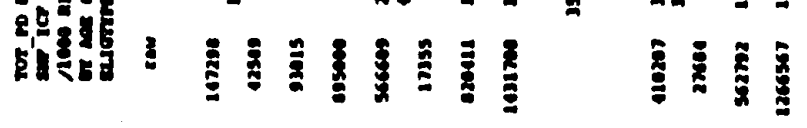

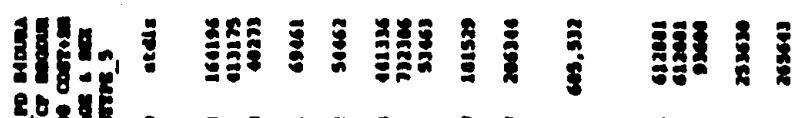
ถ⿻ำ

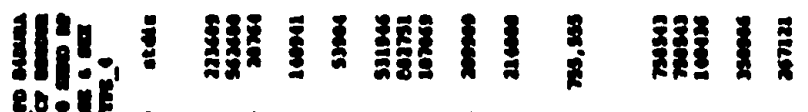

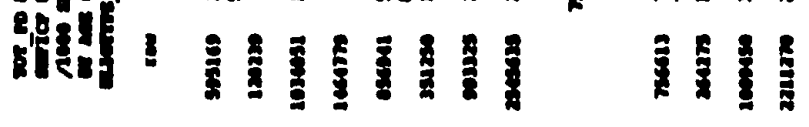

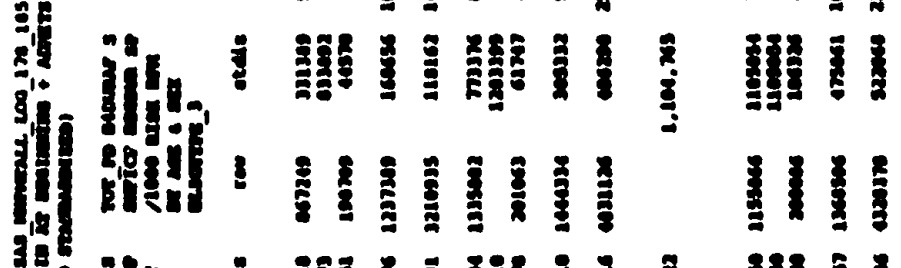
施

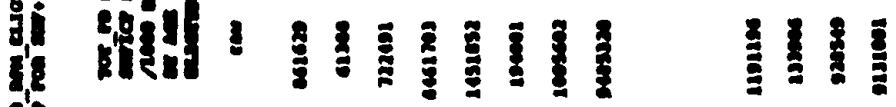

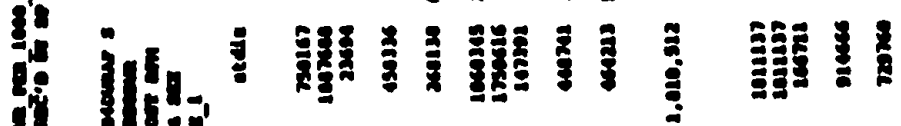
ใ

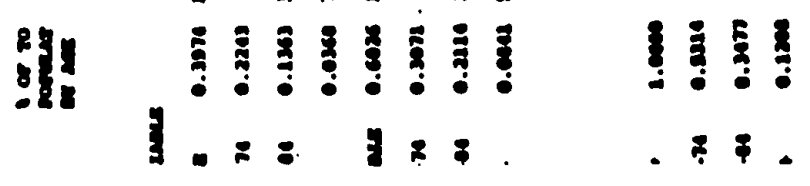



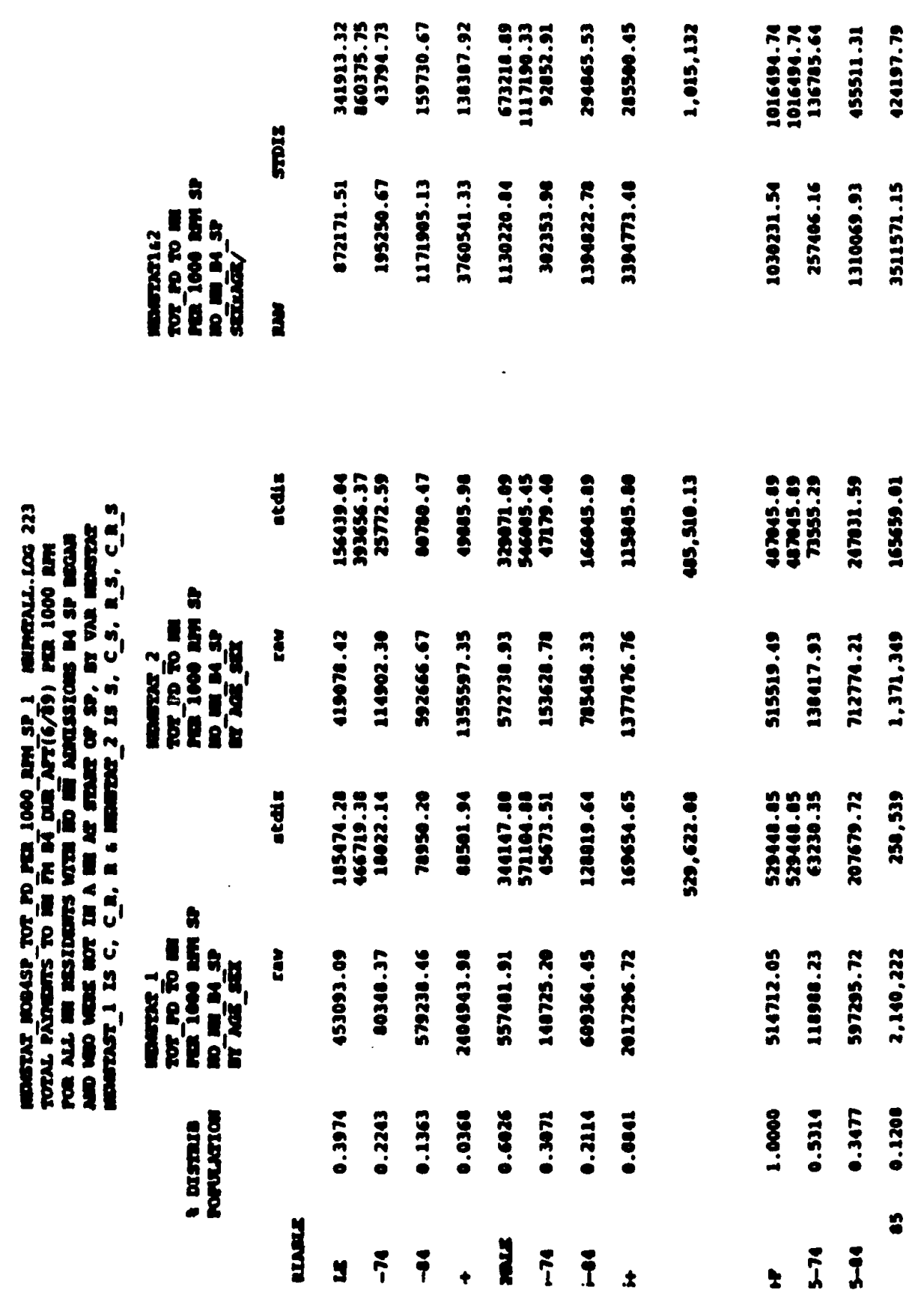


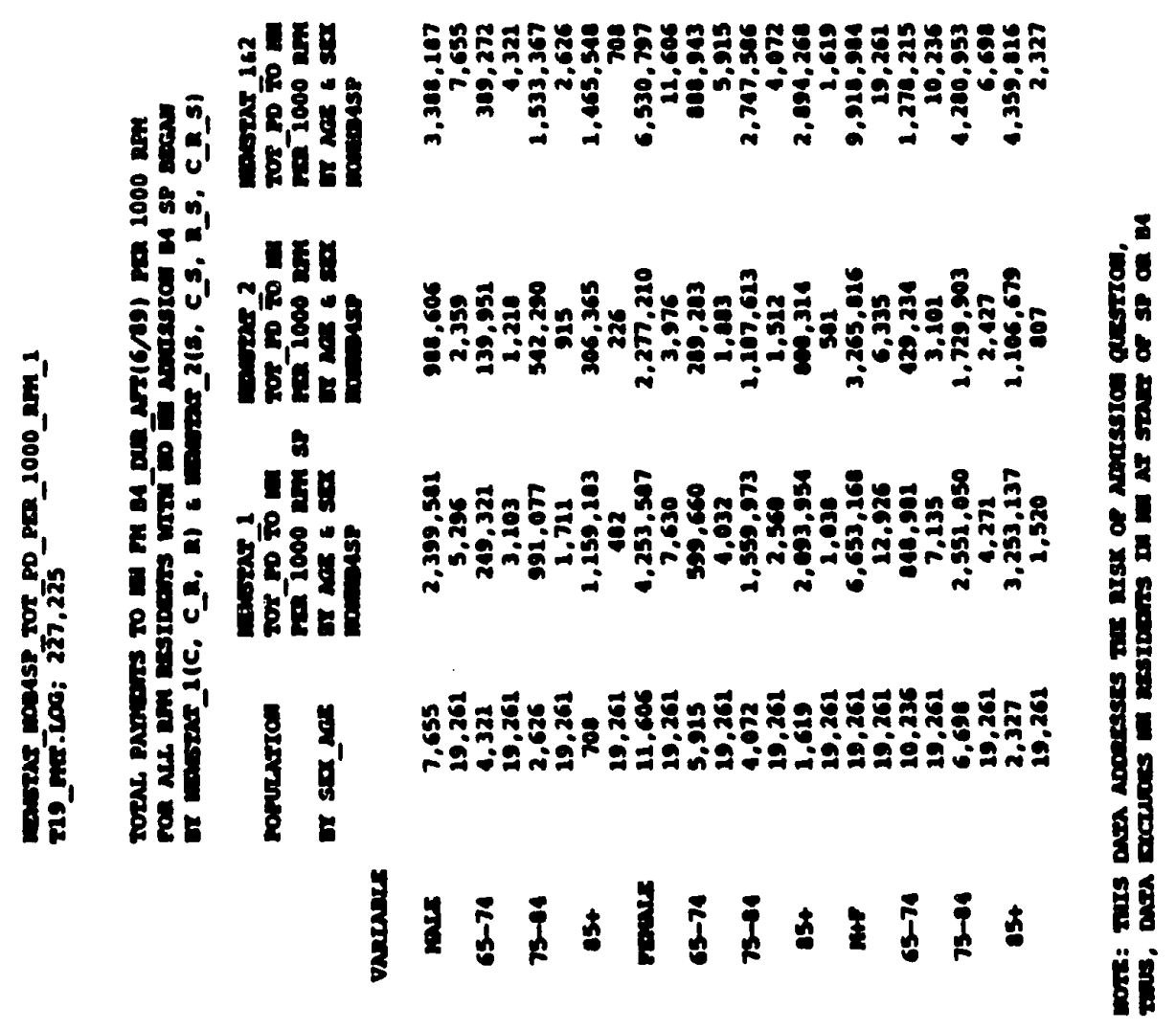



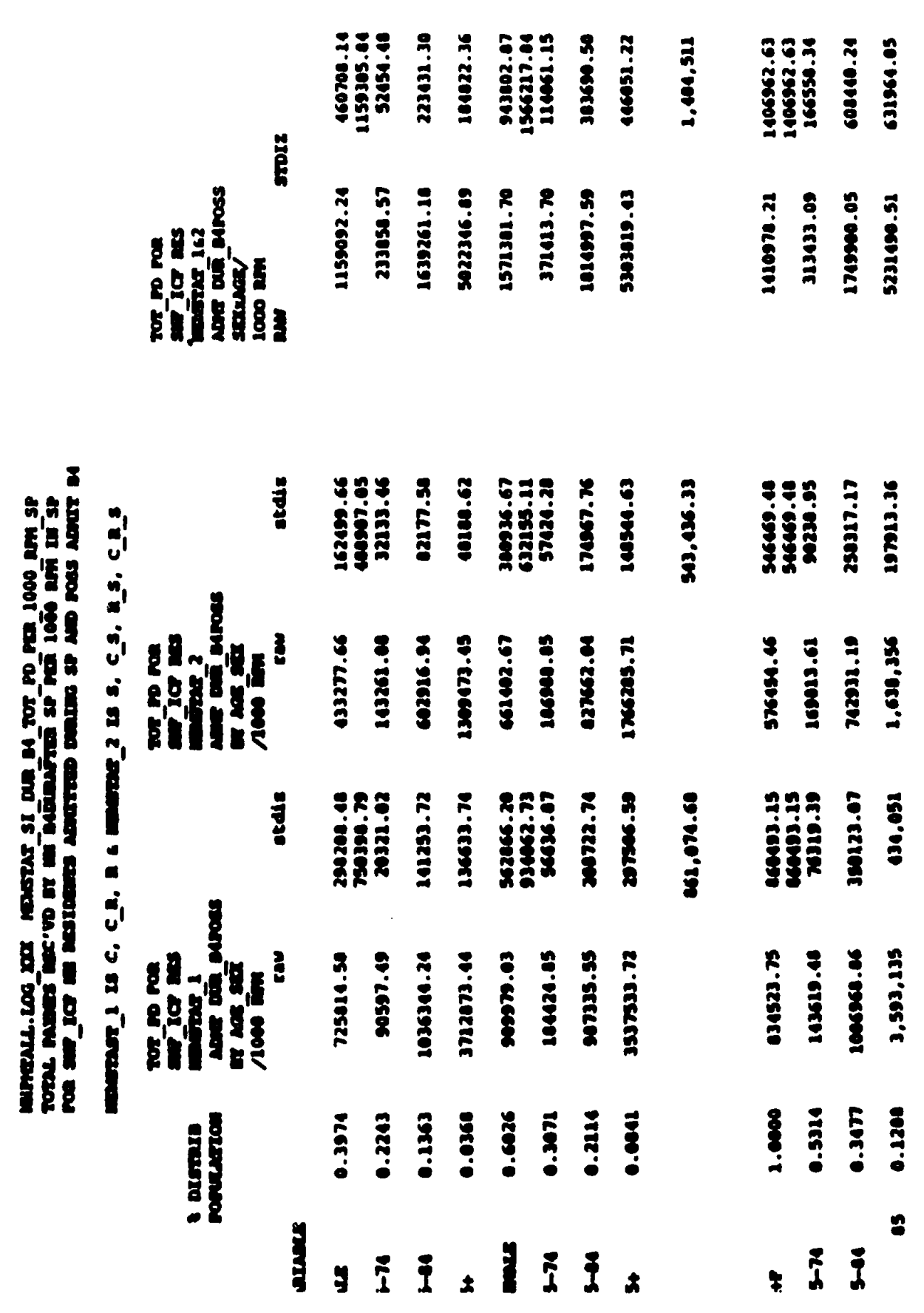


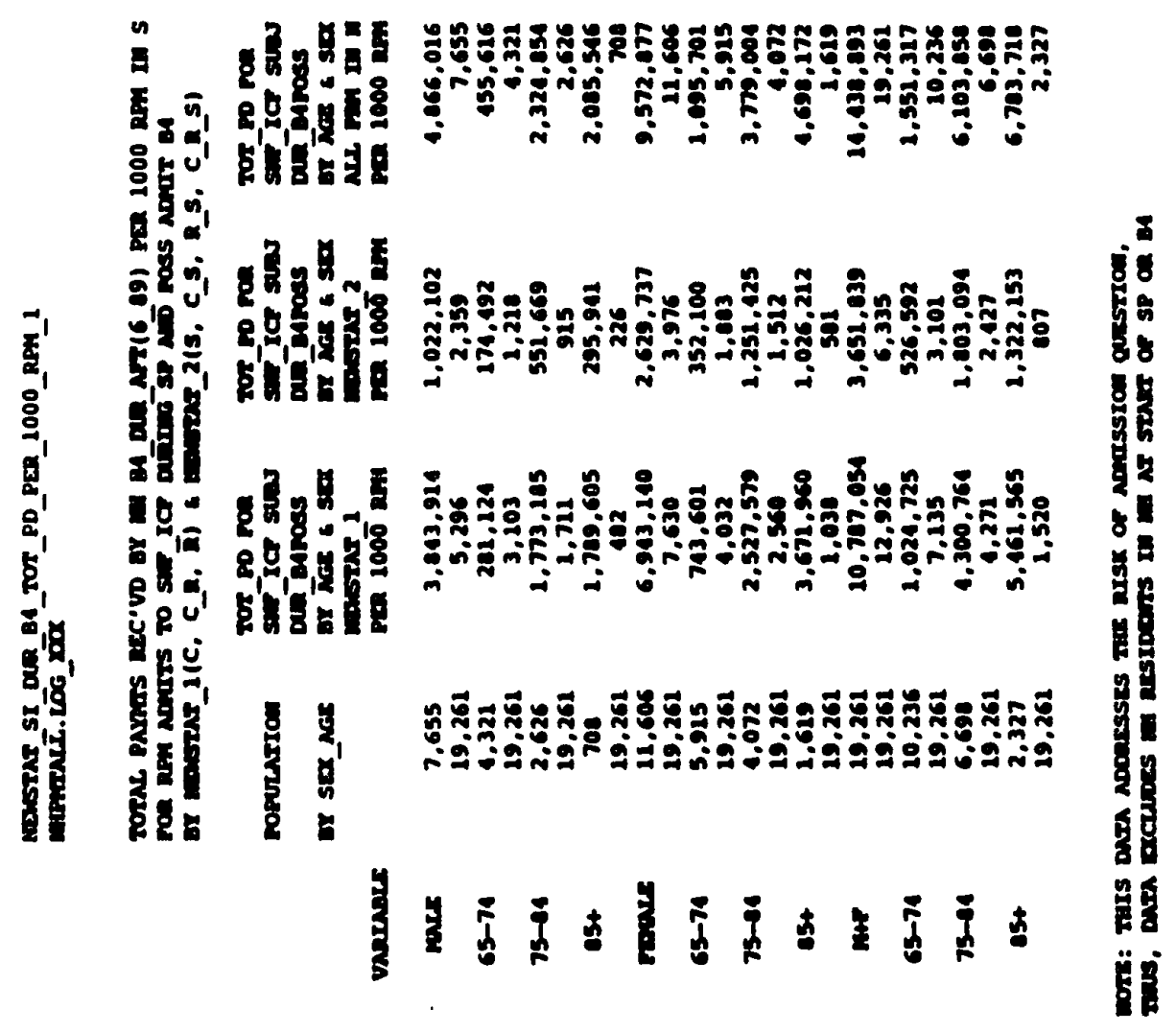




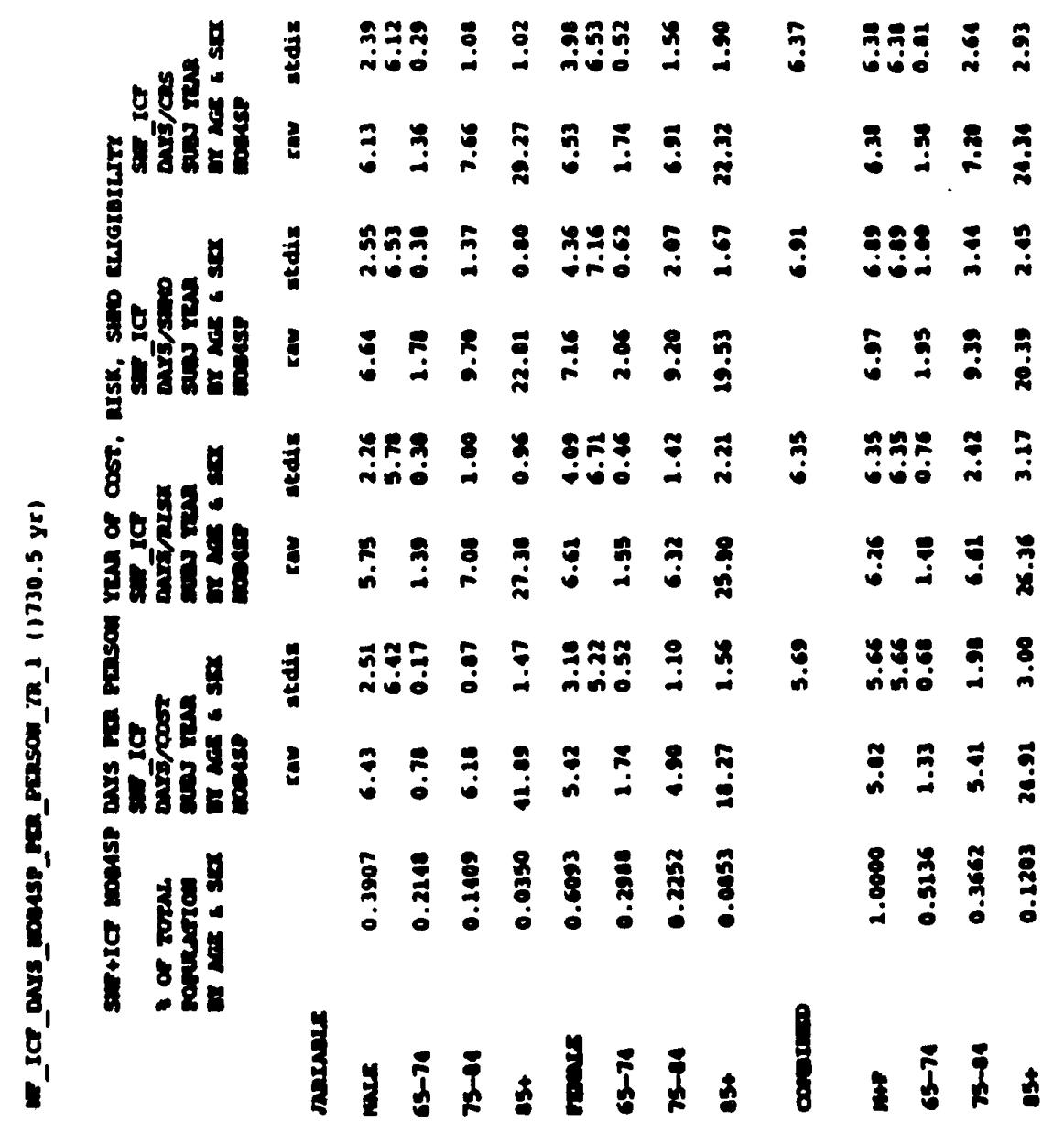




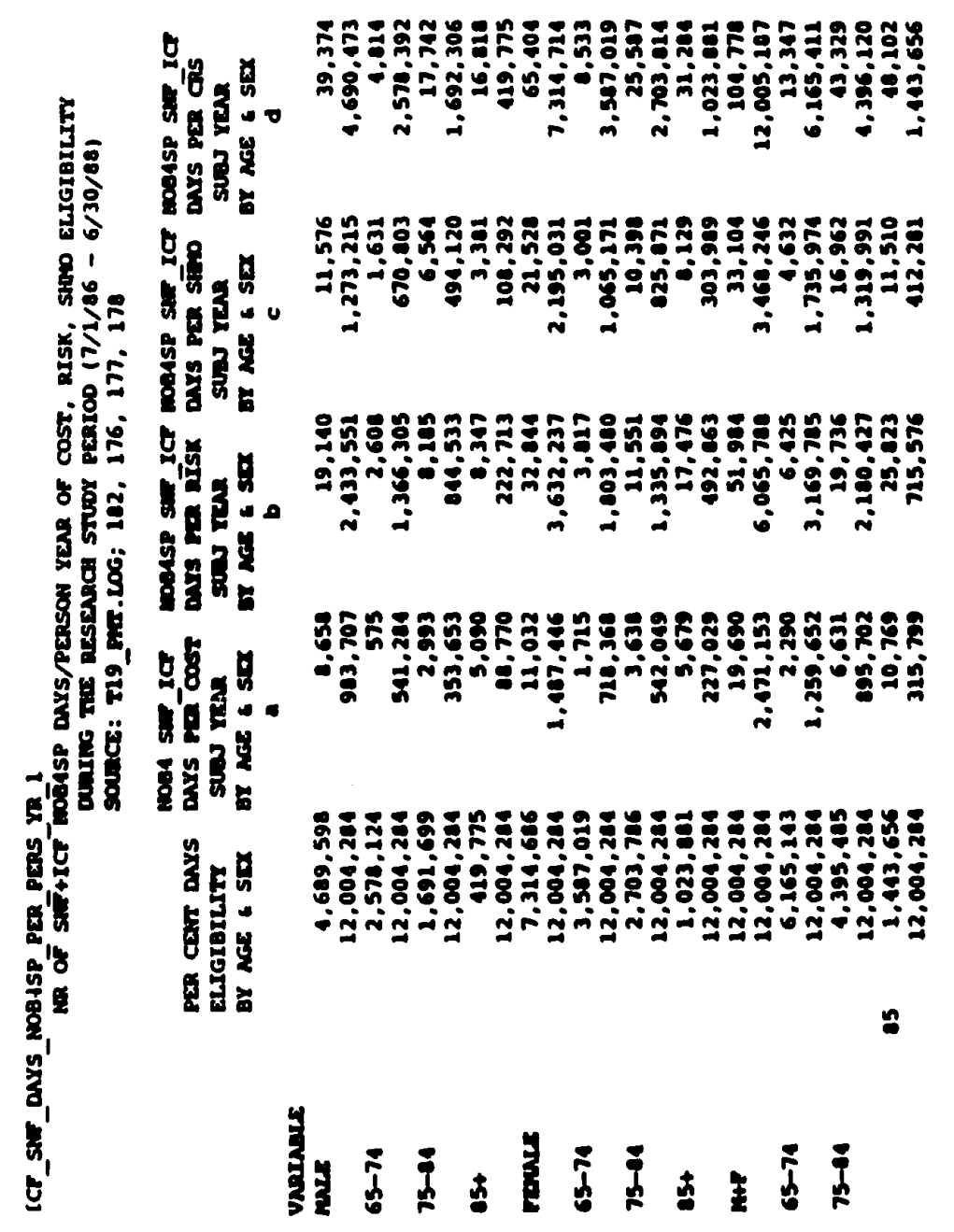




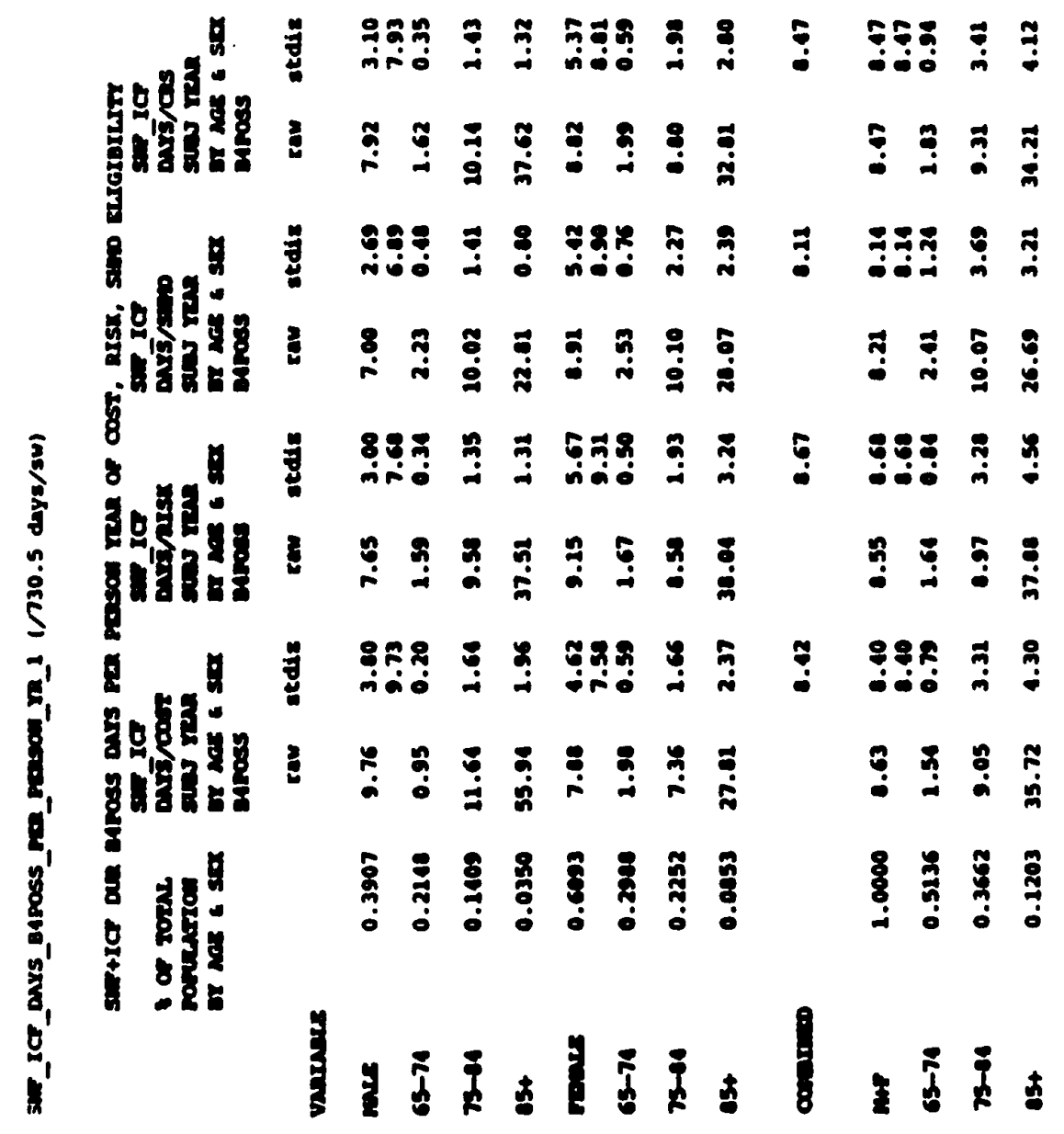




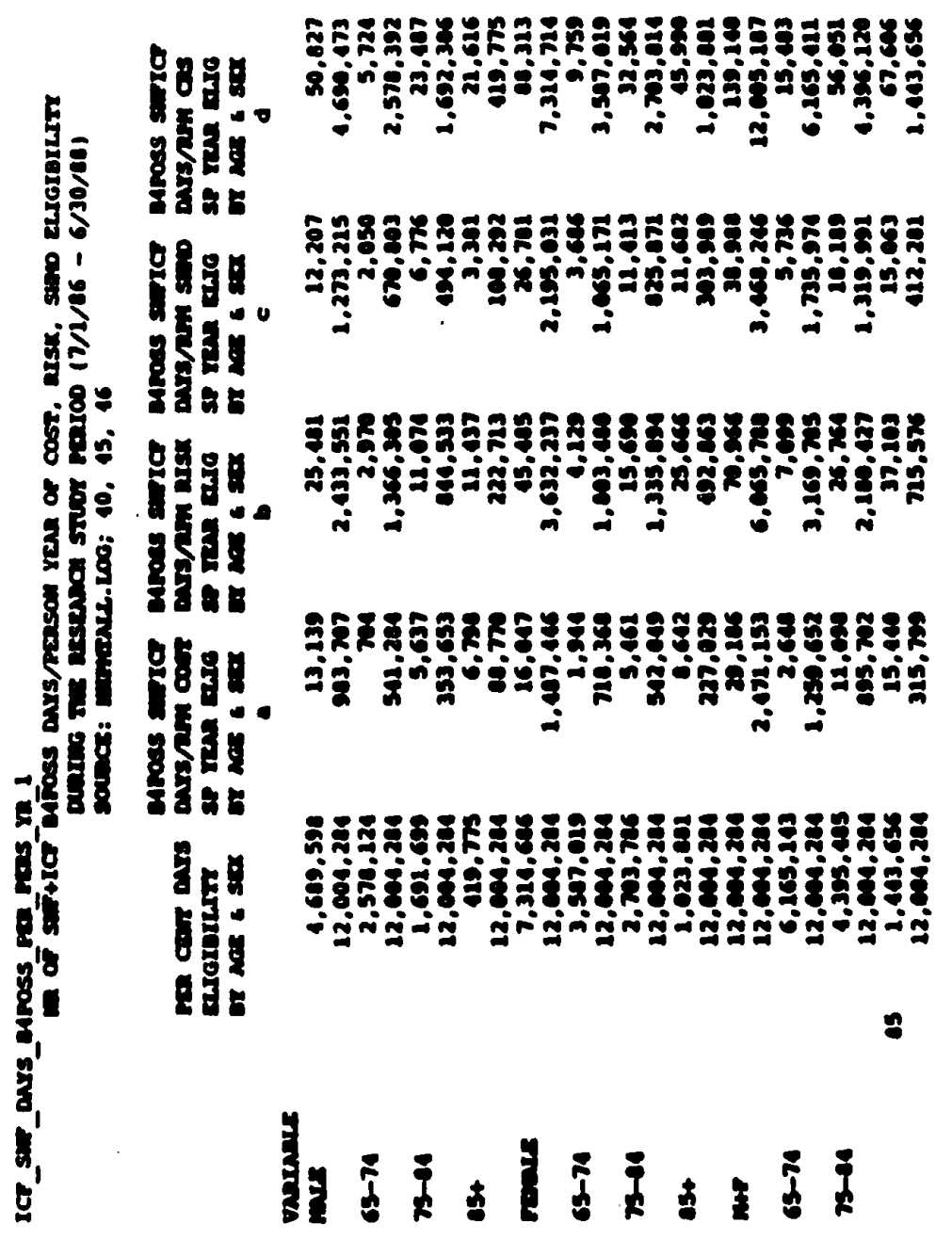




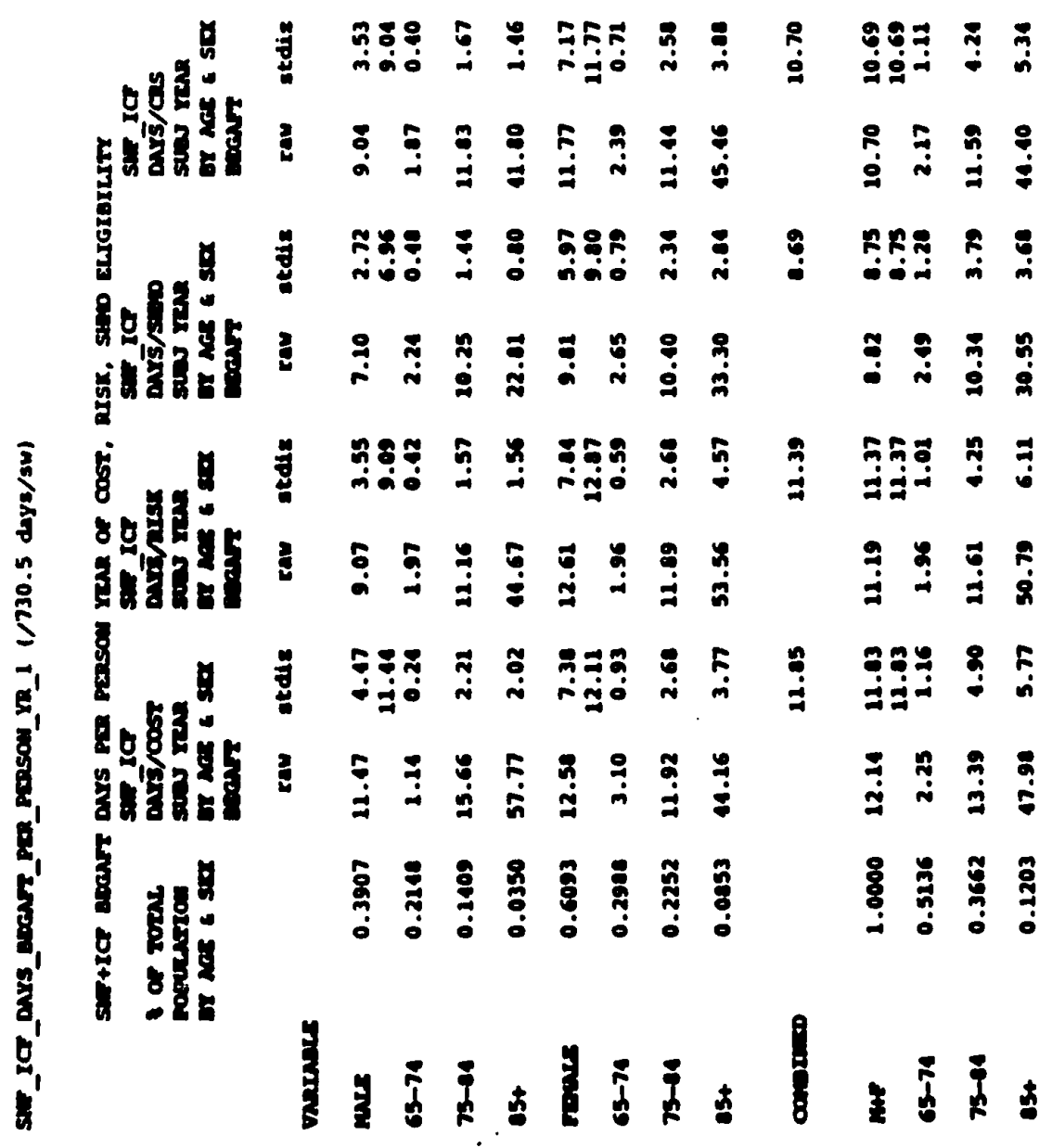




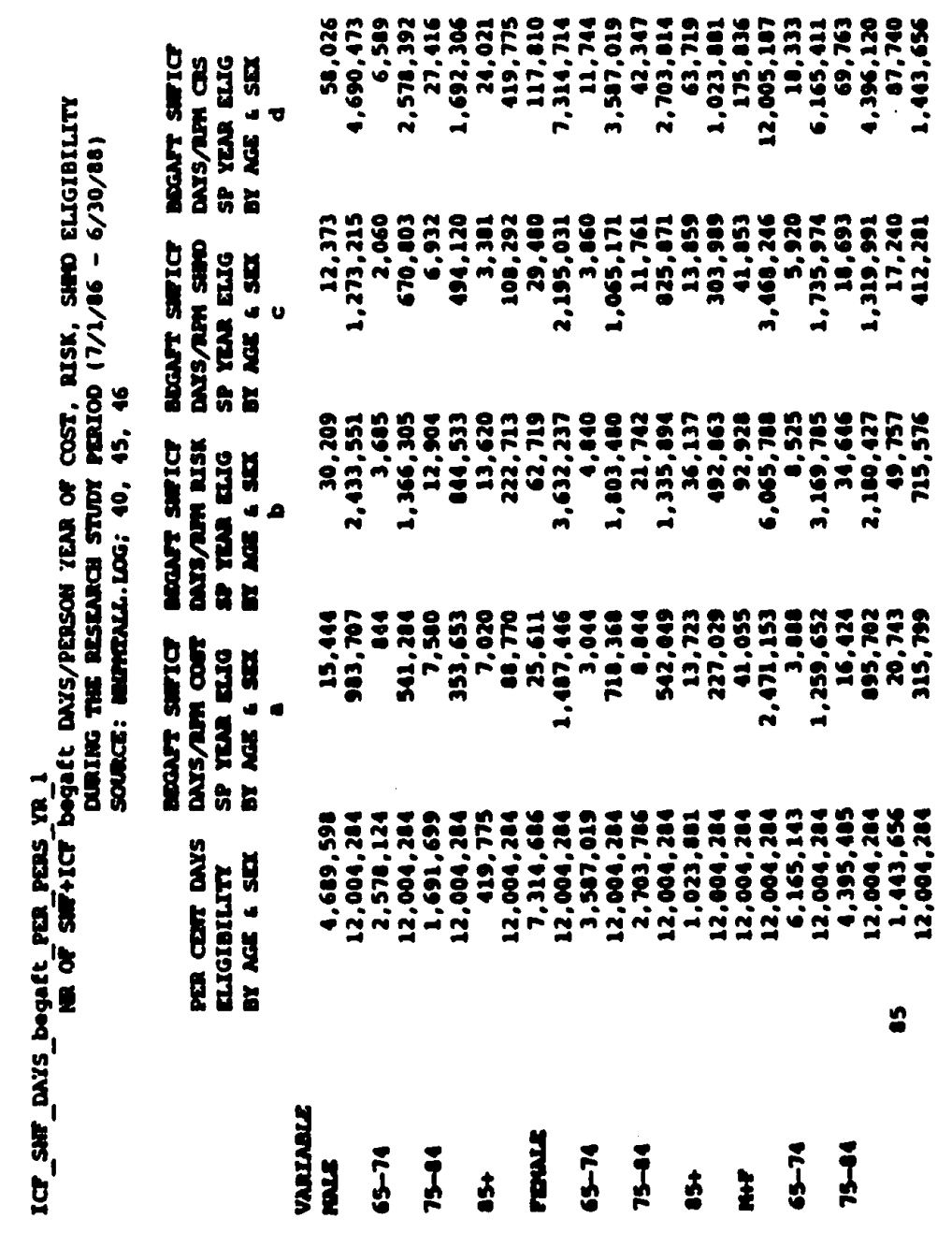




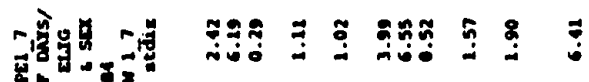

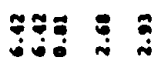
हैe

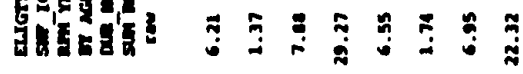

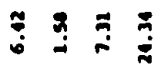

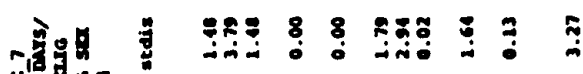

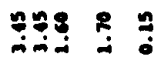

ตำ

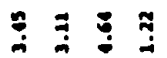

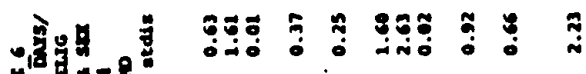

จุด

topin

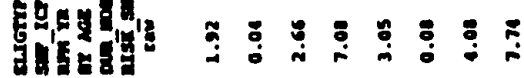

ำ

8ิ

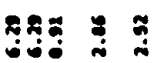

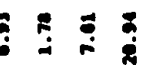

มฺุ

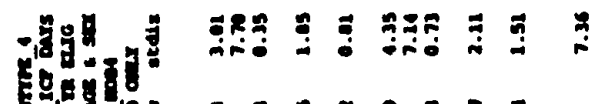

कृष्य

เัญ

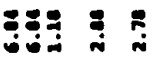

类

ced

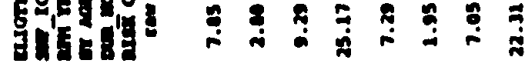

จุด

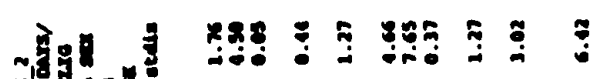

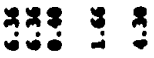

F :

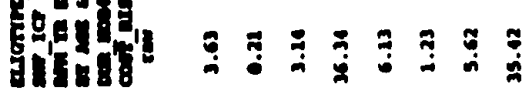

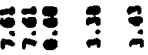

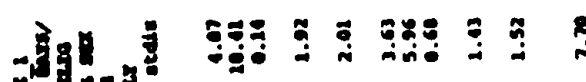

$\underset{x}{x}$

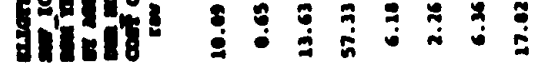

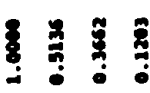

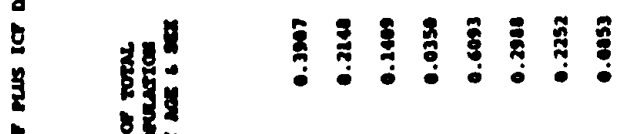

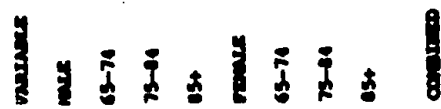

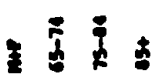




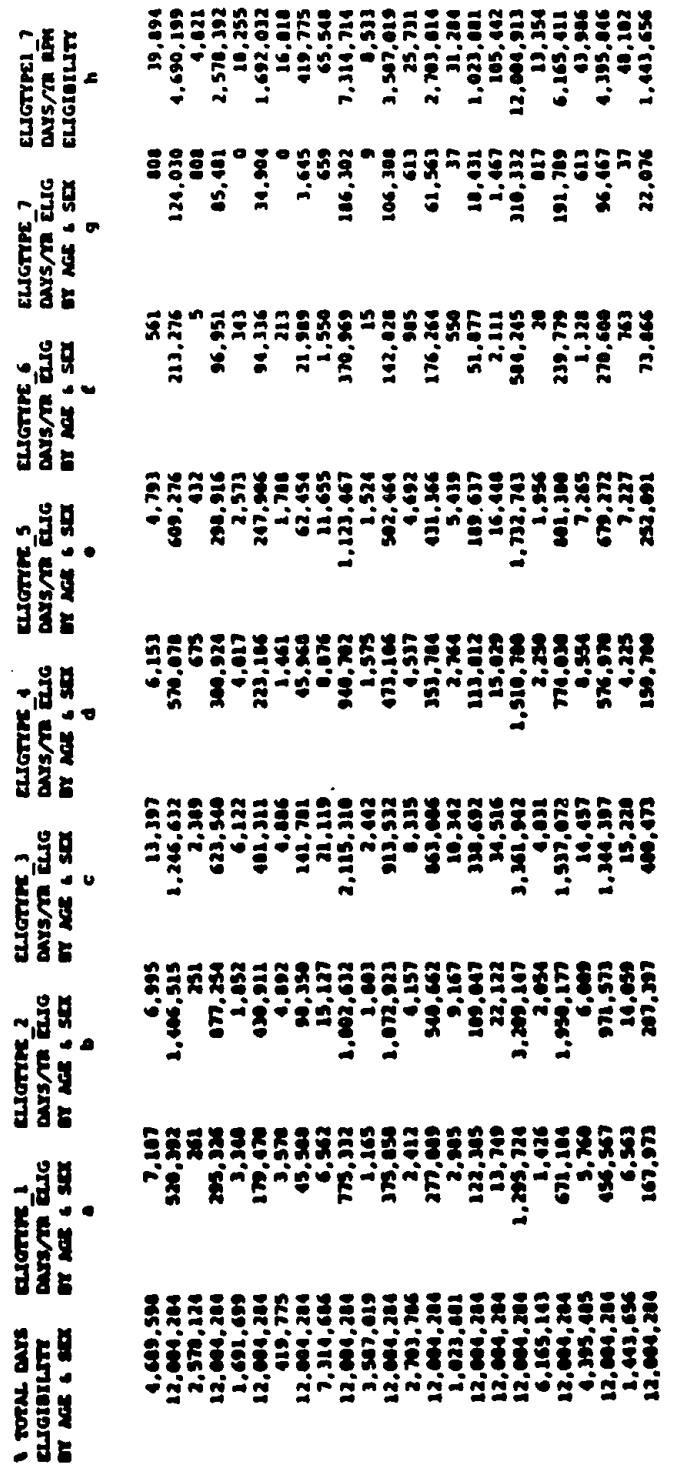

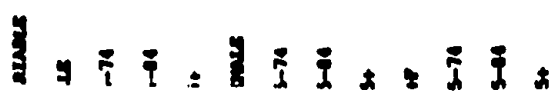




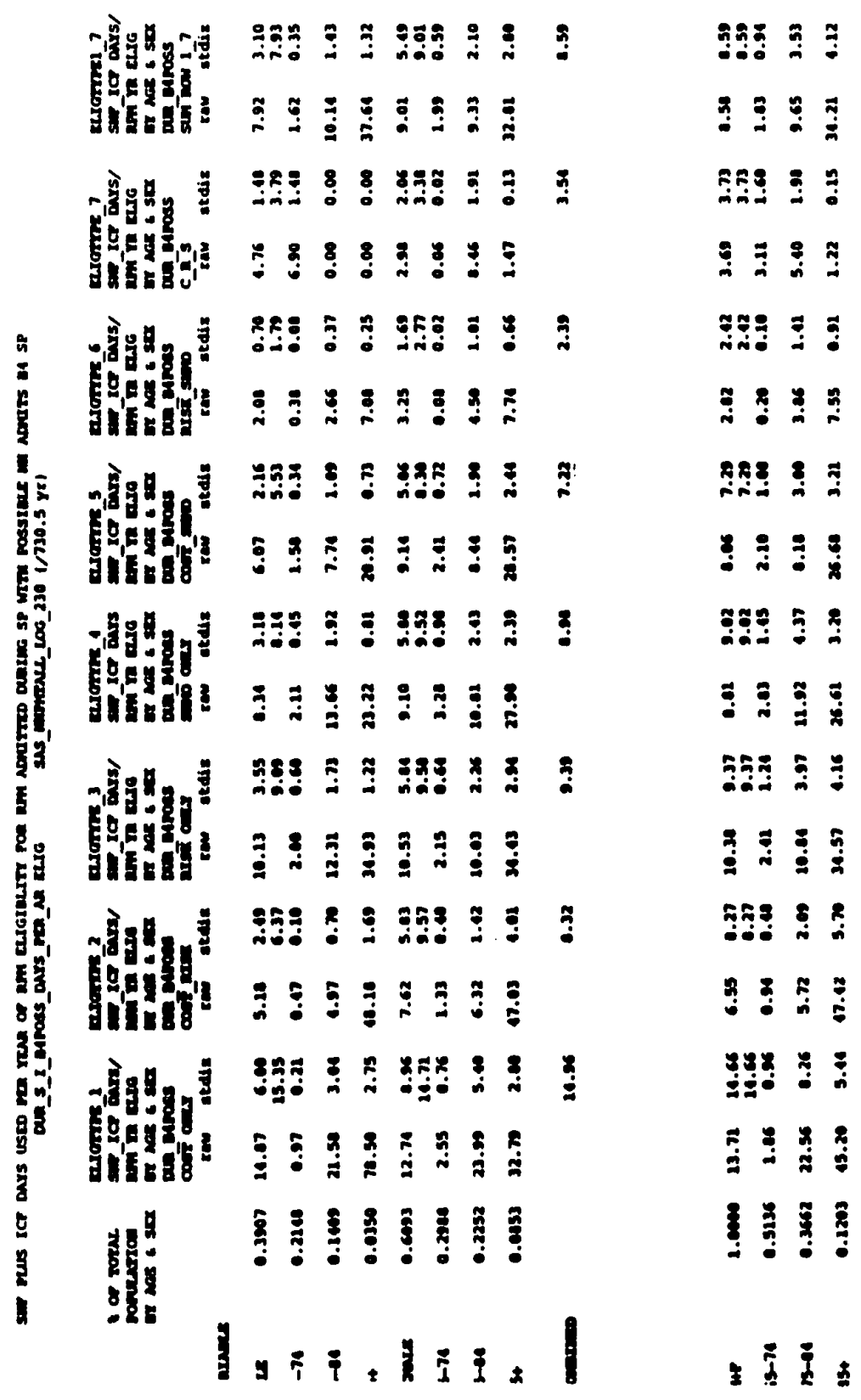




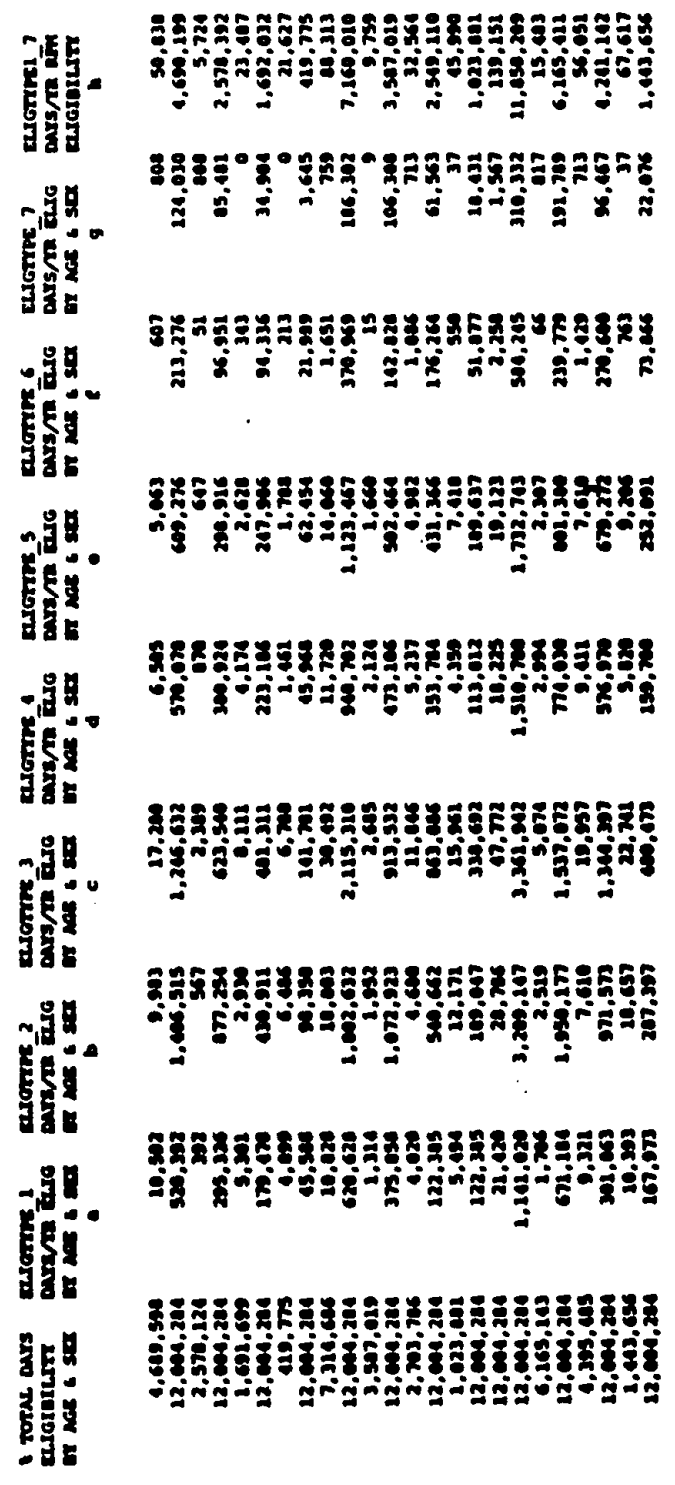

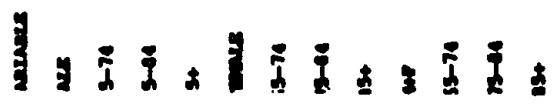




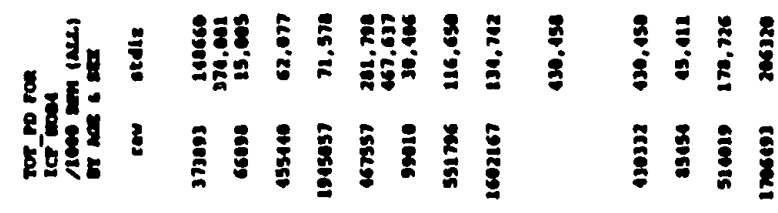

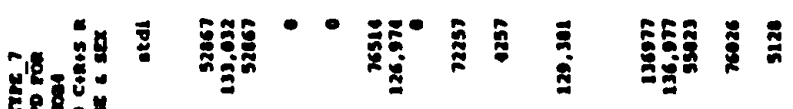

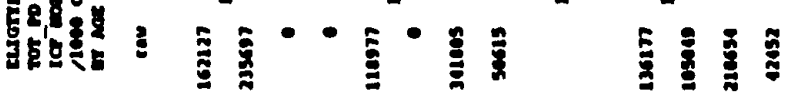

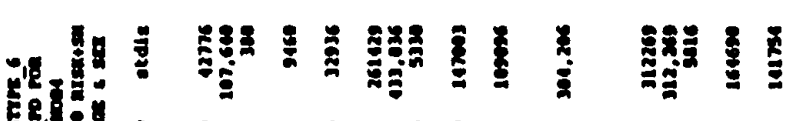

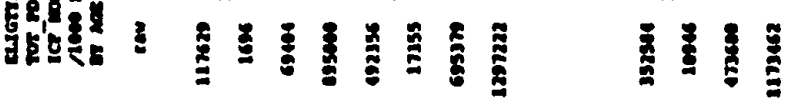

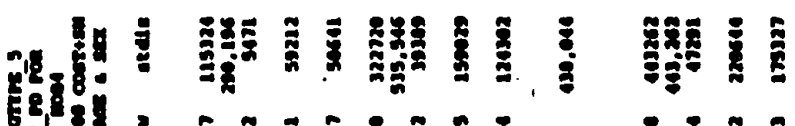

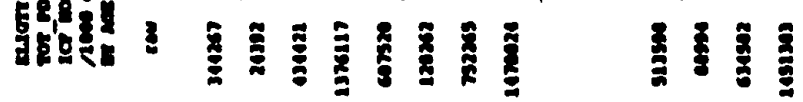
ह

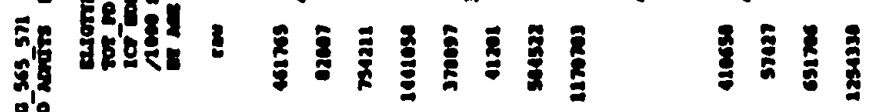
แด้

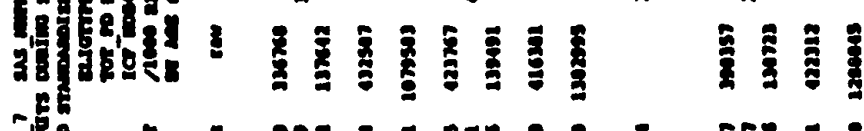

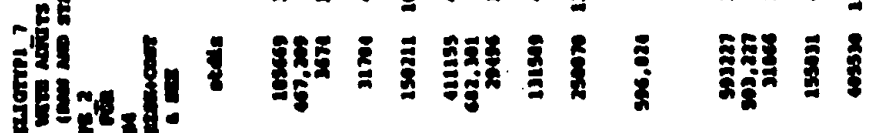

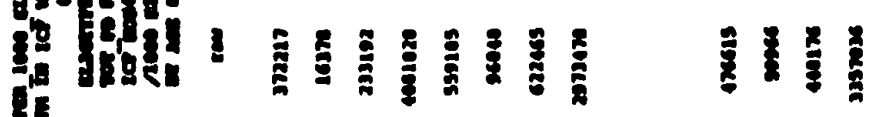
and

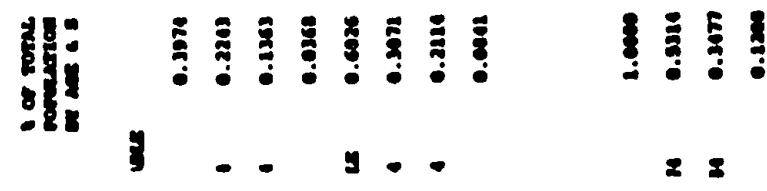




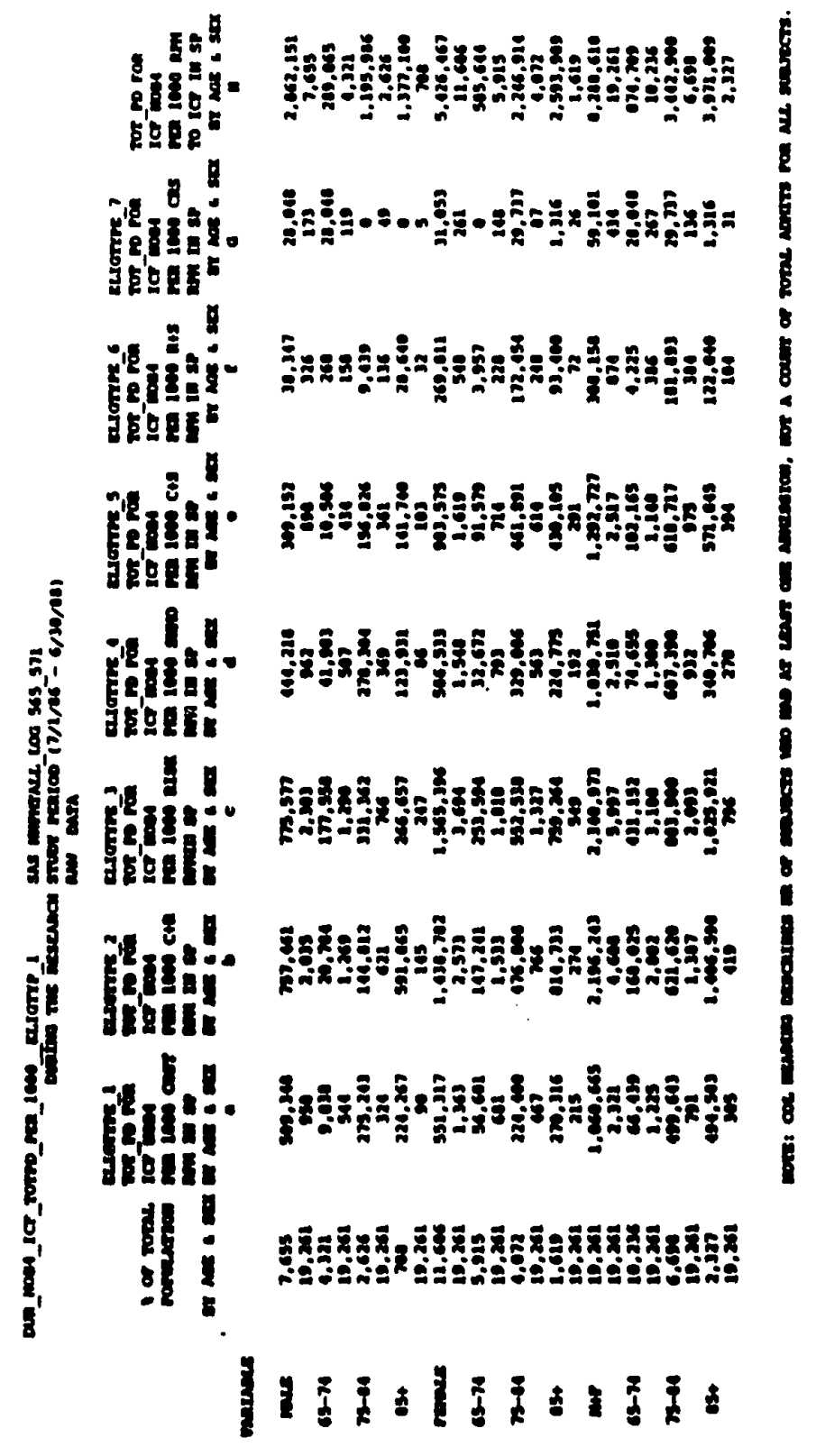




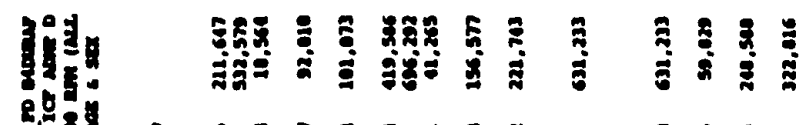

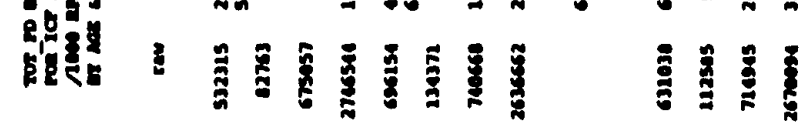

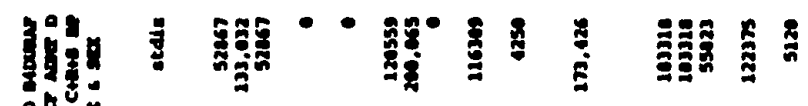

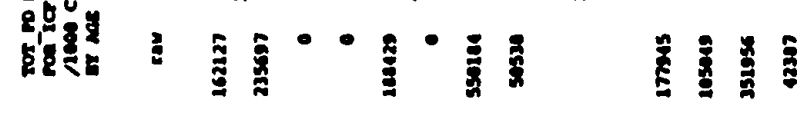

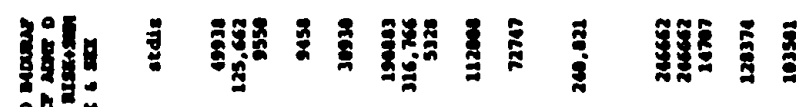
:

?!I

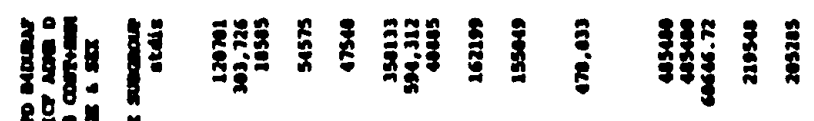

喵!

气 ไif

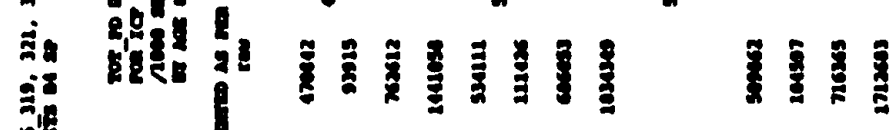

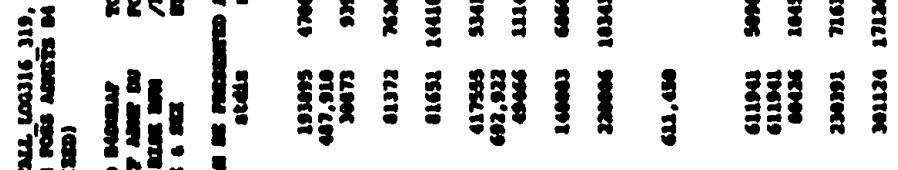

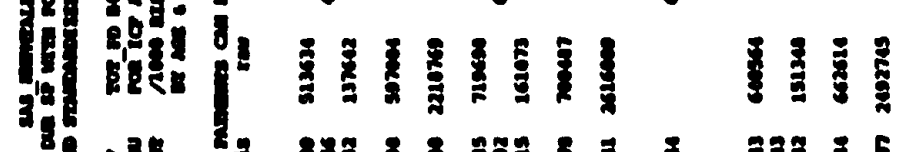

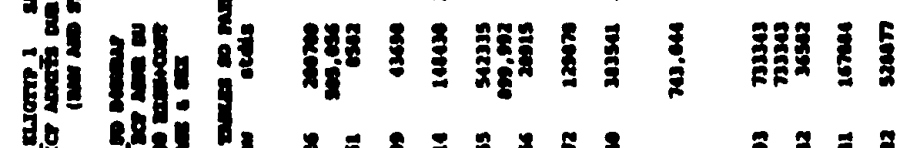

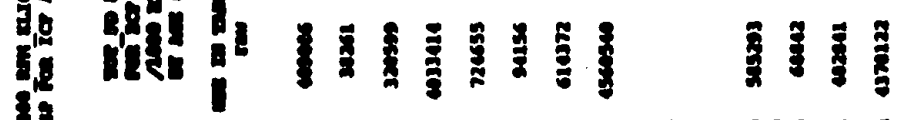

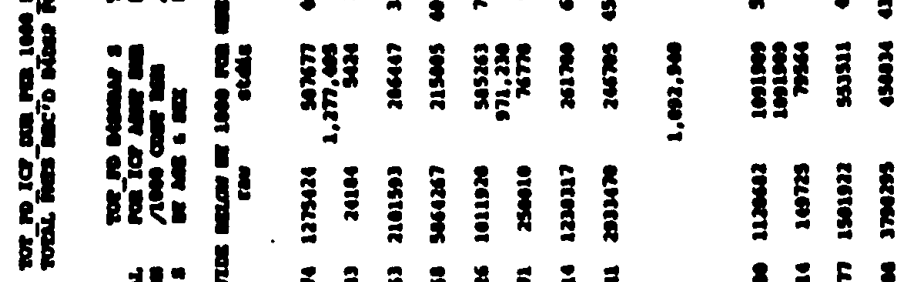

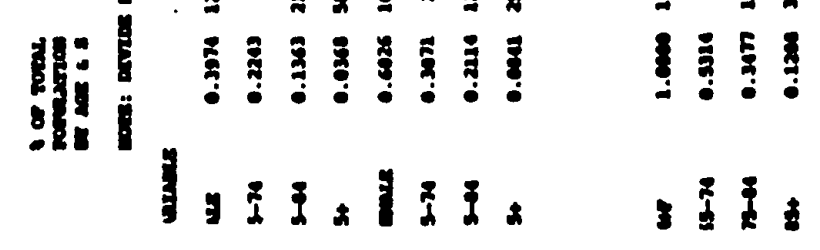




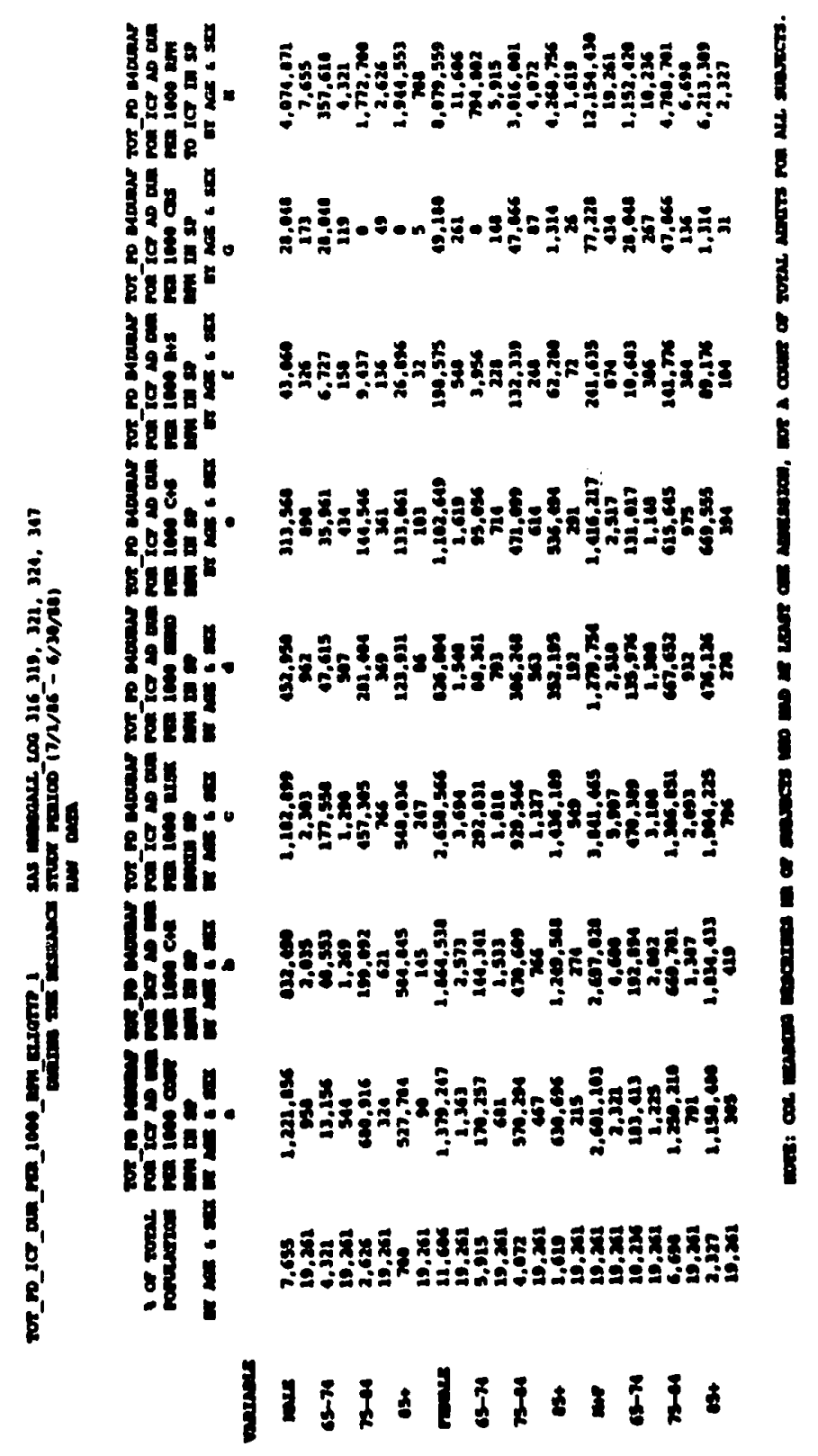


APPENDIX F

FOR CHAPTER VIII 


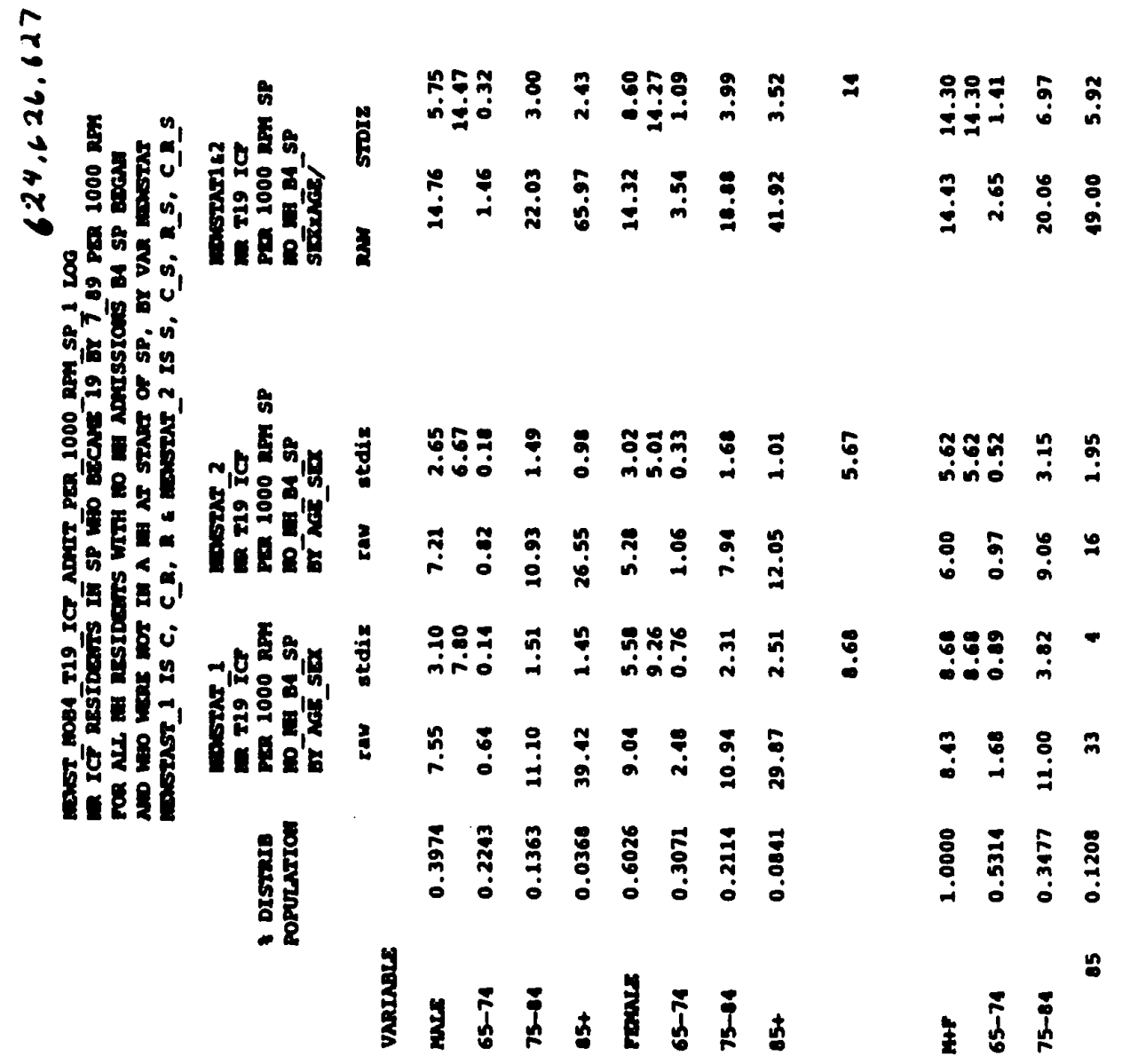




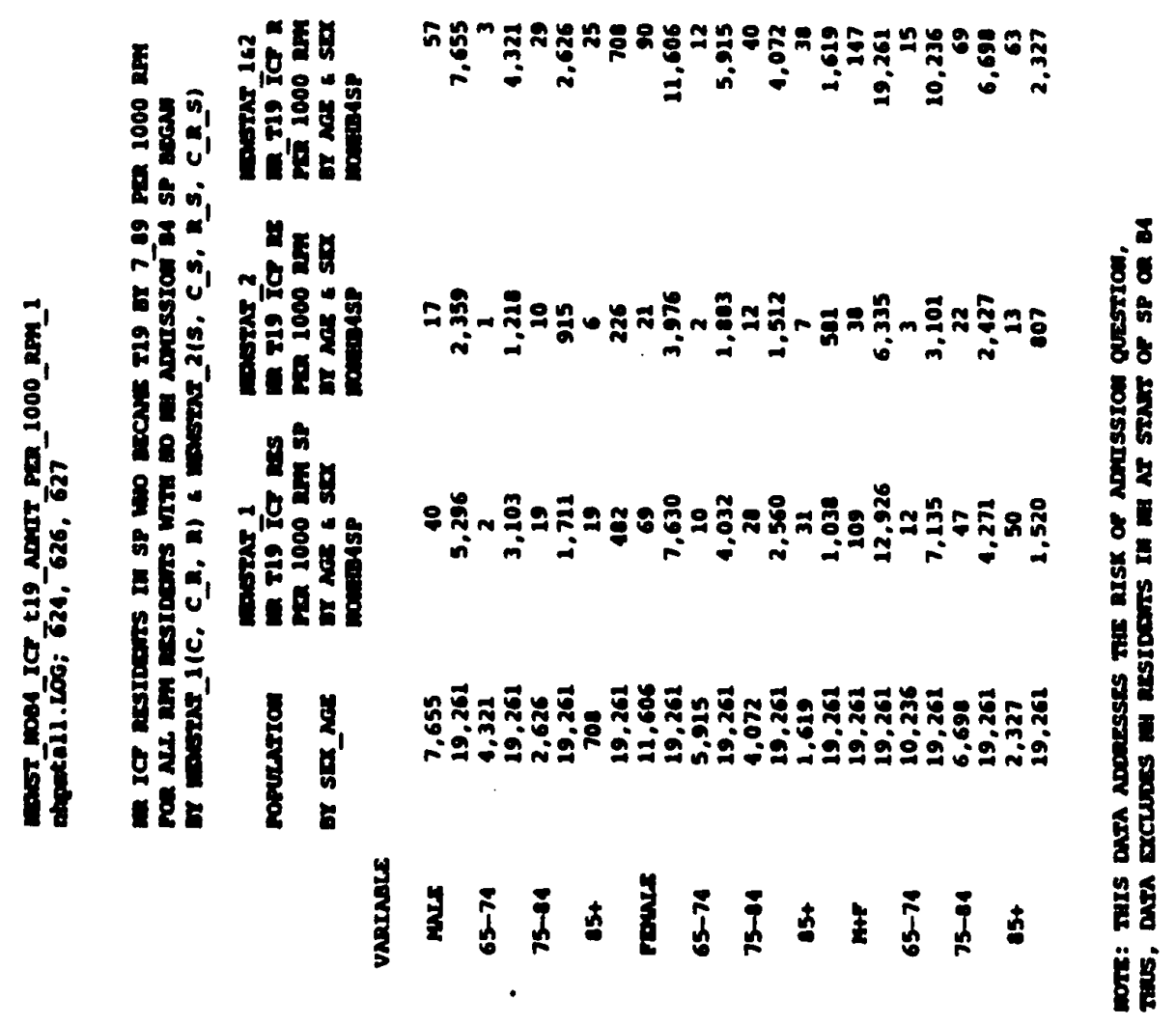




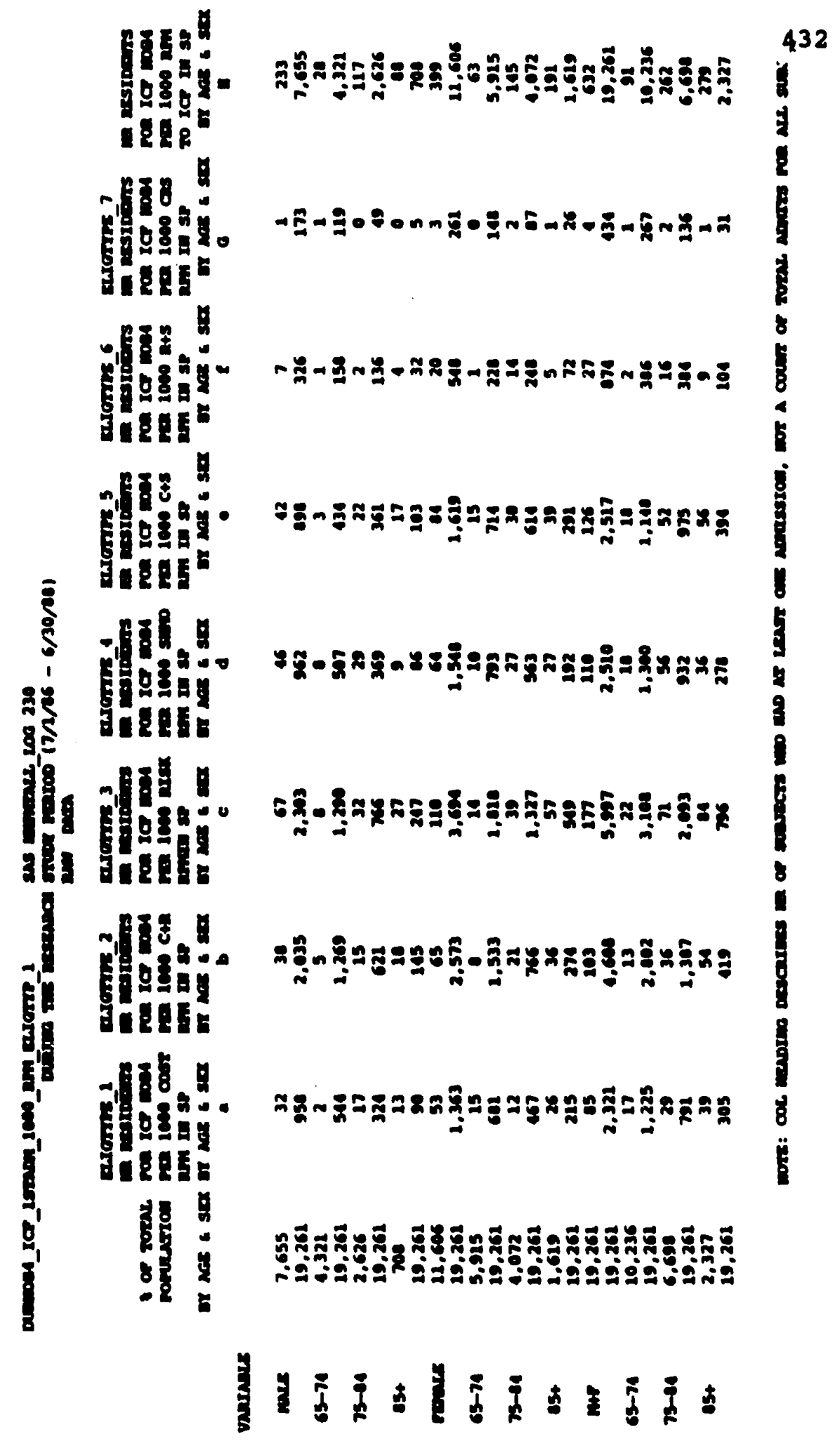




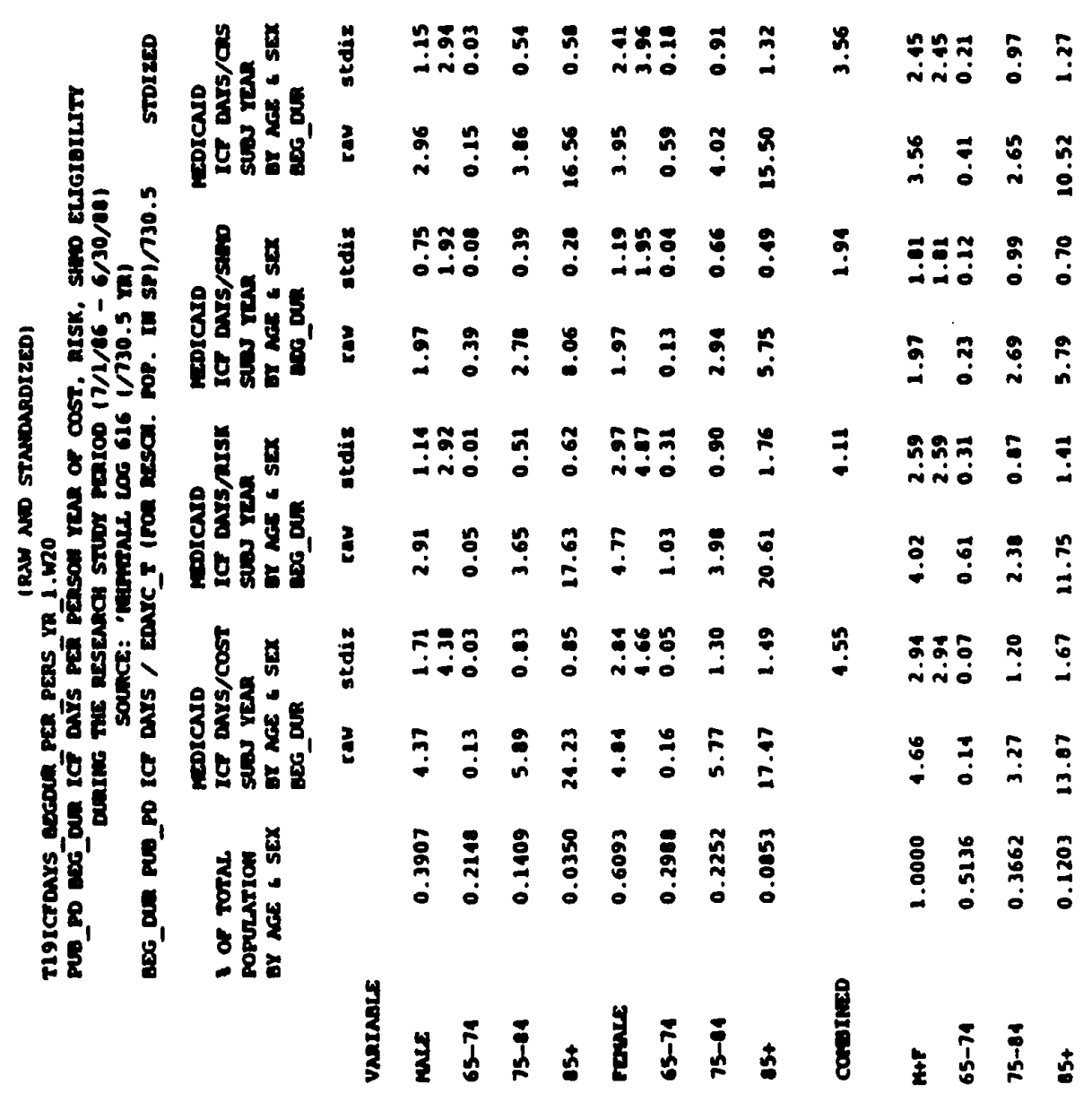




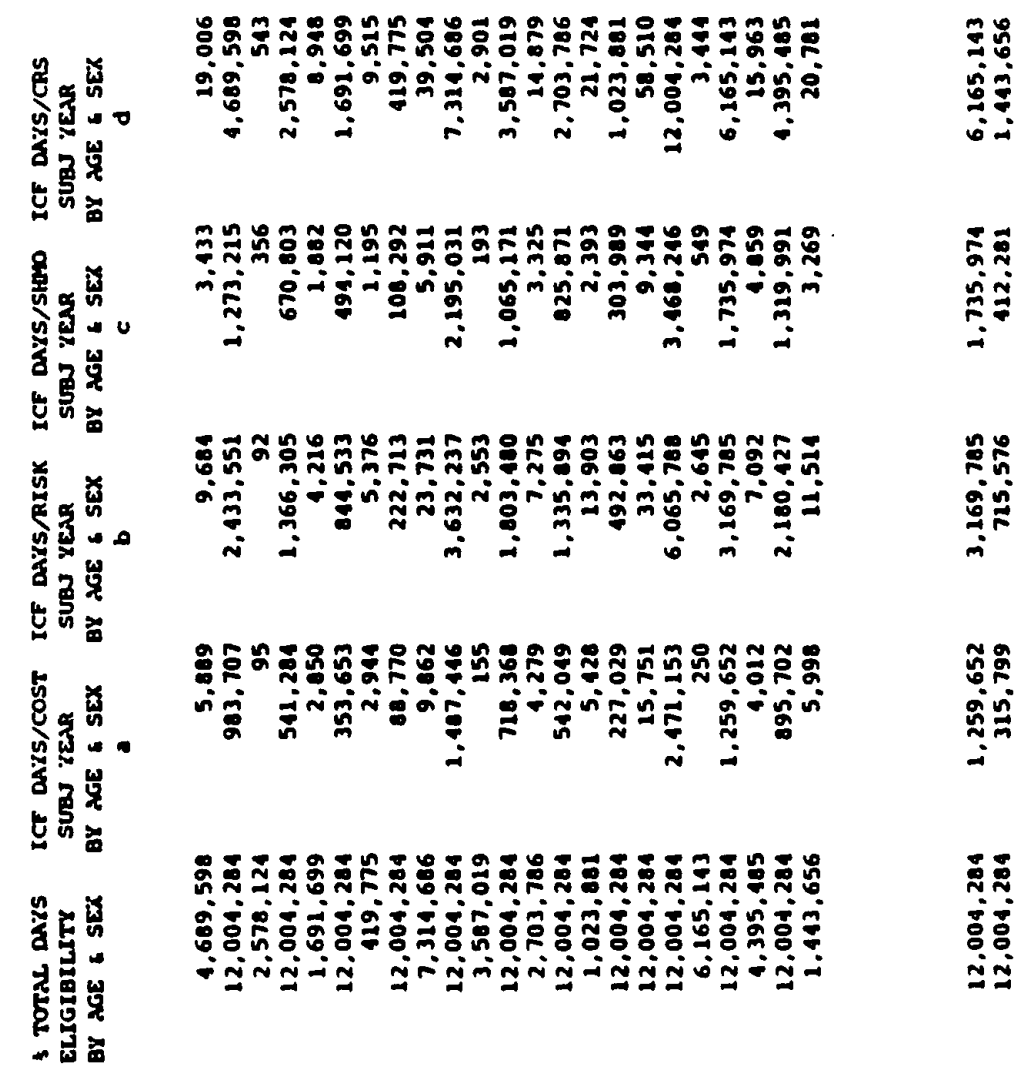

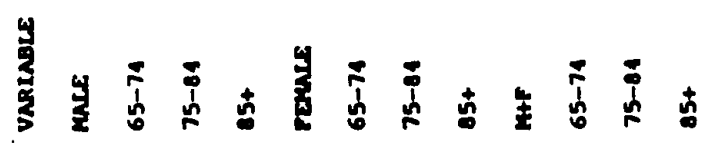




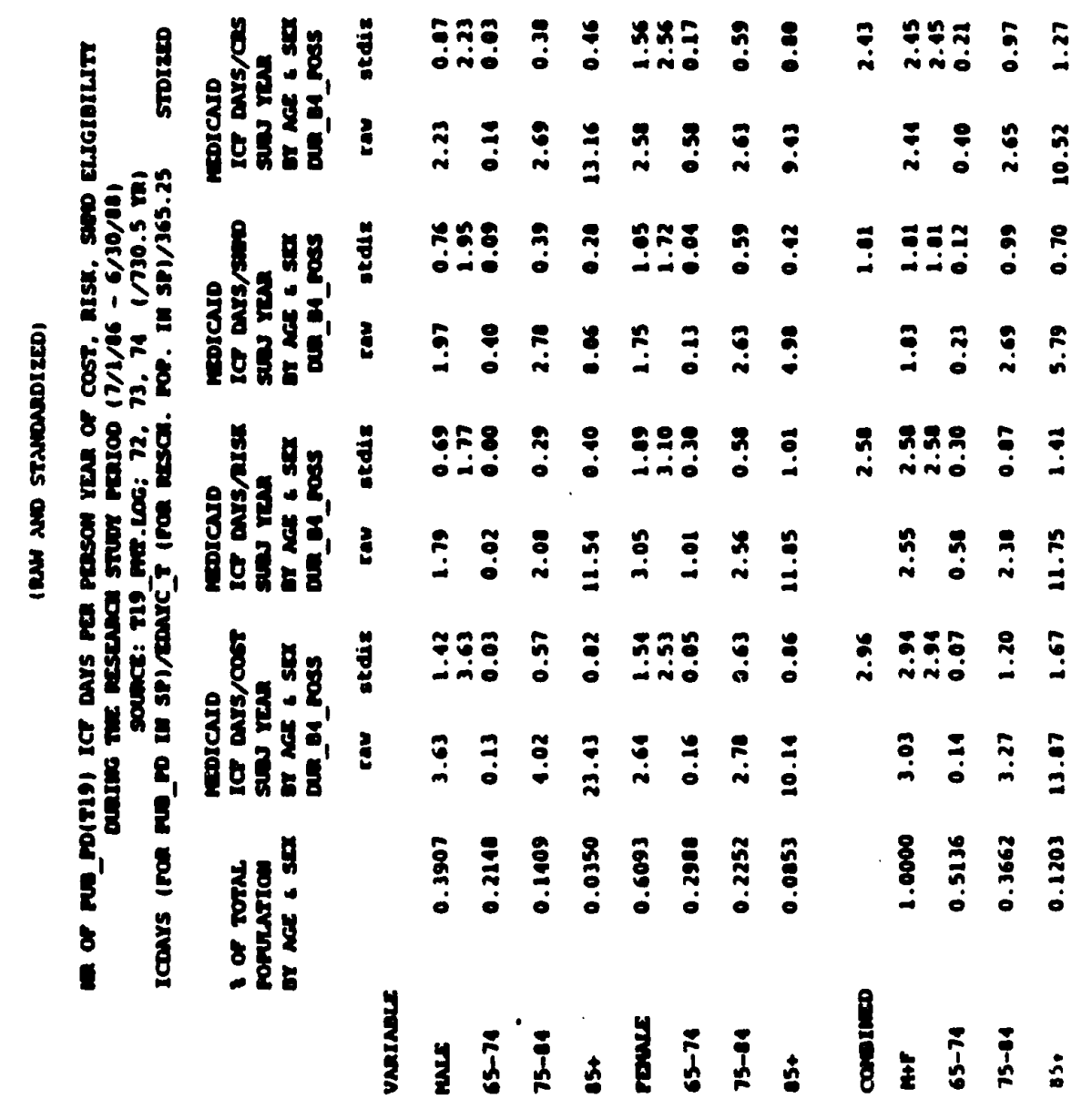




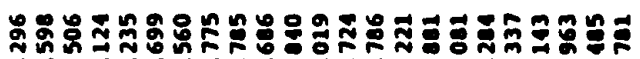

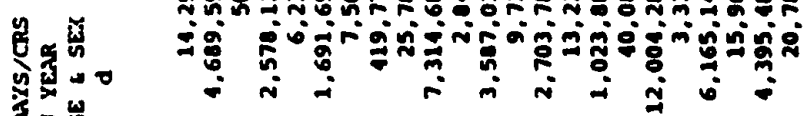

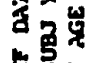

点的染

赛 啳

势政

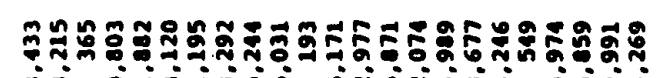

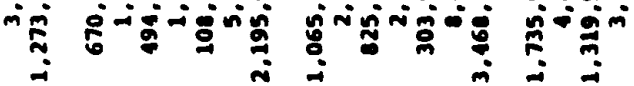

:

בำ

的要

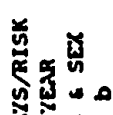

ริ)

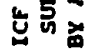

尊总

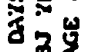

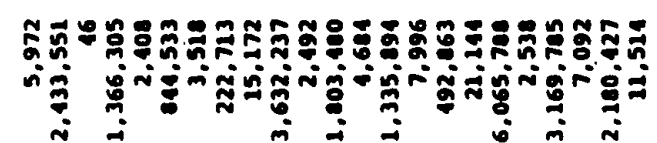

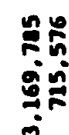

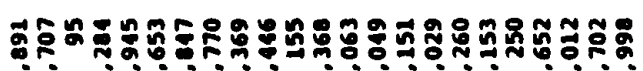

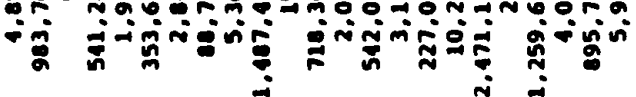

踏

影枈

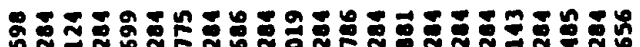

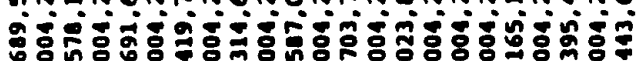

作

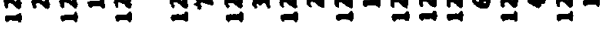



لَّ

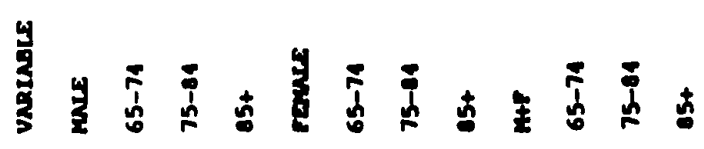



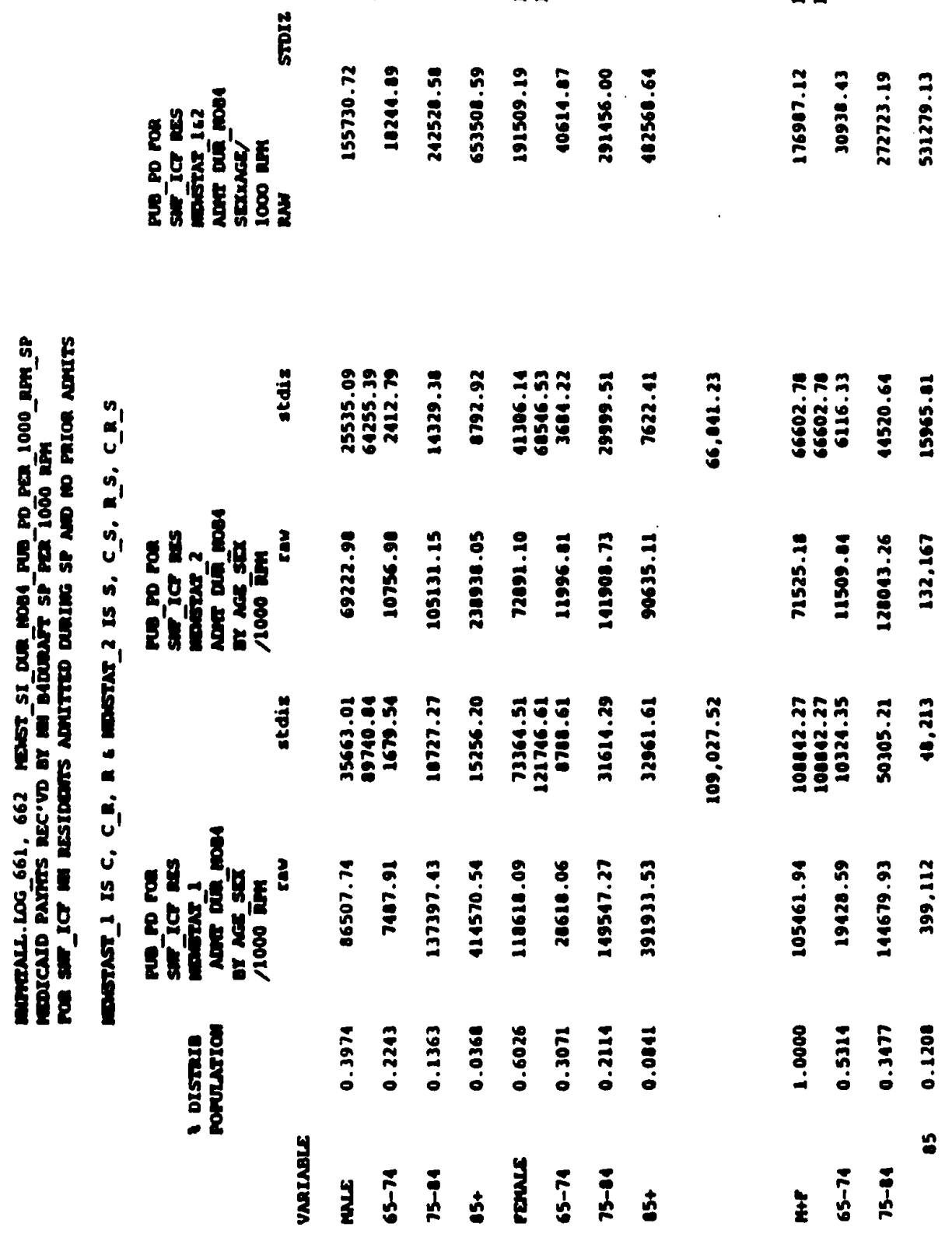


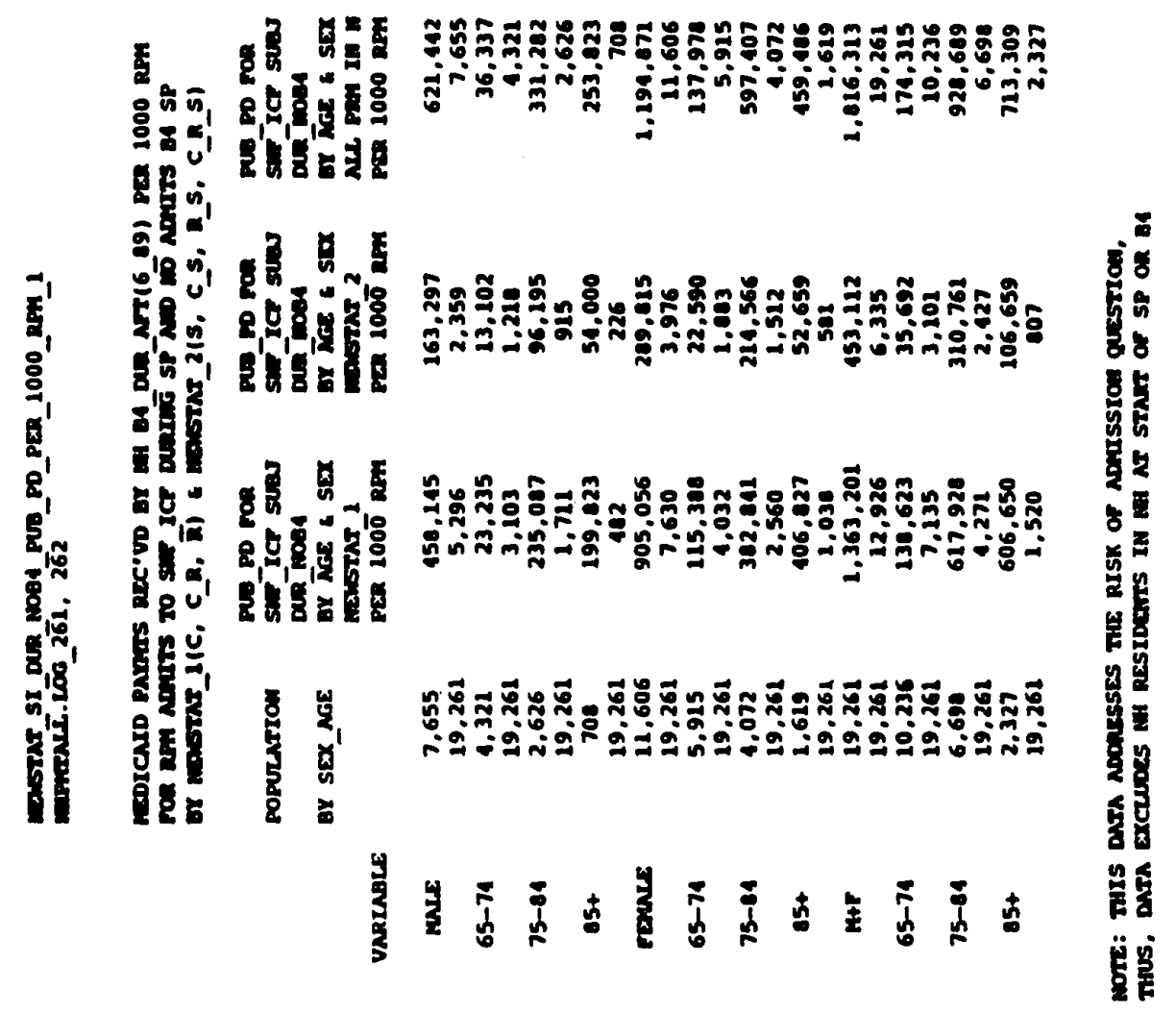




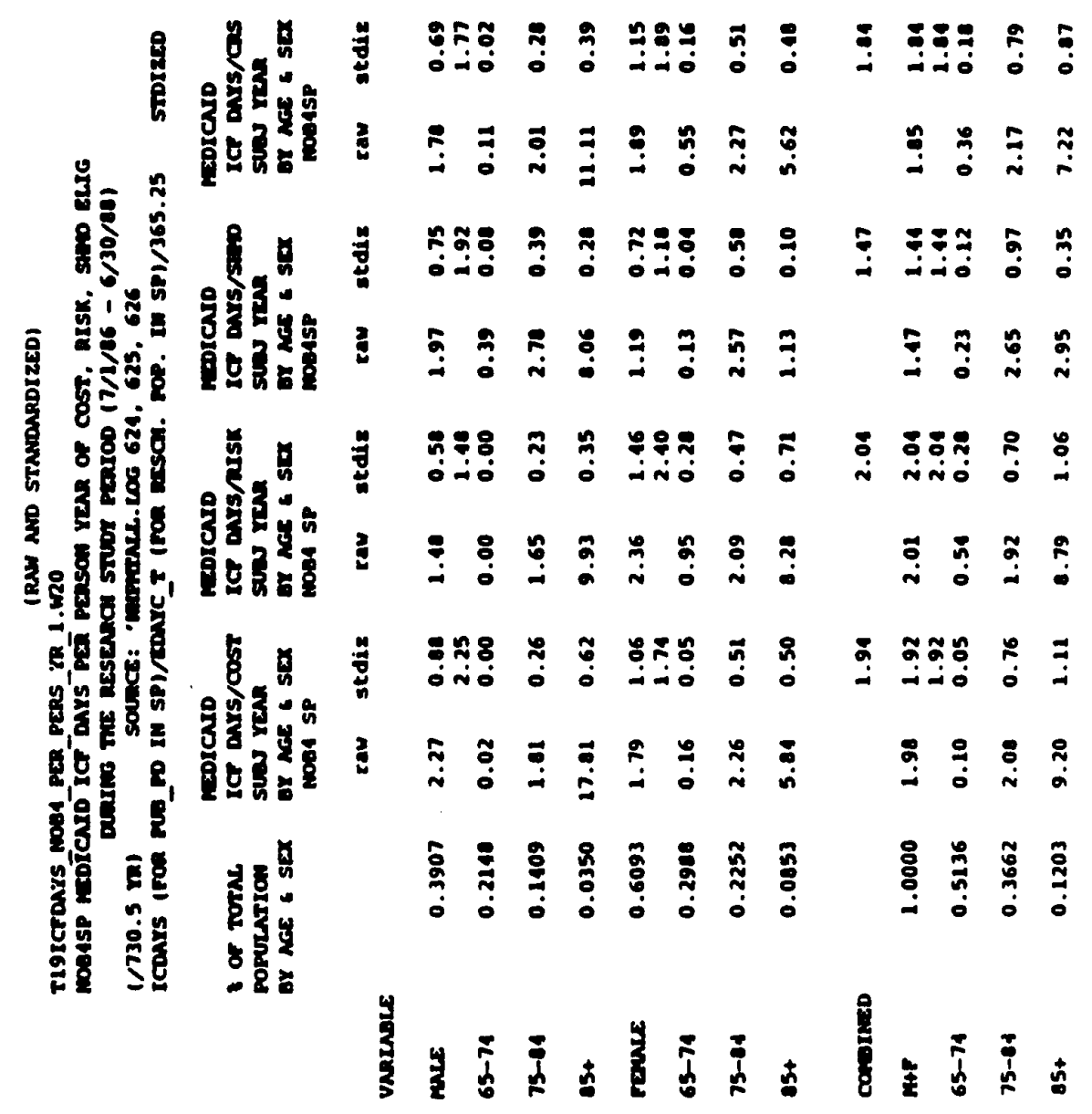




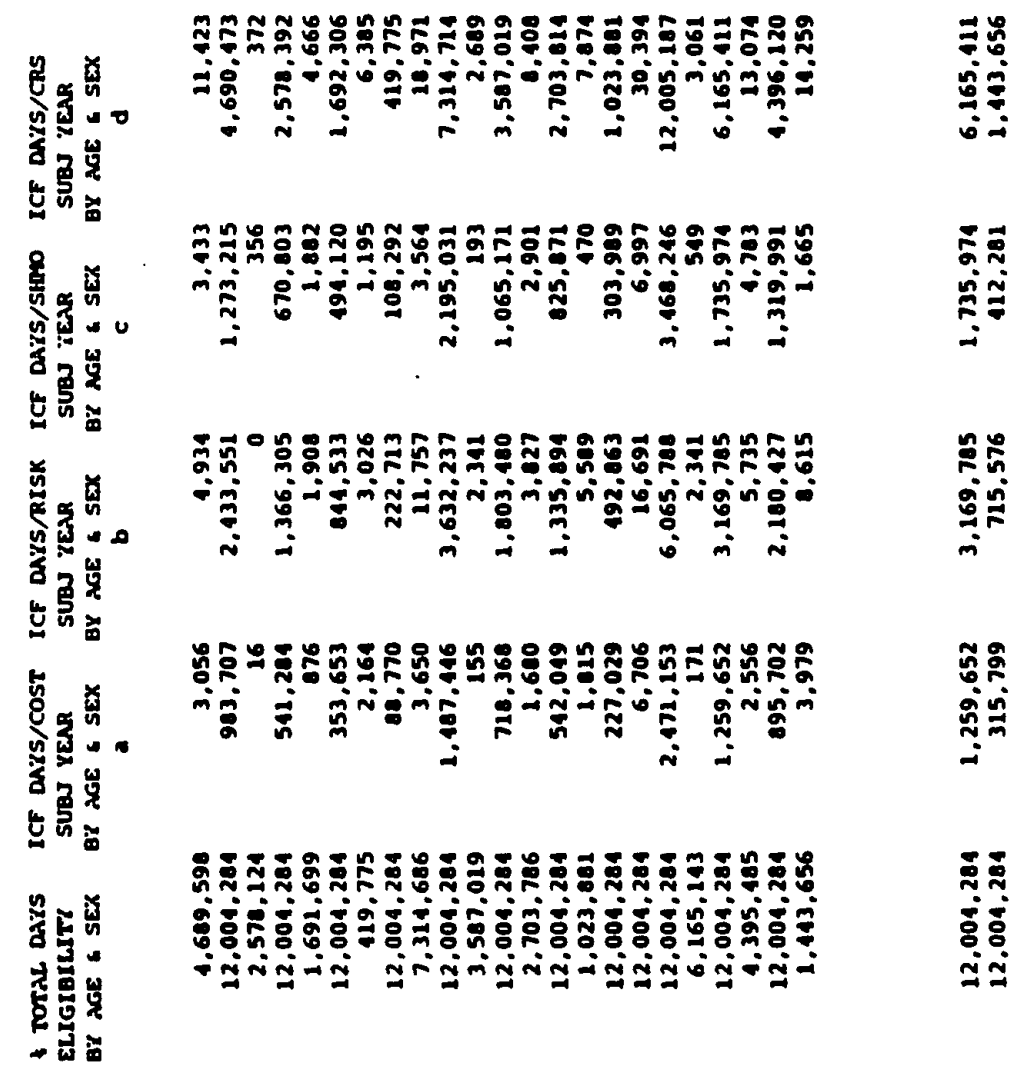

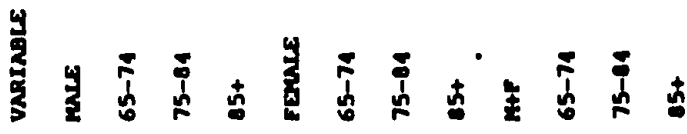




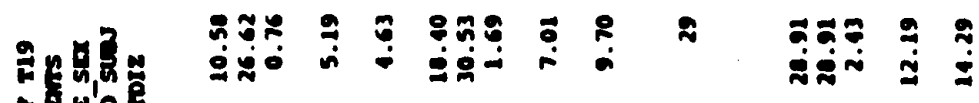
0848

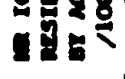

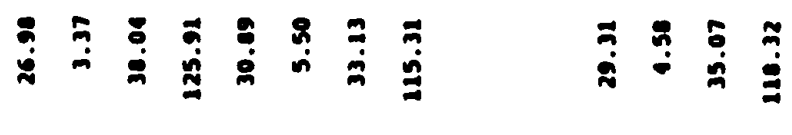

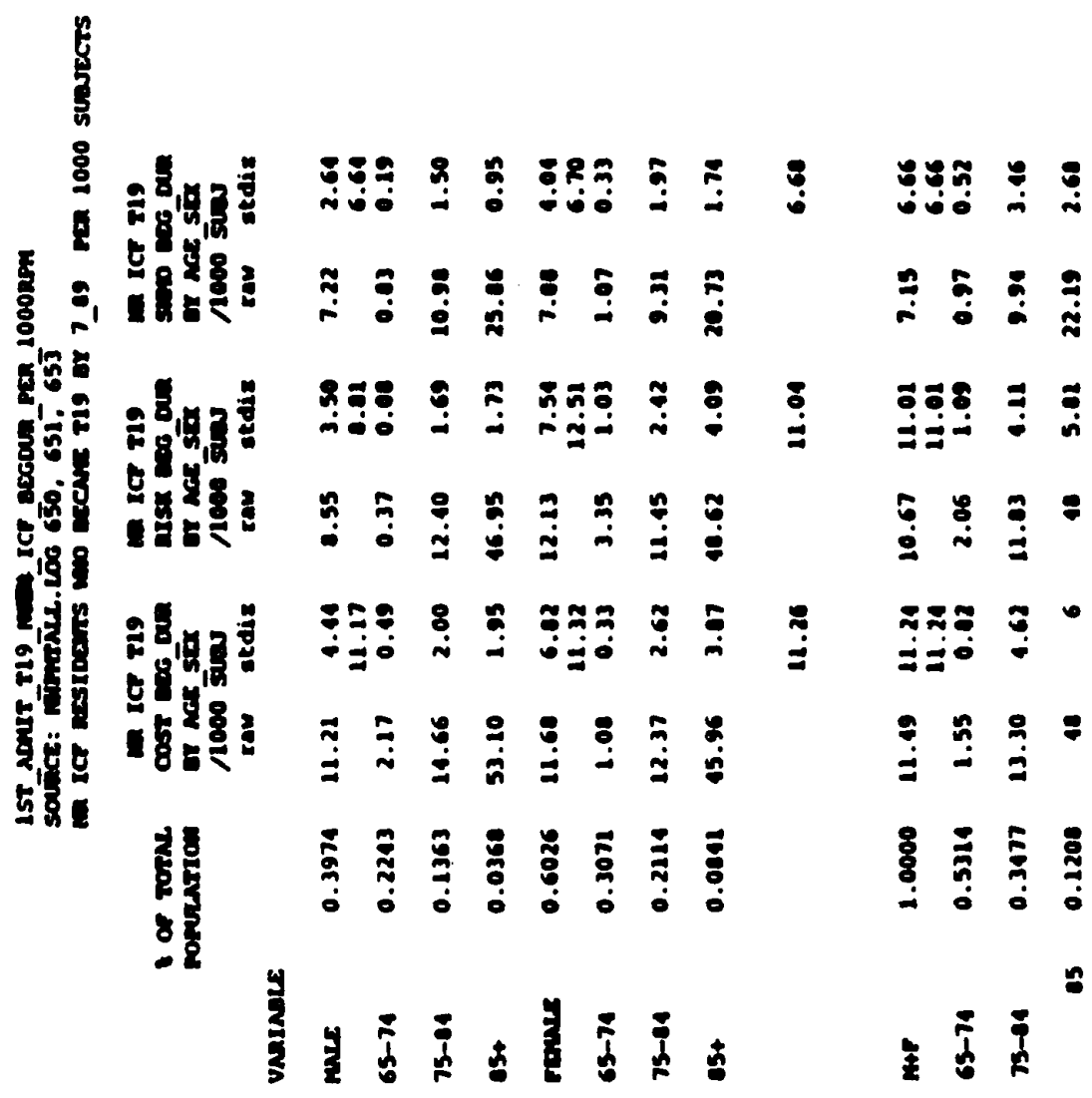




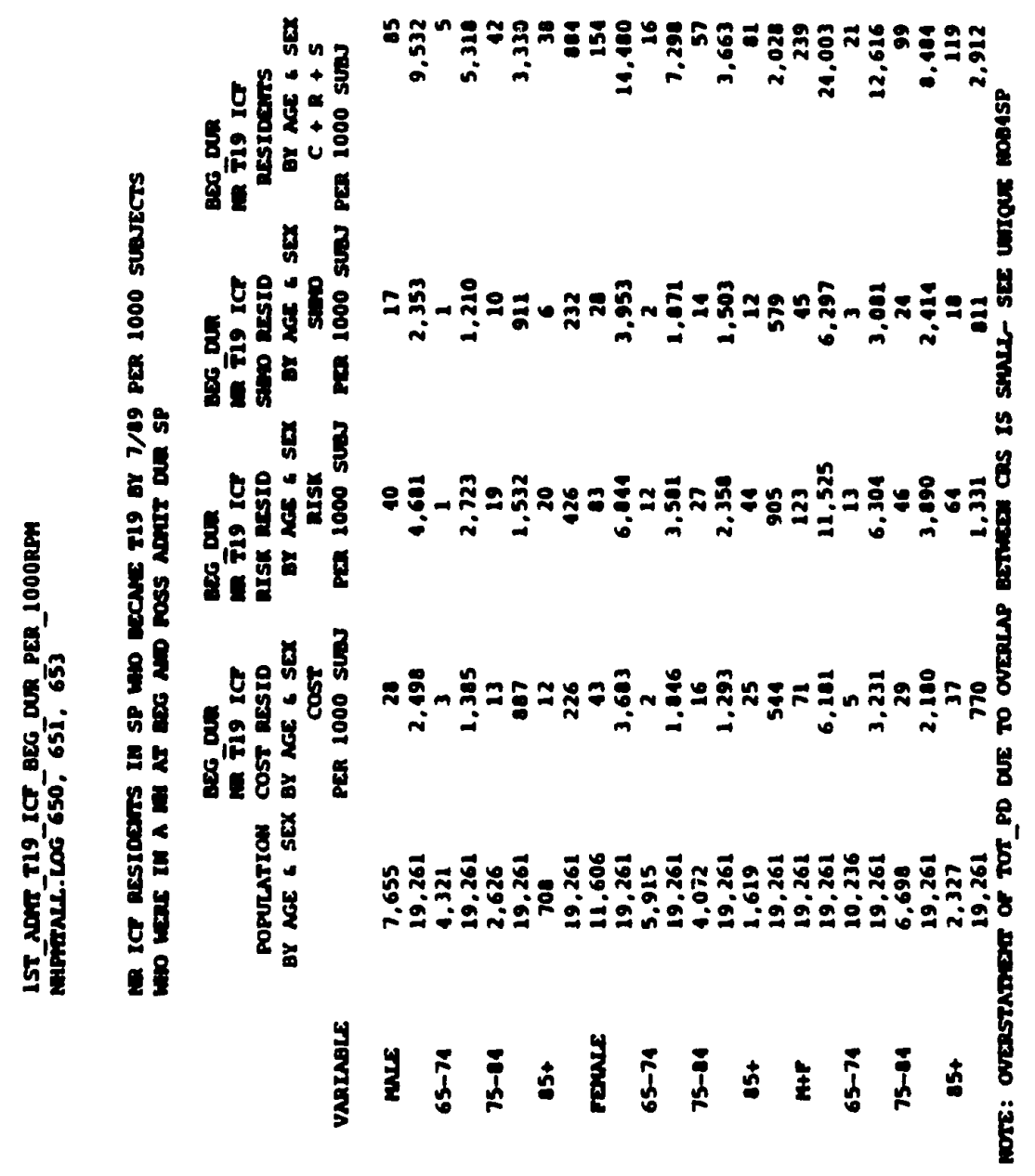




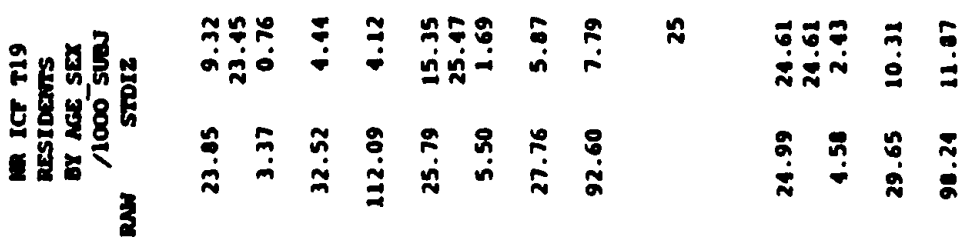

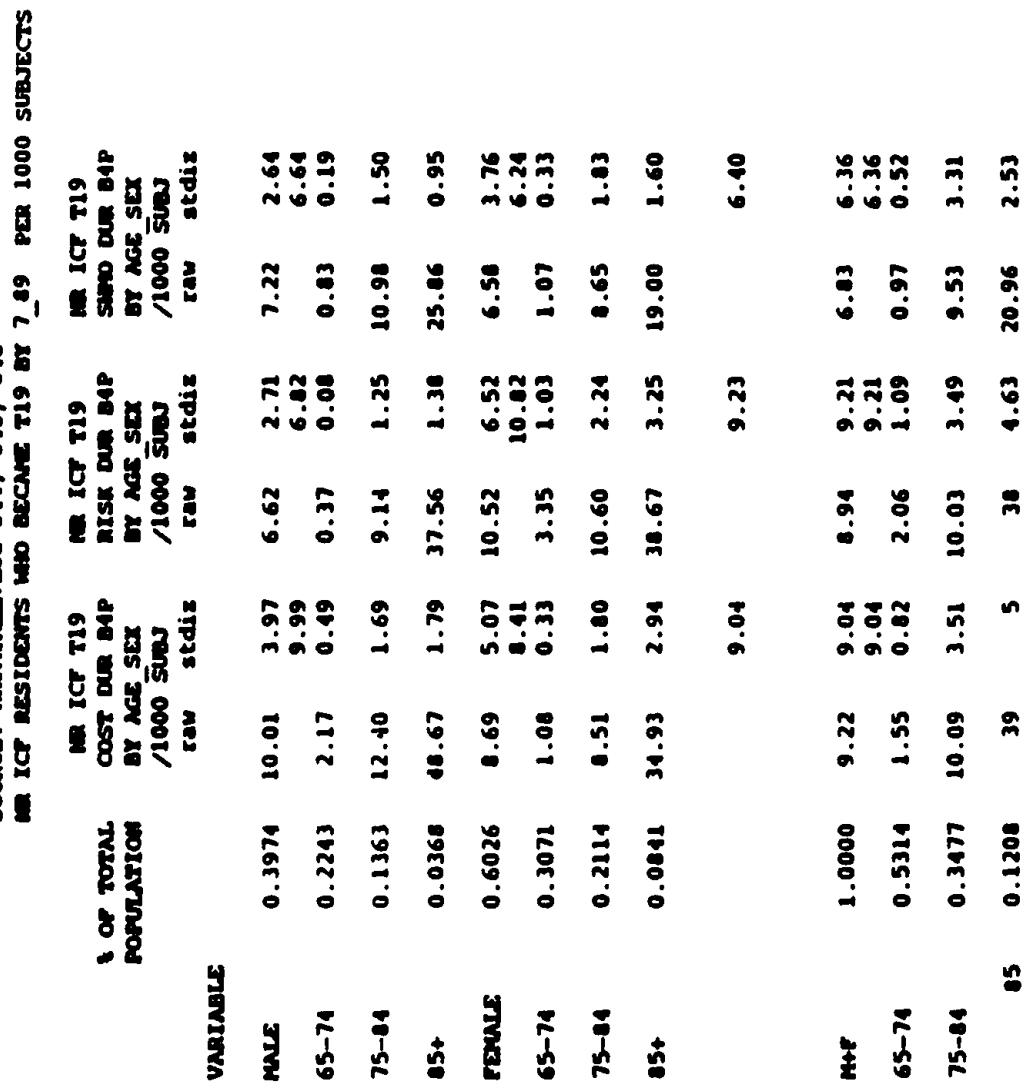




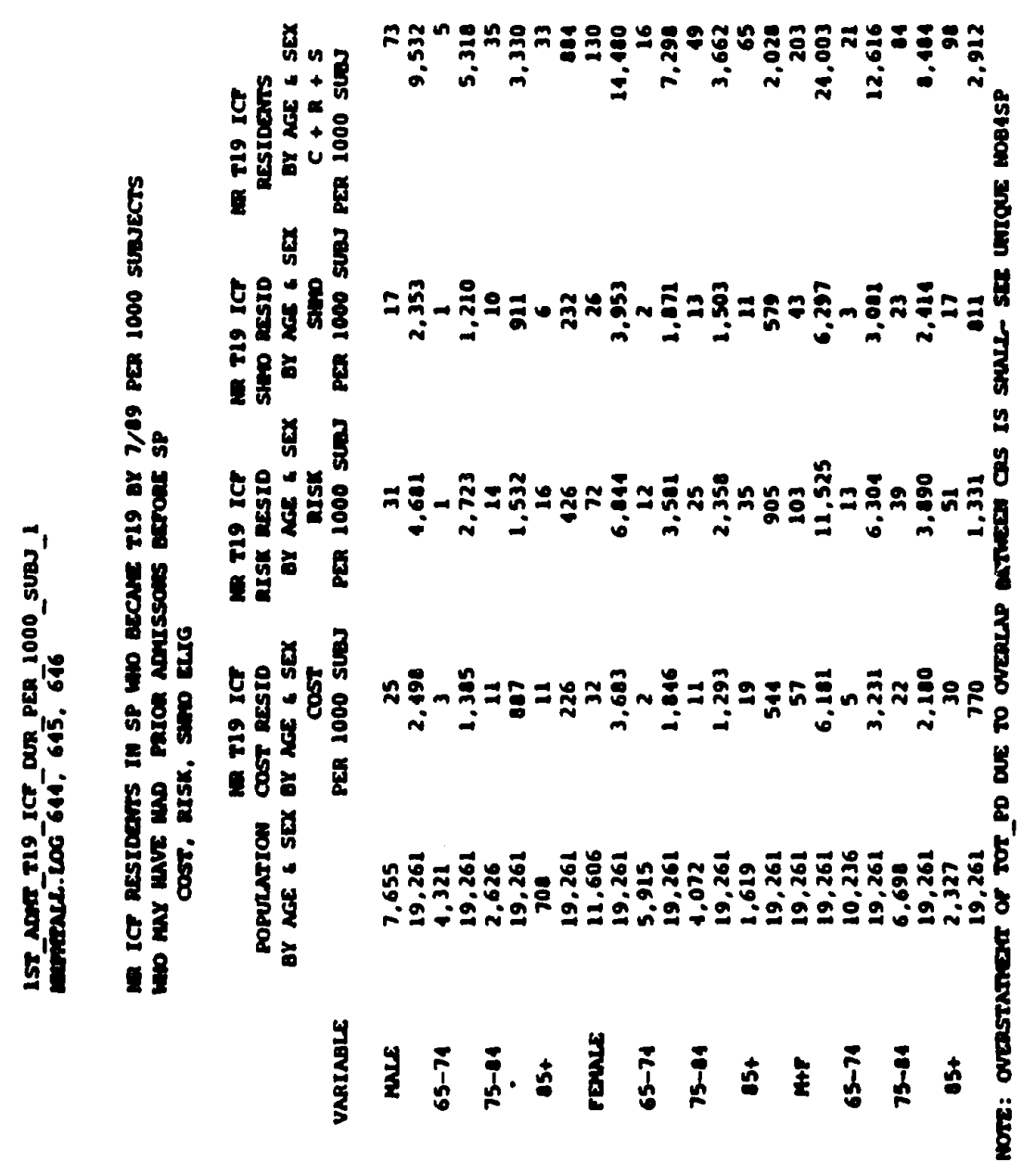




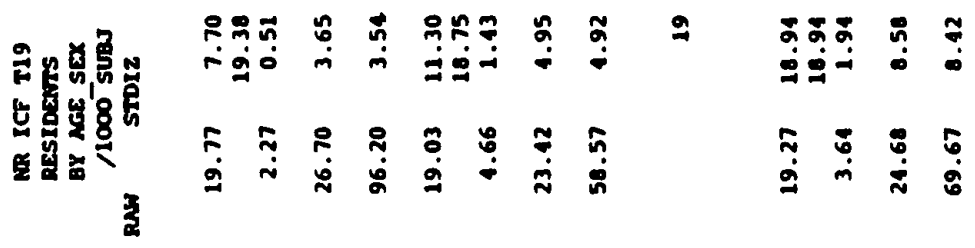

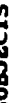

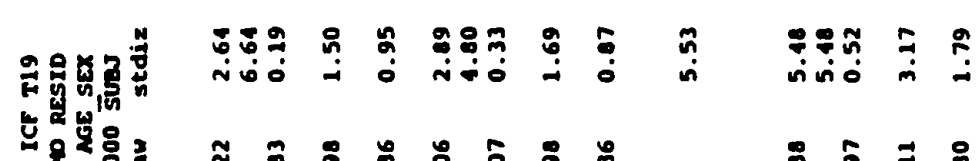

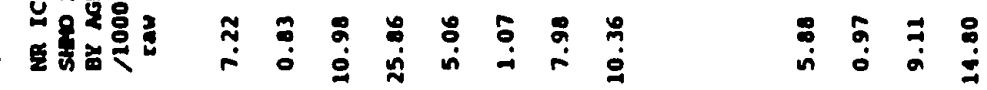

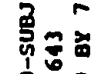

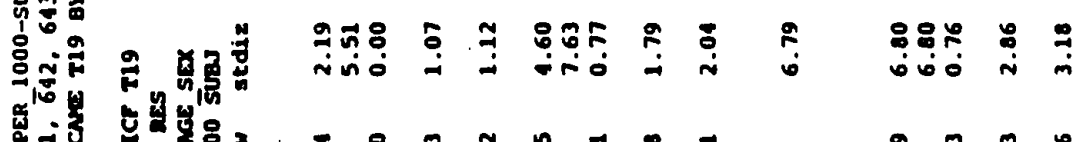

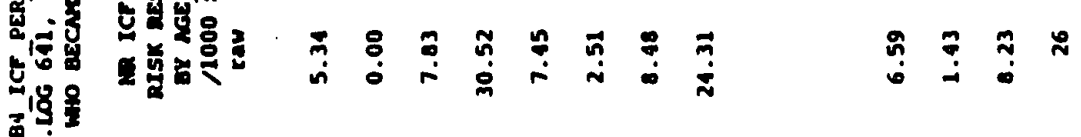

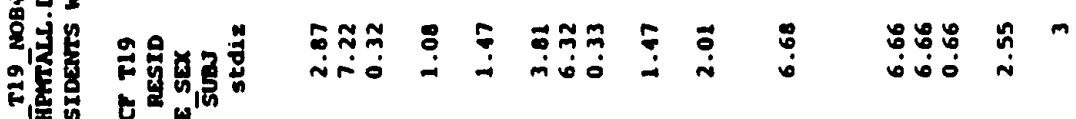

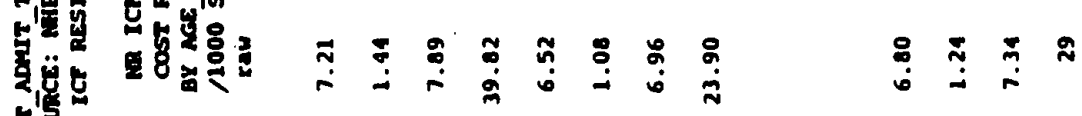
案总

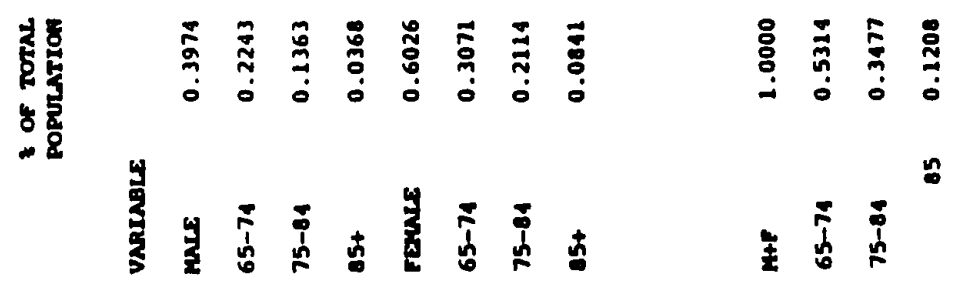




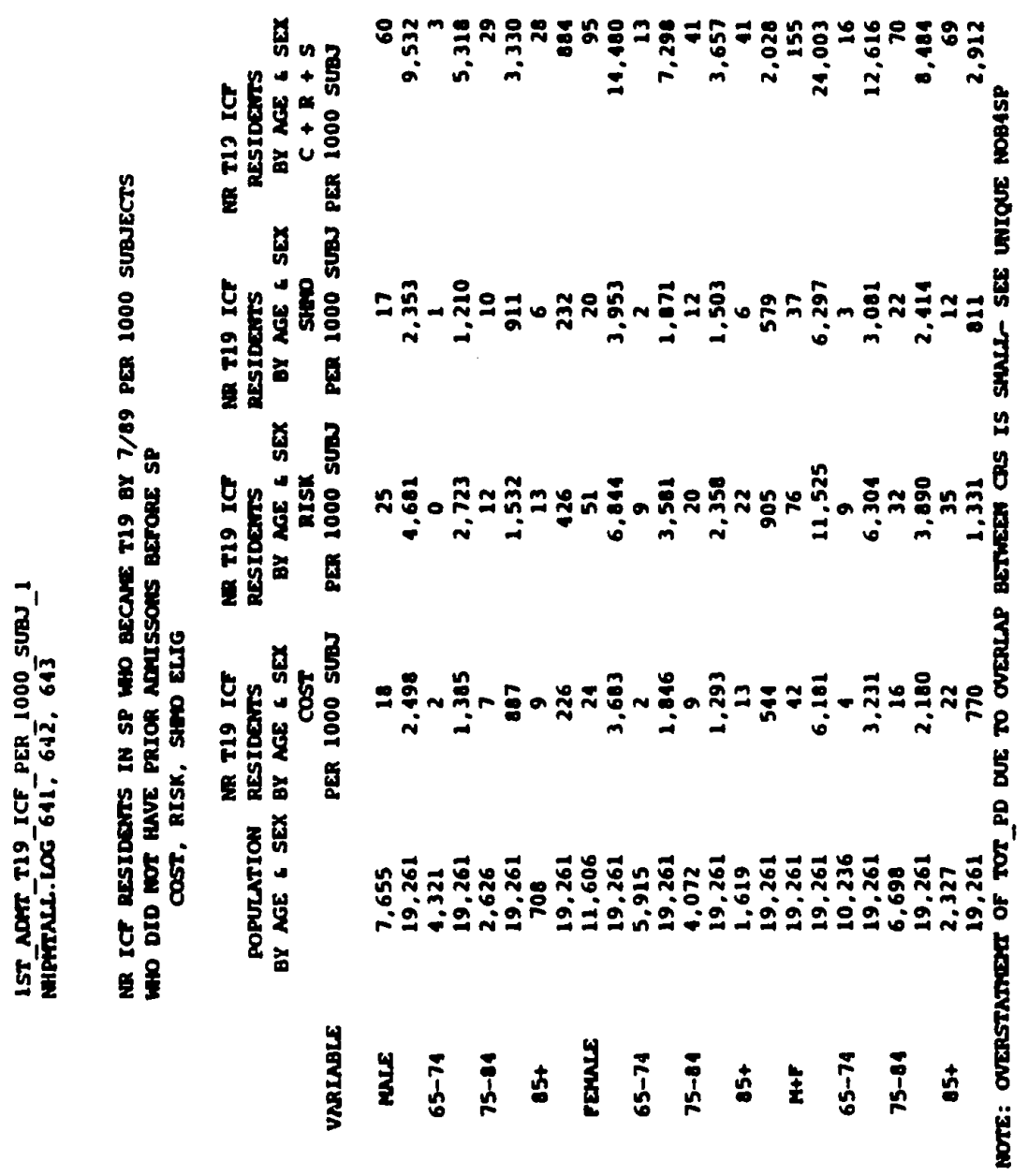



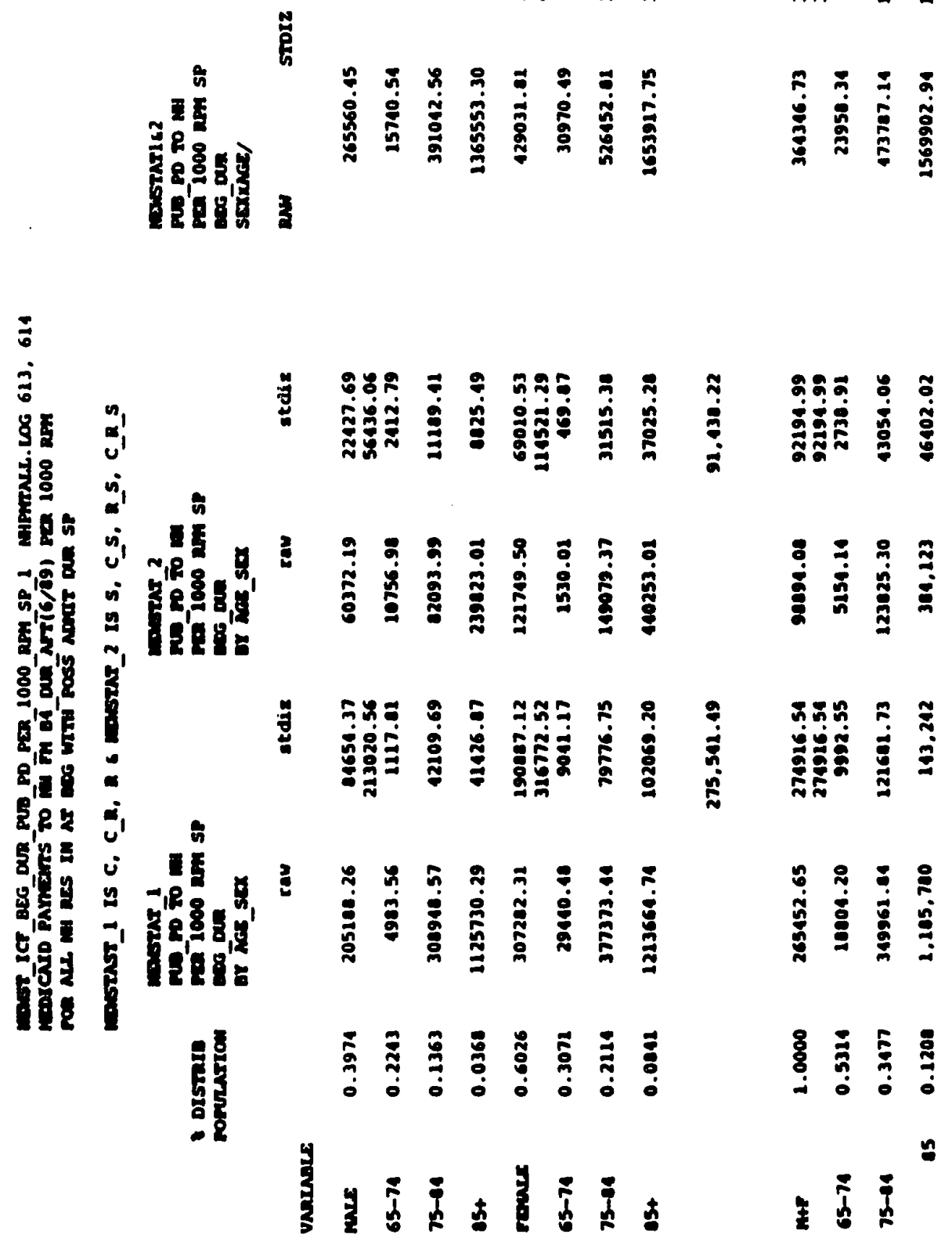


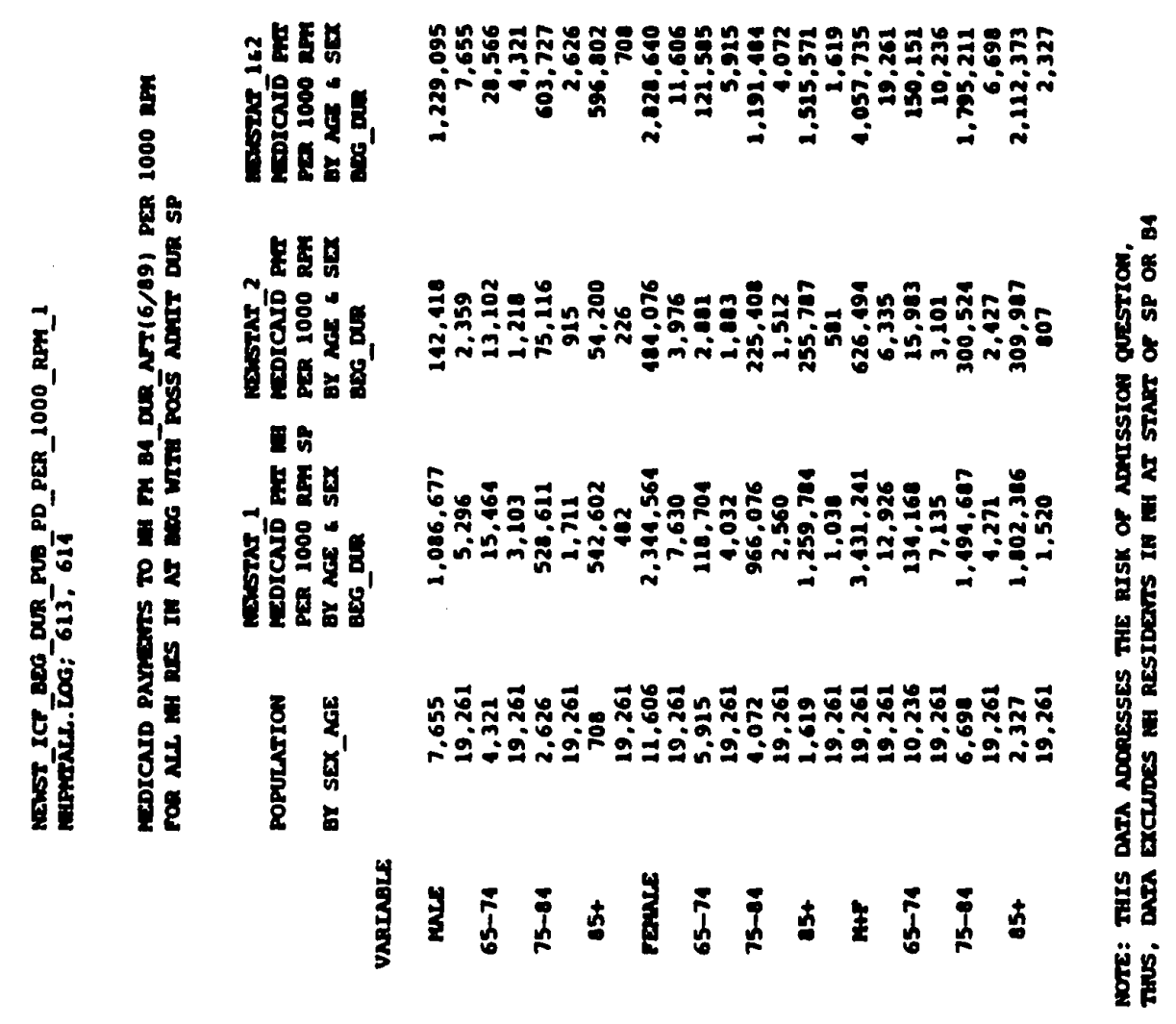



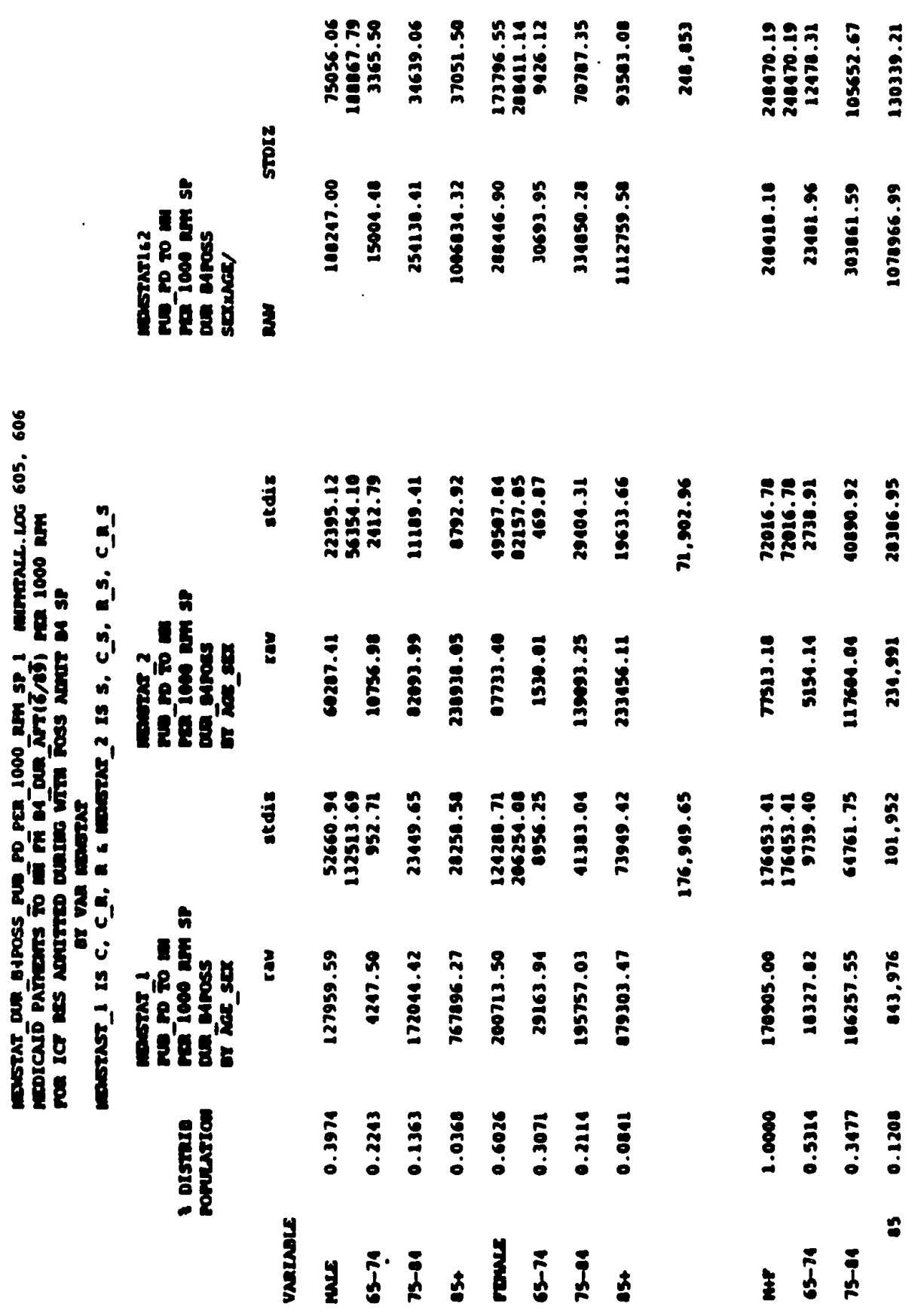


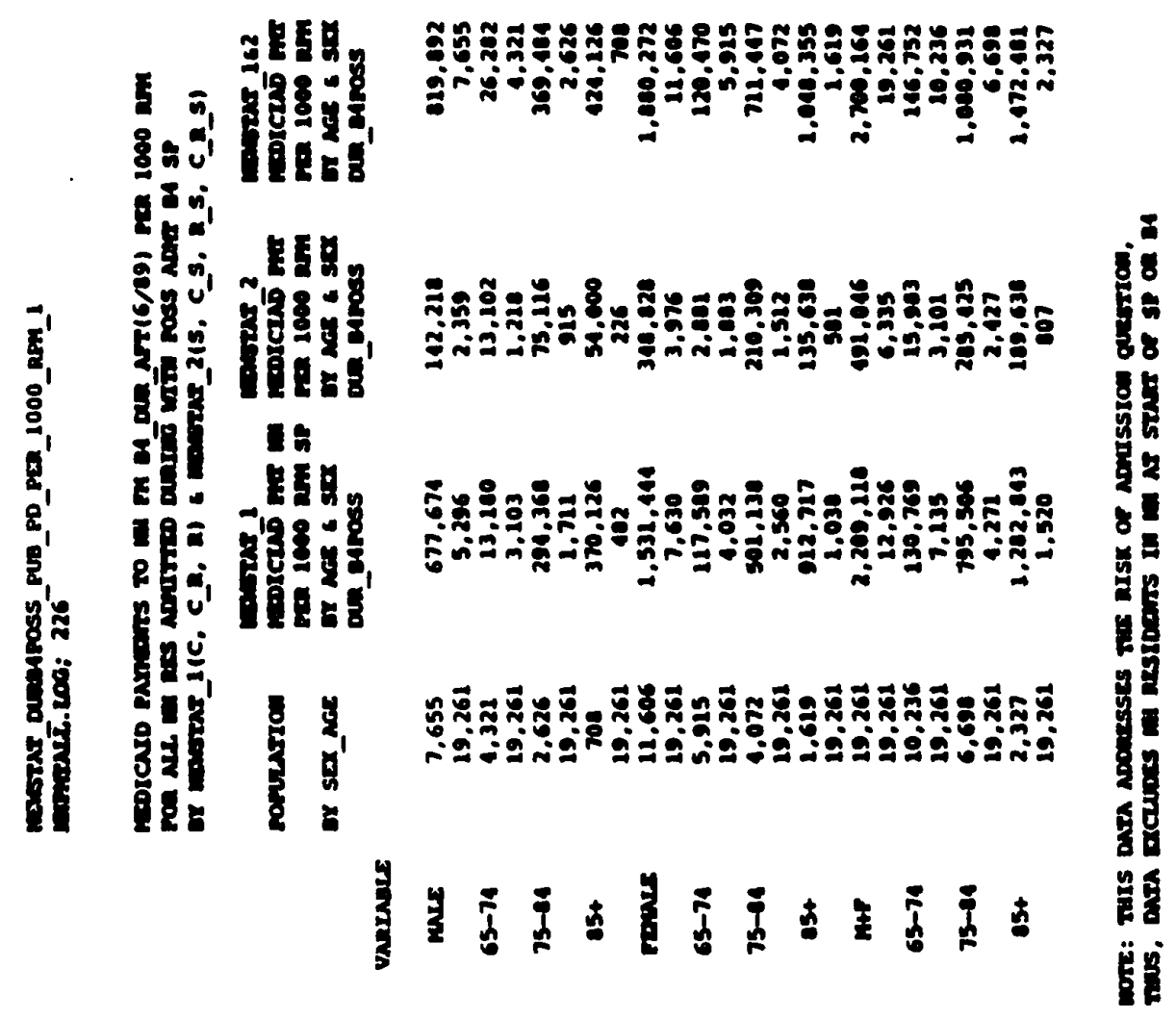




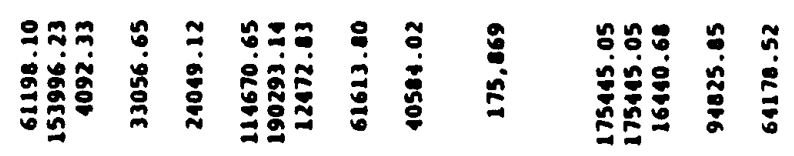

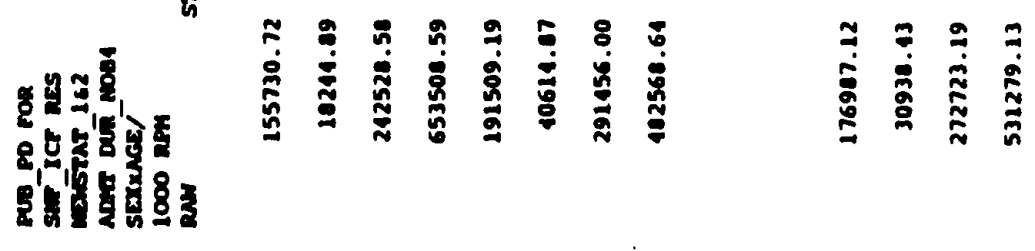

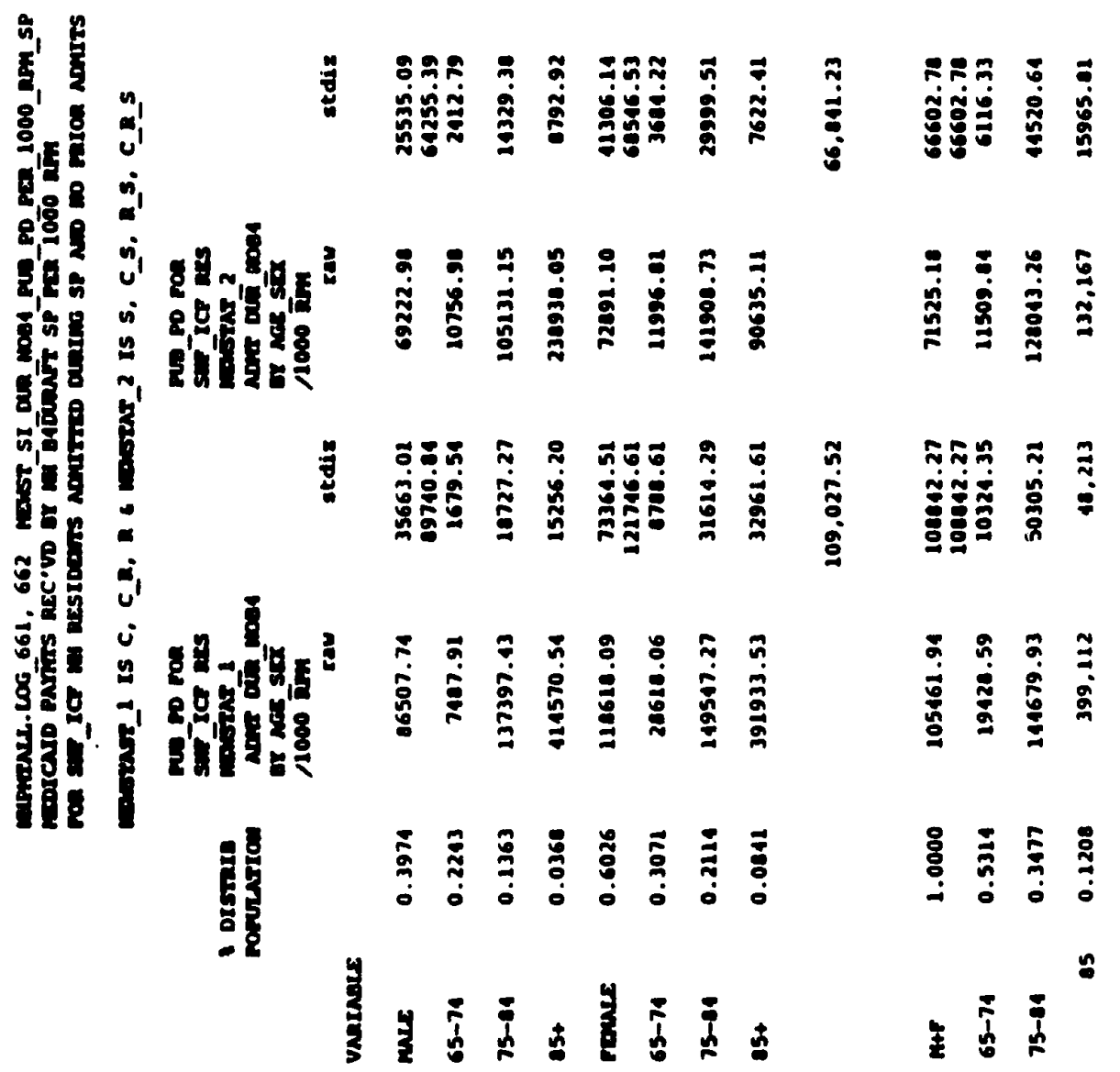




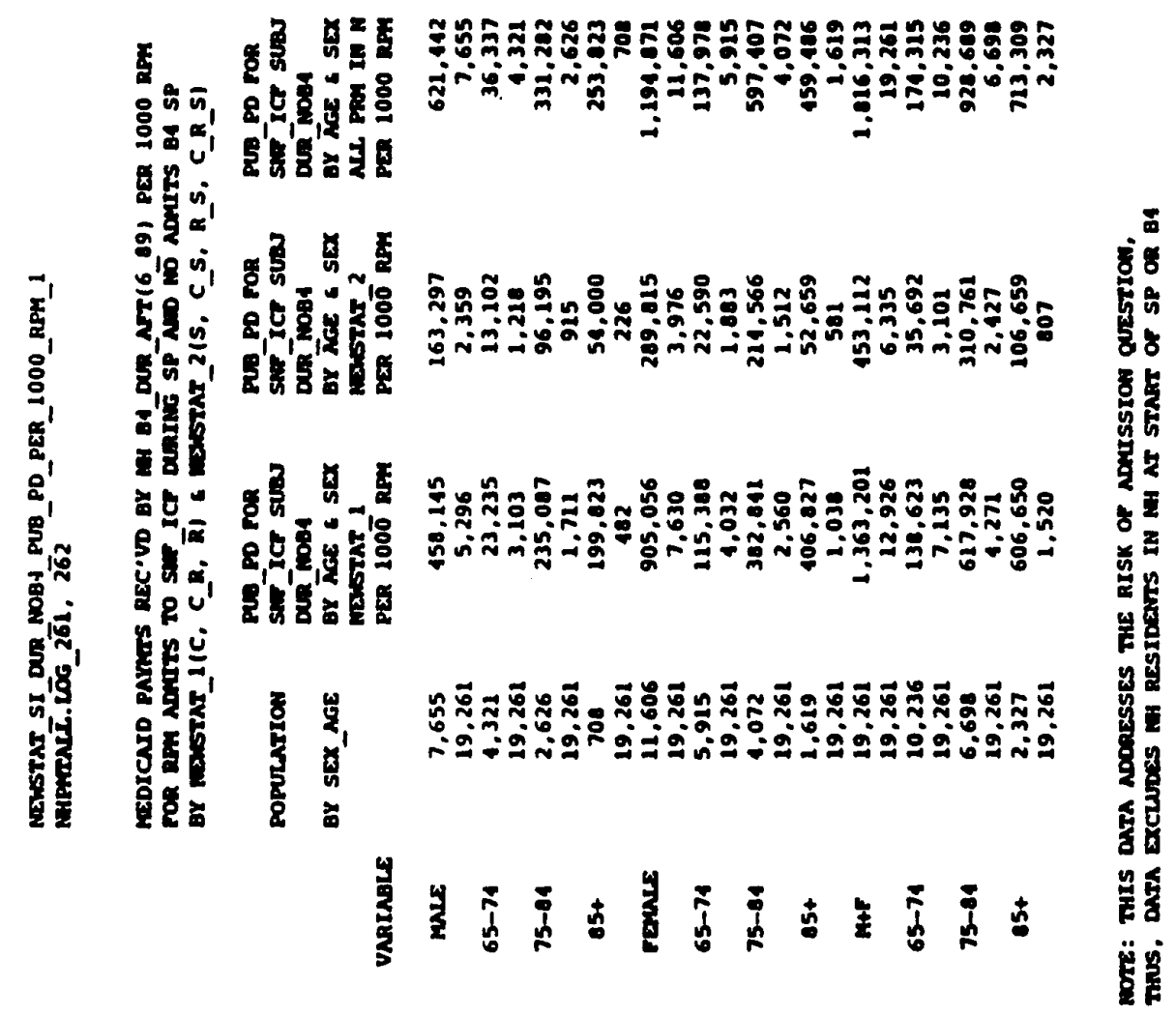




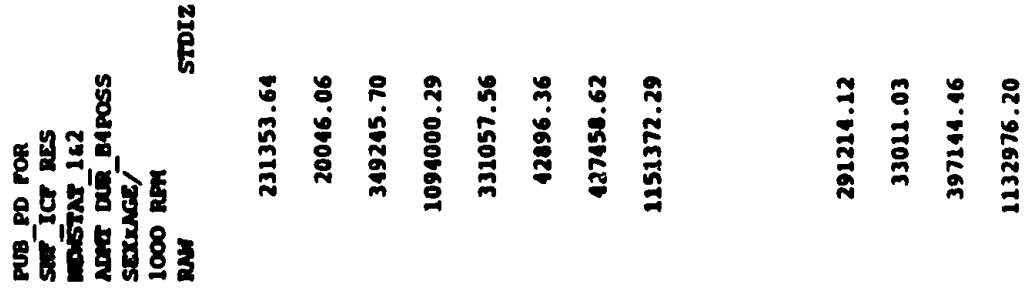

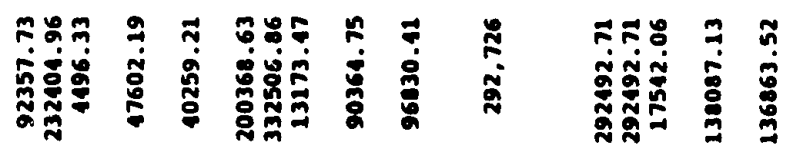

䓪

용용

mathen

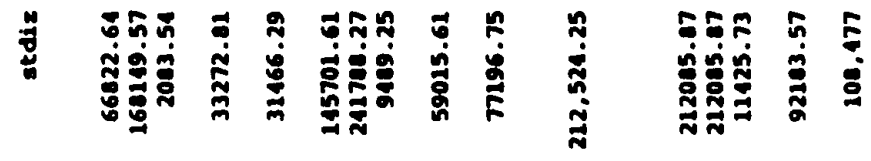

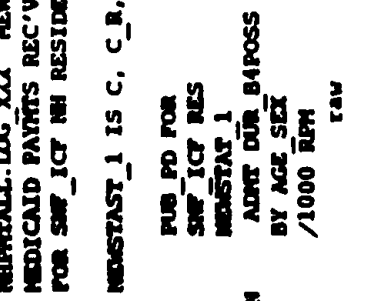

IInחIIIII

II

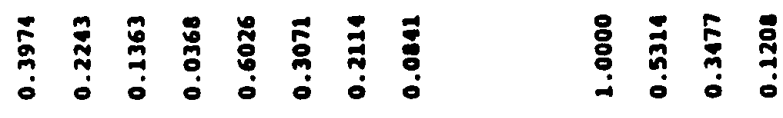

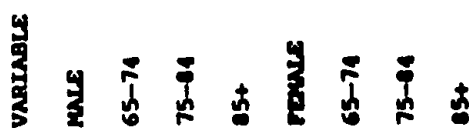

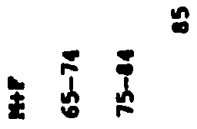




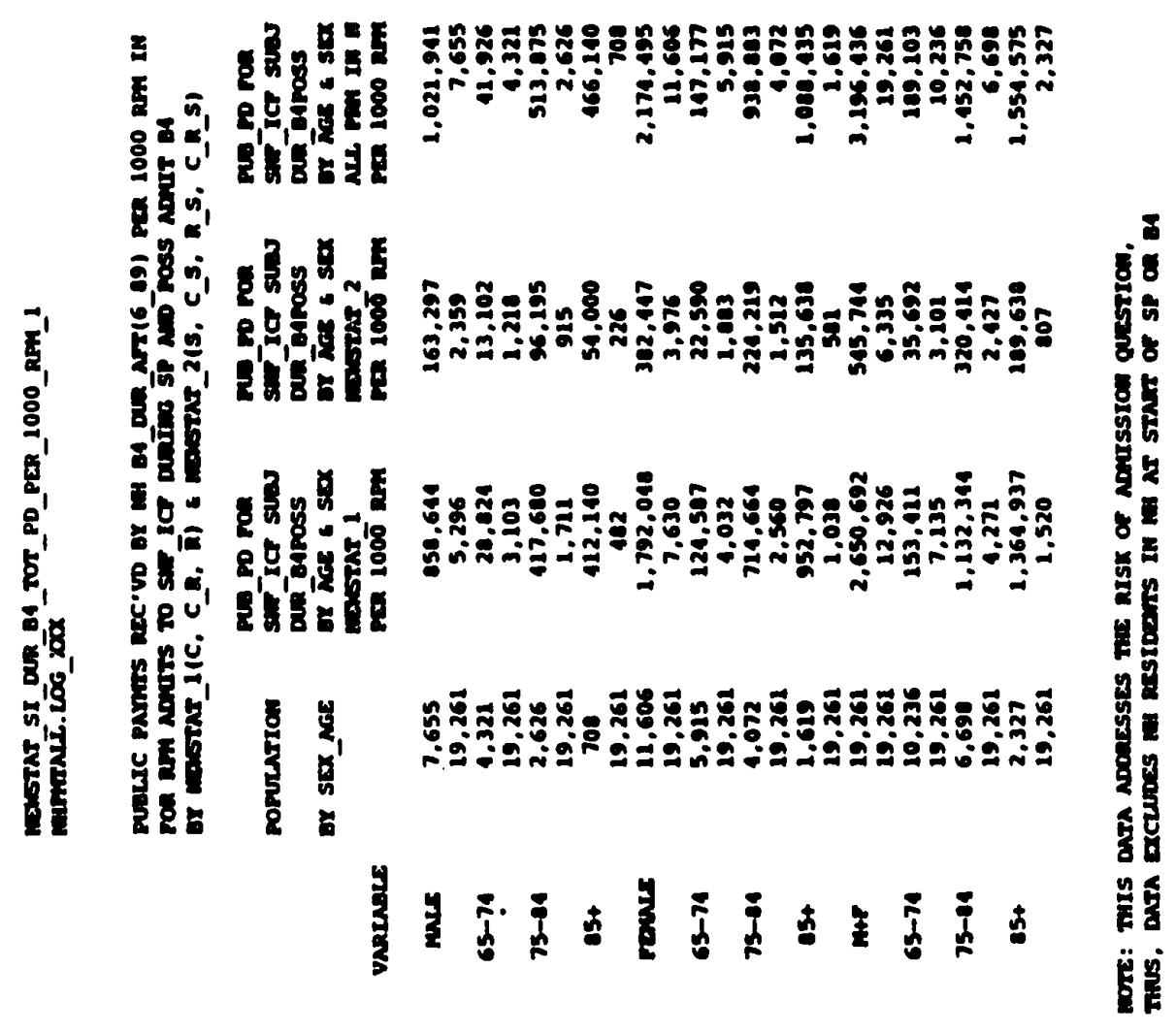



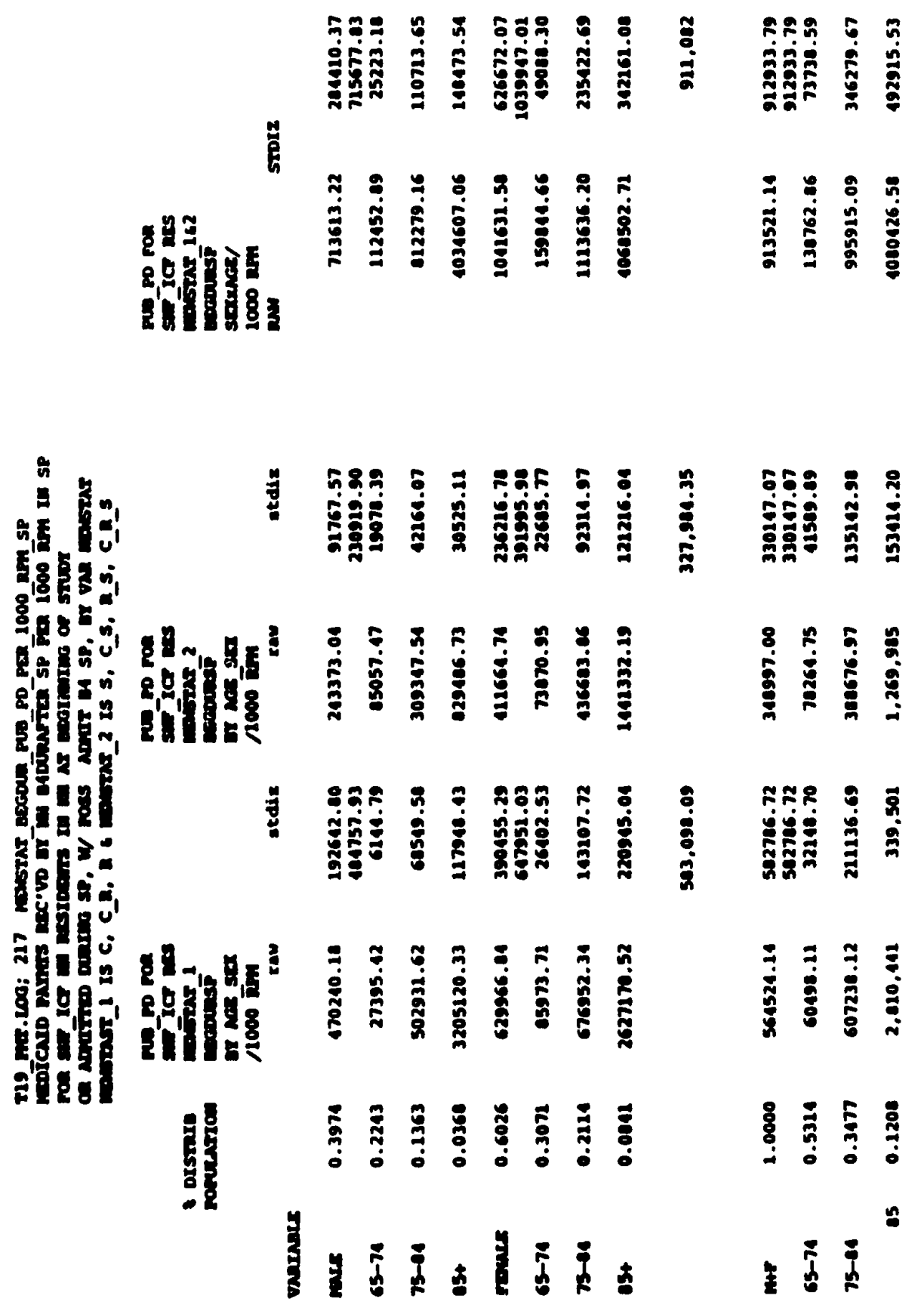


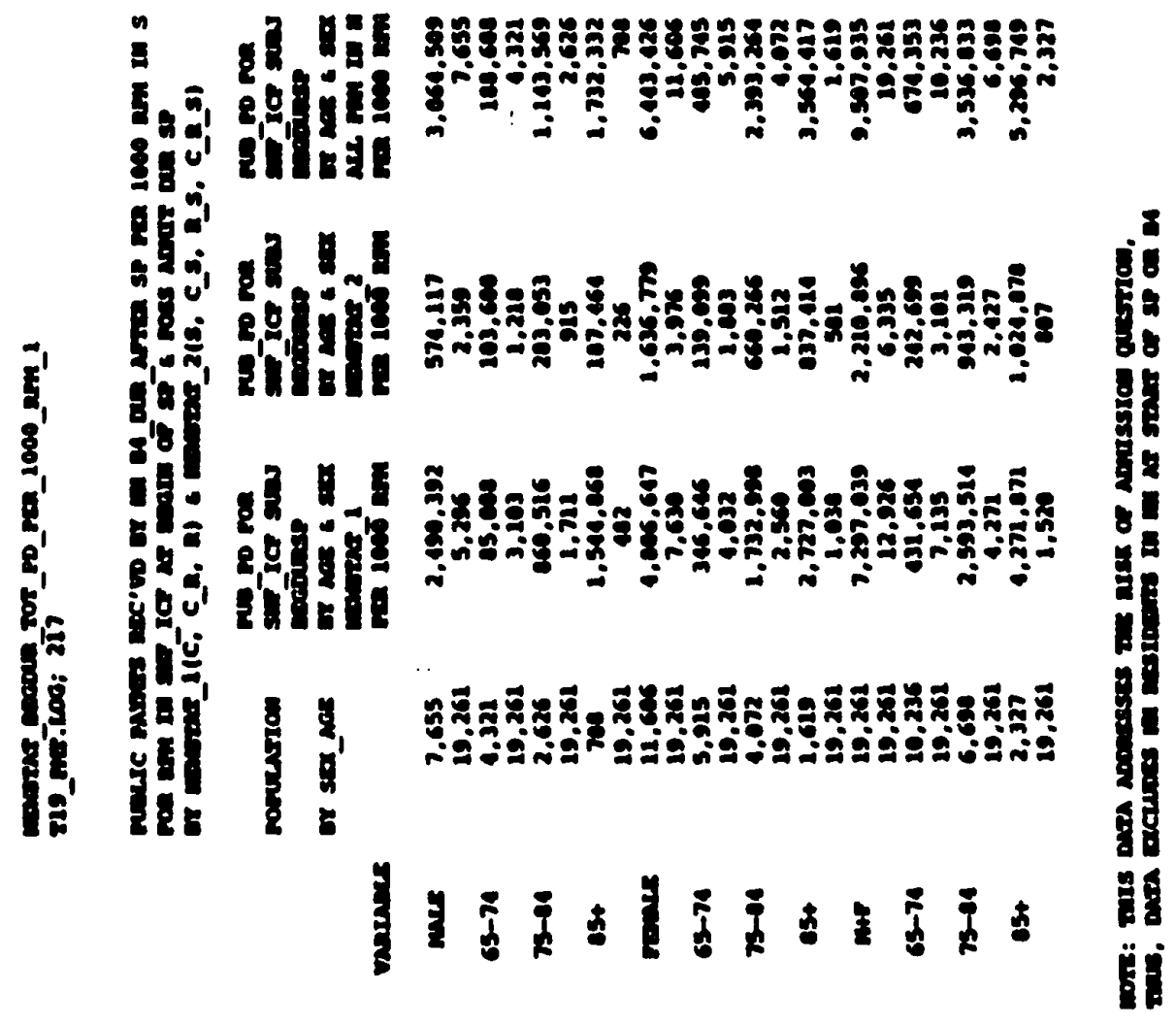




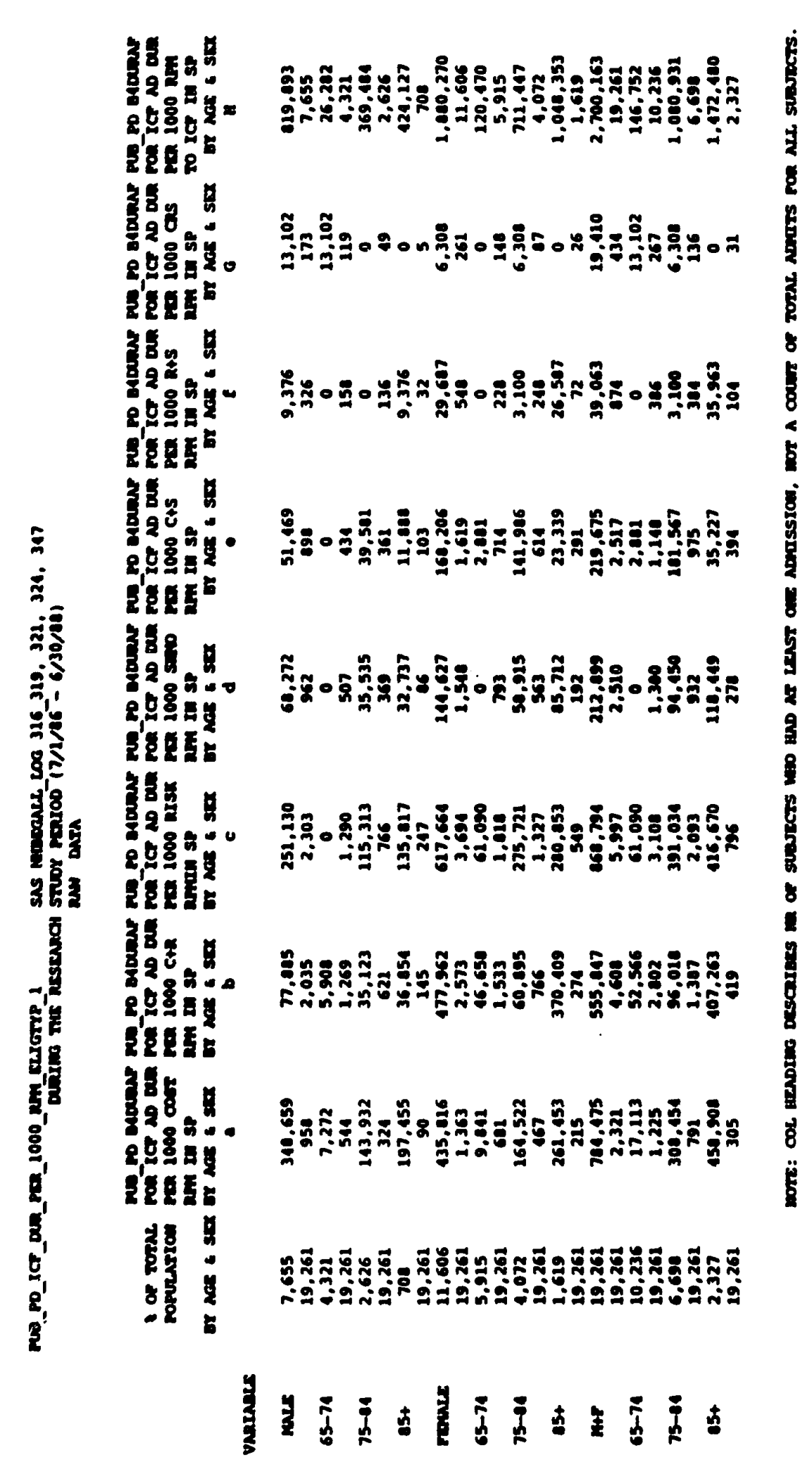

457 


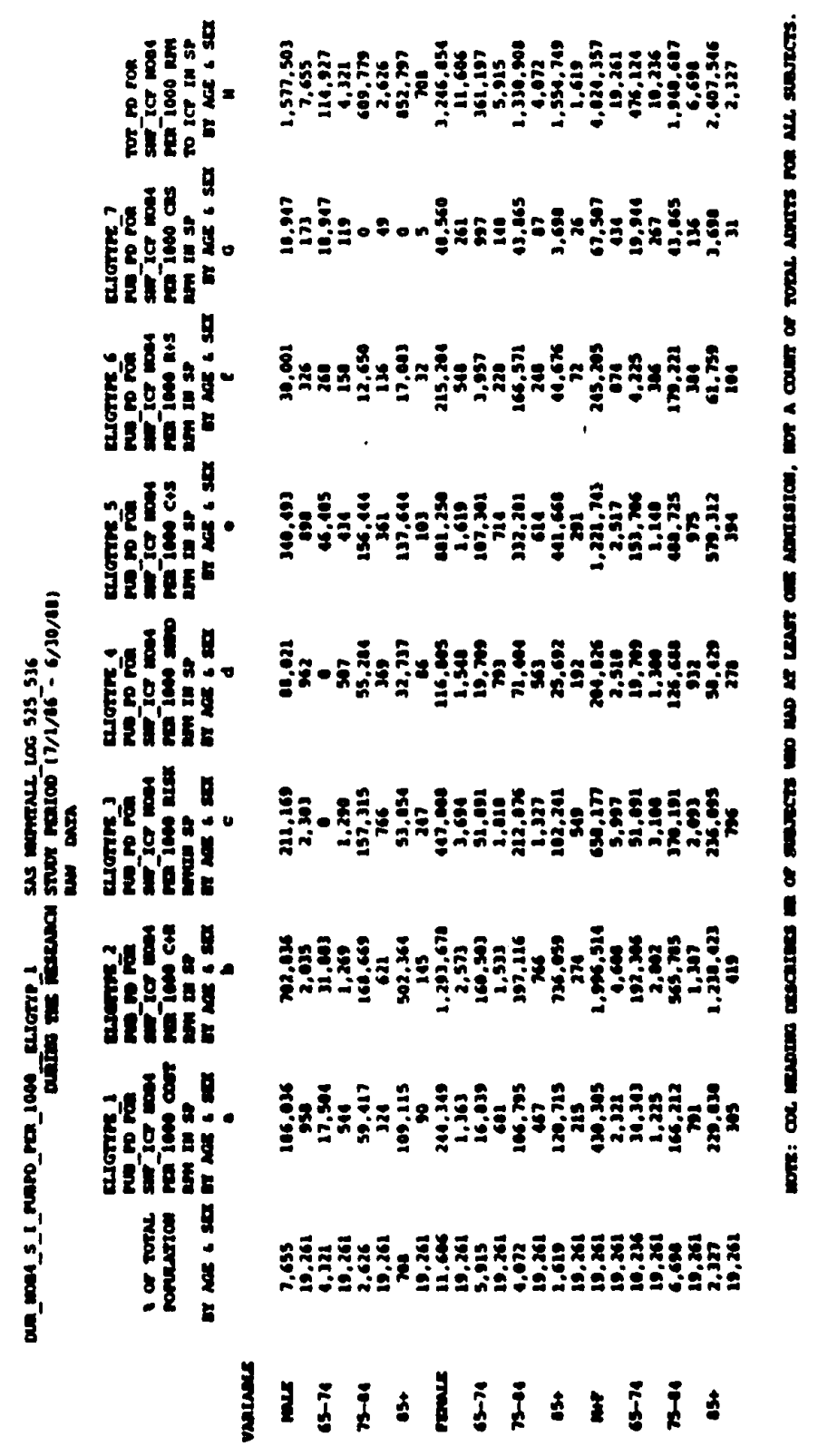




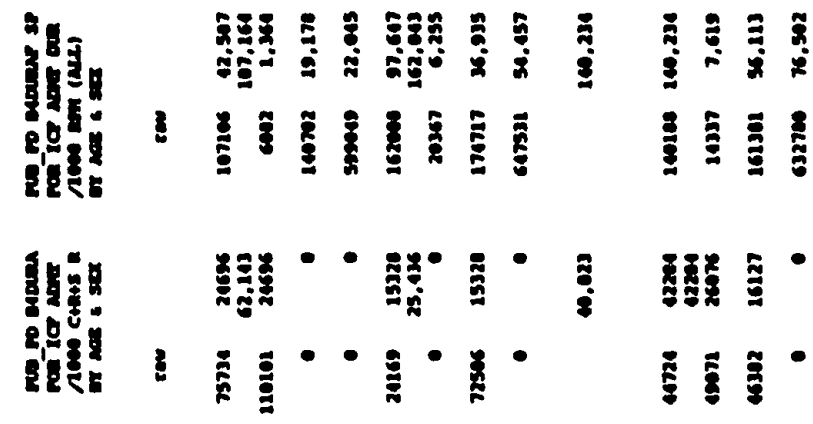

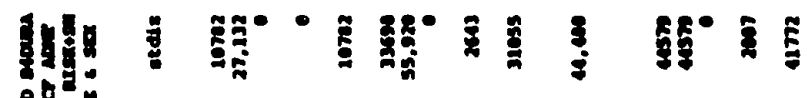

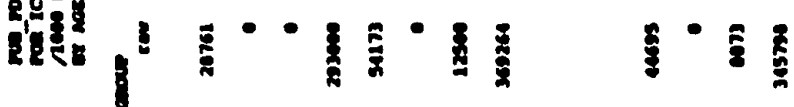

แแ! hy!n!

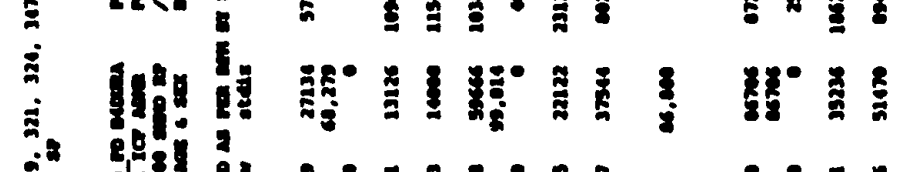

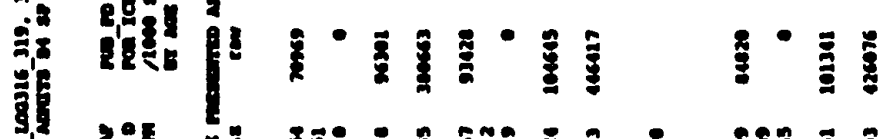
High

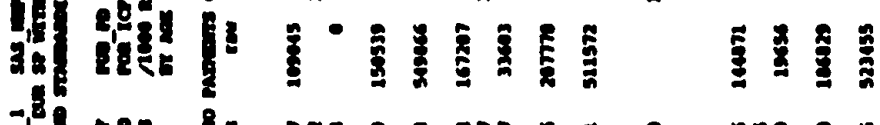
Clif!

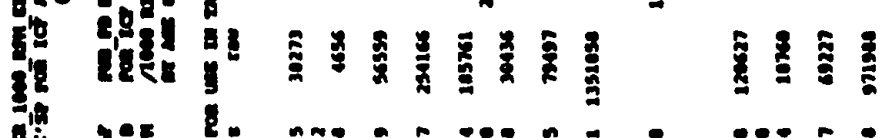

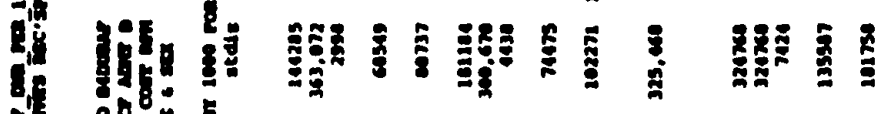

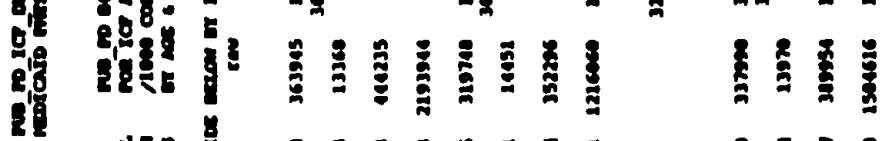

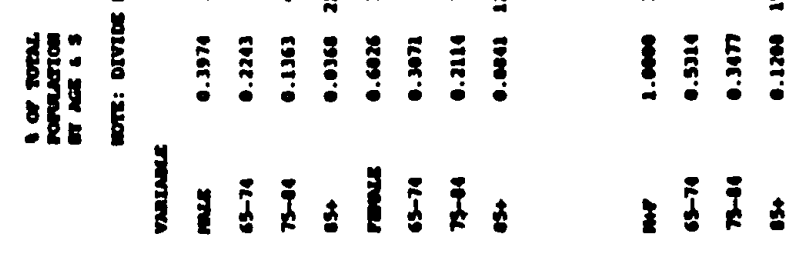




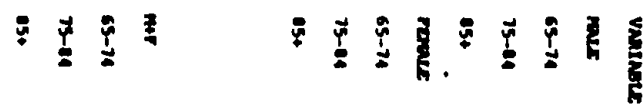

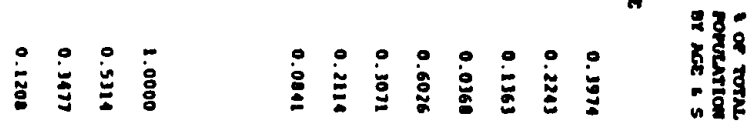

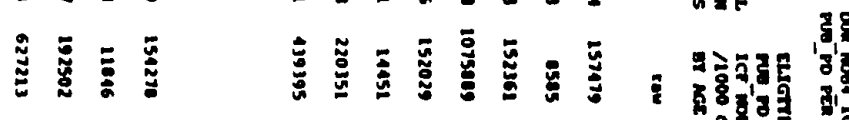

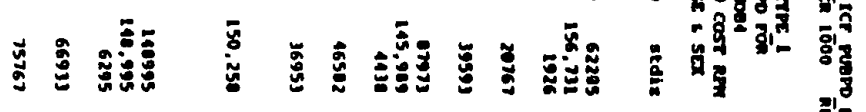

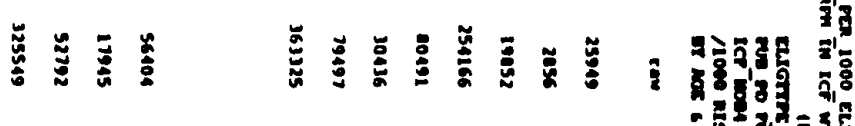

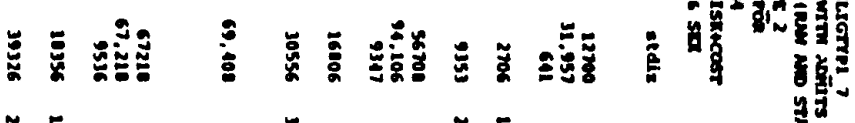

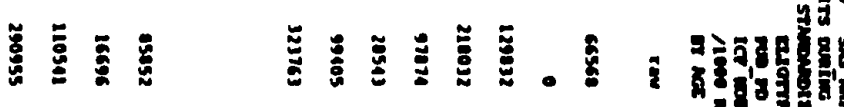

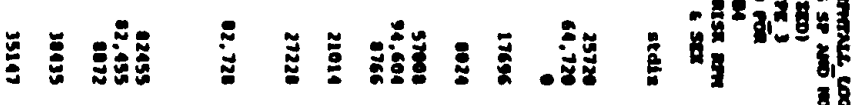

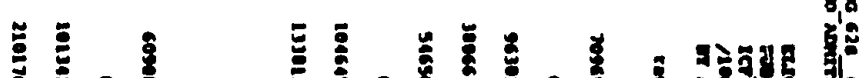

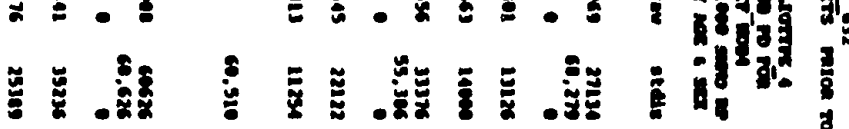

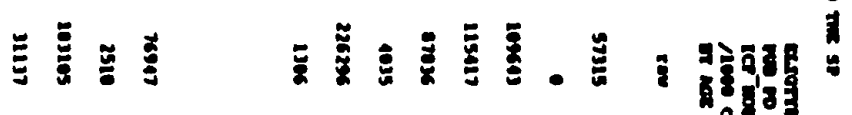

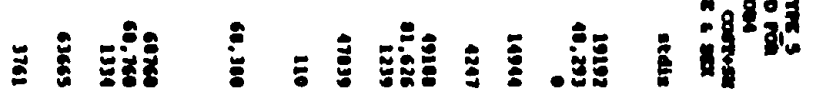

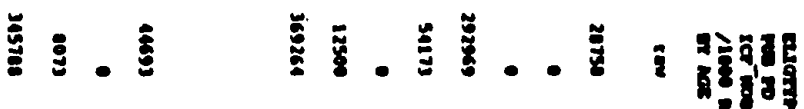

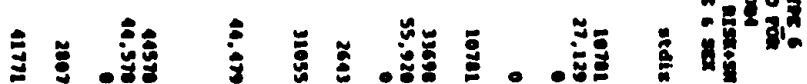

..

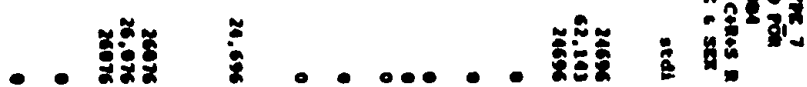

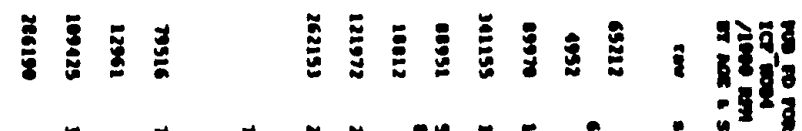

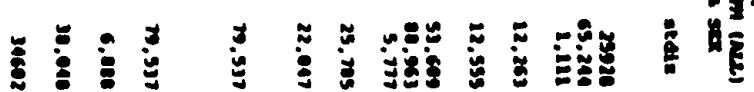




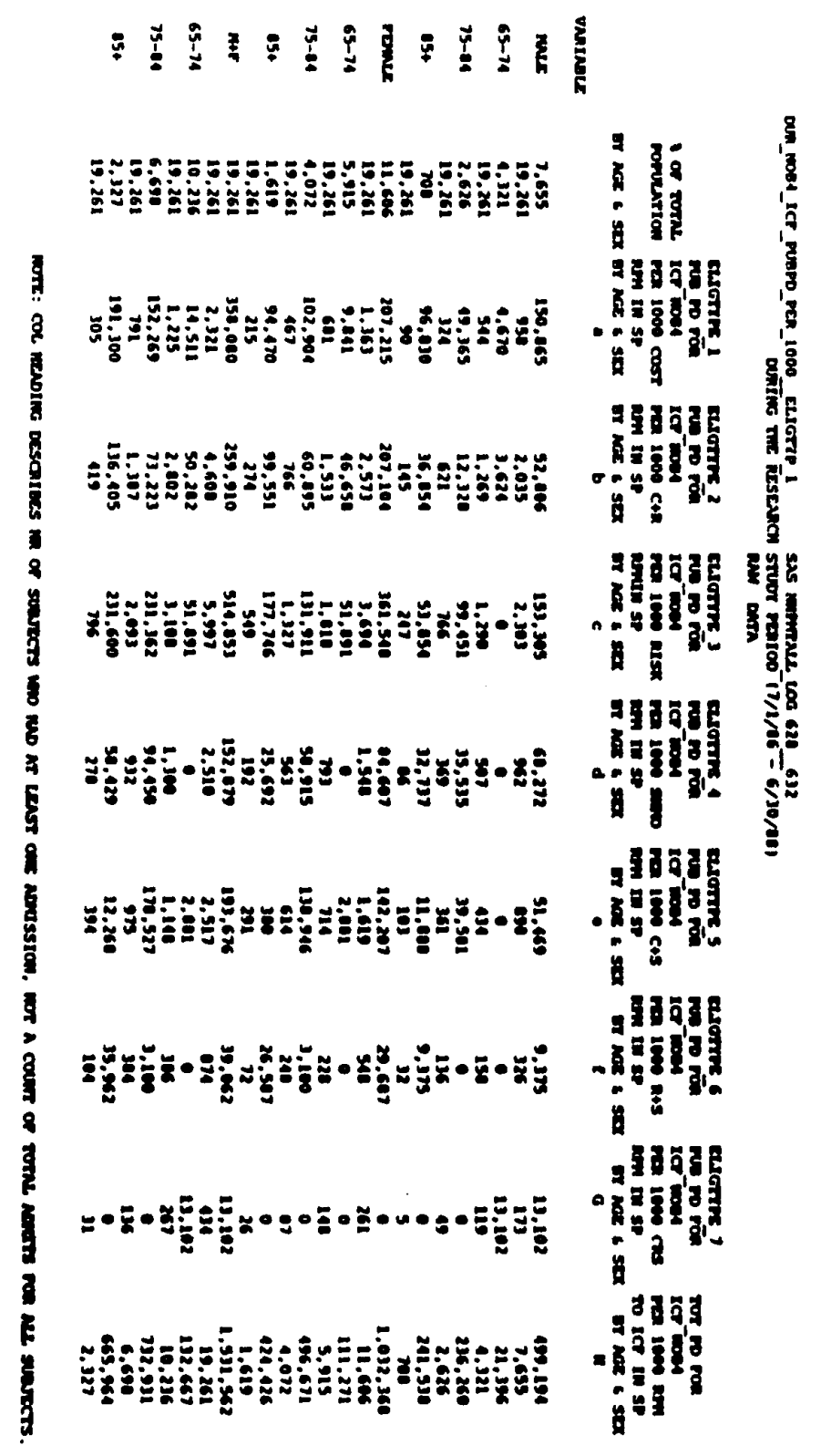




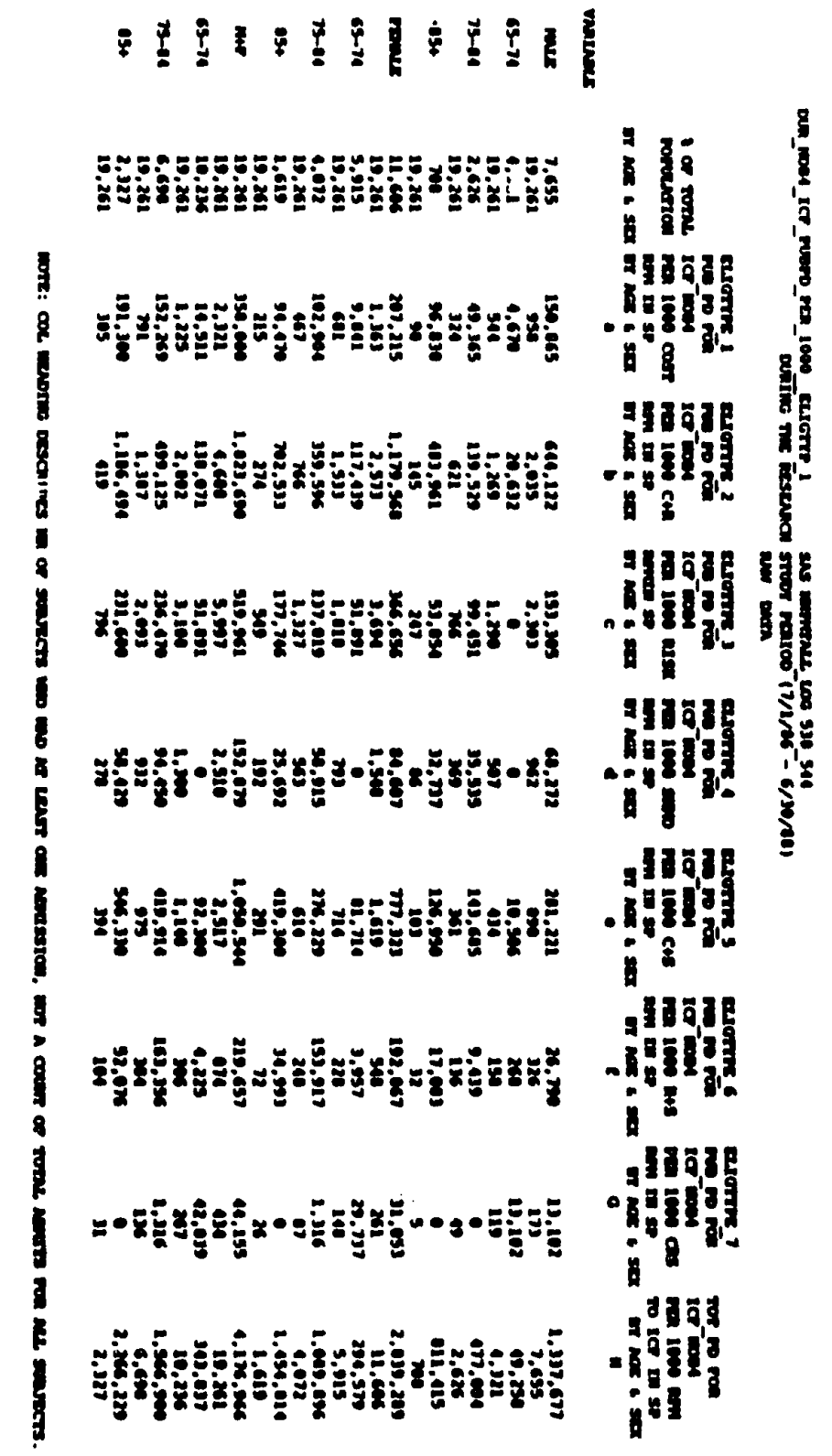




$$
\begin{aligned}
& \text { จำ }
\end{aligned}
$$

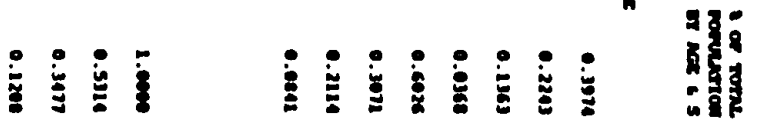

$$
\begin{aligned}
& \text { บ1 }
\end{aligned}
$$

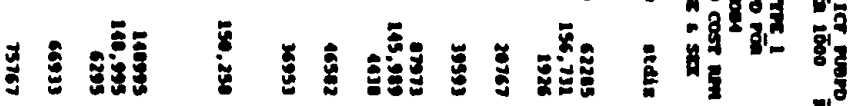

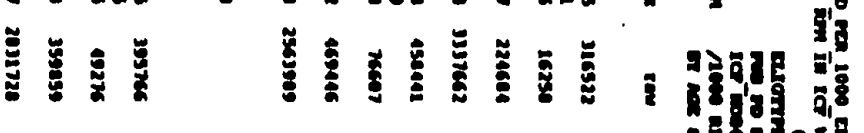

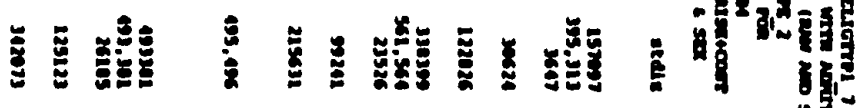

$$
\begin{aligned}
& \text { II II In }
\end{aligned}
$$

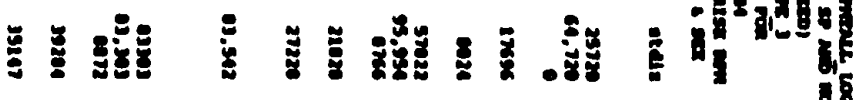

$$
\begin{aligned}
& \text { II.1 II. }
\end{aligned}
$$

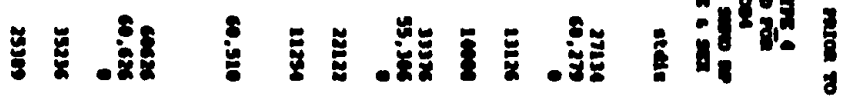

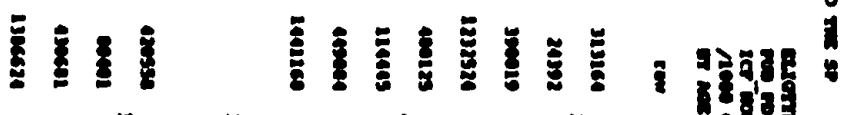

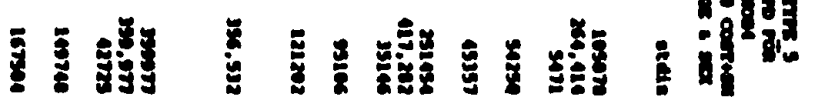

$$
\begin{aligned}
& \text { UI! IIIIII) }
\end{aligned}
$$

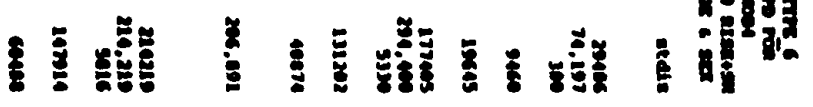

$$
\begin{aligned}
& .11 \text {. }
\end{aligned}
$$

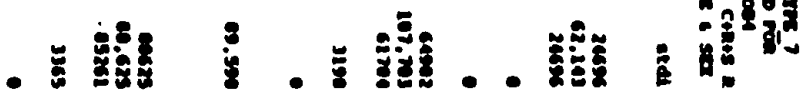

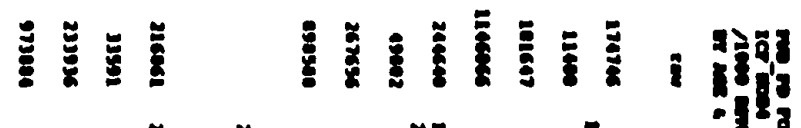

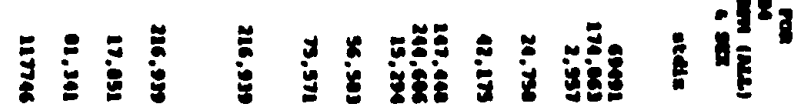




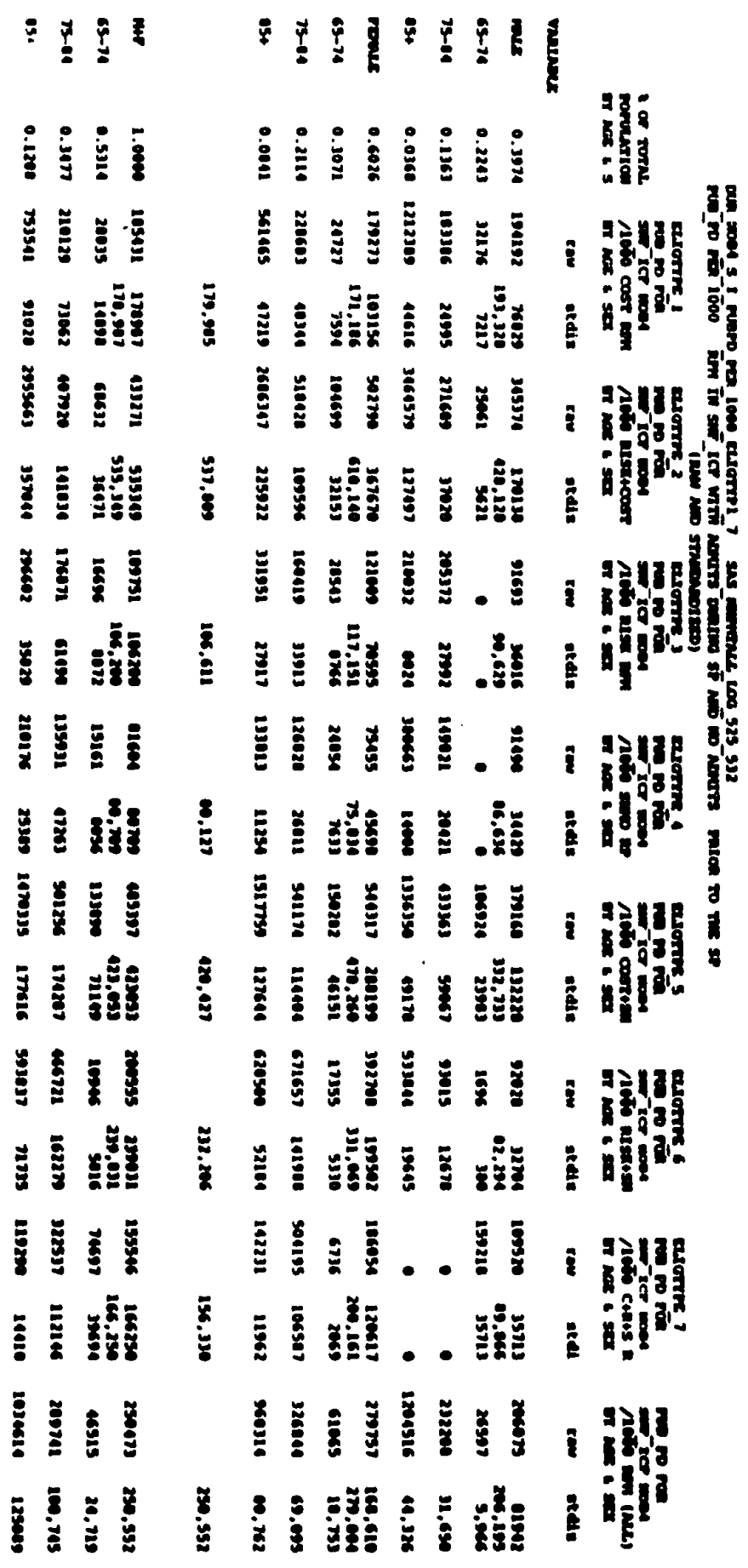


465

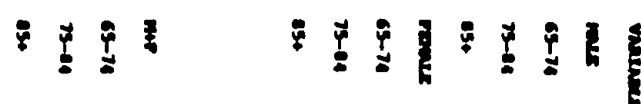

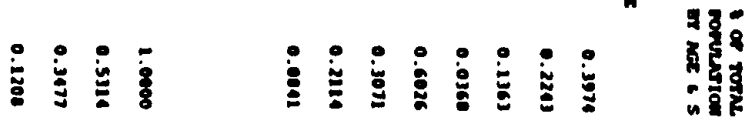

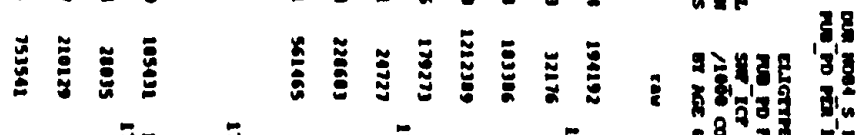

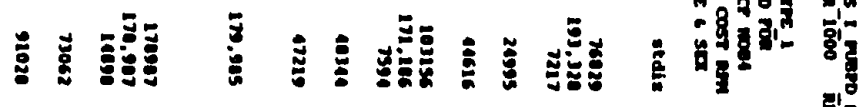

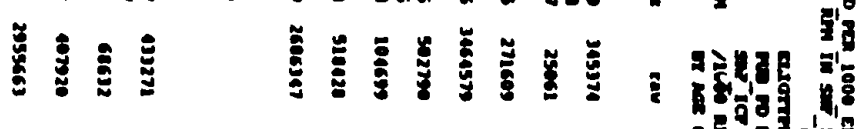

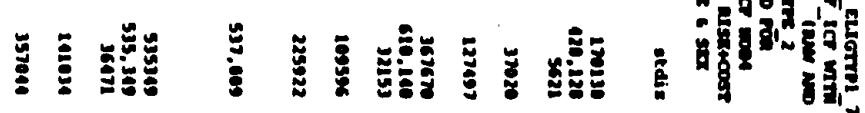

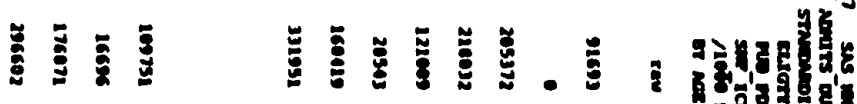

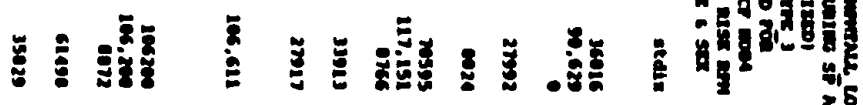

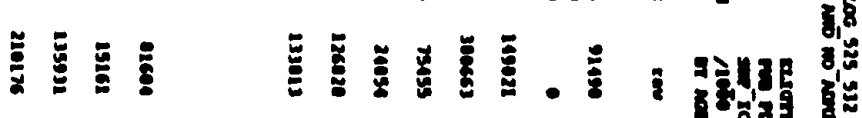

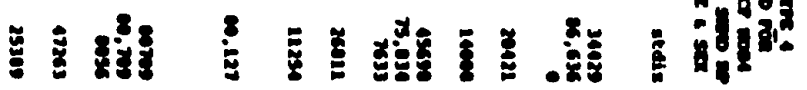

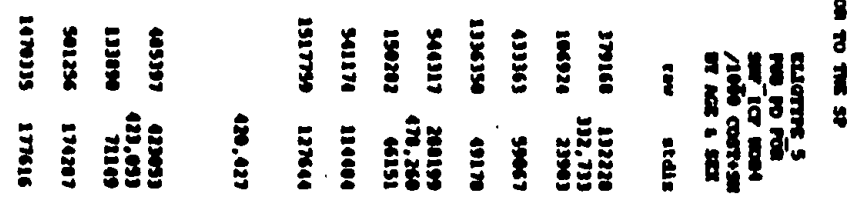

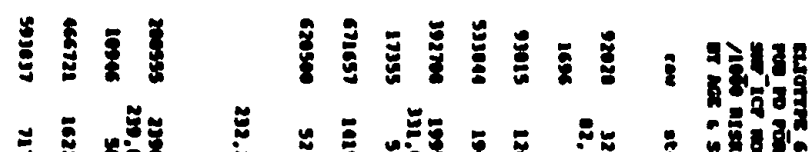

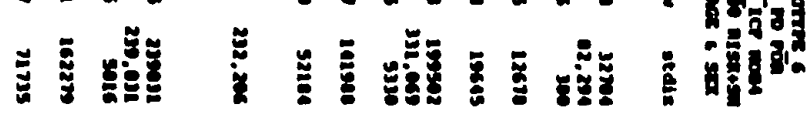

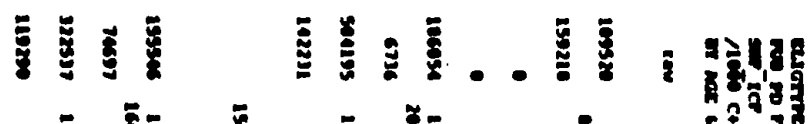

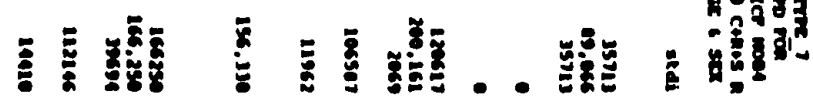

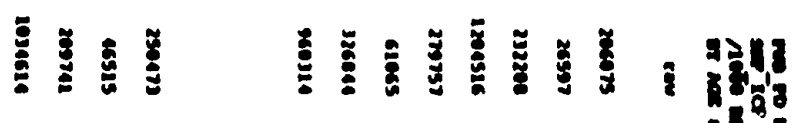

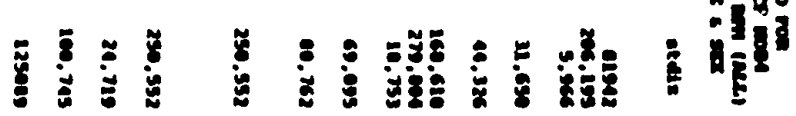




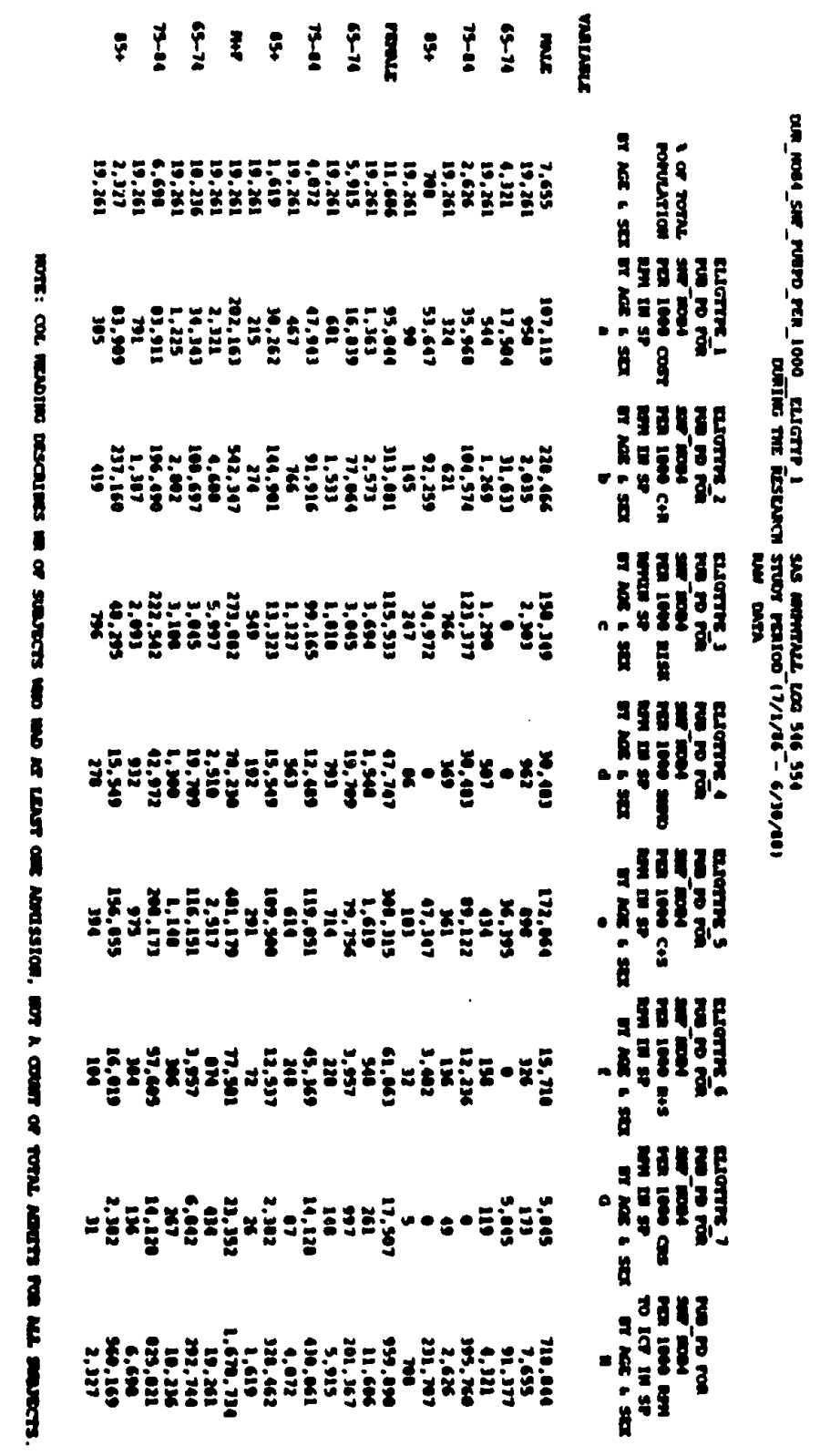




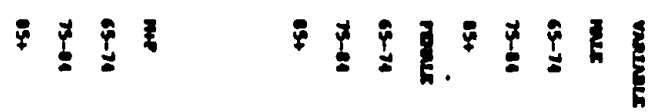

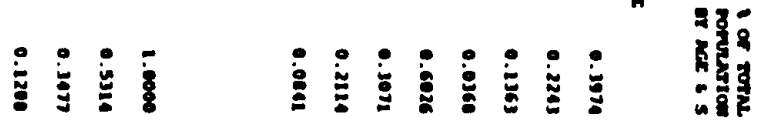

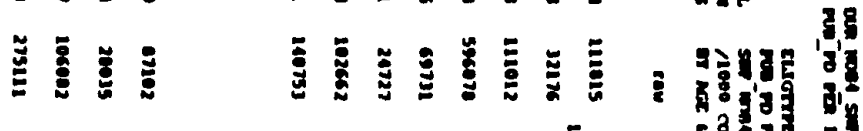

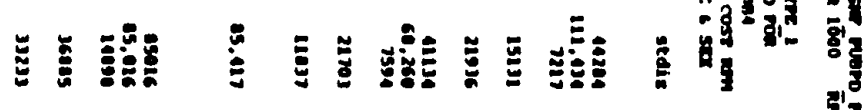

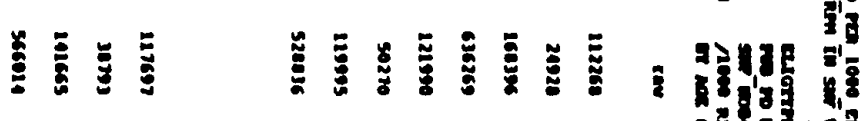

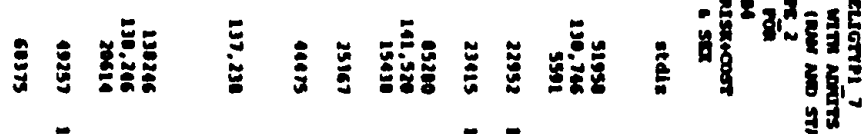

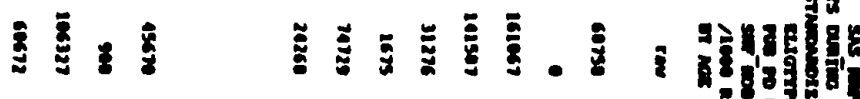

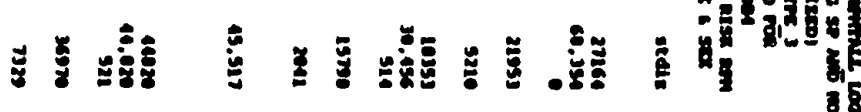

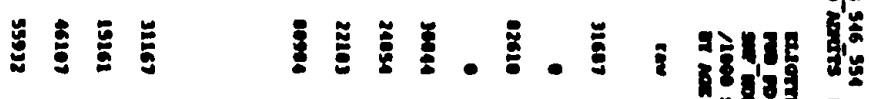

ง

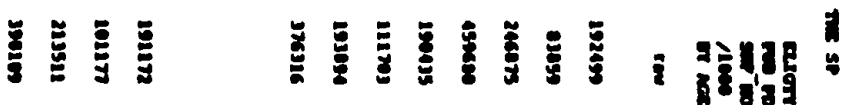

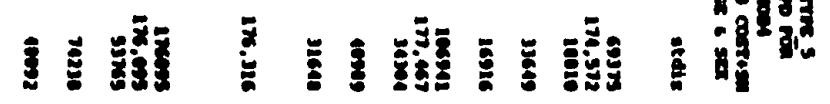

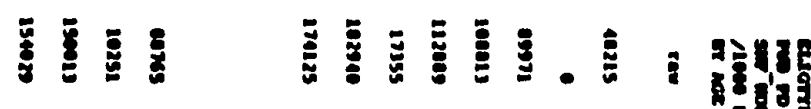

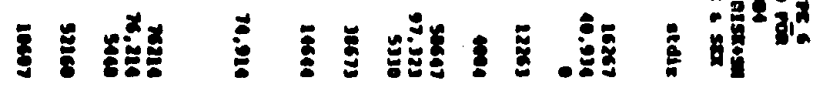

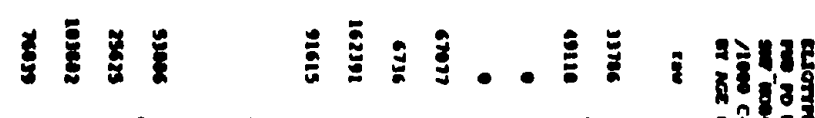

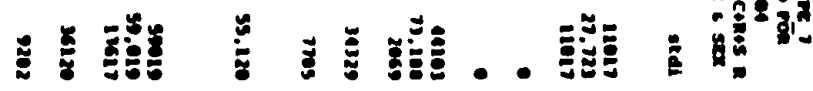

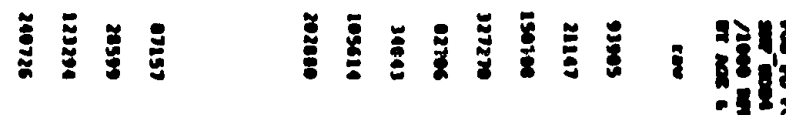

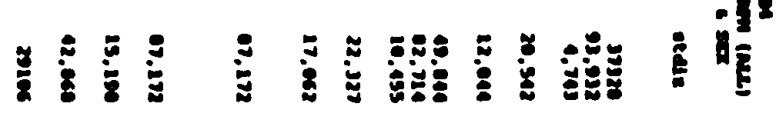




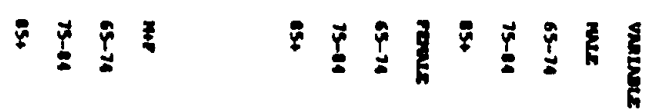

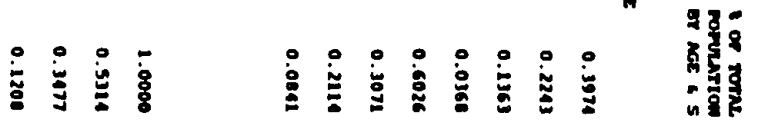

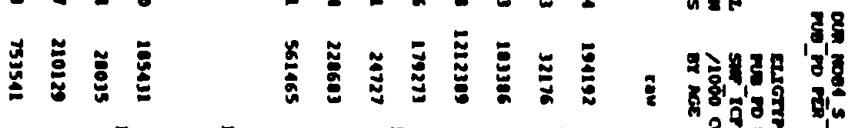

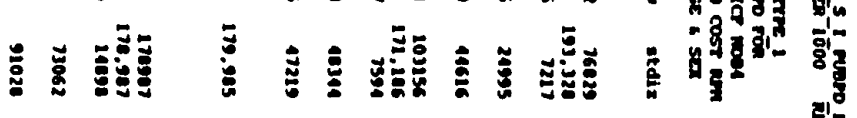

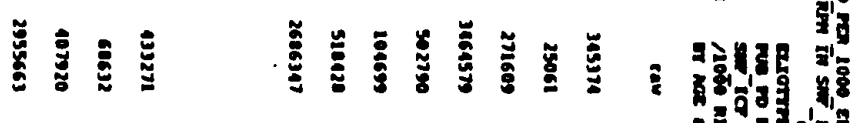

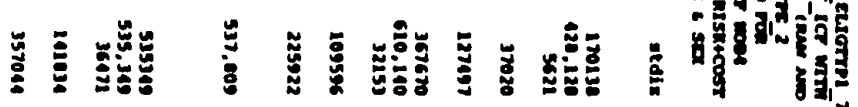

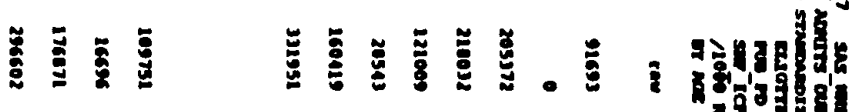

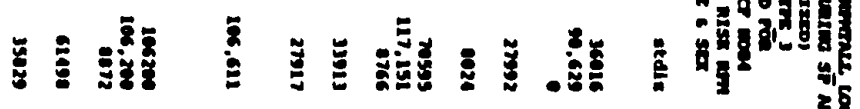

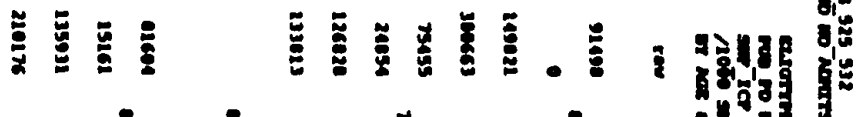

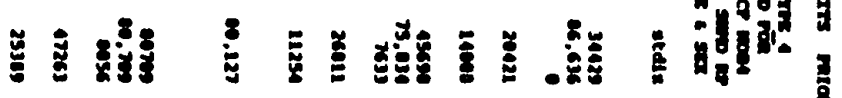

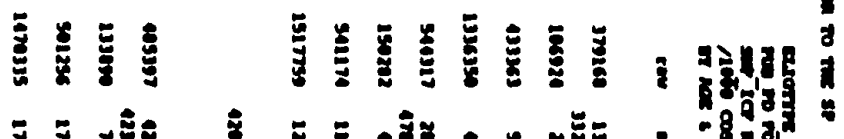

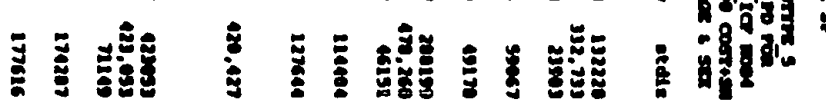

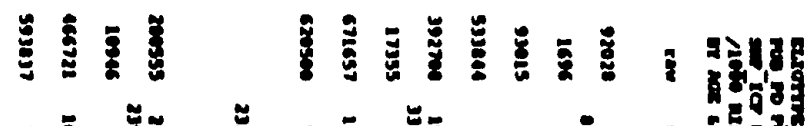

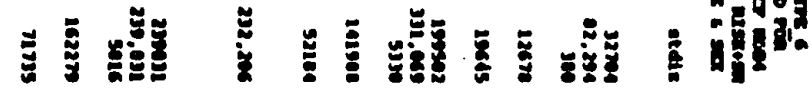

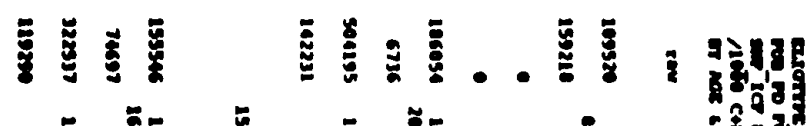

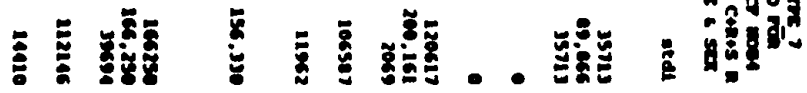

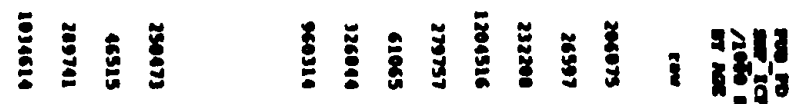

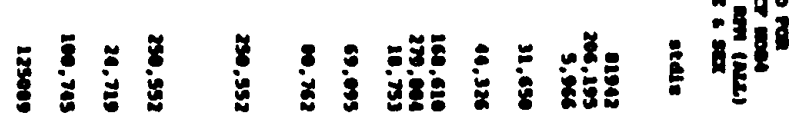

